\title{
Untersuchungen zur Synthese \\ fluoreszenzaktiver aromatischer Polyzyklen \\ durch Palladium-katalysierte Domino-C-H-Aktivierungen
}

\author{
Dissertation \\ zur Erlangung des mathematisch-naturwissenschaftlichen Doktorgrades \\ „Doctor rerum naturalium“ \\ der Georg-August-Universität Göttingen \\ im Promotionsprogramm der \\ Georg-August University School of Science (GAUSS)
}

vorgelegt von

\section{Christoph Eichhorst}

aus Lübeck

Göttingen, 2014 
Betreuungsausschuss:

Prof. Dr. Dr. h.c. L. F. Tietze

Prof. Dr. L. Ackermann

Mitglieder der Prüfungskommission:

Referent: $\quad$ Prof. Dr. Dr. h.c. L. F. Tietze

Koreferent: Prof. Dr. L. Ackermann

Weitere Mitglieder der Prüfungskommission:

Prof. Dr. U. Diederichsen

Prof. Dr. H. Laatsch

Prof. Dr. C. Steinem

Dr. A. Breder

Tag der mündlichen Prüfung: 09. Oktober 2014 
Die vorliegende Arbeit wurde in der Zeit von März 2011 bis September 2014 unter der Leitung von Herrn Prof. Dr. Dr. h.c. L. F. Tietze angefertigt.

Ich danke Herrn Prof. Dr. Dr. h.c. L. F. Tietze

für die interessante Themenstellung, die Bereitstellung der hervorragenden Arbeitsbedingungen und die gewährte Freiheit bei der Gestaltung der Arbeit. Außerdem danke ich sehr herzlich für seine Unterstützung und Förderung sowie sein stetes Interesse am Fortgang der Arbeit. 

Einige Ergebnisse dieser Arbeit wurden bereits veröffentlicht:

„A Fast Way to Fluorescence: A Fourfold Domino Reaction to Condensed Polycylic Compounds”:

L. F. Tietze, C. Eichhorst, T. Hungerland, M. Steinert, Chem. Eur. J. 2014, early view.

„An Efficient Domino Sonogashira/Double Carbopalladation/C-H Activation Leading to Fluorescent Polycyclic Aromatic Hydrocarbons":

L. F. Tietze, C. Eichhorst, Heterocycles 2014, accepted.

Publikationen unter Beteiligung des Autors dieser Arbeit:

„Efficient Synthesis of Helical Tetrasubstituted Alkenes as Potential Molecular Switches: A TwoComponent Palladium-Catalyzed Triple Domino Process":

L. F. Tietze, T. Hungerland, C. Eichhorst, A. Düfert, C. Maaß, D. Stalke, Angew. Chem., Int. Ed. 2013, 52, 3668-3671.

„A facile synthesis of 3-substituted benzofurans and indoles through a palladium-catalyzed domino carbopalladation/CH-activation/isomerization process"

L. F. Tietze, T. Hungerland, J. Ammermann, C. Eichhorst, S. O. Reichmann, D. Stalke, J. Indian Chem. Soc. 2013, 90, 1537-1555. 



\section{Inhalt}

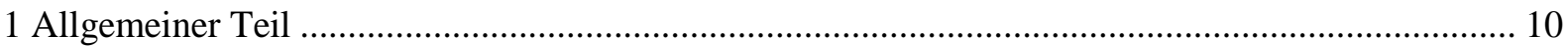

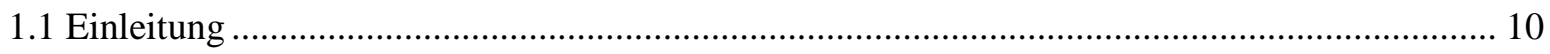

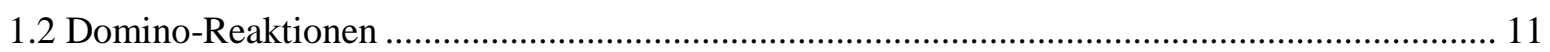

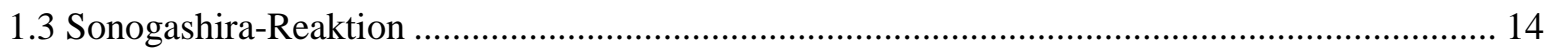

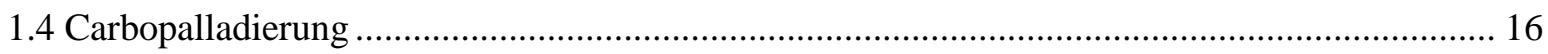

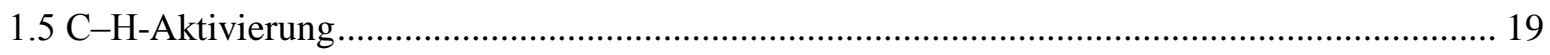

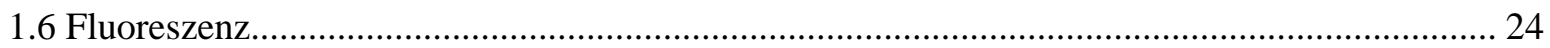

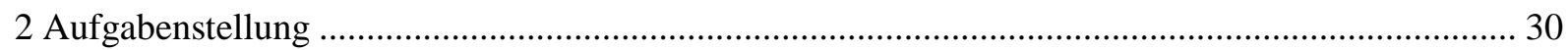

2.1 Bisherige Forschungsergebnisse in der Arbeitsgruppe Tietze .................................................... 30

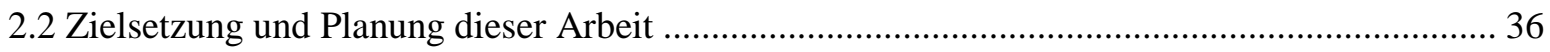

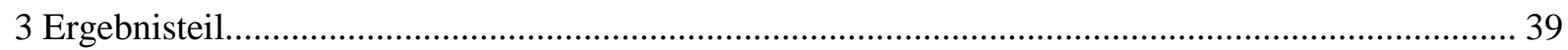

3.1 Untersuchungen zur Synthese von fluoreszenzaktiven Polyzyklen des Typs 107 ..................... 39

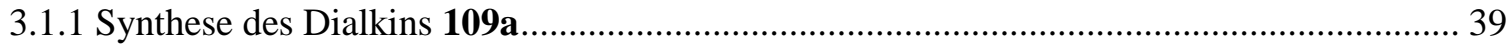

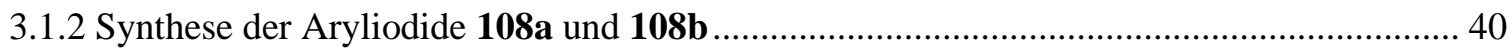

3.1.3 Synthese der Polyzyklen 107a-f durch die Domino-Reaktion ........................................... 41

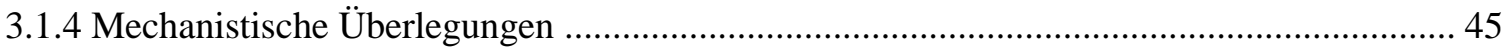

3.1.5 Diskussion ausgewählter analytischer Daten von Verbindung 107b ................................. 46

3.1.6 Untersuchung der Fluoreszenz am Beispiel von Verbindung 107d .................................... 49

3.1.7 Untersuchungen zur Synthese dimerer Strukturen ........................................................... 51

3.2 Untersuchungen zur Synthese von fluoreszenzaktiven Polyzyklen des Typs 110 ..................... 54

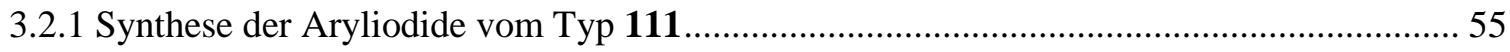

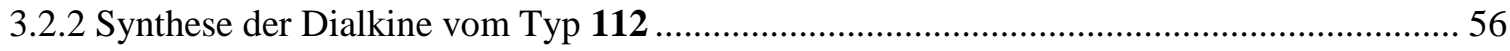

3.2.3 Synthese der Polyzyklen 110a-i durch die Domino-Reaktion ........................................... 57

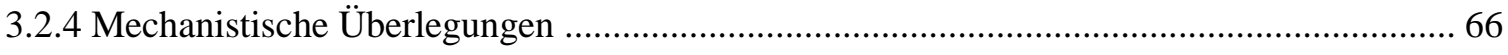

3.2.5 Diskussion ausgewählter analytischer Daten von Verbindung 110a .................................. 68

3.2.6 Untersuchung der Fluoreszenz am Beispiel von Verbindung 110a ................................... 71

3.2.7 Untersuchung der dynamischen Eigenschaften am Beispiel von Verbindung 110a............ 73

3.2.8 Untersuchungen zur Synthese weiterer Aryliodide vom Typ 111 ...................................... 74

3.2.9 Untersuchungen zur Synthese funktionalisierter Fluoreszenzfarbstoffe .............................. 76

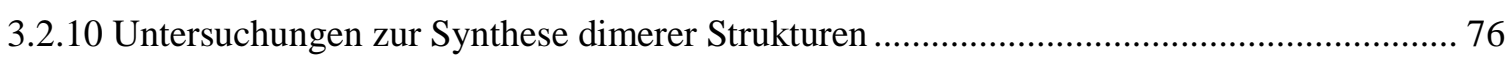

3.3. Untersuchungen zur Synthese von hydrophilen Polyzyklen des Typs 114............................... 78

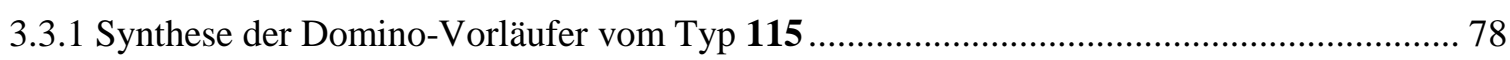

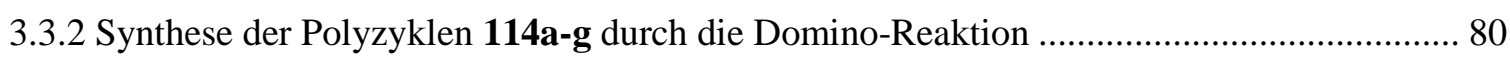

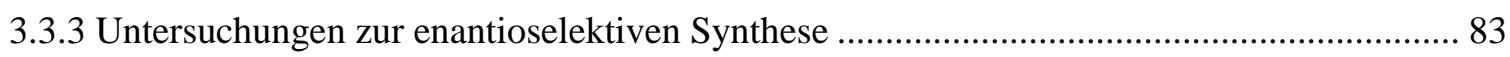


3.4 Untersuchungen zur Synthese von Polyalkenen mit tetrasubstituierten Doppelbindungen ........ 85

3.4.1 Untersuchungen zur Synthese von Dialkenen des Typs 116.............................................. 85

3.4.2 Untersuchungen zur Synthese von Dialkenen des Typs 117............................................ 87

3.4.3 Untersuchungen zur Synthese von Dialkenen des Typs 118............................................... 89

3.4.4 Untersuchungen zur Synthese von Dialkenen des Typs 119............................................ 90

3.4.5 Untersuchungen zur Synthese von Trialkenen des Typs 120 ............................................ 92

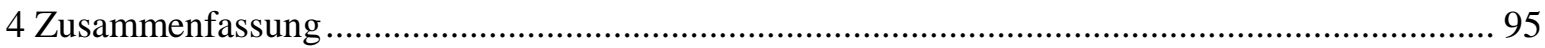

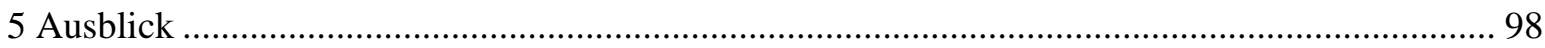

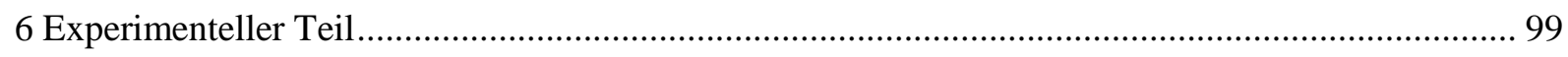

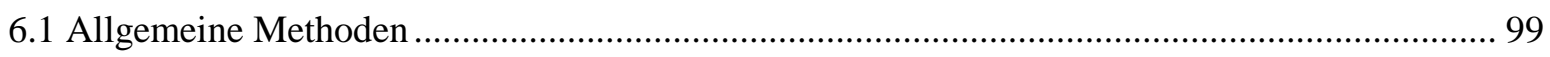

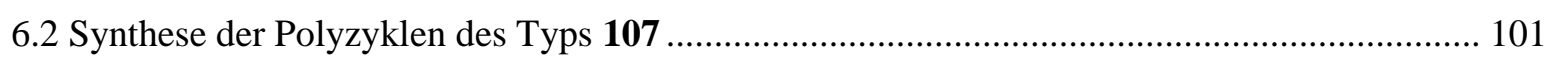

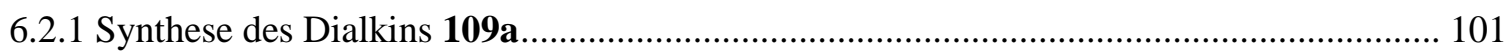

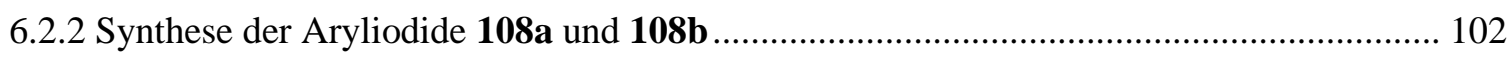

6.2.3 Synthese der Substrate 107a-f durch die Domino-Reaktion ............................................ 106

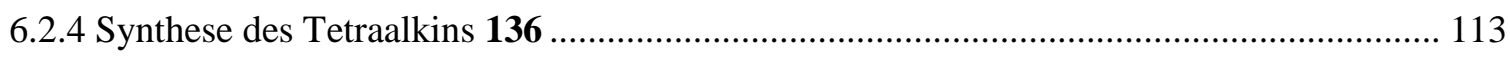

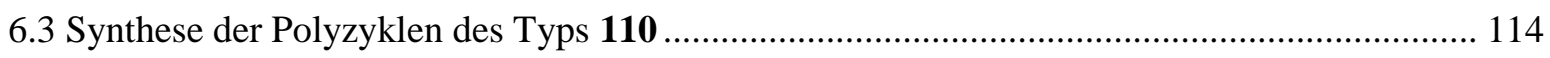

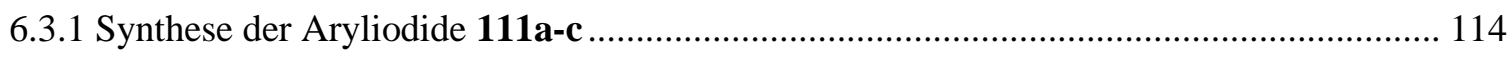

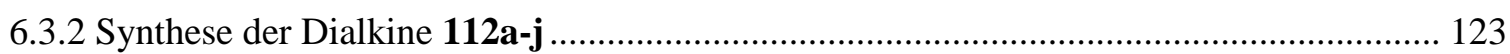

6.3.3 Synthese der Dialkine 151b-k durch die Sonogashira-Reaktion......................................... 152

6.3.4 Synthese der Polyzyklen 110a-i durch die Domino-Reaktion ........................................... 165

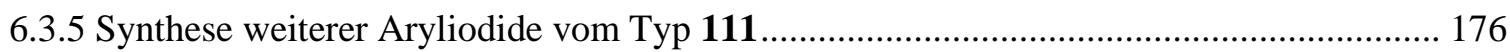

6.3.6 Synthese funktionalisierter Polyzyklen vom Typ 161..................................................... 179

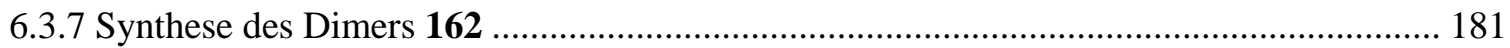

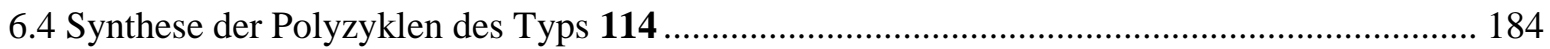

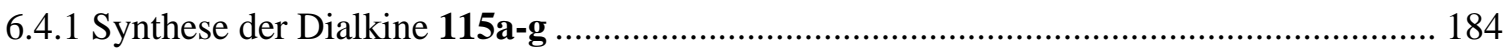

6.4.2 Synthese der Poylyzyklen 114a-g durch die Domino-Reaktion ...................................... 220

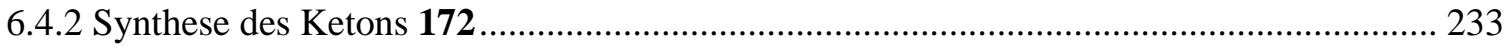

6.5 Untersuchungen zur Synthese der tetrasubstituierten Polyalkene 116-120.............................. 234

6.5.1 Untersuchungen zur Synthese von Dialkenen des Typs 116............................................ 234

6.5.2 Untersuchungen zur Synthese von Dialkenen des Typs 117.......................................... 238

6.5.3 Untersuchungen zur Synthese von Dialkenen des Typs 119........................................... 243

6.5.4 Untersuchungen zur Synthese von Trialkenen des Typs 120.......................................... 251

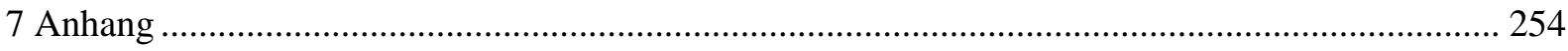

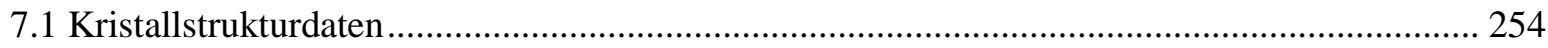

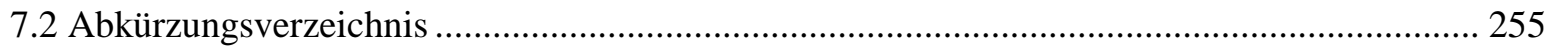

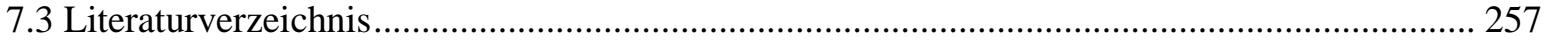




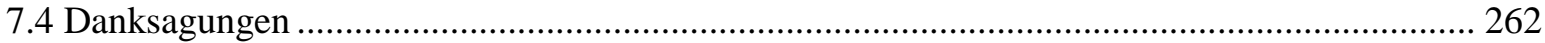

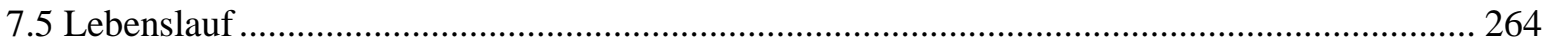




\section{Allgemeiner Teil}

\subsection{Einleitung}

Ein effizienter Weg zum Zielmolekül in möglichst wenigen Einzelschritten und ohne aufwändige Isolierung der jeweiligen Zwischenstufen ist das Ziel jeder organischen Synthese. So sollen komplexe organische Moleküle aus möglichst simplen Vorläufern erzeugt und diese dann in wenigen Reaktionsschritten zu den gewünschten Verbindungen, Naturstoffen oder funktionalen Substanzen, umgewandelt werden. Dies ist nicht nur im Hinblick auf die Ökonomie der Synthese von Belang, da Arbeitsschritte eingespart werden. Auch in ökologischer Hinsicht ist ein solches Ziel wünschenswert, da die Ersparnis an Arbeitsaufwand einhergeht mit der Ersparnis an Reagenzien, Lösungsmitteln und Energie, die für mehrere Reaktionen und die erforderlichen Aufarbeitungsschritte notwendig wären. Eine Möglichkeit, zu einer solchen verkürzten Synthese zu gelangen, stellen Domino-Reaktionen dar. Sie erlauben es, mehrere Reaktionen in einer zu kombinieren, wobei keine intermediäre Aufarbeitung notwendig ist. Damit vereinfacht sich die Synthese um einen oder mehrere Schritte und die geforderte Einsparung von Arbeitsaufwand und Material kann erreicht werden.

Eine Weiterentwicklung der Effizienz dieser Methode kann durch übergangsmetallkatalysierte Domino-Reaktionen erfolgen. Da Katalysatoren nur in geringen Mengen benötigt werden, verringert deren Einsatz den Materialverbrauch um ein Vielfaches. Zudem eröffnen gerade übergangsmetallkatalysierte Reaktionen den Zugang zu vielfältigen Transformationen, welche die Synthese neuer, komplexer Strukturen ermöglichen.

Im Bereich der übergangsmetallkatalysierten Reaktionen spielen insbesondere $\mathrm{C}-\mathrm{H}$-Aktivierungen eine Schlüsselrolle. Im Gegensatz zu den herkömmlichen Kreuzkupplungsreaktionen ermöglichen sie die Knüpfung von Kohlenstoff-Kohlenstoff-Bindungen ohne die vorherige Funktionalisierung eines der Kupplungspartner.

Alle erwähnten Konzepte zeichnen sich durch ihre Effizienz aus. Demnach ist es erstrebenswert, eine Kombination dieser Methoden zu erreichen und über eine Domino-C-H-Aktivierung zur Synthese von Naturstoffmotiven oder funktionalen Materialien zu gelangen.

In der Forschungsgruppe Tietze konnten hierzu bereits entsprechende Erfolge in der Synthese tetrasubstituierter Alkene erzielt werden, die als potenzielle molekulare Schalter von besonderem Interesse sind. ${ }^{1-3}$ Daneben ermöglichten Domino-C-H-Aktivierungen einen synthetischen Zugang zu Indolen und Benzofuranen ${ }^{4}$ sowie zu Acenaphthylenen. ${ }^{5}$

Ziel dieser Arbeit war es, Domino-C-H-Aktivierungen zu entwickeln, die zu fluoreszenzaktiven aromatischen Polyzyklen führen. Des Weiteren sollte die Möglichkeit einer Synthese helikaler tetrasubstituierter Polyalkene über diese Methode untersucht werden. 


\subsection{Domino-Reaktionen}

Domino-Reaktionen stellen eine effektive Methode dar, um aus einfachen Grundbausteinen in möglichst wenigen Schritten komplexe Strukturen zu erzeugen, wobei eine Aufarbeitung der intermediär entstehenden Verbindungen entfällt.

Nach L. F. Tietze ist eine Domino-Reaktion definiert als „die Durchführung zweier oder mehrerer bindungsbildender Transformationen unter identischen Reaktionsbedingungen, in der die letzte Transformation jeweils an der im vorangegangenen Teilschritt entstandenen Funktionalität erfolgt.“ 6 Demnach unterscheidet sich der Begriff der Domino-Reaktion von dem ebenfalls häufig verwendeten Terminus der Tandem-Reaktion, die nicht dem Grundgedanken des Domino-Konzepts genügt, wonach jeder Schritt des Domino-Prozesses erst durch die ihm vorausgehende Transformation ermöglicht wird. Auch die Bezeichnung Kaskaden-Reaktion ist irreführend, da sie für zahlreiche andere chemische Prozesse verwendet wird (z.B. photochemische Reaktionen).

Die Verwendung von Domino-Reaktionen in der organischen Synthese bietet gegenüber herkömmlichen Methoden einige Vorteile: Wenn die Ausgangsstoffe des Domino-Prozesses leicht herzustellen sind, können über die Zusammenfassung von zwei oder mehr Reaktionsschritten die jeweiligen Einzelreaktionen samt Aufarbeitung eingespart und somit die Syntheseroute verkürzt werden. Damit ist das Konzept der Domino-Reaktion sowohl in ökonomischer als auch in ökologischer Hinsicht gewinnbringend. Hinzu kommt, dass die Bildung komplexer Strukturen aus einfachen Substraten ein wünschenswertes Syntheseziel darstellt, wobei die Einführung verschiedenster Funktionalitäten über einen einzelnen Prozess eine ebenso anspruchsvolle wie elegante Methode ist. Je mehr bindungsbildende Einzelschritte in einen Domino-Prozess integriert werden können, umso vorteilhafter ist dabei die Domino-Reaktion.

Eine genauere Klassifizierung der Domino-Reaktionen erfolgt über die Art der Einzelschritte. Besteht ein Domino-Prozess aus Einzelreaktionen desselben Typs, so spricht man von einer Homo-DominoReaktion. Sind die Einzelreaktionen verschiedener Art, so handelt es sich um eine Hetero-DominoReaktion. Beispiele für Typen von Einzelreaktionen, die in einer Domino-Reaktion kombiniert werden können, sind: kationische, anionische, radikalische, perizyklische oder auch übergangsmetallkatalysierte Reaktionen. In der Forschungsgruppe Tietze liegt der Fokus der aktuellen Forschung vor allem auf Palladium-katalysierten Domino-Reaktionen.

Domino-Reaktionen sind kein künstliches Konzept: In der Natur finden sich zahlreiche Beispiele für die Umsetzung von chemischen Reaktionen, die zu einem Domino-Prozess zusammengefasst sind. So enthalten unter anderem die Biosynthesen zahlreicher Alkaloide sowie des Porphyrin-Derivats Uroporphyrinogen III (2) (einem Vorläufer u.a. des Häms und des Chlorophylls) allesamt natürliche Domino-Reaktionen (Abbildung 1). ${ }^{7,8}$ 
HOOC

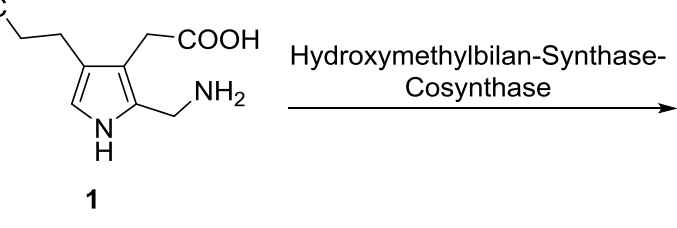

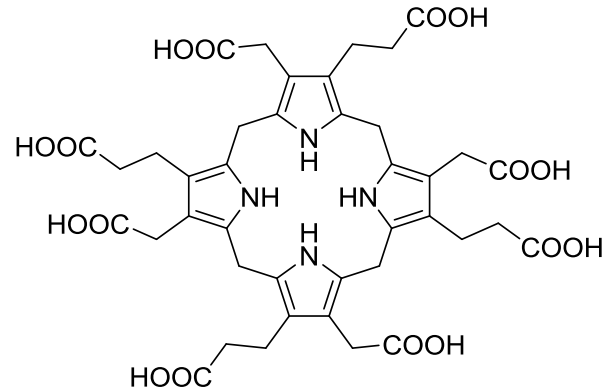

2

Abbildung 1: Biosynthese von Uroporphyrinogen III (2). ${ }^{7,8}$

Als Beispiel einer Domino-Reaktion in der Totalsynthese sei hier die von Pattenden et al. entwickelte radikalvermittelte Domino-Zyklisierung konjugierter Elektrophore vom Typ 8 genannt, welche als Bausteine in der Synthese von Steroiden und anderen annelierten Polyzyklen dienen können (Abbildung 2). ${ }^{9}$

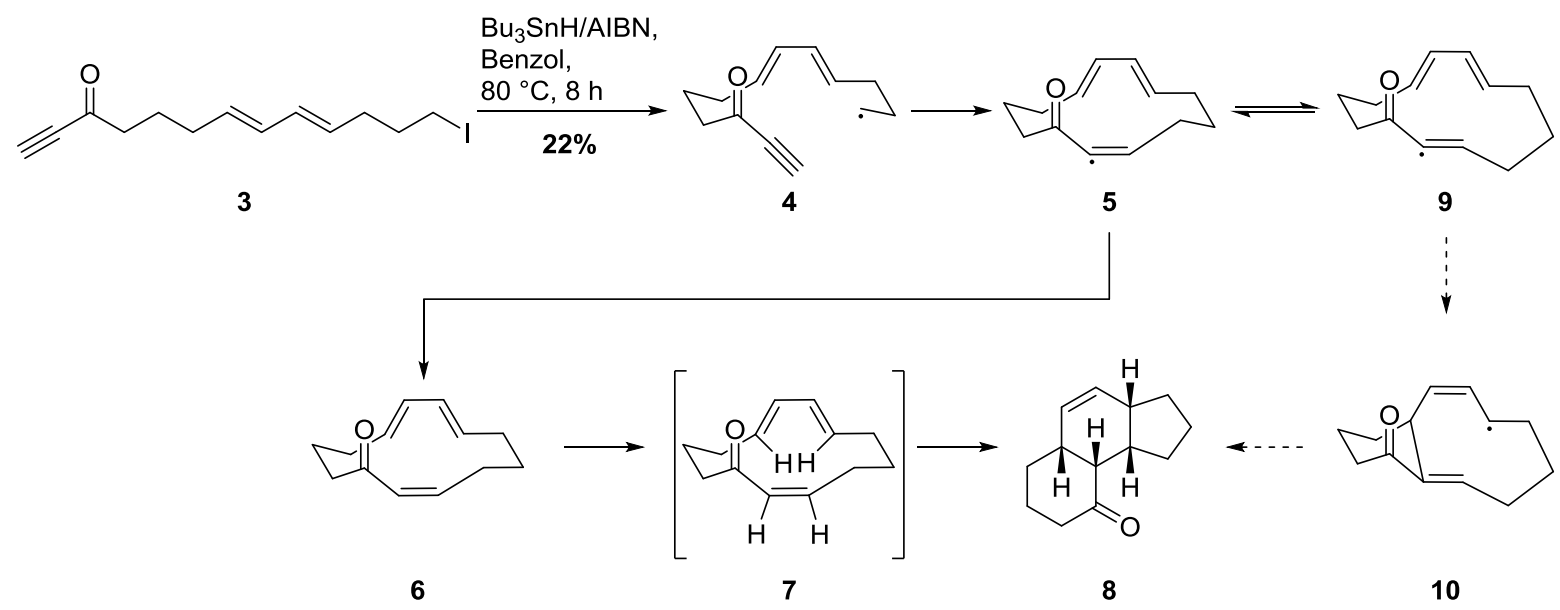

Abbildung 2: Domino-Zyklisierung zur Synthese von Steroidbausteinen des Typs 8. ${ }^{9}$

Die Reaktion beginnt mit der Bildung des Radikals 4, ausgelöst durch den Radikalstarter $\mathrm{AIBN} / \mathrm{Bu}_{3} \mathrm{SnH}$. In einer 13-endo-dig-Zyklisierung wird aus Verbindung 4 Radikal 5 erzeugt, welches nach Quenchen des Radikals zu Alkatrien 6 reagiert. In einer in situ erfolgenden intramolekularen Diels-Alder-Reaktion wird in 22\% Ausbeute ausschließlich das cis,syn,cis-Diastereomer 8 über den endo-artigen Übergangszustand 7 gebildet. Eine ebenfalls denkbare radikalvermittelte Domino-6-exotrig/5-exo-trig-Zyklisierung von 5, welche über die Radikale $\mathbf{9}$ und $\mathbf{1 0}$ ebenfalls zu Produkt $\mathbf{8}$ führt, ist aufgrund dieser Diastereoselektivität unwahrscheinlich. ${ }^{8,9}$

In der Forschungsgruppe Tietze liegt der Fokus neuerer Arbeiten auf Palladium-katalysierten DominoReaktionen. So konnten eine Reihe von Naturstoffen und deren Analoga sowie funktionale organische Moleküle wie tetrasubstitutierte Alkene über entsprechende Domino-Reaktionen synthetisiert werden. Ein Beispiel ist die 2014 publizierte Synthese der Naturstoffe (-)-Blennolid C (17) und 
(-)-Gonytolid C (18), welche beide über eine Domino-Wacker/Carbonylierung/Methoxylierung zugänglich gemacht wurden (Abbildung 3 ). ${ }^{10}$
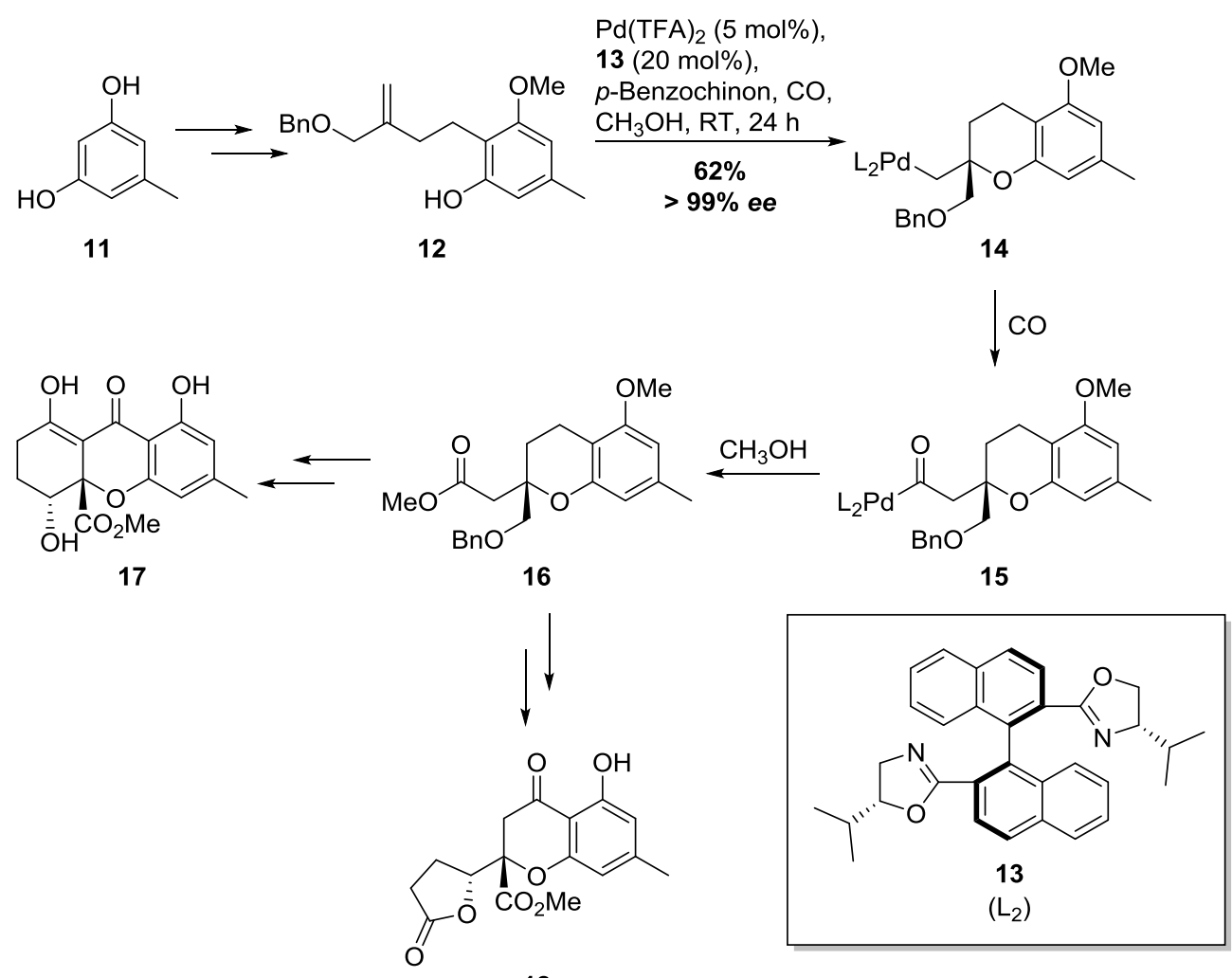

18

Abbildung 3: Synthese von (-)-Blennolid C (17) und (-)-Gonytolid C (18) über eine Domino-Wacker/ Carbonylierung/Methoxylierung. ${ }^{10}$

Die Synthese erfolgte ausgehend vom kommerziell erhältlichen Orcinol (11), welches in sechs Stufen zum Domino-Vorläufer 12 umgesetzt wurde. Die Domino-Reaktion wurde mit 5 mol\% Pd(TFA) $)_{2}$ und $20 \mathrm{~mol} \%$ des (S,S)-BOXAX-Liganden $\mathbf{1 3}$ in Methanol unter einer CO-Atmosphäre bei Raumtemperatur durchgeführt. Als Reoxidans für den Wacker-Prozess diente para-Benzochinon. Der Domino-Mechanismus beginnt mit einer Wacker-Oxidation des Domino-Vorläufers 12 zu Heterozyklus 14. Eine anschließende CO-Insertion liefert Carbonyl-Intermediat 15, welches mit Methanol zu Methylester 16 reagiert. 16 wiederum diente als Ausgangsmaterial sowohl für die Synthese von (-)-Blennolid C (17) als auch von (-)-Gonytolid C (18). Bemerkenswert ist die hohe Enantioselektivität der Reaktion: Die Untersuchung verschiedener Derivate des (S,S)-BOXAXLiganden ergab, dass 62\% Ausbeute und über 99\% Enantiomerenüberschuss erzielt werden konnten, wenn das iso-Propyl-Analogon 13 verwendet wurde. 


\subsection{Sonogashira-Reaktion}

Die Sonogashira-(Hagihara-)Reaktion gehört zur Klasse der Kreuzkupplungsreaktionen. Innerhalb dieser Gruppe von Reaktionen stellt sie eine Ausnahme dar, da sich die Sonogashira-Reaktion ausschließlich zum Kuppeln von Alkinen mit aromatischen und olefinischen (Pseudo-)Halogeniden eignet und damit im Gegensatz zu anderen Kreuzkupplungsreaktionen eine beschränkte Substratbreite besitzt.

1975 veröffentlichten Sonogashira et al. eine Variante der Castro-Stephens-Kupplung, die mit nur katalytischen Mengen einer Kupfer-(I)-Verbindung sowie katalytischen Mengen an Palladium(II) die Kupplung von terminalen Alkinen mit Arylhalogeniden ermöglichte. Damit entfiel der zuvor nötige stöchiometrische Einsatz der extrem stoßempfindlichen, explosiven Kupferacetylide. ${ }^{11}$ Daneben zeichnet sich die Sonogashira-Reaktion durch eine Reihe weiterer Vorteile aus: So sind in den meisten Fällen Umsetzungen bei Raumtemperatur möglich, womit eine extrem hohe Toleranz der Reaktion gegenüber funktionalen Gruppen einhergeht. Ferner werden nur geringe Katalysatorbeladungen benötigt und auch große Ansätze lassen sich noch in guten Ausbeuten umsetzen. Des Weiteren ist die Reaktion relativ unempfindlich gegenüber Feuchtigkeit und zum Teil auch gegenüber Luft. Einschränkungen ergeben sich lediglich durch unreaktive Arylhalogenide und sterisch überfrachtete Alkine, die bei höheren Temperaturen umgesetzt werden müssen. Bei der kupferkatalysierten Variante können außerdem Nebenreaktionen wie die Glaser-Kupplung auftreten. ${ }^{12}$

Der Mechanismus der Sonogashira-Kreuzkupplung ähnelt dem der anderen Kreuzkupplungsreaktionen (Abbildung 4). Aus einer Palladium(0)- oder Palladium(II)-Verbindung (üblicherweise $\operatorname{Pd}\left(\mathrm{PPh}_{3}\right)_{4}$ oder $\left.\quad \mathrm{PdCl}_{2}\left(\mathrm{PPh}_{3}\right)_{2}\right)$ wird zunächst die aktive Palladium(0)Katalysatorspezies gebildet. Es folgt die oxidative Addition an ein Aryl- oder Alkenyl-Halogenid. Die daraus entstehende Palladium(II)-Verbindung reagiert in einem Transmetallierungsschritt mit einem Kupferacetylid zum entsprechenden Palladium(II)-Alkinyl-Komplex, welcher nach reduktiver Eliminierung das Kupplungsprodukt freigibt. Die verbleibende Palladium(0)-Spezies wiederum steht für weitere Transformationen zur Verfügung. Das Kupferacetylid wird durch die in-situ-Umsetzung eines terminalen Alkins mit einer Aminbase $\left(\mathrm{HNEt}_{2}, \mathrm{NEt}_{3},(i \mathrm{Pr})_{2} \mathrm{NEt}\right.$ o.ä.) und katalytischen Mengen Kupfer(I)-Bromid oder -Iodid gewonnen. Möglich ist auch eine kupferfreie Transformation, wobei hier eine stärkere Base erforderlich ist, um das terminale Alkin zu deprotonieren (z.B. $\left.(n \mathrm{Bu})_{4} \mathrm{NOAc}\right)$. Die Sonogashira-Reaktion liefert die besten Ergebnisse mit Aryliodiden und -triflaten. Bromide und Chloride sind weniger reaktiv und erfordern höhere Temperaturen. Diese Chemoselektivität kann von Vorteil sein, da sie die Umsetzung von aromatischen Iodiden und Triflaten in Gegenwart von Bromiden und Chloriden erlaubt. Wie bereits erwähnt, zeichnet sich die Sonogashira-Reaktion durch eine hohe Toleranz vieler verschiedener funktionaler Gruppen aus. Diese spiegelt sich auch in der Natur des Alkin-Kupplungspartners wider, der sowohl Alkyl- und Aryl- als auch Alkenyl-Reste tragen 
kann. Auch eine Umsetzung mit silylgeschützten Alkinen ist möglich, sodass nach einer möglichen Entschützung ein terminales Alkin zur Verfügung steht.

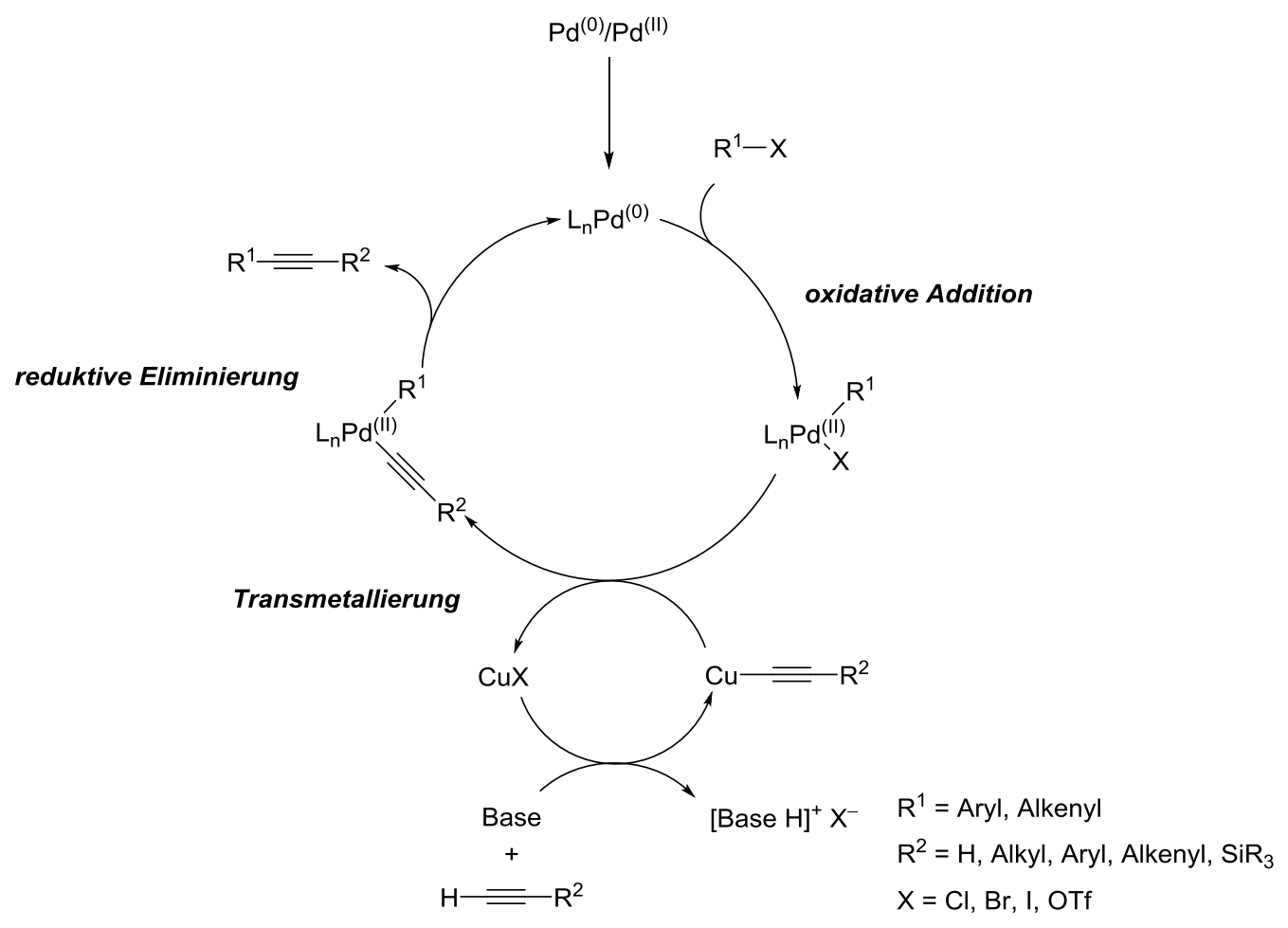

Abbildung 4: Mechanismus der Sonogashira-Kreuzkupplung. ${ }^{12}$

Eine interessante Anwendung der Sonogashira-Kupplung findet sich in den Arbeiten von Müller et al. ${ }^{13}$ Über eine Eintopf-Multikomponenten-Reaktion mit einer Sequenz aus Sonogashiraund Glaser-Kupplungen sowie anschließender Zyklisierung konnten einfache Aryliodide (20) mit Trimetylsilylacetylen (19) über Dialkin-Intermediate des Typs 22 in 2,5-disubstituierte Furane (21) umgewandelt werden (Abbildung 5).
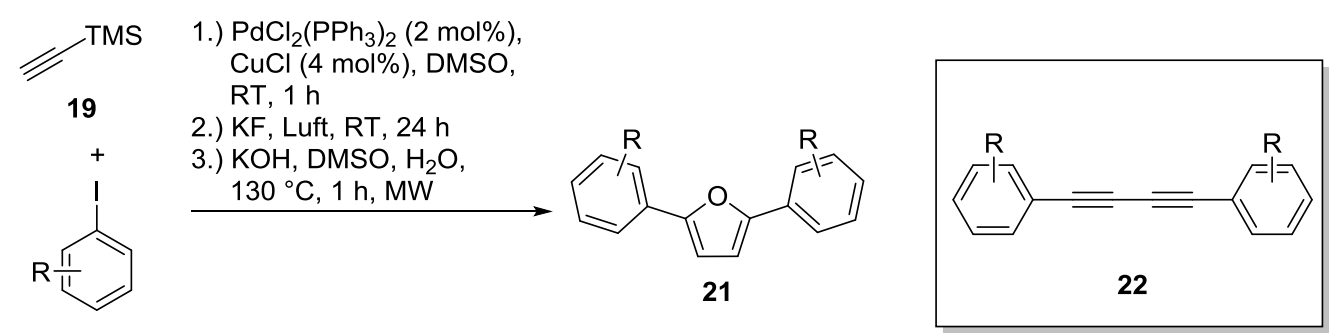

20

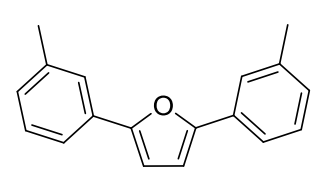

21a

$(65 \%)$<smiles>c1ccc2c(-c3ccc(-c4cc5ccccc5c5ccccc45)o3)cccc2c1</smiles>

21b

$(51 \%)$<smiles>c1csc(-c2ccc(-c3cccs3)o2)c1</smiles>

21c

$(31 \%)$<smiles>Fc1ccc(-c2ccc(-c3ccc(F)cc3)o2)cc1</smiles>

21c

$(25 \%)$

Abbildung 5: Synthese von 2,5-disubstituierten Furanen des Typs 21 über eine Eintopf-Sonogashira-GlaserZyklisierungssequenz (gezeigt ist eine Auswahl der synthetisierten Verbindungen). ${ }^{13}$ 


\subsection{Carbopalladierung}

Ein interessantes Instrument für C-C-Bindungsknüpfungen und vor allem Zyklisierungsreaktionen stellt die Carbopalladierung dar. Bei diesem Reaktionstypus handelt es sich um die Addition einer Palladium-Kohlenstoff-Spezies an eine C-C-Mehrfachbindung (Abbildung 6). ${ }^{14}$

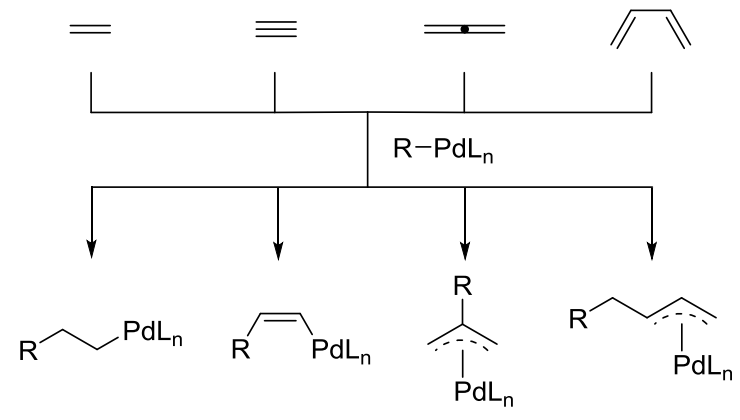

Abbildung 6: Addition von Palladium-Kohlenstoff-Verbindungen an C-C-Mehrfachbindungen (Carbopalladierung).

In der Folge kann eine Reihe weiterer Umsetzungen der entstehenden Palladium-Spezies erfolgen (Abbildung 7): So ist die direkte Umsetzung mit einem Nukleophil durchführbar (A), ebenso wie eine Reaktion mit Carbonylverbindungen (B) und weiteren $\mathrm{C}-\mathrm{C}-$ Mehrfachbindungen (C). Aber auch Eliminierungen einer Palladium-Hydrid-Spezies (D) sind möglich, sofern ein $\beta$-syn-ständiges Wasserstoffatom existiert (Heck-Reaktion). Des Weiteren bietet das Konzept der Domino-Reaktion die Möglichkeit, andere Palladium-katalysierte Transformationen (z.B. Kreuzkupplungen) auf die Carbopalladierung folgen zu lassen (E).

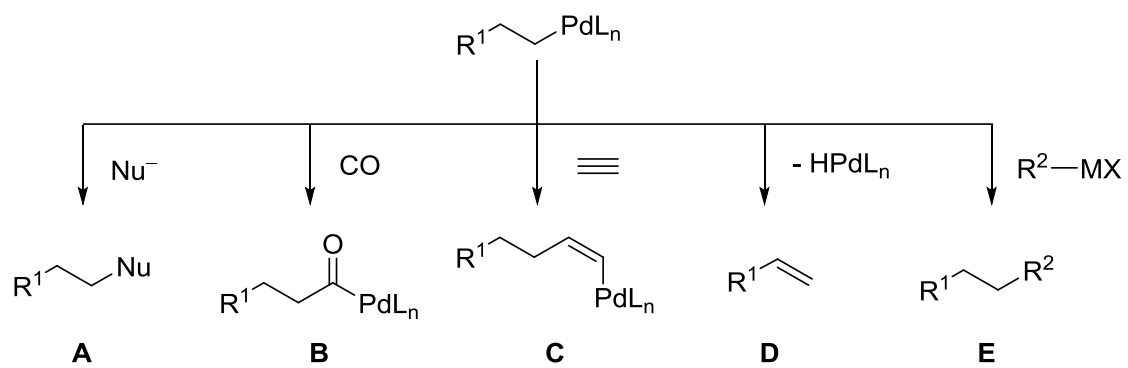

Abbildung 7: Mögliche Reaktionen infolge einer Carbopalladierung (vereinfachte Darstellung).

Der Mechanismus der Carbopalladierung erfolgt in einer strikten syn-Addition der Palladium-Spezies in die Kohlenstoff-Kohlenstoff-Bindung. Erklärt werden kann diese Selektivität, die insbesondere bei der Carbopalladierung von Alkinen von Bedeutung ist, durch einen konzertierten Mechanismus, bei dem ein unbesetztes dsp-Hybridorbital des Palladiums mit dem $\pi$-Orbital der C-C-Doppel-oder Dreifachbindung wechselwirkt. Gleichzeitig kommt es zur Interaktion des $\sigma$-Orbitals der PalladiumKohlenstoff-Bindung mit dem antibindenden $\pi^{*}$-Orbital der Mehrfachbindung. Der Vorgang findet unter Erhalt der Stereoselektivität statt (Abbildung 8). ${ }^{15}$ 


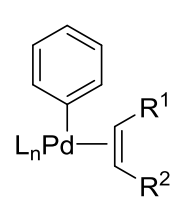

23

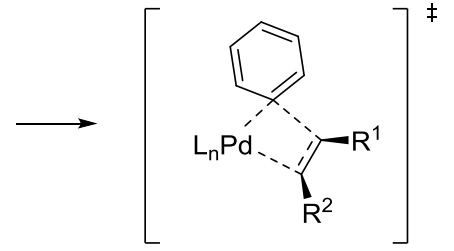

24

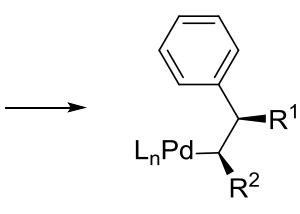

25

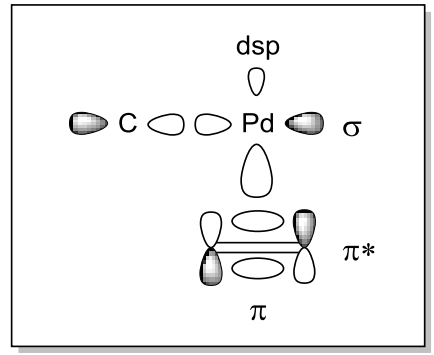

Abbildung 8: Erhalt der Steroselektivität bei Carbopalladierungen. ${ }^{15}$

Die Regioselektivität der Carbopalladierung ist schwer vorherzusagen und hängt stark von Substituenteneffekten ab. Dabei spielen sowohl elektronische als auch sterische Faktoren eine Rolle. Eine vorhersagbare Selektivität gibt es hingegen bei intramolekularen Carbopalladierungen, die den Baldwin-Regeln zu Zyklisierungen unterliegen: In Abhängigkeit von der Größe des gebildeten Ringes kommt es entweder zu einer exo-trig- oder aber einer endo-trig-Zyklisierung (Abbildung 9).

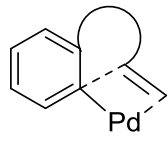

26

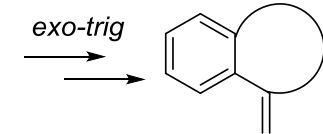

27

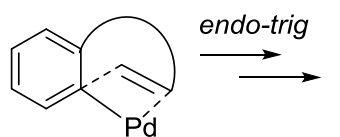

28

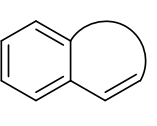

29

Abbildung 9: Mögliche Ringbildungen infolge einer intramolekularen Zyklisierung. ${ }^{15}$

Während kleine bis mittlere Ringe dem exo-trig-Mechanismus von 26 zu 27 folgen, läuft die endotrig-Zyklisierung von 28 zu 29 aufgrund der Spannung, die durch die Bildung der Z-Doppelbindung entsteht, erst bei größeren Ringen ab. ${ }^{15,16}$

Von besonderem Interesse ist auch die Carbopalladierung von Alkinen, da diese den syn-selektiven Aufbau tetrasubstituierter Alkene ermöglicht. Gerade intramolekulare Reaktionen dieses Typs sind von Bedeutung, da hierbei zudem eine regioselektive Umsetzung erfolgt. Wie bereits in Abbildung 7 beschrieben, kann der Carbopalladierung außerdem eine große Anzahl an weiteren Palladiumkatalysierten Reaktionen folgen. Diese Tatsache bietet die Möglichkeit zur Durchführung von Palladium-katalysierten Domino-Reaktionen mit einer oder mehreren Carbopalladierungen, der sich weitere Transformationen anschließen.

Ein besonders eindrucksvolles Beispiel einer solchen Domino-Reaktion lieferten Bäckvall et al. mit einer Domino-Carbozyklisierungs/Alkinylierungsreaktion (Abbildung 10). ${ }^{17}$ Hierbei beginnt der Mechanismus mit einer Insertion der katalytisch aktiven Palladium-Spezies in die Doppelbindung des Allens in Ausgangsstoff 30 und führt somit zu Dienyl-Palladium-Verbindung 31. Diese reagiert in einer zweiten, intramolekularen Carbopalladierung mit der Dreifachbindung in 31, wodurch die VinylPalladium-Spezies 32 entsteht, welche nach Reaktion mit Alkin 33 und abschließender reduktiver Eliminierung zu Zielverbindung 34 umgesetzt wird. Die Transformation wurde unter oxidativen Bedingungen in einer Sauerstoffatmoshäre mit Benzochinon sowie Cobalt-Salophen als Reoxidantien durchgeführt. Damit konnte die katalytisch aktive Palladium(II)-Spezies wiedergewonnen und ein 
Katalysezyklus etabliert werden. Die Reaktion wurde mit einer Reihe von Substraten durchgeführt, wobei unter modifizierten Bedingungen sogar interne Alkine als Substrate verwendet werden konnten. Insgesamt wurden so 23 Beispielverbindungen in Ausbeuten von bis zu 92\% hergestellt.

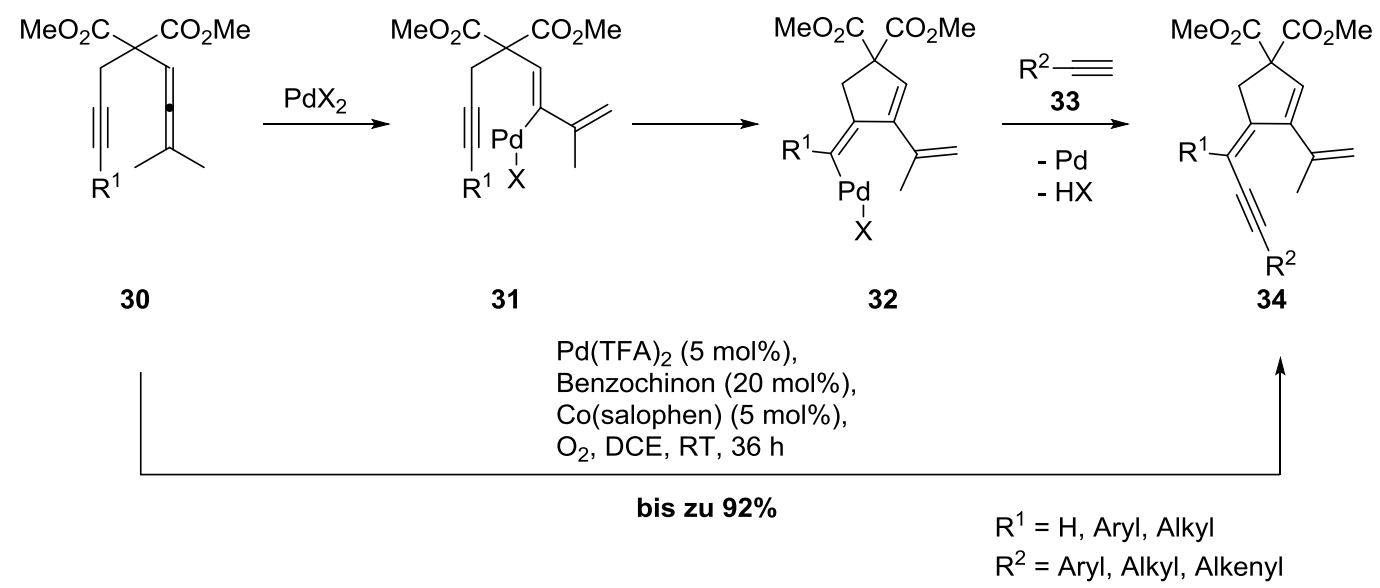

Abbildung 10: Domino-Carbozyklisierung/Alkinylierung nach Bäckvall. ${ }^{17}$

Das Beispiel verdeutlicht die Möglichkeit, mehrere Carbopalladierungen zu einem Domino-Prozess zusammenzufassen und diese Sequenz mit einer beliebigen Palladium-katalysierten Transformation zu beenden. Wie in Abbildung 10 dargestellt, konnte hierbei eine Dreifach-Domino-Reaktion, bestehend aus zwei Carbopalladierungen und einer terminierenden Palladium-katalysierten Alkinylierung, entwickelt werden. 


\subsection{C-H-Aktivierung}

Unter dem Begriff der C-H-Aktivierung werden verschiedene Prozesse zusammengefasst, wie etwa die Funktionalisierung von Olefinen, die Aktivierung von $\mathrm{C}-\mathrm{H}$-Bindungen an $\mathrm{sp}^{3}$-hybridisierten Kohlenstoffatomen und die direkte Arylierung, also die C-C-Bindungsknüpfung unter Beteiligung von aromatischen Kohlenstoffatomen. In diesem Kapitel soll vor allem letztere beschrieben werden.

Die direkte Aktivierung von $\mathrm{C}-\mathrm{H}$-Bindungen ist von großem synthetischem Wert für die organische Chemie, da sie die unvermittelte Transformation relativ einfacher Substrate erlaubt. So muss bei einer herkömmlichen Kreuzkupplungsreaktion eine vorherige Funktionalisierung der eingesetzten Verbindungen vorgenommen werden, etwa, indem die entsprechende Organometallkomponente hergestellt wird. Dieser Schritt entfällt bei der C-H-Aktivierung und das Substrat kann direkt umgesetzt werden (Abbildung 11). Durch die Ersparnis von zusätzlichen Funktionalisierungs- und Aufarbeitungsschritten ist die C-H-Aktivierung damit sowohl ökonomisch als auch ökologisch deutlich eleganter als die herkömmliche Kreuzkupplungsreaktion. ${ }^{18}$

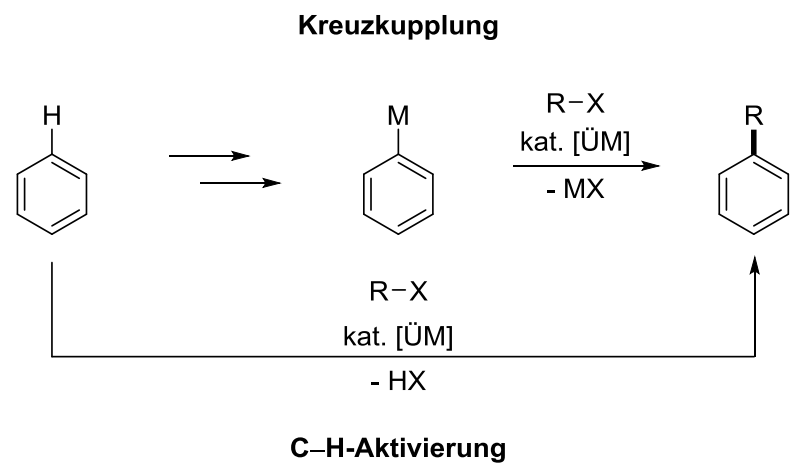

Abbildung 11: Vorzüge der C-H-Aktivierung gegenüber der klassischen Kreuzkupplung.

Eines der ersten Beispiele einer solchen C-H-Aktivierung wurde 1967 von Moritani und Fujiwara mit der Palladium-katalysierten Olefinierung von Benzol vorgestellt. ${ }^{19}$ Seither hat das Interesse an übergangsmetallkatalysierten Aktivierungen von $\mathrm{C}-\mathrm{H}$-Bindungen stetig zugenommen.

Die mechanistischen Aspekte des C-H-Aktivierungsprozesses sind noch nicht vollständig geklärt, da es offenbar Unterschiede in Abhängigkeit von Substrat, Katalysatormetall, Ligand, Lösungsmittel und allgemeinen Reaktionsbedingungen zu geben scheint. Für die Palladium-katalysierte direkte Arylierung werden hauptsächlich fünf mögliche Mechanismen diskutiert (Abbildung 12): So ist neben einem elektrophilen aromatischen Substitutionsmechanismus (A) auch ein $\mathrm{S}_{\mathrm{E}}$ 3-Prozess (B) denkbar. Des Weiteren könnte die Aktivierung auch über eine $\sigma$-Bindungsmetathese $(C)$ oder einen der HeckReaktion ähnelnden Mechanismus, bestehend aus einer Insertion des Palladiums in eine Doppelbindung des Benzolrings sowie anschließender Eliminierung einer Palladium-Hydrid-Spezies, ablaufen (D). Daneben wird auch eine oxidative Addition (E) diskutiert. ${ }^{20}$ 


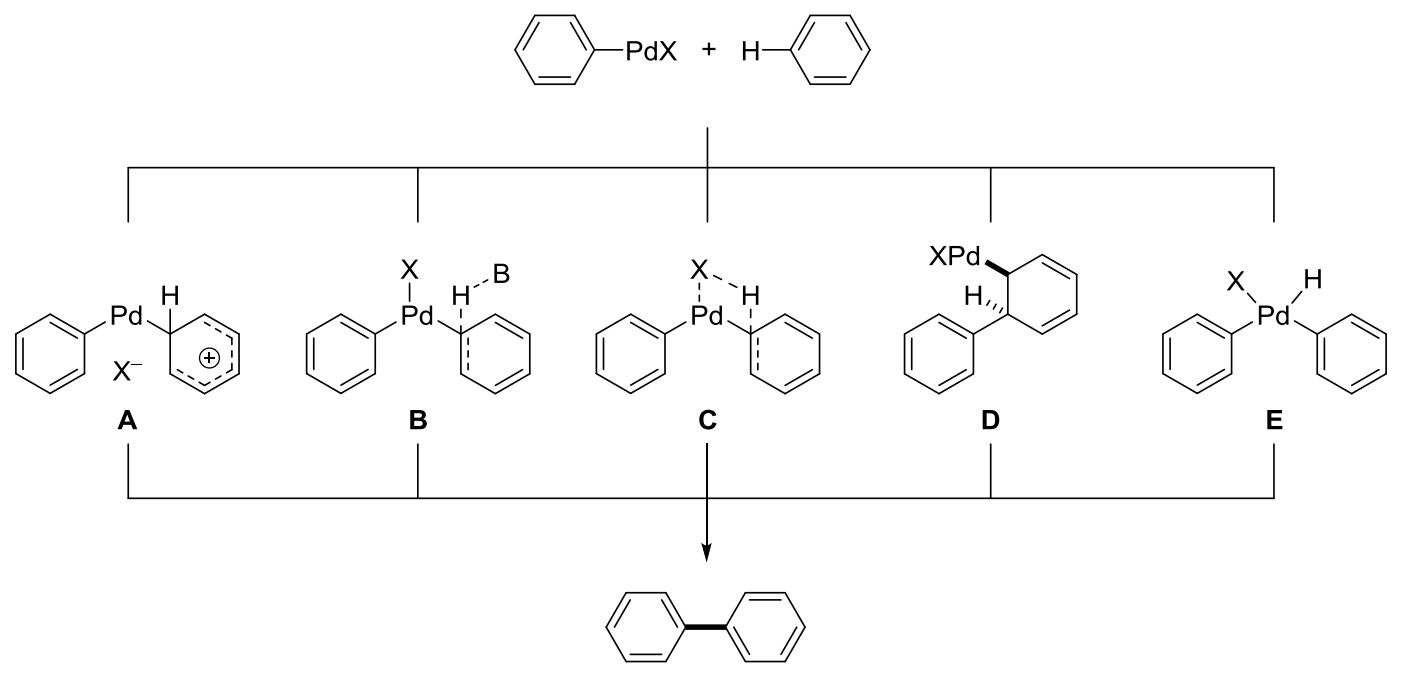

Abbildung 12: Diskutierte Mechanismen einer Palladium-katalysierten direkten Arylierung. ${ }^{20}$

Untersuchungen zeigen, dass die Mechanismen einer konzertierten aromatischen Substitution $\left(\mathrm{S}_{\mathrm{E}} 3\right)$ sowie einer $\sigma$-Bindungsmetathese wahrscheinlich sind. ${ }^{18}$ So konnte der Heck-artige Prozess nach den Ergebnissen von entsprechenden intramolekularen Zyklisierungsreaktionen nicht bestätigt werden. ${ }^{21}$ Auch der Ablauf einer oxidativen Addition mit einer intermediär entstehenden Palladium(IV)Verbindung wird mittlerweile weitgehend ausgeschlossen. ${ }^{18}$

Deuterierungsexperimente lieferten zudem Hinweise darauf, dass auch eine elektrophile aromatische Substitution weniger wahrscheinlich ist. So ergab die intramolekulare Arylierung von Verbindung 35 ein Gemisch aus dem Produkt der C-D-Aktivierung (36) und dem deuterierten Analogon (36-d $\mathbf{1}$ ), welches aus der entsprechenden $\mathrm{C}-\mathrm{H}$-Aktivierung resultiert (Abbildung 13). ${ }^{22}$ Der daraus berechnete kinetische Isotopeneffekt mit einem Wert von $k_{H} / k_{D}=4.25$ liefert einen Anhaltspunkt dafür, dass der C-H-Bindungsbruch den geschwindigkeitsbestimmenden Schritt der Arylierung darstellt und somit entweder eine $\sigma$-Bindungsmetathese oder ein $\mathrm{S}_{\mathrm{E}}$ 3-Prozess ablaufen. Ein $\mathrm{ArS}_{\mathrm{E}}$-Mechanismus kommt aus ebendiesen Gründen nicht infrage. Daneben lieferten Untersuchungen von Substituenteneffekten Gemische von Regioisomeren, die sich nicht mit den Erkenntnissen zur Regioselektivität von elektrophilen aromatischen Substitutionen in Einklang bringen lassen. ${ }^{22}$

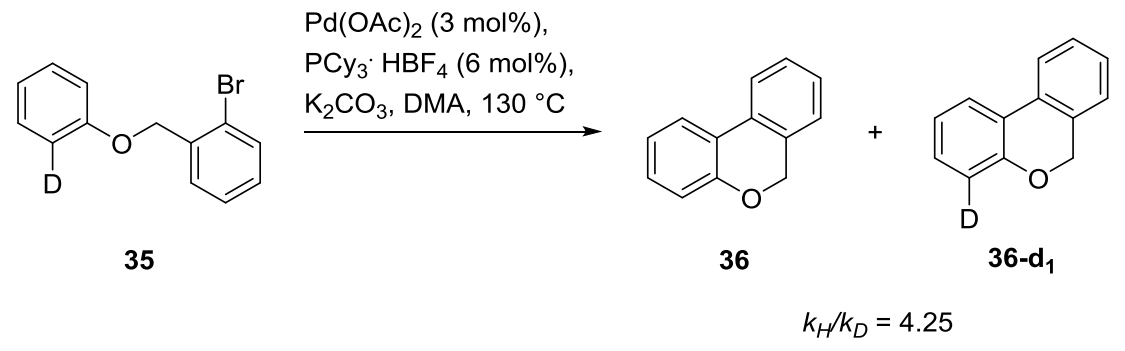

Abbildung 13: Deuterierungsexperiment zur mechanistischen Untersuchung der direkten Arylierung. ${ }^{22}$

In Abhängigkeit von den gewählten Reaktionsbedingungen wird auch ein basengestützer $\mathrm{C}-\mathrm{H}$ Aktivierungsprozess diskutiert. In einem konzertierten Metallierungs-Deprotonierungs-Prozess 
(concerted metalation/deprotonation, CMD) wird hierbei das Proton des Aromaten unter Mitwirkung von Metall und eingesetzter Base (vorzugsweise Carbonat) abstrahiert (Abbildung 14). Der Mechanismus entspricht dabei ausdrücklich nicht dem der elektrophilen aromatischen Substitution.

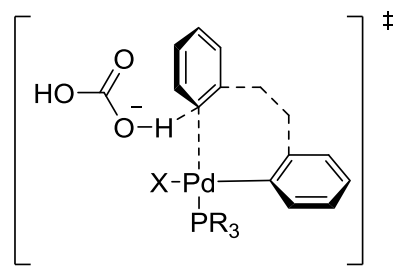

37

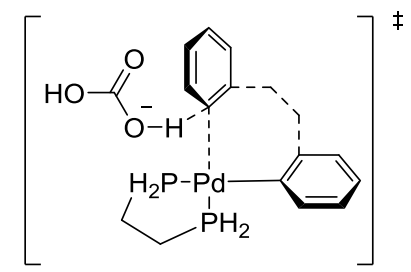

38

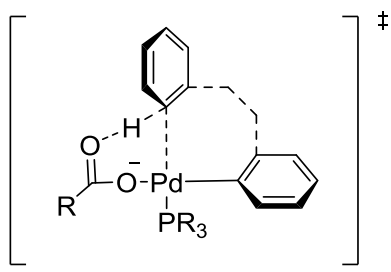

39

Abbildung 14: Basengestützte Mechanismen der C-H-Aktivierung. ${ }^{18}$

Diskutiert werden vor allem zwei grundlegende Mechanismen. Zum einen ist ein $\mathrm{C}-\mathrm{H}-$ Aktivierungsprozess denkbar, bei dem in einem intermolekularen Protonen-Abstraktionsmechanismus das verwendete Carbonat als externe Base wirkt (37). ${ }^{18,23,24}$ Dieser Ablauf wird auch für sterisch anspruchsvolle sowie bidentate Phosphinliganden angenommen $(38) .{ }^{25}$ Zum anderen ist es auch möglich, dass das Carbonat als interne Base an das Palladium koordiniert und in einer intramolekularen Abstraktion die C-H-Bindung aktiviert (39). Dieser Prozess wird in ähnlicher Form auch für Acetate und Pivalate als Basen angenommen, wobei in letzterem Fall umstritten ist, ob das Pivalat nicht lediglich die Funktion eines Protonenshuttles zur Unterstützung des schlecht löslichen Carbonats übernimmt. ${ }^{26-29}$

Problematisch bei Arylierungen ist die Herstellung einer Regioselektivität des $\mathrm{C}-\mathrm{H}$ Aktivierungsprozesses. In Abhängigkeit von der Art der Umsetzung stehen verschiedene Ansätze zur Verfügung, um eine Regioselektivität zu erreichen. Die einfachste Möglichkeit ist die Durchführung intramolekularer C-H-Aktivierungen. Diese führen zu einer Zyklisierung und begrenzen daher ganz natürlich das Spektrum der möglichen Produkte. Des Weiteren ist die Ausnutzung sterischer Faktoren eine Möglichkeit. So können sterisch anspruchsvolle Gruppen am Substrat oder am Katalysatorkomplex eine Benachteiligung von schwerer zugänglichen Positionen bewirken. Ähnlich verhält es sich mit der Ausnutzung von elektronischen Faktoren: Die Wahl geeigneter Substituenten am Benzolring der C-H-Aktivierung ermöglicht eine gewisse Selektivität bei der Produktbildung. ${ }^{18}$ Dennoch sind diese Methoden in Hinblick auf die nur eingeschränkte Substratbreite eher unelegant. Einen Ausweg bietet die Verwendung von dirigierenden Gruppen. Hierbei handelt es sich in der Regel um Funktionalitäten mit Lewis-basischen Heteroatomen, die das Katalysatormetall koordinieren und somit in die räumliche Nähe der zu aktivierenden C-H-Bindung bringen. Üblicherweise werden orthodirigierende Gruppen eingesetzt, die eine $\mathrm{C}-\mathrm{H}$-Aktivierung in benachbarter Position bewirken. Hierbei handelt es sich um entsprechende Sauerstoff- oder Stickstoffverbindungen (Phenole, Carbamate, Pyridine etc.). ${ }^{30,31}$ Sanford et al. gelang es zum Beispiel, in einer Palladium-katalysierten Arylierung 
selektiv die $\mathrm{C}-\mathrm{H}$-Bindung in ortho-Position zur jeweiligen dirigierenden Gruppe zu aktivieren (Abbildung 15). ${ }^{32}$<smiles>O=C1OCCN1c1ccccc1</smiles>

40

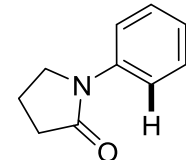

41<smiles>CC(=O)Nc1ccccc1</smiles>

42<smiles>CC(=O)N1CCc2ccccc21</smiles>

43<smiles>c1ccc(-c2ccc3ccccc3n2)cc1</smiles>

44

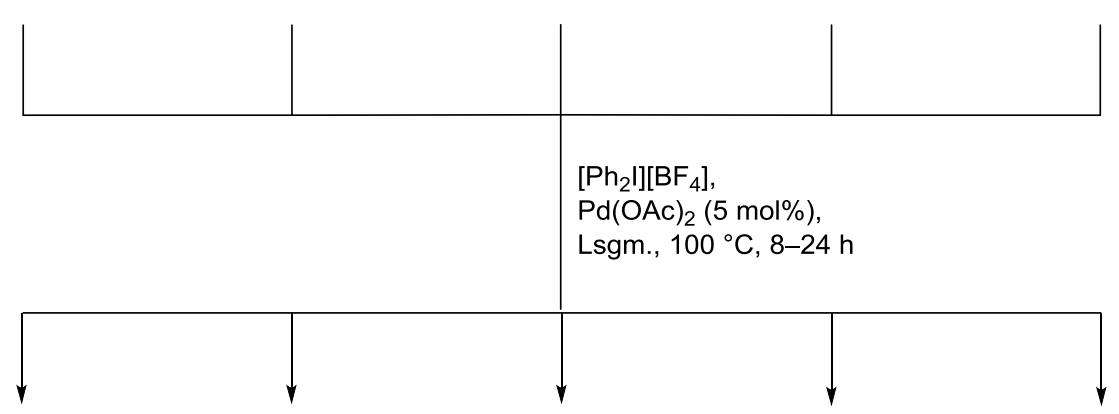<smiles>O=C1OCCN1c1ccccc1-c1ccccc1</smiles>

45

(83\%)

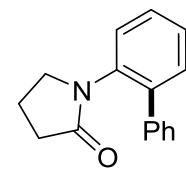

46

(75\%)

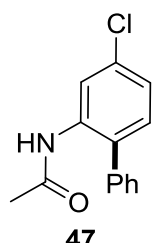

(67\%)<smiles>CC(=O)N1CCc2cccc(-c3ccccc3)c21</smiles>

48

(49\%)<smiles>c1ccc(-c2cccc3ccccc23)cc1</smiles>

49

(58\%)

Abbildung 15: Regioselektive C-H-Aktivierung unter Verwendung ortho-dirigierender Gruppen. ${ }^{32}$

Wie in der Abbildung veranschaulicht, führen diverse dirigierende Gruppen zu einer erfolgreichen selektiven C-H-Aktivierung. So konnten sowohl Oxazolidinon (40) und Pyrrolidinon (41) als auch freie (42) und annelierte (43) Amide eingesetzt werden, deren Heteroatome das Palladium koordinierten und dirigierten. Auch aromatische Stickstoffverbindungen in der entsprechenden Position (44) führten zu einer selektiven Arylierung in moderaten Ausbeuten.

Als Beispiel für eine Anwendung der C-H-Funktionalisierung in der Totalsynthese sei hier die von Lautens et al. 2014 entwickelte Synthese von (+)-Linoxepin (61) genannt (Abbildung 16). ${ }^{33}$ Schlüsselschritt dieser Synthese ist eine Variante der Catellani-Reaktion, bei der es zu einer Norbornen-gestützten Palladium-katalysierten C-H-Aktivierung kommt. ${ }^{34,35}$ Nach einer oxidativen Addition der katalytisch aktiven Palladium(0)-Spezies an Substrat 50 kommt es zur Insertion der daraus entstehenden Palladium-Verbindung in die Doppelbindung des Norbornen-Moleküls (51). Da kein $\beta$-ständiges Wasserstoffatom in der geeigneten Ausrichtung für eine $\beta$-Hydrid-Eliminierung vorliegt, kann die resultierende Spezies 52 in einem intramolekularen elektrophilen Angriff des Palladiums zu Palladazyklus $\mathbf{5 3}$ reagieren, welcher ebenfalls eine Eliminierung des Palladiums erschwert. Der folgende mechanistische Ablauf ist umstritten: Nach Catellani reagiert Verbindung $\mathbf{5 3}$ in einer weiteren oxidativen Addition mit Alkylbromid 54 zu Palladium(IV)-Intermediat 55, welches nach reduktiver Eliminierung bei gleichzeitiger ortho-Alkylierung und anschließender Decarbopalladierung von Norbornen zu Molekül 56 umgesetzt wird (Pfad A). ${ }^{34,35}$ Gemäß Echavarren 
wird 56 dagegen über den zweikernigen Palladiumkomplex 57 erzeugt (Pfad B). ${ }^{36}$ Beide Routen enden mit einer Heck-Reaktion, welche 56 mit tert-Butylacrylat 58 kuppelt und nach reduktiver Eliminierung Alken 60 freigibt. Dieses diente als Vorläufer für die Synthese von (+)-Linoxepin (61). Besonders bemerkenswert ist hierbei die Regioselektivität der $\mathrm{C}-\mathrm{H}$-Aktivierung, die unter gewöhnlichen Bedingungen nicht zu erreichen wäre. In diesem Fall wird sie durch die Verwendung von Norbornen (51) als Shuttle bewirkt, welches das katalytisch aktive Palladium an die gewünschte Position bringt und nach einer Retro-Carbopalladierung wieder zur Verfügung steht.

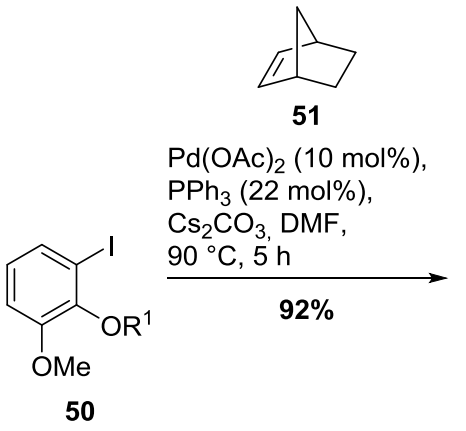

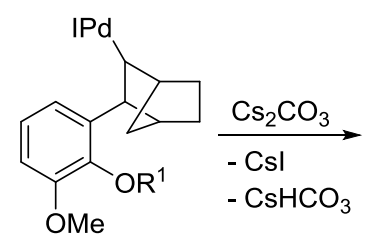

52

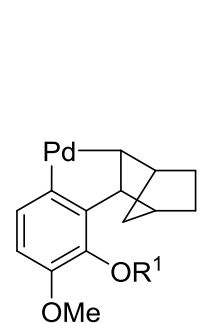

53

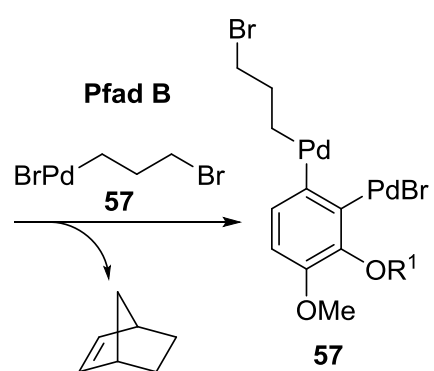

51

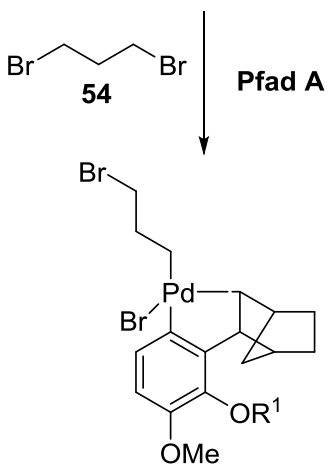

55

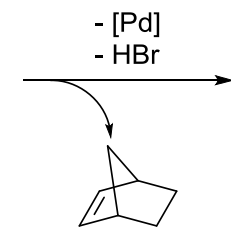

51

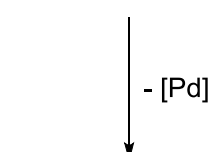

$\mathrm{Br}$

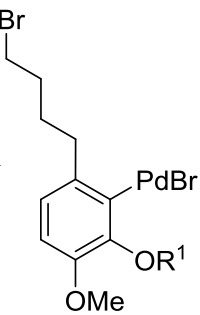

56

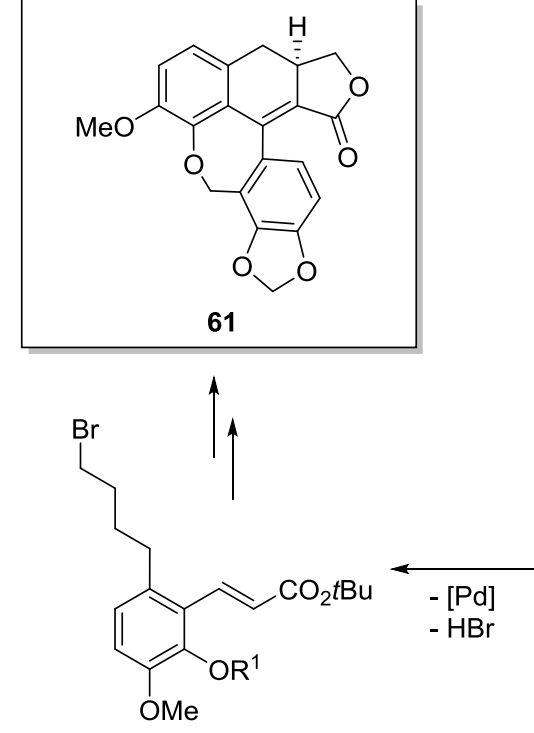

60

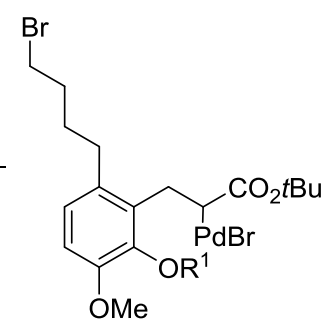

59

$\mathrm{CO}_{2} \mathrm{tBu}$

58

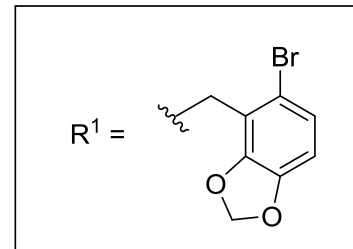

Abbildung 16: Synthese von (+)-Linoxepin (61) über eine Norbornen-gestützte C-H-Aktivierung. ${ }^{33}$ 


\subsection{Fluoreszenz}

Die Bestrahlung eines Moleküls mit Licht einer spezifischen Frequenz kann die Überführung des Moleküls in einen angeregten Zustand bewirken. Spontane und durch einfallendes Licht hervorgerufene stimulierte Emission können in Abhängigkeit von den jeweiligen Übergangswahrscheinlichkeiten eine Relaxation in den energetischen Grundzustand bewirken. Diese Relaxation kann auf verschiedene Arten erfolgen, wie das modifizierte Jablonski-Diagramm in Abbildung 17 zeigt.

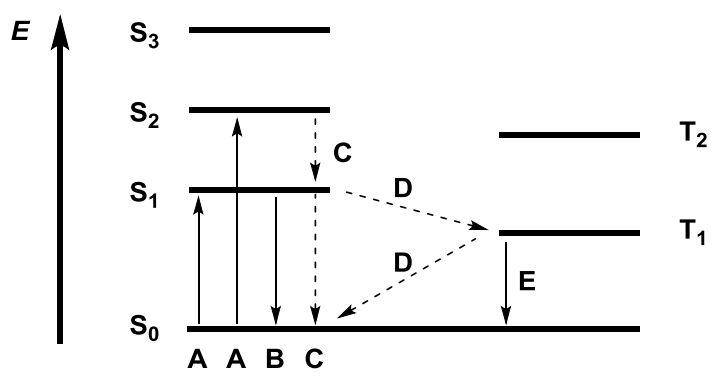

Abbildung 17: Modifiziertes Jablonski-Diagramm zur Darstellung verschiedener energetischer Übergänge (A: Absorption, B: Fluoreszenz, C: innere Konversion, D: Interkombination, E: Phosphoreszenz). ${ }^{37}$

Demnach sind nach einer Absorption (A) von Licht Übergänge vom Grundzustand $\mathrm{S}_{0}$, der in der Regel ein Singulettzustand ist, in höhere Singulettzustände (z.B. $S_{1}, S_{2}, \ldots$ ) möglich, welche die angeregten Zustände darstellen. Für die Relaxation in den Grundzustand ergeben sich verschiedene Möglichkeiten: So kann sie durch Emission von Strahlung erfolgen, welche als Fluoreszenz sichtbar wird (B). Eine weitere Möglichkeit ist der strahlungslose Übergang. Dieser kann zwischen Energiezuständen gleicher Spinmultiplizität stattfinden und wird als innere Konversion (internal conversion, C) bezeichnet. Möglich ist auch ein Übergang zwischen Zuständen unterschiedlicher Multiplizität (D). Diese sogenannte Interkombination (intersystem crossing) bezeichnet einen eigentlich verbotenen Übergang mit Spinumkehr, der das Molekül aus seinem Singulett- in einen angeregten Triplettzustand $T_{1}$ überführt. Aus diesem kann das System dann durch strahlungslose Interkombination (D) oder die Emission von Strahlung in Form von Phosphoreszenz (E) in den Grundzustand $S_{0}$ relaxieren. Diese Übergänge sind allesamt spinverboten, weswegen die Phosphoreszenz im Vergleich zur Fluoreszenz schwächer und zeitlich verzögert auftritt. ${ }^{37}$ Eine weitere Möglichkeit ist die Übertragung der überschüssigen Energie auf ein Akzeptormolekül, die strahlungslos abläuft. Das Akzeptormolekül wiederum kann unter anderem durch Fluoreszenz wieder in den Grundzustand relaxieren. Dieses Phänomen des sogenannten Förster-Resonanzergietransfers (FRET) wird vor allem zur Bestimmung molekularer Abstände genutzt. ${ }^{38}$

Anwendung findet das Phänomen der Fluoreszenz vor allem im Bereich des Imaging, also der Visualisierung von Zellkompartimenten oder anderen biologischen Systemen durch 
Fluoreszenzfarbstoffe zur Untersuchung von biologischen und physiologischen Prozessen auf zellularer Ebene.

Dabei hängt die Verwendbarkeit eines Fluorophors von seinen Eigenschaften ab. Von Bedeutung für die Fluoreszenzspektroskopie sind vor allem folgende physikalische Parameter: ${ }^{39}$

- Die Absorptions- und die Emissionswellenlänge des Farbstoffs

- Die Differenz aus beiden (der sogenannte Stokes-Shift)

- Die Lebensdauer der Fluoreszenz

- Die Quantenausbeute (der Quotient aus emittierten und absorbierten Photonen)

- Die Photostabilität des Fluorophors

Die Wellenlänge der Absorption und Emission spielt eine entscheidende Rolle für die Eignung des Fluorophors als Fluoreszenzmarker. Grundsätzlich sind längerwellig absorbierende und emittierende Farbstoffe von größerem Interesse für biologische Imaging-Methoden, da das energiearme Licht tiefer in das Gewebe eindringt und zudem eine störende Autofluoreszenz der Zellbestandteile vermieden wird. Dagegen kann das Wissen über den Stokes-Shift Grundlage für die Untersuchung mittels StokesShift-Spektroskopie sein, die eine exakte Methode zur Bestimmung von Fluorophoren in komplexen Mischungen darstellt. ${ }^{40}$ Parameter wie die Fluoreszenzlebensdauer und die von ihr abhängige Quantenausbeute sind für die Durchführung von Fluorescence Lifetime Imaging Microscopy (FLIM) sowie von Fluoreszenz-Polarisationsmessungen von Bedeutung. Diese Methoden liefern deutlich genauere und weiterreichende Aussagen über die zu untersuchenden Abläufe in Organismen als gewöhnliche Fluoreszenzmessungen. Die Photostabilität wiederum beschreibt die Anfälligkeit des Moleküls für Photobleaching-Reaktionen, welche das Molekül und seine Fluoreszenzeigenschaften zerstören und von der Struktur des Fluorophors und den experimentellen Bedingungen abhängen. In Messmethoden wie FRAP (Fluorescence Recovery after Photobleaching) ist Photobleaching ausdrücklich erwünscht und wird zur Untersuchung der Mobilität in Membranen verwendet. ${ }^{38}$

Im Folgenden werden typische Klassen von Fluoreszenzfarbstoffen kleiner Molekülgröße vorgestellt, die in biologischen Untersuchungen Anwendung finden. Als erstes Beispiel seien die CumarinDerivate genannt, deren Grundgerüst in zahlreichen Naturstoffen und Pharmazeutika wiederzufinden ist. Eine typische Synthese erfolgt über eine Pechmann-Kondensation zwischen Resorcin (62) und $\beta$ Ketoester 63 (Abbildung 18). ${ }^{41}$

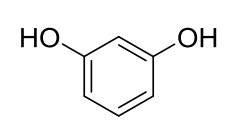

62

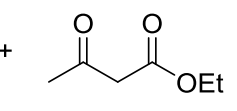

63

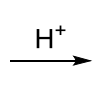

(1)

Abbildung 18: Typische Synthese von Cumarin-Grundgerüsten. ${ }^{41}$ 
Das entstehende 4-Methyl-7-hydroxycumarin (64) absorbiert im UV-Bereich $(\lambda=360 \mathrm{~nm})$ und emittiert blaues Licht $(\lambda=450 \mathrm{~nm}){ }^{39}$ Modifizierte Strukturen wie 7-Aminocumarin können die problematische pH-Sensitivität von $\mathbf{6 4}$ mindern und bilden zudem den Ausgangspunkt für das Labeling von Proteinen und die Verwendung als fluorophore Enzymsubstrate. ${ }^{42-44}$

Eine weitere Klasse von Fluorophoren stellen die sogenannten BODIPY-Farbstoffe (boron dipyrromethene) dar, welche auf einem heterozyklischen Grundgerüst basieren. Die Synthese erfolgt hierbei über eine Vilsmeier-Haack-analoge Kupplung der Pyrrole 65 und 66, welche nach Umsetzung mit $\mathrm{BF}_{3}$-Diethyletherat zu BODIPY-Derivat 67 reagieren (Abbildung 19). ${ }^{45}$

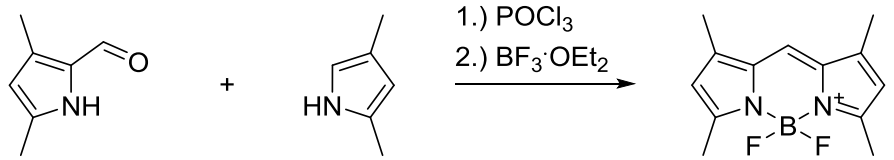

65
66
67

Abbildung 19: Synthese von BODIPY-Derivaten. ${ }^{45}$

Die Absorptionsbande von Verbindung 67 liegt bei $\lambda=505 \mathrm{~nm}$, die der Emission bei $\lambda=516 \mathrm{~nm}$. Die Wellenlängen können zu deutlich längerwelligen Bereichen verschoben werden, wenn etwa das verbrückende Kohlenstoffatom durch einen Stickstoff ersetzt wird. Ein bathochromer Effekt ergibt sich auch durch die Substitution der Fluor-Liganden am Bor durch Benzolringe. ${ }^{39}$ BODIPY-Farbstoffe zeichnen sich vor allem durch ihre Ladungsneutralität und die Unempfindlichkeit der Fluoreszenzaktivität gegen Umwelteinflüsse aus, weswegen sie oft in Membranen zum Einsatz kommen. Weitere Anwendungsgebiete sind die Verwendung als Donoren in FRET oder BRET (Biolumineszenz-Resonanzenergietransfer). ${ }^{46}$

Ein Klassiker unter den Fluororeszenzfarbstoffen sind die Fluoresceine. In der ursprünglichen Synthese wird Fluorescein (69) über eine Reaktion zwischen Phthalsäureanhydrid (68) und Resorcin (69) in einer Schmelze aus Zink(II)-Chlorid hergestellt (Abbildung 20). ${ }^{47}$ Mildere Bedingungen führen über eine Umsetzung in Säure zu Fluorescein. ${ }^{48}$<smiles></smiles>

68<smiles>Oc1cccc(O)c1</smiles>

62
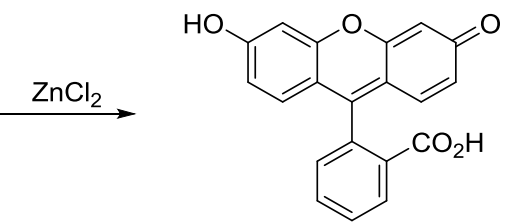

69

Abbildung 20: Ursprüngliche Synthese von Fluorescein (69). ${ }^{47}$

Fluorescein (69) absorbiert Licht bei $\lambda=491 \mathrm{~nm}$ und emittiert bei $\lambda=510 \mathrm{~nm}$. Die Wellenlängen können durch Modifikationen in den längerwelligen Bereich verschoben werden, allerdings geschieht dies oft auf Kosten der Quantenausbeute, so zum Beispiel bei dem Fluorescein-Derivat Rose Bengal. Erfolgreicher ist dagegen die Einführung annelierter Benzolringe, ebenso wie die Substitution des Xanthen-Sauerstoffs durch eine Dimethyl-Kohlenstoff- oder Dimethyl-Silicium-Funktionalität, die 
starke bathochrome Effekte hervorrufen. Fluoresceine können außerdem leicht funktionalisiert werden, um sie mit biologisch aktiven Molekülen zu koppeln. ${ }^{39}$ Sie werden daher unter anderem als Enzym-Substrate verwendet. ${ }^{49}$ Des Weiteren dienen sie in der retinalen Angiographie als Marker. ${ }^{50}$ Rhodamine können als die Amin-Gegenstücke des Fluoresceins betrachtet werden. Im Gegensatz zu diesem sind sie deutlich photostabiler, weniger $\mathrm{pH}$-sensitiv und zudem in ihren Emissionseigenschaften besser $\mathrm{zu}$ steuern. ${ }^{39}$ Die ursprüngliche Synthese des Prototyps Sulforhodamin B (72) erfolgt mittels einer Kondensation von Aminophenol 70 mit Benzaldehyd 71 und anschließender Oxidation mit Eisen(III)-Chlorid (Abbildung 21). ${ }^{51}$
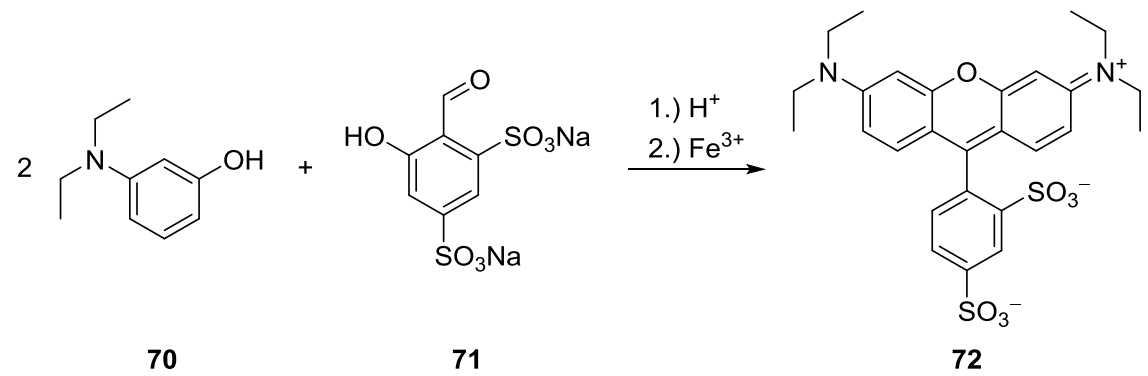

Abbildung 21: Ursprüngliche Synthese von Sulforhodamin B (72). ${ }^{51}$

Neuere Synthesen erfolgen direkt aus Fluorescein-Derivaten über eine Buchwald-HartwigKreuzkupplung. ${ }^{52}$ Sulforhodamin B (72) absorbiert im grünen $(\lambda=565 \mathrm{~nm})$ und emittiert im gelben $(\lambda=586 \mathrm{~nm})$ Spektralbereich. Die Einführung von Carboxyl- anstelle von Sulfonyl-Gruppen erlaubt eine Verwendung als Label und führt zudem zu einem leichten hypsochromen Effekt, ebenso wie Modifikationen an der Aminoalkyl-Kette. Desgleichen kann ähnlich wie beim Fluorescein der Xanthen-Sauerstoff durch Kohlenstoff-, Silicium-, Germanium- und Tellur-Gruppen ersetzt und damit eine Farbvertiefung erreicht werden. ${ }^{39}$ Verwendung finden Rhodamin-Farbstoffe unter anderem als Label in der Fluoreszenzmikroskopie sowie in der Untersuchung von Enzymaktivitäten. ${ }^{53,54}$

Strukturelle Analoga der Fluoresceine und Rhodamine sind die Phenoxazine, die ebenfalls ein Xanthen-Grundgerüst aufweisen und durch rotverschobene Fluoreszenzspektren auffallen, die zudem stark von der Polarität des Mediums abhängen. Der einfachste Vertreter dieser Stoffklasse ist das Resorufin (74). Dieses wird über eine Kondensation von Nitrosoresorcin 73 mit Resorcin (62) gewonnen (Abbildung 22). ${ }^{55}$

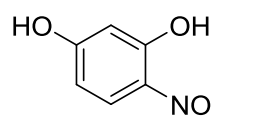

73<smiles>Oc1cccc(O)c1</smiles>

62

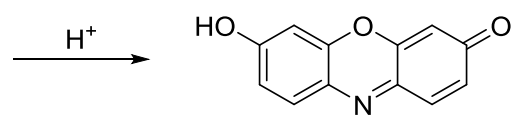

74

Abbildung 22: Synthese von Resorufin (74) als Prototyp der Phenoxazine. ${ }^{55}$

Resorufin absorbiert bei $\lambda=572 \mathrm{~nm}$ und emittiert gelbes Licht bei $\lambda=585 \mathrm{~nm}$. Die für die Analoga typische Rotverschiebung kann wie bei Fluoresceinen und Rhodaminen über AlkylaminoSubstituenten oder annelierte Bezolringe erreicht werden. Außerdem ermöglicht eine Dimethyl- 
Kohlenstoff-Gruppe anstelle des Xanthen-Sauerstoffs auch hier einen bathochromen Effekt. ${ }^{39}$ Bei dem Derivat Nil-Rot zeigt sich zudem ein hypsochromer Effekt in unpolaren Medien, weswegen dieser Farbstoff für Untersuchungen von Lipid-Membranen verwendet wird. ${ }^{56}$ Weitere Anwendungsbereiche sind auch hier die Untersuchung von Enzym-Aktivitäten sowie die histologische Forschung. ${ }^{57,58}$ Cyanine sind die sechste Klasse der Fluoreszenzfarbstoffe und vor allem wegen ihrer großen Extinktionswerte, der gut modulierbaren Strukturen und ihrer extremen Photostabilität beliebt. Ein Beispiel ist der Farbstoff Cy3 (77), welcher über eine Kondensation zwischen Indolinium-Salz 75 und Triethylorthoformat (76) erzeugt werden kann (Abbildung 23). ${ }^{59}$

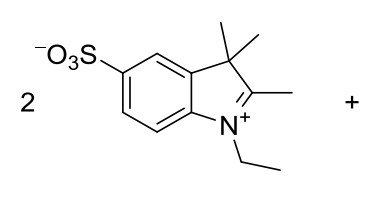

75<smiles>CCOC(OCC)OCC</smiles>

76

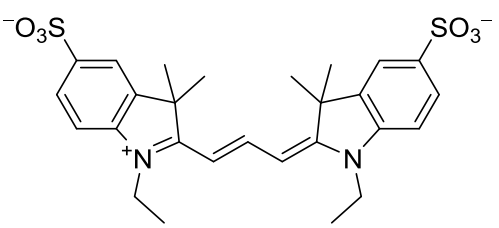

77

Abbildung 23: Synthese von Cyanin-Farbstoff Cy3 (77). ${ }^{59}$

Neuere Darstellungsmethoden erfolgen über eine Festphasensynthese. Cy3 absorbiert bei $\lambda=554 \mathrm{~nm}$ und emittiert gelbes Licht bei $\lambda=568 \mathrm{~nm}$, wobei diese Werte durch die Verlängerung oder Verkürzung der zentralen Polyen-Kette moduliert werden können. Ein ähnlich effektiver Weg ist außerdem die Synthese von Cumarin-, Fluorescein- oder Rhodamin-Hybriden, die eine Übertragung der Modulierungsmöglichkeiten dieser Verbindungen auf die Cyanine erlauben. Somit können auch hier enorme bathochrome Effekte erzielt werden. ${ }^{39}$ Wegen ihrer strukturellen Variabilität werden Cyanin-Farbstoffe vor allem im Labeling von Zellmembranen oder DNA-Strängen eingesetzt. ${ }^{60-62}$

Ein weiterer Anwendungsbereich für fluoreszente Moleküle ist die Verwendung als chemische Sensoren. Hierbei handelt es sich um Moleküle, die nach einer chemischen Reaktion mit dem nachzuweisenden Stoff (z.B. Ionen) eine (optisch) wahrnehmbare Veränderung eingehen. Die bekanntesten Vertreter dieser Gruppe sind die pH-Indikatoren, jedoch wurden, auch auf der Basis der bereits erwähnten Farbstoffe, chemische Sensoren entwickelt, die auch andere Ionen nachweisen können. So gelang es Guo et al. 2011, einen Sensor für Cyanid-Ionen zu synthetisieren (Abbildung 24). ${ }^{63}$

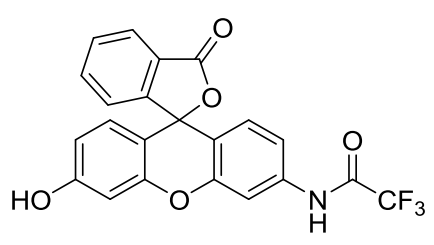

78

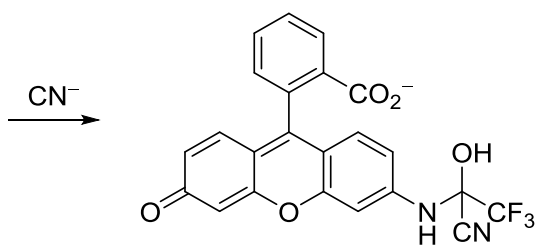

79

Abbildung 24: Maßgebliche chemische Reaktion des Sensors für Cyanid-Ionen. ${ }^{63}$ 
Dieser ist in seiner Spirolactonform (78) nicht fluoreszent und farblos. Die Zugabe von Cyanid-Ionen in einem Methanol-Wasser-Gemisch führt dagegen zu einer chemischen Reaktion zwischen dem Cyanid-Ion und der Carbonyl-Gruppe in 78, nach der das gebildete Addukt in einer Ringöffnungsreaktion zum fluoreszenzaktiven Rhodamin-Derivat 79 umlagert. Der Sensor ist äußerst selektiv für Cyanid-Ionen, andere Anionen lösen keine oder nur geringe Fluoreszenz aus. 


\section{Aufgabenstellung}

\subsection{Bisherige Forschungsergebnisse in der Arbeitsgruppe Tietze}

In der Forschungsgruppe Tietze wurden verschiedene Ansätze verfolgt, um über DominoCarbopalladierung/C-H-Aktivierungsreaktionen funktionale organische Moleküle herzustellen. Dabei standen vor allem tetrasubstituierte Alkene mit helikaler Chiralität im Fokus, die als mögliche molekulare Schalter dienen können. Aber auch andere Verbindungsklassen wurden erfolgreich synthetisiert, wie im Folgenden erläutert wird.

In den Arbeiten von F. Lotz gelang die Synthese von Acenaphthylenen des Typs 83 über eine Domino-Carbopalladierung/C-H-Aktivierung, die ausgehend von Alkin $\mathbf{8 0}$ erfolgte (Abbildung 25). ${ }^{5,64}$<smiles>OC1COc2ccc3ccccc3c2C1=C1c2ccccc2Oc2ccccc21</smiles>

84

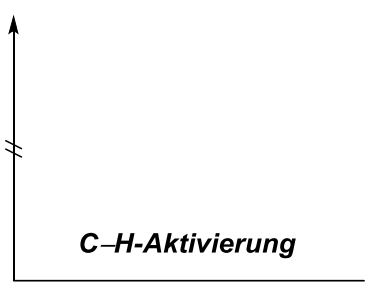<smiles>OCCOc1ccc2ccccc2c1OCC#Cc1ccccc1Oc1ccccc1</smiles>

$82(10 \mathrm{~mol} \%)$,

LiOAC, $(n \mathrm{Bu})_{4} \mathrm{NOAC}$ $\mathrm{DMF} / \mathrm{CH}_{3} \mathrm{CN} / \mathrm{H}_{2} \mathrm{O}$, $100-140{ }^{\circ} \mathrm{C}, 2-46 \mathrm{~h}$

bis zu $98 \%$

80

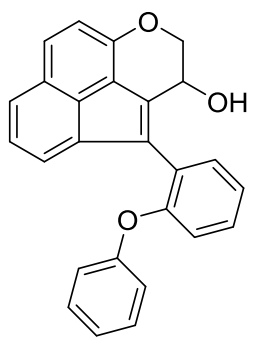

83

Carbopalladierung<smiles>O=C(C(=C1c2c(ccc3ccccc23)OCC1O)c1ccccc1Oc1ccccc1)c1ccccc1</smiles>

81

\section{C-H-Aktivierung}

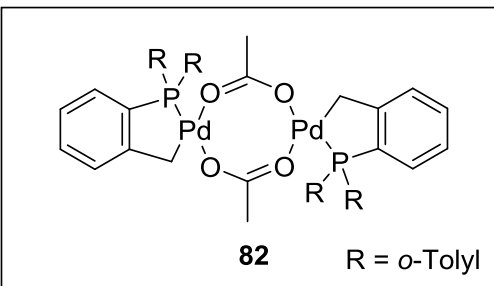

Abbildung 25: Synthese von Acenaphthylenen des Typs 83. ${ }^{5,64}$

Die Umsetzung wurde mit dem von Herrmann und Beller entwickelten Palladazyklus 82 mit LiOAc und $(n \mathrm{Bu})_{4} \mathrm{NOAc}$ als Base und einem Lösungsmittelgemisch aus DMF, Acetonitril und Wasser bei Temperaturen von 100 bis $140{ }^{\circ} \mathrm{C}$ in der Mikrowelle oder im Ölbad durchgeführt. ${ }^{65}$ Die DominoReaktion führte über eine Carbopalladierung zunächst zu 81 und schließlich mittels einer C-HAktivierung am Naphthyl-Substituenten zu Acenaphthylen 83. Eine ebenfalls denkbare $\mathrm{C}-\mathrm{H}-$ Aktivierung am unteren Phenoxy-Substituenten von 81 zum tetrasubstituierten Alken 84 wurde 
dagegen nicht beobachtet. Unter Verwendung der genannten Bedingungen konnten verschieden substituierte Acenaphthylene in Ausbeuten von bis zu 98\% synthetisiert werden.

Die Synthese von tetrasubstituierten Alkenen gelang schließlich über eine DominoCarbopalladierung/Heck-Reaktion. Ausgehend von Verbindung 85, bei der die untere Phenyl- durch eine Cyclohexenyl-Einheit substituiert wurde, erfolgte die Umsetzung über Carbopalladierungsprodukt 86 zum Cyclohexenyl-basierten tetrasubstitierten Alken 87 in bis zu 84\% Ausbeute (Abbildung 26).

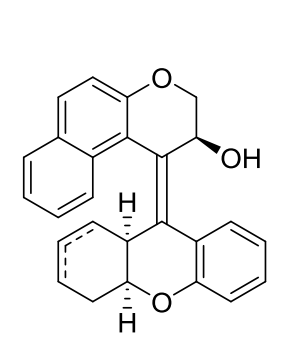

87

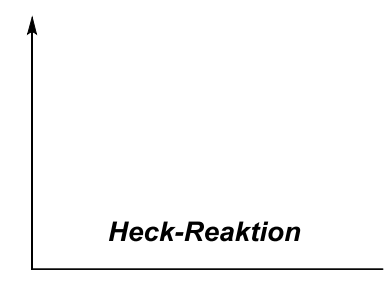

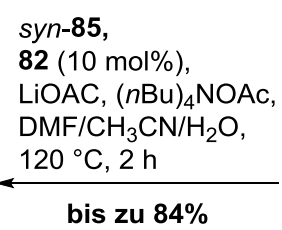

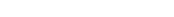

.

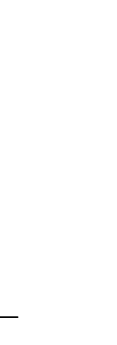

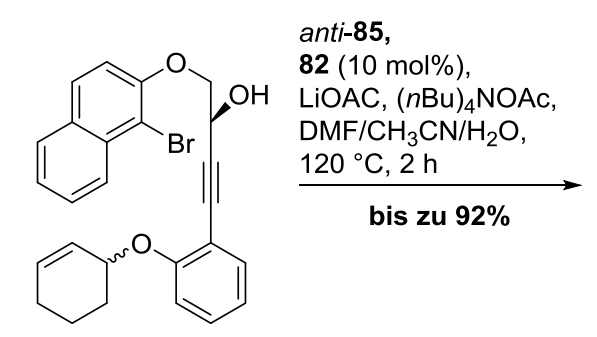

(rac) -85

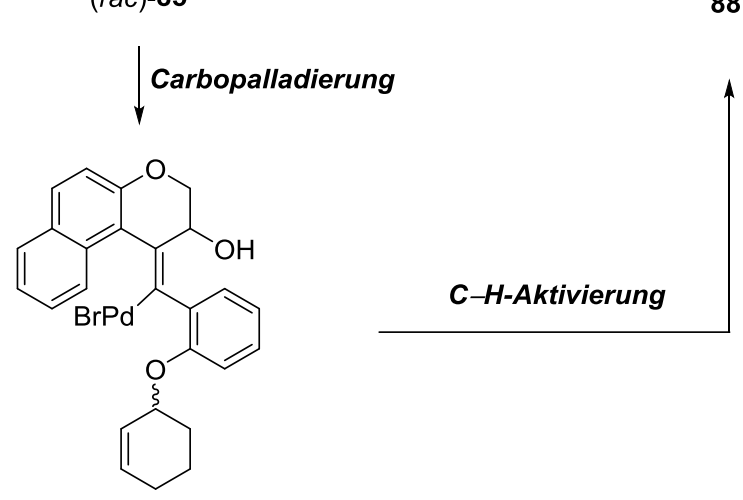

$($ rac)-86

Abbildung 26: Synthese von tetrasubstituierten Alkenen des Typs 87 und Acenaphthylenen des Typs $88 .^{64}$

Interessanterweise gelang die Transformation jedoch ausschließlich mit dem syn-Diastereomer der Ausgangsverbindung 85. Die anti-Verbindung führte dagegen unter identischen Reaktionsbedingungen zum Acenaphthylen 88, ein Produkt der bereits beschriebenen DominoCarbopalladierung/C-H-Aktivierung. Dieses Ergebnis deutet auf einen diastereodiskriminierenden Verlauf der Domino-Reaktion hin. Für einen effektiven und praktischen Zugriff auf tetrasubstituierte Alkene erwies sich dieser Ansatz jedoch als ungeeignet, da Produkt 87 Isomerisierungen der Doppelbindung im Cyclohexenyl-System unterlag und die Reaktion folglich zu Produktgemischen führte.

In den Arbeiten von M. A. Düfert wurde ebenfalls versucht, tetrasubstituierte Alkene über eine Domino-Carbopalladierung/C-H-Aktivierung zu erhalten (Abbildung 27). ${ }^{66}$ Dabei sollte die C-HAktivierung am Naphthylring von Ausgangsverbindung 89 erfolgen, wohingegen die oxidative Addition am unteren Phenyl-Substituenten stattfinden sollte. Eine Bildung des entsprechenden Acenaphthylens wäre damit nicht mehr möglich. Die Reaktion wurde mit $\operatorname{Pd}(\mathrm{OAc})_{2}$ als Katalysator 
und $\left[\mathrm{Pd} t \mathrm{Bu}_{4}\right]\left[\mathrm{HBF}_{4}\right]$ als Ligand mit $\mathrm{K}_{2} \mathrm{CO}_{3}$ als Base in Toluol bei $100{ }^{\circ} \mathrm{C}$ durchgeführt. Durch eine $\beta$ Hydrid-Eliminierung des Palladiums im Anschluss an die Carbopalladierung kam es jedoch lediglich zur Bildung des Vinylketons 90. Das erwünschte Produkt 84 wurde hingegen nicht beobachtet.<smiles>OC1COc2ccc3ccccc3c2C1=C1c2ccccc2Oc2ccccc21</smiles>

84<smiles>OC(C#Cc1ccccc1Oc1ccccc1Br)COc1ccc2ccccc2c1</smiles>

89

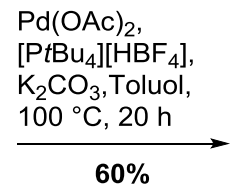

$60 \%$<smiles>O=C(C=C1c2ccccc2Oc2ccccc21)COc1ccc2ccccc2c1</smiles>

90

Abbildung 27: Untersuchungen zur Domino-Reaktion von $89 .{ }^{66}$

Da die Synthese der entsprechenden tetrasubstituierten Alkene über eine DominoCarbopalladierung/C-H-Aktivierung an der Selektivität der C-H-Aktivierung scheiterte, wurde eine alternative Synthesestrategie erarbeitet: Anstelle der bisher verwendeten unfunktionalisierten DominoVorläufer wurden nun Stannane des Typs 91 eingesetzt, um über eine DominoCarbopalladierung/Stille-Reaktion die erwünschten Strukturen vom Typ 84 zu erhalten. Die DominoReaktion erfolgte unter Verwendung von $\mathrm{Pd}_{2} \mathrm{dba}_{3}$ und $\left[\mathrm{Pd} t \mathrm{Bu}_{4}\right]\left[\mathrm{HBF}_{4}\right]$ als Katalysatorsystem mit Cäsiumfluorid als Base in 1,4-Dioxan bei $80^{\circ} \mathrm{C}$. Die entsprechenden tetrasubstituierten Alkene vom Typ 84 konnten in bis zu 70\% Ausbeute erhalten werden (Abbildung 28). ${ }^{66-68}$<smiles>CCCCCC(O)COc1ccccc1C#CC(O)COc1cccc2ccccc12</smiles>

91 Carbopalladierung<smiles>CCC(C)c1ccccc1Oc1ccccc1/C(=C1\c2c(ccc3ccccc23)OCC1Br)C1CCC(O)CO1</smiles>

92
$\mathrm{Pd}_{2} \mathrm{dba}_{3}(5 \mathrm{~mol} \%)$, [ $\left.\mathrm{PtBu}_{4}\right]\left[\mathrm{HBF}_{4}\right](10 \mathrm{~mol} \%)$, CsF, 1,4-Dioxan, $80^{\circ} \mathrm{C}, 18 \mathrm{~h}$

bis zu $70 \%$<smiles>OC1COc2ccc3ccccc3c2C1=C1c2ccccc2Oc2ccccc21</smiles>

84

Stille-Reaktion

Abbildung 28: Synthese tetrasubstituierter Alkene des Typs 84 über eine Domino-Carbopalladierung/StilleReaktion. ${ }^{66-68}$ 
In weiteren Untersuchungen konnte zudem nachgewiesen werden, dass die Domino-Reaktion diastereoselektiv erfolgt. Dieses wird der Vorkoordination des Palladiums durch die Hydroxyl-Gruppe zugeschrieben.

In den Arbeiten von T. Hungerland wurde die Domino-Carbopalladierung/C-H-Aktivierung erstmalig erfolgreich zur Synthese von tetrasubstituierten Alkenen eingesetzt: Die Substitution des Naphthylrings durch einen einfachen Benzolring ermöglichte die $\mathrm{C}-\mathrm{H}$-Aktivierung am unteren aromatischen System, da eine Reaktion zum sonst erhaltenen Acenaphthylen nicht möglich war. Die Reaktion erfolgte ausgehend von Alkin 93, welches unter Einfluss von $20 \mathrm{~mol} \% \mathrm{Pd}(\mathrm{OAc})_{2}$ und 1.00 Äq. $\mathrm{PPh}_{3}$ mit $\mathrm{K}_{2} \mathrm{CO}_{3}$ als Base in DMF über Carbopalladierungsprodukt 94 zum tetrasubstituierten Alken 95 reagierte. Über diese Methode konnte eine Reihe von Derivaten synthetisiert werden, die neben verschiedenen Substituenten auch unterschiedliche Ringgrößen des oberen Cycloalkyl-Systems enthielten. Die Reaktion verlief in guten bis exzellenten Ausbeuten von bis zu 99\% (Abbildung 29). 2,3,69

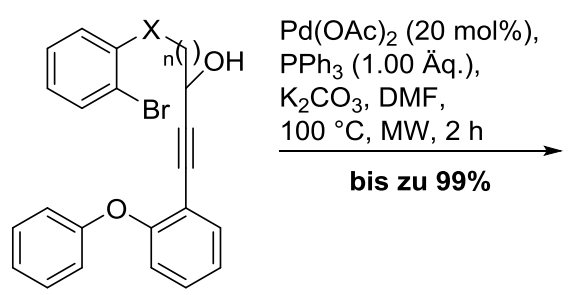

93

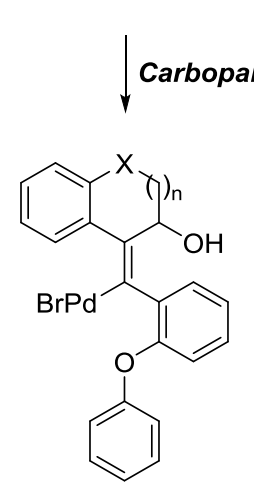

94

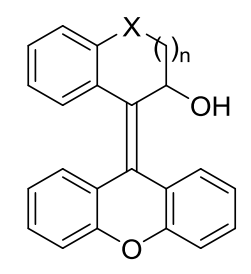

95

Abbildung 29: Domino-Carbopalladierung/C-H-Aktivierung zur Synthese tetrasubstituierter Alkene des Typs $95^{2,3,69}$

In einem Versuch, auch Naphthyl-basierte tetrasubstituierte Alkene über eine Domino-C-HAktivierung zu synthetisieren, wurde ein gegenüber den Arbeiten von M. A. Düfert modifiziertes System verwendet, das im oberen Teil des Systems einen weiteren Benzolring enthielt, um die ansonsten auftretende $\beta$-Hydrid-Eliminierung zu unterbinden (vgl. Abbildung 27).

Die Synthese über die bereits bekannte Domino-Carbopalladierung/C-H-Aktivierung gelang in guten Ausbeuten. In einer Erweiterung des Domino-Prozesses konnte zusätzlich die vorausgehende Sonogashira-Reaktion in die Domino-Transformation integriert werden. Über die resultierende 
Zweikomponenten-Domino-Sonogashira/Carbopalladierung/C-H-Aktivierungsreaktion wurden unter Verwendung von $\mathrm{Pd}(\mathrm{OAc})_{2}$ als Katalysator, $\mathrm{PPh}_{3}$ als Ligand und $(n \mathrm{Bu})_{4} \mathrm{NOAc}$ als Base tetrasubstituierte Alkene des Typs 100 in Ausbeuten von bis zu 96\% synthetisiert (Abbildung 30). ${ }^{1,69}$<smiles>C#Cc1ccccc1Oc1ccccc1Br</smiles>

97 Sonogashira-Reaktion<smiles>Brc1ccccc1Oc1ccccc1C#Cc1c(Oc2ccccc2)ccc2ccccc12</smiles>

98
$\mathrm{Pd}(\mathrm{OAc})_{2}(20 \mathrm{~mol} \%)$ $\mathrm{PPh}_{3}\left(1.00 \mathrm{Äq}\right.$.), $(n \mathrm{Bu})_{4} \mathrm{NOAc}$, DMF, $120^{\circ} \mathrm{C}, 4 \mathrm{~h}, \mathrm{MW}$

bis zu $96 \%$

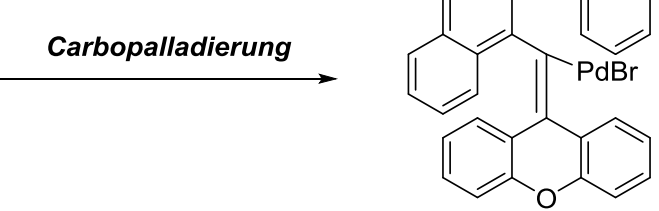

99

Abbildung 30: Synthese von tetrasubstituierten Alkenen des Typs 100 über eine Domino-Sonogashira/ Carbopalladierung/C-H-Aktivierung. ${ }^{1,69}$

Die Substratbreite dieser Domino-Reaktion wurde in der Masterarbeit von J. Ammermann zur Synthese von heterozyklischen Verbindungen des Typs 106 erweitert. ${ }^{4,70}$
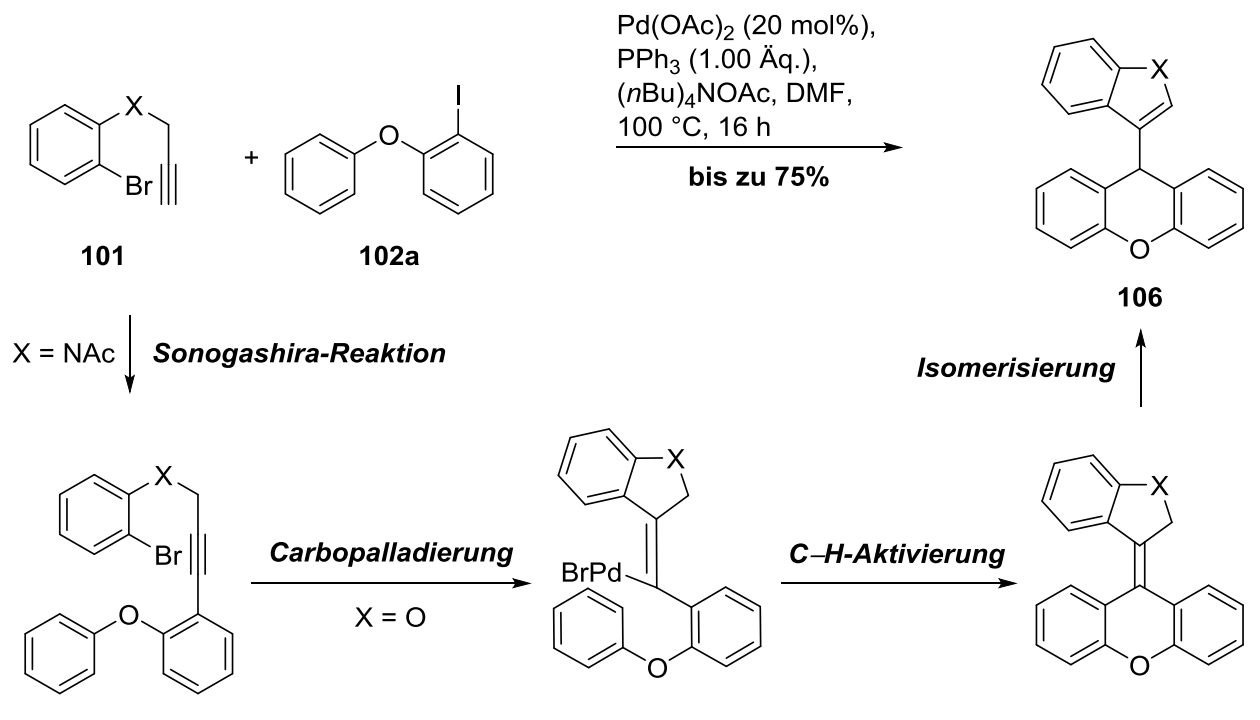

103

104

105

Abbildung 31: Synthese heterozyklischer Verbindungen des Typs 106 über eine Domino-Sonogashira/ Carbopalladierung/C-H-Aktivierung/Isomerisierungsreaktion. ${ }^{4,70}$ 
In dieser Sequenz schloss sich der Domino-C-H-Aktivierung eine Dopplelbindungsisomerisierung an, da die tetrasubstituierten Alkene $\mathbf{1 0 5}$ sich auf diesem Wege zu aromatisierten Strukturen des Typs 106 stabilisierten (Abbildung 31). Die Domino-C-H-Aktivierung/Isomerisierung wurde ausschließlich bei heterozyklischen Fünfringen beobachtet. In Ausbeuten von bis zu 75\% konnten unter den bereits von T. Hungerland verwendeten Reaktionsbedingungen Indole des Typs 106 hergestellt werden. Die entsprechenden Benzofurane wurden über einen Domino-Carbopalladierung/C-HAktivierung/Isomerisierungsprozess unter Ausschluss der Sonogashira-Reaktion synthetisiert. 


\subsection{Zielsetzung und Planung dieser Arbeit}

Aufbauend auf den vorangegangenen Forschungsergebnissen sollte eine Ausdehnung des DominoCarbopalladierung/C-H-Aktivierungsprozesses auf die effektive Synthese weiterer funktionaler Moleküle vorgenommen werden. Domino-Reaktionen stellen eine elegante Methode dar, um aus einfachen Vorläufern komplexe Strukturen zu erzeugen. Eine in den Domino-Prozess eingeschlossene $\mathrm{C}-\mathrm{H}-$ Aktivierung ist gegenüber herkömmlichen Kreuzkupplungsreaktionen noch eleganter, da keine vorhergehenden Funktionalisierungen nötig sind und damit noch einfachere Vorläufer Verwendung finden können. Je mehr Schritte in den Domino-Prozess integriert werden, umso effizienter und synthetisch anspruchsvoller ist dieser. In dieser Arbeit sollten deswegen erweiterte DominoSonogashira/Zweifach-Carbopalladierung/C-H-Aktivierungs-Reaktionen entwickelt und diese zur Synthese von fluoreszenzaktiven polyzyklischen Kohlenwasserstoffen verwendet werden.

Zum einen sollten dabei Xanthen-Derivate des Typs 107 synthetisiert werden, die durch einen entsprechenden Domino-Sonogashira/Zweifach-Carbopalladierung/C-H-Aktivierungs-Prozess $\quad \mathrm{zu}-$ gänglich wären. In insgesamt vier Schritten würden vier neue Bindungen geknüpft und drei Ringsysteme geformt werden. Für die Domino-Reaktion schienen die Aryliodide vom Typ 108 und das Dialkin 109a geeignet, wobei Substratbreite und Limitation der Reaktion durch Variation der Substituenten am Aryliodid bestimmt werden sollten (Abbildung 32).
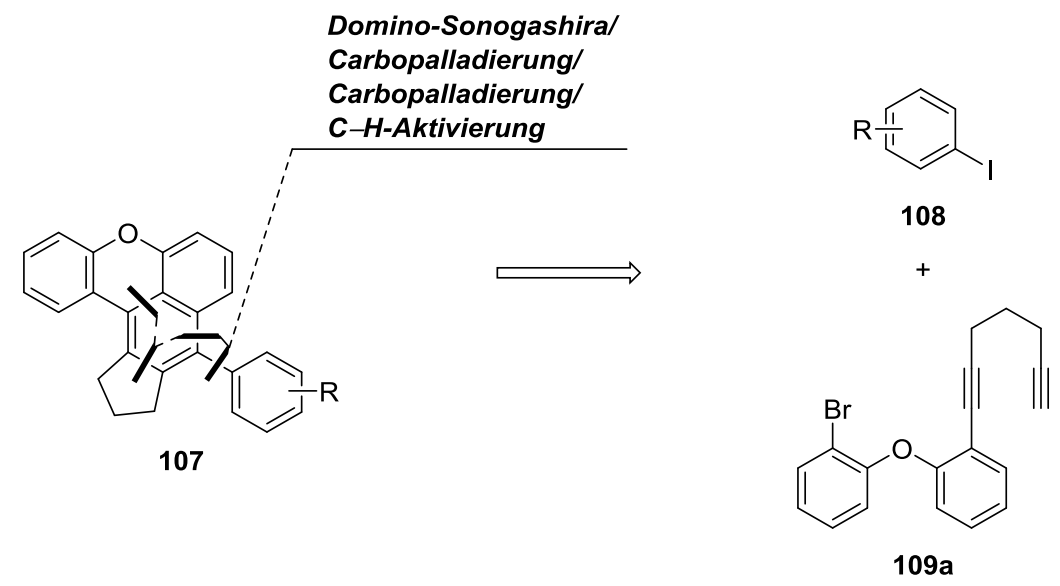

Abbildung 32: Retrosynthetischer Ansatz zum Aufbau von fluoreszenzaktiven aromatischen Polyzyklen des
Typs 107.

Nach erfolgter Synthese würden Untersuchungen zur Fluoreszenz der hochkonjugierten Zielverbindungen erfolgen. Des Weiteren sollte auch die Möglichkeit einer Synthese von dimeren Analoga des Moleküls 107 analysiert werden, die über eine zweifache Domino-Sonogashira/ZweifachCarbopalladierung/C-H-Aktivierung erfolgen könnte. Dimere Domino-Prozesse wurden bislang nur selten berichtet, bieten jedoch äußerst effiziente Ansätze für die Synthese pseudosymmetrischer Naturstoffe oder Polymere. 
Um die Fluoreszenzaktivität zu intensivieren und zudem die Absorption in längerwellige Bereiche des Vis-Bereichs zu verschieben, sollte eine Domino-Sonogashira/Zweifach-Carbopalladierung/C-HAktivierung zur Synthese von Polyzyklen des Typs 110 entwickelt werden. Die gebildeten XanthenDerivate würden zwei annellierte Naphthylringe enthalten, wodurch ein bathochromer Effekt hervorgerufen werden sollte (Abbildung 33). Damit böte die Domino-Reaktion einen Zugang zu noch komplexeren hochkonjugierten Strukturen. Bei der geplanten Reaktion würden insgesamt vier neue Bindungen sowie drei neue Ringsysteme in einem Reaktionsprozess gebildet werden.

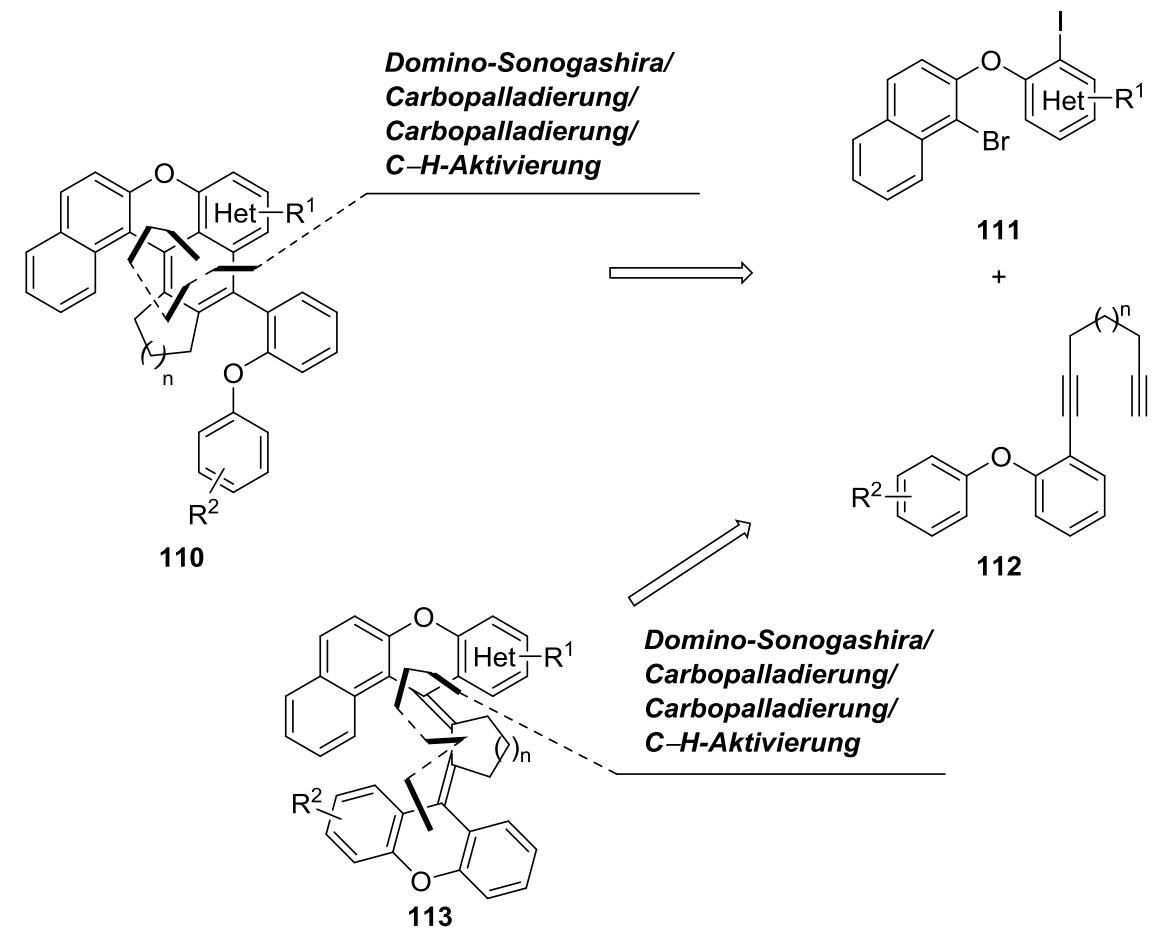

Abbildung 33: Retrosynthetischer Ansatz zur Synthese von fluoreszenzaktiven Polyzyklen des Typs 110 sowie tetrasubstituierten Dialkenen des Typs 113.

Eine Variation der Substrate sollte auch hier Aufschluss über die Toleranz der Domino-Reaktion gegenüber verschiedenen Funktionalitäten und Substituenten sowie deren Einfluss auf die Fluoreszenzeigenschaften von $\mathbf{1 1 0}$ geben.

Denkbar wäre auch die Bildung von tetrasubstituierten Dialkenen des Typs $\mathbf{1 1 3}$ aus identischen Ausgangsverbindungen. Diese Strukturen sind aufgrund ihrer helikalen Chiralität von besonderem Interesse in der Anwendung als molekulare Schalter. Gleichzeitig wäre der Aufbau von zwei konjugierten tetrasubstituierten Doppelbindungen in einer vierfachen Domino-Reaktion ein erstrebenswertes Syntheseziel, da sich somit der Zugang zu weiteren komplexen Strukturen eröffnen würde.

In einem anderen Ansatz zur Synthese von hochkonjugierten aromatischen Polyzyklen sollte zudem versucht werden, hydrophile Derivate vom Typ 114 herzustellen. Diese sollten über eine bessere 
Löslichkeit als die bereits beschriebenen Polyzyklen verfügen und zudem eine größere Modifizierbarkeit über die Hydroxyl-Gruppen aufweisen. Außerdem würde das Vorliegen von zwei Stereozentren im Substrat den enantio- und diasteroselektiven Aufbau der Produkte ermöglichen und interessante Aufschlüsse über die Stereochemie der zentralen Domino-Reaktion zulassen. Die Synthese von 114 wurde über eine Domino-Zweifach-Carbopalladierung/C-H-Aktivierung angestrebt (Abbildung 34).

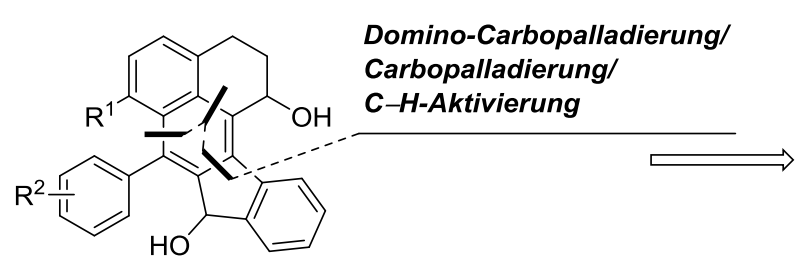

114

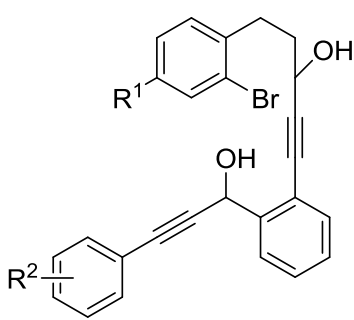

115

Abbildung 34: Retrosynthetischer Ansatz zur Synthese von hydrophilen Polyzyklen des Typs 114.

Neben der Synthese von fluorophoren polyzyklischen Kohlenwasserstoffen sollten auch Untersuchungen zur Herstellung tetrasubstituierter Dialkene durch entsprechende DominoCarbopalladierung/C-H-Aktivierungs- bzw. (116), -Heck- (117) oder -Kreuzkupplungsreaktionen (118) erfolgen. Auch wäre eine Erweiterung der Domino-Reaktion um eine zusätzliche Carbopalladierung von synthetischem Interesse, die dann in einer Domino-Sonogashira/DreifachCarbopalladierung/C-H-Aktivierung zu tetrasubstituierten Trialkenen des Typs 119 führen würde (Abbildung 35).
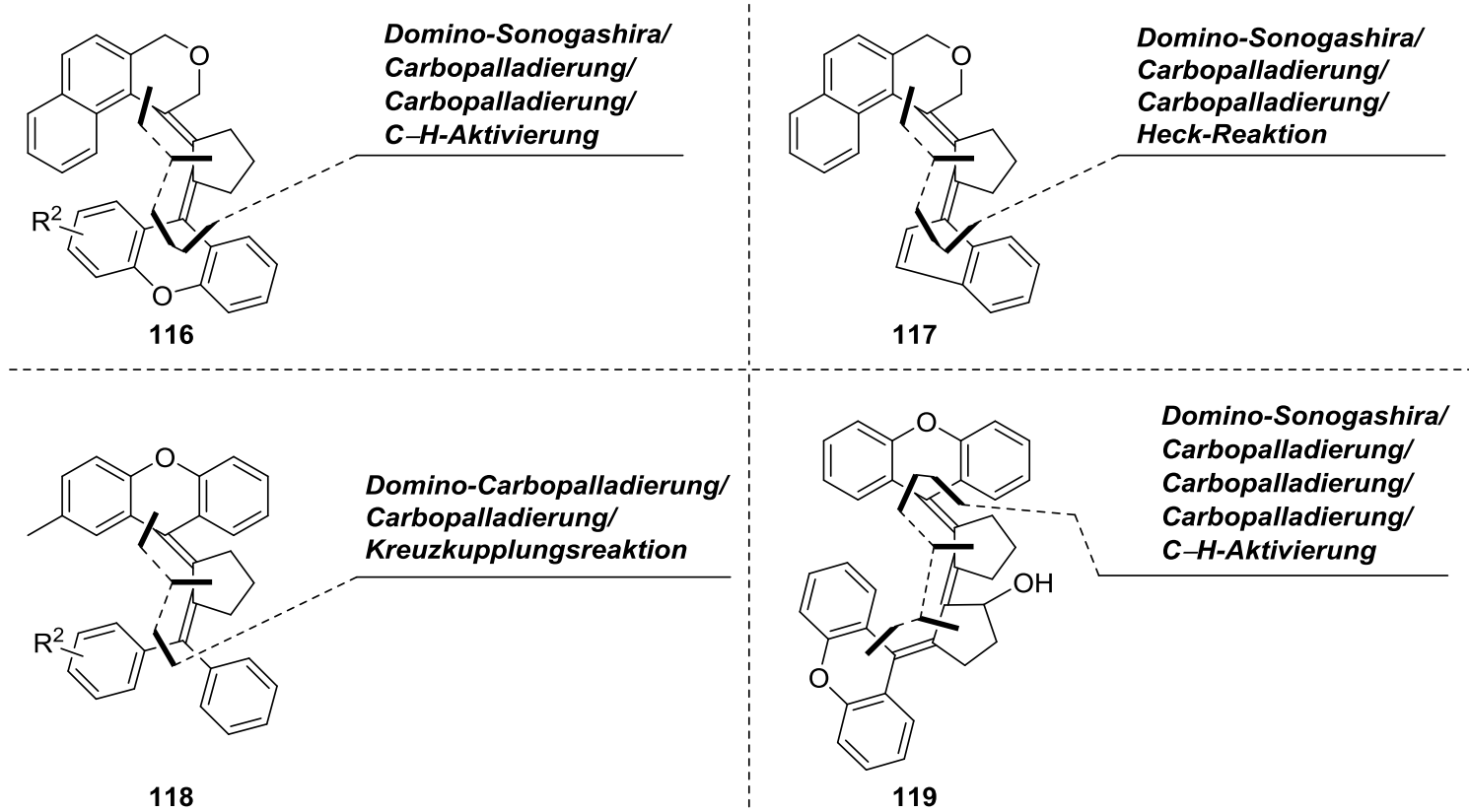

Abbildung 35: Untersuchungen zur Synthese tetrasubstituierter Dialkene (116-118) und Trialkene (119). 


\section{Ergebnisteil}

\subsection{Untersuchungen zur Synthese von fluoreszenzaktiven Polyzyklen des Typs 107}

Bisherige Forschungsbestrebungen in der Abteilung Tietze hatten sich auf Dreifach-DominoReaktionen beschränkt. Im Rahmen dieser Arbeit sollten daher Zweikomponenten-Vierfach-DominoSonogashira/Zweifach-Carbopalladierung/C-H-Aktivierungsreaktionen zur Synthese von hochkonjugierten Polyzyklen des Typs 107 entwickelt werden. Als Ausgangsverbindungen schienen die Aryliodide vom Typ 108 sowie das Dialkin 109a geeignet (Abbildung 36).
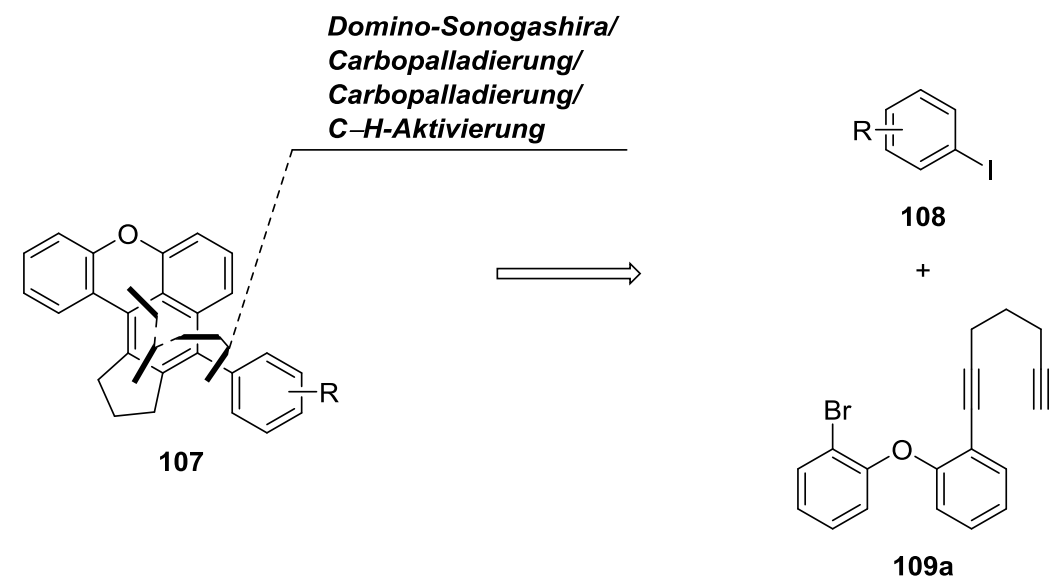

Abbildung 36: Retrosynthetischer Ansatz zur Synthese von fluoreszenzaktiven Polyzyklen des Typs 107.

Dabei wurden verschiedene Derivate des Aryliodids 108 eingesetzt, um die Toleranz der DominoReaktion gegenüber verschiedenen funktionellen Gruppen und den Einfluss dieser Gruppen auf die Reaktionsergebnisse zu untersuchen.

\subsubsection{Synthese des Dialkins 109a}

Die Synthese des Domino-Vorläufers 109a erfolgte über einen im Arbeitskreis Tietze bekannten Ansatz. ${ }^{66}$ Dieser beginnt mit einer nukleophilen aromatischen Substitution zwischen 2-Bromphenol (120a) und 2-Fluornitrobenzol (121a), die in quantitativer Ausbeute zu Biarylether 122a umgesetzt wurden. Dieser wurde in einer anschließenden Reduktion der Nitrogruppe in das Amin 123a überführt, welches in $86 \%$ Ausbeute erhalten werden konnte. In einer Sandmeyer-Reaktion nach Bedingungen von Knochel et al. wurde Aryliodid 124a in $87 \%$ Ausbeute aus 123a erzeugt. ${ }^{71}$ Verbindung 124a wurde abschließend in einer Sonogashira-Reaktion mit $\mathrm{PdCl}_{2}\left(\mathrm{PPh}_{3}\right)_{2}$ und $\operatorname{Kupfer}(\mathrm{I})$ Iodid als Katalysatorsystem und einem Überschuss an 1,6-Heptadiin (5 Äq.) in Triethylamin als 
Lösungsmittel zum erwünschten Dialkin 109a umgesetzt. Die Transformation erfolgte in $72 \%$ Ausbeute für die Sonogashira-Reaktion sowie in einer sehr guten Gesamtausbeute von 54\% über vier Stufen (Abbildung 37).<smiles>Oc1ccccc1Br</smiles>

$120 \mathrm{a}$<smiles>O=[N+]([O-])c1ccccc1F</smiles>

$121 \mathrm{a}$

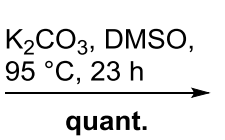
quant.<smiles>O=[N+]([O-])c1ccccc1Oc1ccccc1Br</smiles>

$122 a$
$\mathrm{Zn}$, konz. $\mathrm{HCl}$,

konz. AcOH, EtOAc,

$\underset{86 \%}{\stackrel{0{ }^{\circ} \mathrm{C} \rightarrow \mathrm{RT}, 15 \mathrm{~min}}{\longrightarrow}}$

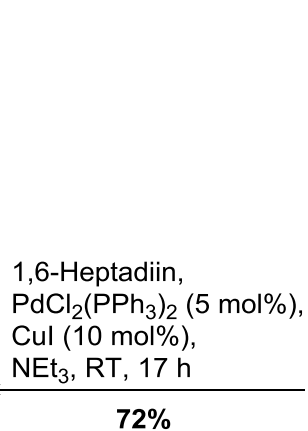<smiles>Nc1ccccc1Oc1ccccc1Br</smiles>

123a<smiles>C#CCCCC#Cc1ccccc1Oc1ccccc1Br</smiles>

109a

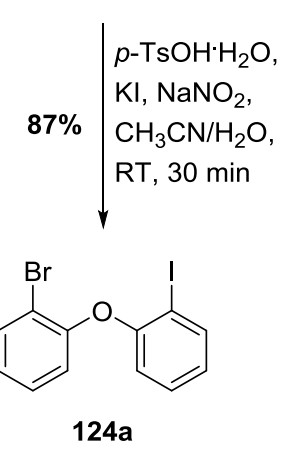

Abbildung 37: Synthese des Dialkins 109a.

\subsubsection{Synthese der Aryliodide 108a und 108b}

Die Synthese der Aryliodide 108a und 108b als Kupplungspartner für die Domino-Reaktion erfolgte ebenfalls über literaturbekannte Darstellungen. Aryliodid 108a wurde wegen der sterischen und elektronischen Eigenschaften des Naphthyl-Systems gewählt. Des Weiteren sollte hiermit die Möglichkeit der Bildung einer Dialken-Struktur untersucht werden (vgl. Mechanismus Abbildung 43). Zur Synthese erfolgte zunächst eine Iodierung des kommerziell erhältlichen Naphthols $\mathbf{1 2 5}$ unter Verwendung von Kaliumiodid, Wasserstoffperoxid und konzentrierter Schwefelsäure in Methanol bei $0{ }^{\circ} \mathrm{C}$. Das entsprechende Iodnaphthol $\mathbf{1 2 6}$ wurde in $72 \%$ Ausbeute erhalten und in einer anschließenden nukleophilen aromatischen Substitution mit 4-Fluornitrobenzol (121b) zum Biarylether 108a in 78\% Ausbeute umgesetzt (Abbildung 38). ${ }^{69,72}$

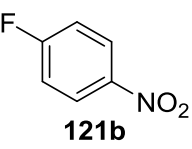

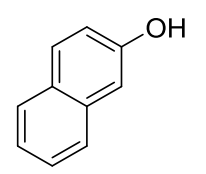

125

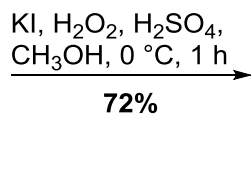

$72 \%$

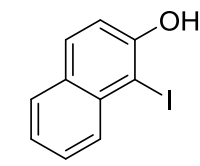

126

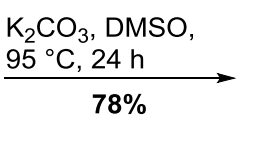

$78 \%$<smiles>O=[N+]([O-])c1ccc(Oc2ccc3ccccc3c2I)cc1</smiles>

$108 a$

Abbildung 38: Synthese des Aryliodid-Bausteins 108a. ${ }^{69,72}$ 
Als weiterer Synthesebaustein wurde das Aryliodid 108b mit einer Vinyl-Gruppe gewählt. Diese sollte zur Untersuchung der Selektivität der terminalen $\mathrm{C}-\mathrm{H}$-Aktivierung gegenüber inter- und intramolekularen Heck-Reaktionen dienen, die durch die enthaltene terminale Doppelbindung möglich waren (vgl. Abbildung 41). Die Synthese begann mit einer Oxidation des kommerziell erhältlichen Benzylalkohols 127 mittels $\mathrm{MnO}_{2}$ in Dichlormethan, welche in 77\% Ausbeute zum Benzaldehyd 128 führte. Eine anschließende Wittig-Reaktion mit Methyltriphenylphosphonium-Bromid und n-Butyllithium als Base führte zum Alken $\mathbf{1 0 8 b}$, das aufgrund seiner Instabilität direkt in der DominoReaktion eingesetzt werden musste. Die Ausbeute der Wittig-Reaktion betrug $81 \%$ (Abbildung 39). ${ }^{73,74}$

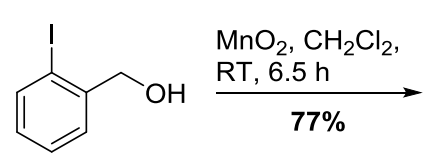

127

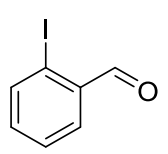

128
1.) $\mathrm{MePPh}_{3}{ }^{+} \mathrm{Br}^{-}, n$-BuLi, $\mathrm{THF}, 0^{\circ} \mathrm{C}, 15 \mathrm{~min}$ 2.) $128, \mathrm{THF}, 0^{\circ} \mathrm{C}, 15 \mathrm{~h}$ $\mathbf{8 1 \%}$

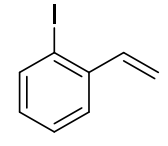

$108 b$

Abbildung 39: Synthese des Aryliodids 108b. ${ }^{73,74}$

Die ebenfalls eingesetzten Vorläufer $\mathbf{1 0 8 c}-\mathbf{e}$ waren kommerziell erhältlich. Auf die Synthese von Aryliodid 108f wird in Kapitel 3.2.2 (vgl. Abbildung 54) näher eingegangen (Abbildung 40).

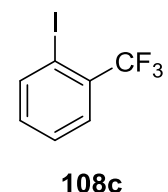

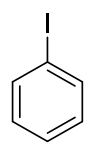

$108 d$<smiles>Cc1cc(C)c(C)c(C)c1C</smiles>

$108 \mathrm{e}$<smiles>COc1ccccc1Oc1ccccc1I</smiles>

$108 f$

Abbildung 40: Weitere Aryliodid-Vorläufer für die Domino-Reaktion.

\subsubsection{Synthese der Polyzyklen 107a-f durch die Domino-Reaktion}

Die Ausbeuten der Domino-Reaktion mit den Modellsubstraten Aryliodid 108a und Dialkin 109a, die zu Polyzyklus 107a führte, wurden zunächst durch den Einsatz verschiedener Liganden optimiert. Die besten Ausbeuten lieferte mit $81 \%$ eine Kombination von $10 \mathrm{~mol} \% \mathrm{Pd}(\mathrm{OAc})_{2}$ mit der fünffachen Menge an Triphenylphosphin sowie $(n \mathrm{Bu})_{4} \mathrm{NOAc}$ als Base in DMF als Lösungsmittel bei $100{ }^{\circ} \mathrm{C}$, die in Eintrag 6 von Tabelle 1 hervorgehoben ist.

$\mathrm{Zu}$ Beginn der Optimierungen wurden $\mathrm{Pd}(\mathrm{OAc})_{2}$ und Triphenylphosphin im Verhältnis 1:5 bei $20 \mathrm{~mol} \%$ Katalysatorbeladung mit der Base $(n \mathrm{Bu})_{4} \mathrm{NOAc}$ in DMF als Lösungsmittel bei $100^{\circ} \mathrm{C}$ verwendet. Diese Bedingungen hatten sich in Vorgängerarbeiten bewährt. ${ }^{69}$ Das gewünschte Produkt 107a wurde hierbei in 60\% Ausbeute erhalten (Tabelle 1, Eintrag 1). Ein Austausch von $\mathrm{PPh}_{3}$ durch andere Liganden führte $\mathrm{zu}$ geringeren Ausbeuten. So wurde bei der Verwendung des elektronenreichen Liganden $\mathrm{PCy}_{3}$ 107a in nur 53\% Ausbeute isoliert (Eintrag 2). Der sterisch 
anspruchsvolle und elektronenreiche SPhos-Ligand führte wie auch die ionische Spezies

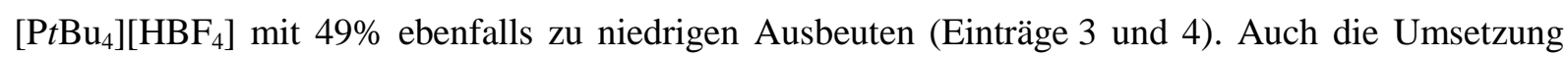
mit dem bidentaten dppe-Liganden ergab geringere Ausbeuten. Hier wurden nur $16 \%$ von 107a gebildet (Eintrag 5). Damit führte der bereits in früheren Arbeiten erfolgreich eingesetzte Triphenylphosphin-Ligand $\mathrm{zu}$ den besten Ergebnissen. Weitere Versuche widmeten sich einer Reduktion der Katalysatorbeladung. Dabei konnte die Ausbeute deutlich gesteigert werden, wenn $10 \mathrm{~mol} \% \mathrm{Pd}(\mathrm{OAc})_{2}$ mit Triphenylphosphin im etablierten Verhältnis von 1:5 eingesetzt wurden. Hierbei wurde eine sehr gute Ausbeute von 81\% Ausbeute erreicht (Eintrag 6). Dagegen ergaben geringere Katalysatormengen keine weitere Erhöhung der Ausbeute. Dennoch konnten sowohl mit $5 \mathrm{~mol} \%$ als auch mit $1 \mathrm{~mol} \% \mathrm{Pd}(\mathrm{OAc})_{2}$ und der jeweils fünffachen Menge an $\mathrm{PPh}_{3}$ mit $67 \%$ bzw. 66\% Ausbeute immer noch bessere Ergebnisse erzielt werden als mit 20 mol\% Katalysatorbeladung (Einträge 7 und 8).

Tabelle 1: Optimierung der Reaktionsbedingungen. ${ }^{[a]}$

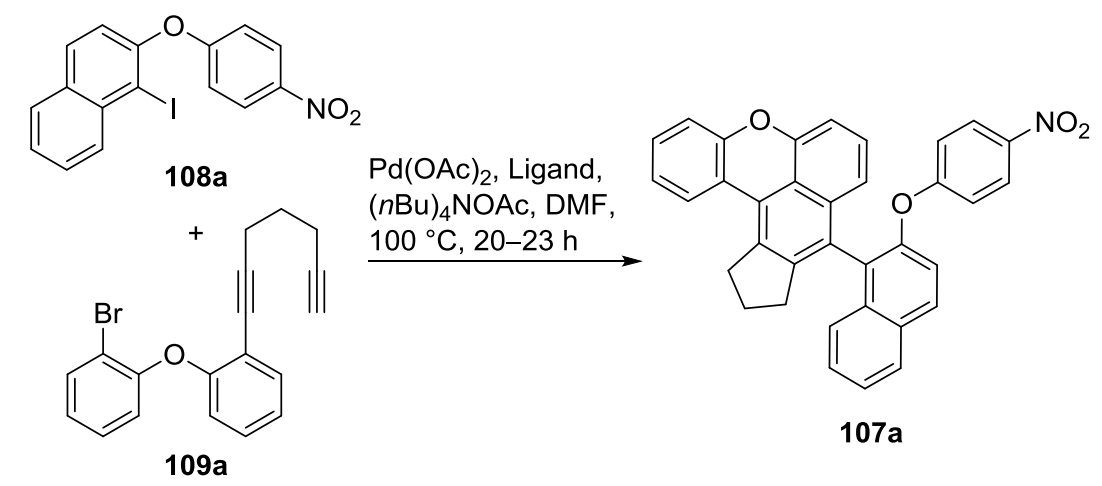

\begin{tabular}{ccccc}
\hline Eintrag & Pd(OAc) $)_{2}[\mathbf{m o l} \%]$ & Ligand & Ligand [mol\%] & Ausbeute [\% $]^{[\mathbf{b}]}$ \\
\hline 1 & 20 & $\mathrm{PPh}_{3}$ & 100 & 60 \\
2 & 20 & $\mathrm{PCy}_{3}$ & 100 & 53 \\
3 & 20 & $\mathrm{SPhos}$ & 100 & 49 \\
4 & 20 & {$\left[\mathrm{PtBu}_{4}\right]\left[\mathrm{BF}_{4}\right]$} & 100 & 49 \\
5 & 20 & $\mathrm{dppe}$ & 50 & 16 \\
$\mathbf{6}$ & $\mathbf{1 0}$ & $\mathbf{P P h}_{3}$ & $\mathbf{5 0}$ & $\mathbf{8 1}$ \\
7 & 5 & $\mathrm{PPh}_{3}$ & 25 & 67 \\
8 & 1 & $\mathrm{PPh}_{3}$ & 5 & 66 \\
\hline
\end{tabular}

[a] 108a (1.00 Äq.), 109a (1.05 Äq.), Pd(OAc $)_{2}$, Ligand, $(n B u)_{4} \mathrm{NOAc}\left(6.00\right.$ Äq.), DMF, $100{ }^{\circ} \mathrm{C}$, 20-23 h; [b] Isolierte Ausbeute nach Säulenchromatographie.

Die auf diese Weise optimierten Bedingungen (vgl. Tabelle 1, Eintrag 6) wurden schließlich für die Synthese weiterer Derivate des Polyzyklus 107 verwendet. Dabei wurden verschiedene Substituenten am Aryliodid 108 eingesetzt, die sowohl elektronisch als auch sterisch veränderte Eigenschaften des Aryliodids bewirkten. Die Umsetzung von Dialkin 109a mit dem vinyl-substituierten Aryliodid 108b 
lieferte das entsprechende Domino-Produkt 107b in einer Ausbeute von 54\%. Interessant ist, dass keine entsprechenden Nebenprodukte in signifikanter Menge beobachtet wurden, die aus ebenfalls denkbaren inter- und intramolekularen (Domino-)Heck-Reaktionen resultieren könnten (Abbildung 41). Besonderes Augenmerk wurde dabei auf die mögliche intramolekulare DominoSonogashira/Zweifach-Carbopalladierung/Heck-Reaktion gelegt, welche die Bildung von hochinteressanten tetrasubstituierten Dialkenen des Typs 129 zur Folge hätte. Das entsprechende Produkt wurde jedoch nicht beobachtet. Demnach scheint die Domino-C-H-Aktivierung der DominoHeck-Reaktion gegenüber bevorzugt zu sein. Die mehrheitliche Umsetzung zum Domino-C-HAktivierungsprodukt 107b zeigte außerdem, dass auch mögliche intermolekulare Heck-Reaktionen, wie zum Beispiel die Dimerisierung von 108b zu Verbindung 130, der einleitenden SonogashiraReaktion des Domino-Prozesses gegenüber benachteiligt sind. Lediglich die gegenüber den anderen Substraten leicht verminderte Ausbeute liefert einen Hinweis darauf, dass derartige Nebenreaktionen in Anteilen eine Rolle spielen könnten.

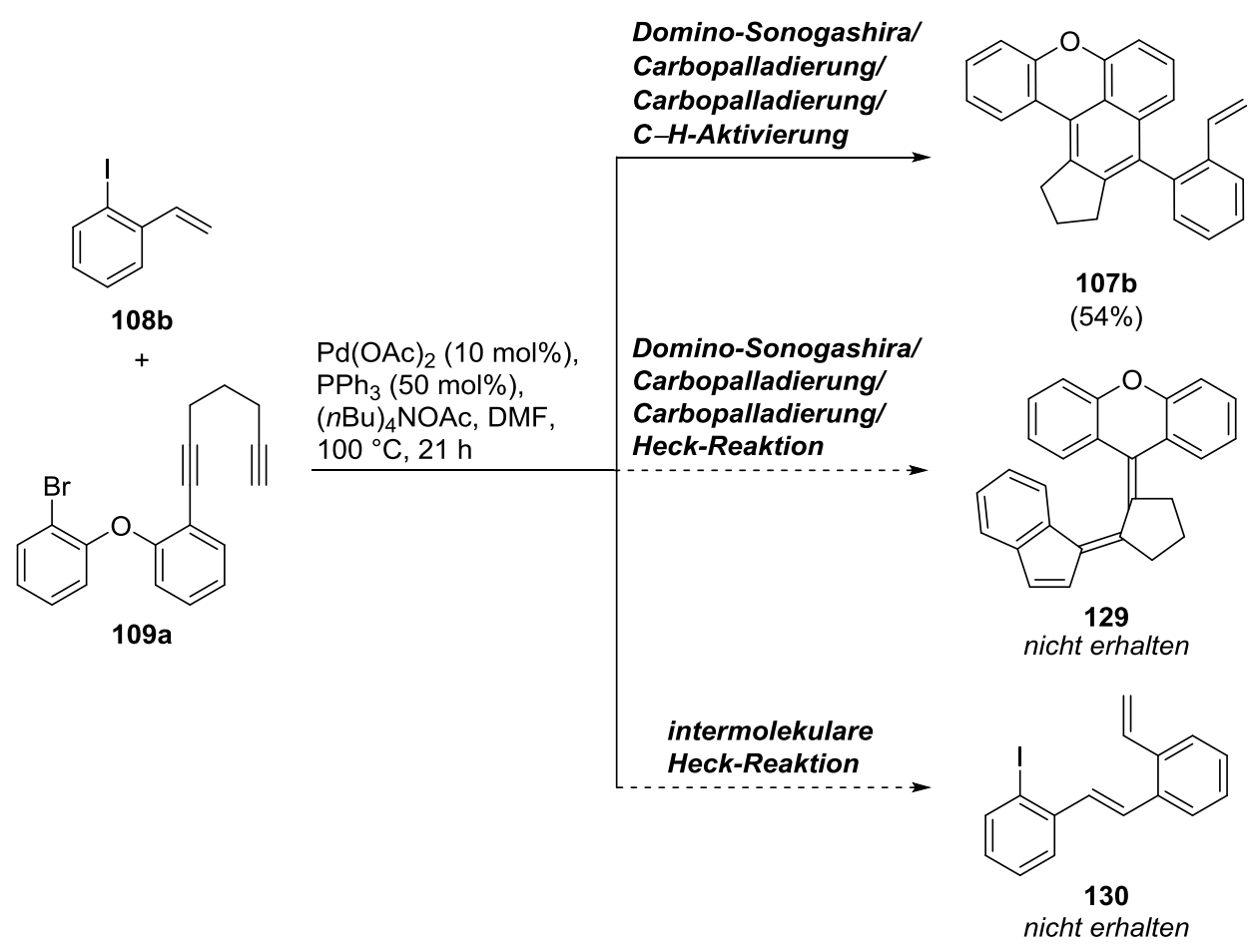

Abbildung 41: Reaktion des vinyl-substituierten Aryliodids 108b mit 109a sowie mögliche Nebenreaktionen.

Als weiteres Aryliodid wurde 108c umgesetzt, das eine elektronenziehende $\mathrm{CF}_{3}$-Gruppe enthielt. In diesem Fall konnte mit 97\% Ausbeute der beste Wert für die hier untersuchte Domino-Reaktion erzielt werden. Auch das elektronisch neutrale Iodbenzol (108d) lieferte mit 90\% exzellente Ausbeuten bei einer Verwendung als Reaktionspartner im Vierfach-Domino-Prozess. Zur Untersuchung der Auswirkung von sterischen Faktoren auf das Iodatom des Aryliodids wurde außerdem Derivat 108e umgesetzt. Hier ist die Halogen-Funktionalität durch zwei benachbarte Methylgruppen abgeschirmt. Zudem sorgen die insgesamt vier Methylsubstituenten an 108e für einen gewissen 
Elektronenreichtum. Die Umsetzung erfolgte zu 64\%, womit die Ausbeute geringer war als für das unsubstituierte Substrat 107d. Dies kann sowohl elektronische als auch sterische Gründe haben. Um diese Ursachen näher zu bestimmen, wurde ebenfalls das elektronenreiche Aryliodid 108f umgesetzt, welches mit einer Methoxyphenoxy-Gruppe in ortho-Position substituiert war. Das entsprechende Domino-Produkt 107f wurde in 80\% Ausbeute isoliert; ein Ergebnis, das sterische Ursachen für die verminderte Ausbeute von 107e nahelegt. Insgesamt scheinen jedoch elektronenarme und elektrisch neutrale Aryliodide etwas bessere Ausbeuten zu liefern als ihre elektronenreichen Analoga, wie die guten Ergebnisse für die Domino-Produkte 107a, 107c und 107d zeigen. Dies scheint vor allem für eine bevorzugte Umsetzung elektronenarmer Derivate im Sonogashira-Prozess zu sprechen, da nur in diesem die elektronische Struktur des Aryliodids eine signifikante Rolle spielen dürfte. Bemerkenswert ist die enorme Toleranz der Domino-Reaktion gegenüber verschiedensten funktionellen Gruppen. So scheinen problematische Substrate wie Nitro- und Vinylgruppen keinen nennenswerten Nebenreaktionen zu unterliegen.

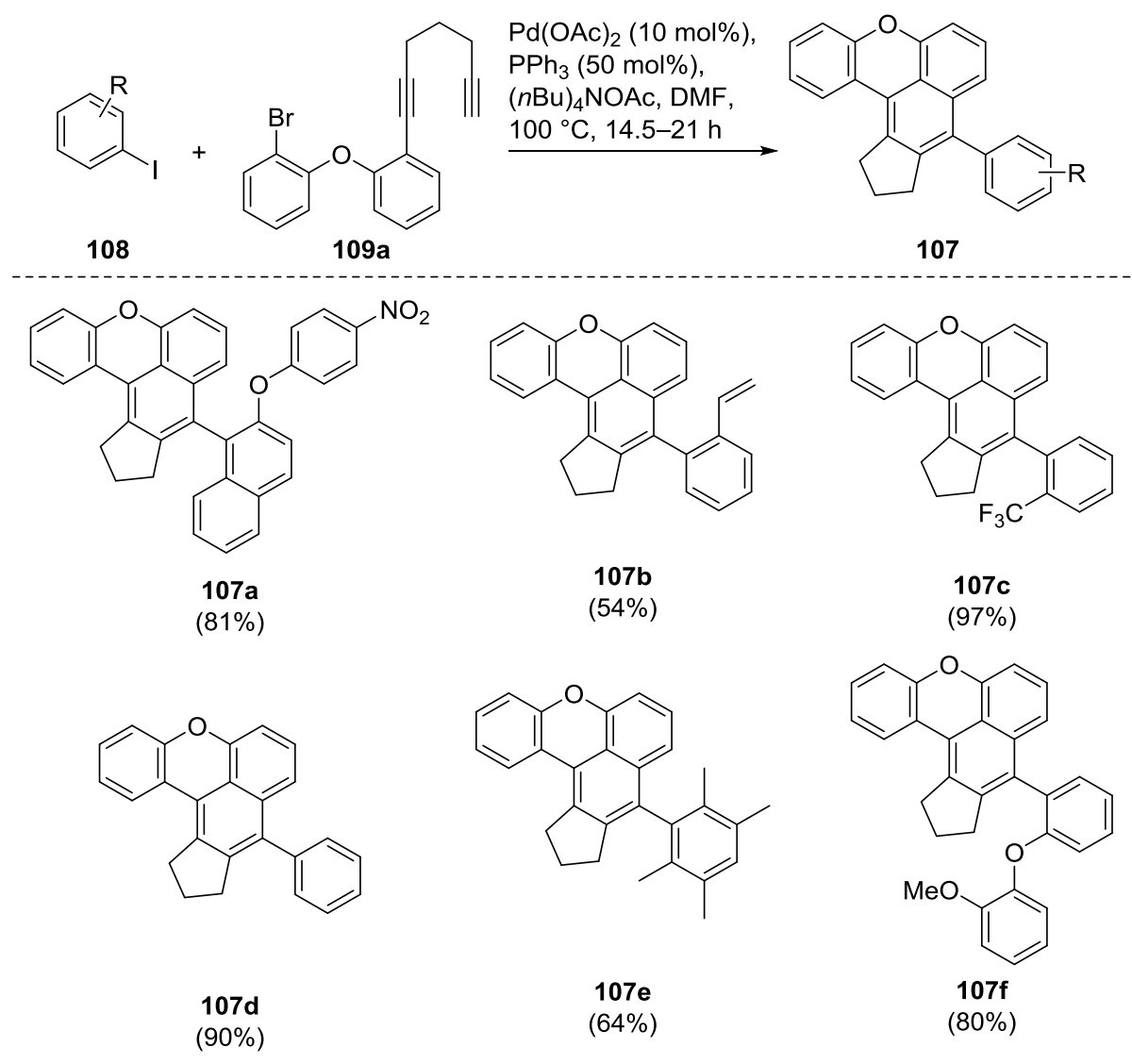

Abbildung 42: Produkte der Domino-Sonogashira/Zweifach-Carbopalladierung/C-H-Aktivierung. 


\subsubsection{Mechanistische Überlegungen}

Die Produkte des Typs 107 werden vermutlich in einer Domino-Sonogashira/ZweifachCarbopalladierung/C-H-Aktivierung gebildet (Abbildung 43). Nach einer Sonogashira-Reaktion, die Aryliodid 108a mit Dialkin 109a zu Dialkin 131a kuppelt, kommt es anschließend zur ersten Carbopalladierung. Bei dieser wird intramolekular ein Sechsring geformt, die ebenfalls enthaltene Vinyl-Palladium-Funktionalität in Intermediat 132a steht als reaktives Zentrum für weitere Reaktionen zur Verfügung.

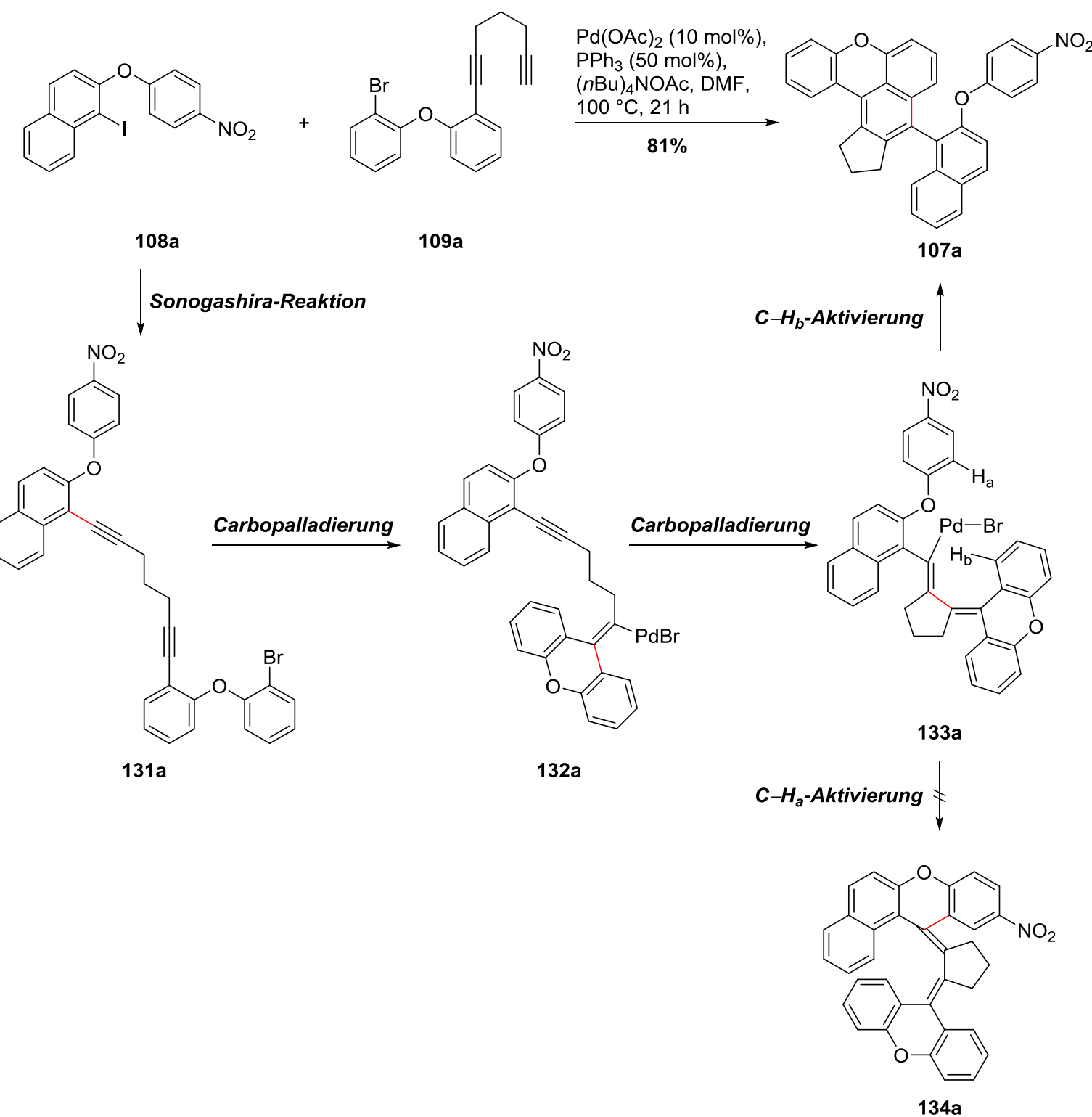

Abbildung 43: Postulierter Mechanismus der Domino-Sonogashira/Zweifach-Carbopalladierung/C-HAktivierung (Liganden und beteiligte Basen wurden der Übersichtlichkeit halber weggelassen).

In einem nächsten Schritt, der zweiten Carbopalladierung, findet eine weitere Zyklisierung statt, bei der ein Cyclopentan-Ring gebildet wird (Intermediat 133a). Dieses Molekül enthält ebenfalls eine 
reaktive Vinyl-Palladium-Spezies, welche zum Abschluss der Reaktion eine C-H-Aktivierung eingeht. Bei Aktivierung von Proton $\mathrm{H}_{\mathrm{b}}$ führt diese zur Bildung des aromatischen Produkts 107a. $\mathrm{Zu}$ beachten ist, dass die in den zwei Carbopalladierungen gebildeten Doppelbindungen Teil des aromatischen Systems werden, indem sie einen annelierten Benzolring bilden. Diese Aromatisierung scheint Grundlage der enormen Triebkraft dieser Reaktion zu sein. Eine ebenfalls denkbare Aktivierung des Protons $\mathrm{H}_{\mathrm{a}}$, die zum vollständig substituierten Alken 134a führt, ist demnach aus energetischen Gründen unwahrscheinlich und konnte auch nicht beobachtet werden. Für den $\mathrm{C}-\mathrm{H}-$ Aktivierungsprozess wird eine Acetat-vermittelte Protonenabstraktion angenommen, wie sie bereits in Abbildung 14 vorgestellt wurde. Es konnten keine der postulierten Zwischenprodukte oder gequenchten Derivate der intermediären Palladium-Spezies isoliert werden.

\subsubsection{Diskussion ausgewählter analytischer Daten von Verbindung 107b}

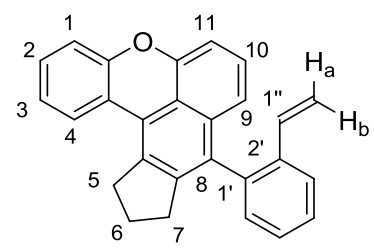

$107 \mathrm{~b}$

Alle isolierten Verbindungen wurden mittels gängiger Analysemethoden charakterisiert. Exemplarisch sollen im Folgenden die analytischen Daten von Verbindung 107b diskutiert werden. Die in Benzol- $\mathrm{d}_{6}$ aufgenommenen ${ }^{1} \mathrm{H}$ - und ${ }^{13} \mathrm{C}$-NMR-Spektren sind in Abbildung 44 und Abbildung 45 dargestellt. Eine genauere Zuordnung der Signale erfolgte über entsprechende zweidimensionale NMR-Spektren (COSY, HSQC und HMBC) sowie über die unterschiedlichen Intensitäten der Signale im ${ }^{13} \mathrm{C}-\mathrm{NMR}$, womit quartäre von anderen Kohlenstoffatomen unterschieden werden konnten. Im ${ }^{1} \mathrm{H}-\mathrm{NMR}$ Spektrum resonieren die Protonen des aliphatischen Fünfrings bei $\delta=1.69,2.51$ und $2.93 \mathrm{ppm}$. Dabei werden die Protonen der Methylen-Gruppe in 6-Position bei $\delta=1.69 \mathrm{ppm}$ als Pseudo-Pentett abgebildet, während die Signale der Wasserstoffatome $7-\mathrm{H}_{2}$ und $5-\mathrm{H}_{2}$ aufgrund der Nähe zum aromatischen System weiter zu tiefem Feld verschoben bei $\delta=2.51$ und $2.93 \mathrm{ppm}$ als Dublett von Dubletts bzw. Triplett von Dubletts vorzufinden sind. Eine exaktere Auflösung des Kopplungsmusters war aufgrund der sehr kleinen Fernkopplungen nicht möglich. Die Protonen der terminalen Doppelbindung resonieren, durch den Anisotropiekegel der Doppelbindung beeinflusst, bei $\delta=4.90$ ppm (2' '- $\left.\mathrm{H}_{\mathrm{a}}\right)$ bzw. $\delta=5.90 \mathrm{ppm}\left(2\right.$ ''- $\left.\mathrm{H}_{\mathrm{b}}\right)$ jeweils als Dublett von Dubletts. Zu unterscheiden sind sie dabei deutlich anhand ihrer jeweiligen Kopplungskonstanten. Zunächst koppeln die beiden geminalen Protonen 2"'- $\mathrm{H}_{\mathrm{a}}$ und 2"'- $\mathrm{H}_{\mathrm{b}}$ mit einer Kopplungskonstante von $J=1.2 \mathrm{~Hz}$ miteinander, die in beiden Signalen wiederzufinden ist. Während 2' $-\mathrm{H}_{\mathrm{a}}$ in einer typischen $Z$-Kopplung von $J=10.9 \mathrm{~Hz}$ mit 1'’-H koppelt, findet sich im Signal von 2''- $\mathrm{H}_{\mathrm{b}}$ eine charakteristische E-Kopplung von $J=17.5 \mathrm{~Hz}$. Dementsprechend resoniert der vicinale Kopplungspartner 1'-H als stark aufgespaltenes 
Dublett von Dubletts bei $\delta=6.51 \mathrm{ppm}$. Tieffeldverschoben folgen die Signale der aromatischen Protonen $3-\mathrm{H}$ und $9-\mathrm{H}$, die als Multiplett bei $\delta=6.88-6.94 \mathrm{ppm} \mathrm{zu}$ finden sind. Noch weiter entschirmt sind die anderen Protonen des Xanthen-Gerüsts: So resonieren 2-H, 10-H und 11- $\mathrm{H}$ alle im Bereich von $\delta=6.96-7.02 \mathrm{ppm}$, ihre Signale überlagern sich zu einem Multiplett. Das Signal von $1^{\circ}$-H kann dagegen als Dublett von Dubletts bei $\delta=7.09 \mathrm{ppm}$ beobachtet werden kann. Die Tieffeldverschiebung der Signale dieser Protonen resultiert vermutlich aus der Nähe zum elektronenziehenden Sauerstoffatom. Weiter zu tiefem Feld verschoben resoniert Proton 6'-H, welches als Dublett von Dubletts bei $\delta=7.12 \mathrm{ppm}$ zu sehen ist und wahrscheinlich durch sterische und anisotrope Effekte des benachbarten Polyzyklus entschirmt wird. Als Multiplett bei $\delta=7.18-7.25 \mathrm{ppm}$ sind die Signale der Protonen 3'-H und 5'-H zu beobachten. Am weitesten ins Tieffeld verschoben sind die Signale von 4'-H und 4-H, welche jeweils als Dublett von Dubletts bei $\delta=7.68$ bzw. $7.77 \mathrm{ppm}$ resonieren. Die Signale der Protonen des vinyl-substituierten Benzolrings sind dabei vermutlich durch den entschirmenden Effekt der konjugierten Doppelbindung zu tiefem Feld verschoben, während 4-H aller Wahrscheinlichkeit nach durch den benachbarten Benzolring beeinflusst wird.

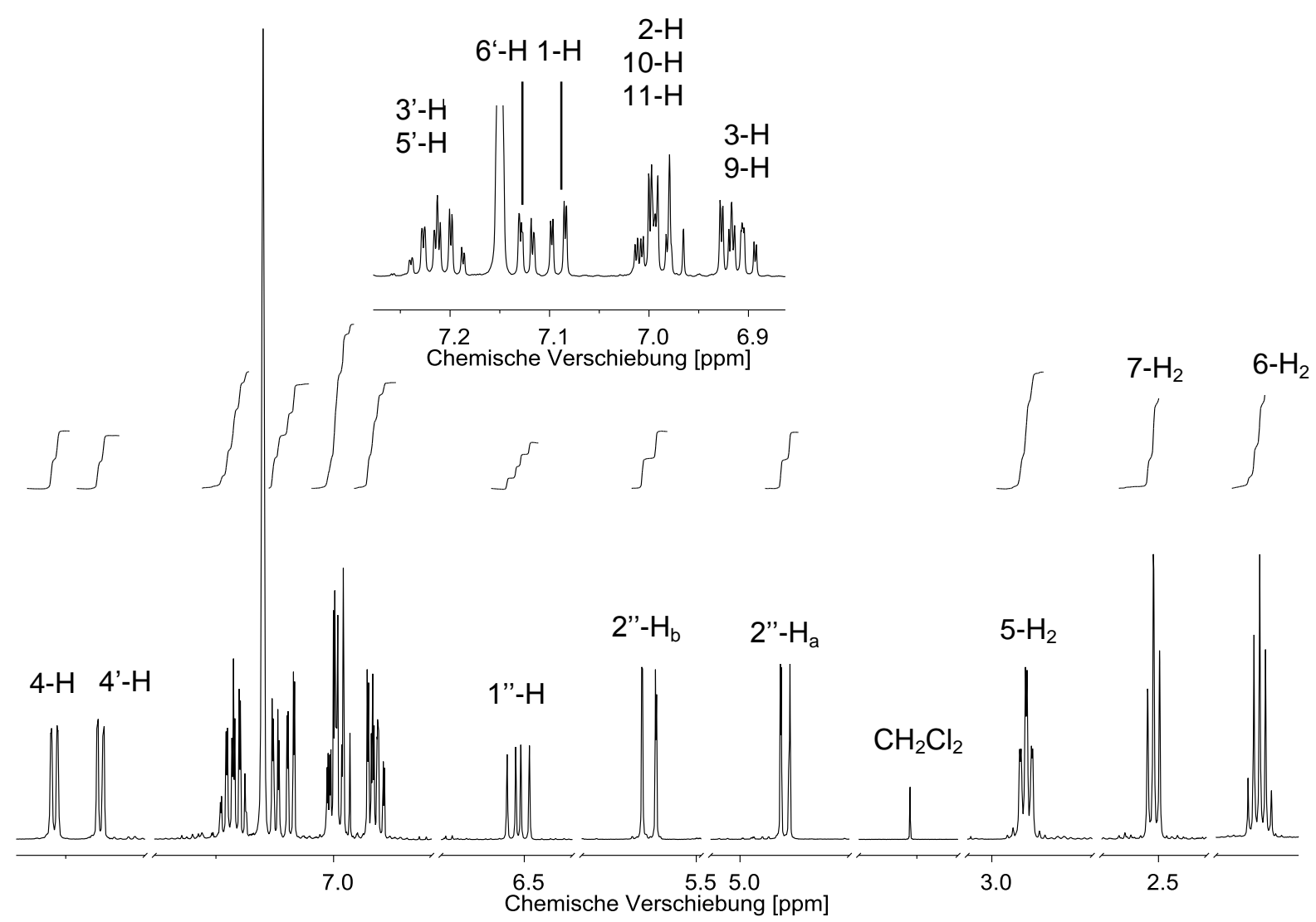

Abbildung 44: ${ }^{1} \mathrm{H}-\mathrm{NMR}-$ Spektrum von Verbindung 107b in $\mathrm{C}_{6} \mathrm{D}_{6}$ bei $600 \mathrm{MHz}$.

Das ${ }^{13}$ C-NMR-Spektrum von $\mathbf{1 0 7 b}$ weist im Hochfeldbereich zunächst die Signale der Kohlenstoffatome des aliphatischen Fünfrings auf, die bei $\delta=25.5$ (C-6), 32.1 (C-7) und $35.4 \mathrm{pm}$ (C-5) resonieren. Dabei sind C-5 und C-7 vermutlich durch die Nähe zum aromatischen System stärker entschirmt als C-6. Es folgt das Signal des aromatischen Kohlenstoffatoms des Naphthylrings 
C-9 bei $\delta=108.0 \mathrm{ppm}$. Dieses unterliegt als Brückenkopfatom zwischen zwei aromatischen Ringen aufgrund der erhöhten Ladungsdichte einer leichten Hochfeldverschiebung. Zudem scheint der Anisotropiekegel der nahen Doppelbindung die Verschiebung des Signals von C-9 zu beeinflussen. Bei ähnlicher Verschiebung resoniert das Doppelbindungskohlenstoffatom C-2", welches bei $\delta=114.6 \mathrm{ppm}$ zu finden ist und wahrscheinlich durch den mesomeren Effekt des konjugierten Benzolrings eine höhere Ladungsdichte besitzt. Die Xanthen-Kohlenstoffatome C-1 und C-11 resonieren bei $\delta=117.1 \mathrm{bzw}$. $118.6 \mathrm{ppm}$. Hier polarisiert das benachbarte Sauerstoffatom vermutlich die aromatischen Kohlenstoffbindungen und schirmt die beiden Signale dementsprechend ab. Nicht exakt zuordenbar waren die Signale der Brückenkopfatome C-4b und C-8b, die bei $\delta=122.3$ und $122.4 \mathrm{ppm}$ zu beobachten sind. Bei $\delta=123.0 \mathrm{ppm}$ sind die Signale für C-3 der Xanthen-Einheit sowie für das Brückenkopfatom C-4a zu finden. Die aromatischen Kohlenstoffatome von C-4', C-10 und C-4 resonieren dagegen bei $\delta=125.5,126.9$ und $127.1 \mathrm{ppm}$. Die Signale bei $\delta=127.9$ und $128.4 \mathrm{ppm}$ entsprechen den Atomen C-3' und C-5', wobei die genaue Zuordnung nicht möglich war.

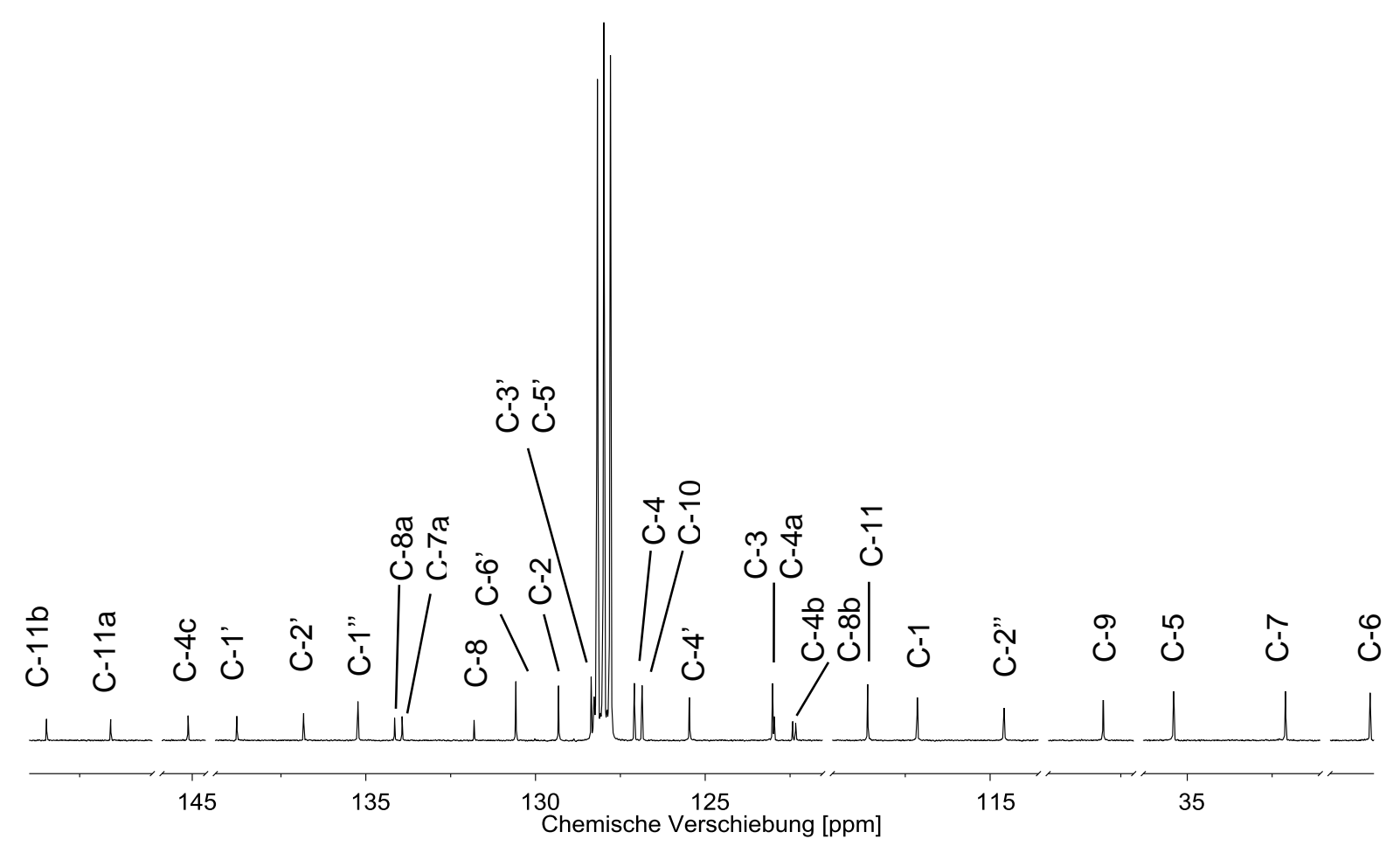

Abbildung 45: ${ }^{13} \mathrm{C}-\mathrm{NMR}$-Spektrum von Verbindung 107b in $\mathrm{C}_{6} \mathrm{D}_{6}$ bei $126 \mathrm{MHz}$.

Diese Signale unterlagen alle weniger starken Effekten durch benachbarte Gruppen. Bei $\delta=129.3$, 130.6 und $131.8 \mathrm{ppm}$ sind die Resonanzen der Atome C-2, C-6' und C-8 zu beobachten. Die Brückenkopfatome C-7a und C-8a wiederum resonieren bei $\delta=133.9$ und $134.2 \mathrm{ppm}$. Hier könnte wie bei C-6' und C-8 der entschirmende Einfluss des jeweils benachbarten aromatischen Systems eine Rolle spielen. Es folgt das Signal des Doppelbindungsatoms C-1", welches aufgrund des mesomeren Efekts wegen der Nähe zum Aromaten weit im Tieffeld bei $\delta=135.2$ ppm resoniert. Ebenso stark 
entschirmt sind die benachbarten aromatischen Kohlenstoffatome C-2' und C-1', deren Signale bei $\delta=136.8$ und 138.8 ppm zu finden sind. Auch hier spielen vermutlich entschirmende Effekte, die von Doppelbindung und Xanthen-Gerüst ausgehen, eine Rolle. Eine ähnliche Verschiebung weist auch das Brückenkopfatom C-4c auf, welches bei $\delta=145.1 \mathrm{ppm}$ resoniert. Die stärkste Tieffeldverschiebung besitzen die Signale der Biaryletherkohlenstoffe C-11a und C-11b, die bei einer charakteristischen Verschiebung von $\delta=151.6$ bzw. $153.5 \mathrm{ppm}$ zu beobachten sind. Sie werden durch das elektronegative Sauerstoffatom stark entschirmt.

Die molekulare Masse von 107b konnte per EI-MS- und ESI-HRMS-Messung bewiesen werden. Im EI wurde der Molekülionen-Peak mit einem Masse-zu-Ladungsverhältnis von $m / z=360.1$ und einer Intensität von $100 \%$ gefunden. Im ESI-HRMS konnte die kalkulierte exakte Masse von $m / z=360.1509$ durch eine gefundene Masse von $m / z=360.1499$ für das Addukt $[\mathrm{M}+\mathrm{H}]^{+}$bestätigt werden.

Im IR-Spektrum sind vor allem die charakteristischen Signale der Valenzschwingung der C-CDoppelbindung bei $\tilde{v}=1625 \mathrm{~cm}^{-1}$ zu sehen. Daneben zeigen sich bei $\tilde{v}=1500$ bis $1600 \mathrm{~cm}^{-1}$ auch die Banden der Streckschwingungen der aromatischen C-C-Doppelbindungen. Zwischen $\tilde{v}=1300$ und $1100 \mathrm{~cm}^{-1}$ sind zudem die C-O-Valenzschwingungen des aromatischen Ethers abgebildet. Im Bereich von weniger als $\tilde{v}=900 \mathrm{~cm}^{-1}$ sind die typischen Resonanzen von Schwingungen im aromatischen Gerüst zu erkennen. Diese sind stark abhängig vom Substitutionsmuster der in 107b enthaltenen Benzolringe. Auch die C-H-Streckschwingung der terminalen Doppelbindung resoniert in diesem Bereich.

Während bei $\lambda=222$ und $254 \mathrm{~nm}$ im UV/Vis-Spektrum Absorptionsbanden erkennbar sind, die auch bei einfachen aromatischen Verbindungen aufgrund der $\pi \rightarrow \pi^{*}$-Übergänge nachweisbar sind, sind vor allem die Absorptionsbanden bei $\lambda=352,366$ und $382 \mathrm{~nm}$ charakteristisch für diese Verbindungsklasse: Diese Absorption bei längeren Wellenlängen konnte bei den jeweiligen DominoVorläufern nicht beobachtet werden. Sie resultiert vor allem aus der Erweiterung des konjugierten aromatischen Systems in der Domino-Reaktion und der damit einhergehenden Annäherung von HOMO und LUMO, welche die $\pi \rightarrow \pi^{*}$-Übergänge auch bei größeren Wellenlängen ermöglicht. Wie im folgenden Kapitel beschrieben, sind diese Wellenlängen von besonderem Interesse für die Messung der Fluoreszenzspektren.

\subsubsection{Untersuchung der Fluoreszenz am Beispiel von Verbindung 107d}

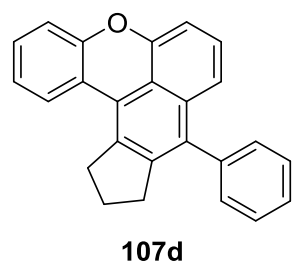

$107 d$ 
Mit Ausnahme von Molekül 107a, welches vermutlich wegen der Nitrogruppe nicht fluoreszent ist, weisen alle synthetisierten Moleküle Fluoreszenzaktivität auf. Diese resultiert wahrscheinlich aus dem in den Verbindungen enthaltenen Xanthen-Gerüst, welches in ähnlicher Form in klassischen Fluoreszenzfarbstoffen wie Fluoresceinen und Rhodaminen $\mathrm{zu}$ finden ist (vgl. Abbildungen 20 und 21). Am Beispiel von Verbindung 107d sollen im Folgenden die Fluoreszenzeigenschaften dieser Verbindungsklasse untersucht werden.

Das UV/Vis-Spektrum von Molekül 107d weist neben den auch bei den Vorläufermolekülen zu findenden Absorptionsbanden bei $\lambda=223,256$ und $261 \mathrm{~nm}$ zusätzlich Banden bei über $\lambda=350 \mathrm{~nm}$ auf. Diese treten auch bei den Molekülen 107a-f in Erscheinung (Abbildung 46). Demnach scheint die Fluoreszenz aus Anregungen in ebendiesem Wellenlängenbereich zu resultieren. Dem folgend wurden Fluoreszenzmessungen unter anderem bei Anregungen im Bereich von $\lambda=352$ bzw. $367 \mathrm{~nm}$ durchgeführt. Bei beiden Anregungswellenlängen konnte eine scharfe Emissionsbande beobachtet werden, deren Maximum eindeutig zu erkennen war. Eine Anregung bei $\lambda=367 \mathrm{~nm}$ führt hierbei zu einer klar erkennbaren Emissionsbande mit einem Maximum bei $\lambda=436 \mathrm{~nm}$. Demnach ergibt sich ein Stokes-Shift von $69 \mathrm{~nm}$ für eine Anregung bei $\lambda=367 \mathrm{~nm}$. Die Verbindung absorbiert also im UVund emittiert im blauen Bereich des Lichtspektrums (Abbildung 47).

Die Fluoreszenzeigenschaften der Domino-Produkte ähneln damit denen von einfachen CumarinAnaloga. So absorbiert das eingangs beschriebene 4-Methyl-7-hydroxycumarin (64, vgl. Abbildung 18) UV-Licht bei $\lambda=360 \mathrm{~nm}$ und emittiert blaues Licht bei $\lambda=450 \mathrm{~nm}$. Verwendung finden derartige Verbindungen im Labeling von Proteinen und als fluorophore Substrate von Enzymen. Dies könnte auch ein mögliches Anwendungsziel der hier synthetisierten Produkte sein, die zudem weitaus weniger $\mathrm{pH}$-sensitiv sind als viele Cumarin-Derivate.

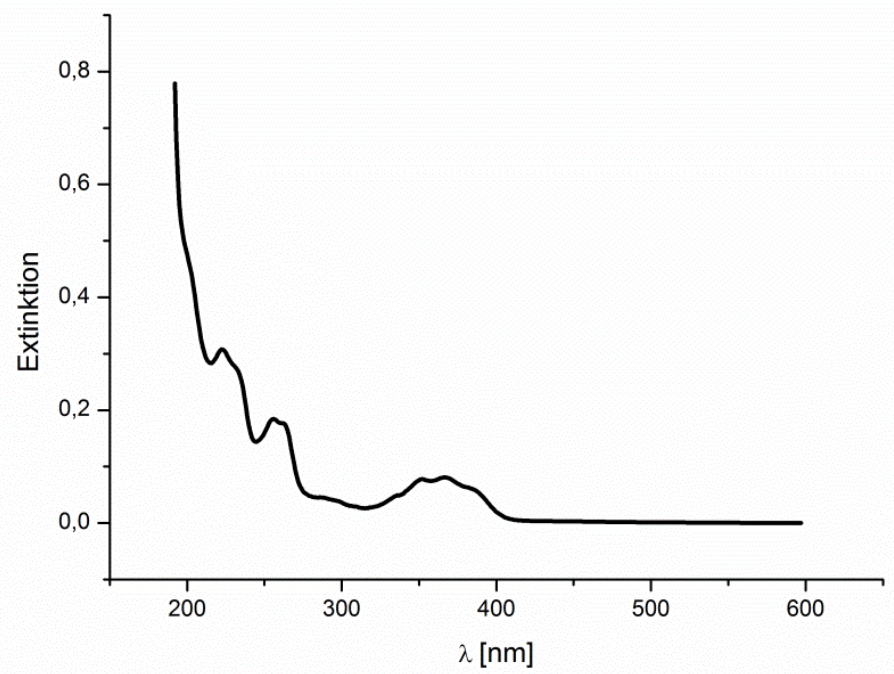

Abbildung 46: UV/Vis-Spektrum von Verbindung 107d in Acetonitril. 


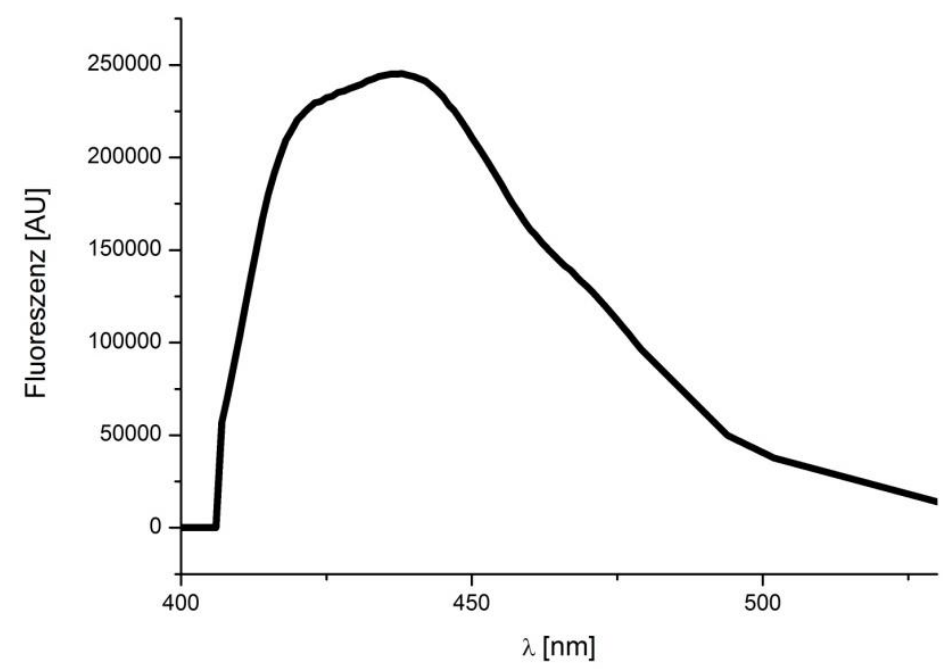

Abbildung 47: Fluoreszenzspektrum von Verbindung 107d in Acetonitril (Anregungswellenlänge: $\lambda=364 \mathrm{~nm}$ ).

\subsubsection{Untersuchungen zur Synthese dimerer Strukturen}

In einer Ergänzung zu der bereits vorgestellten Domino-Sonogashira/Zweifach-Carbopalladierung/ C-H-Aktivierung sollten außerdem dimere Strukturen aufgebaut werden, die aufgrund des vergrößerten aromatischen Systems längerwelliges Licht emittieren könnten als Verbindungen des Typs 107. Die Synthese sollte über eine zweifache Domino-Sonogashira/ZweifachCarbopalladierung/C-H-Aktivierung erfolgen (Abbildung 48).

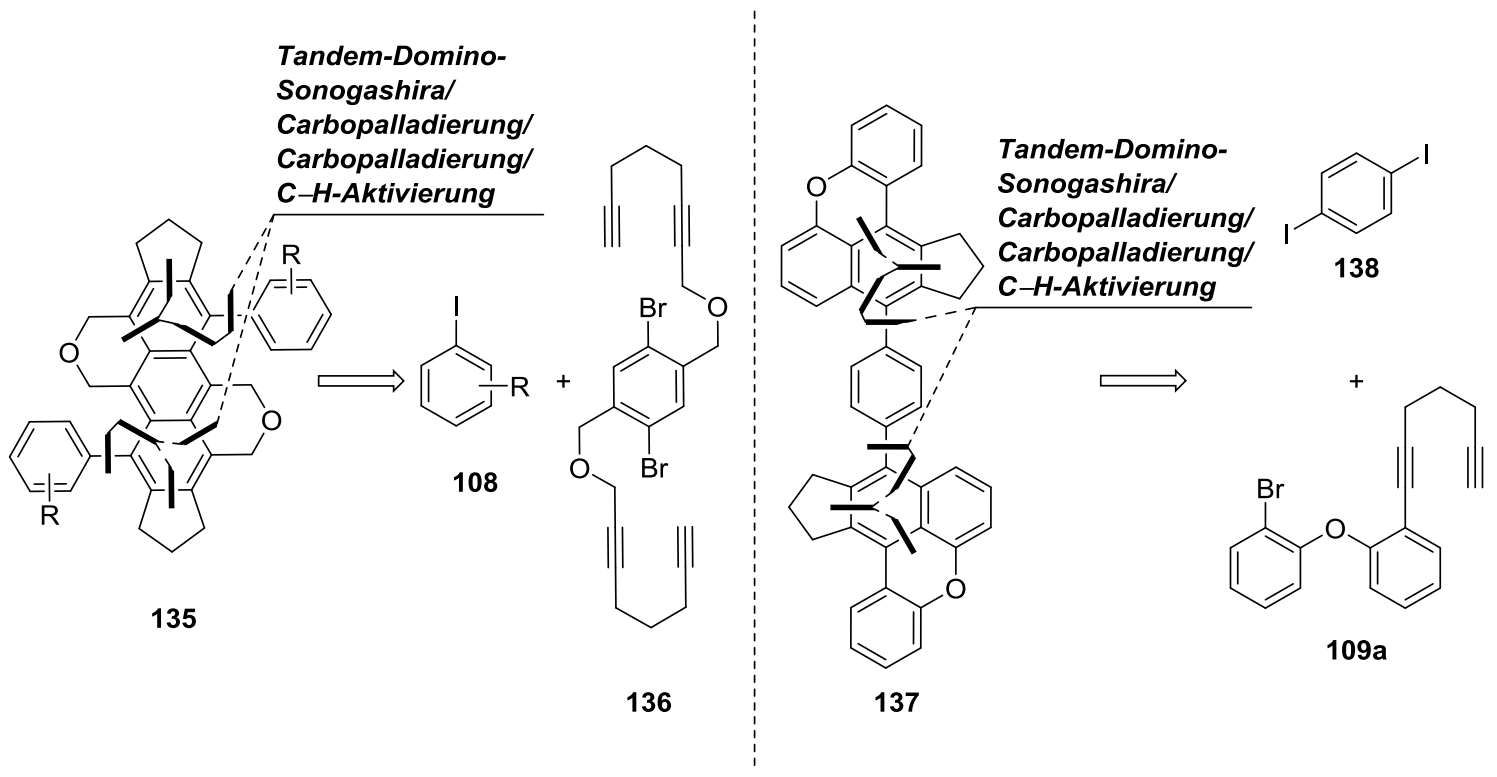

Abbildung 48: Retrosynthetischer Ansatz zum Aufbau der polyzyklischen Verbindungen 135 und 137.

Eine solche Reaktion stellt ein seltenes Beispiel für eine dimere Domino-Reaktion dar, die vor allem als effizientes Synhesewerkzeug dienen könnte. Schließlich verbindet sie das Konzept der Tandemmit dem der Domino-Reaktion und erlaubt dadurch den eleganten Aufbau von 
(pseudo-)symmetrischen Strukturen. Diese kommen in zahleichen Naturstoffmotiven und anderen interessanten Verbindungen vor, die über eine derartige Tandem-Domino-Reaktion leicht zugänglich wären. Zwei verschiedene Syntheserouten wurden verfolgt: Die Umsetzung von Aryliodiden des Typs 108 mit Bis(dialkinen) des Typs 136 sollte den Zugriff auf Polyzyklus 135 ermöglichen. In einem anderen Ansatz sollte Dialkin 109a mit 1,4-Diiodbenzol (138) zum Dimer 137 umgesetzt werden.
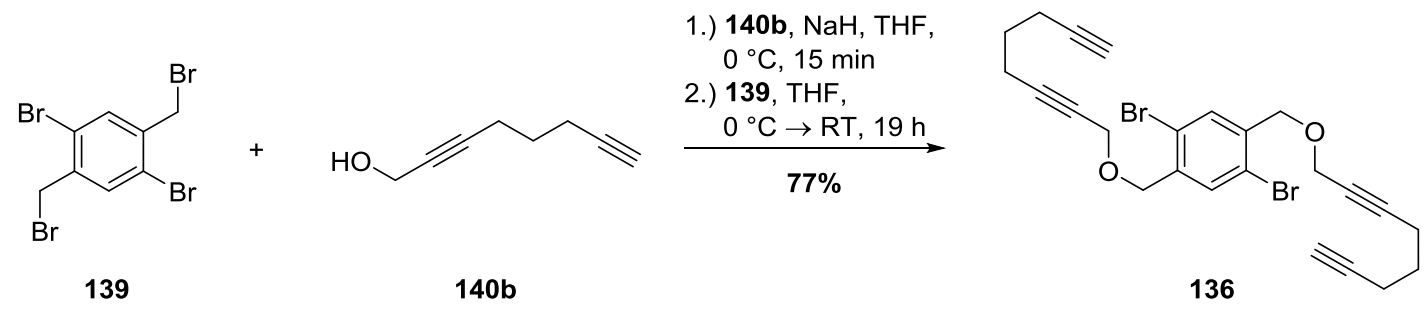

Abbildung 49: Synthese von Bis(dialkin) 136.

Die Synthese des Bis(dialkins) 136 erfolgte ausgehend vom literaturbekannten Tetrabromid 139, welches im Arbeitskreis zur Verfügung stand. ${ }^{75}$ In einer dimeren nukleophilen Substitution zwischen 139 und dem ebenfalls literaturbekannten Alkohol 140b wurde 136 in 77\% Ausbeute gewonnen (Abbildung 49). ${ }^{76}$ Die Synthese von 140b wird in Kapitel 3.4.2 (vgl. Abbildung 81) erläutert.

Die Umsetzung von 136 mit Aryliodiden vom Typ 108 in der Tandem-Domino-Reaktion zum Polyzyklus 135 wurde mit den etablierten Bedingungen mit $\mathrm{Pd}(\mathrm{OAc})_{2}$ und Triphenylphosphin als Katalysatorsystem und $(n \mathrm{Bu})_{4} \mathrm{NOAc}$ als Base sowie DMF als Lösungsmittel bei $100{ }^{\circ} \mathrm{C}$ durchgeführt. Dabei wurden verschiedene Aryliode als Kupplungspartner eingesetzt (Tabelle 2).

Tabelle 2: Untersuchungen zur Tandem-Domino-Sonogashira/Zweifach-Carbopalladierung/C-H-Aktivierung.
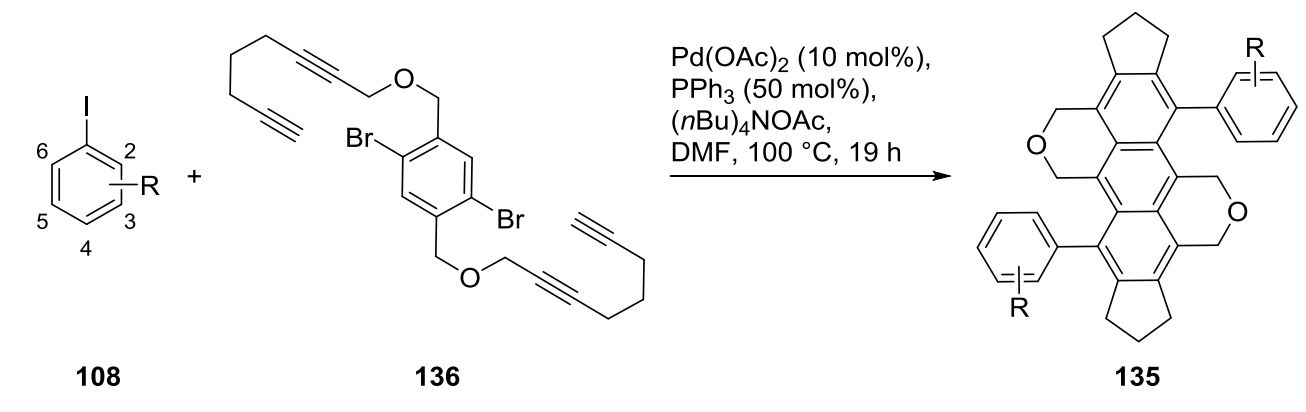

\begin{tabular}{ccccc}
\hline Eintrag & $\mathbf{1 0 8}$ & $\mathbf{R}$ & Zielprodukt $^{\text {Ergebnis }}$ & \\
\hline 1 & $\mathbf{1 0 8 c}$ & $2-\mathrm{CF}_{3}$ & $\mathbf{1 3 5 a}$ & $\mathbf{1 4 1}$ (quant. $^{[b]}$ \\
2 & $\mathbf{1 0 8 d}$ & $\mathrm{H}$ & $\mathbf{1 3 5 b}$ & Produktgemisch \\
3 & $\mathbf{1 0 8 e}$ & $2,3,5,6-\mathrm{Me}_{4}$ & $\mathbf{1 3 5} \mathbf{c}$ & Produktgemisch \\
4 & $\mathbf{1 0 8 g}$ & $4-\mathrm{Me}$ & $\mathbf{1 3 5 d}$ & Produktgemisch \\
5 & $\mathbf{1 0 8 h}$ & $2-\mathrm{Ph}$ & $\mathbf{1 3 5 e}$ & Produktgemisch \\
\hline
\end{tabular}

[a] 136 (1.00 Äq.), 108 (2.40 Äq.), $\mathrm{Pd}(\mathrm{OAc})_{2}(10 \mathrm{~mol} \%), \mathrm{PPh}_{3}(50 \mathrm{~mol} \%),(n \mathrm{Bu})_{4} \mathrm{NOAc}(6.00$ Äq.), DMF, $100{ }^{\circ} \mathrm{C}, 19 \mathrm{~h}$; [b] vgl. Abbildung 50. 
Die Untersuchungen führten jedoch in keinem Fall zum erwünschten Produkt. Im Fall von Substrat 108c wurde lediglich das Produkt der dimeren Sonogashira-Reaktion 141 in quantitativer Ausbeute erhalten (Tabelle 2, Eintrag 1; Abbildung 50).

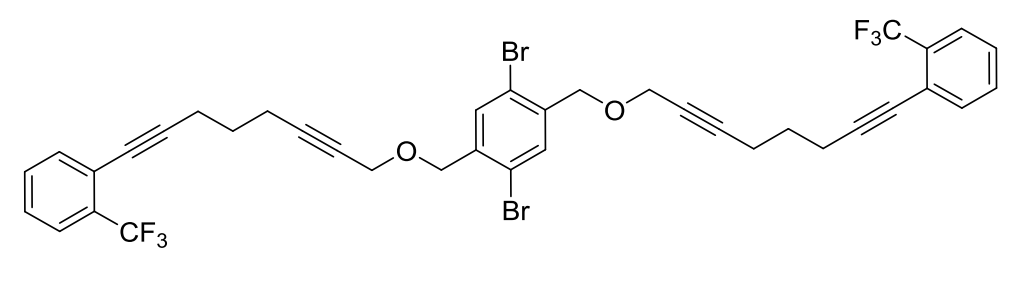

141

Abbildung 50: Erhaltenes Produkt der Umsetzung von 108c mit 136 unter Domino-Bedingungen.

Damit konnte zumindest ein postuliertes Zwischenprodukt des Mechanismus der Domino-Reaktion nachgewiesen und der vermutete Beginn des Domino-Prozesses mit der Sonogashira-Reaktion bestätigt werden. Die anderen Umsetzungen führten dagegen $\mathrm{zu}$ Produktgemischen, wie im Dünnschichtchromatogramm zu sehen war, und wurden daher nicht weiter untersucht.

Die Reaktion von Dialkin 109a mit 1,4-Diiodbenzol (138) führte ebenfalls nicht zum erwünschten Ergebnis. Stattdessen konnten auch hier nur zahlreiche Produkte im Dünnschichtchromatogramm detektiert werden (Abbildung 51), weswegen auf eine nähere Untersuchung verzichtet wurde. Möglicherweise kam es auch bei dieser Transformation zum nur unvollständigen Ablauf der DominoReaktion und damit zur Bildung entsprechender Zwischenprodukte.
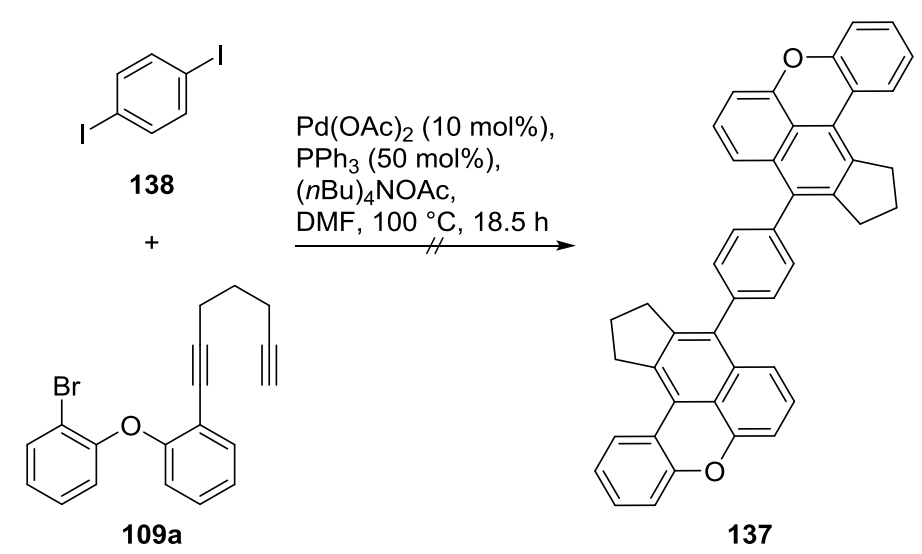

Abbildung 51: Untersuchungen zur Synthese von Polyzyklus 137 über eine Tandem-Domino-Sonogashira/ Zweifach-Carbopalladierung/C-H-Aktivierung. 


\subsection{Untersuchungen zur Synthese von fluoreszenzaktiven Polyzyklen des Typs 110}

Um die Fluoreszenzeigenschaften der synthetisierten Polyzyklen vom Typ 107 zu optimieren, indem beispielsweise die Emission in längerwellige Bereiche verschoben und die Fluoreszenzintensität verstärkt wurde, sollten auch Polyzyklen des Typs 110 synthetisiert werden (Abbildung 52).

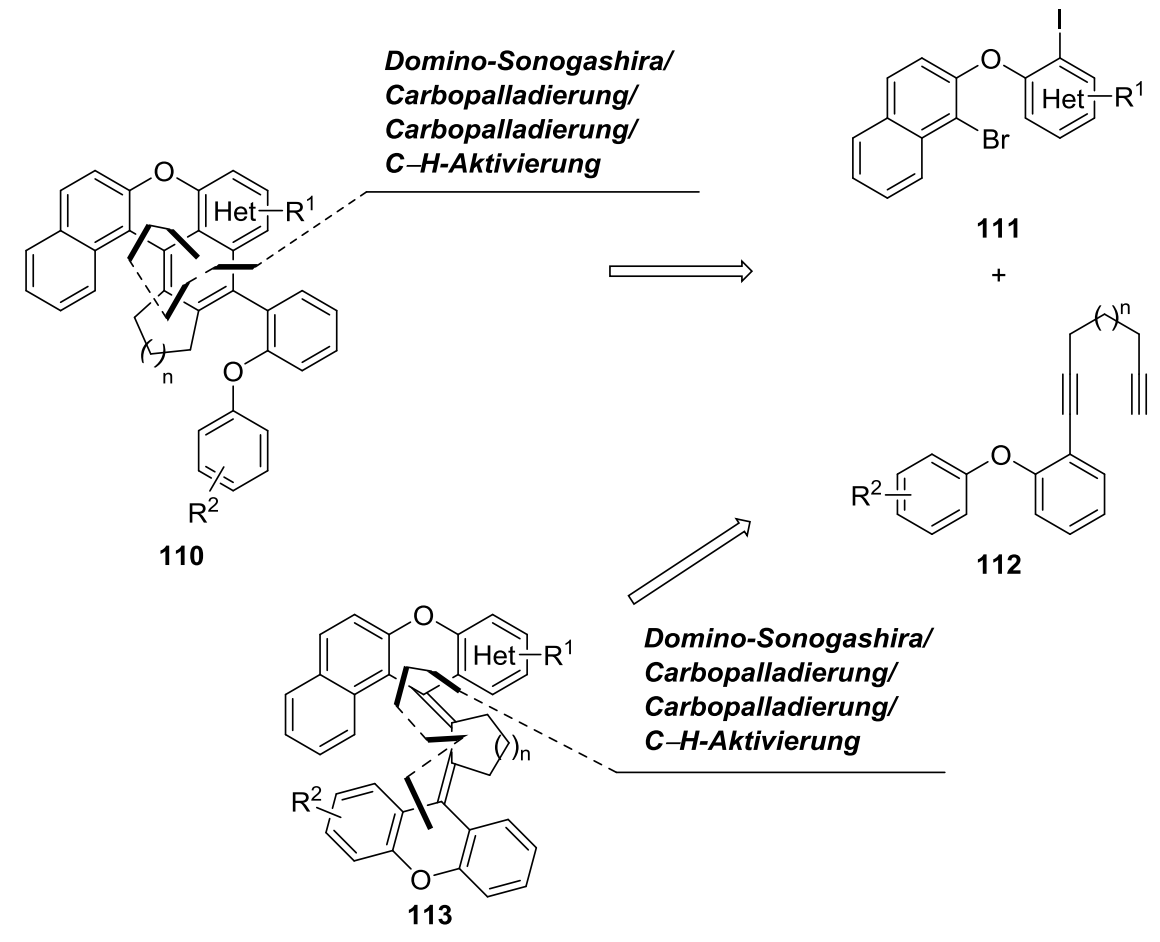

Abbildung 52: Retrosynthetischer Ansatz zur Synthese von Polyzyklen des Typs 110.

Diese enthielten einen zusätzlichen konjugierten Napthylring, der verbesserte Emissionseigenschaften gegenüber den Polyzyklen vom Typ 107 bewirken sollte. Auch hier erfolgte die Darstellung über eine vierfache Domino-Sonogashira/Zweifach-Carbopalladierung/C-H-Aktivierungs-Reaktion, die vier neue Bindungen sowie drei neue Ringe in einem Reaktionsprozess generieren sollte. Ausgangsverbindungen waren Aryliodide des Typs 111 sowie Dialkine vom Typ 112. Des Weiteren sollte die Möglichkeit der Synthese von tetrasubstituierten Dialkenen des Typs 113 untersucht werden. Diese würde ebenfalls durch eine Domino-Sonogashira/Zweifach-Carbopalladierung/C-HAktivierung erfolgen, wobei die $\mathrm{C}-\mathrm{H}$-Aktivierung am unteren Ring erfolgen müsste. Derartige Strukturen sind aufgrund der Tatsache, dass zwei tetrasubstituierte Doppelbindungen in einem Schritt aufgebaut werden, von besonderem synthetischem Interesse. Außerdem besitzen sie aufgrund ihrer helikalen Struktur ein Potenzial in der Anwendung als molekulare Schalter. Modifizierte Reaktionsbedingungen sowie unterschiedliche Substitutionsmuster sollten einen Zugang zu 113 ermöglichen. 


\subsubsection{Synthese der Aryliodide vom Typ 111}

Die Aryliodide 111a-c wurden über ein im Arbeitskreis Tietze etabliertes Protokoll synthetisiert (Abbildung 53). ${ }^{66}$
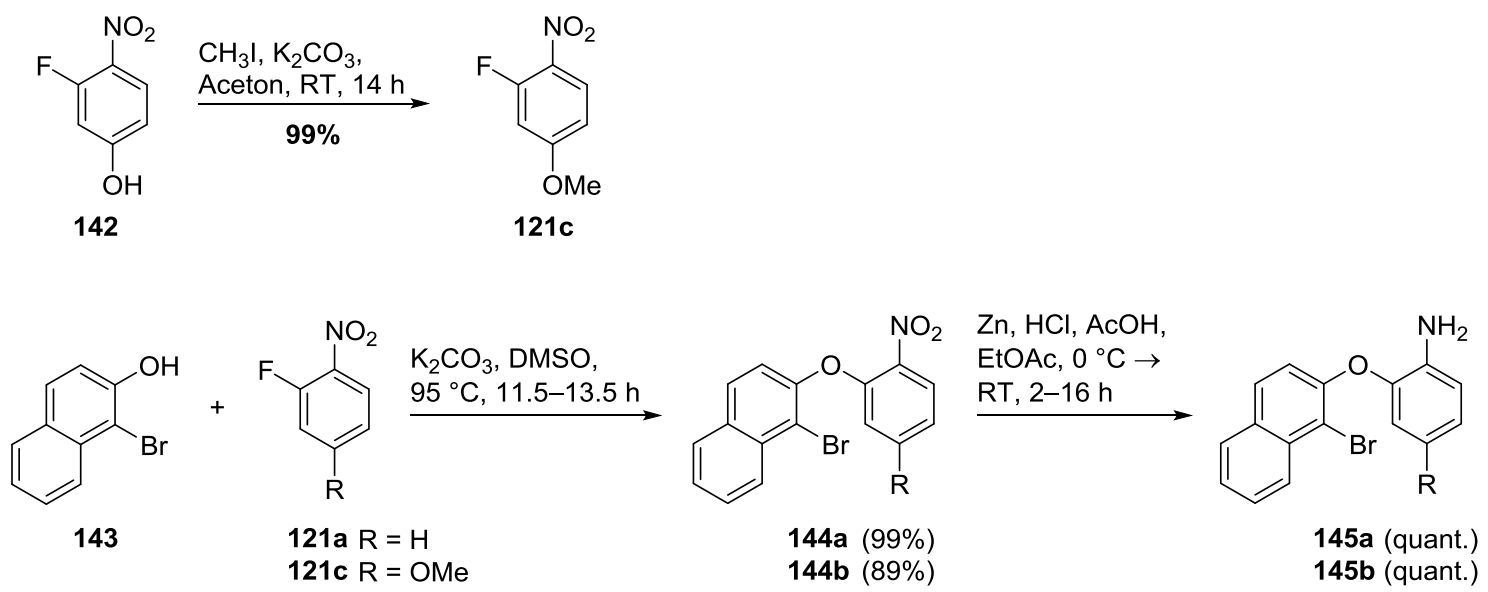

$p$ - $\mathrm{TsOH} \cdot \mathrm{H}_{2} \mathrm{O}$ $\mathrm{KI}, \mathrm{NaNO}_{2}$ $\mathrm{CH}_{3} \mathrm{CN} / \mathrm{H}_{2} \mathrm{O}$ RT, 3-14 h
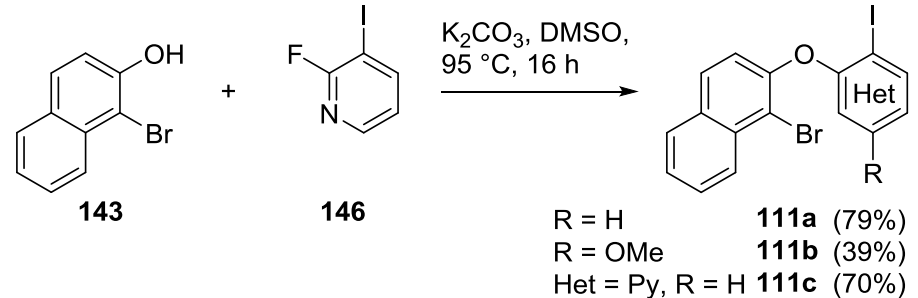

Abbildung 53: Synthese der Aryliodide vom Typ 111.

Zunächst wurde der für die Synthese eines Methoxy-substituierten Aryliodids benötigte Fluornitroaromat 121c mittels einer nukleophilen Substitution hergestellt. ${ }^{77}$ Dazu wurde Fluornitrophenol 142 unter basischen Bedingungen mit Methyliodid umgesetzt. Die Synthese der Aryliodide 111a und 111b begann mit einer nukleophilen aromatischen Substitution zwischen Naphthol 143 und den Fluornitrobenzolen 121a und 121c in DMSO bei $95{ }^{\circ} \mathrm{C}$ in 99 bzw. 89\% Ausbeute. Es folgte eine Reduktion der Nitrogruppe zu den entsprechenden Aminen 145a und 145b mithilfe von Zink in konzentrierter Salz- und Essigsäure, die in beiden Fällen quantitativ ablief. Eine Sandmeyer-Reaktion unter Bedingungen nach Knochel et al. lieferte schließlich die Aryliodide 111a und 111b in 79 bzw. 39\% Ausbeute. ${ }^{71}$ Das elektronenarme Aryliodid 111c konnte direkt über die nukleophile aromatische Substitution aus dem entsprechenden Fluoriodaromaten 146 dargestellt werden. Die Umsetzung erfolgte dabei in $70 \%$ Ausbeute. 


\subsubsection{Synthese der Dialkine vom Typ 112}

Die Synthese der Dialkine 112a-g erfolgte über ein ähnliches Protokoll. In einer nukleophilen aromatischen Substitution wurden zunächst verschiedene Phenole 147b-g mit Fluornitrobenzol 121a gekuppelt (Abbildung 54). Die Synthese erfolgte in Ausbeuten von $88 \%$ bis zu quantitativem Umsatz. Anschließend wurde die Nitrogruppe mit Zink in konzentrierter Salz- und Essigsäure zur Aminofunktion reduziert, wobei die entsprechenden Amine 149b-g in über 90\% Ausbeute erhalten wurden.
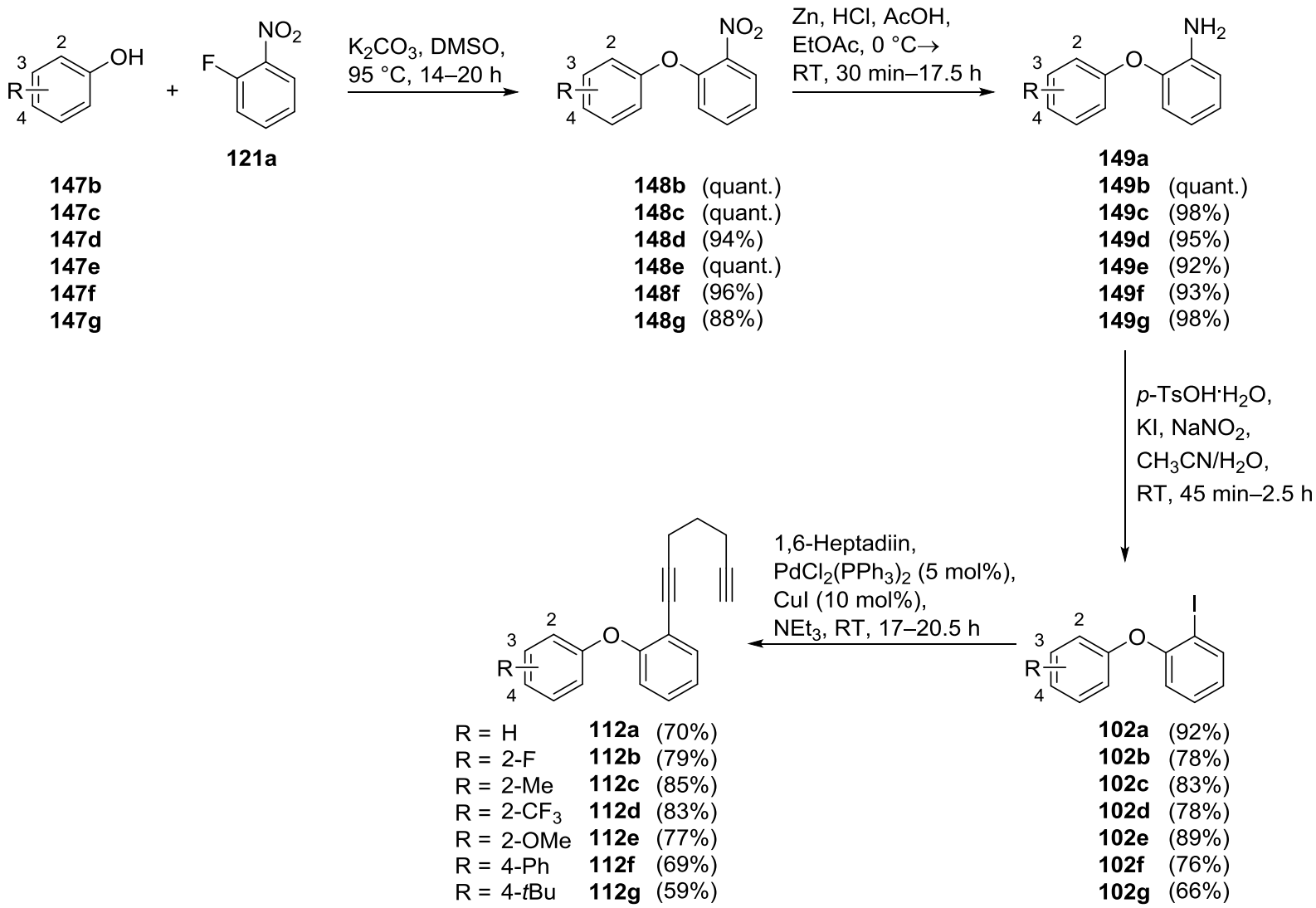

Abbildung 54: Synthese der Dialkine 112a-g.

Die nachfolgende Sandmeyer-Reaktion nach Bedingungen von Knochel et al. in wässrigem Acetonitril mit Kaliumiodid und Natriumnitrit als Reagenzien und para-Toluolsulfonsäure als Säure führte zu den entsprechenden Aryliodiden 102a-g in 66 bis 92\% Ausbeute. ${ }^{71}$ Das unsubstituierte Phenoxyanilin 149a war kommerziell erhältlich und wurde direkt in der Sandmeyer-Reaktion umgesetzt. Es folgte eine Sonogashira-Reaktion, welche die Aryliodide 102a-g mit überschüssig eingesetztem 1,6-Heptadiin (5-7 Äq.) kuppelte. Als Katalysatorsystem wurden $\mathrm{PdCl}_{2}\left(\mathrm{PPh}_{3}\right)_{2}$ und Kupfer(I)-Iodid in Triethylamin verwendet. Die entsprechenden Dialkine 112a-g konnten dabei in 59 bis $85 \%$ Ausbeute gewonnen werden. 
Außerdem sollte der im Produkt auftretende Cyclopentyl-Ring durch unterschiedlich große aliphatische Ringe ersetzt werden. Dazu wurde Aryliodid 102a mit Diinen von unterschiedlicher Kettenlänge gekuppelt. Die Bedingungen dieser Sonogashira-Reaktion entsprachen denen für die Kupplung der Aryliodide mit 1,6-Heptadiin. Die verschiedenen Dialkine 112h-j, die zu Vier-, Sechsund Sieben-Ringen im Produkt führen sollten, konnten in guten Ausbeuten von 61 bis $68 \%$ erhalten werden (Abbildung 55).
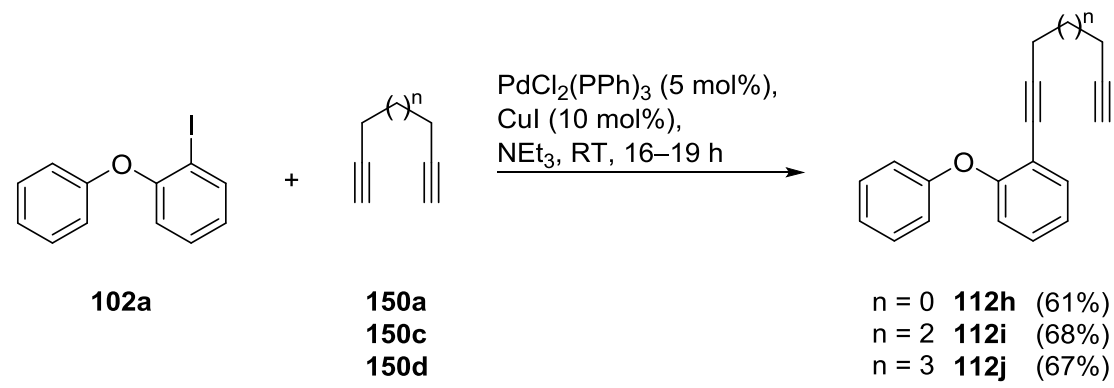

Abbildung 55: Synthese der Dialkine 112h-j.

\subsubsection{Synthese der Polyzyklen 110a-i durch die Domino-Reaktion}

Die Ausbeuten der Domino-Reaktion mit den Modellsubstraten Aryliodid 111a und Dialkin 112a, die zu Polyzyklus 110a führte, wurden zunächst durch den Einsatz verschiedener Liganden optimiert. Die besten Ausbeuten lieferte mit $73 \%$ dabei eine Kombination von 1 mol\% $\mathrm{Pd}(\mathrm{OAc})_{2}$ mit der fünffachen Menge an Triphenylphosphin sowie $(n \mathrm{Bu})_{4} \mathrm{NOAc}$ als Base in DMF als Lösungsmittel bei $100{ }^{\circ} \mathrm{C}$, die in Eintrag 8 von Tabelle 3 hervorgehoben ist.

$\mathrm{Zu}$ Beginn der Optimierungsexperimente wurden die Reaktionsbedingungen probiert, die sich bereits in Vorgängerarbeiten als effektiv erwiesen hatten und auch bei der Synthese der bereits vorgestellten Polyzyklen vom Typ 107 eingesetzt wurden. ${ }^{69}$ Mit 10 mol\% $\mathrm{Pd}(\mathrm{OAc})_{2}$ und Triphenylphosphin im Verhältnis 1:5 als Katalysatorsystem und $(n \mathrm{Bu})_{4} \mathrm{NOAc}$ als Base in DMF bei $100{ }^{\circ} \mathrm{C}$ konnte Verbindung 110a in 45\% Ausbeute isoliert werden, wenn 111a und 112a im Verhältnis 1:1.4 eingesetzt wurden (Tabelle 3, Eintrag 1). Ein umgekehrtes Verhältnis mit einem Überschuss an Aryliodid 111a von 1.1 Äquivalenten lieferte dagegen eine gesteigerte Ausbeute von $62 \%$ unter ansonsten identischen Reaktionsbedingungen (Eintrag 2). Diese Reaktionsbedingungen wurden daher mit anderen Liganden getestet, wobei keiner zu ähnlich hohen Ausbeuten wie Triphenylphosphin führte. So wurden mit der ionischen Spezies $\left[\mathrm{P} \mathrm{Bu}_{4}\right]\left[\mathrm{HBF}_{4}\right]$ ebenso wie mit dem elektronenreichen $\mathrm{PCy}_{3}$ nur $11 \%$ an 110a erhalten. (Einträge 3 und 4). Das $N$-heterocyclische Carben (NHC) $\mathrm{IPr} \cdot \mathrm{HCl}$ ergab nur 20\% Ausbeute (Eintrag 5). Mit dem elektronenarmen $\mathrm{P}(4-\mathrm{Cl}-\mathrm{Ph})_{3}$-Liganden wurden 57\% Ausbeute erzielt (Eintrag 6). Dieser ist dem Triphenylphosphin strukturell ähnlich, womit dieser Liganden-Typus die besten Ergebnisse zu liefern scheint. Weitere Untersuchungen wurden daher mit Triphenylphosphin als Liganden durchgeführt. Zunächst wurde die Katalysatorbeladung herabgesetzt. 
Eine Umsetzung mit 5 mol\% $\mathrm{Pd}(\mathrm{OAc})_{2}$ führte zu einer leicht erhöhten Ausbeute von 66\% (Eintrag 7). Demgegenüber konnten sogar $73 \%$ Ausbeute erzielt werden, wenn 1 mol\% Katalysator verwendet wurden (Eintrag 8). Eine weitere Absenkung der $\mathrm{Pd}(\mathrm{OAc})_{2}$-Menge auf $0.5 \mathrm{~mol} \%$ ergab dagegen eine geringere Ausbeute von 58\% (Eintrag 9). Des Weiteren konnte bewiesen werden, dass ein Verhältnis von Metallsalz zu Ligand von 1:5 von Vorteil ist, da ein Verhältnis von 1:2 zu etwas geringeren 66\% Ausbeute führte (Eintrag 10). Zusätzlich wurde gezeigt, dass ein Ligand notwendig ist, da eine Umsetzung ausschließlich mit $\mathrm{Pd}(\mathrm{OAc})_{2}$ nur 28\% Ausbeute ergab (Eintrag 11). Eine Erhöhung der Ansatzgröße wiederum war mit einer niedrigeren Ausbeute verbunden: Eine Umsetzung größerer Mengen an Aryliodid 111a und Dialkin 112a (1.1 mmol statt $0.11 \mathrm{mmol}$ Ansatzgröße) führte nur zu 40\% zu Produkt 110a (Eintrag 12).

Tabelle 3: Optimierung der Reaktionsbedingungen. ${ }^{[a]}$

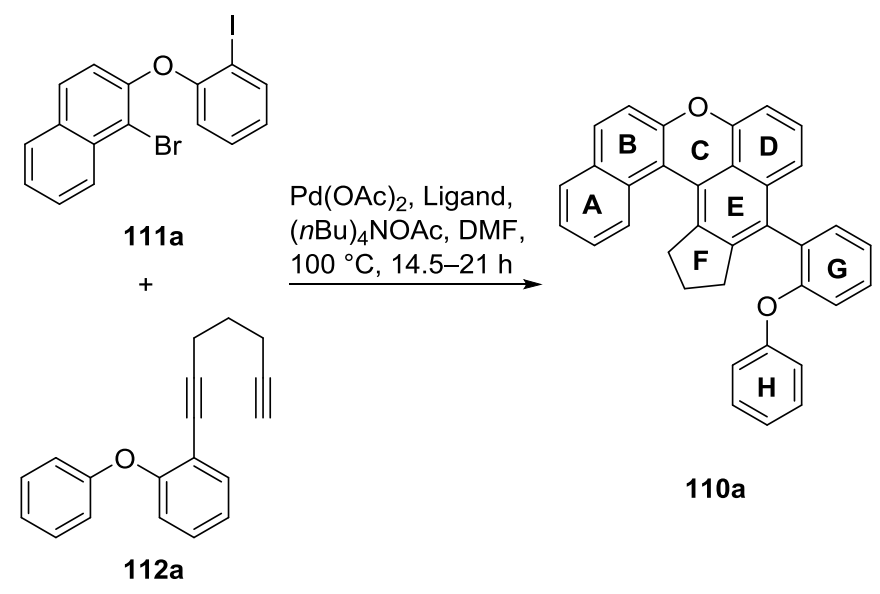

\begin{tabular}{ccccc}
\hline Eintrag & Pd(OAc $)_{2}[\mathbf{m o l} \%]$ & Ligand & Ligand $[\mathbf{m o l} \%]$ & Ausbeute $[\%]^{[\mathrm{b}]}$ \\
\hline $1^{[\mathrm{cc}}$ & 10 & $\mathrm{PPh}_{3}$ & 50 & 45 \\
2 & 10 & $\mathrm{PPh}_{3}$ & 50 & 62 \\
3 & 10 & {$\left[\mathrm{P}(t \mathrm{Bu})_{4}\right]\left[\mathrm{HBF}_{4}\right]$} & 50 & 11 \\
4 & 10 & $\mathrm{PCy}_{3}$ & 50 & 11 \\
5 & 10 & $\mathrm{PPr} \cdot \mathrm{HCl}$ & 50 & 20 \\
6 & 10 & $\mathrm{P}(4-\mathrm{Cl}-\mathrm{Ph})_{3}$ & 50 & 57 \\
7 & 5 & $\mathrm{PPh}_{3}$ & 25 & 66 \\
8 & 1 & $\mathbf{P P h}_{3}$ & $\mathbf{5}$ & $\mathbf{7 3}$ \\
9 & 0.5 & $\mathrm{PPh}_{3}$ & 2.5 & 58 \\
10 & 1 & $\mathrm{PPh}_{3}$ & 2 & 66 \\
11 & 1 & - & - & 28 \\
$12^{[\mathrm{d}]}$ & 1 & $\mathrm{PPh}_{3}$ & 5 & 40 \\
\hline
\end{tabular}

[a] 111a (1.1 Äq.), 112a (1.0 Äq.), Pd(OAc) $)_{2}$, Ligand, $(n \mathrm{Bu})_{4} \mathrm{NOAc}\left(5\right.$ Äq.), DMF, $100{ }^{\circ} \mathrm{C}, 14.5-21 \mathrm{~h}$; [b] Isolierte Ausbeute nach Säulenchromatographie; [c] wie in [a], außer: 111a (1.0 Äq.), 112a (1.4 Äq.), $(n \mathrm{Bu})_{4}$ NOAc (6 Äq.); [d] wie in Eintrag 8, außer: $1.1 \mathrm{mmol}$ Ansatz (anstelle von $0.11 \mathrm{mmol}$ ). 
Die somit optimierten Bedingungen wurden nun zur Synthese der verschiedenen Substrate verwendet (Tabelle 4).

Tabelle 4: Substratbreite der Vierfach-Domino-Reaktion. ${ }^{[a]}$

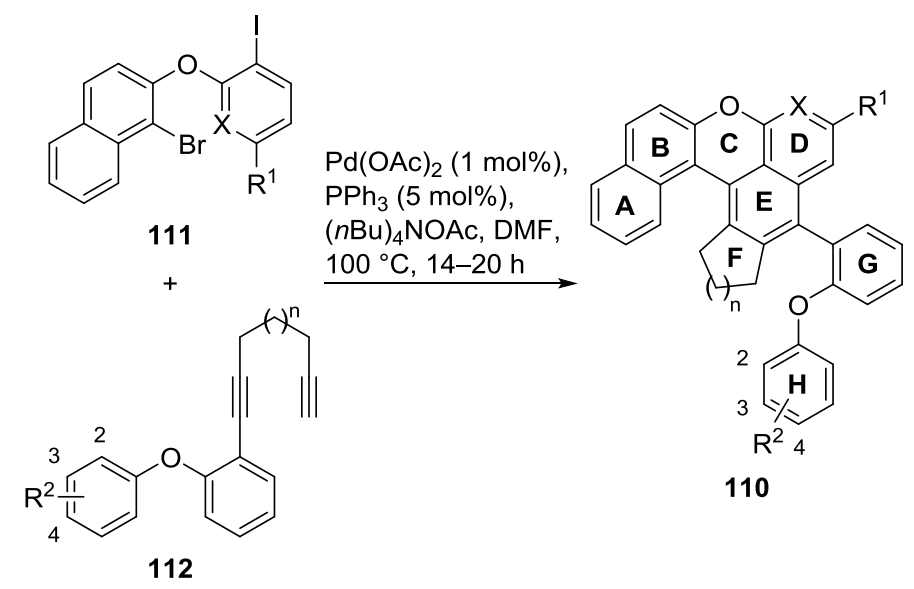

\begin{tabular}{cccccccc}
\hline Eintrag & Substrate & $\mathbf{R}^{\mathbf{1}}$ & $\mathbf{R}^{\mathbf{2}}$ & $\mathbf{X}$ & $\mathbf{n}$ & Produkte & ${\text { Ausbeute [\% }]^{[\mathbf{b}]}}$ \\
\hline 1 & $\mathbf{1 1 1 c}, \mathbf{1 1 2 a}$ & $\mathrm{H}$ & $\mathrm{H}$ & $\mathrm{N}$ & 1 & $\mathbf{1 1 0 b}$ & 89 \\
$2^{[\mathrm{c}]}$ & $\mathbf{1 1 1 b}, \mathbf{1 1 2 a}$ & $\mathrm{OMe}$ & $\mathrm{H}$ & $\mathrm{CH}$ & 1 & $\mathbf{1 1 0 c}$ & 29 \\
3 & $\mathbf{1 1 1 a}, \mathbf{1 1 2 b}$ & $\mathrm{H}$ & $2-\mathrm{F}$ & $\mathrm{CH}$ & 1 & $\mathbf{1 1 0 d}$ & 67 \\
4 & $\mathbf{1 1 1 a}, \mathbf{1 1 2 c}$ & $\mathrm{H}$ & $2-\mathrm{Me}$ & $\mathrm{CH}$ & 1 & $\mathbf{1 1 0 e}$ & 40 \\
5 & $\mathbf{1 1 1 a}, \mathbf{1 1 2 d}$ & $\mathrm{H}$ & $2-\mathrm{CF}_{3}$ & $\mathrm{CH}$ & 1 & $\mathbf{1 1 0 f}$ & 35 \\
6 & $\mathbf{1 1 1 a}, \mathbf{1 1 2} \mathrm{H}$ & $\mathrm{H}$ & $2-\mathrm{OMe}$ & $\mathrm{CH}$ & 1 & $\mathbf{1 1 0 g}$ & 22 \\
7 & $\mathbf{1 1 1 a}, \mathbf{1 1 2 f}$ & $\mathrm{H}$ & $4-\mathrm{Ph}$ & $\mathrm{CH}$ & 1 & $\mathbf{1 1 0 h}$ & 40 \\
8 & $\mathbf{1 1 1 a}, \mathbf{1 1 2 g}$ & $\mathrm{H}$ & $4-t \mathrm{Bu}$ & $\mathrm{CH}$ & 1 & $\mathbf{1 1 0 i}$ & 37 \\
$9^{[\mathrm{d}]}$ & $\mathbf{1 1 1 a}, \mathbf{1 1 2 i}$ & $\mathrm{H}$ & $\mathrm{H}$ & $\mathrm{CH}$ & 2 & $\mathbf{1 1 0 k}$ & Spuren \\
$10^{[\mathrm{d}]}$ & $\mathbf{1 1 1 a}, \mathbf{1 1 2 j}$ & $\mathrm{H}$ & $\mathrm{H}$ & $\mathrm{CH}$ & 3 & $\mathbf{1 1 0 1}$ & Spuren \\
$11^{[\mathrm{d}]}$ & $\mathbf{1 1 1 a}, \mathbf{1 1 2 b}$ & $\mathrm{H}$ & $2-\mathrm{F}$ & $\mathrm{CH}$ & 1 & $\mathbf{1 1 0 d}$ & 23 \\
$12^{[\mathrm{d}]}$ & $\mathbf{1 1 1 a}, \mathbf{1 1 2}$ & $\mathrm{H}$ & $2-\mathrm{OMe}$ & $\mathrm{CH}$ & 1 & $\mathbf{1 1 0 g}$ & Spuren \\
$13^{[\mathrm{d}]}$ & $\mathbf{1 1 1 a}, \mathbf{1 1 2 f}$ & $\mathrm{H}$ & $4-\mathrm{Ph}$ & $\mathrm{CH}$ & 1 & $\mathbf{1 1 0 h}$ & Spuren \\
$14^{[\mathrm{d}]}$ & $\mathbf{1 1 1 a}, \mathbf{1 1 2 g}$ & $\mathrm{H}$ & $4-t \mathrm{Bu}$ & $\mathrm{CH}$ & 1 & $\mathbf{1 1 0}$ & Spuren \\
\hline
\end{tabular}

[a] Alkin 112 (1.0 Äq.), Aryliodid 111 (1.1 Äq.), Pd(OAc) 2 (1 mol\%), $\mathrm{PPh}_{3}(5 \mathrm{~mol} \%),(n \mathrm{Bu})_{4} \mathrm{NOAc}(5.0$ Äq.), DMF, $100^{\circ} \mathrm{C}, 14-20 \mathrm{~h}$; [b] Isolierte Ausbeute nach Säulenchromatographie; [c] wie in [a], außer: 111 (1.0 Äq.), $\mathrm{Pd}(\mathrm{OAc})_{2}(20 \mathrm{~mol} \%), \mathrm{PPh}_{3}$ (1.0 Äq.), $(n \mathrm{Bu})_{4} \mathrm{NOAc}$ (5.2 Äq.); [d] wie in [a], außer: 111 (1.2 Äq.), $\operatorname{Pd}(\mathrm{OAc})_{2}(20 \mathrm{~mol} \%), \mathrm{PPh}_{3}\left(1.0\right.$ Äq.), $(n \mathrm{Bu})_{4} \mathrm{NOAc}(6.0$ Äq.).

Die erhaltenen Ergebnisse mit stark variierenden Ausbeuten lassen darauf schließen, dass die DominoReaktion enorm vom Substitutionsmuster der Reaktanden beeinflusst wird. So konnte Verbindung 110b mit einem Pyridinsystem in Ring D in 89\% Ausbeute gewonnen werden und lieferte damit die beste Ausbeute aller Substrate (Eintrag 1). Produkt 110c mit einer Methoxy-Gruppe im oberen D-Ring wurde dagegen in nur 29\% Ausbeute isoliert (Eintrag 2), wenn die Reaktion mit einer erhöhten Katalysatorbeladung von $20 \mathrm{~mol} \% \quad \mathrm{Pd}(\mathrm{OAc})_{2} \quad$ durchgeführt wurde. Auch verschiedene 
Substitutionsmuster am H-Ring führten zu sehr unterschiedlichen Ergebnissen nach ihrer Umsetzung in der Domino-Reaktion. Das fluorierte Derivat 110d, in ortho-Position substituiert, konnte in 68\% Ausbeute erhalten werden (Eintrag 3), während 110e mit einer ortho-Methyl-Gruppe zu $40 \%$ isoliert wurde (Eintrag 4). 110f mit einer $\mathrm{CF}_{3}$-Gruppe in 2-Position konnte in moderaten $35 \%$ Ausbeute erhalten werden (Eintrag 5). Der entsprechende Methoxy-substituierte Vorläufer 112e lieferte das Domino-Produkt 110g dagegen in nur 20\% Ausbeute, ein Ergebnis, das vermutlich mit der geringen Reinheit des Dialkin-Vorläufers erklärt werden kann (Eintrag 6). Die Verbindungen 110h und 110i mit einer para-Phenyl- bzw. para-tert-Butyl-Gruppe als Substituenten konnten in moderaten 40 bzw. 37\% Ausbeute gewonnen werden (Einträge 7 und 8). Nur in Spuren wurden hingegen die Derivate 110k und 1101 detektiert, die größere aliphatische F-Ringe enthielten (Einträge 9 und 10). Diese beiden Substanzen konnten lediglich im Massenspektrum sowie im ${ }^{1} \mathrm{H}-\mathrm{NMR}-\mathrm{Spektrum}$ des Rohprodukts nachgewiesen werden.

Demnach scheinen zusätzliche funktionelle Gruppen am H-Ring der Reaktion hinderlich zu sein, was mit dem gesteigerten sterischen Anspruch des Dialkin-Kupplungspartners zusammenhängen könnte, der damit unzugänglicher wird. Die relativ gute Ausbeute beim Fluor-Derivat 110c ließe sich demnach mit der geringen Größe des Fluoratoms und der damit einhergehenden geringeren sterischen Hinderung erklären. Die Substrate mit größeren F-Ringen sind vermutlich aufgrund der geringeren konformativen Stabilität von Sechs- und Siebenringen schwerer zugänglich.

Um die Ausbeuten zu verbessern, wurden ausgewählte Derivate mit einer höheren Katalysatorbeladung von $20 \mathrm{~mol} \%$ umgesetzt. Dieses führte jedoch im Falle des ortho-FluorSubstituenten sowie des ortho-Methoxy-Substituenten in Ring H zu deutlich geringeren Ausbeuten als eine Umsetzung mit $1 \mathrm{~mol} \% \mathrm{Pd}(\mathrm{OAc})_{2}$ (Einträge 11 und 12). Das Gleiche galt auch für die Produkte mit para-Phenyl- und para-tert-Butyl-Gruppen, die beide nur in geringen Mengen detektiert werden konnten (Einträge 13 und 14). Somit scheint eine große Katalysatormenge hinderlich zu sein und den Ablauf der Domino-Reaktion durch die hohe Konzentration an reaktivem Katalysatormetall zu stören.

Aufgrund der mehrheitlich eher moderaten Ausbeuten bei der Domino-Sonogashira/ZweifachCarbopalladierung/C-H-Aktivierung wurde die Sonogashira-Reaktion vom Domino-Prozess abgetrennt. Die isolierten Produkte der Sonogashira-Reaktion wurden in einer anschließenden Domino-Zweifach-Carbopalladierung/C-H-Aktivierung umgesetzt. Die Reaktionsbedingungen der Sonogashira-Reaktion entsprachen den bisher für diese Reaktion verwendeten Bedingungen mit $\mathrm{PdCl}_{2}\left(\mathrm{PPh}_{3}\right)_{2}$ und Kupfer(I)-Iodid als Katalysatorsystem in Triethylamin als Lösungsmittel (Tabelle 5). Die Umsetzung erfolgte bei $60{ }^{\circ} \mathrm{C}$. Die Ausbeuten lagen größtenteils bei über $60 \%$. So wurde Produkt 151b mit einem Pyridin-System in Ring D in 79\% Ausbeute gewonnen (Eintrag 1). Methoxy-Derivat 151c konnte in 67\% Ausbeute erhalten werden (Eintrag 2). Die Produkte mit verschiedenen Substituenten am H-Ring wurden ebenfalls in guten Ausbeuten erhalten. So wurde das ortho-Fluor-substituierte Produkt 151d in 77\% Ausbeute isoliert (Eintrag 3), während das entsprechende ortho-Methyl-Derivat 151e in 66\% Ausbeute gewonnen wurde (Eintrag 4). Die 
ortho- $\mathrm{CF}_{3^{-}}$und ortho-Methoxy-Derivate $\mathbf{1 5 1 f}$ und $\mathbf{1 5 1 g}$ wurden zu 69 bzw. $71 \%$ umgesetzt (Einträge 5 und 6). Besonders gute Ausbeuten lieferten die para-Phenyl- und para-tert-Butyl-Analoga 151h und 151i: Sie konnten in 96 bzw. 90\% Ausbeute gewonnen werden (Einträge 7 und 8). Die Dialkine mit unterschiedlicher Länge des Alkyl-Spacers wurden ebenfalls in der SonogashiraReaktion umgesetzt. Das Derivat mit dem entsprechenden Ethyl-Spacer 151j wurde in 76\% Ausbeute gewonnen (Eintrag 9). Derivat 151k mit einem Butyl-Spacer konnte zu 85\% umgesetzt werden (Eintrag 10).

Tabelle 5: Substratbreite der Sonogashira-Reaktion. ${ }^{[a]}$

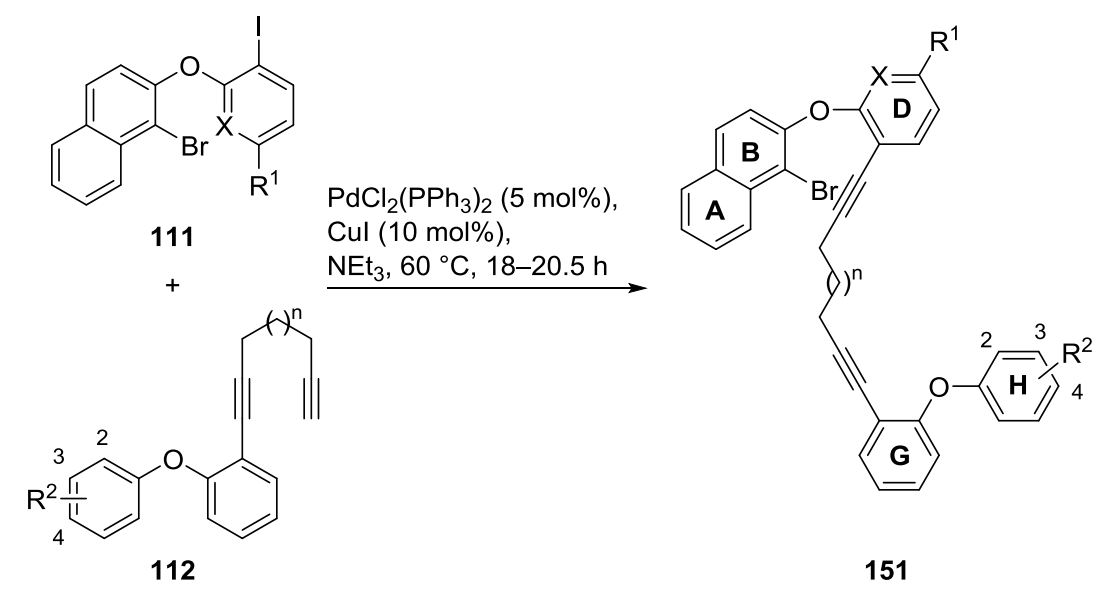

\begin{tabular}{|c|c|c|c|c|c|c|c|}
\hline Eintrag & Substrate & $\mathbf{R}^{1}$ & $\mathbf{R}^{2}$ & $\mathbf{X}$ & $\mathbf{n}$ & Produkt & Ausbeute $[\%]^{[b]}$ \\
\hline 1 & 111c, 112a & $\mathrm{H}$ & $\mathrm{H}$ & $\mathrm{N}$ & 1 & 151b & 79 \\
\hline 2 & $111 b, 112 a$ & $\mathrm{OMe}$ & $\mathrm{H}$ & $\mathrm{CH}$ & 1 & $151 \mathrm{c}$ & 67 \\
\hline 3 & $111 a, 112 b$ & $\mathrm{H}$ & $2-\mathrm{F}$ & $\mathrm{CH}$ & 1 & 151d & 77 \\
\hline 4 & $111 a, 112 c$ & $\mathrm{H}$ & 2-Me & $\mathrm{CH}$ & 1 & $151 \mathrm{e}$ & 66 \\
\hline 5 & $111 a, 112 d$ & $\mathrm{H}$ & $2-\mathrm{CF}_{3}$ & $\mathrm{CH}$ & 1 & $151 f$ & 69 \\
\hline 6 & $111 a, 112 e$ & $\mathrm{H}$ & 2-OMe & $\mathrm{CH}$ & 1 & $151 \mathrm{~g}$ & 71 \\
\hline 7 & 111a, 112f & $\mathrm{H}$ & 4-Ph & $\mathrm{CH}$ & 1 & $151 \mathrm{~h}$ & 96 \\
\hline 8 & $111 \mathrm{a}, 112 \mathrm{~g}$ & $\mathrm{H}$ & $4-t \mathrm{Bu}$ & $\mathrm{CH}$ & 1 & $151 \mathrm{i}$ & 90 \\
\hline 9 & $111 \mathrm{a}, 112 \mathrm{~h}$ & $\mathrm{H}$ & $\mathrm{H}$ & $\mathrm{CH}$ & 0 & $151 j$ & 76 \\
\hline 10 & 111a, 112i & $\mathrm{H}$ & $\mathrm{H}$ & $\mathrm{CH}$ & 2 & $151 k$ & 85 \\
\hline
\end{tabular}

[a] 111 (1.00 Äq.), 112 (1.10 Äq.), $\mathrm{PdCl}_{2}\left(\mathrm{PPh}_{3}\right)_{2}(5 \mathrm{~mol} \%), \mathrm{CuI}(10 \mathrm{~mol} \%), \mathrm{NEt}_{3}, 60^{\circ} \mathrm{C}, 18-20.5 \mathrm{~h}$; [b] Isolierte Ausbeute nach Säulenchromatographie; [c] wie in [a], außer: 112 (1.25 Äq.).

Die Ergebnisse der Sonogashira-Reaktion weisen insofern eine gewisse Konsistenz auf, als dass die Ausbeuten der am H-Ring para-substituierten Derivate sich von denen der anderen Umsetzungen abheben. Ursache könnte die bereits für den Vierfach-Domino-Prozess vermutete geringere sterische Hinderung am Dialkin-Vorläufer 112 sein, über die diese Derivate zweifellos verfügen. Diese Vermutung wird auch durch die etwas verbesserten Ausbeuten beim Fluor-substituierten SonogashiraProdukt 151d gestützt, das die geringste Größe aller am H-Ring ortho-substituierten Derivate aufweist 
und damit den geringsten sterischen Effekt ausübt. Die unterschiedlichen Ausbeuten für 151b und 151c mit einem Pyridin-Ring bzw. einem Methoxy-Substituenten am oberen D-Ring lassen dagegen auf elektronische Effekte schließen: Vermutlich sind elektronenarme Aryliodide in der SonogashiraReaktion leichter zugänglich. Diese Erkenntnis deckt sich mit den Ergebnissen der Umsetzungen zu Polyzyklen des Typs 107 (vgl. Kapitel 3.1.3, Abbildung 42)

Die somit erhaltenen Sonogashira-Produkte wurden anschließend in einer Domino-ZweifachCarbopalladierung/C-H-Aktivierung umgesetzt. Die Ergebnisse in Tabelle 6 zeigen, dass dabei zum Teil deutlich bessere Ausbeuten erhalten wurden als mit dem Domino-Sonogashira/ZweifachCarbopalladierung/C-H-Aktivierungsprozess. Die Umsetzungen erfolgten mit 20 mol\% $\mathrm{Pd}(\mathrm{OAc})_{2}$ und 1.00 Äq. Triphenylphosphin, womit Bedingungen verwendet wurden, die sich in Vorgängerarbeiten für diesen Reaktionstyp bewährt hatten. ${ }^{69,70}$ Das Pyridin-Derivat 110b wurde in $79 \%$ Ausbeute erhalten (Eintrag 1), womit die Ausbeute hinter den 89\% der Vierfach-Domino-Reaktion zurückblieb. Das am D-Ring Methoxy-substituierte Domino-Produkt 110c wurde in 54\% Ausbeute gewonnen (Eintrag 2). Dies ist eine deutliche Steigerung gegenüber den im Vierfach-Domino-Prozess erhaltenen 29\% Ausbeute. Die unterschiedlichen Substitutionsmuster am H-Ring ergaben im Dreifach-DominoProzess fast alle deutlich bessere Ausbeuten. Die ortho-Fluor- und ortho-Methyl-substituierten Polyzyklen 110d und 110e wurden zu 57\% (67\%) bzw. 81\% (40\%) umgesetzt (Einträge 3 und 4). Damit war die Ausbeute für das fluorierte 110d im Dreifach-Prozess etwas schlechter als in der vierfachen Domino-Reaktion. Das ortho-Methyl-substituierte Produkt 110e hingegen konnte in deutlich verbesserten $81 \%$ Ausbeute isoliert werden (Eintrag 4). Produkt 110f, das ortho- $\mathrm{CF}_{3}$ substituiert war, lieferte mit 53\% (statt 35\%) Ausbeute ebenfalls bessere Ergebnisse im DreifachDomino-Prozess (Eintrag 5). In 53\% (22\%) Ausbeute wurde das entsprechende Methoxy-Derivat 110g isoliert (Eintrag 6). Mit 53\% (40\%) Ausbeute konnte auch die para-Phenyl-substituierte Verbindung 110h in besseren Ausbeuten erhalten werden (Eintrag 7), wie auch der tert-Butyl-substituierte Polyzyklus 110i, der in einer Ausbeute von 68\% (37\%) isoliert wurde (Eintrag 8). Die Produkte mit aliphatischen F-Ringen unterschiedlicher Größe konnten dagegen nicht oder nur in Spuren gewonnen werden. Während Produkt 110j mit einem Vierring gar nicht nachgewiesen werden konnte (Eintrag 9), war das entsprechende Sechsring-Derivat 110k nur in Spuren zu finden (Eintrag 10).

Zur Optimierung der Reaktionsbedingungen wurde die Domino-Reaktion exemplarisch mit einer Katalysatorbeladung von $1 \mathrm{~mol} \%$ durchgeführt. Dabei konnte das tert-Butyl-substituierte Derivat 110i in einer exzellenten Ausbeute von 99\% isoliert werden (Eintrag 11).

Die mehrheitlich besseren Ausbeuten für den Dreifach-Domino-Prozess legen die Vermutung nahe, dass die Sonogashira-Reaktion die entscheidende Ursache für die moderaten Ausbeuten in der Vierfach-Domino-Reaktion ist. Wahrscheinlich beruhen diese Unterschiede in der Ausbeute auf sterischen Einflüssen der Substituenten, die sowohl in der Vierfach- als auch in der isolierten Sonogashira-Reaktion eine Rolle zu spielen scheinen. Die Ergebnisse des dreifachen DominoProzesses lassen solche Schlussfolgerungen dagegen nicht zu. Womöglich sind hier elektronische 
Einflüsse dominierend, wobei interessanterweise sowohl elektronenreiche als auch -arme Substrate zu verringerten Ausbeuten führten und nur die relativ elektronisch neutralen Substrate 110e und 110i bessere Ergebnisse lieferten.

Tabelle 6: Substratbreite der Dreifach-Domino-Reaktion. ${ }^{[a]}$
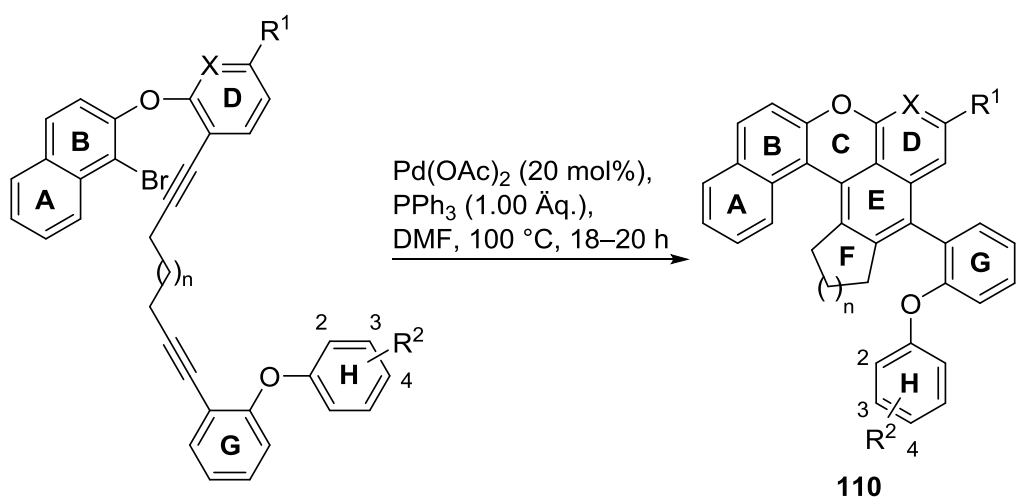

151

\begin{tabular}{cccccccc}
\hline Eintrag & Substrate & $\mathbf{R}^{\mathbf{1}}$ & $\mathbf{R}^{\mathbf{2}}$ & $\mathbf{X}$ & $\mathbf{n}$ & Produkte & ${\text { Ausbeute [\% }]^{[\mathbf{b}]}}$ \\
\hline 1 & $\mathbf{1 5 1 b}$ & $\mathrm{H}$ & $\mathrm{H}$ & $\mathrm{N}$ & 1 & $\mathbf{1 1 0 b}$ & 79 \\
2 & $\mathbf{1 5 1 c}$ & $\mathrm{OMe}$ & $\mathrm{H}$ & $\mathrm{CH}$ & 1 & $\mathbf{1 1 0 c}$ & 54 \\
3 & $\mathbf{1 5 1 d}$ & $\mathrm{H}$ & $2-\mathrm{F}$ & $\mathrm{CH}$ & 1 & $\mathbf{1 1 0 d}$ & 57 \\
4 & $\mathbf{1 5 1 e}$ & $\mathrm{H}$ & $2-\mathrm{Me}$ & $\mathrm{CH}$ & 1 & $\mathbf{1 1 0 e}$ & 81 \\
5 & $\mathbf{1 5 1}$ & $\mathrm{H}$ & $2-\mathrm{CF}_{3}$ & $\mathrm{CH}$ & 1 & $\mathbf{1 1 0 f}$ & 53 \\
6 & $\mathbf{1 5 1 g}$ & $\mathrm{H}$ & $2-\mathrm{OMe}$ & $\mathrm{CH}$ & 1 & $\mathbf{1 1 0 g}$ & 53 \\
7 & $\mathbf{1 5 1 h}$ & $\mathrm{H}$ & $4-\mathrm{Ph}$ & $\mathrm{CH}$ & 1 & $\mathbf{1 1 0 h}$ & 53 \\
8 & $\mathbf{1 5 1 i}$ & $\mathrm{H}$ & $4-t \mathrm{Bu}$ & $\mathrm{CH}$ & 1 & $\mathbf{1 1 0 i}$ & 68 \\
9 & $\mathbf{1 5 1 j}$ & $\mathrm{H}$ & $\mathrm{H}$ & $\mathrm{CH}$ & 0 & $\mathbf{1 1 0 j}$ & - \\
10 & $\mathbf{1 5 1 k}$ & $\mathrm{H}$ & $\mathrm{H}$ & $\mathrm{CH}$ & 2 & $\mathbf{1 1 0 k}$ & Spuren \\
$11^{[\mathrm{c}]}$ & $\mathbf{1 5 1 i}$ & $\mathrm{H}$ & $4-t \mathrm{Bu}$ & $\mathrm{CH}$ & 1 & $\mathbf{1 1 0 i}$ & 99 \\
\hline
\end{tabular}

[a] 151 (1.00 ̈̈q.), Pd(OAc) $)_{2}(20 \mathrm{~mol} \%), \mathrm{PPh}_{3}\left(1.00\right.$ Äq.), $(n \mathrm{Bu})_{4} \mathrm{NOAc}(3.00$ Äq. $), \mathrm{DMF}, 100{ }^{\circ} \mathrm{C}, 18-20 \mathrm{~h}$; [b] Isolierte Ausbeute nach Säulenchromatographie; [c] 151 (1.00 Äq.), $\mathrm{Pd}(\mathrm{OAc})_{2}(1 \mathrm{~mol} \%), \mathrm{PPh}_{3}(5 \mathrm{~mol} \%)$, $(n \mathrm{Bu})_{4} \mathrm{NOAc}\left(3.00\right.$ Äq.), DMF, $100^{\circ} \mathrm{C}, 19.5 \mathrm{~h}$.

Am oberen D-Ring wiederum scheint der elektronenarme Pyridin-Ring reaktiver zu sein als das entsprechende Methoxy-substituierte Analogon. Bestätigt werden konnten die geringen Ausbeuten des Vierfach-Domino-Prozesses hingegen für die Substrate mit vergrößerten aliphatischen F-Ringen: Sie konnten auch in der dreifachen Domino-Reaktion nur in Spuren nachgewiesen werden. Bemerkenswert ist die Tatsache, dass sowohl im Drei- als auch im Vierfach-Prozess geringere Katalysatormengen bessere Ausbeuten liefern. Dies könnte auch die schlechteren Ausbeuten für 110b und 110d im Dreifach-Domino-Prozess erklären, die womöglich wie auch die übrigen Substrate bei einer Umsetzung mit $1 \mathrm{~mol} \% \mathrm{Pd}(\mathrm{OAc})_{2}$ bessere Ergebnisse liefern würden. Weitere Experimente in dieser Richtung wurden jedoch aus Zeitmangel nicht vorgenommen. 
Interessant ist auch ein Vergleich der Ausbeuten des Vierfach-Domino-Prozesses mit den Ergebnissen, welche sich insgesamt in den zwei Stufen der Kombination aus Sonogashira- und Dreifach-DominoReaktion ergeben. Hierdurch lässt sich untersuchen, ob es effizient ist, in der Synthese der Polyzyklen vom Typ 110 die Sonogashira-Reaktion abzutrennen oder die Umsetzung über den Vierfach-Prozess durchzuführen. Für das Pyridin-Derivat 110b „rechnet“ sich dieser zusätzliche Aufwand einer separaten Sonogashira-Reaktion nicht, wie die höheren Ausbeuten im Vierfach-Prozess belegen. Gleiches gilt für das fluorierte Molekül 110d, welches ebenfalls in besseren Ausbeuten erhalten wurde, wenn der Vierfach-Prozess zur Synthese eingesetzt wurde. Beim am D-Ring Methoxysubstituierten Derivat 110c ergibt sich eine leicht bessere Ausbeute von 36\% des kombinierten Sonogashira-Dreifach-Domino-Prozesses gegenüber $29 \%$ Ausbeute für die Vierfach-DominoReaktion. Gleiches gilt für die am H-Ring ortho-Methyl und ortho- $\mathrm{CF}_{3}$-substituierten Substrate 110e und 110f: Mit 53 bzw. 37\% Ausbeute in der Kombination von Sonogashira- und Dreifach-DominoReaktion sind auch hier die Verbesserungen gering, wenn man die 40 bzw. 35\% Ausbeute im Vierfach-Domino-Prozess gegenüberstellt. Deutlicher ist die Verbesserung beim ortho-Methoxysubstituierten Molekül 110g: Mit 38\% Ausbeute über zwei Stufen liegt das Ergebnis von Sonogashiraund Dreifach-Domino-Transformation fast 20\% über dem der Umsetzung im Vierfach-Prozess (22\% Ausbeute). Die para-Phenyl-substituierte Verbindung 110h konnte nach der Kombination aus Sonogashira- und Dreifach-Domino-Reaktion ebenfalls in höheren Ausbeuten von 51\% isoliert werden. Verglichen mit den 40\% Ausbeute im Vierfach-Domino-Prozess ist diese Steigerung jedoch eher moderat. Besonders interessant sind die Ergebnisse für die Herstellung von 110i mit einer paraständigen tert-Butyl-Gruppe: Hier wurden mit den optimierten Bedingungen mit Abstand die besten Ergebnisse im Dreifach-Domino-Prozess erzielt. Dementsprechend effizient ist eine Umsetzung in der Kombination aus Sonogashira- und Dreifach-Domino-Reaktion, die zu 89\% Ausbeute über zwei Stufen führt. Verglichen damit fallen die 37\% Ausbeute im Vierfach-Prozess deutlich geringer aus.

Somit scheint die Substitution der Vierfach-Domino-Reaktion durch eine Kombination aus Sonogashira- und Dreifach-Domino-Prozess in den meisten Fällen nicht zu so stark verbesserten Ausbeuten zu führen, dass sich der zusätzliche Aufwand lohnen würde. Werden jedoch die optimierten Bedingungen des Dreifach-Prozesses verwendet, sind die Ausbeutengewinne enorm, wie das Beispiel 110 zeigt. Hier scheint die getrennte Durchführung von Sonogashira-Reaktion und DominoTransformation von Vorteil zu sein.

Zusammenfassend konnten neun Polyzyklen des Typs 110 in Ausbeuten von bis zu 99\% synthetisiert werden. Dazu wurden sowohl eine vierfache Domino-Sonogashira/Zweifach-Carbopalladierung/C-HAktivierung (Pfad A) als auch eine dreifache Domino-Zweifach-Carbopalladierung/C-H-Aktivierung (Pfad B) eingesetzt (Abbildung 56). 
<smiles>[R1]c1ccc(I)c(Oc2ccc3ccccc3c2Br)c1</smiles>

111<smiles></smiles>

$\mathrm{Pd}(\mathrm{OAc})_{2}(1 \mathrm{~mol} \%)$

$\mathrm{PPh}_{3}(5 \mathrm{~mol} \%)$,

$(n \mathrm{Bu})_{4} \mathrm{NOAc}$,

$\mathrm{DMF}, 100^{\circ} \mathrm{C}$,

$14.5-20 \mathrm{~h}$

$\mathrm{Pd}(\mathrm{OAc})_{2}(20 \mathrm{~mol} \%)$, $\mathrm{PPh}_{3}(1.00$ Äq.), $(n \mathrm{Bu})_{4} \mathrm{NOAc}$

DMF, $100^{\circ} \mathrm{C}$,

18-20 h

B
A

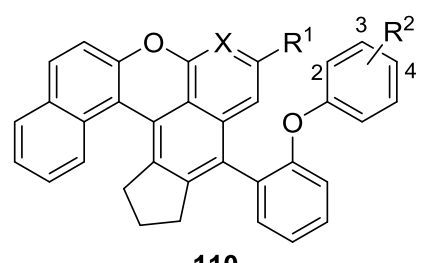

110

151<smiles></smiles>

110a

A

$73 \%$<smiles></smiles>

110d

A
B<smiles>COc1ccccc1Oc1ccccc1-c1c2c(c3c4c(cccc4oc4ccc5ccccc5c14)CCC3)CCC2</smiles>

A

$22 \%$

$53 \%$

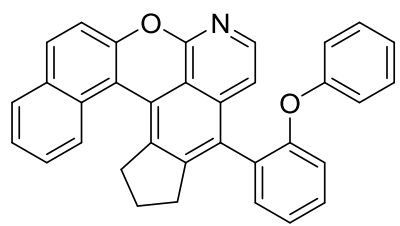

110b

$89 \%$

$79 \%$

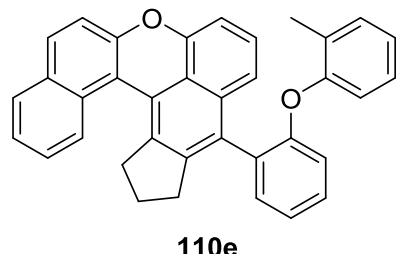

$40 \%$

$81 \%$

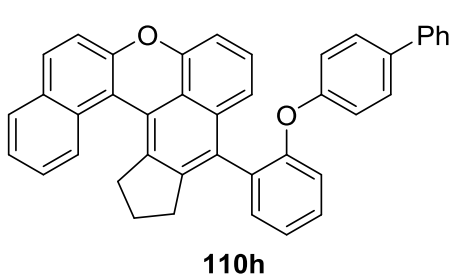

$40 \%$

$53 \%$<smiles>COc1cc2c3c(c(-c4ccccc4Oc4ccccc4)c4c(c3c1)CCC4)-c1c(ccc3ccccc13)O2</smiles>

$110 \mathrm{c}$

$29 \%{ }^{1}$

$54 \%$<smiles>FC(F)(F)c1ccccc1Oc1ccccc1-c1c2c(c3c4c(cccc14)Oc1ccc4ccccc4c1-3)CCC2</smiles>

$110 f$

$35 \%$

$53 \%$

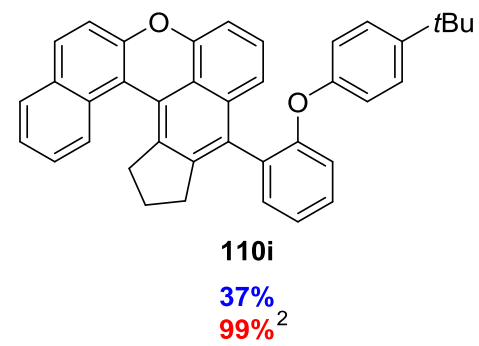

${ }^{1} \mathrm{Pd}(\mathrm{OAc})_{2}$ (20 mol\%), $\mathrm{PPh}_{3}$ (1.00 Äq.)

${ }^{2} \mathrm{Pd}(\mathrm{OAc})_{2}$ (1 $\left.\mathrm{mol} \%\right), \mathrm{PPh}_{3}(5 \mathrm{~mol} \%)$

Abbildung 56: Substratbreite der Drei- und Vierfach-Domino-Reaktion. 
Molekül 110a konnte kristallisiert und mittels Röntgenstrukturanalyse charakterisiert werden. Die Konnektivität der Verbindung wurde somit in der Festphase belegt (Abbildung 57). Unter reproduzierbaren Bedingungen wurden hierzu $5 \mathrm{mg}$ von 110a in $5 \mathrm{~mL}$ Diethylether gelöst, welcher langsam in einer $n$-Hexan-Atmosphäre evaporiert wurde. Die Bedingungen waren auf alle Derivate außer 110g übertragbar.

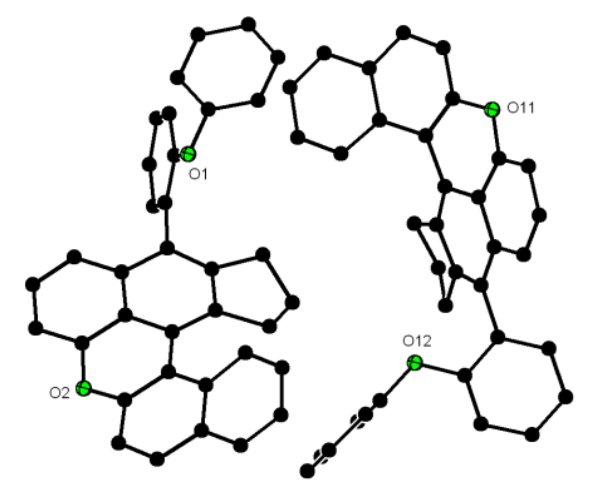

Abbildung 57: Kristallstruktur von 110a.

Im Einkristall sind den Ergebnissen der Strukturanalyse zufolge zwei Moleküle in einer triklinen Elementarzelle eingeschlossen. Wie Abbildung $57 \mathrm{zu}$ entnehmen ist, weist das zentrale Xanthenanaloge Gerüst mit den beiden Naphthylringen eine leichte Krümmung auf, die zu einer helikalen Verdrillung dieses Molekülteils führt. Deutlich zu erkennen ist auch die Envelope-Konformation des aliphatischen Cyclopentan-Rings. Eine weitere interessante Eigenschaft kommt durch die Biarylachse zwischen Xanthen-Gerüst und unterer Biphenylether-Einheit zustande: Offenbar existiert hier eine freie Drehbarkeit der beiden Molekülteile um diese Achse. Die daraus resultierenden dynamischen Effekte werden in Kapitel 3.2.7 diskutiert.

Durch die Kristallstruktur wurde Gewissheit erhalten, dass die Struktur dem Polyzyklus 110a und nicht dem tetrasubstituierten Dialken 113a entspricht. Die Bildung von 113a wurde bei keinem der untersuchten Substrate beobachtet und konnte auch durch veränderte Reaktionsbedingungen nicht herbeigeführt werden. So lieferten sowohl alternative Liganden (vgl. Tabelle 3) als auch Umsetzungen über $10 \mathrm{~h}$ bei $40,60,140$ und $160{ }^{\circ} \mathrm{C}$ in der Mikrowelle ausschließlich 110a. Diese Reaktionen wurden zudem bei Dunkelheit durchgeführt. Somit konnte auch ein Lichteinfluss auf die Doppelbildungsisomerisierung ausgeschlossen werden, die im postulierten Mechanismus als ausschlaggebend für die exklusive Bildung von 110a angenommen wird (vgl. folgendes Kapitel).

\subsubsection{Mechanistische Überlegungen}

Vermutlich folgt der Mechanismus der Domino-Sonogashira/Zweifach-Carbopalladierung/C-HAktivierung weitgehend dem für diese Reaktionen postulierten Muster (Abbildung 58): Zunächst kommt es zu einer Sonogashira-Reaktion, die Aryliodid 111a mit Dialkin 112a zum Produkt 151a kuppelt. 


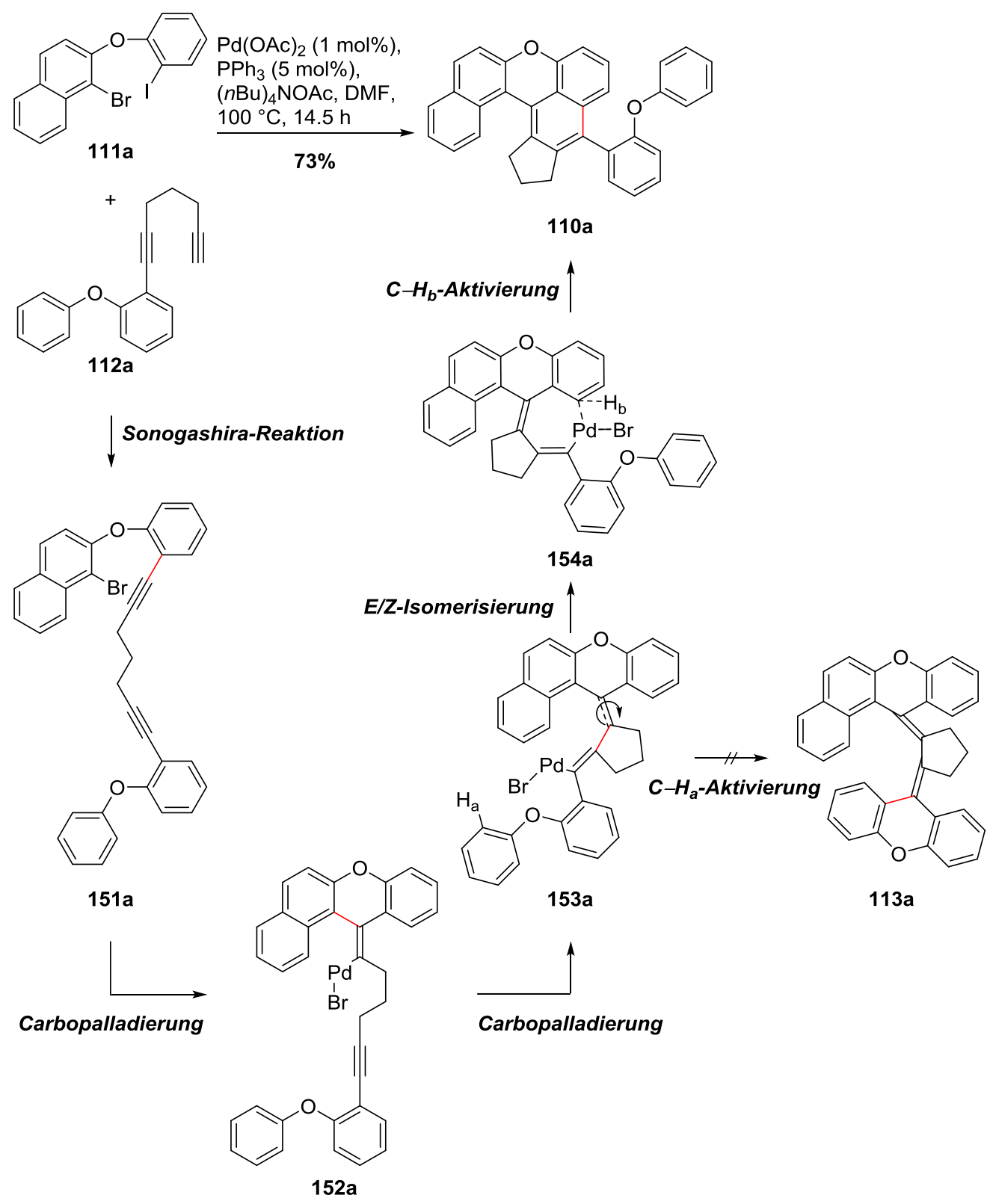

Abbildung 58: Postulierter Mechanismus der Domino-Sonogashira/Zweifach-Carbopalladierung/C-HAktivierung (Liganden und beteiligte Basen wurden der Übersichtlichkeit halber weggelassen).

Nach der oxidativen Addition und anschließender Carbopalladierung wird Vinyl-Palladium-Spezies 152a mit einem in der intramolekularen Carbopalladierung gebildeten Heterozyklus erhalten. In einer zweiten Carbopalladierung wird Dialken-Spezies 153a gebildet, die einen aliphatischen Fünfring beinhaltet. Im Folgenden kommt es jedoch nicht zu einer C-H-Aktivierung an Proton $\mathrm{H}_{\mathrm{a}}$ und damit zur Bildung des Dialkens 113a. Stattdessen unterläuft Dialken-Intermediat 153a wahrscheinlich eine E/Z-Isomerisierung zu 154a. Diese bringt das an 154a gebundene Palladium in die räumliche Nähe des oberen Benzolrings und ermöglicht eine $\mathrm{C}-\mathrm{H}$-Aktivierung an Wasserstoffatom $\mathrm{H}_{\mathrm{b}}$ die mit einhergehender Aromatisierung zu Polyzyklus 110a führt. Vermutlich ist die Bildung von 110a wegen ebendieser Aromatisierung energetisch bevorzugt. Für die C-H-Aktivierung kann aufgrund des 
beteiligten Acetats ein entsprechender basengestützter Mechanismus angenommen werden (vgl. Abbildung 14).

\subsubsection{Diskussion ausgewählter analytischer Daten von Verbindung 110a}

Im Folgenden sollen die analytischen Daten von Verbindung 110a diskutiert werden. Die NMRSpektren wurden wegen dynamischer Effekte der Verbindung bei erhöhten Temperaturen aufgenommen, um gut aufgelöste Signale zu erhalten. Über COSY-, HSQC- und HMBC-Spektren konnten die Signale den Atomen im Molekül zugeordnet werden.

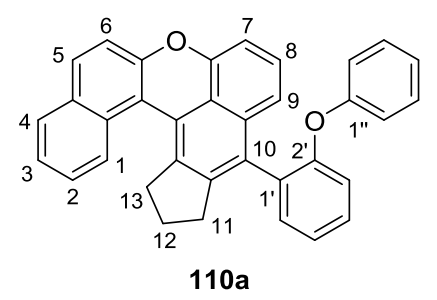

Das ${ }^{1} \mathrm{H}$-NMR-Spektrum (Abbildung 59) wurde in $\mathrm{C}_{2} \mathrm{D}_{2} \mathrm{Cl}_{4}$ gemessen. Die Verbindung wies starke dynamische Effekte auf, die sich in Form von verbreiterten Signalen im NMR-Spektrum äußerten. Deshalb wurde das ${ }^{1} \mathrm{H}-\mathrm{NMR}$-Spektrum bei $100{ }^{\circ} \mathrm{C}$ aufgenommen, um scharfe Koaleszenzsignale zu erhalten.
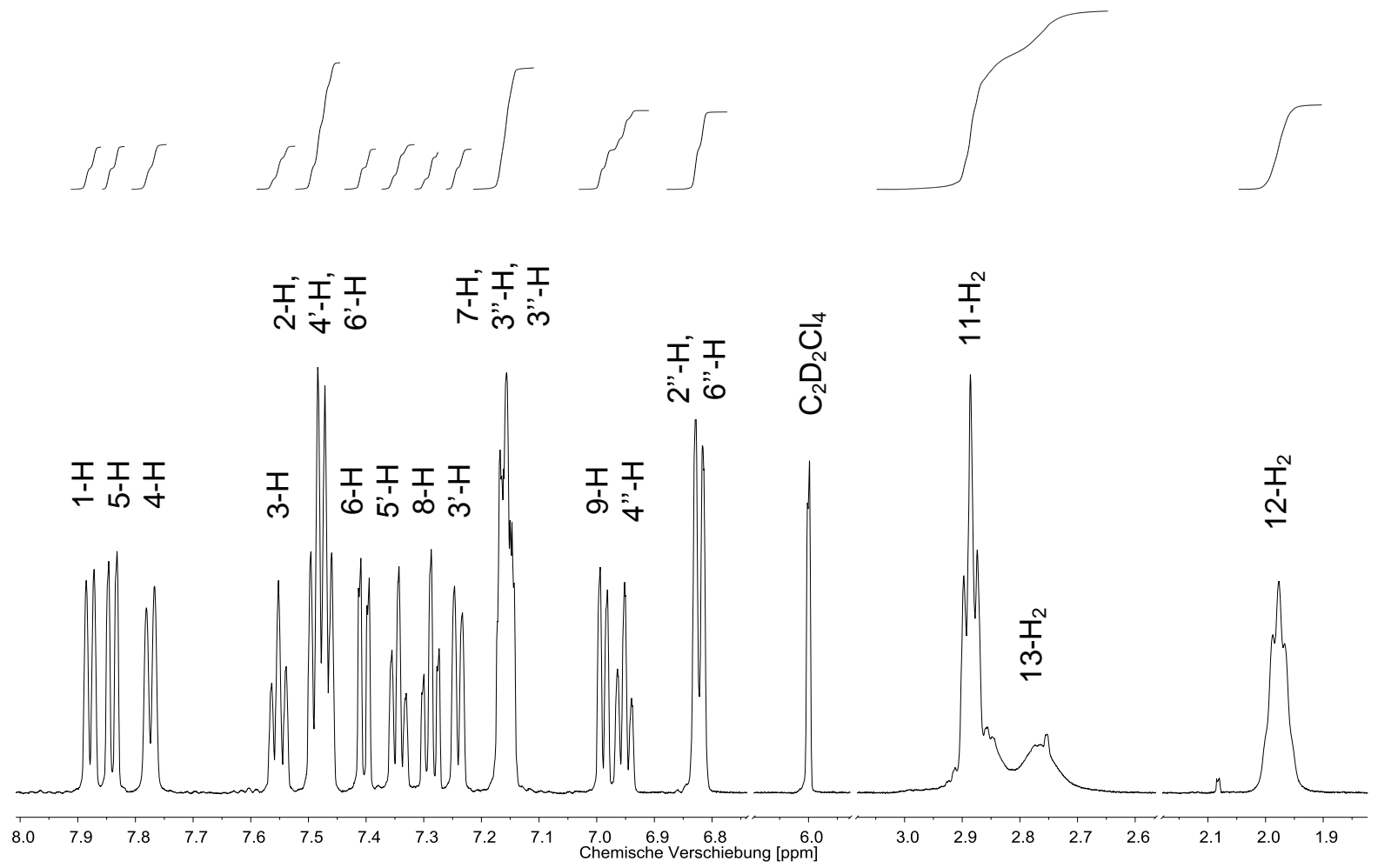

Abbildung 59: ${ }^{1} \mathrm{H}-\mathrm{NMR}-$ Spektrum von Verbindung 110a in $\mathrm{C}_{2} \mathrm{D}_{2} \mathrm{Cl}_{4}$ bei $100{ }^{\circ} \mathrm{C}$ und $600 \mathrm{MHz}$. 
Im Spektrum resonieren im Hochfeldbereich die Signale der Methylengruppen des aliphatischen Fünfrings, $12-\mathrm{H}_{2}$ und $13-\mathrm{H}_{2}$, bei $\delta=1.90-2.05$ und $2.70-2.89 \mathrm{ppm}$, die wegen der dynamischen Effekte nur als Multipletts zu erkennen sind. Die Resonanzen Protonen der Methylengruppe in 11-Position sind als Dublett von Dubletts bei $\delta=2.88 \mathrm{ppm} \mathrm{zu}$ beobachten. Die Signale der aromatischen Wasserstoffatome wurden ab $\delta=6.82 \mathrm{ppm}$ detektiert, wo die chemisch äquivalenten Protonen 2"'-H und 6"'-H als Dublett resonieren. Das Signal des Protons 4"'-H desselben Ringes ist bei $\delta=6.95 \mathrm{ppm}$ als Triplett zu sehen. Vermutlich unterliegen diese Protonen am wenigsten dem Einfluss benachbarter aromatischer Ringe und sind daher weniger stark entschirmt. Mit 9-H folgt bei $\delta=6.99 \mathrm{ppm}$ das Signal eines Wasserstoffatoms des Xanthen-Gerüsts, welches als Dublett identifiziert wurde. Auch dieses Atom wird von sterischen und anisotropen Effekten benachbarter aromatischer Ringe wahrscheinlich weniger beeinflusst als die anderen. Als Multiplett bei $\delta=7.11-$ $7.20 \mathrm{ppm}$ resonieren die Protonen des Xanthen-Gerüsts 7-H sowie des Phenoxy-Ringes 3"'-H und 5"'-H. Weiter im Tieffeld bei $\delta=7.24 \mathrm{ppm}$ ist das Signal des Protons 3'-H des biarylisch an das Xanthen-Gerüst gebundenen Benzolringes zu finden, welches durch Kopplung mit 4'-H zum Dublett aufspaltet. Bei $\delta=7.28$ ppm ist als Dublett von Dubletts das Signal von 8-H zu erkennen. Proton 5'-H resoniert als Triplett bei $\delta=7.34 \mathrm{ppm}$, während 6-H als Bestandteil des Naphthylsystems als Dublett von Dubletts bei $\delta=7.40 \mathrm{ppm}$ zu finden ist. Es folgen die Protonen 2-H, 4'-H und 6'-H, die trotz ihrer Inäquivalenz als Pseudo-(Dublett von Dubletts) zu erkennen sind. Die Signale des über eine Biarylbrücke gebundenen Benzolrings werden dabei vermutlich durch den Einfluss der benachbarten Ringe des Xanthen-analogen aromatischen Systems entschirmt. Die Protonen 3-H und 4-H des Naphthylsystems wiederum resonieren als Triplett bzw. Dublett bei $\delta=7.55$ bzw. 7.77 ppm weit ins Tieffeld verschoben. Dies gilt auch für die ebenfalls zum Naphthylsystem gehörenden Wasserstoffatome 5-H und 1-H, deren Signale bei $\delta=7.84$ bzw. $7.88 \mathrm{ppm}$ zu finden sind und die als Dublett von Dubletts bzw. einfaches Dublett resonieren. Im Napthylsystem ist der Ringstromeffekt stärker als bei einfachen Aromaten. Daher werden die Protonen dieses aromatischen Systems am stärksten entschirmt.

Im ${ }^{13} \mathrm{C}$-NMR-Spektrum (Abbildung 60), welches ebenfalls bei $100{ }^{\circ} \mathrm{C}$ aufgenommen wurde, resonieren im Hochfeldbereich die Signale der Kohlenstoffatome des aliphatischen Fünfrings C-12, C-11 und C-13 bei $\delta=26.3,32.6$ und 36.5 ppm. Das Signal des Atoms C-9, welches zum XanthenGerüst gehört, ist bei $\delta=106.6 \mathrm{ppm}$ zu finden. Ebenfalls relativ weit zu hohen Feld verschoben sind das Brückenkopfatome C-13d, C-6 und C-7 bei 115.8, 116.8 und 117.7 ppm. Diese Kohlenstoffatome unterliegen wahrscheinlich dem polarisierenden Einfluss des nahegelegenen Ether-Sauerstoffs, der eine erhöhte Ladungsdichte in diesen Positionen verursacht. Es folgt das Signal der chemisch äquivalenten C-Atome C-2'" und C-6', die zur Phenoxy-Einheit von 110a gehören und bei $\delta=118.3 \mathrm{ppm}$ resonieren. Auch diese besitzen eine erhöhte Ladungsdichte aufgrund des nahen Sauerstoffatoms und sind daher stärker abgeschirmt. Das Signal von C-3' wiederum ist bei $\delta=119.5 \mathrm{ppm} \mathrm{zu}$ finden, während das des Brückenkopfatoms C-4a bei $\delta=121.8 \mathrm{ppm}$ abgebildet 
wird. Bei $\delta=122.4,123.4$ und 124.0 ppm resonieren die Kohlenstoffe C-4', C-5' und C-2. Es folgen C-3 und C-13b, deren Signale sich bei $\delta=124.8$ ppm überlagern. Die C-Atome des Xanthen-Gerüsts C-8, C-4 und C-1 sind den Signalen bei $\delta=125.3,125.5$ und 127.6 ppm zuzuordnen. Die Kohlenstoffe des zweiten Naphthylsystems C-6c sowie die des verknüpften Benzolringes C-4'/C-6' resonieren bei $\delta=128.3,128.5$ und $132.0 \mathrm{ppm}$, wobei die Signale bei $\delta=128.5$ und $132.0 \mathrm{ppm}$ nicht exakt den Atomen C-4' und C-6' zugeordnet werden konnten. Bei $\delta=128.8$ ppm ist das Signal der chemisch äquivalenten Atome C-3' ' und C-5' 'zu finden. Diese besitzen wie auch C-4' und C-6' aufgrund des nahegelegenen polarisierenden Ether-Sauerstoffs eine geringere Ladungsdichte und sind daher stärker entschirmt. C-5 und Brückenkopfatom C-13c resonieren beide bei $\delta=129.5 \mathrm{ppm}$. Es folgen die Atome der Biarylbrücke C-1' und C-10 bei $\delta=130.5$ und $131.0 \mathrm{ppm}$. Weitere Signale von Brückenatomen sind bei $\delta=133.1,135.4$ und 145.4 zu finden, die den Atomen C-9a, C-10a und C-13a entsprechen. Letztere sind dabei nicht eindeutig zuzuordnen. Am weitesten im Tieffeld resonieren die Kohlenstoffe der enthaltenen Biaryletherbrücken. So befinden sich die Signale von $6 \mathrm{~b}$ und $6 \mathrm{a}$ des Xanthen-Gerüsts bei $\delta=150.7$ und 153.3 ppm, während C-2' und C-1', bei $\delta=155.0$ und $157.2 \mathrm{ppm}$ resonieren. Hier macht sich ebenfalls der entschirmende Effekt der benachbarten Sauerstoffatome bemerkbar.

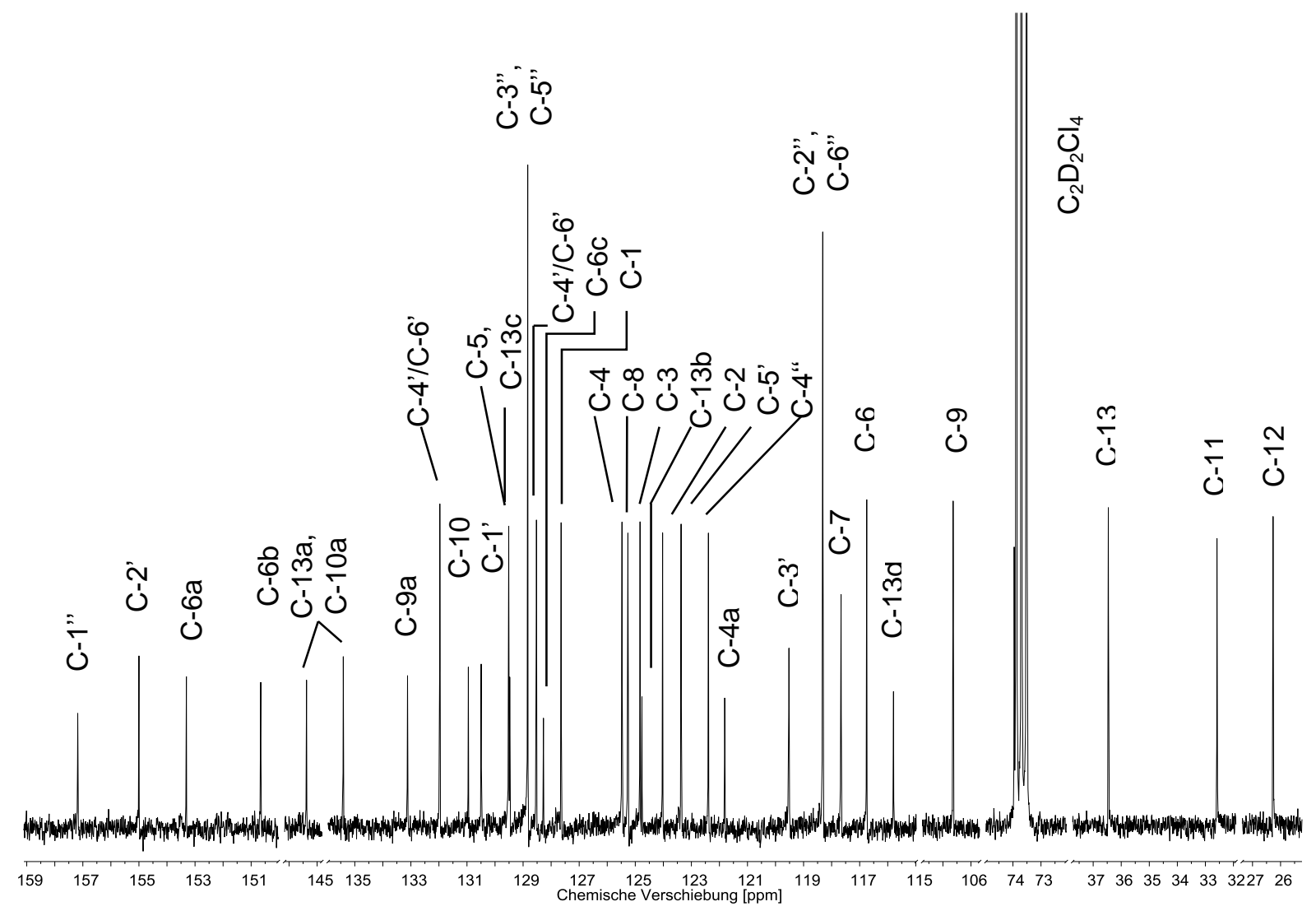

Abbildung 60: ${ }^{13} \mathrm{C}-\mathrm{NMR}$-Spektrum von Verbindung 110a in $\mathrm{C}_{2} \mathrm{D}_{2} \mathrm{Cl}_{4}$ bei $100{ }^{\circ} \mathrm{C}$.

Im ESI- und ESI-HRMS-Spektrum konnten zudem molekulare Masse und Summenformel von 110a bestätigt werden. Bei $m / z=476.2$ und 499.2 wurden die Signale des Molekülions $[\mathrm{M}]^{+}$sowie seines Natrium-Addukts $[\mathrm{M}+\mathrm{Na}]^{+}$gefunden. Im ESI-HRMS wurde zudem die Summenformel $\mathrm{C}_{35} \mathrm{H}_{24} \mathrm{O}_{2}$ 
bestätigt, die einer berechneten Masse von $m / z=476.1771$ für das Molekülion entspricht. Gefunden wurde eine Masse von $m / z=476.1767$.

Im IR-Spektrum sind besonders die Absorptionsbanden bei $\tilde{v}=1251,1263,1251,1236$ und $1217 \mathrm{~cm}^{-1}$ charakteristisch, da sie den Schwingungen der aromatischen Ether-Bindungen zugeordnet werden können. Daneben ist bei $\tilde{v}=1572 \mathrm{~cm}^{-1}$ die Resonanz der Valenzschwingung der aromatischen $\mathrm{C}-\mathrm{C}$ Bindungen zu erkennen. Auch die Streckschwingungen der aromatischen C-H-Bindungen sind durch Banden bei $\tilde{v}=810,747,735$ und $690 \mathrm{~cm}^{-1}$ vertreten.

Im UV/Vis-Spektrum sind zunächst Absorptionsbanden bei $\lambda=214$ und $266 \mathrm{~nm}$ erkennbar, die $\pi \rightarrow \pi^{*}$ Übergängen in einfachen aromatischen Systemen entsprechen. Charakteristisch für die Verbindungen dieses Typs sind aber vor allem die Banden bei $\lambda=326,353$ und $397 \mathrm{~nm}$. Sie resultieren vermutlich aus $\pi \rightarrow \pi^{*}$-Übergängen im vergrößerten aromatischen System, die durch die Annäherung von HOMO und LUMO in konjugierten Systemen ermöglicht werden. Eine genauere Untersuchung erfolgt im folgenden Kapitel.

\subsubsection{Untersuchung der Fluoreszenz am Beispiel von Verbindung 110a}

Alle Moleküle des Typs 110 weisen Fluoreszenzeigenschaften auf, die sich in Lichtemissionen im selben Wellenlängenbereich äußern. Auch bei dieser Verbindungsklasse entspringt diese Fluoreszenz vermutlich dem Xanthen-Gerüst, welches Rhodamin- und Fluorescein-Farbstoffen relativ ähnlich ist (vgl. Abbildungen 20 und 21). Durch eine Vergrößerung des aromatischen Systems gegenüber den Verbindungen vom Typ 107 sind Absorption und Emission in den Bereich längerer Wellenlängen verschoben.

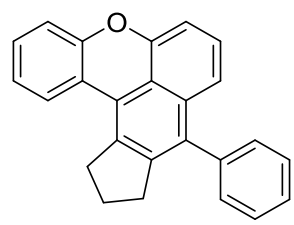

$107 d$

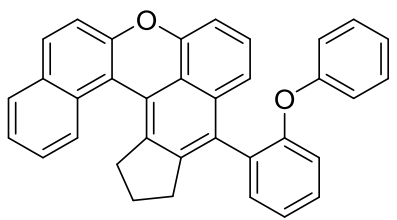

$110 a$

Grundlage der Fluoreszenzmessungen war das UV/Vis-Spektrum von Verbindung 110a (Abbildung 61). Gegenüber den Vorläufermolekülen weist die Verbindung neue, stark zu längeren Wellenlängen verschobene Absorptionsbanden auf, unter anderem bei $\lambda=326,353$ und $397 \mathrm{~nm}$. Die Wellenlänge bei $\lambda=397 \mathrm{~nm}$ besitzt darunter die stärkste Absorptionsbande und wurde bei der Fluoreszenzmessung als Einstrahlungswellenlänge verwendet. So konnte nach Bestrahlung der Substanz mit Licht von $\lambda=397 \mathrm{~nm}$ Wellenlänge ein starkes Fluoreszenzsignal detektiert werden (Abbildung 62). Das Emissionsmaximum liegt bei $\lambda=481 \mathrm{~nm}$. Damit ergibt sich ein Stokes-Shift von $84 \mathrm{~nm}$, der größer ist als die $72 \mathrm{~nm}$ bei Verbindung 107d. Des Weiteren liegen die Wellenlängen der Absorption und Emission von 110a näher am bzw. weiter im Vis-Bereich: 110a absorbiert violettes 
Licht und emittiert im Bereich zwischen blauem und grünem Licht, während 107d Licht im UVBereich bei $\lambda=367 \mathrm{~nm}$ absorbiert und blaues Licht bei $\lambda=439 \mathrm{~nm}$ emittiert.

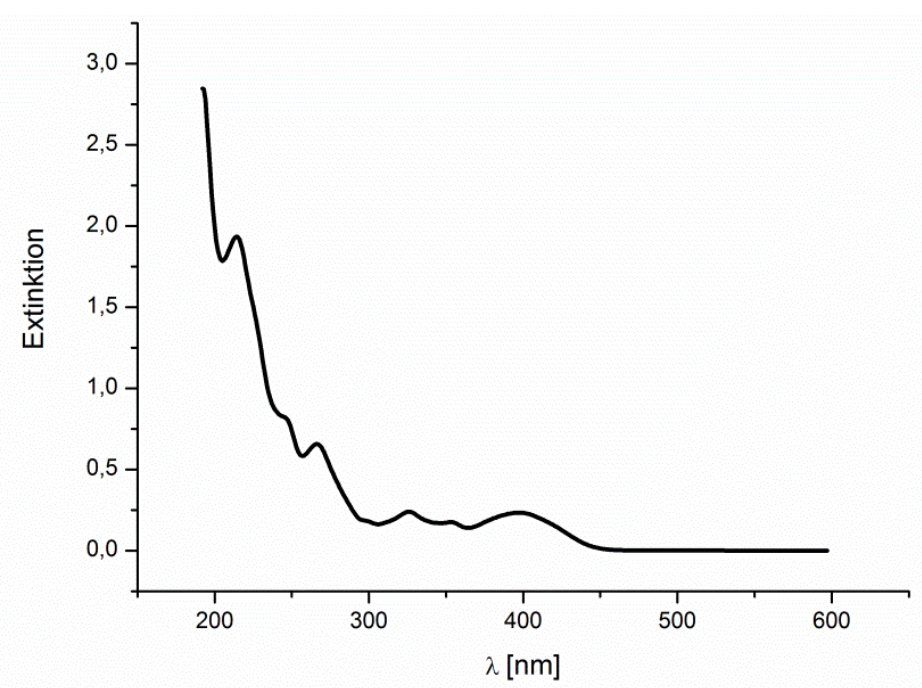

Abbildung 61: UV/Vis-Spektrum von Verbindung 110a in Acetonitril.

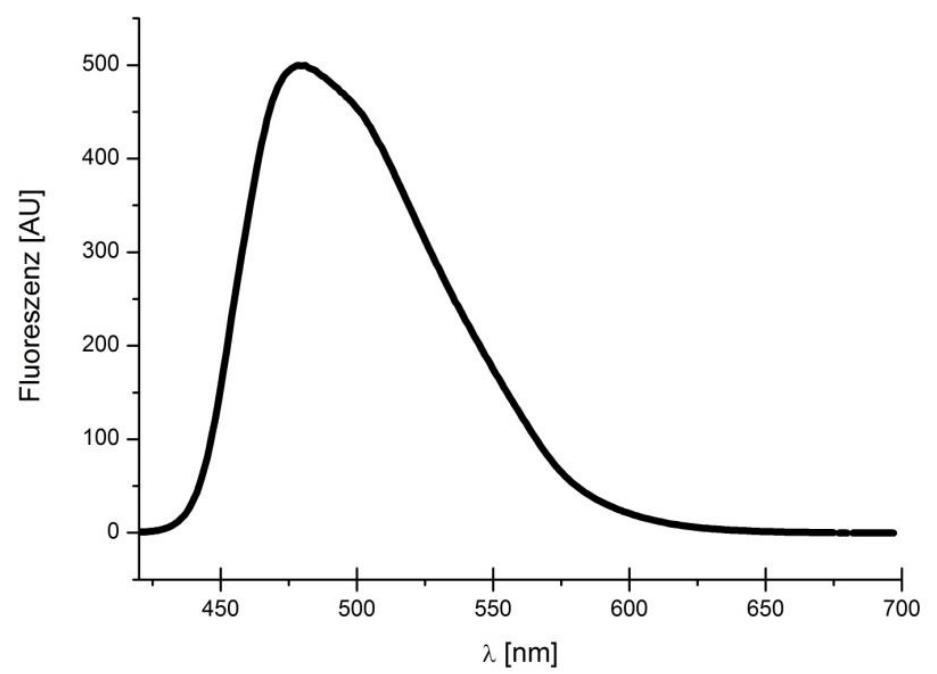

Abbildung 62: Fluoreszenzspektrum von Verbindung 110a in Acetonitril (Anregungswellenlänge: $\lambda=397 \mathrm{~nm}$; $\left.c=3.43 \times 10^{-4} \mathrm{~mol} / \mathrm{L}\right)$.

Die Wellenlängen der Absorption und Emission entsprechen ebenfalls ungefähr denen einfacher Cumarin-Derivate (vgl. Abbildung 18), wobei sie etwas weiter im Vis-Bereich liegen. Damit wären Moleküle des Typs $\mathbf{1 1 0}$ möglicherweise ebenso als Fluoreszenzmarker für Proteine oder als fluorophore Substrate für Enzyme von Interesse. Dazu wären weitere Funktionalisierungen vonnöten. Ebenso wäre auch eine Verschiebung der Fluoreszenzemission in noch längerwellige Bereiche von Interesse. Beides soll in den Kapiteln 3.2.8 und 3.2.9 näher erläutert werden. 


\subsubsection{Untersuchung der dynamischen Eigenschaften am Beispiel von}

\section{Verbindung 110a}

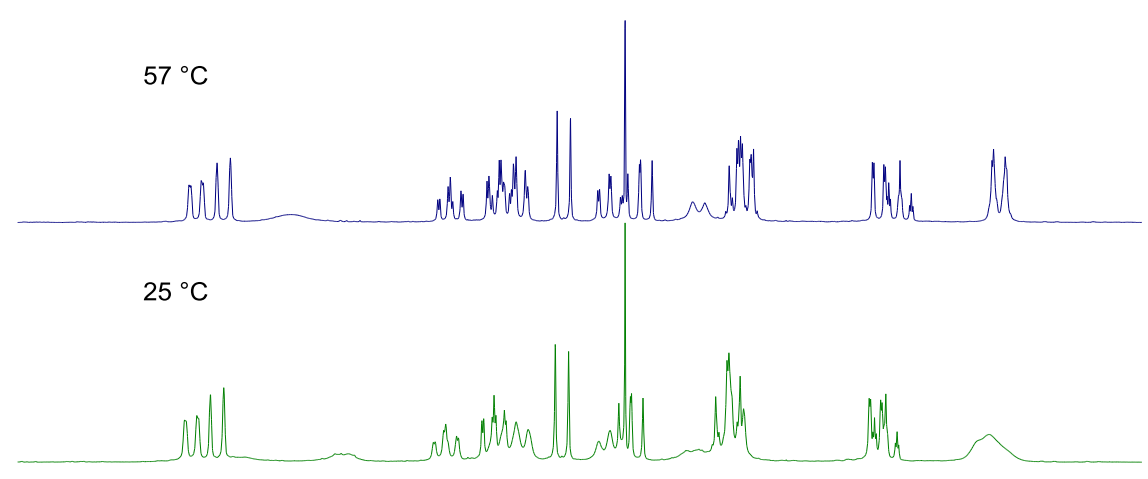

$-37^{\circ} \mathrm{C}$

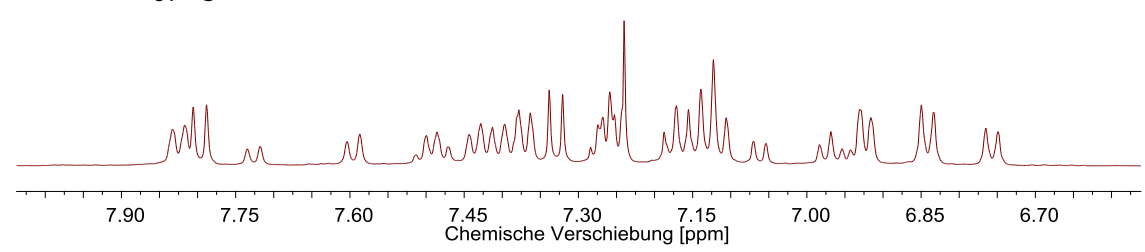

Abbildung 63: ${ }^{1} \mathrm{H}-\mathrm{NMR}-$ Spektrum von Verbindung 110a in $\mathrm{CDCl}_{3}$ bei verschiedenen Temperaturen (aromatischer Bereich).

Die Verbindungen des Typs 110 weisen allesamt eine strukturelle Labilität auf, die sich in dynamischen Effekten in den NMR-Spektren äußert. So konnten bei verschiedenen Temperaturen verschiedene Signalschärfen detektiert werden (Abbildung 63). Bei Raumtemperatur sind zum Teil unscharfe Signale zu beobachten, während bei $57^{\circ} \mathrm{C}$ Koaleszenzsignale erkennbar sind. Diese spalten bei einer Temperatur von $-37{ }^{\circ} \mathrm{C}$ in die den jeweiligen Konformeren entsprechenden Signale auf. Dieses Vorhandensein zweier Konformationen resultiert vermutlich aus der Rotation um die BiarylAchse in den Molekülen des Typs 110 (Abbildung 64, vgl. auch Kristallstruktur Abbildung 57).

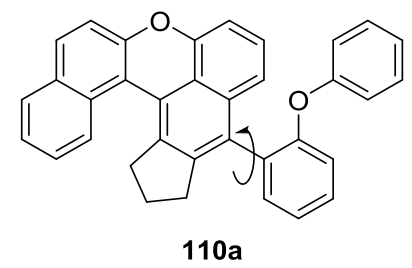

Abbildung 64: Rotation um die Biarylachse am Beispiel von Verbindung 110a.

Um die Energiebarriere dieser Rotation zu untersuchen, wurden entsprechende NMR-Experimente durchgeführt. Dazu wurden NOESY-Messungen bei verschiedenen Mischzeiten durchgeführt $\left(10^{-2} \mathrm{msec}\right.$ (Referenzzeit); $125 \mathrm{msec}, 250 \mathrm{msec}, 500 \mathrm{msec}, 1000 \mathrm{msec}$ ). Anschließend wurden die Amplituden der Diagonal- und Kreuz-Peaks der Signale bei $\delta=1.6$ und $2.1 \mathrm{ppm}$ bestimmt und diese benutzt, um mittels des Programms „Exsycalc“ der Firma „MestreLab Research“ die entsprechenden 
Geschwindigkeitskonstanten $k$ des Austauschs zwischen den Signalen zu bestimmen (eventuelle dynamische Effekte durch den aliphatischen Fünfring wurden dabei vernachlässigt). Über die EyringGleichung wurden anschließend die Übergangsenergien $\Delta G^{\ddagger}$ berechnet (Tabelle 6). Die Berechnungen ergaben einen Durchschnittswert von $\Delta G^{\ddagger}=69.7 \pm 2.3 \mathrm{~kJ} / \mathrm{mol}$. Damit liegt die Energiebarriere der Rotation in einem Bereich, der bei Raumtemperatur keine Auflösung der Signale der beiden Konformere im ${ }^{1}$ H-NMR zulässt. Dieses konnte durch die Temperatur-Experimente bestätigt werden (vgl. Abbildung 63).

Tabelle 6: Berechnung der Übergangsenergien $\Delta G^{\ddagger}$.

\begin{tabular}{|c|c|c|c|}
\hline Eintrag & Mischzeit [msec] & $k\left[\sec ^{-1}\right]$ & $\Delta G^{\ddagger}[\mathrm{kJ} / \mathrm{mol}]$ \\
\hline 1 & 1000 & 0.74 & 72.9 \\
\hline 2 & 500 & 1.88 & 70.6 \\
\hline 3 & 250 & 4.41 & 68.5 \\
\hline 4 & 125 & 9.06 & 66.8 \\
\hline
\end{tabular}

Eyring-Gleichung: $\Delta G^{\neq}=\frac{k_{B} T}{h}-R T \ln K$

$k_{B}$ : Boltzmann-Konstante; $h$ : Plancksches Wirkungsquantum; $T$ : Temperatur; $R$ : universelle Gaskonstante; $K$ : Gleichgewichtskonstante (abhängig von $k$ )

\subsubsection{Untersuchungen zur Synthese weiterer Aryliodide vom Typ 111}

Im Rahmen der Synthese der fluoreszenzaktiven Farbstoffe des Typs $\mathbf{1 1 0}$ sollten auch weitere Aryliodide vom Typ 111 hergestellt werden, um das Substratspektrum der Domino-Reaktion zu erweitern. Zum einen sollte ein Fluoreszenzfarbstoff mit vergrößertem aromatischem System synthetisiert werden, das zu einem bathochromen Effekt bei der Fluoreszenz führen sollte. Deshalb wurde zunächst das kommerziell erhältliche Phenanthrol 155 in einer literaturbekannten Reaktion selektiv mit $N$-Bromsuccinimid (NBS) zu 156 bromiert (Abbildung 65). ${ }^{78}$

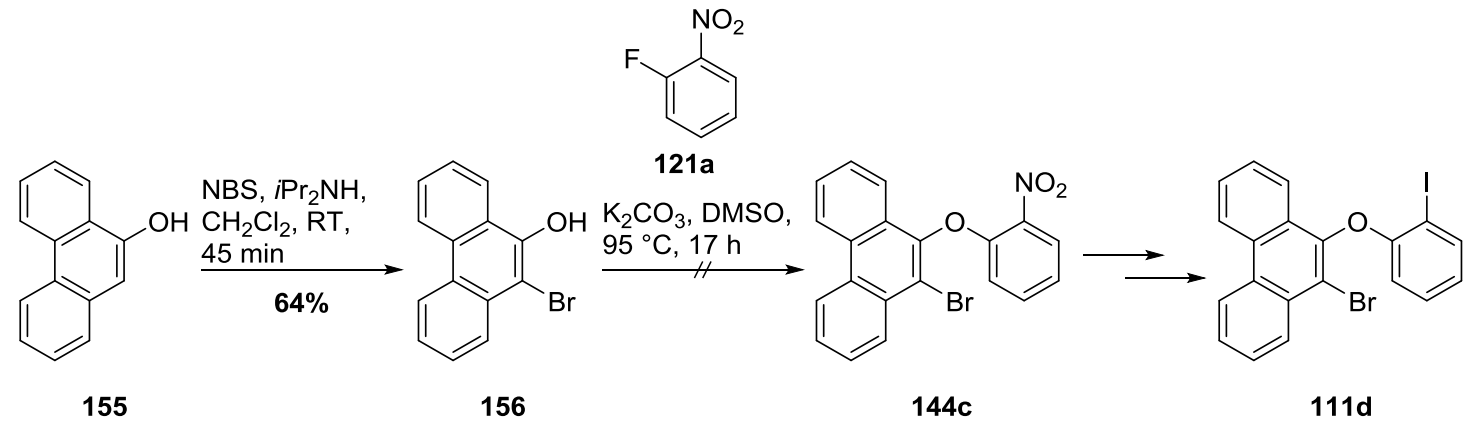

Abbildung 65: Untersuchung zur Synthese des Aryliodids 111d. 
Die nachfolgende Kopplung mit Fluornitrobenzol 121a, die unter den bereits beschriebenen Bedingungen zum Biarylether 144c führen sollte, gelang jedoch nicht. Vermutlich war das im Verlauf der Substitution gebildete Phenanthrolat-Ion zu stark stabilisiert, um weitere Reaktionen einzugehen. Die Synthese von Aryliodid 111d gelang daher nicht.

In weiteren Experimenten wurde Synthese des Aryliodids 111e mit einer Methylen-Sauerstoff-Brücke durchgeführt. Die Domino-Reaktion dieser Verbindung mit dem Dialkin 112a könnte zu Polyzyklus 110n mit einem Oxepin-Ring führen (Abbildung 66).

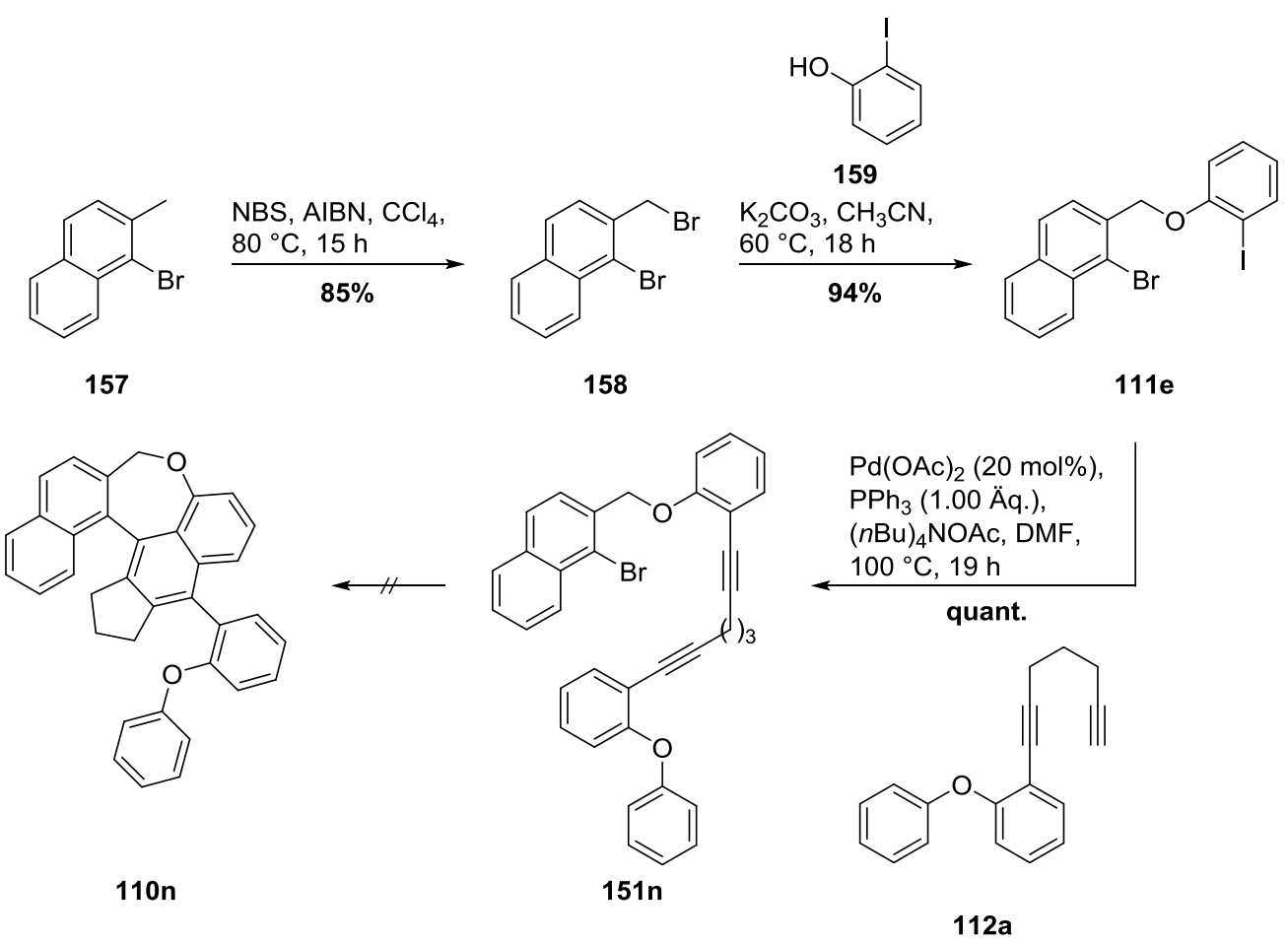

Abbildung 66: Untersuchungen zur Synthese von Siebenring-Derivaten der Polyzyklen (110n).

Die Synthese des Aryliodids 111e begann mit der Bromierung des kommerziell erhältlichen Naphthalin-Derivats 157 mit $N$-Bromsuccinimid (NBS) unter Bildung des Dibromids 158 in 85\% Ausbeute. ${ }^{79}$ Dieses wurde anschließend in einer nukleophilen aromatischen Substitution mit dem kommerziell erhältlichen 2-Iodphenol (159) zu Aryliodid 111e in 94\% Ausbeute gekoppelt. Eine Umsetzung zum Domino-Produkt 110n unter den bisher verwendeten Bedingungen gelang dagegen nicht. Stattdessen wurde das entsprechende Sonogashira-Produkt 151n in quantitativer Ausbeute erhalten. Eine Umsetzung dieses Produkts unter den Bedingungen für die Dreifach-Domino-Reaktion mit $20 \mathrm{~mol} \% \mathrm{Pd}(\mathrm{OAc})_{2}$ und 1.00 Äq. $\mathrm{PPh}_{3}$ mit $(n \mathrm{Bu})_{4} \mathrm{NOAc}$ als Base in DMF führte jedoch auch bei erhöhten Temperaturen von $120{ }^{\circ} \mathrm{C}$ nicht zum gewünschten Domino-Produkt 110n. Stattdessen wurde nach dünnschichtchromatographischer Analyse nur ein Produktgemisch erhalten. 


\subsubsection{Untersuchungen zur Synthese funktionalisierter Fluoreszenzfarbstoffe}

Weitere Untersuchungen befassten sich mit der Funktionalisierung der Fluoreszenzfarbstoffe des Typs 110. Um eine Anbindung des Fluorophors an eine zu untersuchende Verbindung zu ermöglichen, ist eine entsprechende Funktionalisierung des Fluorophors vonnöten. Als Ausgangsstoff schien der Farbstoff 110c geeignet. Die Spaltung des in 110c enthaltenen Methylethers erfolgte mit einem Überschuss an Natriumthioethanolat in DMF bei hohen Temperaturen (Abbildung 67). Da die nachfolgende phenolische Spezies 160 vermutlich aufgrund ihrer geringen Stabilität in Lösung nicht in reiner Form isoliert werden konnte, wurde sie direkt weiter umgesetzt. Es folgte eine Veresterung mit Trifluormethansulfonsäure-Anhydrid, deren Zielverbindung 161 in $46 \%$ Ausbeute über zwei Stufen erhalten wurde.
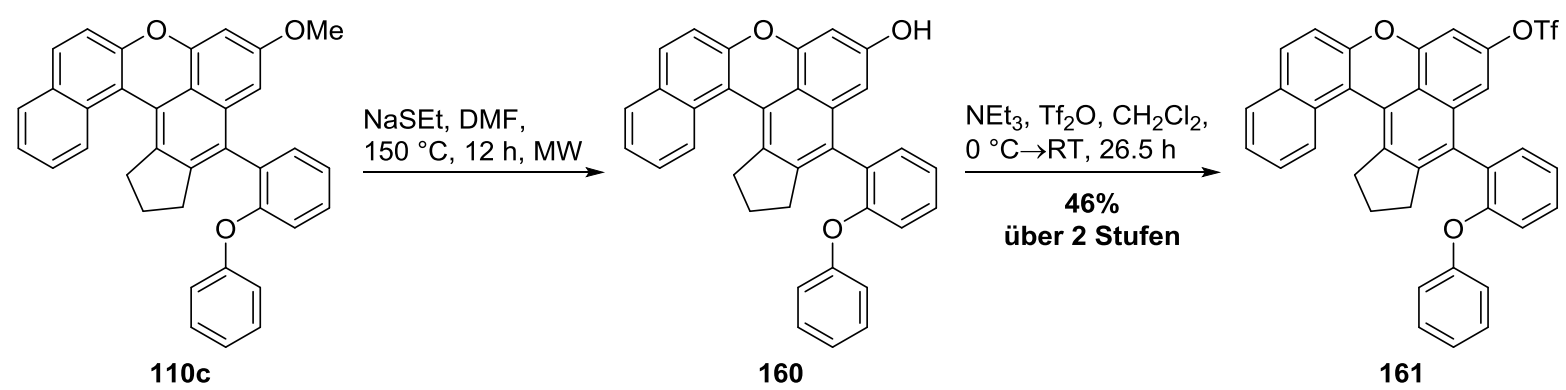

Abbildung 67: Funktionalisierung von Verbindung 110c.

Somit wurden die Voraussetzungen für weitergehende Funktionalisierungen geschaffen. Zum einen wurde gezeigt, dass die Hydroxyl-Gruppe in $\mathbf{1 6 0}$ weiter funktionalisiert werden kann, womit eine Anbindung des Fluorophors beispielsweise an $\mathrm{zu}$ untersuchende biologisch aktive Substrate ermöglicht wird. Zum anderen eröffnet das synthetisierte Triflat die Möglichkeit zur Funktionalisierung von 161, etwa durch Kreuzkupplungsreaktionen.

\subsubsection{Untersuchungen zur Synthese dimerer Strukturen}
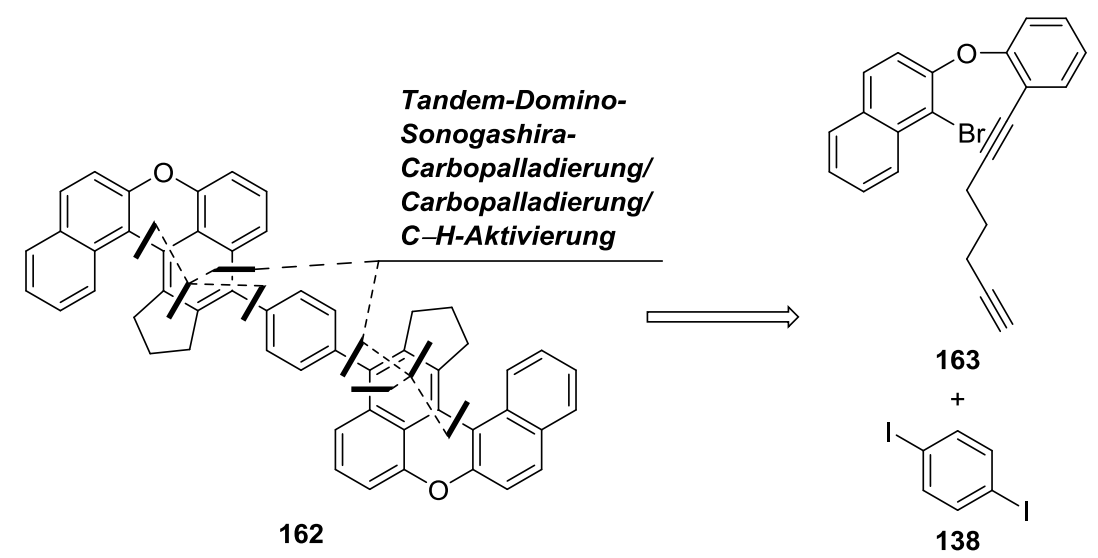

Abbildung 68: Retrosynthetischer Ansatz zur Synthese von Verbindung 162. 
Die entwickelte Domino-Sonogashira/Zweifach-Carbopalladierung/C-H-Aktivierung von Aryliodiden 111 und Dialkinen 112 zu Polyzyklen des Typs 110 sollte auch über Zweifach-Reaktionen zur Bildung dimerer Strukturen des Typs 162 eingesetzt werden (Abbildung 68). Retrosynthetisch lässt sich 162 hierbei auf Diiodbenzol 138 und Dialkin 163 zurückführen.

1,4-Diiodbenzol (138) war kommerziell verfügbar. Die Synthese des Dialkins 163 erfolgte über eine Sonogashira-Reaktion, die das bereits synthetisierte Aryliodid 111a mit einem Überschuss 1,6-Heptadiin (7 Äq.) kuppelte (Abbildung 69). Das Produkt 163 wurde dabei in quantitativer Ausbeute in einer Reinheit von etwa 90\% erhalten.
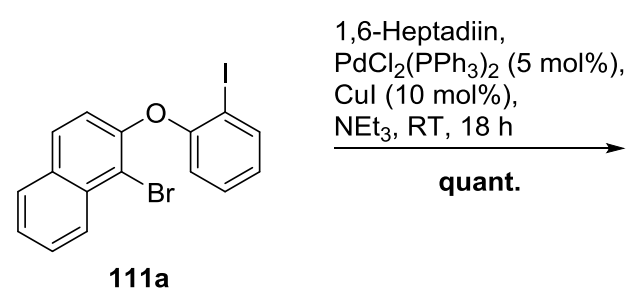

quant.

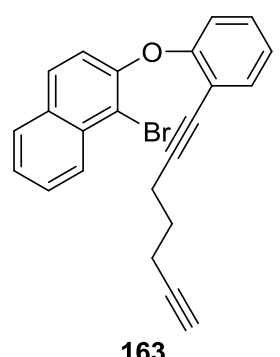

163

Abbildung 69: Synthese des Dialkins 163.

Die anschließende Tandem-Domino-Reaktion erfolgte unter den bereits bekannten Bedingungen mit $10 \mathrm{~mol} \% \mathrm{Pd}(\mathrm{OAc})_{2}, 50 \mathrm{~mol} \%$ Triphenylphosphin sowie $(n \mathrm{Bu})_{4} \mathrm{NOAc}$ als Base bei $100{ }^{\circ} \mathrm{C}$ in DMF (Abbildung 70). Dabei konnte Zielverbindung 162 in 51\% Ausbeute erhalten werden. Ein Nachweis der Verbindung gelang jedoch aufgrund der mäßigen Löslichkeit bisher nur im EI-MS und EI-HRMS.
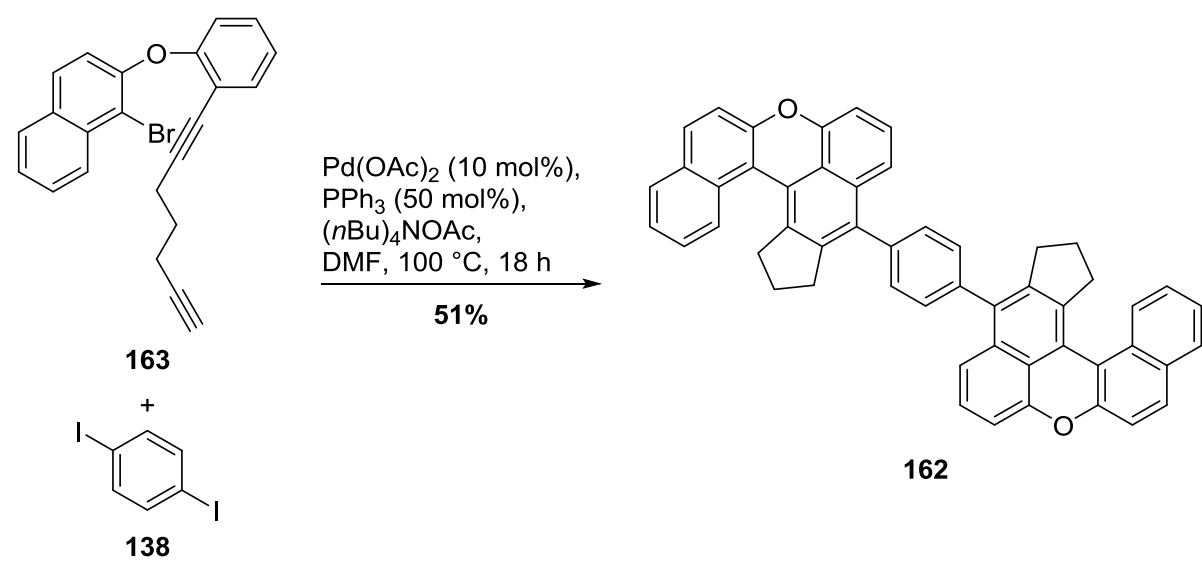

Abbildung 70: Synthese des Polyzyklus 162 über eine dimere Tandem-Domino-Sonogashira/ZweifachCarbopalladierung/C-H-Aktivierung.

Die Synthese von 162 stellt eines der wenigen Beispiele dar, in denen die Konzepte der Tandem- und der Domino-Reaktion miteinander kombiniert werden. Das Potenzial für die effektive Synthese von (pseudo-)symmetrischen Naturstoffen und funktionellen Materialien ist enorm, da in diesem Fall in einem Reaktionsablauf acht Bindungen geknüpft sowie sechs Ringe geformt werden. Das Projekt der Tandem-Domino-Reaktion wird im Arbeitskreis Tietze derzeit weiter ausgebaut (vgl. Ausblick). 


\subsection{Untersuchungen zur Synthese von hydrophilen Polyzyklen des Typs 114}

Im Rahmen dieser Arbeit sollten außerdem hydrophile Polyzyklen des Typs $\mathbf{1 1 4}$ synthetisiert werden, die aufgrund der beiden Hydroxylgruppen eine bessere Löslichkeit besitzen sollten. Alle bisher synthetisierten Verbindungen waren lediglich in unpolareren Lösungsmitteln löslich; für eine Anwendung in biologisch aktiven Systemen ist jedoch eine bessere Löslichkeit in polaren Lösungsmitteln vonnöten. Außerdem sollte eine Funktionalisierung der Moleküle erleichtert werden. Da durch die Hydroxylgruppen zudem stereogene Zentren eingeführt würden, könnten sie den Zugang zu einer enantioselektiven Synthese ermöglichen. Zentraler Bestandteil der Synthese war eine Domino-Zweifach-Carbopalladierung/C-H-Aktivierungs-Reaktion, die wie in den vorherigen Untersuchungen $\mathrm{zu}$ einem Domino-Sonogashira/Zweifach-Carbopalladierung/C-H-AktivierungsProzess erweitert werden sollte (Abbildung 71).

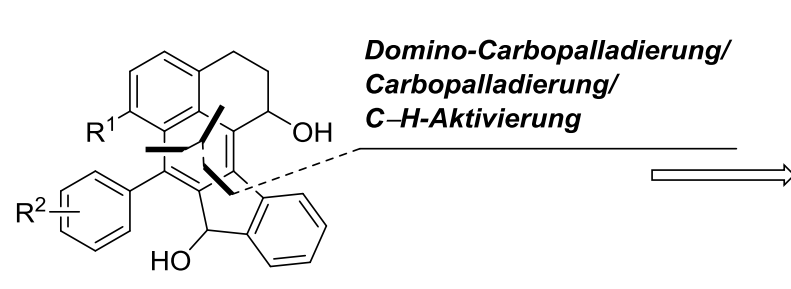

114

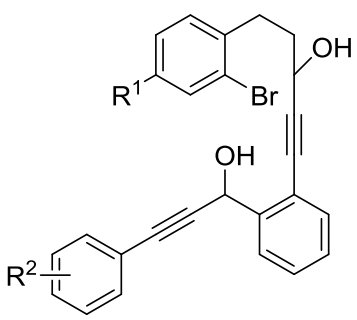

115

Abbildung 71: Retrosynthetischer Ansatz für die Synthese hydrophiler Polyzyklen des Typs 114.

\subsubsection{Synthese der Domino-Vorläufer vom Typ 115}

Die Synthese der Dialkin-Vorläufer vom Typ 115 begann mit einer Sonogashira-Reaktion, die den kommerziell erhältlichen Benzaldehyd $\mathbf{1 6 4}$ in einer literaturbekannten Umsetzung in das entsprechende TMS-geschützte Alkin-Derivat 165 überführte (Abbildung 72).$^{80}$ Dieses konnte mit kommerziell erhältlichen $(\mathrm{R}=\mathrm{H}, 4-\mathrm{Me}, 4-\mathrm{F}, 4-\mathrm{OMe})$ oder im Arbeitskreis verfügbaren $(\mathrm{R}=2-\mathrm{OPh})$ Phenylacetylenen vom Typ 166 gekuppelt werden, indem diese mit $n$-Butyllithium deprotoniert und an die Aldehyd-Funktion von 165 addiert wurden. Die Addition lieferte die entsprechenden Propargylalkohole 167a-e in bis zu 96\% Ausbeute. Eine anschließende TBS-Schützung von 167a-e war notwendig, da der komplett entschützte Propargylalkohol mit freiem Alkohol und freier Alkinfunktion nicht stabil war. Die Schützung verlief unproblematisch in exzellenten Ausbeuten von 86 bis 98\%. Im Anschluss konnte das Alkin im entstandenen Produkt 168a-e entschützt werden, indem es in basischem Methanol umgesetzt wurde. Die somit gebildeten terminalen Alkine 169a-e wurden in 80 bis $97 \%$ Ausbeute isoliert. 
<smiles>O=Cc1ccccc1Br</smiles>

TMS-Acetylen, $\mathrm{PdCl}_{2}\left(\mathrm{PPh}_{3}\right)_{2}(2 \mathrm{~mol} \%)$, Cul $(2 \mathrm{~mol} \%)$, $\underset{68 \%}{\mathrm{NEt}_{3}, 100{ }^{\circ} \mathrm{C}, 17 \mathrm{~h}}$ 164<smiles>CC#Cc1ccccc1C=O</smiles>

165
1.) $166, n-B u L i, T H F$, $-78^{\circ} \mathrm{C}, 15 \mathrm{~min} \rightarrow$ $\mathrm{RT}, 105 \mathrm{~min}$

2.) $165, \mathrm{THF},-78^{\circ} \mathrm{C} \rightarrow \mathrm{RT}$, über Nacht

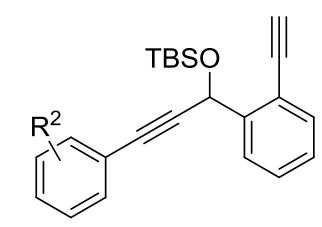

$\mathrm{R}=\mathrm{H}$

169a $(93 \%)$

$\mathrm{R}=4-\mathrm{Me} \quad 169 \mathrm{~b}(94 \%)$

$\mathrm{R}=4-\mathrm{F} \quad 169 \mathrm{c}(90 \%)$

$\mathrm{R}=4-\mathrm{OMe} 169 \mathrm{~d}(80 \%)$

$\mathrm{R}=2-\mathrm{OPh} 169 \mathrm{e}(97 \%)$
$\mathrm{K}_{2} \mathrm{CO}_{3}, \mathrm{CH}_{3} \mathrm{OH}$ RT, 17-19 h

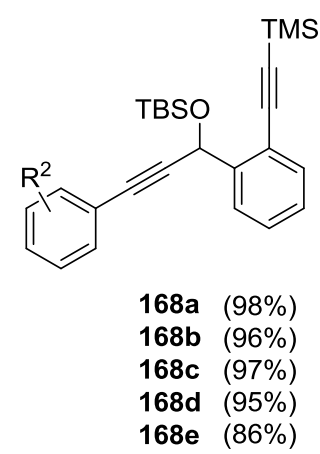

Abbildung 72: Synthese der Dialkine 169a-e.

In einer nun folgenden Addition wurden die Alkine 169a-e mit den im Arbeitskreis verfügbaren Aldehyden 170a-c verknüpft (Abbildung 73). ${ }^{80}$ Dazu wurden die Alkine zunächst mit LiHMDS deprotoniert. Dabei diente zugesetztes Triethylamin zur Deaggregation der entstehenden LithiumKomplexe.
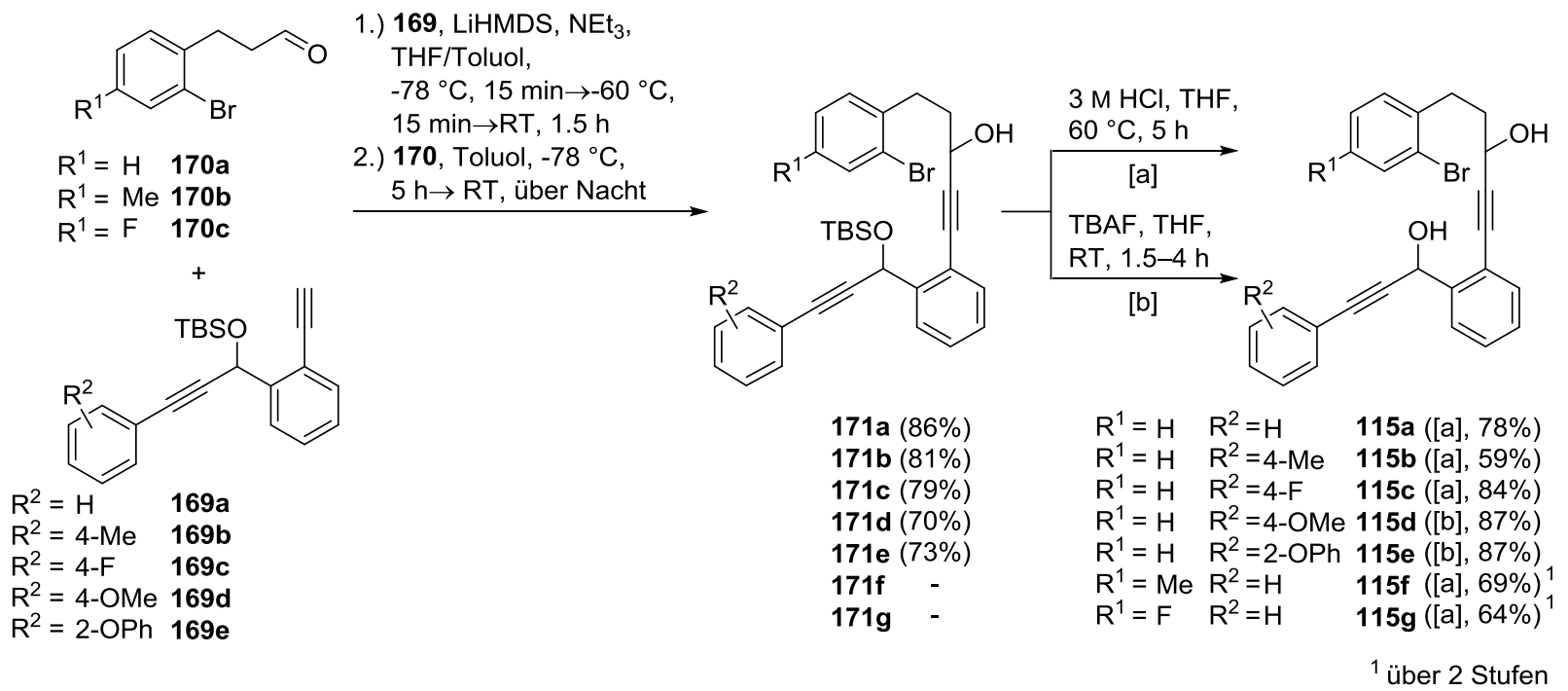

Abbildung 73: Synthese der Dialkine 115a-g.

Im Anschluss daran wurde eine Lösung der Aldehyde langsam über eine Spritzenpumpe hinzugefügt. Die entsprechenden Propargylalkohole 171a-g konnten in bis zu 86\% Ausbeute isoliert werden. Eine Entschützung der TBS-Schutzgruppe unter Verwendung von $3 \mathrm{M}$ Salzsäure bzw. TBAF lieferte schließlich die sieben unterschiedlich substituierten Domino-Vorläufer 115a-g in Ausbeuten von 59 bis $87 \%$. 
Die Diole 115a-g wurden jeweils als Gemisch zweier Diastereomere im Verhältnis 1:1 erhalten, die säulenchromatographisch nicht getrennt werden konnten. Daher erfolgten weitere Untersuchungen zunächst mit den Diastereomerengemischen.

\subsubsection{Synthese der Polyzyklen 114a-g durch die Domino-Reaktion}

Die Ausbeuten der Domino-Reaktion mit dem Modellsubstrat 115e, die zu Polyzyklus 114e führte, wurden zunächst durch den Einsatz verschiedener Reaktionsbedingungen optimiert. Die besten Ausbeuten lieferte mit über $40 \%$ eine Kombination von $20 \mathrm{~mol} \% \mathrm{Pd}(\mathrm{OAc})_{2}$ mit $50 \mathrm{~mol} \% \mathrm{PCy}_{3}$ sowie $\mathrm{K}_{2} \mathrm{CO}_{3}$ als Base in DME als Lösungsmittel bei $80{ }^{\circ} \mathrm{C}$ (Eintrag 15, Tabelle 7).

Tabelle 7: Optimierung der Reaktionsbedingungen. ${ }^{[a]}$

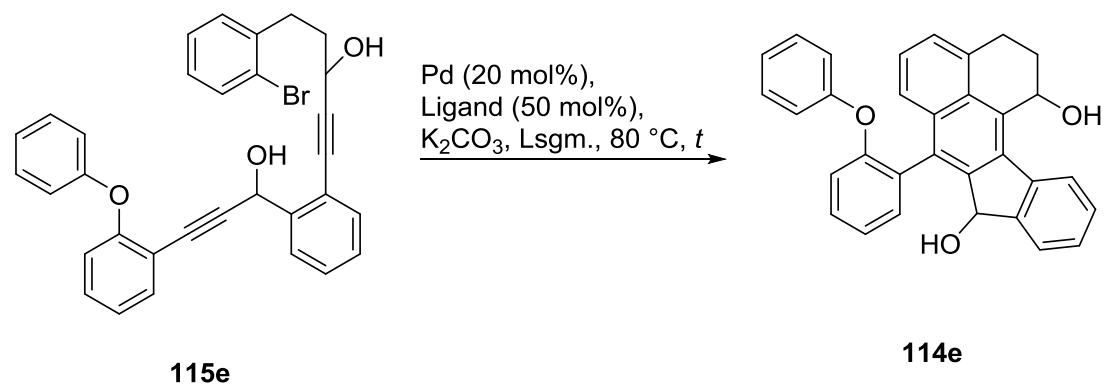

\begin{tabular}{|c|c|c|c|c|c|}
\hline Eintrag & Pd-Quelle & Ligand & Lsgm. & $t[\mathbf{h}]$ & Ausbeute $[\%]^{[b]}$ \\
\hline 1 & $\mathrm{Pd}(\mathrm{OAc})_{2}$ & $\mathrm{PPh}_{3}$ & DMF & 3 & $20-30$ \\
\hline $2^{[\mathrm{c}]}$ & 82 & - & DMF, $\mathrm{CH}_{3} \mathrm{CN}, \mathrm{H}_{2} \mathrm{O}, 5: 5: 1$ & 4 & 20 \\
\hline 3 & $\mathrm{Pd}(\mathrm{OAc})_{2}$ & $\mathrm{P}(o-\mathrm{Tol})_{3}$ & DMF & 3 & $20-30$ \\
\hline 4 & $\mathrm{Pd}(\mathrm{OAc})_{2}$ & 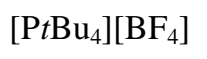 & DMF & 3 & $20-30$ \\
\hline 5 & $\mathrm{Pd}(\mathrm{OAc})_{2}$ & $t \mathrm{Bu}-\mathrm{XPhos}$ & DMF & 3 & $20-30$ \\
\hline 6 & $\mathrm{Pd}(\mathrm{OAc})_{2}$ & DPE-Phos & DMF & 3 & $20-30$ \\
\hline 7 & $\mathrm{Pd}(\mathrm{OAc})_{2}$ & XantPhos & DMF & 3 & - \\
\hline 8 & $\mathrm{Pd}(\mathrm{OAc})_{2}$ & SPhos & DMF & 3 & $30-40$ \\
\hline $9^{[\mathrm{d}]}$ & $\mathrm{Pd}(\mathrm{OAc})_{2}$ & dppe & DMF & 3 & $30-40$ \\
\hline $10^{[\mathrm{d}]}$ & $\mathrm{Pd}(\mathrm{OAc})_{2}$ & dppp & DMF & 3 & $30-40$ \\
\hline $11^{[\mathrm{d}]}$ & $\mathrm{Pd}(\mathrm{OAc})_{2}$ & dppp & 1,4-Dioxan & 6 & Unv. Umsatz \\
\hline $12^{[\mathrm{d}]}$ & $\mathrm{Pd}(\mathrm{OAc})_{2}$ & dppp & $\mathrm{CH}_{3} \mathrm{CN}$ & 3 & - \\
\hline $13^{[\mathrm{d}]}$ & $\mathrm{Pd}(\mathrm{OAc})_{2}$ & dppp & Toluol & 3 & - \\
\hline $14^{[\mathrm{d}]}$ & $\mathrm{Pd}(\mathrm{OAc})_{2}$ & dppp & DME & 3 & 40 \\
\hline 15 & $\operatorname{Pd}(\mathrm{OAc})_{2}$ & $\mathbf{P C y}_{3}$ & DME & 8 & $>40$ \\
\hline
\end{tabular}

[a] 115e (1.00 Äq.), Pd (20 mol\%), Ligand (50 mol\%), $\mathrm{K}_{2} \mathrm{CO}_{3}$ (3.00 Äq.), Lsgm., $80^{\circ} \mathrm{C}, t$; [b] ungefähre Ausbeute des Rohprodukts laut ${ }^{1} \mathrm{H}-\mathrm{NMR}-$ Analyse; [c] wie in [a], außer: LiOAc (4.00 Äq.), $(n \mathrm{Bu})_{4} \mathrm{NOAc}$ (3.00 Äq.), DMF/CH ${ }_{3} \mathrm{CN} / \mathrm{H}_{2} \mathrm{O} 5: 5: 1,120{ }^{\circ} \mathrm{C}, 4$ h, MW; [d] wie in [a], außer: Ligand (0.3 Äq.). 
Zunächst wurden die bereits in Vorgängerarbeiten erfolgreich für Domino-Carbopalladierung/C-HAktivierungs-Reaktionen eingesetzten Bedingungen mit einem Katalysatorsystem aus $\operatorname{Pd}(\mathrm{OAc})_{2}$ und Triphenylphosphin mit $\mathrm{K}_{2} \mathrm{CO}_{3}$ als Base in DMF verwendet (Eintrag 1). ${ }^{69}$ Diese führten jedoch lediglich zu Rohausbeuten zwischen 20 und 30\%. Die ebenfalls für derartige Domino-Sequenzen in Vorgängerarbeiten eingesetzten Bedingungen für eine Umsetzung mit dem von Herrmann und Beller entwickelten Palladazyklus 82 lieferten sogar nur 20\% Rohausbeute (Eintrag 2). ${ }^{64,65,69}$ Alternative Liganden wie Tris(ortho-Tolyl)-Phosphin und die ionische Spezies $\left[\mathrm{P} t \mathrm{Bu}_{4}\right]\left[\mathrm{BF}_{4}\right]$ führten mit $\mathrm{Pd}(\mathrm{OAc})_{2}$ in DMF mit $\mathrm{K}_{2} \mathrm{CO}_{3}$ als Base zu vergleichbaren Ausbeuten zwischen 20 und 30\% (Einträge 3 und 4). Gleiches galt für eine Auswahl der von Buchwald et al. entwickelten, kommerziell erhältlichen Phosphinliganden, $t \mathrm{Bu}$-XPhos und DPE-Phos (Einträge 5 und 6), während der ebenfalls zu dieser Ligandengruppe gehörende XantPhos-Ligand keinen Umsatz lieferte (Eintrag 7). Dagegen konnten mit dem SPhos-Liganden leicht erhöhte Ausbeuten von 30 bis $40 \%$ erzielt werden (Eintrag 8). Ähnliche Ausbeuten lieferten die bidentaten Liganden dppe und dppp (Einträge 9 und 10). Letzterer wurde für weitere Optimierungsexperimente in anderen Lösungsmitteln verwendet. Bei einer Umsetzung des Substrats in 1,4-Dioxan verblieb auch bei verdoppelter Reaktionszeit noch Ausgangsstoff im Reaktionsgemisch, ein Ergebnis, das sonst nicht beobachtet wurde (Eintrag 11). In Acetonitril und Toluol wurde das Substrat hingegen unter den gegeben Bedingungen zersetzt (Einträge 12 und 13). Bessere Ausbeuten lieferte eine Umsetzung in 1,2-Dimethoxyethan (DME), welche zu fast $40 \%$ Rohausbeute führte (Eintrag 14). Demgegenüber leicht verbesserte Ergebnisse wurden zudem mit dem elektronenreichen $\mathrm{PCy}_{3}$-Liganden erzielt (Eintrag 15).

Tabelle 8: Optimierung der Reaktionsbedingungen an Substrat 115a.

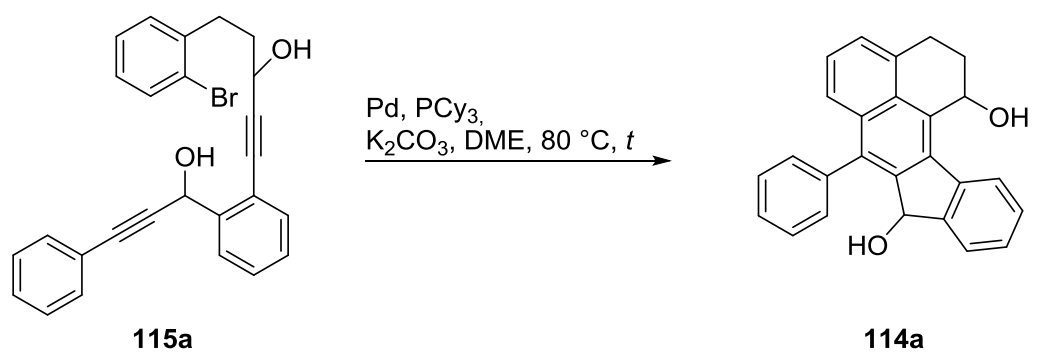

\begin{tabular}{cccccc}
\hline Eintrag & Pd-Quelle & Pd [mol\%] & Ligand [mol\%] & $\boldsymbol{t}[\mathbf{h}]$ & Ausbeute [\%] $^{[\mathbf{b}]}$ \\
\hline 1 & $\mathrm{Pd}(\mathrm{OAc})_{2}$ & 20 & 40 & 4 & $50^{[\mathrm{c}]}$ \\
$\mathbf{2}$ & Pd(TFA) 2 & $\mathbf{2 0}$ & $\mathbf{4 0}$ & $\mathbf{6}$ & $\mathbf{5 2}^{[\mathrm{cc}]}$ \\
3 & $\mathrm{Pd}(\mathrm{TFA})_{2}$ & 15 & 40 & 4 & 29 \\
4 & $\mathrm{Pd}_{2}(\mathrm{TFA})_{2}$ & 10 & 40 & 4 & 30 \\
5 & $\mathrm{Pd}(\mathrm{TFA})_{2}$ & 5 & 40 & 4 & 33 \\
\hline
\end{tabular}

[a] 115a (1.00 Äq.), Pd (20 mol\%), Ligand (50 mol\%), $\mathrm{K}_{2} \mathrm{CO}_{3}$ (3.00 Äq.), Lsgm., $80^{\circ} \mathrm{C}, \quad t ; \quad[\mathrm{b}]$ ungefähre Ausbeute des Rohprodukts laut ${ }^{1} \mathrm{H}-\mathrm{NMR}$-Analyse; [c] isolierte Reinausbeute. 
Da die moderaten Ausbeuten möglicherweise mit dem Substrat zusammenhingen, wurde die DominoReaktion auch in einer Umsetzung mit dem einfacheren Substrat 115a durchgeführt (Tabelle 8). Das resultierende Produkt 114a konnte unter Verwendung der optimierten Bedingungen (vgl. Tabelle 7, Eintrag 15) in 50\% Ausbeute isoliert werden (Eintrag 1). Leicht verbesserte Ausbeuten ergaben sich durch die Verwendung der aktiveren Palladium-Spezies Pd(TFA) $)_{2}$, die 114a in 52\% Ausbeute lieferte (Eintrag 2). In weiteren Untersuchungen wurde versucht, die Menge an Katalysator zu reduzieren. Dabei konnten weder der Einsatz von 15 mol\% (29\% Ausbeute) noch von 10 mol\% (30\% Ausbeute) oder 5 mol\% (33\% Ausbeute) eine Verbesserung der Ausbeuten bewirken (Einträge 3-5).

Die optimierten Reaktionsbedingungen aus Eintrag 2 (Tabelle 8) wurden im Folgenden zur weiteren Umsetzung der im Vorigen synthetisierten Substrate 115a-g verwendet (Abbildung 74).

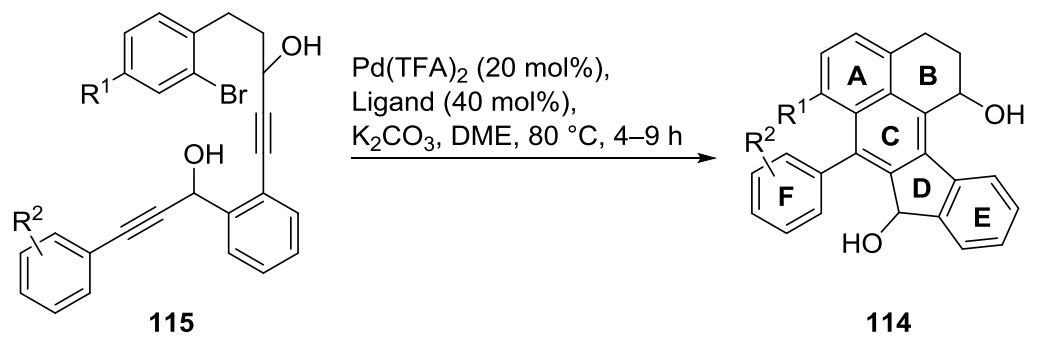

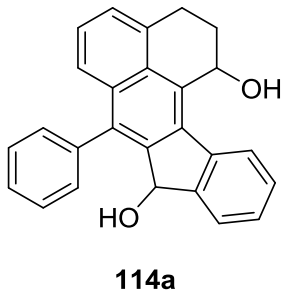

(52\%)

(d.r.: 1:1.7)<smiles>COc1ccc(-c2c3c4cccc5c(c-4c2C(O)c2ccccc2-5)CCC3O)cc1</smiles>

114d

(26\%)

(d.r.: 1:3.6)<smiles>Cc1ccc(-c2c3c4cccc5c(c-4c2C(O)CC5)C(O)c2ccccc2-3)cc1</smiles>

$114 \mathrm{~b}$

(29\% (33\% brsm.))

(d.r.: 1:1.2)<smiles></smiles>

$114 f$

$(44 \%)$

(d.r.: 1.5:1)<smiles>OC1CCc2cccc3c(-c4ccc(F)cc4)c4c(c1c23)-c1ccccc1C4O</smiles>

114c

(25\%)

(d.r.: 1:1.3)<smiles>OC1CCc2ccc(F)c3c(-c4ccccc4)c1c1c(c23)C(O)c2ccccc2-1</smiles>

$114 \mathrm{~g}$

(31\%)

(d.r.: 1:1.2)

Abbildung 74: Produkte der Domino-Zweifach-Carbopalladierung/C-H-Aktivierungs-Reaktion.

Dazu wurden auch die entsprechenden Diastereomerengemische im Verhältnis 1:1 eingesetzt. Die Ausbeuten der weiteren Verbindungen lagen allesamt unter dem Ergebnis des unsubstituierten 
Substrats. So konnte Verbindung 114b mit einer para-Methylgruppe am unteren F-Ring in 29\% Ausbeute gewonnen werden, während die entsprechenden para-Fluor- und para-Methoxy-Derivate 114c und 114d 25 bzw. 26\% Ausbeute lieferten. Die Derivate mit funktionellen Gruppen am oberen A-Ring konnten in etwas höheren Ausbeuten isoliert werden. So wurde Produkt 114f in $44 \%$ Ausbeute und Polyzyklus 114g in 31\% Ausbeute gewonnen. Alle Reaktionen liefen in einer geringen (d.r.: 1:1.2, 114b) bis moderaten (d.r.: 1:3.6, 114d) Diastereoselektivität ab, deren Ursache bislang unbekannt ist. Erfreulicherweise waren alle Diastereomerengemische säulenchromatographisch trennbar.

\subsubsection{Untersuchungen zur enantioselektiven Synthese}

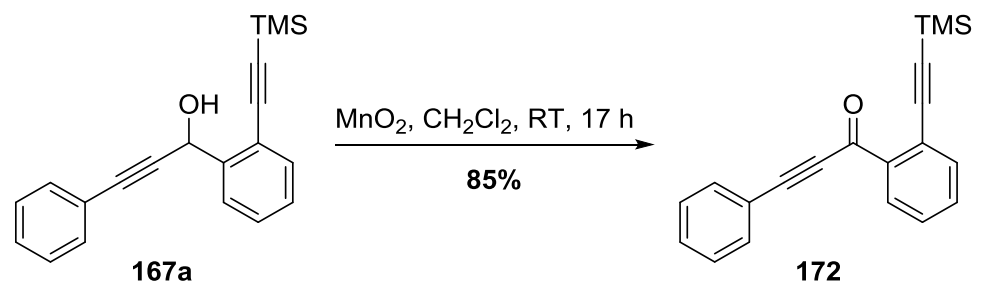

Abbildung 75: Oxidation von 167a zum Keton 172.

Die moderaten Ausbeuten für die Domino-Reaktion haben ihre Ursache vermutlich in der Tatsache, dass Isomerengemische eingesetzt wurden, deren diastereomere Bestandteile wahrscheinlich unterschiedliche Reaktivitäten aufweisen. Zudem ist ein Nachteil, dass die Auswertung und Aufarbeitung der Domino-Reaktion durch die Verwendung dieser Diastereomerengemische erschwert wird. Dementsprechend sollte in einem nächsten Schritt eine diastereo- oder enantioselektive Synthese ermöglicht werden. Als Modellsubstrat wurde hierfür Propargylalkohol 167a gewählt. Dieser wurde zunächst zum Keton 172 oxidiert, um dieses anschließend enantioselektiv zu reduzieren (Abbildung 75).

Für die enantioselektive Reduktion von 172 wurden verschiedene Bedingungen untersucht (Tabelle 9). Die Reduktion mit dem von Corey et al. entwickelten CBS-Reagenz führte zu lediglich $18 \%$ des $(S)$ Enantiomers von 167a. ${ }^{81}$ Der Enantiomerenüberschuss betrug moderate 16\% (Eintrag 1). Eine Umsetzung mit dem von Noyori et al. entwickelten Ruthenium-Katalysator führte lediglich zur Zersetzung des Substrats (Einträge 3 und 4), ${ }^{82}$ während eine Reduktion mit $\mathrm{BH}_{3}$ und dem enantiomerenreinen Liganden (-)- $\alpha$-Pinen nur in Spuren zum gewünschten Alkohol 167a reagierte (Einträge 5 und 6). 
Tabelle 9: Enantioselektive Reduktion von Keton 172.
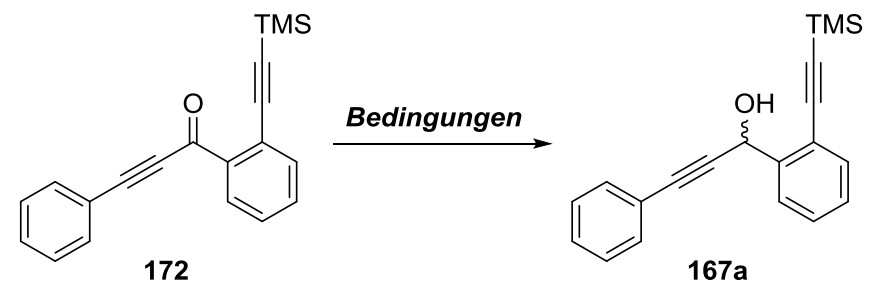

\begin{tabular}{|c|c|c|}
\hline Eintrag & Bedingungen & Ausbeute $[\%]^{[b]}$ \\
\hline 1 & $\begin{array}{l}\mathrm{CBS} \text { Reagenz } \\
\text { Zugabe von } 172 \text { über } 6 \mathrm{~h} \text {, dann }-40{ }^{\circ} \mathrm{C}, 22 \mathrm{~h}\end{array}$ & $\begin{array}{c}18 \%(S)-\mathbf{1 6 7 a} \\
(16 \% e e)\end{array}$ \\
\hline 2 & $\begin{array}{l}\text { Noyori-Kat. }{ }^{[\mathrm{b}]} \text { (0.05 Äq., } 19 \text { h, dann } 0.10 \text { Äq., } 21 \text { h), } \mathrm{HCO}_{2} \mathrm{H}(8.00 \text { Äq.), } \\
\mathrm{NEt}_{3} \text { (3.20 Äq.), } \mathrm{CH}_{2} \mathrm{Cl}_{2}, \mathrm{RT}\end{array}$ & $\begin{array}{c}\text { TMS-Entschützung } \\
\text { und } \\
\text { Zersetzung }\end{array}$ \\
\hline 3 & Noyori-Kat. ${ }^{[\mathrm{b}]}$ (0.05 Äq.), $\mathrm{HCO}_{2} \mathrm{H} / \mathrm{NEt}_{3} 2.5: 1, \mathrm{RT}, 19.5 \mathrm{~h}$ & $\begin{array}{c}\text { TMS-Entschützung } \\
\text { und } \\
\text { Zersetzung }\end{array}$ \\
\hline 4 & Noyori-Kat. $^{[\mathrm{b}]}$ (0.05 Äq.), KOH, $i \mathrm{PrOH} / \mathrm{CH}_{3} \mathrm{CN} 10: 1, \mathrm{RT}$ & Produktgemisch \\
\hline 5 & $\begin{array}{l}\mathrm{BH}_{3} \cdot \mathrm{THF}\left(2.00 \text { Äq.), (-)- } \alpha \text {-Pinen (4.00 Äq.), THF, }-30{ }^{\circ} \mathrm{C}, 21 \mathrm{~h}\right. \\
\text { 172, THF über } 4 \mathrm{~h} \text {, dann } 18.5 \mathrm{~h},-30{ }^{\circ} \mathrm{C}\end{array}$ & Spuren \\
\hline 6 & $\begin{array}{l}\mathrm{BH}_{3} \cdot \mathrm{THF}\left(2.00 \text { Äq.), (-)- } \alpha \text {-Pinen (4.00 Äq.), THF, } 0{ }^{\circ} \mathrm{C}, 2 \mathrm{~h}\right. \\
\text { 172, THF über } 30 \text { min, dann } 65 \text { h, r.t. }\end{array}$ & Spuren \\
\hline
\end{tabular}

[a] $(S)$-2-Methyl-CBS-oxazaborolidin; ${ }^{81}[\mathrm{~b}] \mathrm{RuCl}\left(p\right.$-Cymol)-[R, $R$-Tos-DPEN] (Noyori-Katalysator). ${ }^{82}$

Eine enantio- oder diastereoselektive Synthese konnte somit nicht verfolgt werden. Von weiteren Untersuchungen wurde abgesehen. 


\subsection{Untersuchungen zur Synthese von Polyalkenen mit tetrasubstituierten Doppelbindungen}

In Anlehnung an die Arbeiten von M. A. Düfert und T. Hungerland sollten über Palladium-katalysierte Domino-Reaktionen auch Polyalkene der Typen 116-120 als mögliche molekulare Schalter synthetisiert werden. ${ }^{66,69}$ Dazu wurden verschiedene Ansätze gewählt (Abbildung 76).

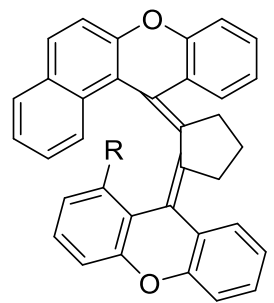

116

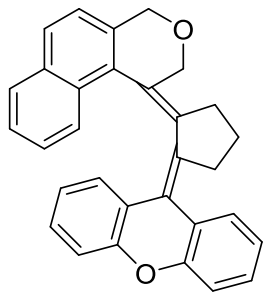

117

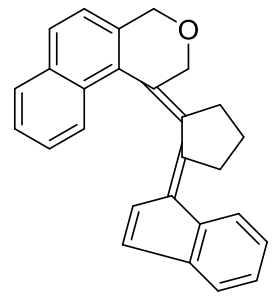

118<smiles>Cc1ccc2c(c1)C(=C1CCCC1=C(c1ccccc1)c1ccccc1)c1ccccc1O2</smiles>

119

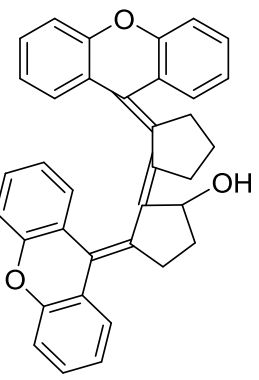

120

Abbildung 76: Verschiedene Polyalkene als Syntheseziele.

\subsubsection{Untersuchungen zur Synthese von Dialkenen des Typs 116}

Zunächst wurde die Einführung ortho-dirigierender Gruppen untersucht, die eine selektive C-H-Aktivierung ermöglichen sollten. Grundlage war dabei das bereits in Kapitel 3.2 untersuchte System. Hier erfolgte die terminierende $\mathrm{C}-\mathrm{H}$-Aktivierung der Domino-Reaktion am oberen aromatischen System (vgl. Mechanismus Abbildung 58). Durch ortho-dirigierende Gruppen sollte diese Selektivität dahingehend beeinflusst werden, dass die terminierende $\mathrm{C}-\mathrm{H}$-Aktivierung nun am unteren Benzolring stattfand (Abbildung 77).
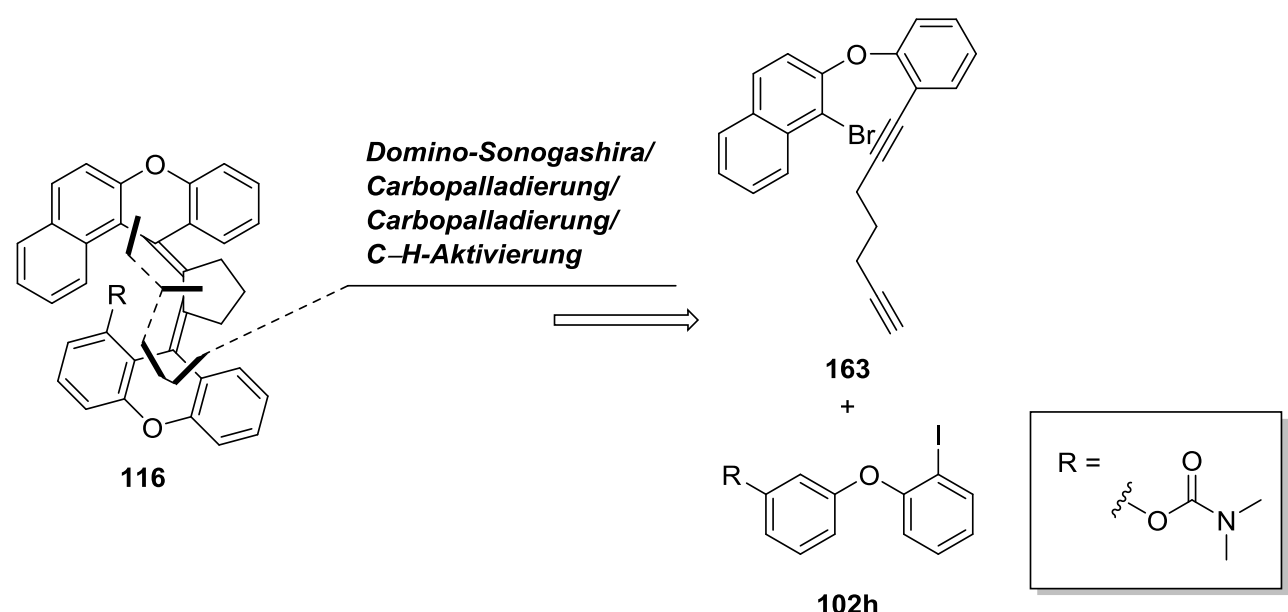

$102 \mathrm{~h}$

Abbildung 77: Retrosynthetischer Ansatz zur Synthese tetrasubstituierter Polyalkene des Typs 116. 
Als Vorläufer der angestrebten Domino-Sonogashira/Zweifach-Carbopalladierung/C-H-Aktivierung wurden Dialkin 163 sowie Aryliodid 102h eingesetzt. Das Aryliodid enthielt dabei eine Dimethylcarbamoyl-Gruppe, die eine ortho-selektive C-H-Aktivierung ermöglichen sollte.

Die Synthese von Aryliodid $\mathbf{1 0 2}$ h erfolgte über die bereits bekannte Sequenz aus nukleophiler aromatischer Substitution, Reduktion der Nitrogruppe und anschließender Sandmeyer-Reaktion ${ }^{71}$ in einer Gesamt-Ausbeute von 40\%. Der danach isolierte Methylether 173 wurde schließlich durch Umsetzung mit $\mathrm{BBr}_{3}$ in $65 \%$ Ausbeute gespalten, um das freie Phenol 174 zu erhalten. Dieses konnte durch Umsetzung mit Dimethylcarbamoylchlorid (175) unter Einführung der entsprechenden orthodirigierenden Gruppe funktionalisiert werden (Abbildung 78).

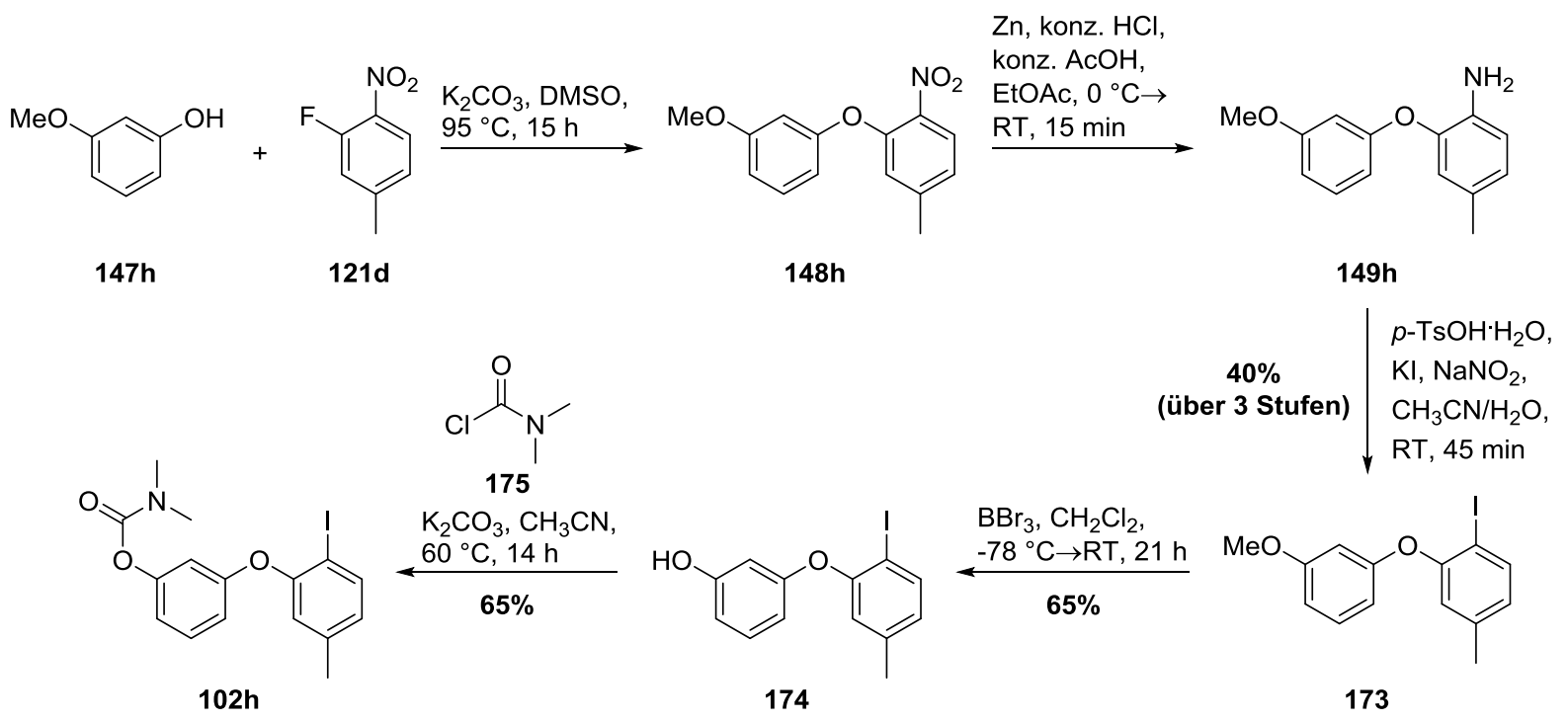

Abbildung 78: Synthese des Aryliodids 102h.

Die Umsetzung von Dialkin 163 mit Aryliodid 102h in der Domino-Sonogashira/ZweifachCarbopalladierung/C-H-Aktivierung erfolgte unter den etablierten Bedingungen mit $\operatorname{Pd}(\mathrm{OAc})_{2}$ und $\mathrm{PPh}_{3}$ als Katalysatorsystem und $(n \mathrm{Bu})_{4} \mathrm{NOAc}$ als Base (Abbildung 79 ).<smiles>C#CCCCC#Cc1ccccc1Oc1ccc2ccccc2c1Br</smiles>

163<smiles>[R]c1cccc(Oc2ccccc2I)c1</smiles>

$\mathrm{Pd}(\mathrm{OAc})_{2}(10 \mathrm{~mol} \%)$ $\mathrm{PPh}_{3}(50 \mathrm{~mol} \%)$ $(n \mathrm{Bu})_{4} \mathrm{NOAc}$, DMF, $100^{\circ} \mathrm{C}, 18.5 \mathrm{~h}$

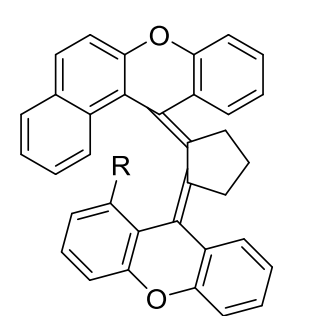

$$
\begin{gathered}
116 \\
\text { nicht erhalten }
\end{gathered}
$$<smiles></smiles>

1100 $(>60 \%)$ $\mathrm{R}=\mathrm{s}_{\text {s. }} \mathrm{O}^{\mathrm{O}}$

Abbildung 79: Umsetzung von 163 und 102h in der Domino-Reaktion. 
Das erwünschte Dialken 116 konnte jedoch nicht erhalten werden. Stattdessen wurde in über 60\% Ausbeute ausschließlich der entsprechende Polyzyklus 110o isoliert, wie ${ }^{1} \mathrm{H}-\mathrm{NMR}$ - und Massenspektren der Rohsubstanz belegten. Weitere ortho-dirigierende Gruppen wurden nicht untersucht.

\subsubsection{Untersuchungen zur Synthese von Dialkenen des Typs 117}

Ein weiterer Ansatz war die Synthese tetrasubstituierter Dialkene vom Typ 117. Diese sollten ebenfalls über eine Domino-Sonogashira/Zweifach-Carbopalladierung/C-H-Aktivierung zwischen Dialkin 176 und Aryliodid 102a hergestellt werden. Im Vergleich zu der entsprechenden DominoReaktion, die zu Polyzyklen des Typs 111 führte (vgl. Kapitel 3.2), wurde hierbei der obere Benzolring weggelassen, um die C-H-Aktivierung an diesem Ring zu unterbinden. Stattdessen sollte eine terminale C-H-Aktivierung am unteren System erfolgen, die Strukturen des Typs 117 zur Folge hätte (Abbildung 80).
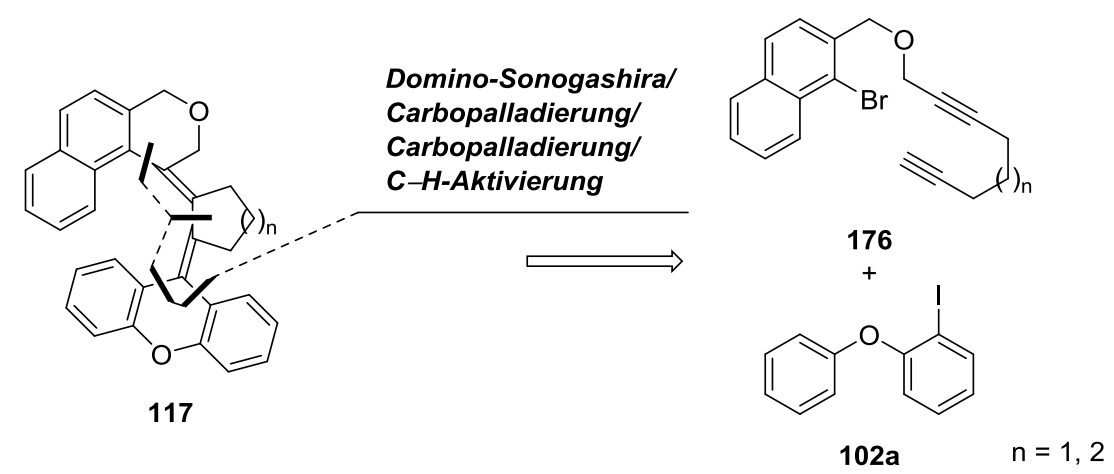

Abbildung 80: Retrosynthetischer Ansatz zur Synthese von tetrasubstituierten Dialkenen des Typs 117.

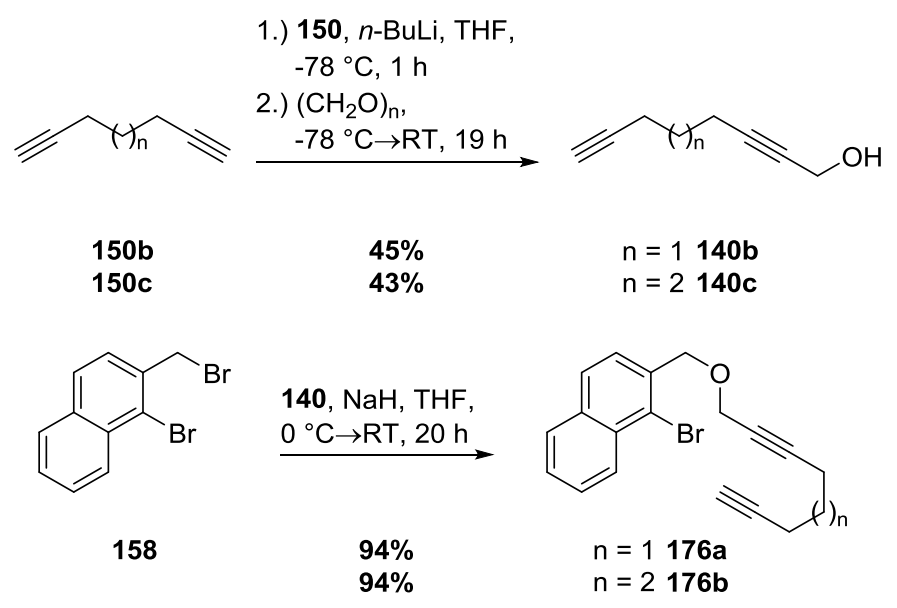

Abbildung 81: Synthese der Dialkine 176a und 176b.

Für die Synthese der oberen Bausteine vom Typ 176 wurden die Dialkine 1,6-Heptadiin (150b) und 1,7-Octadiin (150c) mit $n$-Butyllithium deprotoniert und in moderaten Ausbeuten an Paraformaldehyd 
addiert (Abbildung 81). ${ }^{76}$ Die anschließende Umsetzung der erhaltenen Alkohole 140b und 140c mit Bromid 158 in einer nukleophilen Substitution erfolgte in beiden Fällen in 94\% Ausbeute.

Für die folgende Domino-Sonogashira/Zweifach-Carbopalladierung/C-H-Aktivierung zwischen Dialkin 176a bzw. 176b und Aryliodid 102a wurden die etablierten Bedingungen verwendet. Die Reaktionen führte jedoch nicht zum Dialken 117. Stattdessen wurden Verbindungen des Typs 177 isoliert, die vermutlich in einer Domino-Sonogashira/Zweifach-Carbopalladierung/EliminierungsReaktion entstanden. Dabei wurde 177a in 50\% Ausbeute erhalten; 177b wurde im ${ }^{1} \mathrm{H}-\mathrm{N} M R-$ Spektrum des Rohprodukts nachgewiesen. Zur erwünschten terminalen C-H-Aktivierung kam es auch bei höheren Temperaturen von bis zu $180^{\circ} \mathrm{C}$ nicht (Abbildung 82).

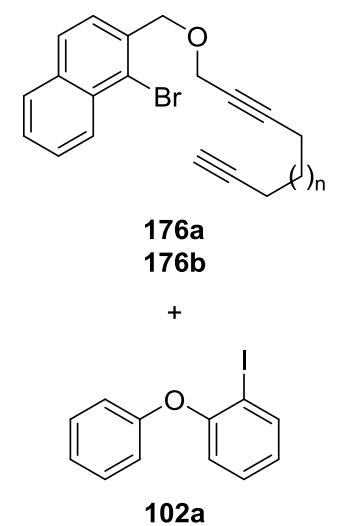

$\mathrm{Pd}(\mathrm{OAc})_{2}(20 \mathrm{~mol} \%)$, $\mathrm{PPh}_{3}(100 \mathrm{~mol} \%)$, $(n \mathrm{Bu})_{4} \mathrm{NOAc}, \mathrm{DMF}$, $100^{\circ} \mathrm{C}, 5 \mathrm{~h}, \mathrm{MW}$
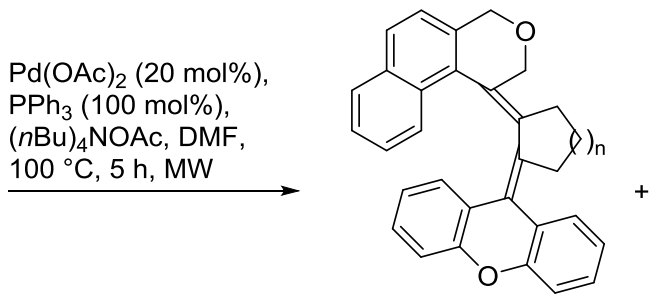

$\begin{array}{ll}\mathrm{n}=1 & 117 \mathrm{a} \\ \mathrm{n}=2 & \mathbf{1 1 7 b}\end{array}$

nicht erhalten<smiles>C(=C1CCCC1)C1=COCc2ccc3ccccc3c21</smiles>

$177 \mathrm{a}(52 \%)$

$177 b$

Abbildung 82: Untersuchungen zur Synthese der tetrasubstituierten Alkene des Typs 117.

Ein weiterer Ansatz war eine Kombination der beiden bisher beschriebenen Strategien, um zu Dialken 117c zu gelangen (Abbildung 83).
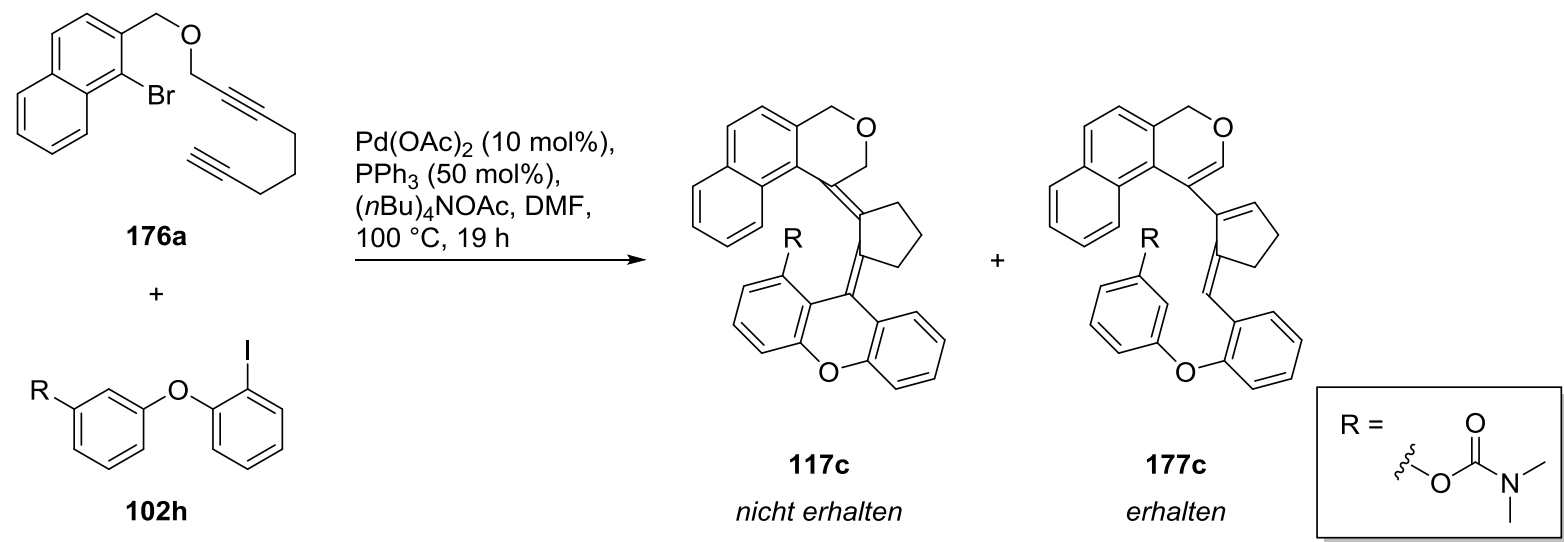

Abbildung 83: Untersuchungen zur Synthese des Dialkens 117c.

Der Einsatz des mit einer ortho-dirigierenden Gruppe funktionalisierten Aryliodids $\mathbf{1 0 2} \mathbf{h}$, um das Palladium in die räumliche Nähe der zu aktivierenden $\mathrm{C}-\mathrm{H}$-Bindung zu bringen, führte jedoch nicht zum gewünschten Dialken 117c, sondern ebenfalls zum entsprechenden Eliminierungsprodukt 177c, 
das im ${ }^{1}$ H-NMR-Spektrum der Rohsubstanz nachgewiesen wurde. Die Ausbeute wurde nicht bestimmt.

\subsubsection{Untersuchungen zur Synthese von Dialkenen des Typs 118}

In einem weiteren Plan wurde versucht, die gewünschten Dialken-Strukturen über eine DominoSonogashira/Zweifach-Carbopalladierung/Heck-Reaktion zu synthetisieren. Die terminale HeckReaktion könnte eine geringere Aktivierungsbarriere aufweisen als die entsprechende $\mathrm{C}-\mathrm{H}$ Aktivierung. Zusätzlich war der untere Vinyl-Baustein kleiner als das sonst verwendete XanthenSystem, womit die zu bildende helikale Struktur weniger sterisch gehindert wäre (Abbildung 84).
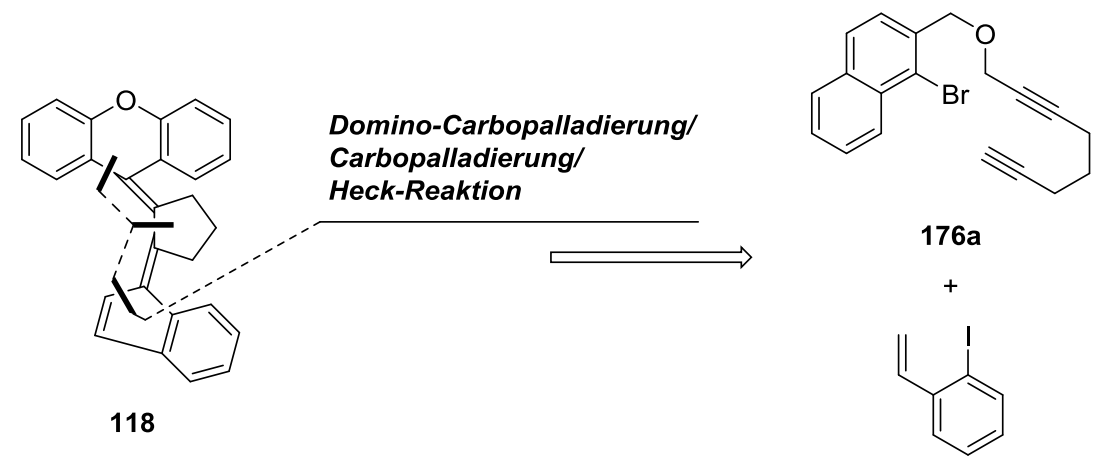

$108 b$

Abbildung 84: Retrosynthetischer Ansatz für die Synthese von tetrasubstituierten Dialkenen des Typs 118.

Die Umsetzung erfolgte über die Kupplung der beiden bereits bekannten Bausteine 176a und 108b unter den etablierten Bedingungen. Wie in Abbildung 85 dargestellt, führte die Domino-Reaktion jedoch nicht zum gewünschten Zielmolekül 118 sondern auch hier zum Eliminierungsprodukt 178, wie das 1H-NMR-Spektrum der Rohsubstanz belegte.

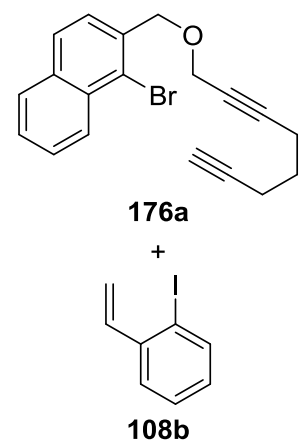

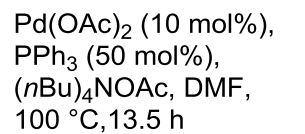
$100{ }^{\circ} \mathrm{C}, 13.5 \mathrm{~h}$

$108 b$

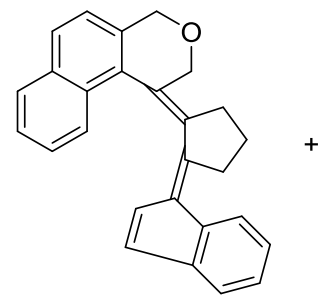

118 nicht erhalten

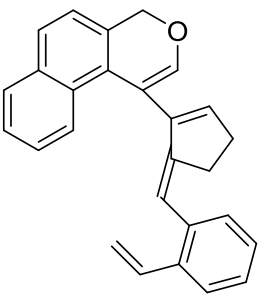

178

$(33 \%)$

Abbildung 85: Untersuchungen zur Synthese von Dialkenen des Typs 118. 


\subsubsection{Untersuchungen zur Synthese von Dialkenen des Typs 119}

Da die Synthese von tetrasubstituierten Dialkenen über eine Domino-C-H-Aktivierung nicht erfolgreich war, wurde versucht, eine entsprechende Domino-Zweifach-Carbopalladierung/ Kreuzkupplungs-Reaktion zur Synthese von Strukturen des Typs 119 einzusetzen. Dazu sollten Dialkine des Typs 179 mit Bor- (terminierende Suzuki-Kupplung) und Zinn-Organylen (terminierende Stille-Kupplung) des Typs 180 gekuppelt werden (Abbildung 86).
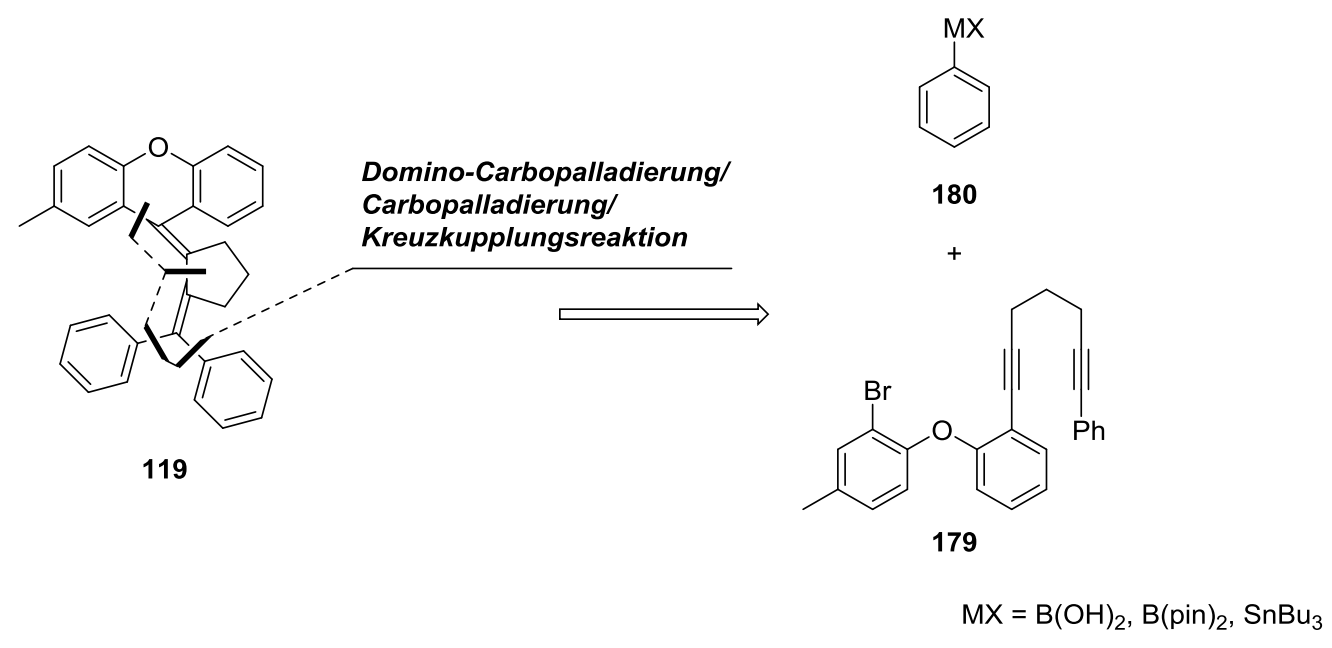

Abbildung 86: Retrosynthetischer Ansatz zur Synthese von Dialkenen des Typs 119.

Die Synthese des Dialkins 179 erfolgte zunächst über die im Arbeitskreis Tietze bekannte übliche Sequenz bestehend aus nukleophiler aromatischer Substitution, Reduktion der Nitro-Gruppe und nachfolgender Sandmeyer-Reaktion ${ }^{71}$ (Abbildung 87). ${ }^{66}$

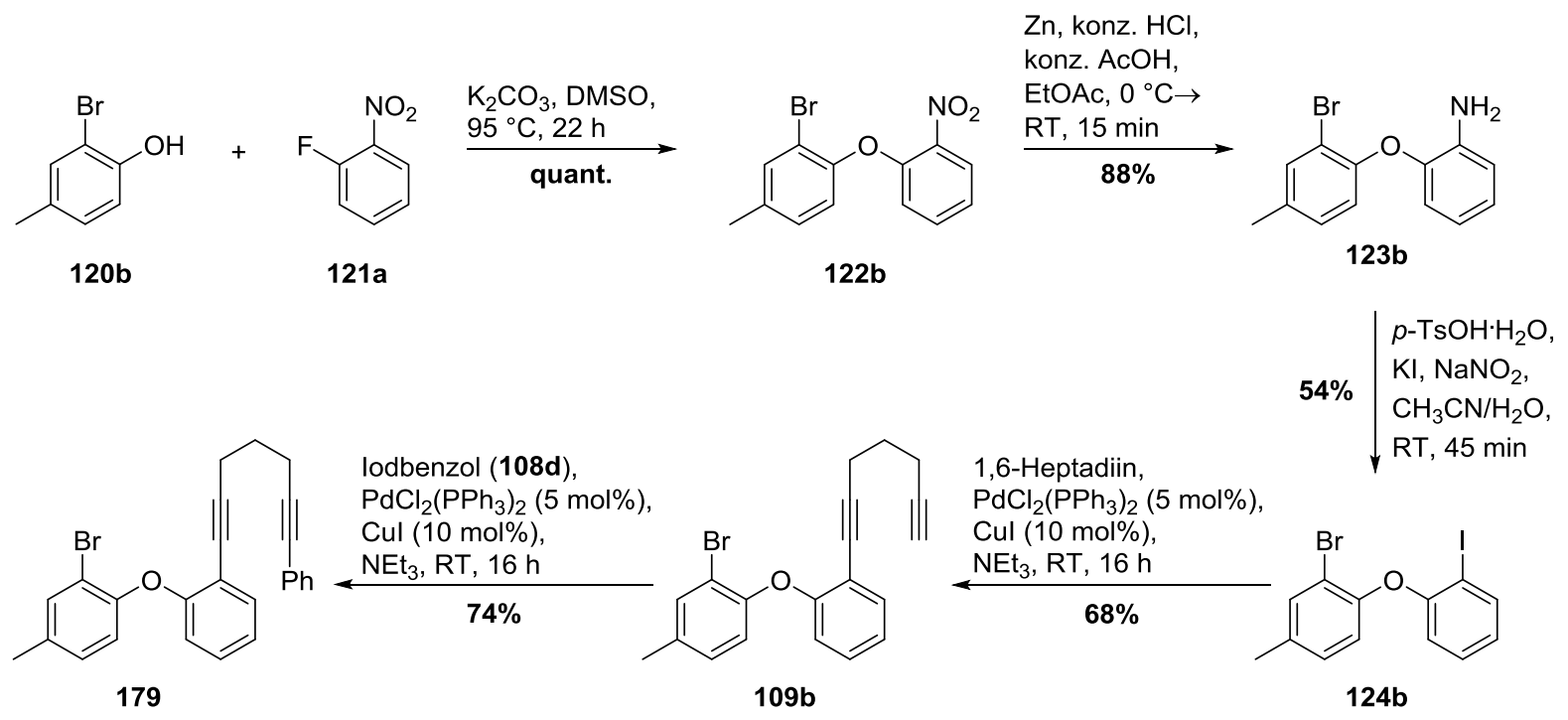

Abbildung 87: Synthese des Dialkins 179. 
Im Anschluss wurde das erhaltene Aryliodid 124b in einer Sonogashira-Reaktion mit 1,6-Heptadiin (5 Äq.) gekuppelt. Das resultierende Dialkin 109b wurde in einer Ausbeute von 68\% isoliert und in einer weiteren Sonogashira-Reaktion mit Iodbenzol (108d) zum Zielmolekül 179 umgesetzt.

Tabelle 10: Untersuchungen zur Synthese von tetrasubstituierten Dialkenen des Typs 119.
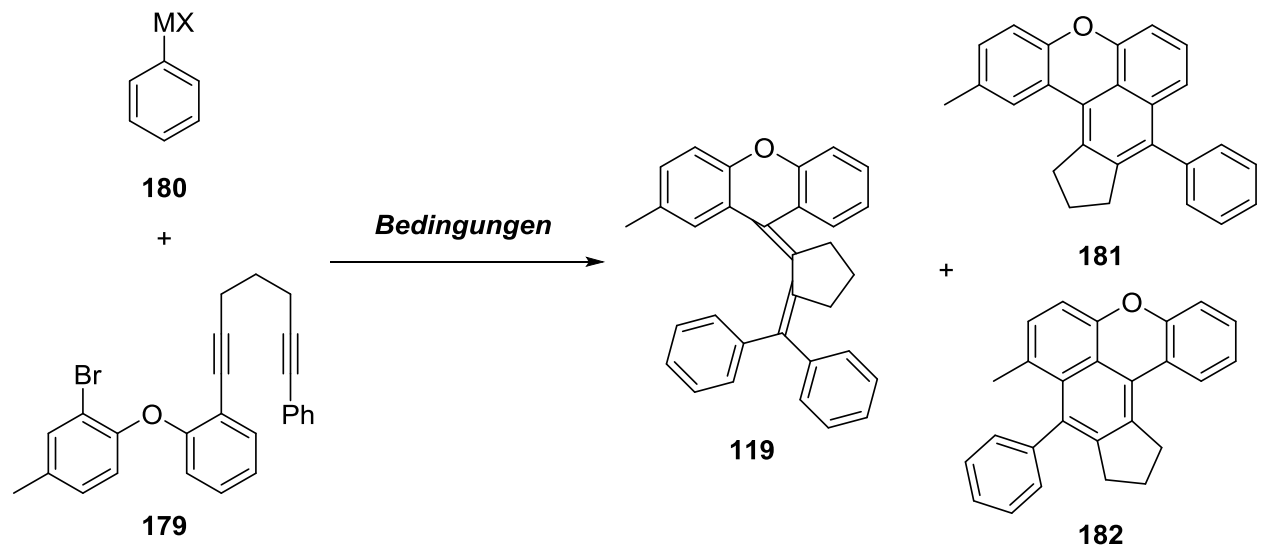

\begin{tabular}{|c|c|c|c|}
\hline Eintrag & 180 & Bedingungen & Ergebnis \\
\hline 1 & $\mathrm{PhB}(\mathrm{OH})_{2}$ & $\begin{array}{l}180 \text { (1.50 Äq.), } \mathrm{Pd}(\mathrm{OAc})_{2}(10 \mathrm{~mol} \%), \mathrm{PPh}_{3} \\
(50 \mathrm{~mol} \%), \mathrm{CsF}(3.5 \text { Äq. }), 1,4-\text { Dioxan, } 90^{\circ} \mathrm{C}, 17.5 \mathrm{~h}\end{array}$ & $\begin{array}{r}181 \& 182 \\
\text { (quant.) }^{[\mathrm{a}]}\end{array}$ \\
\hline 2 & $\mathrm{PhB}($ pin $)$ & $\begin{array}{l}180 \text { (1.50 Äq.), } \mathrm{Pd}(\mathrm{OAc})_{2}(10 \mathrm{~mol} \%), \mathrm{PPh}_{3} \\
(50 \mathrm{~mol} \%), \mathrm{CsF}(3.5 \text { Äq. }), 1,4-\text { Dioxan, } 90^{\circ} \mathrm{C}, 17.5 \mathrm{~h}\end{array}$ & 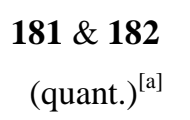 \\
\hline 3 & $\mathrm{PhSnBu}_{3}$ & $\begin{array}{l}180 \text { (1.50 Äq.), } \mathrm{Pd}_{2}(\mathrm{dba})_{3}(5 \mathrm{~mol} \%),\left[\mathrm{P}\left(t \mathrm{Bu}_{4}\right)\right]\left[\mathrm{HBF}_{4}\right] \\
(10 \mathrm{~mol} \%), \mathrm{CsF} \text { (3.5 Äq.), 1,4-Dioxan, } 90{ }^{\circ} \mathrm{C}, 17.5 \mathrm{~h}\end{array}$ & $181 \& 182^{[b]}$ \\
\hline
\end{tabular}

[a] Isolierte Rohausbeute nach Säulenchromatographie (für Produktgemisch); [b] laut DC-Analyse.

Dialkin 179 wurde nun mit den Organometallverbindungen vom Typ 180 in der entsprechenden Domino-Zweifach-Carbopalladierung/Kreuzkupplungs-Reaktion umgesetzt. Dazu wurden literaturbekannte Bedingungen für Domino-Carbopalladierung/Suzuki- ${ }^{84,85}$ bzw. DominoCarbopalladierung/Stille-Kupplungen ${ }^{86,87}$ eingesetzt, die in der Vergangenheit auch in der Arbeitsgruppe Tietze Verwendung fanden (Tabelle 10) ${ }^{66,70}$ Die Transformationen führten jedoch nicht zum erwünschten Domino-Kreuzkupplungsprodukt $\mathbf{1 1 9}$ sondern zur Zielverbindung der entsprechenden Domino-Zweifach-Carbopalladierung/C-H-Aktivierung 181 sowie zum ebenfalls in dieser Reaktion gebildeten Regioisomer 182. Demnach war die intramolekulare C-H-Aktivierung der intermolekularen Kreuzkupplungs-Reaktion gegenüber bevorzugt.

Eine weitere Strategie sah daher vor, die entsprechenden Dialkene über eine intramolekulare DominoZweifach-Carbopalladierung/Kreuzkupplungs-Reaktion zu synthetisieren. Dazu sollte der bereits bekannte Dialkin-Baustein 109a mit einem Bor- oder Zinn-Substituenten funktionalisiert werden 
(Abbildung 88). Zunächst wurde die terminale Alkinfunktion in 109a mit TMSCl in 93\% Ausbeute zu Verbindung 183 silylgeschützt. Eine anschließende Funktionalisierung zum Pinacolboran 184 mittels einer Miyaura-Borylierung nach modifizierten Bedingungen von Buchwald et al. gelang jedoch nicht. ${ }^{88}$ Daher wurde das geschützte Dialkin 183 mit tert-Buthyllithium lithiiert und anschließend mit Tributylstannylchlorid stannyliert, um eine Domino-Stille-Kupplung zu ermöglichen. Die nachfolgende TMS-Entschützung in basischem Methanol lieferte Dialkin 185 in 34\% Ausbeute über zwei Stufen. Die anschließende Addition von $\mathbf{1 8 5}$ an Paraformaldehyd ergab jedoch keinen Umsatz.

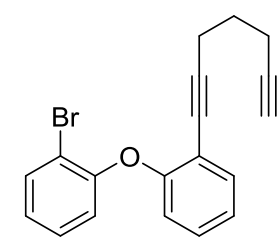

$109 a$
1.) LiHMDS, THF, $-78^{\circ} \mathrm{C}, 1.5 \mathrm{~h}$

2.) $\mathrm{TMSCl},-78^{\circ} \mathrm{C} \rightarrow$ $\mathrm{RT}, 20 \mathrm{~h}$

$93 \%$

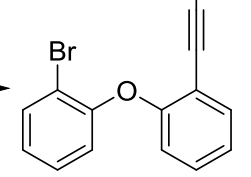

183

1.) $183, t-B u L i, T H F$, $-78^{\circ} \mathrm{C}, 45 \mathrm{~min}$

2.) $\mathrm{SnBu}{ }_{3} \mathrm{Cl},-78{ }^{\circ} \mathrm{C}$ $\rightarrow \mathrm{RT}, 16 \mathrm{~h}$

3.) $\mathrm{K}_{2} \mathrm{CO}_{3}, \mathrm{CH}_{3} \mathrm{OH} / \mathrm{CH}_{2} \mathrm{Cl}_{2}$ TMS $\mathrm{RT}, 24 \mathrm{~h}$

$34 \%$

über 2 Stufen<smiles>C#CCCCC#Cc1ccccc1Oc1ccccc1SCCC</smiles>

185 $\mathrm{B}_{2} \mathrm{pin}_{2}, \mathrm{Pd}(\mathrm{OAc})_{2}(10 \mathrm{~mol} \%)$,
SPhos $(25 \mathrm{~mol} \%), \mathrm{K}_{3} \mathrm{PO}_{4}$,
THF/H $\mathrm{H}, 70{ }^{\circ} \mathrm{C}, 16 \mathrm{~h}$

1.) LiHMDS, THF, $-78{ }^{\circ} \mathrm{C}, 1 \mathrm{~h}$

2.) $\left(\mathrm{CH}_{2} \mathrm{O}\right)_{n},-78^{\circ} \mathrm{C} \rightarrow \mathrm{RT}, 17.5 \mathrm{~h}$<smiles>CC#CCCCC#Cc1ccccc1Oc1ccccc1B1OC(C)(C)C(C)(C)O1</smiles>

184<smiles>c1ccc2c(c1)COCC2=C1CCCC1=C1c2ccccc2Oc2ccccc21</smiles>

188

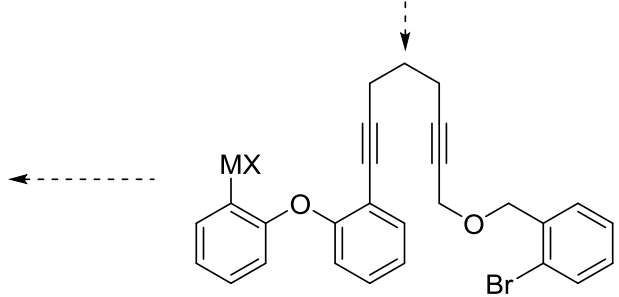

187

Abbildung 88: Untersuchungen zur Synthese von Dialkenen des Typs 188.

Die Synthese der angestrebten Domino-Vorläufer 187 blieb somit ohne Erfolg und eine Umsetzung in der Domino-Zweifach-Carbopalladierung/Kreuzkupplungsreaktion, die zu Dialken 188 führen könnte, konnte nicht untersucht werden.

\subsubsection{Untersuchungen zur Synthese von Trialkenen des Typs 120}

In einem weiteren Ansatz sollten Trialkene des Typs $\mathbf{1 2 0}$ synthetisiert werden. In einer postulierten Domino-Sonogashira/Dreifach-Carbopalladierung/C-H-Aktivierung sollte die Aromatisierung vermieden werden, die bei der Domino-Sonogashira/Zweifach-Carbopalladierung/C-H-Aktivierung auftrat. Des Weiteren war die helikale Dialken-Struktur, die in den vorigen Untersuchungen 
synthetisiert werden sollte, möglicherweise sterisch zu stark gehindert. Durch eine Erweiterung der Helix um eine Alken-Einheit sollte diese sterische Hinderung gemindert werden (Abbildung 89).

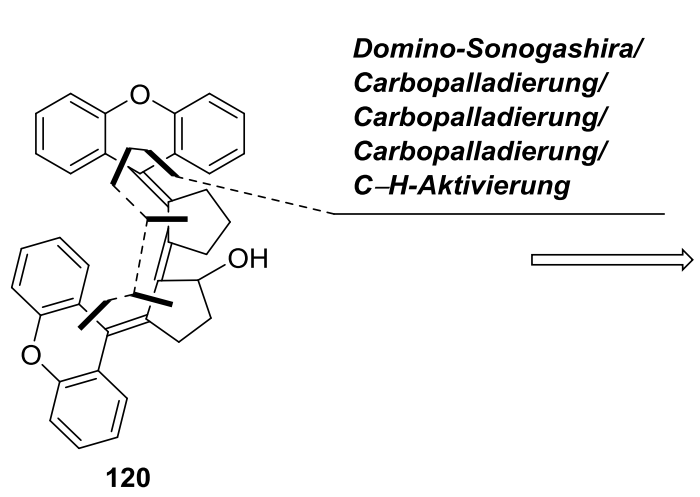

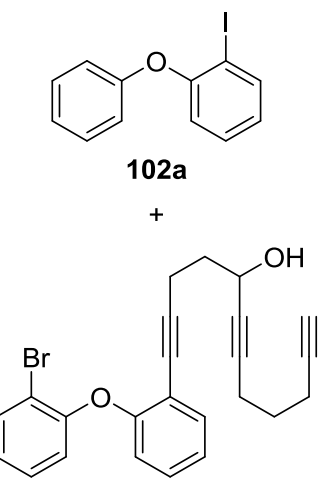

189

Abbildung 89: Retrosynthetischer Ansatz zur Synthese von Trialkenen des Typs 120.

Retrosynthetisch kann 120 auf das bereits bekannte Aryliodid 102a und Trialkin 189 zurückgeführt werden. Die Synthese von 189 erfolgte ausgehend von Aryliodid 124a, welches in einer SonogashiraReaktion mit Pentinol 190 gekuppelt wurde (Abbildung 90). Der entstehende Alkohol 191 wurde mit Dess-Martin-Periodinan (DMP) in 96\% Ausbeute zu Aldehyd 192 oxidiert. Es folgte eine Addition von 1,6-Heptadiin an 193, die in 52\% Ausbeute zu Trialkin 189 führte.

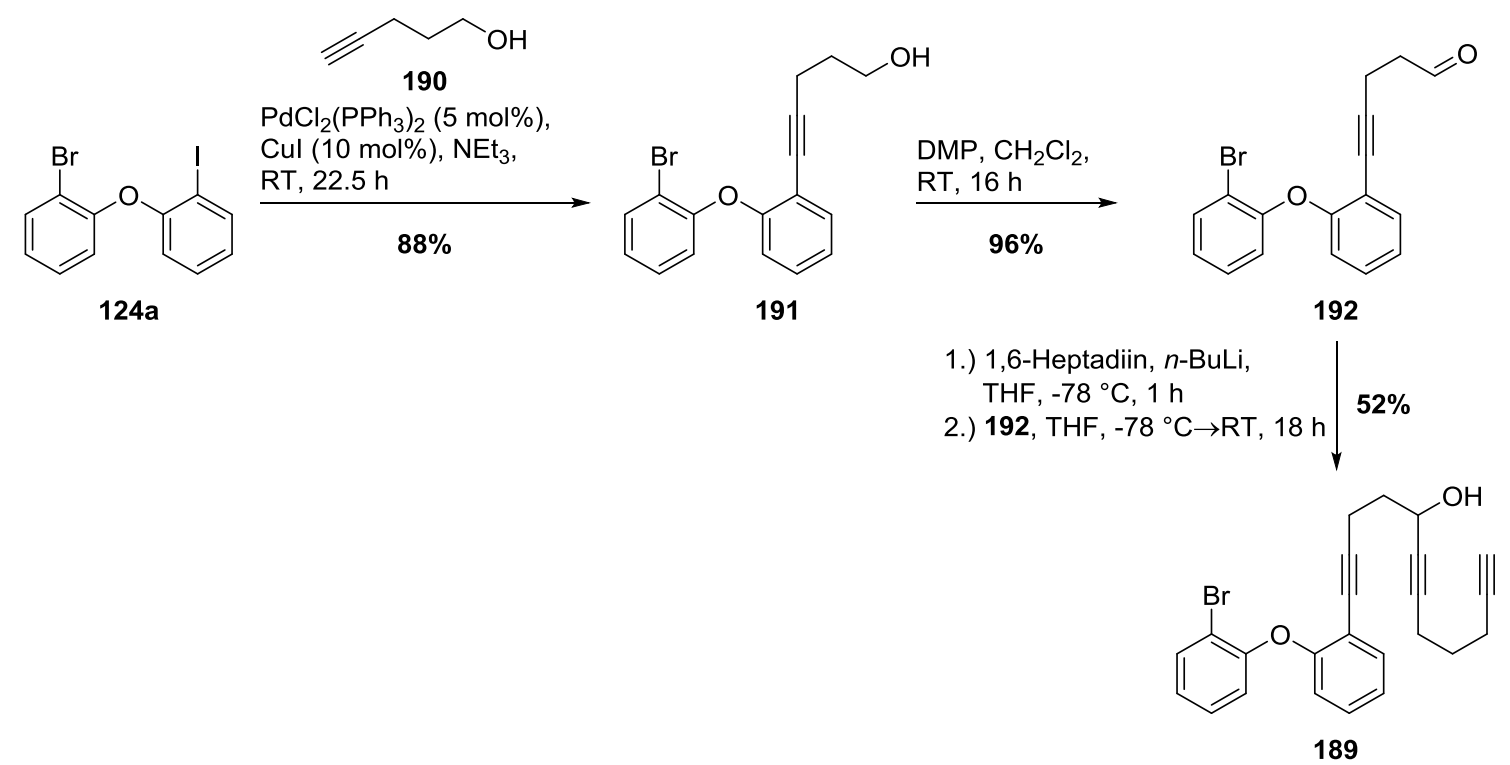

Abbildung 90: Synthese des Trialkins 189.

Die Domino-Sonogashira/Dreifach-Carbopalladierung/C-H-Aktivierung zwischen Aryliodid 102a und Trialkin 189 wurde unter den üblichen Bedingungen mit $\mathrm{Pd}(\mathrm{OAc})_{2}$ als Katalysator, Triphenylphosphin als Ligand und $(n \mathrm{Bu})_{4} \mathrm{NOAc}$ als Base durchgeführt (Abbildung 91). Die Umsetzung führte jedoch nicht zum gewünschten Trialken 120. Stattdessen ließen sich zwei Produkte isolieren, deren genaue Struktur nicht aufgeklärt werden konnte. Vermutlich handelt es sich um 
Eliminierungsprodukte. Es wird davon ausgegangen, dass sie den Verbindungen ähneln, die bereits in den Untersuchungen zur Synthese von Dialkenen beobachtet wurden.
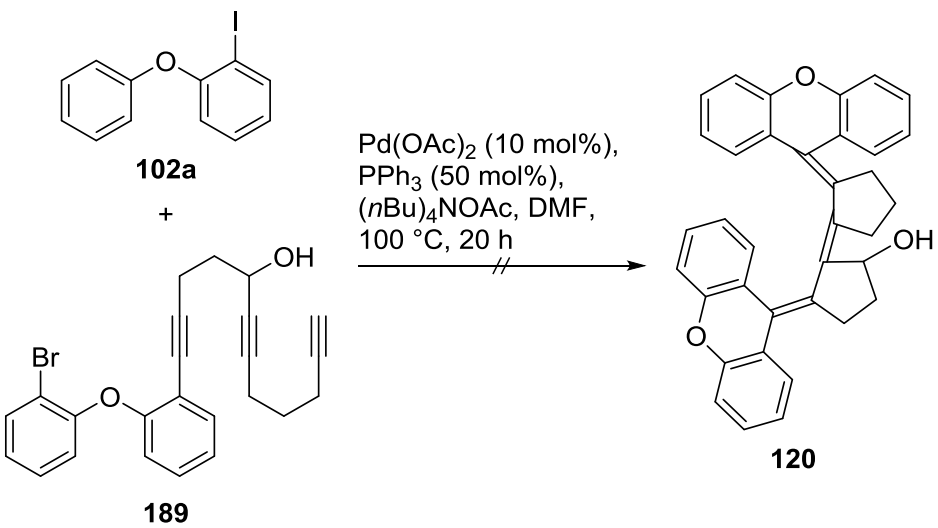

Abbildung 91: Untersuchungen zur Synthese des Trialkens 120.

Keine der sechs untersuchten Strategien zur Synthese von vollständig substituierten Polyalkenen war erfolgreich. Die Strategien beruhten sowohl auf einer Senkung der Energiebarriere für die gewünschte terminale $\mathrm{C}-\mathrm{H}$-Aktivierung und einer Vermeidung der unerwünschten $\mathrm{C}-\mathrm{H}$-Aktivierung durch Eliminierung des oberen aromatischen Rings als auch auf einer Minderung der sterischen Hinderung im Produkt sowie einer Substitution der terminalen C-H-Aktivierung durch Suzuki- oder StilleKreuzkupplungsreaktionen. Vermutlich war die entstehende Helix sterisch zu stark gehindert, um gebildet zu werden. 


\section{Zusammenfassung}

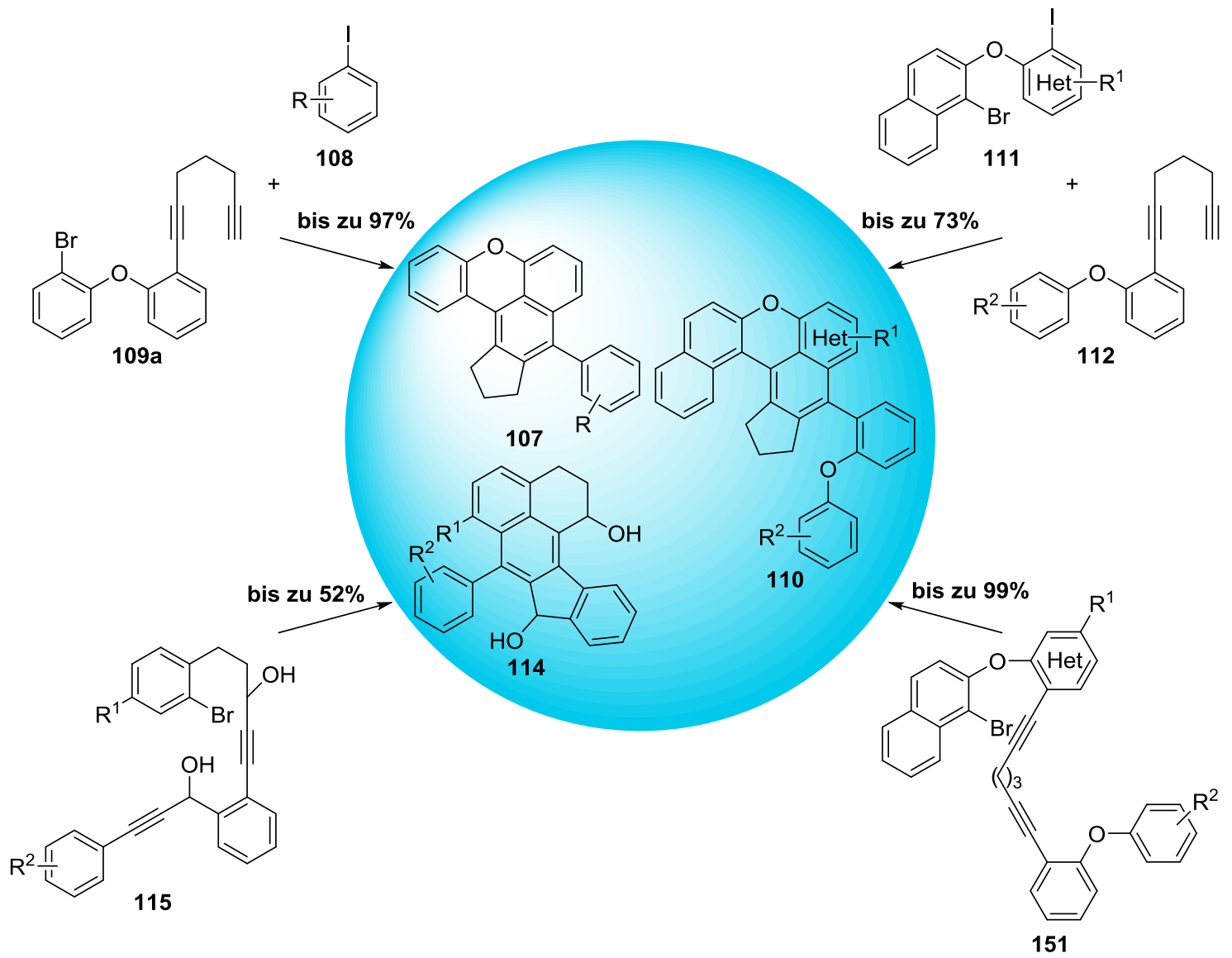

Abbildung 92: Zusammenfassung der gelungenen Synthesen von Polyzyklen der Typen 107, 110 und 114.

Das Ziel dieser Arbeit war die Synthese unterschiedlicher Polyzyklen 107, 110, und 114 durch Domino-Reaktionen mit einem terminalen $\mathrm{C}-\mathrm{H}$-Aktivierungsschritt. Dieses Ziel konnte erreicht werden. Durch Vierfach-Domino-Reaktionen unter Einsatz eines Zweikomponenten-DominoSonogashira/Zweifach-Carbopalladierung/C-H-Aktivierungs-Prozesses ließen sich aus 108 und 109a die Verbindungen 107 sowie aus 111 und 112 die Strukturen 110 herstellen. Zudem konnten durch eine Domino-Zweifach-Carbopalladierung/C-H-Aktivierung aus 115 die Verbindungen 114 aufgebaut werden.

Im Einzelnen gelang die Synthese von fluoreszenzaktiven Polyzyklen des Typs $\mathbf{1 0 7}$ ausgehend von Aryliodiden 108 sowie Dialkin 109a über eine Palladium-katalysierte Domino-Sonogashira-ZweifachCarbopalladierung/C-H-Aktivierungs-Reaktion in Ausbeuten von bis zu 97\%. Die besten Ausbeuten lieferten elektronenarme und elektrisch neutrale Aryliodide vom Typ 108 mit Ausbeuten von über 90\%. Elektronenreiche Aryliodide waren ebenfalls in nur leicht reduzierten Ausbeuten zu erhalten. Etwas geringere Ausbeuten wurden mit sterisch gehinderten Aryliodiden erhalten. Des Weiteren konnte auch ein Aryliodid mit einer reaktiven Vinyl-Gruppe in moderaten Ausbeuten von 54\% umgesetzt werden 
Auch fluoreszenzaktive Polyzyklen vom Typ 110, die ein vergrößertes konjugiertes System aufweisen, ließen sich in einer Domino-Sonogashira/Zweifach-Carbopalladierung/C-H-AktivierungsReaktion aufbauen. In diesem vierfachen Domino-Prozess wurden Aryliodide 111 und Dialkine 112 in Ausbeuten von bis zu $89 \%$ isoliert. Noch höhere Ausbeuten konnten in einer dreifachen DominoZweifach-Carbopalladierung/C-H-Aktivierungs-Reaktion von Dialkinen des Typs 151 unter Ausschluss der Sonogashira-Reaktion erhalten werden. Hierbei wurden Polyzyklen des Typs 110 in exzellenten Ausbeuten von bis zu 99\% im isolierten Domino-Prozess synthetisiert. Betrachtet man die Gesamtausbeute von Sonogashira- und anschließendem Dreifach-Domino-Prozess, so ergeben sich im günstigsten Fall Ausbeuten von bis zu 89\% über zwei Stufen. Besonders gute Ausbeuten lieferten im Vierfach-Domino-Prozess unsubstituierte bzw. heterozyklische Derivate sowie sterisch weniger gehinderte Dialkine vom Typ 112. Im Dreifach-Domino-Prozess konnten vor allem durch den Einsatz elektronisch neutraler Dialkine gute Ausbeuten erzielt werden.

Sowohl die Verbindungen des Typs 107 als auch des Typs 110 sind fluoreszenzaktiv. Die Absorptionsmaxima liegen im Bereich von $\lambda=450 \mathrm{~nm}$ mit Stokes-Shifts von $69 \mathrm{bzw} .84 \mathrm{~nm}$ für den Fluoreszenzübergang. Die Verbindungen des Typs 110 zeigen außerdem interessante dynamische Effekte in den NMR-Spektren. Diese liegen der eingeschränkten Rotation um die im System vorhandene Biarylachse zugrunde. Die Energiebarriere für diese Rotation wurde in 2D-NOESYExperimenten bestimmt.

Die Synthese von hydrophilen Polyzyklen des Typs 114 erfolgte über eine Domino-ZweifachCarbopalladierung/C-H-Aktivierung in Ausbeuten von bis zu 52\% mit dem Diastereomerengemisch von Edukten des Typs 115. Eine enantio- oder diasteroselektive Synthese gelang dagegen aufgrund der fehlgeschlagenen Synthese der Ausgangsstoffe nicht.

Ein weiterer Schwerpunkt dieser Arbeit war die Synthese zweifach- oder dreifach tetrasubstituierter helikaler Alkene 116-120 (Abbildung 93).

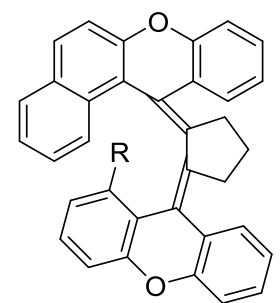

116

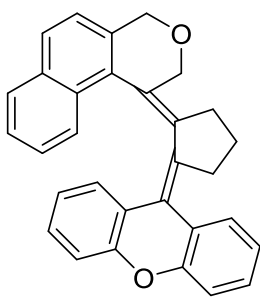

117

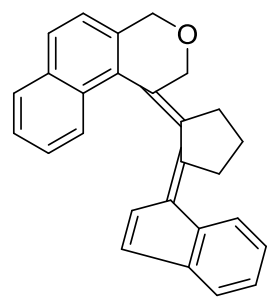

118

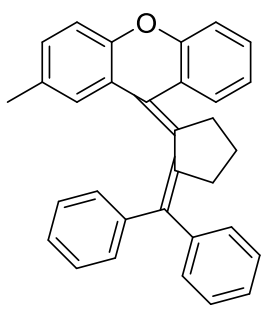

119

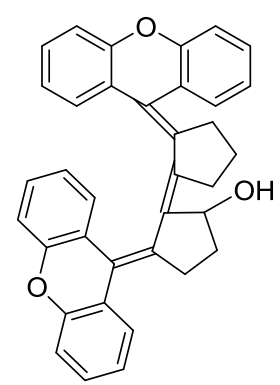

120

Abbildung 93: Vollständig substituierte Alkene als potentielle Syntheseziele.

Dazu wurden verschiedene Strategien verfolgt, wie eine Domino-Sonogashira/ZweifachCarbopalladierung-C-H-Aktivierung (116, 117), eine Domino-Sonogashira/Zweifach- 
Carbopalladierung-Heck-Reaktion (118), eine Domino-Zweifach-Carbopalladierung/KreuzkupplungsReaktion (119) und eine Domino-Sonogashira/Dreifach-Carbopalladierung-C-H-Aktivierung (120). Leider kam es in allen Fällen im letzten Schritt des Domino-Prozesses entweder zu einer Eliminierung oder einer alternativen C-H-Aktivierung, sodass die Bildung der gewünschten Produkte nicht beobachtet werden konnte. 


\section{Ausblick}

In der im Rahmen dieser Arbeit betreuten Masterarbeit von B. Waldecker wurde ein neuer Ansatz gewählt, um zweifach tetrasubstituierte Dialkene herzustellen. ${ }^{89}$ Demnach sollten dimere Strukturen vom Typ 194 und 196 in einer zweifachen Domino-Carbopalladierung/C-H-Aktivierung synthetisiert werden, bei der die jeweiligen tetrasubstituierten Doppelbindungen parallel zueinander aufgebaut werden. Die Synthese von 194 und 196 gelang in Ausbeuten von bis zu 98\% (Abbildung 94).
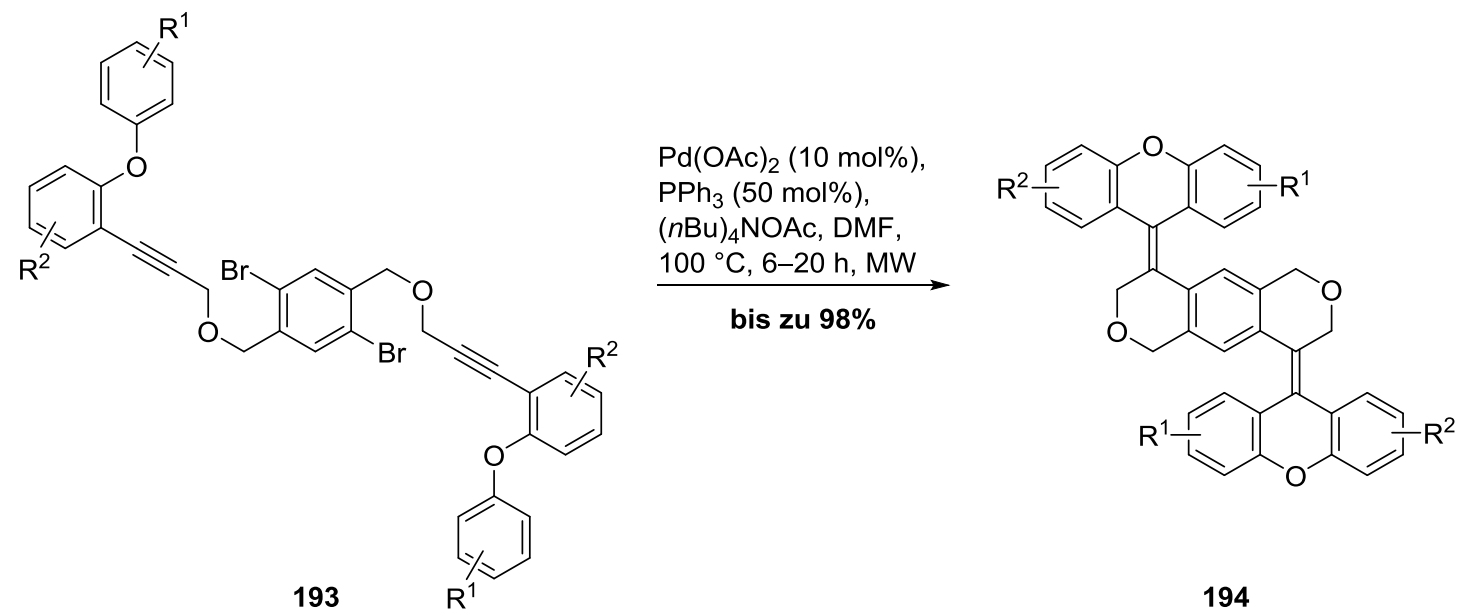

194<smiles>[R]c1ccccc1Oc1ccccc1C#CCOCc1cc(Br)c(COCC#Cc2ccccc2Oc2cccc([R])c2[R])cc1Br</smiles>

195
$\mathrm{Pd}(\mathrm{OAc})_{2}(10 \mathrm{~mol} \%)$

$\mathrm{PPh}_{3}(50 \mathrm{~mol} \%)$,

$(n \mathrm{Bu})_{4} \mathrm{NOAc}, \mathrm{DMF}$,

$100{ }^{\circ} \mathrm{C}, 12 \mathrm{~h}, \mathrm{MW}$

bis zu $98 \%$<smiles>[R][R]1ccc2c(c1)Oc1ccccc1C2=C1COCc2cc3c(cc21)C(=C1COCc2ccc([R])cc21)COC3</smiles>

196

Abbildung 94: Synthese von zweifach tetrasubstituierten Dialkenen des Typs 194 und 196 über eine zweifache Domino-Carbopalladierung/C-H-Aktivierung. ${ }^{89}$ 


\section{Experimenteller Teil}

\subsection{Allgemeine Methoden}

Experimentelle Methoden: Alle luft- und wasserempfindlichen Reaktionen wurden unter einer Argon-Atmosphäre in ausgeheizten Glasgeräten durchgeführt und die Reagenzien über eine Spritze oder Transferkanüle hinzugefügt. Alle Lösungsmittel wurden über 3-Ångström-Molekularsieb getrocknet, welches zuvor in einem Vakuumtrockenschrank „Vacutherm 6025“ der Firma „Heraeus Instruments" bei $100{ }^{\circ} \mathrm{C}$ in vacuo aktiviert wurde. Für Palladium-katalysierte Domino-Reaktionen verwendetes DMF wurde durch wiederholtes Evakuieren des Lösungsmittelkolbens entgast. Alle kommerziell erhältlichen Reagenzien wurden ohne weitere Reinigung verwendet. Kommerziell erhältliches $(n \mathrm{Bu})_{4} \mathrm{NOAc}$ wurde zur Sicherstellung der Trockenheit in einer Trockenbox „LABmaster sp“ der Firma „MBraun“ gelagert und abgewogen.

Chromatographie: Dünnschichtchromatographische Untersuchungen wurden auf vorgefertigten Kieselgelplatten der Firma „Merck“ (TLC Silica gel $60 \mathrm{~F}_{254}$ ) durchgeführt. Säulenchromatographische Reinigungen erfolgten mit Kieselgel der Firma „Merck“ (Geduran ${ }^{\circledR}$ Si60, $\varnothing=32-64 \mu \mathrm{m}$ ). Dünnschichtchromatographie auf Reverse-Phase-Kieselgel ( $\left.\mathrm{RP}-\mathrm{SiO}_{2}\right)$ erfolgte auf Kieselgelplatten der Firma „Merck“ (TLC silica gel RP-18 F F54 $_{4}$. Säulenchromatographische Reinigungen mit Reverse-Phase-Kieselgel wurden mit Kieselgel der Firma „Knauer“ $(\varnothing=32-45 \mu \mathrm{m})$ durchgeführt.

NMR-Spektroskopie: NMR-Spektren wurden mit den Geräten „Mercury-300“, „Unity-300“, „Inova-500“ und „Inova-600“ der Firma „Varian“ und dem Gerät „AMX-300“ der Firma „Bruker“ aufgenommen. Die chemische Verschiebung $\delta$ ist in ppm angegeben bezogen auf Tetramethylsilan (TMS). Die Lösungsmittelsignale dienten als Referenz. Die Kopplungskonstanten $J$ sind in Hertz angegeben. Die Multiplizitäten von Signalen erster Ordnung wurden wie folgt bezeichnet: s (Singulett), $s_{b r}$ (breites Singulett), d (Dublett), t (Triplett), q (Quartett), p (Pentett), dd (Dublett von Dubletts) usw. Signale höherer Ordnung wurden als m (Multiplett) bzw. $\mathrm{m}_{\mathrm{C}}$ (zentriertes Multiplett) bezeichnet.

IR-Spektroskopie: IR-Spektren wurden mit einem Spektrometer „FT/IR-4100“ der Firma „JASCO“ aufgenommen. Alle Substanzen wurden direkt auf eine ATR-Einheit aufgetragen.

UV/Vis-Spektroskopie: UV/Vis-Spektren wurden mit einem Spektrometer „V-630“ der Firma „JASCO“ aufgenommen.

Massen-Spektrometrie: ESI-MS- und ESI-HRMS-Spektren wurden mit einem Spektrometer „Apex IV“ der Firma „Bruker Daltronik“, EI-MS und EI-HRMS mit einem Spektrometer „MAT 95“ der Firma „Finnigan“ aufgenommen. 
Fluoreszenzspektroskopie: Fluoreszenzspektren wurden mit einem Fluorimeter „FP 6500” der Firma „JASCO“ aufgenommen. Lichtquelle: $150 \mathrm{~W}$, Xe-Lampe; photometrisches System: monochromatisches Licht; Monochromator: Signal-zu-Rauschen-Verhältnis: 200:1 (Raman-Bande von Wasser, $350 \mathrm{~nm}$ Anregungswellenlänge, $2 \mathrm{sec}$ Response-Zeit, $5 \mathrm{~nm}$ Bandbreite); Auflösung: $1 \mathrm{~nm}$, Wellenlängen-Genauigkeit: $+/-1.5 \mathrm{~nm}$; Reproduzierbarkeit: $+/-0.3 \mathrm{~nm}$; Detektor: Photomultiplier tube.

Röntgenkristallographie: Ein Einkristall von 110a wurde auf einem Diffraktometer „IPDS II” (Graphit-monochromatisierte Mo-K $\alpha$-Strahlung, $\lambda=0.71073 \AA$ ) der Firma „STOE” untersucht. Die Messung erfolgte mittels $\omega$-Scans bei $-140{ }^{\circ} \mathrm{C}$. Die Struktur wurde über direkte Methoden bestimmt (SHELXS) und mit dem Programm „SHELXL-97“ gegen $\mathrm{F}^{2}$ unter Verwendung aller Reflexionen verfeinert. ${ }^{90}$ Alle Nicht-Wasserstoffatome wurden mit anisotropen Auslenkungsparametern verfeinert. Waserstoffatome wurden an den berechneten Positionen platziert und den isotropen Auslenkungsparametern von 1.2/1.5 $\mathrm{U}_{\mathrm{eq}}$ zugeordnet. Flächenindizierte Absorptionskorrekturen wurden numerisch mit dem Programm „X-RED“ der Firma „STOE“ durchgeführt.

Mikrowellenreaktionen: Reaktionen in der Mikrowelle wurden in einem Reaktor vom Typ „Initiator“ der Firma „Biotage“ durchgeführt (Absorptionslevel: very high (bei DMF als Lösungsmittel), Pre-Stirring: $30 \mathrm{sec}$, Fixed Hold Time: On). 


\subsection{Synthese der Polyzyklen des Typs 107}

\subsubsection{Synthese des Dialkins 109a}

\section{1-Brom-2-(2-(hepta-1,6-diin-1-yl)phenoxy)benzol (109a) $)^{\mathrm{a}}$}

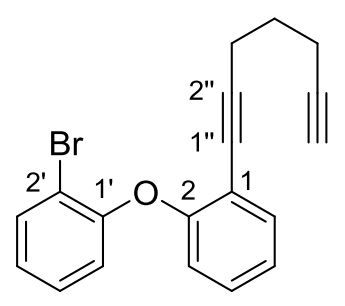

Eine Mischung von 1-Iod-2-(2-bromphenoxy)benzol (124a) (656 mg, $1.75 \mathrm{mmol}, 1.00$ Äq.),

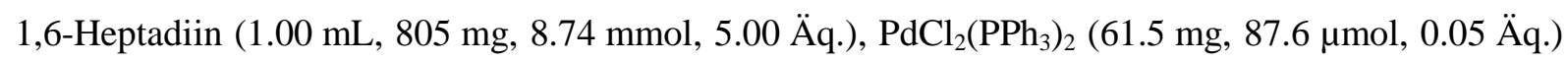
und $\mathrm{CuI}\left(33.5 \mathrm{mg}, 176 \mu \mathrm{mol}, 0.10\right.$ Äq.) in entgastem $\mathrm{NEt}_{3}(8.5 \mathrm{~mL})$ wurde $17 \mathrm{~h}$ bei RT gerührt. Das Lösungsmittel wurde in vacuo entfernt. Säulenchromatographische Reinigung $\left(\mathrm{SiO}_{2}, n\right.$-Pentan/ $\mathrm{CH}_{2} \mathrm{Cl}_{2}$ 5:1) lieferte Dialkin 109a als gelbes Öl (427 mg, $1.26 \mathrm{mmol}, 72 \%)$.

DC: $R_{f}=0.29\left(n-\mathrm{Pentan} / \mathrm{CH}_{2} \mathrm{Cl}_{2}, 5: 1\right)$.

UV/Vis $\left(\mathrm{CH}_{3} \mathrm{CN}\right): \lambda_{\max }(\mathrm{nm})(\lg \varepsilon)=252(4.207), 282(3.450)$.

IR (ATR): $\tilde{v}\left(\mathrm{~cm}^{-1}\right)=3294,1568,1485,1470,1440,1253,1228,1196,1157,1104,1045,1029,871$, $798,748,630$.

${ }^{1} \mathbf{H}-\mathbf{N M R}\left(600 \mathrm{MHz}, \mathrm{CDCl}_{3}\right): \delta(\mathrm{ppm})=1.65\left(\mathrm{p}, J=7.0 \mathrm{~Hz}, 2 \mathrm{H}, 4{ }^{\prime}{ }^{\prime}-\mathrm{H}_{2}\right), 1.92(\mathrm{t}, J=2.6 \mathrm{~Hz}, 1 \mathrm{H}$,

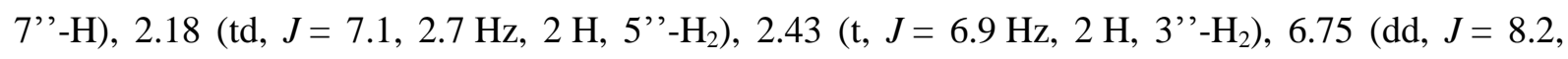
$1.5 \mathrm{~Hz}, 1 \mathrm{H}, 6$ '-H), 6.86-7.00 (m, 2 H, 5-H, 3'-H), 7.08 (td, J = 7.5, $1.2 \mathrm{~Hz}, 1 \mathrm{H}, 4-\mathrm{H}), 7.15-7.27$ (m, 2 H, 4'-H, 5'-H), 7.44 (dd, J= 7.6, 1.7 Hz, 1 H, 3-H), 7.60 (dd, J= 7.9, 1.6 Hz, 1 H, 6-H).

${ }^{13}$ C-NMR (126 MHz, $\left.\mathrm{CDCl}_{3}\right): \delta(\mathrm{ppm})=17.4$ (C-5’'), 18.6 (C-3’'), 27.4 (C-4’’), 68.7 (C-7’'), 76.3 (C-6’'), 83.7 (C-2'”), 94.7 (C-1'”), 113.5 (C-2'), 116.5 (C-1), 118.5 (C-6'), 119.5 (C-3'), 124.0, 124.1 (C-4, C-5), 128.4, 129.0 (C-4', C-5'), 133.6, 133.7 (C-3, C-6), 154.2 (C-1'), 156.5 (C-2).

MS (ESI): $m / z(\%)=341.0(40)[\mathrm{M}+\mathrm{H}]^{+}, 356.1(56)\left[\mathrm{M}+\mathrm{NH}_{4}\right]^{+}, 363.0(100)[\mathrm{M}+\mathrm{Na}]^{+}$.

HRMS (ESI): $m / z=$ gef.: 339.0378 , ber.: $339.0379\left[\mathrm{M}\left({ }^{79} \mathrm{Br}\right)+\mathrm{H}\right]^{+}$; gef.: 341.0354 , ber.: 341.0359 $\left[\mathrm{M}\left({ }^{81} \mathrm{Br}\right)+\mathrm{H}\right]^{+}$.

\section{$\mathbf{C}_{19} \mathrm{H}_{15} \mathrm{BrO}$ (339.23).}

${ }^{a}$ Für eine Synthesevorschrift für 124a siehe u.a.: C. Eichhorst, Diplomarbeit, Georg-August-Universität, Göttingen, 2010. 


\subsubsection{Synthese der Aryliodide 108a und 108b}

\section{1-Iod-2-naphthol (126)}<smiles>Oc1ccc2ccccc2c1I</smiles>

Eine Lösung von konz. $\mathrm{H}_{2} \mathrm{SO}_{4}\left(5.5 \mathrm{~mL}, 10 \mathrm{~g}, 104 \mathrm{mmol}, 1.5\right.$ Äq.) in $\mathrm{CH}_{3} \mathrm{OH}(200 \mathrm{~mL})$ wurde bei $0{ }^{\circ} \mathrm{C}$ mit 2-Naphthol (125) (10.0 g, 69.4 mmol, 1.00 Äq.), KI (11.5 g, 69.4 mmol, 1.00 Äq.) und 30\%iger wässr. $\mathrm{H}_{2} \mathrm{O}_{2}$-Lösung (37.4 mL, $139 \mathrm{mmol}, 2.00$ Äq.) versetzt und $1 \mathrm{~h}$ bei $0{ }^{\circ} \mathrm{C}$ gerührt. Nach Zugabe von ges. wässr. $\mathrm{NaHSO}_{3}$-Lösung $(200 \mathrm{~mL})$ und $\mathrm{H}_{2} \mathrm{O}(100 \mathrm{~mL})$ wurde die Mischung mit $\mathrm{CH}_{2} \mathrm{Cl}_{2}$ extrahiert $(300 \mathrm{~mL})$, die organische Phase wurde mit $\mathrm{H}_{2} \mathrm{O}(300 \mathrm{~mL})$ gewaschen, über $\mathrm{Na}_{2} \mathrm{SO}_{4}$ getrocknet, filtriert und das Lösungsmittel in vacuo entfernt. Säulenchromatographische Reinigung $\left(\mathrm{SiO}_{2}, n\right.$-Pentan/ $\left.\mathrm{CH}_{2} \mathrm{Cl}_{2}, 5: 1\right)$ lieferte Iodid $\mathbf{1 2 6}$ als gelben Feststoff (13.5 g, $\left.50.0 \mathrm{mmol}, 72 \%\right)$.

DC: $R_{f}=0.25\left(n-\operatorname{Pentan} / \mathrm{CH}_{2} \mathrm{Cl}_{2}, 5: 1\right)$.

UV/Vis $\left(\mathrm{CH}_{3} \mathrm{CN}\right): \lambda_{\max }(\mathrm{nm})(\lg \varepsilon)=231$ (4.772), 282 (3.744), 294 (3.673), 324 (3.408), 334 (3.476).

IR (ATR): $\tilde{v}\left(\mathrm{~cm}^{-1}\right)=3233,1621,1599,1494,1344,1206,806,742$.

${ }^{1}$ H-NMR $\left(300 \mathrm{MHz}, \mathrm{CDCl}_{3}\right): \delta(\mathrm{ppm})=5.77(\mathrm{~s}, 1 \mathrm{H}, \mathrm{OH}), 7.24(\mathrm{~d}, J=8.9 \mathrm{~Hz}, 1 \mathrm{H}, 3-\mathrm{H}), 7.36(\mathrm{ddd}$, $J=8.1,6.9,1.1 \mathrm{~Hz}, 1 \mathrm{H}, 5-\mathrm{H}), 7.53(\mathrm{ddd}, J=8.4,6.9,1.3 \mathrm{~Hz}, 1 \mathrm{H}, 6-\mathrm{H}), 7.69-7.75(\mathrm{~m}, 2 \mathrm{H}, 7-\mathrm{H}, 8-\mathrm{H})$ $7.91(\mathrm{dd}, J=8.5,0.9 \mathrm{~Hz}, 1 \mathrm{H}, 4-\mathrm{H})$.

${ }^{13}$ C-NMR $\left(126 \mathrm{MHz}, \mathrm{CDCl}_{3}\right): \delta(\mathrm{ppm})=86.2(\mathrm{C}-1), 116.4(\mathrm{C}-3), 124.2(\mathrm{C}-6), 128.2(\mathrm{C}-5), 128.3$ (C-7), 129.7 (C-4a), 130.2 (C-8), 130.6 (C-4), 134.8 (C-8a), 153.7 (C-2).

MS (EI): $m / z=143.0(25)[\mathrm{M}-\mathrm{I}]^{+}, 270.0(100)[\mathrm{M}]^{+}$.

HRMS (EI): $m / z=$ gef.: 269.9533, ber.: $269.9542[\mathrm{M}]^{+}$.

$\mathbf{C}_{10} \mathbf{H}_{7} \mathbf{I O}(270.07)$

\footnotetext{
${ }^{\text {b }}$ Für Originalvorschrift siehe: T. Kometani, D. S. Watt, T. Ji, T. Fitz, J. Org. Chem. 1985, 50, 5384-5387. Experimentelle Daten aus: T. Hungerland, Dissertation, Georg-August-Universität, Göttingen, 2013; M. A. Düfert, Dissertation, Georg-August-Universität, Göttingen, 2010.
} 


\section{1-Iod-2-(4-nitrophenoxy)naphthalin (108a)}

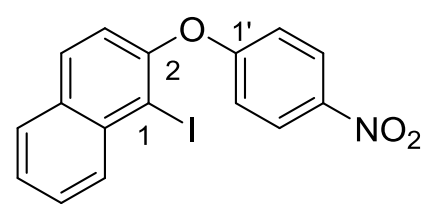

Ein Gemisch aus 1-Iod-2-naphthol (126) (5.00 g, 18.5 mmol, 1.00 Äq.) und $\mathrm{K}_{2} \mathrm{CO}_{3}$ (7.67 g, $55.5 \mathrm{mmol}, 3.00$ Äq.) in DMSO $(50 \mathrm{~mL})$ wurde $15 \mathrm{~min}$ bei $95{ }^{\circ} \mathrm{C}$ gerührt. 4-Fluornitrobenzol (121b) (1.96 mL, $2.61 \mathrm{~g}, 18.5 \mathrm{mmol}, 1.00$ Äq.) wurde hinzugefügt und die Reaktionsmischung $24 \mathrm{~h}$ bei $95{ }^{\circ} \mathrm{C}$ gerührt. Nach Zugabe von $\mathrm{H}_{2} \mathrm{O}(200 \mathrm{~mL})$ wurde die Mischung mit MTBE extrahiert $(4 \times 200 \mathrm{~mL})$, die vereinigten organischen Phasen wurden über $\mathrm{Na}_{2} \mathrm{SO}_{4}$ getrocknet, filtriert und das Lösungsmittel in vacuo entfernt. Umkristallisation aus $n$-Hexan $(300 \mathrm{~mL})$ und $\mathrm{CH}_{2} \mathrm{Cl}_{2}(100 \mathrm{~mL})$ lieferte Biarylether 108a als braunen Feststoff (5.69 g, $14.5 \mathrm{mmol}, 78 \%$ ).

DC: $R_{f}=0.19\left(n-P e n t a n / \mathrm{CH}_{2} \mathrm{Cl}_{2}, 5: 1\right)$.

UV/Vis $\left(\mathrm{CH}_{3} \mathrm{CN}\right): \lambda_{\max }(\mathrm{nm})(\lg \varepsilon)=228$ (4.686), $291(4.245)$.

IR (ATR): $\tilde{v}\left(\mathrm{~cm}^{-1}\right)=1583,1504,1484,1331,1237,1108,842,798,746$.

${ }^{1} \mathbf{H}-\mathbf{N M R}\left(300 \mathrm{MHz}, \mathrm{CDCl}_{3}\right): \delta(\mathrm{ppm})=6.96-7.01\left(\mathrm{~m}, 2 \mathrm{H}, 2^{\prime}-\mathrm{H}, 6^{\prime}-\mathrm{H}\right), 7.21(\mathrm{~d}, J=8.7 \mathrm{~Hz}, 1 \mathrm{H}$, 3-H), 7.56 (t, $J=7.5 \mathrm{~Hz}, 1 \mathrm{H}, 6-\mathrm{H}), 7.64(\mathrm{t}, J=7.7 \mathrm{~Hz}, 1 \mathrm{H}, 7-\mathrm{H}), 7.85(\mathrm{~d}, J=8.7 \mathrm{~Hz}, 1 \mathrm{H}, 5-\mathrm{H}), 7.90$ (d, $J=8.7 \mathrm{~Hz}, 1 \mathrm{H}, 4-\mathrm{H}), 8.21$ (m, 3 H, 8-H, 3'-H, 5'-H).

${ }^{13}$ C-NMR (126 MHz, CDCl $): \delta(\mathrm{ppm})=94.1(\mathrm{C}-1), 116.6$ (C-2', C-6'), 120.3 (C-3), 126.0 (C-3', C-5'), 126.6 (C-6), 128.4 (C-5), 128.6 (C-7), 131.2 (C-4), 131.9 (C-4a), 132.0 (C-8), 135.7 (C-8a), 142.9 (C-4'), 152.6 (C-2), 162.4 (C-1').

MS (EI): $m / z=218.1(43)\left[\mathrm{M}-\mathrm{I}-\mathrm{NO}_{2}\right]^{+}, 263.1(6)[\mathrm{M}-\mathrm{I}]^{+}, 391.0(100)[\mathrm{M}]^{+}$.

HRMS (EI): $m / z=$ gef.: 390.9689 , ber.: $390.9705[\mathrm{M}]^{+}$.

$\mathbf{C}_{16} \mathrm{H}_{10} \mathrm{INO}_{3}$ (391.16).

${ }^{\mathrm{c}}$ Originalvorschrift und Experimentelle Daten aus: T. Hungerland, Dissertation, Georg-August-Universität, Göttingen, 2013. 


\section{2-Iodbenzaldehyd (128) ${ }^{\mathrm{d}}$}

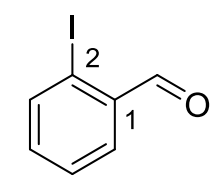

$\mathrm{MnO}_{2}$ (12.3 g, $141 \mathrm{mmol}, 30.0$ Äq.) wurde portionsweise zu einer Lösung von 2-Iodbenzylalkohol (127) (1.10 g, 4.70 mmol, 1.00 Äq.) in $\mathrm{CH}_{2} \mathrm{Cl}_{2}(50 \mathrm{~mL})$ hinzugefügt und die Reaktionsmischung $6.5 \mathrm{~h}$ bei RT gerührt. Säulenfiltration $\left(\mathrm{SiO}_{2}\right.$, EtOAc) lieferte Aldehyd 128 als gelbes Öl (841 mg, $3.62 \mathrm{mmol}, 77 \%)$.

DC: $R_{f}=0.13(n-$ Pentan).

${ }^{1}$ H-NMR $\left(300 \mathrm{MHz}, \mathrm{CDCl}_{3}\right): \delta(\mathrm{ppm})=7.21-7.34(\mathrm{~m}, 1 \mathrm{H}, 4-\mathrm{H}), 7.45(\mathrm{tt}, J=7.7,1.0 \mathrm{~Hz}, 1 \mathrm{H}, 5-\mathrm{H})$, $7.86(\mathrm{dd}, J=7.8,1.8 \mathrm{~Hz}, 1 \mathrm{H}, 3-\mathrm{H}), 7.93(\mathrm{dd}, J=7.9,1.1 \mathrm{~Hz}, 1 \mathrm{H}, 6-\mathrm{H}), 10.05(\mathrm{~d}, J=0.8 \mathrm{~Hz}, 1 \mathrm{H}$, $\mathrm{CHO})$.

${ }^{13} \mathbf{C}-\mathrm{NMR}\left(126 \mathrm{MHz}, \mathrm{CDCl}_{3}\right): \delta(\mathrm{ppm})=100.6(\mathrm{C}-2), 128.7,130.2(\mathrm{C}-4, \mathrm{C}-5), 135.1(\mathrm{C}-1), 135.4$, 140.6 (C-3, C-6), 195.7 (CHO).

HRMS (ESI): $m / z=$ gef.: 232.9457 , ber.: $232.9458[\mathrm{M}+\mathrm{H}]^{+}$.

$\mathbf{C}_{7} \mathbf{H}_{5} \mathbf{I O}(232.02)$.

\footnotetext{
${ }^{\mathrm{d}}$ Für Originalvorschrift siehe: W. S. Rapson, R. G. Shuttleworth, J. Chem. Soc. 1941, 487-490.
} 


\section{1-Iod-2-vinylbenzol (108b) $)^{\mathrm{e}}$}

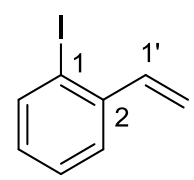

$n$-Butyllithium (2.5 M in $n$-Hexan, $1.59 \mathrm{~mL}, 3.98 \mathrm{mmol}, 1.10$ Äq.) wurde bei $0{ }^{\circ} \mathrm{C}$ zu einer Suspension von $\mathrm{MePPh}_{3}{ }^{+} \mathrm{Br}^{-}(1.55 \mathrm{~g}, \quad 4.34 \mathrm{mmol}, \quad 1.20 \mathrm{Äq}$.$) in \mathrm{THF}(60 \mathrm{~mL})$ hinzugetropft. Die Reaktionsmischung wurde $15 \mathrm{~min}$ bei $0{ }^{\circ} \mathrm{C}$ gerührt, eine Lösung von 2-Iodbenzaldehyd (128) (841 mg, $3.62 \mathrm{mmol}, 1.00$ Äq.) in THF (10 mL) wurde hinzugefügt und die Reaktionsmischung über $15 \mathrm{~h}$ auf RT erwärmt. Nach Zugabe von $\mathrm{H}_{2} \mathrm{O}(100 \mathrm{~mL})$ wurde die Mischung mit $\mathrm{CH}_{2} \mathrm{Cl}_{2}$ extrahiert $(3 \times 100 \mathrm{~mL})$, die vereinigten organischen Phasen über $\mathrm{Na}_{2} \mathrm{SO}_{4}$ getrocknet, filtriert und das Lösungsmittel in vacuo entfernt. Säulenchromatographische Reinigung $\left(\mathrm{SiO}_{2}, n\right.$-Pentan) lieferte das polymerisationsempfindliche Alken 108b als farbloses Öl (678 mg, 2.95 mmol, 81\%).

DC: $R_{f}=0.68(n$-Pentan).

UV/Vis $\left(\mathrm{CH}_{3} \mathrm{CN}\right): \lambda_{\max }(\mathrm{nm})(\lg \varepsilon)=221(4.264), 245(4.041)$.

IR (ATR): $\tilde{v}\left(\mathrm{~cm}^{-1}\right)=3057,2921,1951,1915,1836,1802,1623,1582,1556,1461,1433,1412,1275$, 1202, 1009, 982, 914, 760, 726, 646, 570.

${ }^{1} \mathbf{H}-\mathbf{N M R}\left(300 \mathrm{MHz}, \mathrm{CDCl}_{3}\right): \delta(\mathrm{ppm})=5.31\left(\mathrm{dd}, J=10.9,1.0 \mathrm{~Hz}, 1 \mathrm{H}, 2^{\prime}-\mathrm{H}_{\mathrm{a}}\right), 5.62(\mathrm{dd}, J=17.3$, $\left.1.0 \mathrm{~Hz}, 1 \mathrm{H}, 2^{\prime}-\mathrm{H}_{\mathrm{b}}\right), 6.83-6.90$ (m, $\left.1 \mathrm{H}\right), 6.90-6.97$ (m, $\left.1 \mathrm{H}\right)(4-\mathrm{H}, 5-\mathrm{H}), 7.30$ (dddd, $J=7.8,7.3,1.3$, $\left.0.6 \mathrm{~Hz}, 1 \mathrm{H}, 1^{\prime}-\mathrm{H}\right), 7.50$ (dd, $\left.J=7.8,1.7 \mathrm{~Hz}, 1 \mathrm{H}, 6-\mathrm{H}\right), 7.82$ (dd, $\left.J=8.0,1.3 \mathrm{~Hz}, 1 \mathrm{H}, 3-\mathrm{H}\right)$.

${ }^{13}$ C-NMR $\left(126 \mathrm{MHz}, \mathrm{CDCl}_{3}\right): \delta(\mathrm{ppm})=99.6,116.8,126.3,128.3,129.2,139.4,140.6,140.7$.

MS (EI): $m / z(\%)=77.0(61)\left[\mathrm{C}_{6} \mathrm{H}_{5}\right]^{+}, 230.0(100)[\mathrm{M}]^{+}$.

$\mathbf{C}_{8} \mathbf{H}_{7} \mathbf{I}(230.05)$.

${ }^{\text {e }}$ Für Originalvorschrift siehe: M. R. Acheson, G. C. M. Lee, J. Chem. Soc., Perkin Trans. 1 1987, 11, 23212332. 


\subsubsection{Synthese der Substrate 107a-f durch die Domino-Reaktion}

Allgemeine Vorschrift für die Synthese der Produkte 107a-f (Vierfach-Domino-Reaktion):

Ein Gemisch aus Aryliodid 108 (1.00 Äq.), Dialkin 109a (1.09 Äq.), Pd(OAc) $)_{2}\left(0.10\right.$ Äq.), $\mathrm{PPh}_{3}$ (0.50 Äq.) und $(n \mathrm{Bu})_{4} \mathrm{NOAc}\left(6.00\right.$ Äq.) in entgastem DMF $(3 \mathrm{~mL})$ wurde $14.5-21 \mathrm{~h}$ bei $100{ }^{\circ} \mathrm{C}$ gerührt. Die Mischung wurde durch $\mathrm{SiO}_{2}$ filtriert, mit EtOAc gespült und das Lösungsmittel in vacuo entfernt. Säulenchromatographische Reinigung $\left(\mathrm{SiO}_{2}\right)$ lieferte Domino-Produkt 107 als gelben Feststoff.

Tabelle 11: Synthese der Domino-Produkte 107a-f.

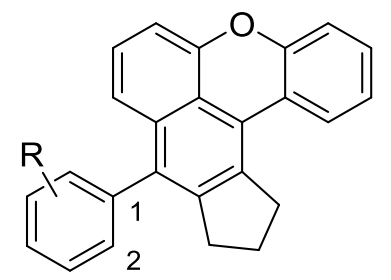

\begin{tabular}{ccccc}
\hline Eintrag & Aryliodid & Produkt & Ausbeute [mg] & Ausbeute [\%] \\
\hline $1^{[\mathrm{a}]}$ & $\mathbf{1 0 8 a}$ & $\mathbf{1 0 7 a}$ & 79.9 & 81 \\
$2^{[\mathrm{b}]}$ & $\mathbf{1 0 8 b}$ & $\mathbf{1 0 7 b}$ & 42.5 & 54 \\
3 & $\mathbf{1 0 8 c}$ & $\mathbf{1 0 7 c}$ & 45.9 & 97 \\
4 & $\mathbf{1 0 8 d}$ & $\mathbf{1 0 7 d}$ & 35.3 & 90 \\
5 & $\mathbf{1 0 8 e}$ & $\mathbf{1 0 7 e}$ & 29.4 & 64 \\
6 & $\mathbf{1 0 8 f}$ & $\mathbf{1 0 7 f}$ & 43.1 & 80 \\
\hline
\end{tabular}

[a] 109a (1.05 ̈̈q.), DMF (5 mL); [b] 109a (1.20 Äq.). 


\section{9-(2-(4-Nitrophenoxy)naphthalin-1-yl)-11,12-dihydro-10H-inden[6,5,4-kl]xanthen (107a)}

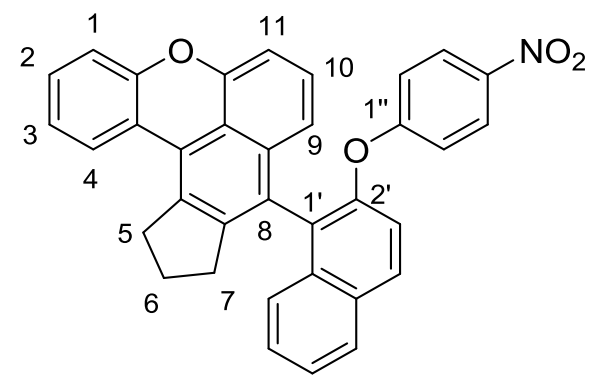

DC: $R_{f}=0.40(n$-Pentan/EtOAc, 20:1).

UV/Vis $\left(\mathrm{CH}_{3} \mathrm{CN}\right): \lambda_{\max }(\mathrm{nm})(\lg \varepsilon)=193$ (4.935), 223 (4.378), 256 (3.884), 286 (3.687), 350 (3.496), 367 (3.486).

IR (ATR): $\tilde{v}\left(\mathrm{~cm}^{-1}\right)=1580,1509,1485,1463,1442,1338,1307,1279,1240,1166,1128,1110,1066$, $1045,1028,1012,958,860,852,836,821,808,768,761,750,734,703,688,667,645,629,618,530$, $522,514$.

${ }^{1} \mathbf{H}-\mathrm{NMR}\left(600 \mathrm{MHz}, \mathrm{C}_{6} \mathrm{D}_{6}\right): \delta(\mathrm{ppm})=1.58-1.70\left(\mathrm{~m}, 2 \mathrm{H}, 6-\mathrm{H}_{2}\right), 2.33(\mathrm{ddd}, J=16.2,8.4,6.2 \mathrm{~Hz}, 1 \mathrm{H}$, 7- $\left.\mathrm{H}_{\mathrm{a}}\right), 2.50\left(\mathrm{dt}, J=15.9,7.9 \mathrm{~Hz}, 1 \mathrm{H}, 7-\mathrm{H}_{\mathrm{b}}\right), 2.87\left(\mathrm{dt}, J=15.6,7.5 \mathrm{~Hz}, 1 \mathrm{H}, 5-\mathrm{H}_{\mathrm{a}}\right), 2.94(\mathrm{ddd}, J=15.8$, 8.2, 5.9 Hz, $\left.1 \mathrm{H}, 5-\mathrm{H}_{\mathrm{b}}\right), 6.37$ (d, $J=9.2 \mathrm{~Hz}, 2 \mathrm{H}, 2$ ''-H, 6'- $\left.-\mathrm{H}\right), 6.77$ (dd, $\left.J=7.9,1.6 \mathrm{~Hz}, 1 \mathrm{H}, 11-\mathrm{H}\right)$, 6.84-6.92 (m, 3 H, 3-H, 9-H, 10-H), 6.97 (ddd, $J=8.3,7.3,1.4 \mathrm{~Hz}, 1 \mathrm{H}, 2-\mathrm{H}), 7.04$ (dd, $J=8.3$, $1.3 \mathrm{~Hz}, 1 \mathrm{H}, 1-\mathrm{H}), 7.06$ (d, $\left.J=9.0 \mathrm{~Hz}, 1 \mathrm{H}, 3^{\prime}-\mathrm{H}\right), 7.14$ (m $\left.\mathrm{C}, 1 \mathrm{H}, 7^{\prime}-\mathrm{H}\right), 7.26$ (ddd, J = 8.1, 6.8, $1.2 \mathrm{~Hz}$, $1 \mathrm{H}, 6$ ''-H), 7.43 (dd, $\left.J=8.5,1.1 \mathrm{~Hz}, 1 \mathrm{H}, 8^{\prime}-\mathrm{H}\right), 7.59$ (d, $J=9.2 \mathrm{~Hz}, 2 \mathrm{H}, 3$ '’-H, 5'’-H), 7.67 (d, J = $\left.8.9 \mathrm{~Hz}, 1 \mathrm{H}, 4^{\prime}-\mathrm{H}\right), 7.71$ (d, $\left.J=8.4 \mathrm{~Hz}, 1 \mathrm{H}, 4-\mathrm{H}\right), 7.73$ (d, $\left.J=8.4 \mathrm{~Hz}, 1 \mathrm{H}, 5^{\prime}-\mathrm{H}\right)$.

${ }^{13}$ C-NMR (126 MHz, $\left.\mathrm{C}_{6} \mathrm{D}_{6}\right): \delta(\mathrm{ppm})=25.5$ (C-6), 32.2 (C-7), 35.3 (C-5), 108.1 (C-9), 117.0 (C-2”, C-6”), 117.2 (C-1), 118.2 (C-11), 120.7 (C-3'), 122.4, 122.6 (C-4a), 123.1 (C-3), 123.3 (C-4b), 125.5 (C-3', C-5'), 125.7, 126.1 (C-6', C-8'), 127.0, 127.1, 127.6 (C-4, C-10, C-7'), 127.9 (C-1'), 128.3, 128.6 (C-5'), 129.6 (C-2), 130.2 (C-4'), 132.0 (C-4'a), 133.9 (C-8'a, C-4c/C-7a), 134.1 (C-8a), 143.0 (C-1'), 146.4 (C-4c/C-7a), 150.2 (C-2'), 151.7 (C-11a), 153.4 (C-11b), 162.7 (C-1).

Weitere Signale konnten nicht zugeordnet werden.

MS (ESI): $m / z(\%)=544.2(41)[\mathrm{M}+\mathrm{Na}]^{+}$.

HRMS (ESI): $m / z=$ gef.: 544.1505 , ber.: $544.1519[\mathrm{M}+\mathrm{Na}]^{+}$.

$\mathrm{C}_{35} \mathrm{H}_{23} \mathrm{NO}_{4}$ (521.64). 
9-(2-Vinylphenyl)-11,12-dihydro-10H-inden[6,5,4-kl]xanthen (107b)

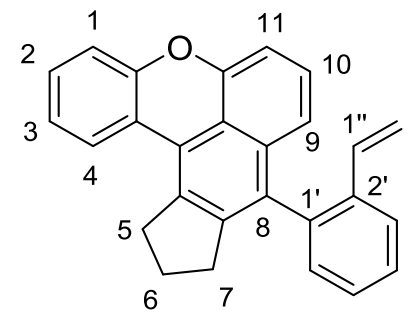

DC: $R_{f}=0.24\left(n-\mathrm{Pentan} / \mathrm{CH}_{2} \mathrm{Cl}_{2}, 20: 1\right)$.

UV/Vis $\left(\mathrm{CH}_{3} \mathrm{CN}\right): \lambda_{\max }(\mathrm{nm})(\lg \varepsilon)=199$ (4.475), 222 (4.307), 254 (4.142), 352 (3.685), 366 (3.702), 383 (3.569).

IR (ATR): $\tilde{v}\left(\mathrm{~cm}^{-1}\right)=2953,2935,2922,2846,1625,1593,1584,1562,1479,1459,1442,1432,1386$, 1370, 1341, 1306, 1277, 1260, 1243, 1230, 1209, 1196, 1119, 1105, 985, 912, 866, 810, 764, 744, 732, $704,678,650,645,634$.

${ }^{1}$ H-NMR $\left(600 \mathrm{MHz}, \mathrm{C}_{6} \mathrm{D}_{6}\right): \delta(\mathrm{ppm})=1.69\left(\mathrm{p}, J=7.5 \mathrm{~Hz}, 2 \mathrm{H}, 6-\mathrm{H}_{2}\right), 2.51(\mathrm{dd}, J=8.1,7.1 \mathrm{~Hz}, 2 \mathrm{H}$, 7- $\left.\mathrm{H}_{2}\right), 2.93\left(\mathrm{td}, J=7.3,1.9 \mathrm{~Hz}, 2 \mathrm{H}, 5-\mathrm{H}_{2}\right), 4.90\left(\mathrm{dd}, J=10.9,1.2 \mathrm{~Hz}, 1 \mathrm{H}, 2\right.$ ''- $\left.\mathrm{H}_{\mathrm{a}}\right), 5.60(\mathrm{dd}, J=17.5$, $1.2 \mathrm{~Hz}, 1 \mathrm{H}, 2$ ' '- $\mathrm{H}_{\mathrm{b}}$ ), 6.51 (dd, $J=17.5,11.0 \mathrm{~Hz}, 1 \mathrm{H}, 1$ '’-H), 6.88-6.94 (m, $\left.2 \mathrm{H}, 3-\mathrm{H}, 9-\mathrm{H}\right), 6.96-7.02$ (m, 3 H, 2-H, 10-H, 11-H), 7.09 (dd, $J=8.2,1.4 \mathrm{~Hz}, 1 \mathrm{H}, 1-\mathrm{H}), 7.12$ (dd, $J=7.2,1.7 \mathrm{~Hz}, 1 \mathrm{H}, 6$ '-H), 7.18-7.25 (m, 2 H, 3'-H, 5'-H), 7.68 (dd, $\left.J=7.6,1.6 \mathrm{~Hz}, 1 \mathrm{H}, 4^{\prime}-\mathrm{H}\right), 7.77$ (dd, $J=7.9,1.4 \mathrm{~Hz}, 1 \mathrm{H}$, $4-\mathrm{H})$.

${ }^{13}$ C-NMR (126 MHz, $\left.\mathrm{C}_{6} \mathrm{D}_{6}\right): \delta(\mathrm{ppm})=25.5(\mathrm{C}-6), 32.1$ (C-7), 35.4 (C-5), 108.0 (C-9), 114.6 (C-2'”), 117.1 (C-1), 118.6 (C-11), 122.3, 122.4 (C-4b, C-8b), 123.0 (C-3, C-4a), 125.5 (C-4'), 126.9 (C-10), 127.1 (C-4), 127.9 (C-3'/C-5'), 128.4 (C-3'/C-5'), 129.3 (C-2), 130.6 (C-6'), 131.8 (C-8), 133.9 (C-7a), 134.2 (C-8a), 135.2 (C-1'’), 136.8 (C-2'), 138.8 (C-1'), 145.1 (C-4c), 151.6 (C-11a), 153.5 (C-11b).

MS (EI): $m / z(\%)=360.1(100)[\mathrm{M}]^{+}$.

HRMS (ESI): $m / z=$ gef.: 360.1499 , ber.: $360.1509[\mathrm{M}+\mathrm{H}]^{+}$.

$\mathbf{C}_{27} \mathbf{H}_{20} \mathrm{O}$ (360.46). 


\section{9-(2-(Trifluormethyl)phenyl)-11,12-dihydro-10H-inden[6,5,4-kl]xanthen (107c)}

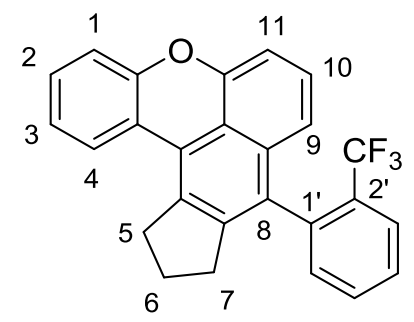

DC: $R_{f}=0.28\left(n-\mathrm{Pentan} / \mathrm{CH}_{2} \mathrm{Cl}_{2}, 10: 1\right)$.

UV/Vis $\left(\mathrm{CH}_{3} \mathrm{CN}\right): \lambda_{\max }(\mathrm{nm})(\lg \varepsilon)=223$ (4.637), 254 (4.450), 262 (4.439), 286 (3.731), 296 (3.661), 307 (3.517), 350 (4.073), 365 (4.093), 381 (3.951).

IR (ATR): $\tilde{v}\left(\mathrm{~cm}^{-1}\right)=1601,1587,1486,1444,1388,1311,1280,1259,1173,1131,1118,1105,1066$, 1052, 1036, 812, 766, 746, 734, 683, 661, 647, 635, 598.

${ }^{1} \mathbf{H}-\mathbf{N M R}\left(600 \mathrm{MHz}, \mathrm{C}_{6} \mathrm{D}_{6}\right): \delta(\mathrm{ppm})=1.63-1.72\left(\mathrm{~m}, 1 \mathrm{H}, 6-\mathrm{H}_{\mathrm{a}}\right), 1.72-1.82\left(\mathrm{~m}, 1 \mathrm{H}, 6-\mathrm{H}_{\mathrm{b}}\right), 2.37(\mathrm{ddd}$, $\left.J=15.9,8.5,6.2 \mathrm{~Hz}, 1 \mathrm{H}, 5-\mathrm{H}_{\mathrm{a}}\right), 2.58-2.64\left(\mathrm{~m}, 1 \mathrm{H}, 5-\mathrm{H}_{\mathrm{b}}\right), 2.85-2.97\left(\mathrm{~m}, 2 \mathrm{H}, 7-\mathrm{H}_{2}\right), 6.75(\mathrm{~d}, J=$ $8.0 \mathrm{~Hz}, 1 \mathrm{H}, 9-\mathrm{H}), 6.87-6.90$ (m, $1 \mathrm{H}, 3-\mathrm{H}), 6.91$ (dd, $J=7.7,1.1 \mathrm{~Hz}, 1 \mathrm{H}, 11-\mathrm{H}), 6.95$ (d, $J=7.4 \mathrm{~Hz}$, 1 H, 6'-H), 6.97-7.04 (m, 3 H, 2-H, 10-H, 4'-H), 7.07 (dd, J= 8.1, 1.4 Hz, 1 H, 1-H), 7.10-7.19 (m, 1 H, 5'-H), 7.62 (d, $\left.J=7.7 \mathrm{~Hz}, 1 \mathrm{H}, 3^{\prime}-\mathrm{H}\right), 7.73$ (dd, $\left.J=8.0,1.0 \mathrm{~Hz}, 1 \mathrm{H}, 4-\mathrm{H}\right)$.

${ }^{13} \mathrm{C}-\mathrm{NMR}\left(126 \mathrm{MHz}, \mathrm{C}_{6} \mathrm{D}_{6}\right): \delta(\mathrm{ppm})=25.7(\mathrm{C}-6), 32.1(\mathrm{C}-7), 35.3(\mathrm{C}-5), 107.9(\mathrm{C}-11), 117.1(\mathrm{C}-1)$, 118.5 (C-9), 122.1 (C-8b), 122.8 (C-4a), 123.0 (C-3, C-4b), $123.6\left(\mathrm{CF}_{3}\right), 126.7$ (C-10/C-4', C-3'), 127.2 (C-4), 127.8 (C-10/C-4'), 129.4 (C-2), 129.8 (C-2'), 130.3 (C-8), 132.2 (C-6'), 132.3 (C-5'), 133.7 (C-7a), 134.4 (C-8a), 139.0 (C-1'), 145.0 (C-4c), 151.4 (C-11a), 153.5 (C-11b).

${ }^{13}$ C-Multipletts konnten nicht zugeordnet werden.

${ }^{19}$ F-NMR $\left(282 \mathrm{MHz}, \mathrm{C}_{6} \mathrm{D}_{6}\right): \delta(\mathrm{ppm})=-60.1(\mathrm{~s})$.

MS (EI): $m / z(\%)=402.1(100)[\mathrm{M}]^{+}$.

HRMS (EI): $m / z=$ gef.: 402.1221, ber.: $402.1231[\mathrm{M}]^{+}$.

$\mathbf{C}_{26} \mathbf{H}_{17} \mathbf{F}_{3} \mathbf{O}(402.42)$. 


\section{9-Phenyl-11,12-dihydro-10H-inden[6,5,4-kl]xanthen (107d)}

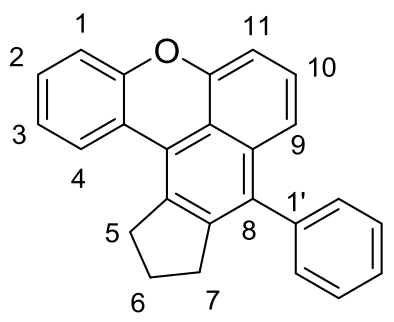

DC: $R_{f}=0.26\left(n-\mathrm{Pentan} / \mathrm{CH}_{2} \mathrm{Cl}_{2}, 10: 1\right)$.

UV/Vis $\left(\mathrm{CH}_{3} \mathrm{CN}\right): \lambda_{\max }(\mathrm{nm})(\lg \varepsilon)=223$ (4.012), 256 (4.379), 261 (3.772), 352 (3.414), 367 (3.431).

IR (ATR): $\tilde{v}\left(\mathrm{~cm}^{-1}\right)=1625,1592,1584,1565,1485,1460,1437,1390,1369,1342,1306,1281,1261$, 1228, 1210, 1118, 1071, 1065, 865, 839, 808, 755, 741, 726, 704, 645, 633, 615, 537.

${ }^{1} \mathbf{H}-\mathrm{NMR}\left(600 \mathrm{MHz}, \mathrm{C}_{6} \mathrm{D}_{6}\right): \delta(\mathrm{ppm})=1.69\left(\mathrm{p}, J=7.5 \mathrm{~Hz}, 2 \mathrm{H}, 6-\mathrm{H}_{2}\right), 2.57\left(\mathrm{t}, J=7.6 \mathrm{~Hz}, 2 \mathrm{H}, 7-\mathrm{H}_{2}\right)$, 2.94 (t, $\left.J=7.3 \mathrm{~Hz}, 2 \mathrm{H}, 5-\mathrm{H}_{2}\right), 6.88-6.93$ (m, $\left.1 \mathrm{H}, 3-\mathrm{H}\right), 6.94$ (dd, $\left.J=7.5,1.0 \mathrm{~Hz}, 1 \mathrm{H}, 11-\mathrm{H}\right), 6.97-$ 7.01 (m, 1 H, 2-H), 7.00-7.03 (m, 1 H, 10-H), 7.09 (dd, $J=8.1,1.3 \mathrm{~Hz}, 1 \mathrm{H}, 1-\mathrm{H}), 7.19-7.23$ (m, 2 H, 9-H, 4'-H), 7.25 (dt, J = 7.6, 1.7 Hz, 2 H, 2'-H, 6'-H), 7.28-7.32 (m, 2 H, 3'-H, 5'-H), 7.78 (dd, J = 7.9, $0.9 \mathrm{~Hz}, 1 \mathrm{H}, 4-\mathrm{H})$.

${ }^{13} \mathrm{C}-\mathrm{NMR}\left(126 \mathrm{MHz}, \mathrm{C}_{6} \mathrm{D}_{6}\right): \delta(\mathrm{ppm})=25.7(\mathrm{C}-6), 32.3(\mathrm{C}-7), 35.4(\mathrm{C}-5), 107.9(\mathrm{C}-11), 117.1(\mathrm{C}-1)$, 118.5 (C-9), 122.2 (C-4b), 122.5 (C-8b), 123.0 (C-4a), 123.1 (C-3), 126.6 (C-10), 127.1, 127.3 (C-4, C-4'), 128.9 (C-3', C-5'), 129.3 (C-2), 130.0 (C-2', C-6'), 133.4 (C-8), 134.1 (C-7a, C-8a), 140.2 (C-1'), 144.4 (C-4c), 151.6 (C-11a), 153.5 (C-11b).

MS (EI): $m / z(\%)=334.1(100)[\mathrm{M}]^{+}$.

HRMS (EI): $m / z=$ gef.: 334.1343 , ber.: $334.1358[\mathrm{M}]^{+}$.

$\mathbf{C}_{25} \mathbf{H}_{18} \mathrm{O}(334.42)$. 


\section{9-(2,3,5,6-Tetramethylphenyl)-11,12-dihydro-10H-inden[6,5,4- $k l]$ xanthen $(107 \mathrm{e})$}

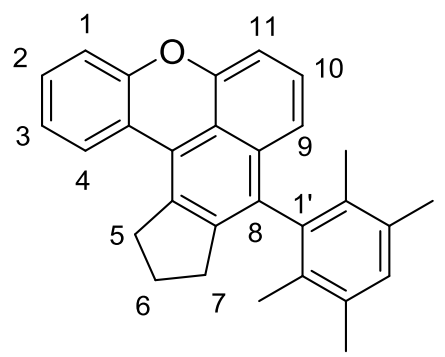

DC: $R_{f}=0.28\left(n-\mathrm{Pentan} / \mathrm{CH}_{2} \mathrm{Cl}_{2}, 10: 1\right)$.

UV/Vis $\left(\mathrm{CH}_{3} \mathrm{CN}\right): \lambda_{\max }(\mathrm{nm})(\lg \varepsilon)=221(4.000), 254$ (3.725), 262 (3.700), 351 (3.381), 364 (3.400), $379(3.260)$.

IR (ATR): $\tilde{v}\left(\mathrm{~cm}^{-1}\right)=1584,1462,1442,1381,1366,1343,1307,1282,1270,1258,1210,1114,1005$, $806,760,743,729,583$.

${ }^{1} \mathbf{H}-\mathrm{NMR}\left(600 \mathrm{MHz}, \mathrm{C}_{6} \mathrm{D}_{6}\right): \delta(\mathrm{ppm})=1.74\left(\mathrm{p}, J=7.4 \mathrm{~Hz}, 2 \mathrm{H}, 6-\mathrm{H}_{2}\right), 1.87\left(\mathrm{~s}, 6 \mathrm{H}, 2{ }^{\prime}-\mathrm{CH}_{3}, 6\right.$ '- $\left.\mathrm{CH}_{3}\right)$, $2.21\left(\mathrm{~s}, 6 \mathrm{H}, 3^{\prime}-\mathrm{CH}_{3}, 5^{\prime}-\mathrm{CH}_{3}\right), 2.46\left(\mathrm{t}, J=7.6 \mathrm{~Hz}, 2 \mathrm{H}, 7-\mathrm{H}_{2}\right), 2.98\left(\mathrm{t}, J=7.3 \mathrm{~Hz}, 2 \mathrm{H}, 5-\mathrm{H}_{2}\right), 6.90-6.95$ (m, 2 H, 3-H, 9-H), 6.96-7.03 (m, 3 H, 2-H, 10-H, 11-H), 7.05 (s, 1 H, 6'-H), 7.11 (dd, J = 8.1, $1.3 \mathrm{~Hz}$, $1 \mathrm{H}, 1-\mathrm{H}), 7.80(\mathrm{dd}, J=7.9,1.0 \mathrm{~Hz}, 1 \mathrm{H}, 4-\mathrm{H})$.

${ }^{13} \mathbf{C}-\mathbf{N M R}\left(126 \mathrm{MHz}, \mathrm{C}_{6} \mathrm{D}_{6}\right): \delta(\mathrm{ppm})=16.3\left(2^{\prime}-\mathrm{CH}_{3}, 6{ }^{\prime}-\mathrm{CH}_{3}\right), 20.3\left(3^{\prime}-\mathrm{CH}_{3}, 5^{\prime}-\mathrm{CH}_{3}\right), 25.6$ (C-6), 32.0 (C-7), 35.5 (C-5), 108.1 (C-9), 117.1 (C-1), 117.9 (C-11), 121.9 (C-4b), 122.5 (C-8b), 123.0 (C-3), 123.2 (C-4a), 126.9, 127.0 (C-4, C-10), 129.2 (C-2), 131.1 (C-4'), 132.2 (C-2', C-6'), 133.4 (C-8), 133.8 (C-8a), 134.1 (C-7a, C-3', C-5'), 138.8 (C-1'), 144.4 (C-4c), 151.8 (C-11a), 153.5 (C-11b).

MS (EI): $m / z(\%)=390.2(100)[\mathrm{M}]^{+}$.

HRMS (ESI): $m / z=$ gef.: 390.1980 , ber.: $390.1984[\mathrm{M}+\mathrm{Na}]^{+}$.

$\mathbf{C}_{29} \mathbf{H}_{26} \mathbf{O}(390.53)$. 


\section{9-(2-(2-Methoxyphenoxy)phenyl)-11,12-dihydro-10H-inden[6,5,4-kl]xanthen (107f)}

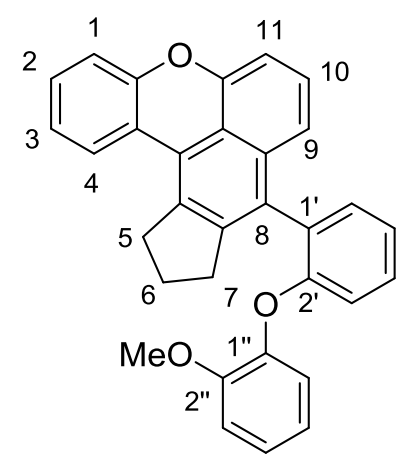

DC: $R_{f}=0.46\left(n-\mathrm{Pentan} / \mathrm{CH}_{2} \mathrm{Cl}_{2}, 2: 1\right)$.

UV/Vis $\left(\mathrm{CH}_{3} \mathrm{CN}\right): \lambda_{\max }(\mathrm{nm})(\lg \varepsilon)=195$ (4.958), 222 (4.711), 256 (4.402), 263 (4.400), 351 (4.047), 367 (4.085), 384 (3.953).

IR (ATR): $\tilde{v}\left(\mathrm{~cm}^{-1}\right)=1599,1584,1573,1497,1482,1456,1442,1391,1373,1343,1305,1259,1229$, $1215,1175,1156,1114,1108,1038,1019,884,810,800,759,731,644,633$.

${ }^{1} \mathbf{H}-\mathbf{N M R}\left(600 \mathrm{MHz}, \mathrm{C}_{6} \mathrm{D}_{6}\right): \delta(\mathrm{ppm})=1.74-1.89\left(\mathrm{~m}, 2 \mathrm{H}, 6-\mathrm{H}_{2}\right), 2.71-2.80\left(\mathrm{~m}, 1 \mathrm{H}, 7-\mathrm{H}_{\mathrm{a}}\right), 2.86-2.94$ (m, $\left.1 \mathrm{H}, 7-\mathrm{H}_{\mathrm{b}}\right), 2.95-3.02\left(\mathrm{~m}, 1 \mathrm{H}, 5-\mathrm{H}_{\mathrm{a}}\right), 3.06-3.13\left(\mathrm{~m}, 1 \mathrm{H}, 5-\mathrm{H}_{\mathrm{b}}\right), 3.11\left(\mathrm{~d}, J=1.6 \mathrm{~Hz}, 3 \mathrm{H}, \mathrm{OCH}_{3}\right)$, $6.42(\mathrm{dt}, J=8.1,1.5 \mathrm{~Hz}, 1 \mathrm{H}, 3$ ''-H), 6.60 (ddt, $J=9.3,7.9,1.6 \mathrm{~Hz}, 1 \mathrm{H}, 5$ '”-H), 6.73-6.78 (m, $1 \mathrm{H}$, 4'”-H), 6.85-6.91 (m, 3 H, 3-H, 3'-H, 6''-H), 6.93-7.02 (m, 3 H, 2-H, 9-H, 5'-H), 7.05-7.12 (m, 3 H, 1-H, 10-H, 4'-H), 7.26 (dt, J= 7.4, 1.6 Hz, 1 H, 6'-H), 7.35 (dt, $J=8.4,1.2 \mathrm{~Hz}, 1 \mathrm{H}, 11-\mathrm{H}), 7.74$ (d, $J=8.0 \mathrm{~Hz}, 1 \mathrm{H}, 4-\mathrm{H})$.

${ }^{13}$ C-NMR $\left(126 \mathrm{MHz}, \mathrm{C}_{6} \mathrm{D}_{6}\right): \delta(\mathrm{ppm})=25.6(\mathrm{C}-6), 32.3(\mathrm{C}-7), 35.5(\mathrm{C}-5), 55.3\left(\mathrm{OCH}_{3}\right), 107.6(\mathrm{C}-9)$, 113.9 (C-3'), 116.5 (C-3/C-3'), 117.0 (C-1), 118.9 (C-11), 121.2 (C-5’'), 122.1 (C-6’'), 122.2 (C-1'), 122.5, 122.6 (C-4b, C-8b), 122.9 (C-3/C-3'), 123.1 (C-4a), 124.9 (C-4'’), 126.2 (C-10), 127.1 (C-4), 128.9, 129.1 (C-2, C-4'), 129.6, 129.7 (C-8, C-5'), 132.1 (C-6'), 134.1 (C-7a), 134.4 (C-8a), 145.4 (C-1'”), 145.7 (C-4c), 151.6 (C-11a), 152.0 (C-2’'), 153.5 (C-11b), 156.7 (C-2').

MS (ESI): $m / z(\%)=457.2(38)[\mathrm{M}+\mathrm{H}]^{+}, 474.2(9)\left[\mathrm{M}+\mathrm{NH}_{4}\right]^{+}, 479.2(17)[\mathrm{M}+\mathrm{Na}]^{+}$.

HRMS (ESI): $m / z=$ gef.: 457.1778, ber.: $457.1798[\mathrm{M}+\mathrm{H}]^{+}$.

$\mathbf{C}_{32} \mathbf{H}_{24} \mathbf{O}_{3}$ (456.54). 


\subsubsection{Synthese des Tetraalkins 136}

\section{1,4-Dibrom-2,5-bis((octa-2,7-diin-1-yloxy)methyl)benzol (136)}

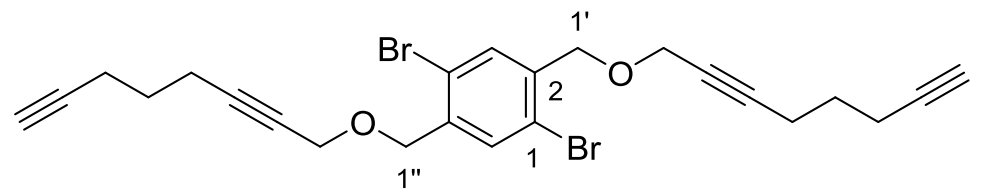

Eine Suspension von NaH (60\% in Mineralöl, 190 mg, 4.74 mmol, 10.0 Äq.) in THF (5 mL) wurde bei $0{ }^{\circ} \mathrm{C}$ mit einer Lösung von Octa-2,7-diin-1-ol (140b) (290 mg, $2.37 \mathrm{mmol}, 5.00 \mathrm{Äq}$.) in THF (5 mL) versetzt und 15 min gerührt. Eine Lösung von 1,4-Dibrom-2,5-bis(brommethyl)benzol (139) (200 mg, $474 \mu \mathrm{mol}, 1.00$ Äq.) in THF (5 mL) wurde hinzugefügt und die Lösung über $19 \mathrm{~h}$ auf RT erwärmt. Nach Zugabe von $\mathrm{H}_{2} \mathrm{O}(20 \mathrm{~mL})$ wurde die Reaktionsmischung mit $\mathrm{CH}_{2} \mathrm{Cl}_{2}$ extrahiert $(3 \times 20 \mathrm{~mL})$, die vereinigten organischen Phasen über $\mathrm{Na}_{2} \mathrm{SO}_{4}$ getrocknet, filtriert und das Lösungsmittel in vacuo entfernt. Säulenchromatographische Reinigung $\left(\mathrm{SiO}_{2}, n\right.$-Pentan/EtOAc, 50:1) lieferte Dialkin 136 als weißen Feststoff (185 mg, $367 \mu \mathrm{mol}, 77 \%$ ).

DC: $R_{f}=0.40(n-$ Pentan/EtOAc, 50:1).

UV/Vis $\left(\mathrm{CH}_{3} \mathrm{CN}\right): \lambda_{\max }(\mathrm{nm})(\lg \varepsilon)=206$ (4.660), 231 (4.092), 274 (3.093).

IR (ATR): $\tilde{v}\left(\mathrm{~cm}^{-1}\right)=3295,2937,2906,2846,1451,1432,1355,1255,1175,1132,1090,1078,1052$, 1023, 949, 884, 633.

${ }^{1} \mathbf{H}$-NMR $\left(300 \mathrm{MHz}, \mathrm{CDCl}_{3}\right): \delta(\mathrm{ppm})=1.73\left(\mathrm{p}, J=7.0 \mathrm{~Hz}, 4 \mathrm{H}, 6{ }^{\prime}-\mathrm{H}_{2}, 6{ }^{\prime}{ }^{-}-\mathrm{H}_{2}\right), 1.95(\mathrm{t}, J=2.6 \mathrm{~Hz}$, $2 \mathrm{H}, 9^{\prime}-\mathrm{H}, 9^{\prime}$ '-H), 2.30 (td, $\left.J=7.0,2.6 \mathrm{~Hz}, 4 \mathrm{H}\right), 2.37$ (tt, $\left.J=7.0,2.1 \mathrm{~Hz}, 4 \mathrm{H}\right)\left(5^{\prime}-\mathrm{H}_{2}, 5^{\prime}\right.$ ' $-\mathrm{H}_{2}, 7^{\prime}-\mathrm{H}_{2}$, 7' '- $\left.\mathrm{H}_{2}\right), 4.23$ (t, $J=2.2 \mathrm{~Hz}, 4 \mathrm{H}, 2^{\prime}-\mathrm{H}_{2}, 2$ ''- $\left.\mathrm{H}_{2}\right), 4.58$ (s, $4 \mathrm{H}, 1$ ' $-\mathrm{H}_{2}, 1$ '’- $\left.\mathrm{H}_{2}\right), 7.64$ (s, $\left.2 \mathrm{H}, 3-\mathrm{H}, 6-\mathrm{H}\right)$.

${ }^{13} \mathbf{C}-\mathrm{NMR}\left(126 \mathrm{MHz}, \mathrm{CDCl}_{3}\right): \delta(\mathrm{ppm})=17.6,17.8$ (C-5', C-7', C-5', C-7' '), 27.4 (C-6', C-6'”), 58.6 (C-2', C-2'”), 68.9 (C-9', C-9'”), 70.0 (C-1', C-1'”), 76.1 (C-8', C-8''), 83.4, 86.5 (C-3', C-4', C-3'”, C-4’’), 121.3 (C-1, C-4), 132.5 (C-3, C-6), 138.1 (C-2, C-5).

MS (ESI): $m / z(\%)=527(24)[\mathrm{M}+\mathrm{Na}]^{+}$.

HRMS (ESI): $m / z=$ gef.: 525.0015 , ber.: $525.0035\left[\mathrm{M}\left({ }^{79} \mathrm{Br}^{79} \mathrm{Br}\right)\right]^{+}$; gef.: 526.9979 , ber.: 527.0016 $\left[\mathrm{M}\left({ }^{79} \mathrm{Br}^{81} \mathrm{Br}\right)\right]^{+}$; gef.: 528.9965, ber.: $528.9995\left[\mathrm{M}\left({ }^{81} \mathrm{Br}^{81} \mathrm{Br}\right)\right]^{+}$.

$\mathrm{C}_{24} \mathrm{H}_{24} \mathrm{Br}_{2} \mathbf{O}_{2}$ (504.26). 


\subsection{Synthese der Polyzyklen des Typs 110}

\subsubsection{Synthese der Aryliodide 111a-c}

\section{1-Brom-2-(2-nitrophenoxy)naphthalin (144a) ${ }^{\mathrm{f}}$}<smiles>O=[N+]([O-])c1ccccc1Oc1ccc2ccccc2c1Br</smiles>

Eine Mischung aus 2-Bromnaphthol (143) (5.00 g, 22.4 mmol, 1.00 Äq.), 2-Fluornitrobenzol (121a) (2.36 mL, 3.16 g, 22.4 mmol, 1.00 Äq.) und $\mathrm{K}_{2} \mathrm{CO}_{3}$ (6.10 g, $44.8 \mathrm{mmol}, 2.00$ Äq.) in DMSO (40 mL) wurde $11.5 \mathrm{~h}$ bei $95{ }^{\circ} \mathrm{C}$ gerührt. Nach Abkühlen auf RT wurde der ausgefallene Feststoff abfitriert, in $\mathrm{CH}_{2} \mathrm{Cl}_{2}$ gelöst, über $\mathrm{Na}_{2} \mathrm{SO}_{4}$ getrocknet, filtriert und das Lösungsmittel in vacuo entfernt. Biarylether 144a wurde als brauner Feststoff erhalten ( $6.26 \mathrm{~g}, 18.2 \mathrm{mmol}, 81 \%)$.

DC: $R_{f}=0.31\left(\mathrm{PE} / \mathrm{Et}_{2} \mathrm{O}, 10: 1\right)$.

UV/Vis $\left(\mathrm{CH}_{3} \mathrm{CN}\right): \lambda_{\max }(\mathrm{nm})(\lg \varepsilon)=228(4.775), 275$ (3.901).

IR (ATR): $\tilde{v}\left(\mathrm{~cm}^{-1}\right)=1605,1584,1517,1457,1340,1235,1160,987,934,861,832,808,777,751$, $738,637,527$.

${ }^{1} \mathbf{H}-\mathrm{NMR}\left(600 \mathrm{MHz}, \mathrm{CDCl}_{3}\right): \delta(\mathrm{ppm})=6.79\left(\mathrm{dd}, J=8.4,1.2 \mathrm{~Hz}, 1 \mathrm{H}\right.$, 6'-H) $^{\prime} 7.18(\mathrm{ddd}, J=8.4,7.3$, $\left.1.2 \mathrm{~Hz}, 1 \mathrm{H}, 4^{\prime}-\mathrm{H}\right), 7.23$ (d, $\left.J=8.9 \mathrm{~Hz}, 1 \mathrm{H}, 3-\mathrm{H}\right), 7.43$ (ddd, $J=8.6,7.3,1.5 \mathrm{~Hz}, 1 \mathrm{H}, 5$ '-H), 7.54 (ddd, $J=8.1,7.0,1.2 \mathrm{~Hz}, 1 \mathrm{H}, 6-\mathrm{H}), 7.64(\mathrm{ddd}, J=8.2,7.0,1.2 \mathrm{~Hz}, 1 \mathrm{H}, 7-\mathrm{H}), 7.85$ (dd, $J=8.4$, $6.5 \mathrm{~Hz}, 2 \mathrm{H}, 4-\mathrm{H}, 5-\mathrm{H}), 8.00$ (dd, $\left.J=8.2,1.6 \mathrm{~Hz}, 1 \mathrm{H}, 3^{\prime}-\mathrm{H}\right), 8.28$ (dd, $\left.J=8.5,1.2 \mathrm{~Hz}, 1 \mathrm{H}, 8-\mathrm{H}\right)$.

${ }^{13} \mathbf{C}-\mathbf{N M R}\left(126 \mathrm{MHz}, \mathrm{CDCl}_{3}\right): \delta(\mathrm{ppm})=114.3(\mathrm{C}-1), 118.6\left(\mathrm{C}^{\prime}{ }^{\prime}\right.$ '), $120.3(\mathrm{C}-3), 122.9\left(\mathrm{C}-4^{\prime}\right), 125.9$ (C-3'), 126.4 (C-6), 126.9 (C-8), 128.1 (C-7), 128.3 (C-5), 129.6 (C-4), 131.9 (C-4a), 133.1 (C-8a), 134.1 (C-5'), 140.4 (C-2'), 149.6 (C-1'), 150.5 (C-2).

MS (ESI): $m / z=361.0(100)\left[\mathrm{M}\left({ }^{79} \mathrm{Br}\right)+\mathrm{NH}_{4}\right]^{+}, 711.0(61)\left[2 \mathrm{M}\left({ }^{79} \mathrm{Br}{ }^{81} \mathrm{Br}\right)+\mathrm{Na}\right]^{+}$.

HRMS (ESI): $m / z=$ gef.: 343.9916, ber.: $343.9917 \quad\left[\mathrm{M}\left({ }^{79} \mathrm{Br}\right)+\mathrm{H}\right]^{+}$; gef.: 345.9892 , ber.: 345.9897 $\left[\mathrm{M}\left({ }^{81} \mathrm{Br}\right)+\mathrm{H}\right]^{+}$; gef.: 361.0175, ber.: $361.0182 \quad\left[\mathrm{M}\left({ }^{79} \mathrm{Br}\right)+\mathrm{NH}_{4}\right]^{+}$, gef.: 363.0155 , ber.: 363.0163 $\left[\mathrm{M}\left({ }^{81} \mathrm{Br}\right)+\mathrm{NH}_{4}\right]^{+}$; gef.: 365.9730 , ber.: $365.9736\left[\mathrm{M}\left({ }^{79} \mathrm{Br}\right)+\mathrm{Na}\right]^{+}$; gef.: 367.9707 , ber.: 367.9716 $\left[\mathrm{M}\left({ }^{81} \mathrm{Br}\right)+\mathrm{Na}\right]^{+}$.

\footnotetext{
${ }_{\mathrm{f}}^{\mathrm{f}}$ Experimentelle Vorschrift aus: M. A. Düfert, Dissertation, Georg-August-Universität, Göttingen, 2010.
} 
$\mathrm{C}_{16} \mathrm{H}_{10} \mathrm{BrNO}_{3}(344.16)$. 


\section{2-((1-Bromnaphthalin-2-yl)oxy)anilin (145a) ${ }^{\mathrm{g}}$}<smiles>Nc1ccccc1Oc1ccc2ccccc2c1Br</smiles>

$\mathrm{Zu}$ einer Lösung von 1-Brom-2-(2-nitrophenoxy)naphthalin (144a) (6.25 g, $18.2 \mathrm{mmol}, 1.00$ Äq.) in EtOAc $(100 \mathrm{~mL})$ wurden bei $0{ }^{\circ} \mathrm{C}$ tropfenweise konz. AcOH $(125 \mathrm{~mL})$, konz. $\mathrm{HCl}(125 \mathrm{~mL})$ und anschließend portionsweise Zinkpulver $(35.7 \mathrm{~g}, \quad 546 \mathrm{mmol}, \quad 30.0$ Äq.) hinzugefügt. Die Reaktionsmischung wurde auf RT erwärmt, $16 \mathrm{~h}$ bei dieser Temperatur gerührt, auf $0{ }^{\circ} \mathrm{C}$ abgekühlt, tropfenweise mit 33\%iger wässr. $\mathrm{NH}_{3}$-Lösung $\left(300 \mathrm{~mL}\right.$ ) versetzt und mit $\mathrm{CH}_{2} \mathrm{Cl}_{2}$ extrahiert $(4 \times 200 \mathrm{~mL})$. Die vereinigten organischen Phasen wurden über $\mathrm{Na}_{2} \mathrm{SO}_{4}$ getrocknet, filtriert und das Lösungsmittel in vacuo entfernt. Amin 145a wurde als braunes Öl (5.57 g, 17.7 mmol, 97\%) in leicht verunreinigter Form erhalten.

DC: $R_{f}=0.14(n-$ Pentan/EtOAc, 50:1).

UV/Vis $\left(\mathrm{CH}_{3} \mathrm{CN}\right): \lambda_{\max }(\mathrm{nm})(\lg \varepsilon)=202$ (4.516), 230 (4.845), 283 (4.000), 292 (3.974), 331 (3.252).

IR (Film): $\tilde{v}\left(\mathrm{~cm}^{-1}\right)=3467,3379,3055,1620,1501,1460,1232,1191,742$.

${ }^{1} \mathbf{H}-\mathrm{NMR}\left(300 \mathrm{MHz}, \mathrm{CDCl}_{3}\right): \delta(\mathrm{ppm})=3.91\left(\mathrm{~s}, 2 \mathrm{H}, \mathrm{NH}_{2}\right), 6.68(\mathrm{ddd}, J=8.0,7.2,1.4 \mathrm{~Hz}, 1 \mathrm{H}, 4$ '-H), $6.76\left(\mathrm{dd}, J=8.0,1.5 \mathrm{~Hz}, 1 \mathrm{H}, 3^{\prime}-\mathrm{H}\right), 6.84$ (dd, $\left.J=7.9,1.5 \mathrm{~Hz}, 1 \mathrm{H}, 6^{\prime}-\mathrm{H}\right), 6.98$ (ddd, $J=7.8,7.3$, $1.6 \mathrm{~Hz}, 1 \mathrm{H}, 5$ '-H), 7.11 (d, $J=8.9 \mathrm{~Hz}, 1 \mathrm{H}, 3-\mathrm{H}), 7.46$ (ddd, $J=8.1,6.9,1.1 \mathrm{~Hz}, 1 \mathrm{H}, 6-\mathrm{H}), 7.60$ (ddd, $J=8.4,6.9,1.3 \mathrm{~Hz}, 1 \mathrm{H}, 7-\mathrm{H}), 7.73(\mathrm{~d}, J=9.0 \mathrm{~Hz}, 1 \mathrm{H}, 4-\mathrm{H}), 7.79(\mathrm{~d}, J=8.1 \mathrm{~Hz}, 1 \mathrm{H}, 5-\mathrm{H}), 8.27$ (d, $J=8.5 \mathrm{~Hz}, 1 \mathrm{H}, 8-\mathrm{H})$.

${ }^{13}$ C-NMR (126 MHz, $\left.\mathrm{CDCl}_{3}\right): \delta(\mathrm{ppm})=111.5(\mathrm{C}-1), 116.4\left(\mathrm{C}^{\prime}{ }^{\prime}\right.$ '), 118.5 (C-3), $118.6\left(\mathrm{C}^{\prime} 4^{\prime}\right), 118.9$ (C-3'), 124.8 (C-5'), 125.3 (C-6), 126.5 (C-8), 127.8 (C-7), 128.1 (C-5), 129.0 (C-4), 131.0 (C-4a), 133.1 (C-8a), 138.1 (C-2'), 143.5 (C-1'), 151.4 (C-2).

MS $(\mathrm{EI}, 70 \mathrm{eV}): \quad m / z(\%)=235.1 \quad(98) \quad[\mathrm{M}-\mathrm{Br}+\mathrm{H}]^{+}, \quad 314.0 \quad(100) \quad\left[\mathrm{M}\left({ }^{79} \mathrm{Br}\right)+\mathrm{H}\right]^{+}, \quad 316.0 \quad(98)$ $\left[\mathrm{M}\left({ }^{81} \mathrm{Br}\right)+\mathrm{H}\right]^{+}$.

HRMS (ESI): $\mathrm{m} / z=$ gef.: 316.0155 , ber.: $316.0147[\mathrm{M}+\mathrm{H}]^{+}$.

$\mathbf{C}_{16} \mathbf{H}_{12} \operatorname{BrNO}$ (314.18).

\footnotetext{
${ }^{\mathrm{g}}$ Experimentelle Vorschrift und Daten aus: M. A. Düfert, Dissertation, Georg-August-Universität, Göttingen,
} 2010. 


\section{1-Brom-2-(2-iodphenoxy)naphthalin (111a) ${ }^{\mathrm{h}}$}

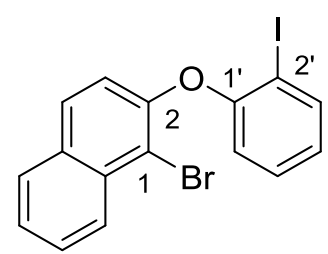

Eine Lösung von 2-((1-Bromnaphthalin-2-yl)oxy)anilin (145a) (5.70 g, $18.1 \mathrm{mmol}, 1.00$ Äq.) und $p$ - $\mathrm{TsOH} \cdot \mathrm{H}_{2} \mathrm{O}\left(10.3 \mathrm{~g}, 54.3 \mathrm{mmol}, 3.00 \mathrm{Äq}\right.$.) in $\mathrm{CH}_{3} \mathrm{CN}(100 \mathrm{~mL})$ wurde tropfenweise mit einer Lösung von KI (6.01 g, $36.2 \mathrm{mmol}, 2.00$ Äq.) und $\mathrm{NaNO}_{2}$ (2.50 g, $36.2 \mathrm{mmol}, 2.00$ Äq.) in $\mathrm{H}_{2} \mathrm{O}$ $(50 \mathrm{~mL})$ versetzt. Die Reaktionsmischung wurde $14 \mathrm{~h}$ bei RT gerührt und nach Zugabe von ges. wässr. $\mathrm{NaHCO}_{3}$-Lösung $(100 \mathrm{~mL})$ und $1 \mathrm{M} \mathrm{Na}_{2} \mathrm{~S}_{2} \mathrm{O}_{3}$-Lösung $(100 \mathrm{~mL})$ mit $\mathrm{CH}_{2} \mathrm{Cl}_{2}$ extrahiert $(3 \times 300 \mathrm{~mL})$. Die vereinigten organischen Phasen wurden über $\mathrm{Na}_{2} \mathrm{SO}_{4}$ getrocknet, filtriert und das Lösungsmittel in vacuo entfernt. Säulenchromatographische Reinigung $\left(\mathrm{SiO}_{2}, n\right.$-Pentan $\left./ \mathrm{CH}_{2} \mathrm{Cl}_{2}, 20: 1\right)$ lieferte Aryliodid 111a als gelben Feststoff (6.08 g, $14.3 \mathrm{mmol}, 79 \%)$.

DC: $R_{f}=0.43\left(n-\mathrm{Pentan} / \mathrm{CH}_{2} \mathrm{Cl}_{2}, 20: 1\right)$.

UV/Vis $\left(\mathrm{CH}_{3} \mathrm{CN}\right): \lambda_{\max }(\mathrm{nm})(\lg \varepsilon)=230(4.814), 283(3.910)$.

IR (ATR): $\tilde{v}\left(\mathrm{~cm}^{-1}\right)=1572,1465,1456,1436,1233,989,934,830,811,747,635,527$.

${ }^{1} \mathbf{H}-\mathrm{NMR}\left(600 \mathrm{MHz}, \mathrm{CDCl}_{3}\right): \delta(\mathrm{ppm})=6.69\left(\mathrm{dt}, J=8.2,1.2 \mathrm{~Hz}, 1 \mathrm{H}, 6^{\prime}-\mathrm{H}\right), 6.86(\mathrm{t}, J=7.6 \mathrm{~Hz}, 1 \mathrm{H}$, 4'-H), 7.09 (dd, $J=8.9,1.0 \mathrm{~Hz}, 1 \mathrm{H}, 3-\mathrm{H}), 7.20-7.27$ (m, $\left.1 \mathrm{H}, 5^{\prime}-\mathrm{H}\right), 7.50$ (t, $\left.J=7.5 \mathrm{~Hz}, 1 \mathrm{H}, 6-\mathrm{H}\right)$, $7.62\left(\mathrm{~m}_{\mathrm{C}}, 1 \mathrm{H}, 7-\mathrm{H}\right), 7.78(\mathrm{~d}, J=8.8 \mathrm{~Hz}, 1 \mathrm{H}, 4-\mathrm{H}), 7.82(\mathrm{~d}, J=8.2 \mathrm{~Hz}, 1 \mathrm{H}, 5-\mathrm{H}), 7.89$ (dd, $J=7.9$, $\left.1.2 \mathrm{~Hz}, 1 \mathrm{H}, 3^{\prime}-\mathrm{H}\right), 8.29$ (d, $\left.J=8.5 \mathrm{~Hz}, 1 \mathrm{H}, 8-\mathrm{H}\right)$.

${ }^{13}$ C-NMR $\left(126 \mathrm{MHz}, \mathrm{CDCl}_{3}\right): \delta(\mathrm{ppm})=87.5$ (C-2'), $113.2(\mathrm{C}-1), 117.6$ (C-6'), 119.8 (C-3), 125.1 (C-4'), 125.8 (C-6), 126.8 (C-8), 127.9 (C-7), 128.2 (C-5), 129.2 (C-4), 129.6 (C-5'), 131.5 (C-4a), 133.2 (C-8a), 140.0 (C-3'), 150.8 (C-1'), 156.4 (C-2).

MS (EI, $70 \mathrm{eV}): \mathrm{m} / z(\%)=218.1(100)[\mathrm{M}-\mathrm{Br}-\mathrm{I}]^{+}, 423.9(73)\left[\mathrm{M}\left({ }^{79} \mathrm{Br}\right)\right]^{+}, 425.9(70)\left[\mathrm{M}\left({ }^{81} \mathrm{Br}\right)\right]^{+}$.

HRMS (EI): $m / z=$ gef.: 423.8957 , ber.: $423.8960\left[\mathrm{M}\left({ }^{79} \mathrm{Br}\right)\right]^{+}$.

$\mathbf{C}_{16} \mathbf{H}_{10}$ BrIO (425.06).

${ }^{\text {h }}$ Experimentelle Vorschrift aus: M. A. Düfert, Dissertation, Georg-August-Universität, Göttingen, 2010. Allgemeine Methode aus: E. A. Krasnokutskaya, N. I. Semenischeva, V. D. Filimonov, P. Knochel, Synthesis 2007, 81-84. 


\section{2-Fluor-4-methoxy-1-nitrobenzol (121c) ${ }^{\mathrm{i}}$}<smiles>COc1ccc([N+](=O)[O-])c(F)c1</smiles>

Eine Mischung aus 3-Fluor-4-nitrophenol (142) (1.50 g, $9.03 \mathrm{mmol}, 1.00 \mathrm{Äq}$ ), $\mathrm{K}_{2} \mathrm{CO}_{3}(2.50 \mathrm{~g}$, $18.1 \mathrm{mmol}, 2.00$ Äq.) und Methyliodid (1.15 mL, $2.50 \mathrm{~g}, 18.1 \mathrm{mmol}, 2.00$ Äq.) in Aceton $(20 \mathrm{~mL})$ wurde $14 \mathrm{~h}$ bei RT gerührt, mit $\mathrm{H}_{2} \mathrm{O}(50 \mathrm{~mL})$ versetzt und mit $\mathrm{CH}_{2} \mathrm{Cl}_{2}$ extrahiert $(2 \times 50 \mathrm{~mL})$. Die vereinigten organischen Phasen wurden über $\mathrm{Na}_{2} \mathrm{SO}_{4}$ getrocknet, filtriert und das Lösungsmittel in vacuo entfernt. Säulenchromatographische Reinigung $\left(\mathrm{SiO}_{2}, n\right.$-Pentan/EtOAc, 10:1) lieferte Methylether 121c als weißen Feststoff (1.53 g, $8.94 \mathrm{mmol}, 99 \%)$.

DC: $R_{f}=0.29(n-$ Pentan/EtOAc, 10:1).

UV/Vis $\left(\mathrm{CH}_{3} \mathrm{CN}\right): \lambda_{\max }(\mathrm{nm})(\lg \varepsilon)=226(3.860), 301(4.013)$.

IR (ATR): $\tilde{v}\left(\mathrm{~cm}^{-1}\right)=1598,1507,1494,1330,1295,1278,1243,1198,1167,1089,1017,954,848$, $827,748,635,624,586$.

${ }^{1} \mathbf{H}-\mathrm{NMR}\left(300 \mathrm{MHz}, \mathrm{CDCl}_{3}\right): \delta(\mathrm{ppm})=3.88\left(\mathrm{~s}, 3 \mathrm{H}, \mathrm{OCH}_{3}\right), 6.64-6.80(\mathrm{~m}, 2 \mathrm{H}, 3-\mathrm{H}, 5-\mathrm{H}), 8.03-8.11$ (m, $1 \mathrm{H}, 6-\mathrm{H})$.

${ }^{13}$ C-NMR $\left(126 \mathrm{MHz}, \mathrm{CDCl}_{3}\right): \delta(\mathrm{ppm})=56.3\left(\mathrm{OCH}_{3}\right), 103.2(\mathrm{~d}, J=24.3 \mathrm{~Hz}, \mathrm{C}-3), 110.4(\mathrm{~d}, J=$ 3.0 Hz, C-6), 127.9 (C-5), 157.5 (d, J=265.1 Hz, C-2), 165.2, 165.3 (C-1, C-4).

${ }^{19}$ F-NMR $\left(282 \mathrm{MHz}, \mathrm{CDCl}_{3}\right): \delta(\mathrm{ppm})=-112.7--112.8(\mathrm{~m}, 2-\mathrm{F})$.

MS (ESI): $m / z(\%)=172.0(48)[\mathrm{M}+\mathrm{H}]^{+}, 194.0(100)[\mathrm{M}+\mathrm{Na}]^{+}$.

HRMS (ESI): $m / z=$ gef.: 172.0405, ber.: $172.0404 \quad[\mathrm{M}+\mathrm{H}]^{+}$; gef.: 189.0669 , ber.: 189.0670 $\left[\mathrm{M}+\mathrm{NH}_{4}\right]^{+}$; gef.: 194.0224 , ber.: $194.0224[\mathrm{M}+\mathrm{Na}]^{+}$.

$\mathrm{C}_{7} \mathrm{H}_{6} \mathrm{FNO}_{3}$ (171.13).

\footnotetext{
${ }^{i}$ Experimentelle Vorschrift aus: M. Koppitz, B. Lindenthal, PCT Int. Appl. 2010, 2010012396.
} 


\section{1-Brom-2-(5-methoxy-2-nitrophenoxy)naphthalin (144b)}<smiles>COc1ccc([N+](=O)[O-])c(Oc2ccc3ccccc3c2Br)c1</smiles>

Eine Mischung aus 2-Bromnaphthol (143) (1.98 g, $8.88 \mathrm{mmol}, \quad 1.00$ Äq.), 2-Fluor-4-methoxy-1-nitrobenzol (121c) (1.52 g, $8.88 \mathrm{mmol}, 1.00$ Äq.) und $\mathrm{K}_{2} \mathrm{CO}_{3} \quad(2.46 \mathrm{~g}$, $17.8 \mathrm{mmol}, 2.00$ Äq.) in DMSO (30 mL) wurde $13 \mathrm{~h}$ bei $95^{\circ} \mathrm{C}$ gerührt. Nach Abkühlen auf RT wurde die Reaktionsmischung in Eiswasser $(100 \mathrm{~mL})$ gegeben, der ausgefallene Feststoff abfiltriert, in $\mathrm{CH}_{2} \mathrm{Cl}_{2}$ gelöst, über $\mathrm{Na}_{2} \mathrm{SO}_{4}$ getrocknet, filtriert und das Lösungsmittel in vacuo entfernt. Säulenchromatographische Reinigung $\left(\mathrm{SiO}_{2}, n\right.$-Pentan/EtOAc, $\left.20: 1 \rightarrow 10: 1\right)$ lieferte Biarylether 144b als gelbes Öl (2.97 g, $7.94 \mathrm{mmol}, 89 \%)$.

DC: $R_{f}=0.23(n$-Pentan/EtOAc, 10:1).

UV/Vis $\left(\mathrm{CH}_{3} \mathrm{CN}\right): \lambda_{\max }(\mathrm{nm})(\lg \varepsilon)=227$ (4.786), 284 (4.111).

IR (ATR): $\tilde{v}\left(\mathrm{~cm}^{-1}\right)=1583,1509,1491,1458,1339,1288,1274,1252,1220,1193,1163,1093,1086$, 1026, 991, 842, 810, 747, 617, 527.

${ }^{1} \mathbf{H}-\mathrm{NMR}\left(600 \mathrm{MHz}, \mathrm{CDCl}_{3}\right): \delta(\mathrm{ppm})=3.71\left(\mathrm{~s}, 3 \mathrm{H}, \mathrm{OCH}_{3}\right), 6.23(\mathrm{~d}, J=2.6 \mathrm{~Hz}, 1 \mathrm{H}, 6$ '-H), $6.65(\mathrm{dd}$, $\left.J=9.2,2.6 \mathrm{~Hz}, 1 \mathrm{H}, 4^{\prime}-\mathrm{H}\right), 7.22(\mathrm{~d}, J=8.8 \mathrm{~Hz}, 1 \mathrm{H}, 3-\mathrm{H}), 7.53(\mathrm{t}, J=7.5 \mathrm{~Hz}, 1 \mathrm{H}, 6-\mathrm{H}), 7.64(\mathrm{t}, J=$ $7.7 \mathrm{~Hz}, 1 \mathrm{H}, 7-\mathrm{H}), 7.84(\mathrm{t}, J=8.3 \mathrm{~Hz}, 1 \mathrm{H}), 7.85(\mathrm{~d}, J=8.3 \mathrm{~Hz}, 1 \mathrm{H})(4-\mathrm{H}, 5-\mathrm{H}), 8.11(\mathrm{~d}, J=9.2 \mathrm{~Hz}$, $\left.1 \mathrm{H}, 3^{\prime}-\mathrm{H}\right), 8.28(\mathrm{~d}, J=8.5 \mathrm{~Hz}, 1 \mathrm{H}, 8-\mathrm{H})$.

${ }^{13} \mathbf{C}-\mathrm{NMR}\left(126 \mathrm{MHz}, \mathrm{CDCl}_{3}\right): \delta(\mathrm{ppm})=56.0\left(\mathrm{OCH}_{3}\right), 104.3(\mathrm{C}-6$ ') 108.0 (C-4'), 114.2 (C-1), 120.2 (C-3), 126.4 (C-6), 126.9 (C-8), 128.1 (C-7), 128.3 (C-4/C-5), 128.4 (C-3'), 129.6 (C-4/C-5), 131.9 (C-4a), 133.1 (C-8a), 133.6 (C-5'), 149.5 (C-1'), 152.9 (C-2), 164.5 (C-2').

MS (ESI): $\quad m / z(\%)=374.0 \quad(57) \quad\left[\mathrm{M}\left({ }^{79} \mathrm{Br}\right)+\mathrm{H}\right]^{+}, \quad 391.0 \quad(40) \quad\left[\mathrm{M}\left({ }^{79} \mathrm{Br}\right)+\mathrm{NH}_{4}\right]^{+}, \quad 771.0 \quad(100)$ $\left[\mathrm{M}\left({ }^{79} \mathrm{Br}\right)+\mathrm{M}\left({ }^{81} \mathrm{Br}\right)+\mathrm{H}\right]^{+}$.

HRMS (ESI): $m / z=$ gef.: 374.0020, ber.: $374.0022\left[\mathrm{M}\left({ }^{79} \mathrm{Br}\right)+\mathrm{H}\right]^{+}$; gef.: 375.9997 , ber.: 376.0003 $\left[\mathrm{M}\left({ }^{81} \mathrm{Br}\right)+\mathrm{H}\right]^{+}$; gef.: 391.0287, ber.: $391.0288 \quad\left[\mathrm{M}\left({ }^{79} \mathrm{Br}\right)+\mathrm{NH}_{4}\right]^{+}$; gef.: 393.0258 , ber.: 393.0268 $\left[\mathrm{M}\left({ }^{81} \mathrm{Br}\right)+\mathrm{NH}_{4}\right]^{+}$; gef.: 395.9835 , ber.: $395.9842 \quad\left[\mathrm{M}\left({ }^{79} \mathrm{Br}\right)+\mathrm{Na}\right]^{+}$; gef.: 397.9818 , ber.: 397.9822 $\left[\mathrm{M}\left({ }^{81} \mathrm{Br}\right)+\mathrm{Na}\right]^{+}$.

$\mathrm{C}_{17} \mathrm{H}_{12} \mathrm{BrNO}_{4}$ (373.00). 


\section{2-((1-Bromnaphthalin-2-yl)oxy)-4-methoxyanilin (145b)}<smiles>COc1ccc(N)c(Oc2ccc3ccccc3c2Br)c1</smiles>

$\mathrm{Zu}$ einer Lösung von 1-Brom-2-(5-methoxy-2-nitrophenoxy)naphthalin (144b) (2.94 g, 7.86 mmol, 1.00 Äq.) in EtOAc $(100 \mathrm{~mL})$ wurden bei $0{ }^{\circ} \mathrm{C}$ tropfenweise konz. AcOH $(50 \mathrm{~mL})$, konz. $\mathrm{HCl}(50 \mathrm{~mL})$ und anschließend portionsweise Zinkpulver (15.5 g, $236 \mathrm{mmol}, 30.0$ Äq.) hinzugefügt. Die Reaktionsmischung wurde auf RT erwärmt, $2 \mathrm{~h}$ bei dieser Temperatur gerührt, auf $0{ }^{\circ} \mathrm{C}$ abgekühlt, tropfenweise mit 33\%iger wässr. $\mathrm{NH}_{3}$-Lösung $\left(200 \mathrm{~mL}\right.$ ) versetzt und mit $\mathrm{CH}_{2} \mathrm{Cl}_{2}$ extrahiert $(3 \times 300 \mathrm{~mL})$. Die vereinigten organischen Phasen wurden über $\mathrm{Na}_{2} \mathrm{SO}_{4}$ getrocknet, filtriert und das Lösungsmittel in vacuo entfernt. Amin 145b wurde als brauner Feststoff (2.70 g, 7.85 mmol, quant.) erhalten und ohne weitere Reinigung weiterverwendet.

DC: $R_{f}=0.30(n-$ Pentan/EtOAc, $5: 1)$.

UV/Vis $\left(\mathrm{CH}_{3} \mathrm{CN}\right): \lambda_{\max }(\mathrm{nm})(\lg \varepsilon)=203$ (4.555), 230 (4.865), 283 (3.925), 294 (3.945).

IR (ATR): $\tilde{v}\left(\mathrm{~cm}^{-1}\right)=1585,1506,1459,1255,1227,1137,1128,1036,991,955,806,745,526$.

${ }^{1} \mathbf{H}-\mathrm{NMR}\left(600 \mathrm{MHz}, \mathrm{CDCl}_{3}\right): \delta(\mathrm{ppm})=3.64\left(\mathrm{~s}, 3 \mathrm{H}, \mathrm{OCH}_{3}\right), 3.70\left(\mathrm{~s}_{\mathrm{br}}, 2 \mathrm{H}, \mathrm{NH}_{2}\right), 6.37(\mathrm{~d}, J=2.7 \mathrm{~Hz}$, $\left.1 \mathrm{H}, 6^{\prime}-\mathrm{H}\right), 6.57$ (dd, $\left.J=8.7,2.7 \mathrm{~Hz}, 1 \mathrm{H}, 4^{\prime}-\mathrm{H}\right), 6.81$ (d, $\left.J=8.7 \mathrm{~Hz}, 1 \mathrm{H}, 3^{\prime}-\mathrm{H}\right), 7.13$ (d, $J=8.9 \mathrm{~Hz}$, $1 \mathrm{H}, 3-\mathrm{H}), 7.46$ (ddd, $J=8.1,6.9,1.1 \mathrm{~Hz}, 1 \mathrm{H}, 6-\mathrm{H}), 7.60$ (ddd, $J=8.3,6.9,1.2 \mathrm{~Hz}, 1 \mathrm{H}, 7-\mathrm{H}), 7.73$ (d, $J=8.9 \mathrm{~Hz}, 1 \mathrm{H}, 4-\mathrm{H}), 7.79(\mathrm{~d}, J=8.1 \mathrm{~Hz}, 1 \mathrm{H}, 5-\mathrm{H}), 8.27$ (dd, $J=8.6,1.0 \mathrm{~Hz}, 1 \mathrm{H}, 8-\mathrm{H})$.

${ }^{13} \mathbf{C}-\mathbf{N M R}\left(126 \mathrm{MHz}, \mathrm{CDCl}_{3}\right): \delta(\mathrm{ppm})=55.8\left(\mathrm{OCH}_{3}\right), 105.5\left(\mathrm{C}^{\prime} 4^{\prime}\right), 109.8\left(\mathrm{C}^{\prime}{ }^{\prime}\right), 111.8(\mathrm{C}-1), 117.4$ (C-3'), 118.7 (C-3), 125.4 (C-6), 126.5 (C-8), 127.8 (C-7), 128.1 (C-5), 129.1 (C-4), 131.1, 131.2 (C-4a, C-2'), 133.1 (C-8a), 144.5 (C-5'), 151.2 (C-2), 153.1 (C-1').

MS (ESI): $m / z(\%)=344.0(100)[\mathrm{M}+\mathrm{H}]^{+}$.

HRMS (ESI): $m / z=$ gef.: 344.0281, ber.: $344.0281\left[\mathrm{M}\left({ }^{79} \mathrm{Br}\right)+\mathrm{H}\right]^{+}$; gef.: 346.0262 , ber.: 346.0261 $\left[\mathrm{M}\left({ }^{81} \mathrm{Br}\right)+\mathrm{H}\right]^{+}$.

$\mathrm{C}_{17} \mathrm{H}_{14} \mathrm{BrNO}_{2}$ (344.02). 


\section{1-Brom-2-(2-iod-5-methoxyphenoxy)naphthalin (111b) $)^{\mathrm{j}}$}<smiles>COc1ccc(I)c(Oc2ccc3ccccc3c2Br)c1</smiles>

Eine Lösung von 2-((1-Bromnaphthalin-2-yl)oxy)-4-methoxyanilin (145b) (2.70 g, 7.84 mmol, 1.00 Äq.) und $p$ - $\mathrm{TsOH} \cdot \mathrm{H}_{2} \mathrm{O}\left(4.47 \mathrm{~g}, 23.5 \mathrm{mmol}, 3.00\right.$ Äq.) in $\mathrm{CH}_{3} \mathrm{CN}(50 \mathrm{~mL})$ wurde tropfenweise mit einer Lösung von KI (2.61 g, 15.7 mmol, 2.00 Äq.) und $\mathrm{NaNO}_{2}$ (1.08 g, 15.7 mmol, 2.00 Äq.) in $\mathrm{H}_{2} \mathrm{O}$ $(25 \mathrm{~mL})$ versetzt. Die Reaktionsmischung wurde $3 \mathrm{~h}$ bei RT gerührt und nach Zugabe von ges. wässr. $\mathrm{NaHCO}_{3}$-Lösung $(50 \mathrm{~mL})$ und $1 \mathrm{M} \mathrm{Na}_{2} \mathrm{~S}_{2} \mathrm{O}_{3}$-Lösung $(50 \mathrm{~mL})$ mit $\mathrm{CH}_{2} \mathrm{Cl}_{2}$ extrahiert $(3 \times 100 \mathrm{~mL})$. Die vereinigten organischen Phasen wurden über $\mathrm{Na}_{2} \mathrm{SO}_{4}$ getrocknet, filtriert und das Lösungsmittel in vacuo entfernt. Säulenchromatographische Reinigung $\left(\mathrm{SiO}_{2}, \quad n\right.$-Pentan/EtOAc, 50:1) und Umkristallision aus $n$-Pentan/ $\mathrm{CH}_{2} \mathrm{Cl}_{2}$ (1:1) lieferten Aryliodid 111b als braunen Feststoff $(1.40 \mathrm{~g}$, $3.08 \mathrm{mmol}, 39 \%)$.

DC: $R_{f}=0.37(n-$ Pentan/EtOAc, 50:1).

UV/Vis $\left(\mathrm{CH}_{3} \mathrm{CN}\right): \lambda_{\max }(\mathrm{nm})(\lg \varepsilon)=203$ (4.573), 231 (4.868), 282 (4.010), 323 (3.240).

IR (ATR): $\tilde{v}\left(\mathrm{~cm}^{-1}\right)=1584,1573,1475,1458,1438,1412,1279,1253,1221,1158,1139,1048,1012$, $992,806,750,643,526$.

${ }^{1} \mathbf{H}-\mathrm{NMR}\left(600 \mathrm{MHz}, \mathrm{CDCl}_{3}\right): \delta(\mathrm{ppm})=3.65\left(\mathrm{~s}, 3 \mathrm{H}, \mathrm{OCH}_{3}\right), 6.28(\mathrm{dd}, J=2.8,1.0 \mathrm{~Hz}, 1 \mathrm{H}, 6$ '-H), $6.48\left(\mathrm{dd}, J=8.8,2.7 \mathrm{~Hz}, 1 \mathrm{H}, 4^{\prime}-\mathrm{H}\right), 7.10(\mathrm{~d}, J=8.8 \mathrm{~Hz}, 1 \mathrm{H}, 3-\mathrm{H}), 7.50(\mathrm{ddd}, J=8.1,6.8,1.1 \mathrm{~Hz}$, $1 \mathrm{H}, 6-\mathrm{H}), 7.62$ (ddd, $J=8.4,6.8,1.2 \mathrm{~Hz}, 1 \mathrm{H}, 7-\mathrm{H}), 7.72(\mathrm{~d}, J=8.7 \mathrm{~Hz}, 1 \mathrm{H}, 3$ '-H), 7.78 (d, $J=$ $8.9 \mathrm{~Hz}, 1 \mathrm{H}, 4-\mathrm{H}), 7.82(\mathrm{~d}, J=8.1 \mathrm{~Hz}, 1 \mathrm{H}, 5-\mathrm{H}), 8.29(\mathrm{~d}, J=8.5 \mathrm{~Hz}, 1 \mathrm{H}, 8-\mathrm{H})$.

${ }^{13}$ C-NMR (126 MHz, CDCl $): \delta(\mathrm{ppm})=55.5\left(\mathrm{OCH}_{3}\right), 76.1(\mathrm{C}-2$ '), $104.6(\mathrm{C}-6$ '), $110.9(\mathrm{C}-4$ ') 113.2 (C-1), 119.7 (C-3), 125.8 (C-6), 126.8 (C-8), 127.9 (C-7), 128.2 (C-3'), 129.2 (C-5), 131.5 (C-4a), 133.2 (C-8a), 139.7 (C-4), 150.7 (C-2), 157.0 (C-1'), 161.2 (C-5').

MS (ESI): $m / z(\%)=454.9(100)[\mathrm{M}+\mathrm{H}]^{+}, 478.9(89)[\mathrm{M}+\mathrm{Na}]^{+}$.

HRMS (ESI): $m / z=$ gef.: 454.9125 , ber.: $454.9138\left[\mathrm{M}\left({ }^{79} \mathrm{Br}\right)+\mathrm{H}\right]^{+}$; gef.: 456.9097 , ber.: 456.9118 $\left[\mathrm{M}\left({ }^{81} \mathrm{Br}\right)+\mathrm{H}\right]^{+}$.

$\mathrm{C}_{17} \mathrm{H}_{12} \mathrm{BrIO}_{2}$ (455.08).

\footnotetext{
${ }^{j}$ Allgemeine Methode aus: E. A. Krasnokutskaya, N. I. Semenischeva, V. D. Filimonov, P. Knochel, Synthesis 2007, 81-84.
} 


\section{2-((1-Bromnaphthalin-2-yl)oxy)-3-iodpyridin (111c)}

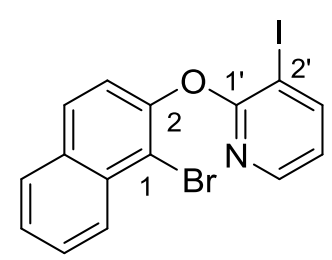

Eine Mischung aus 2-Bromnaphthol (143) (1.83 g, 8.21 mmol, 1.00 Äq.), 2-Fluor-3-iodpyridin (146) (1.83 g, $8.21 \mathrm{mmol}, 1.00$ Äq.) und $\mathrm{K}_{2} \mathrm{CO}_{3}(2.27 \mathrm{~g}, 16.4 \mathrm{mmol}, 2.00$ Äq.) in DMSO (30 mL) wurde $16 \mathrm{~h}$ bei $95^{\circ} \mathrm{C}$ gerührt. Nach Abkühlen auf RT wurde die Reaktionsmischung in Eiswasser $(200 \mathrm{~mL})$ gegeben, der ausgefallene Feststoff abfiltriert und in $\mathrm{CH}_{2} \mathrm{Cl}_{2}$ gelöst. Das Filtrat wurde mit $\mathrm{Et}_{2} \mathrm{O}$ extrahiert $(3 \times 200 \mathrm{~mL})$, die vereinigten organischen Phasen über $\mathrm{Na}_{2} \mathrm{SO}_{4}$ getrocknet, filtriert und das Lösungsmittel in vacuo entfernt. Säulenchromatographische Reinigung $\left(\mathrm{SiO}_{2}, n\right.$-Pentan/MTBE, 10:1) und Umkristallision aus $n$-Pentan $(100 \mathrm{~mL})$ und $\mathrm{CH}_{2} \mathrm{Cl}_{2}(40 \mathrm{~mL})$ lieferten Biarylether 111c als roten Feststoff (2.44 g, $5.73 \mathrm{mmol}, 70 \%)$.

DC: $R_{f}=0.44(n-$ Pentan/MTBE, 10:1).

UV/Vis $\left(\mathrm{CH}_{3} \mathrm{CN}\right): \lambda_{\max }(\mathrm{nm})(\lg \varepsilon)=228(4.828), 281(4.046)$.

IR (ATR): $\tilde{v}\left(\mathrm{~cm}^{-1}\right)=1571,1406,1211,1013,987,935,850,814,788,759,741,527$.

${ }^{1} \mathbf{H}-\mathrm{NMR}\left(600 \mathrm{MHz}, \mathrm{CDCl}_{3}\right): \delta(\mathrm{ppm})=6.75\left(\mathrm{dd}, J=7.6,4.8 \mathrm{~Hz}, 1 \mathrm{H}, 4^{\prime}-\mathrm{H}\right), 7.34(\mathrm{~d}, J=8.8 \mathrm{~Hz}, 1 \mathrm{H}$, 3-H), 7.50 (ddd, $J=8.1,6.9,1.1 \mathrm{~Hz}, 1 \mathrm{H}, 6-\mathrm{H}), 7.59$ (ddd, $J=8.4,6.9,1.3 \mathrm{~Hz}, 1 \mathrm{H}, 7-\mathrm{H}), 7.85\left(\mathrm{~m}_{\mathrm{C}}\right.$, $2 \mathrm{H}, 4-\mathrm{H}, 5-\mathrm{H}), 8.00$ (dd, $\left.J=4.8,1.7 \mathrm{~Hz}, 1 \mathrm{H}, 3^{\prime}-\mathrm{H}\right), 8.17$ (dd, $J=7.6,1.7 \mathrm{~Hz}, 1 \mathrm{H}, 5$ '-H), 8.27 (dq, $J=8.5,0.8 \mathrm{~Hz}, 1 \mathrm{H}, 8-\mathrm{H})$.

${ }^{13}$ C-NMR $\left(126 \mathrm{MHz}, \mathrm{CDCl}_{3}\right): \delta(\mathrm{ppm})=79.6\left(\mathrm{C}^{2}{ }^{\prime}\right), 115.1(\mathrm{C}-1), 120.0\left(\mathrm{C}-4^{\prime}\right), 122.5(\mathrm{C}-3), 126.1$ (C-6), 127.0 (C-8), 127.6 (C-7), 128.2, 128.7 (C-4, C-5), 132.2 (C-4a), 133.0 (C-8a), 146.8 (C-3'), 149.1 (C-5'), 149.2 (C-2), $161.3\left(\mathrm{C}^{\prime} 1^{\prime}\right)$.

MS (ESI): $m / z(\%)=427.9(100)[\mathrm{M}+\mathrm{H}]^{+}$.

HRMS (ESI): $m / z=$ gef.: 425.8981 , ber.: $425.8985\left[\mathrm{M}\left({ }^{79} \mathrm{Br}\right)+\mathrm{H}\right]^{+}$; gef.: 427.8960 , ber.: 427.8965 $\left[\mathrm{M}\left({ }^{81} \mathrm{Br}\right)+\mathrm{H}\right]^{+}$.

$\mathbf{C}_{15} \mathbf{H}_{9}$ BrINO (426.05). 


\subsubsection{Synthese der Dialkine 112a-j}

\section{1-Iod-2-phenoxybenzol (102a) ${ }^{\mathrm{k}}$}<smiles>Ic1ccccc1Oc1ccccc1</smiles>

Eine Lösung von KI (47.9 g, 289 mmol, 2.00 Äq.) und $\mathrm{NaNO}_{2}\left(15.9\right.$ g, $231 \mathrm{mmol}, 2.50$ Äq.) in $\mathrm{H}_{2} \mathrm{O}$ $(400 \mathrm{~mL})$ wurde tropfenweise zu einer Lösung von 2-Phenoxyanilin (149a) (21.4 g, $115 \mathrm{mmol}$, 1.00 Äq.) und $p$-TsOH$\cdot \mathrm{H}_{2} \mathrm{O}\left(65.9 \mathrm{~g}, 346 \mathrm{mmol}, 3.00\right.$ Äq.) in $\mathrm{CH}_{3} \mathrm{CN}(700 \mathrm{~mL})$ gegeben und $45 \mathrm{~min}$ bei RT gerührt. Die Reaktionsmischung wurde mit ges. wässr. $\mathrm{NaHCO}_{3}$-Lösung versetzt, bis pH 9-10 erreicht war und anschließend $1 \mathrm{M} \mathrm{Na}_{2} \mathrm{~S}_{2} \mathrm{O}_{3}$-Lösung $(800 \mathrm{~mL})$ hinzugefügt. Nach Extraktion mit $\mathrm{CH}_{2} \mathrm{Cl}_{2}(5 \times 300 \mathrm{~mL})$ wurden die vereinigten organischen Phasen über $\mathrm{MgSO}_{4}$ getrocknet, filtriert und das Lösungsmittel in vacuo entfernt. Säulenchromatographische Reinigung $\left(\mathrm{SiO}_{2}, n\right.$-Pentan) lieferte Aryliodid 102a als weißen Feststoff (31.3 g, 106 mmol, 92\%).

DC: $R_{f}=0.34(\mathrm{PE})$.

UV/Vis $\left(\mathrm{CH}_{3} \mathrm{CN}\right): \lambda_{\max }(\mathrm{nm})(\lg \varepsilon)=270(3.322), 277$ (3.318).

IR (film): $\tilde{v}\left(\mathrm{~cm}^{-1}\right)=3051,1228$.

${ }^{1} \mathbf{H}-\mathbf{N M R}\left(300 \mathrm{MHz}, \mathrm{CDCl}_{3}\right): \delta(\mathrm{ppm})=6.83-6.90(\mathrm{~m}, 2 \mathrm{H}, 3-\mathrm{H}, 5-\mathrm{H}), 6.94-6.98(\mathrm{~m}, 2 \mathrm{H}, 2$ '- $\mathrm{H}$, 6'-H), 7.10 (dt, $\left.J=7.4,1.0 \mathrm{~Hz}, 1 \mathrm{H}, 4^{\prime}-\mathrm{H}\right), 7.24-7.35$ (m, $\left.3 \mathrm{H}, 4-\mathrm{H}, 3^{\prime}-\mathrm{H}, 5^{\prime}-\mathrm{H}\right), 7.85$ (dd, $J=7.8$, $1.6 \mathrm{~Hz}, 1 \mathrm{H}, 6-\mathrm{H})$.

${ }^{13}$ C-NMR (126 MHz, $\left.\mathrm{CDCl}_{3}\right): \delta(\mathrm{ppm})=88.9(\mathrm{C}-1), 118.4$ (C-2', C-6'), 119.4 (C-3), 123.4 (C-4'), 125.3 (C-5), 129.6 (C-4), 129.8 (C-3', C-5'), 139.9 (C-6), 156.5 (C-1'), 156.8 (C-2).

MS (EI): $m / z(\%)=141.1(38)[\mathrm{M}-\mathrm{I}-\mathrm{CO}]^{+}, 169.1(59)[\mathrm{M}-\mathrm{I}]^{+}, 296.0(100)[\mathrm{M}]^{+}$.

HRMS (EI): $m / z=$ gef.: 295.9699, ber.: $295.9698[\mathrm{M}]^{+}$.

$\mathbf{C}_{12} \mathbf{H}_{9} \mathbf{I O}(296.10)$.

\footnotetext{
${ }^{\mathrm{k}}$ Für Originalvorschrift siehe: M.S. Lesslie, E.E. Turner, J. Chem. Soc. 1932, 281-285. Allgemeine Methode aus: E. A. Krasnokutskaya, N. I. Semenischeva, V. D. Filimonov, P. Knochel, Synthesis 2007, 81-84.

Experimentelle Daten aus: F. Lotz, Dissertation, Georg-August-Universität, Göttingen, 2007.
} 


\section{1-(Hepta-1,6-diin-1-yl)-2-phenoxybenzol (112a)}

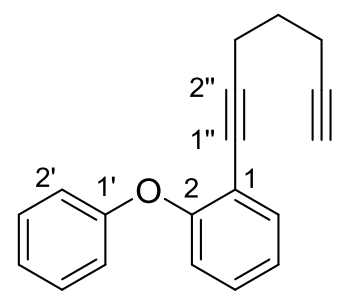

Eine Mischung aus 1-Iod-2-phenoxybenzol (102a) (884 mg, 2.99 mmol, 1.00 Äq.), 1,6-Heptadiin (150b) (1.69 mL, $1.36 \mathrm{~g}, 14.7 \mathrm{mmol}, 5.00$ Äq.), $\mathrm{PdCl}_{2}\left(\mathrm{PPh}_{3}\right)_{2}$ (105 mg, $150 \mu \mathrm{mol}, 0.05$ Äq.) und CuI (56.8 mg, $299 \mu \mathrm{mol}, 0.10$ Äq.) in entgastem $\mathrm{NEt}_{3}(15 \mathrm{~mL})$ wurde $20.5 \mathrm{~h}$ bei RT gerührt. Die Reaktionsmischung wurde durch $\mathrm{SiO}_{2}$ filtriert, mit EtOAc gespült und das Lösungsmittel des Filtrats in vacuo entfernt. Säulenchromatographische Reinigung $\left(\mathrm{SiO}_{2}, n\right.$-Pentan/Toluol 5:1) lieferte Dialkin 112a als gelbes Öl (618 mg, $2.09 \mathrm{mmol}, 70 \%)$.

DC: $R_{f}=0.34(n-$ Pentan/Toluol, 5:1).

UV/Vis $\left(\mathrm{CH}_{3} \mathrm{CN}\right): \lambda_{\max }(\mathrm{nm})(\lg \varepsilon)=241$ (4.222), 252 (4.217), 271 (3.326), 279 (3.354), 288 (3.376).

IR (ATR): $\tilde{v}\left(\mathrm{~cm}^{-1}\right)=1589,1482,1444,1251,1221,1162,867,798,748,689,631$.

${ }^{1} \mathbf{H}-\mathbf{N M R}\left(600 \mathrm{MHz}, \mathrm{CDCl}_{3}\right): \delta(\mathrm{ppm})=1.65\left(\mathrm{p}, J=7.0 \mathrm{~Hz}, 2 \mathrm{H}, 4{ }^{\prime}{ }^{-}-\mathrm{H}_{2}\right), 1.93(\mathrm{t}, J=2.6 \mathrm{~Hz}, 1 \mathrm{H}$, 7'’-H), 2.19 (td, $J=7.1,2.7 \mathrm{~Hz}, 2 \mathrm{H}, 5$ ' '- $\mathrm{H}_{2}$ ), 2.44 (t, $J=6.9 \mathrm{~Hz}, 2 \mathrm{H}, 3$ ' '- $\left.\mathrm{H}_{2}\right), 6.82-7.00$ (m, $3 \mathrm{H}, 3-\mathrm{H}$, 2'-H, 6'-H), 7.01-7.12 (m, 2 H, 5-H, 4'-H), 7.23 (ddd, $J=8.4,7.4,1.8 \mathrm{~Hz}, 1 \mathrm{H}, 4-\mathrm{H}), 7.30$ (dd, $J=$ 8.7, 7.3 Hz, 2 H, 3'-H, 5'-H), 7.44 (dd, $J=7.7,1.7$ Hz, 1 H, 6-H).

${ }^{13}$ C-NMR (126 MHz, $\left.\mathrm{CDCl}_{3}\right): \delta(\mathrm{ppm})=17.4$ (C-5’'), 18.6 (C-3’'), 27.4 (C-4'’), 68.7 (C-7’'), 76.8 (C-6”), 83.7 (C-2'”), 94.2 (C-1'), 116.6 (C-1), 117.8 (C-2', C-6'), 119.7 (C-3), 122.7, 123.6 (C-5, C-4'), 129.0 (C-4), 129.5 (C-3', C-5'), 133.7 (C-6), 157.0 (C-1'), 157.6 (C-2).

MS (ESI): $m / z(\%)=261.1(100)[\mathrm{M}+\mathrm{H}]^{+}, 283.1(83)[\mathrm{M}+\mathrm{Na}]^{+}$.

HRMS (ESI): $m / z=$ gef.: 261.1274, ber.: $261.1274[\mathrm{M}+\mathrm{H}]^{+}$.

$\mathbf{C}_{19} \mathbf{H}_{16} \mathbf{O}(260.33)$. 


\section{1-Fluor-2-(2-nitrophenoxy)benzol (148b) $)^{1}$}<smiles>O=[N+]([O-])c1ccccc1Oc1ccccc1F</smiles>

Eine Mischung aus 2-Fluorphenol (147b) (1.31 mL, 1.59 g, 14.2 mmol, 1.00 Äq.), 2-Fluornitrobenzol (121a) (1.49 mL, $2.00 \mathrm{~g}, 14.2 \mathrm{mmol}, 1.00$ Äq.) und $\mathrm{K}_{2} \mathrm{CO}_{3}$ (3.93 g, $28.4 \mathrm{mmol}, 2.00$ Äq.) in DMSO (30 mL) wurde $14 \mathrm{~h}$ bei $95{ }^{\circ} \mathrm{C}$ gerührt. Nach Abkühlen auf RT wurde die Mischung mit $\mathrm{H}_{2} \mathrm{O}(100 \mathrm{~mL})$ versetzt und mit MTBE extrahiert $(3 \times 100 \mathrm{~mL})$. Die vereinigten organischen Phasen wurden mit ges. wässr. NaCl-Lösung $(150 \mathrm{~mL})$ gewaschen, über $\mathrm{Na}_{2} \mathrm{SO}_{4}$ getrocknet, filtriert und das Lösungsmittel in vacuo entfernt, um Biarylether $\mathbf{1 4 8 b}$ als gelbes Öl (3.56 g, quant.) in leicht verunreinigter Form zu erhalten.

DC: $R_{f}=0.35(n-$ Pentan/EtOAc, 10:1).

UV/Vis $\left(\mathrm{CH}_{3} \mathrm{CN}\right): \lambda_{\max }(\mathrm{nm})(\lg \varepsilon)=257(3.717), 311(3.381)$.

IR (ATR): $\tilde{v}\left(\mathrm{~cm}^{-1}\right)=1594,1525,1495,1479,1457,1347,1262,1225,1185,1162,1103,1088,888$, $849,810,773,739,697,662$.

${ }^{1} \mathbf{H}-\mathrm{NMR}\left(600 \mathrm{MHz}, \mathrm{CDCl}_{3}\right): \delta(\mathrm{ppm})=6.90(\mathrm{dt}, J=8.4,1.1 \mathrm{~Hz}, 1 \mathrm{H}), 7.07-7.23(\mathrm{~m}, 5 \mathrm{H}), 7.47(\mathrm{ddd}$, $J=8.7,7.4,1.7 \mathrm{~Hz}, 1 \mathrm{H}), 7.95(\mathrm{dd}, J=8.1,1.6 \mathrm{~Hz}, 1 \mathrm{H})$.

${ }^{13}$ C-NMR $\left(126 \mathrm{MHz}, \mathrm{CDCl}_{3}\right): \delta(\mathrm{ppm})=117.4(\mathrm{~d}, J=17.9 \mathrm{~Hz}), 118.4,122.2,123.0,125.0(\mathrm{~d}, J=$ $3.9 \mathrm{~Hz}), 125.9,126.2(\mathrm{~d}, J=7.0 \mathrm{~Hz}), 134.2,140.3,142.2(\mathrm{~d}, J=11.7 \mathrm{~Hz}), 150.7,154.0(\mathrm{~d}, J=$ $250.2 \mathrm{~Hz})$.

${ }^{19}$ F-NMR $\left(282 \mathrm{MHz}, \mathrm{CDCl}_{3}\right) \delta(\mathrm{ppm})=-130.1--129.9(\mathrm{~m})$.

MS (ESI): $m / z(\%)=234.0(27)[\mathrm{M}+\mathrm{H}]^{+}, 256.0(100)[\mathrm{M}+\mathrm{Na}]^{+}$.

HRMS (ESI): $\mathrm{m} / z=$ gef.: 234.0567 , ber.: $234.0561[\mathrm{M}+\mathrm{H}]^{+}$.

$\mathrm{C}_{12} \mathrm{H}_{8} \mathrm{FNO}_{3}(233.20)$.

\footnotetext{
${ }^{1}$ Für Originalvorschrift siehe: D. R. Polisetti, PCT Int. Appl. 2004, 2004002481.
} 


\section{2-(2-Fluorphenoxy)anilin (149b) ${ }^{\mathrm{m}}$}<smiles>Nc1ccccc1Oc1ccccc1F</smiles>

Konz. $\mathrm{HCl}(50 \mathrm{~mL})$ und anschließend konz. AcOH $(50 \mathrm{~mL})$ wurden bei $0{ }^{\circ} \mathrm{C}$ tropfenweise zu einer Lösung von 1-Fluor-2-(2-nitrophenoxy)benzol (148b) (3.55 g, $15.2 \mathrm{mmol}, 1.00$ Äq.) in EtOAc (100 mL) hinzugefügt. Zinkpulver (19.8 g, $456 \mathrm{mmol}, 30.0$ Äq.) wurde portionsweise zugegeben, die Lösung auf RT erwärmt und $1 \mathrm{~h}$ bei dieser Temperatur gerührt. Die Reaktionsmischung wurde auf $0{ }^{\circ} \mathrm{C}$ abgekühlt und 33\%ige wässr. $\mathrm{NH}_{3}$-Lösung $(200 \mathrm{~mL})$ tropfenweise hinzugefügt. Die Mischung wurde mit $\mathrm{CH}_{2} \mathrm{Cl}_{2}$ extrahiert $(3 \times 300 \mathrm{~mL})$ und die vereinigten organischen Phasen wurden über $\mathrm{Na}_{2} \mathrm{SO}_{4}$ getrocknet und filtriert. Entfernung des Lösungsmittels in vacuo lieferte Amin 149b als braunes Öl (3.14, quant.) in leicht verunreinigter Form.

DC: $R_{f}=0.42(n-$ Pentan/EtOAc, 20:1).

UV/Vis $\left(\mathrm{CH}_{3} \mathrm{CN}\right): \lambda_{\max }(\mathrm{nm})(\lg \varepsilon)=200$ (4.578), 237 (3.994), 274 (3.411), 291 (3.524).

IR (ATR): $\tilde{v}\left(\mathrm{~cm}^{-1}\right)=1619,1588,1495,1455,1305,1253,1194,1154,1136,1102,1029,886,790$, $738,554$.

${ }^{1} \mathbf{H}-\mathrm{NMR}\left(600 \mathrm{MHz}, \mathrm{CDCl}_{3}\right): \delta(\mathrm{ppm})=3.89\left(\mathrm{~s}_{\mathrm{br}}, 2 \mathrm{H}, \mathrm{NH}_{2}\right), 6.66-6.73(\mathrm{~m}, 1 \mathrm{H}, 4-\mathrm{H}), 6.77(\mathrm{dd}, J=$ 8.0, 1.4 Hz, 1 H, 3-H), 6.84 (dd, $J=7.9,1.5 \mathrm{~Hz}, 1 \mathrm{H}, 6-\mathrm{H}), 6.91-6.98$ (m, $2 \mathrm{H}, 5-\mathrm{H}, 6$ '-H), 7.01-7.07 (m, 2 H, 4'-H, 5'-H), 7.12-7.20 (m, 1 H, 3'-H).

${ }^{13} \mathbf{C}-N M R\left(126 \mathrm{MHz}, \mathrm{CDCl}_{3}\right): \delta(\mathrm{ppm})=116.5(\mathrm{C}-6), 116.6(\mathrm{~d}, J=18.1 \mathrm{~Hz}, \mathrm{C}-3$ ') $, 118.1(\mathrm{C}-3), 118.8$ (C-4), 119.6 (C-5), 123.7 (d, $J=6.8$ Hz, C-4'), 124.3 (d, J=3.8 Hz, C-6'), 124.4 (C-5'), 137.2 (C-1), 143.5 (C-2), 144.2 (d, $J=11.7 \mathrm{~Hz}, \mathrm{C}-1$ ') 153.4 (d, $J=248.1 \mathrm{~Hz}, \mathrm{C}-2$ ').

${ }^{19}$ F-NMR $\left(283 \mathrm{MHz}, \mathrm{CDCl}_{3}\right): \delta(\mathrm{ppm})=-132.6--132.7\left(\mathrm{~m}, 2^{\prime}-\mathrm{F}\right)$.

MS (ESI): $m / z(\%)=204.1(100)[\mathrm{M}+\mathrm{H}]^{+}$.

HRMS (ESI): $m / z=$ gef.: 204.0823, ber.: $204.0819[\mathrm{M}+\mathrm{H}]^{+}$.

$\mathrm{C}_{12} \mathbf{H}_{10}$ FNO (203.21).

${ }^{\mathrm{m}}$ Für Originalvorschrift siehe: D. R. Polisetti, PCT Int. Appl. 2004, 2004002481. 


\section{1-Iod-2-(2-fluorphenoxy)benzol (102b)}<smiles>Fc1ccccc1Oc1ccccc1I</smiles>

Eine Lösung von KI (5.11 g, 30.8 mmol, 2.00 Äq.) und $\mathrm{NaNO}_{2}$ (2.13 g, $30.8 \mathrm{mmol}, 2.00$ Äq.) in $\mathrm{H}_{2} \mathrm{O}$ $(50 \mathrm{~mL})$ wurde zu einer Lösung von 2-(2-Fluorphenoxy)anilin (149b) (3.13 g, 15.4 mmol, 1.00 Äq.) und $p$ - Ts $\mathrm{OH} \cdot \mathrm{H}_{2} \mathrm{O}(8.79 \mathrm{~g}, 46.2 \mathrm{mmol}, 3.00 \mathrm{Äq}$. $)$ in $\mathrm{CH}_{3} \mathrm{CN}(100 \mathrm{~mL})$ getropft und $2 \mathrm{~h}$ bei RT gerührt. Die Reaktionsmischung wurde mit ges. wässr. $\mathrm{NaHCO}_{3}$-Lösung $(100 \mathrm{~mL})$ und ges. wässr. $\mathrm{Na}_{2} \mathrm{~S}_{2} \mathrm{O}_{3}$-Lösung $(100 \mathrm{~mL})$ versetzt und mit $\mathrm{CH}_{2} \mathrm{Cl}_{2}$ extrahiert $(3 \times 300 \mathrm{~mL})$. Die vereinigten organischen Phasen wurden über $\mathrm{Na}_{2} \mathrm{SO}_{4}$ getrocknet, filtriert und das Lösungsmittel in vacuo entfernt. Säulenchromatographische Reinigung $\left(\mathrm{SiO}_{2}, n\right.$-Pentan) lieferte Aryliodid 102b als gelbes Öl (3.78 g, $12.0 \mathrm{mmol}, 78 \%)$.

DC: $R_{f}=0.26(n-$ Pentan $)$.

UV/Vis $\left(\mathrm{CH}_{3} \mathrm{CN}\right): \lambda_{\max }(\mathrm{nm})(\lg \varepsilon)=268(3.470)$.

IR (ATR): $\tilde{v}\left(\mathrm{~cm}^{-1}\right)=1574,1496,1464,1437,1260,1215,1188,1156,1114,1103,934,883,812$, 790, 775, 742, 710, 645, 552, 540 .

${ }^{1} \mathbf{H}-\mathrm{NMR}\left(600 \mathrm{MHz}, \mathrm{CDCl}_{3}\right): \delta(\mathrm{ppm})=6.76(\mathrm{dt}, J=8.1,1.1 \mathrm{~Hz}, 1 \mathrm{H}, 6-\mathrm{H}), 6.84(\mathrm{td}, J=7.6,1.4 \mathrm{~Hz}$, $1 \mathrm{H}, 4-\mathrm{H}), 6.97$ (td, $\left.J=8.0,1.8 \mathrm{~Hz}, 1 \mathrm{H}, 4^{\prime}-\mathrm{H}\right), 7.05-7.14$ (m, 2 H, 3'-H, 6'-H), 7.18 (ddd, $J=10.7$, 7.9, $\left.1.7 \mathrm{~Hz}, 1 \mathrm{H}, 5^{\prime}-\mathrm{H}\right), 7.24$ (ddd, $\left.J=8.0,7.2,1.5 \mathrm{~Hz}, 1 \mathrm{H}, 5-\mathrm{H}\right), 7.84$ (dd, $\left.J=7.8,1.5 \mathrm{~Hz}, 1 \mathrm{H}, 3-\mathrm{H}\right)$.

${ }^{13} \mathbf{C}-\mathbf{N M R}\left(126 \mathrm{MHz}, \mathrm{CDCl}_{3}\right): \delta(\mathrm{ppm})=87.2(\mathrm{C}-1), 117.1(\mathrm{~d}, J=17.9 \mathrm{~Hz}, \mathrm{C}-3$ ') $117.2(\mathrm{C}-6), 121.3$ (C-5'), 124.7 (d, J=3.9 Hz, C-6'), 125.0 (d, J=6.9 Hz, C-4'), 125.1 (C-4), 129.6 (C-5), 139.9 (C-3), 143.5 (d, $J=11.5 \mathrm{~Hz}, \mathrm{C}-1$ ') 153.9 (d, $J=249.2 \mathrm{~Hz}, \mathrm{C}-2$ '), 156.5 (C-2).

${ }^{19}$ F-NMR $\left(282 \mathrm{MHz}, \mathrm{CDCl}_{3}\right): \delta(\mathrm{ppm})=-130.7\left(\mathrm{ddd}, J=10.2,8.1,4.1 \mathrm{~Hz}, 2^{\prime}-\mathrm{F}\right)$.

MS (ESI): $m / z(\%)=336.9(3)[\mathrm{M}+\mathrm{Na}]^{+}$.

HRMS (ESI): $m / z=$ gef.: 336.9491 , ber.: $336.9496[\mathrm{M}+\mathrm{Na}]^{+}$.

$\mathbf{C}_{12} \mathbf{H}_{8}$ FIO (314.09).

${ }^{\text {n }}$ Für Originalvorschrift siehe: R. Sanz, J. Org. Chem. 2006, 71, 6291-6294. Allgemeine Methode aus: E. A. Krasnokutskaya, N. I. Semenischeva, V. D. Filimonov, P. Knochel, Synthesis 2007, 81-84. 


\section{1-(Hepta-1,6-diin-1-yl)-2-(2-fluorphenoxy)benzol (112b)}

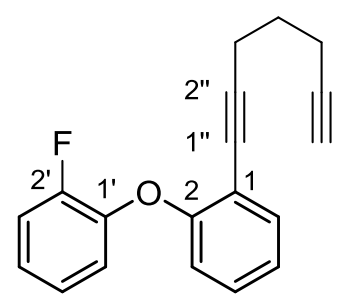

Eine Mischung von 1-Iod-2-(2-fluorphenoxy)benzol (102b) (279 mg, $874 \mu \mathrm{mol}, \quad 1.00$ Äq.), 1,6-Heptadiin (150b) (700 $\mu \mathrm{L}, 564 \mathrm{mg}, 6.12 \mathrm{mmol}, 7.00$ Äq.), $\mathrm{PdCl}_{2}\left(\mathrm{PPh}_{3}\right)_{2}$ (30.7 mg, $43.7 \mu \mathrm{mol}$, 0.05 Äq.) und CuI (10.4 mg, $87.4 \mu \mathrm{mol}, 0.10$ Äq.) in entgastem $\mathrm{NEt}_{3}(8 \mathrm{~mL})$ wurde $17 \mathrm{~h}$ bei $60^{\circ} \mathrm{C}$ gerührt. Die Reaktionsmischung wurde durch $\mathrm{SiO}_{2}$ filtriert, mit EtOAc gespült und das Lösungsmittel des Filtrats in vacuo entfernt. Säulenchromatographische Reinigung $\left(\mathrm{SiO}_{2}, n\right.$-Pentan/Toluol, 5:1) lieferte Dialkin 112b als gelbes Öl (191 mg, $691 \mu \mathrm{mol}, 79 \%)$.

DC: $R_{f}=0.29(n-$ Pentan/Toluol, 5:1).

UV/Vis $\left(\mathrm{CH}_{3} \mathrm{CN}\right): \lambda_{\max }(\mathrm{nm})(\lg \varepsilon)=205$ (4.534), 241 (4.221), 252 (4.232), 287 (3.429), 295 (3.377).

IR (ATR): $\tilde{v}\left(\mathrm{~cm}^{-1}\right)=1498,1485,1445,1432,1262,1205,1107,879,812,794,746,634$.

${ }^{1} \mathbf{H}-\mathbf{N M R}\left(600 \mathrm{MHz}, \mathrm{CDCl}_{3}\right): \delta(\mathrm{ppm})=1.69\left(\mathrm{p}, J=7.0 \mathrm{~Hz}, 2 \mathrm{H}, 4{ }^{\prime}{ }^{-} \mathrm{H}_{2}\right), 1.93(\mathrm{dd}, J=2.9,2.4 \mathrm{~Hz}$,

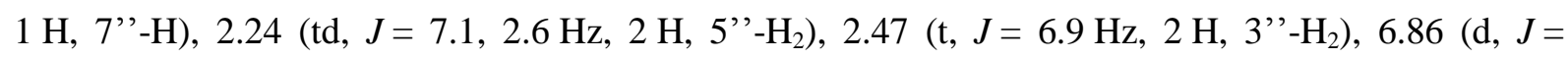
8.2 Hz, 1 H, 6-H), 6.89-6.93 (m, 1 H, 5'-H), 7.01-7.08 (m, 3 H, 4-H, 4'-H, 6'-H), 7.12-7.18 (m, 1 H, 3'-H), 7.21 (ddd, $J=8.1,7.4,1.7 \mathrm{~Hz}, 1 \mathrm{H}, 5-\mathrm{H}), 7.43$ (dd, $J=7.7,1.7 \mathrm{~Hz}, 1 \mathrm{H}, 3-\mathrm{H})$.

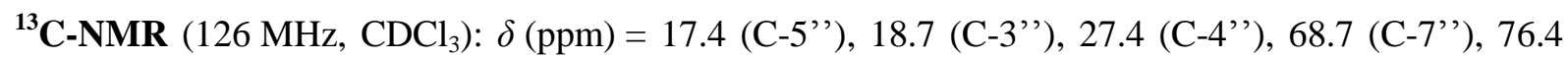
(C-6'), 83.6 (C-2''), 94.4 (C-1''), 115.7 (C-1), 116.8 (d, J=18.1 Hz, C-3'), 118.1 (C-6), 120.4 (C-5'), 123.6 (C-4), 124.1 (d, J=6.8 Hz, C-4'), 124.4 (d, J=3.9 Hz, C-6'), 129.0 (C-5), 133.7 (C-3), 144.5 (d, $J=11.2 \mathrm{~Hz}, \mathrm{C}-1$ '), 153.6 (d, $J=248.3 \mathrm{~Hz}, \mathrm{C}-2$ '), 157.0 (C-2).

${ }^{19}$ F-NMR $\left(282 \mathrm{MHz}, \mathrm{CDCl}_{3}\right): \delta(\mathrm{ppm})=-131.8\left(\mathrm{ddd}, J=10.5,7.6,2.3 \mathrm{~Hz},{ }^{\prime}{ }^{\prime}-\mathrm{F}\right)$.

MS (ESI): $m / z(\%)=279.1(100)[\mathrm{M}+\mathrm{H}]^{+}, 301.1(93)[\mathrm{M}+\mathrm{Na}]^{+}$.

HRMS (ESI): $\mathrm{m} / z=$ gef.: 279.1185 , ber.: $279.1180[\mathrm{M}+\mathrm{H}]^{+}$.

$\mathbf{C}_{19} \mathrm{H}_{15} \mathrm{FO}$ (278.32). 


\section{1-Methyl-2-(2-nitrophenoxy)benzol (148c $)^{\circ}$}<smiles>Cc1ccccc1Oc1ccccc1[N+](=O)[O-]</smiles>

Eine Mischung aus 2-Methylphenol (147c) (1.66 mL, $1.74 \mathrm{~g}, \quad 16.1 \mathrm{mmol}, \quad 1.00$ Äq.), 2-Fluornitrobenzol (121a) (1.70 mL, $2.27 \mathrm{~g}, 16.1 \mathrm{mmol}, 1.00$ Äq.) und $\mathrm{K}_{2} \mathrm{CO}_{3}(4.45 \mathrm{~g}, 32.2 \mathrm{mmol}$, 2.00 Äq.) in DMSO $(40 \mathrm{~mL})$ wurde $20 \mathrm{~h}$ bei $95{ }^{\circ} \mathrm{C}$ gerührt. Nach Abkühlen auf RT wurde die Mischung mit $\mathrm{H}_{2} \mathrm{O}(100 \mathrm{~mL})$ versetzt und mit MTBE $(3 \times 100 \mathrm{~mL})$ extrahiert. Die vereinigten organischen Phasen wurden mit ges. wässr. $\mathrm{NaCl}$-Lösung $(200 \mathrm{~mL})$ gewaschen, über $\mathrm{Na}_{2} \mathrm{SO}_{4}$ getrocknet, filtriert und das Lösungsmittel in vacuo entfernt, um Biarylether 148c als braunes Öl (3.93 g, quant.) in leicht verunreinigter Form zu erhalten.

DC: $R_{f}=0.24(n-$ Pentan/EtOAc, 20:1).

UV/Vis $\left(\mathrm{CH}_{3} \mathrm{CN}\right): \lambda_{\max }(\mathrm{nm})(\lg \varepsilon)=256(3.689), 318(3.414)$.

IR (ATR): $\tilde{v}\left(\mathrm{~cm}^{-1}\right)=1604,1583,1523,1477,1347,1241,1111,884,845,774,758,662$.

${ }^{1} \mathbf{H}-\mathrm{NMR}\left(600 \mathrm{MHz}, \mathrm{CDCl}_{3}\right): \delta(\mathrm{ppm})=2.22\left(\mathrm{~s}, 3 \mathrm{H}, \mathrm{CH}_{3}\right), 6.79(\mathrm{dd}, J=8.4,1.2 \mathrm{~Hz}, 1 \mathrm{H}), 6.92(\mathrm{dd}$, $J=8.0,1.3 \mathrm{~Hz}, 1 \mathrm{H}), 7.07-7.16(\mathrm{~m}, 2 \mathrm{H}), 7.16-7.22(\mathrm{~m}, 1 \mathrm{H}), 7.27(\mathrm{dd}, J=7.5,1.9 \mathrm{~Hz}, 1 \mathrm{H}), 7.42$ (ddd, $J=8.7,7.4,1.6 \mathrm{~Hz}, 1 \mathrm{H}), 7.93(\mathrm{dd}, J=8.2,1.7 \mathrm{~Hz}, 1 \mathrm{H})$.

${ }^{13} \mathbf{C}-\mathrm{NMR}\left(126 \mathrm{MHz}, \mathrm{CDCl}_{3}\right): \delta(\mathrm{ppm})=16.0\left(\mathrm{CH}_{3}\right), 118.3,119.8,122.1,125.2,125.8,127.4,130.1$, $131.8,134.0,140.3,151.2,153.0$.

MS (ESI): $m / z(\%)=230.1(51)[\mathrm{M}+\mathrm{H}]^{+}, 252.1(100)[\mathrm{M}+\mathrm{Na}]^{+}$.

HRMS (ESI): $m / z=$ gef.: 230.0814 , ber.: $230.0812[\mathrm{M}+\mathrm{H}]^{+}$.

$\mathrm{C}_{13} \mathrm{H}_{11} \mathrm{NO}_{3}$ (229.23).

\footnotetext{
${ }^{\circ}$ Für Originalvorschrift siehe: R. Higginbottom, H. Suschitzky, J. Chem. Soc. 1962, 2367-70.
} 


\section{2-(2-Tolyloxy)anilin (149c) $)^{\mathrm{p}}$}<smiles>Cc1ccccc1Oc1ccccc1N</smiles>

Konz. $\mathrm{HCl}(50 \mathrm{~mL})$ und anschließend konz. AcOH $(50 \mathrm{~mL})$ wurden bei $0{ }^{\circ} \mathrm{C}$ tropfenweise zu einer Lösung von 1-Methyl-2-(2-nitrophenoxy)benzol (148c) (3.89 g, $16.1 \mathrm{mmol}, 1.00$ Äq.) in EtOAc (100 mL) gegeben. Zinkpulver (31.6 g, $483 \mathrm{mmol}, 30.0$ Äq.) wurde portionsweise hinzugefügt, die Lösung wurde auf RT erwärmt und $30 \mathrm{~min}$ bei dieser Temperatur gerührt. Die Reaktionsmischung wurde auf $0{ }^{\circ} \mathrm{C}$ gekühlt und $33 \%$ ige wässr. $\mathrm{NH}_{3}$-Lösung $(200 \mathrm{~mL})$ hinzugetropft. Die Reaktionsmischung wurde mit $\mathrm{CH}_{2} \mathrm{Cl}_{2}$ extrahiert $(3 \times 300 \mathrm{~mL})$ und die vereinigten organischen Phasen über $\mathrm{Na}_{2} \mathrm{SO}_{4}$ getrocknet und filtriert. Entfernung des Lösungsmittels in vacuo lieferte Amin 149c als braunes Öl (3.12 g, 15.7 mmol, 98\%) in leicht verunreinigter Form.

DC: $R_{f}=0.59(n-$ Pentan/EtOAc, $10: 1)$.

UV/Vis $\left(\mathrm{CH}_{3} \mathrm{CN}\right): \lambda_{\max }(\mathrm{nm})(\lg \varepsilon)=238(4.002), 291(3.536)$.

IR (ATR): $\tilde{v}\left(\mathrm{~cm}^{-1}\right)=1616,1580,1498,1487,1457,1302,1269,1224,1193,1154,1135,1112,1042$, 1031, 883, 796, 778, 737, 709, 541.

${ }^{1} \mathbf{H}-\mathrm{NMR}\left(600 \mathrm{MHz}, \mathrm{CDCl}_{3}\right): \delta(\mathrm{ppm})=2.29\left(\mathrm{~s}, 3 \mathrm{H}, \mathrm{CH}_{3}\right), 3.79\left(\mathrm{~s}_{\mathrm{br}}, 2 \mathrm{H}, \mathrm{NH}_{2}\right), 6.56-6.72(\mathrm{~m}, 2 \mathrm{H}$, 3-H, 4-H), 6.80 (dd, $J=8.1,1.2 \mathrm{~Hz}, 1 \mathrm{H}, 6$ '-H), 6.82-6.85 (m, $1 \mathrm{H}, 6-\mathrm{H}), 6.92$ (ddd, $J=7.7,5.3$, $3.3 \mathrm{~Hz}, 1 \mathrm{H}, 5-\mathrm{H}), 7.01$ (td, $\left.J=7.4,1.2 \mathrm{~Hz}, 1 \mathrm{H}, 4^{\prime}-\mathrm{H}\right), 7.08-7.15$ (m, $\left.1 \mathrm{H}, 5^{\prime}-\mathrm{H}\right), 7.19-7.28$ (m, $1 \mathrm{H}$, 3 '-H).

${ }^{13}$ C-NMR $\left(126 \mathrm{MHz}, \mathrm{CDCl}_{3}\right): \delta(\mathrm{ppm})=16.3\left(\mathrm{CH}_{3}\right), 116.4(\mathrm{C}-6), 117.5(\mathrm{C}-6$ ') $, 118.1,118.9(\mathrm{C}-3$, C-4'), 123.3 (C-4), 123.8 (C-5), 127.1 (C-5'), 128.8 (C-2'), 131.3 (C-3'), 137.3 (C-1), 144.3 (C-1'), $154.8(\mathrm{C}-2)$.

MS (ESI): $m / z(\%)=200.1(100)[\mathrm{M}+\mathrm{H}]^{+}$.

HRMS (ESI): $m / z=$ gef.: 200.1078, ber.: 200.1070 [M+H] ${ }^{+}$.

$\mathbf{C}_{13} \mathbf{H}_{13} \mathbf{N O}$ (199.25).

${ }^{\mathrm{p}}$ Für Originalvorschrift siehe: R. Higginbottom, H. Suschitzky, J. Chem. Soc. 1962, 2367-70. 


\section{1-Iod-2-(2-tolyloxy)benzol (102c) ${ }^{\mathrm{q}}$}<smiles>Cc1ccccc1Oc1ccccc1I</smiles>

Eine Lösung von KI (5.18 g, 31.2 mmol, 2.00 Äq.) und $\mathrm{NaNO}_{2}$ (2.15 g, 31.2 mmol, 2.00 Äq.) in $\mathrm{H}_{2} \mathrm{O}$ $(50 \mathrm{~mL})$ wurde zu einer Lösung von 2-(2-Tolyloxy)anilin (149c) $(3.11 \mathrm{~g}, 15.6 \mathrm{mmol}, 1.00$ Äq.) und p-TsOH $\cdot \mathrm{H}_{2} \mathrm{O}$ (8.90 g, $46.8 \mathrm{mmol}, 3.00$ Äq.) in $\mathrm{CH}_{3} \mathrm{CN}(100 \mathrm{~mL})$ getropft und $2 \mathrm{~h}$ bei RT gerührt. Die Reaktionsmischung wurde mit ges. wässr. $\mathrm{NaHCO}_{3}$-Lösung $(100 \mathrm{~mL})$ gewaschen und mit $\mathrm{CH}_{2} \mathrm{Cl}_{2}$ extrahiert $(3 \times 300 \mathrm{~mL})$. Die vereinigten organischen Phasen wurden in vacuo eingeengt, in $\mathrm{CH}_{2} \mathrm{Cl}_{2}$ (200 mL) gelöst, mit ges. wässr. $\mathrm{Na}_{2} \mathrm{~S}_{2} \mathrm{O}_{3}$-Lösung $(100 \mathrm{~mL})$ gewaschen, über $\mathrm{Na}_{2} \mathrm{SO}_{4}$ getrocknet, filtriert und das Lösungsmittel in vacuo entfernt. Säulenchromatographische Reinigung $\left(\mathrm{SiO}_{2}\right.$, $n$-Pentan) lieferte Aryliodid 102c als gelbes Öl (4.00 g, $12.9 \mathrm{mmol}, 83 \%)$.

DC: $R_{f}=0.31(n-$ Pentan).

UV/Vis $\left(\mathrm{CH}_{3} \mathrm{CN}\right): \lambda_{\max }(\mathrm{nm})(\lg \varepsilon)=270$ (3.395), 277 (3.399).

IR (ATR): $\tilde{v}\left(\mathrm{~cm}^{-1}\right)=1573,1488,1462,1436,1231,1179,1109,1040,1017,879,778,739,715,645$.

${ }^{1} \mathbf{H}-\mathrm{NMR}\left(600 \mathrm{MHz}, \mathrm{CDCl}_{3}\right): \delta(\mathrm{ppm})=2.24\left(\mathrm{~s}, 3 \mathrm{H}, \mathrm{CH}_{3}\right), 6.65(\mathrm{dd}, J=8.2,1.4 \mathrm{~Hz}, 1 \mathrm{H}, 3-\mathrm{H}), 6.79$ (dd, $J=7.6,1.4 \mathrm{~Hz}, 1 \mathrm{H}, 5-\mathrm{H}), 6.82$ (d, $\left.J=8.4 \mathrm{~Hz}, 1 \mathrm{H}, 6^{\prime}-\mathrm{H}\right), 7.07$ (td, $\left.J=7.4,1.2 \mathrm{~Hz}, 1 \mathrm{H}, 4^{\prime}-\mathrm{H}\right)$, $7.15\left(\mathrm{td}, J=7.7,1.7 \mathrm{~Hz}, 1 \mathrm{H}, 5^{\prime}-\mathrm{H}\right), 7.21$ (ddd, $\left.J=8.7,7.3,1.5 \mathrm{~Hz}, 1 \mathrm{H}, 4-\mathrm{H}\right), 7.23-7.27$ (m, $1 \mathrm{H}$, 3'-H), 7.84 (dd, $J=7.8,1.5 \mathrm{~Hz}, 1 \mathrm{H}, 6-\mathrm{H})$.

${ }^{13}$ C-NMR $\left(126 \mathrm{MHz}, \mathrm{CDCl}_{3}\right): \delta(\mathrm{ppm})=16.5\left(\mathrm{CH}_{3}\right), 87.5(\mathrm{C}-1), 117.1$ (C-3), 119.1 (C-6'), 124.2, 124.4 (C-5, C-4'), 127.1 (C-5'), 129.5 (C-4), 129.6 (C-2'), 131.5 (C-3'), 139.8 (C-6), 154.2 (C-1'), $156.7(\mathrm{C}-2)$.

MS (ESI): $m / z(\%)=333.0(48)[\mathrm{M}+\mathrm{Na}]^{+}$.

HRMS (ESI): $m / z=$ gef.: 332.9741 , ber.: $332.9747[\mathrm{M}+\mathrm{Na}]^{+}$.

$\mathrm{C}_{13} \mathrm{H}_{11} \mathrm{IO}$ (310.13).

${ }^{\mathrm{q}}$ Für Originalvorschrift siehe: J. Baldwin, PCT Int. Appl. 2009, 2009096996. Allgemeine Methode aus: E. A. Krasnokutskaya, N. I. Semenischeva, V. D. Filimonov, P. Knochel, Synthesis 2007, 81-84. 


\section{1-(Hepta-1,6-diin-1-yl)-2-(2-tolyloxy)benzol (112c)}

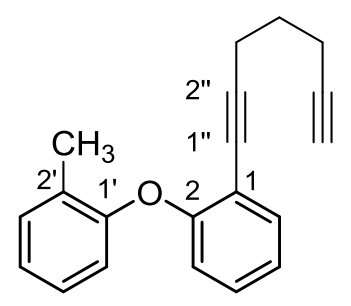

Eine Mischung aus 1-Iod-2-(2-tolyloxy)benzol (102c) (271 mg, $874 \mu$ mol, 1.00 Äq.), 1,6-Heptadiin (150b) (700 $\mu \mathrm{L}, 564$ mg, 6.12 mmol, 7.00 Äq.), $\mathrm{PdCl}_{2}\left(\mathrm{PPh}_{3}\right)_{2}$ (30.7 mg, $43.7 \mu \mathrm{mol}, 0.05$ Äq.) und CuI (10.4 mg, $87.4 \mu \mathrm{mol}, 0.10$ Äq.) in entgastem $\mathrm{NEt}_{3}(8 \mathrm{~mL})$ wurde $17 \mathrm{~h}$ bei $60{ }^{\circ} \mathrm{C}$ gerührt. Die Reaktionsmischung wurde durch $\mathrm{SiO}_{2}$ filtriert, mit EtOAc gespült und das Lösungsmittel des Filtrats in vacuo entfernt. Säulenchromatographische Reinigung $\left(\mathrm{SiO}_{2}, n\right.$-Pentan/Toluol, 5:1) lieferte Dialkin 112c als gelbes Öl (203 mg, $740 \mu \mathrm{mol}, 85 \%)$ in verunreinigter Form.

DC: $R_{f}=0.35(n-$ Pentan/Toluol, 5:1).

UV/Vis $\left(\mathrm{CH}_{3} \mathrm{CN}\right): \lambda_{\max }(\mathrm{nm})(\lg \varepsilon)=206$ (4.532), 241 (4.183), 252 (4.190), 289 (3.492), 297 (3.444).

IR (ATR): $\tilde{v}\left(\mathrm{~cm}^{-1}\right)=3293,1483,1444,1252,1205,1180,1114,875,785,747,633$.

${ }^{1} \mathbf{H}-\mathbf{N M R}\left(600 \mathrm{MHz}, \mathrm{CDCl}_{3}\right): \delta(\mathrm{ppm})=1.70\left(\mathrm{p}, J=7.0 \mathrm{~Hz}, 2 \mathrm{H}, 4{ }^{\prime}{ }^{\prime}-\mathrm{H}_{2}\right), 1.93(\mathrm{t}, J=2.6 \mathrm{~Hz}, 1 \mathrm{H}$, 7' '-H), 2.24 (td, $J=7.1,2.7 \mathrm{~Hz}, 2 \mathrm{H}, 5$ ' '- $\mathrm{H}_{2}$ ), 2.27 (s, $3 \mathrm{H}, \mathrm{CH}_{3}$ ), 2.48 (t, $J=6.9 \mathrm{~Hz}, 2 \mathrm{H}, 3$ '”- $\mathrm{H}_{2}$ ), 6.73 $(\mathrm{dd}, J=8.3,1.1 \mathrm{~Hz}, 1 \mathrm{H}, 3-\mathrm{H}), 6.78\left(\mathrm{dd}, J=8.1,1.2 \mathrm{~Hz}, 1 \mathrm{H}, 6{ }^{\prime}-\mathrm{H}\right), 6.99$ (td, $J=7.5,1.2 \mathrm{~Hz}, 1 \mathrm{H}$, 5-H), 7.02 (dd, $\left.J=7.4,1.2 \mathrm{~Hz}, 1 \mathrm{H}, 4^{\prime}-\mathrm{H}\right), 7.12$ (td, $\left.J=7.9,1.9 \mathrm{~Hz}, 1 \mathrm{H}, 5^{\prime}-\mathrm{H}\right), 7.17$ (ddd, $J=8.2,7.3$, $1.7 \mathrm{~Hz}, 1 \mathrm{H}, 4-\mathrm{H}), 7.22$ (ddd, $\left.J=7.4,1.8,1.0 \mathrm{~Hz}, 1 \mathrm{H}, 3^{\prime}-\mathrm{H}\right), 7.43$ (dd, $\left.J=7.7,1.7 \mathrm{~Hz}, 1 \mathrm{H}, 6-\mathrm{H}\right)$.

${ }^{13}$ C-NMR $\left(126 \mathrm{MHz}, \mathrm{CDCl}_{3}\right): \delta(\mathrm{ppm})=16.2\left(\mathrm{CH}_{3}\right), 17.0$ (C-5'”), 18.7 (C-3"'), 27.5 (C-4”'), 68.7 (C-7'), 76.9 (C-6'), 83.7 (C-2'), 93.8 (C-1'”), 115.4 (C-1), 117.6 (C-3), 118.4 (C-6'), 122.7 (C-5), 123.5 (C-4'), 126.9 (C-5'), 128.9 (C-4), 129.2 (C-2'), 131.3 (C-3'), 133.7 (C-6), 154.9 (C-1'), 157.9 (C-2).

MS (ESI): $m / z(\%)=275.1(100)[\mathrm{M}+\mathrm{H}]^{+}, 297.1(35)[\mathrm{M}+\mathrm{Na}]^{+}$

HRMS (ESI): $m / z=$ gef.: 275.1430 , ber.: $275.1430[\mathrm{M}+\mathrm{H}]^{+}$.

$\mathrm{C}_{20} \mathrm{H}_{18} \mathrm{O}$ (274.14). 


\section{1-Nitro-2-(2-(trifluormethyl)phenoxy)benzol (148d $)^{\mathrm{r}}$}<smiles>O=[N+]([O-])c1ccccc1Oc1ccccc1C(F)(F)F</smiles>

Eine Mischung aus 2-(Trifluormethyl)phenol (147d) (2.30 g, $14.2 \mathrm{mmol}, \quad 1.00$ Äq.), 2-Fluornitrobenzol (121a) (1.49 mL, 2.00 g, $14.2 \mathrm{mmol}, 1.00$ Äq.) und $\mathrm{K}_{2} \mathrm{CO}_{3}$ (3.93 g, $28.4 \mathrm{mmol}$, 2.00 Äq.) in DMSO (30 mL) wurde $14 \mathrm{~h}$ bei $95{ }^{\circ} \mathrm{C}$ gerührt. Nach Abkühlen auf RT wurde die Mischung mit $\mathrm{H}_{2} \mathrm{O}(100 \mathrm{~mL})$ versetzt und mit MTBE extrahiert $(3 \times 100 \mathrm{~mL})$. Die vereinigten organischen Phasen wurden mit ges. wässr. $\mathrm{NaCl}$-Lösung $(150 \mathrm{~mL})$ gewaschen, über $\mathrm{Na}_{2} \mathrm{SO}_{4}$ getrocknet, filtriert und das Lösungsmittel in vacuo entfernt, um Biarylether 148d als gelbes Öl (3.80 g, 13.4 mmol, 94\%) zu erhalten.

DC: $R_{f}=0.43(n-$ Pentan/EtOAc, $10: 1)$.

UV/Vis $\left(\mathrm{CH}_{3} \mathrm{CN}\right): \lambda_{\max }(\mathrm{nm})(\lg \varepsilon)=256(3.755)$.

IR (ATR): $\tilde{v}\left(\mathrm{~cm}^{-1}\right)=1609,1587,1526,1493,1457,1350,1318,1263,1242,1207,1161,1128,1112$, 1089, 1054, 1035, 886, 845, 799, 774, 759, 740, 699, 663, 648.

${ }^{1} \mathbf{H}-\mathrm{NMR}\left(600 \mathrm{MHz}, \mathrm{CDCl}_{3}\right): \delta(\mathrm{ppm})=6.91(\mathrm{~d}, J=8.3 \mathrm{~Hz}, 1 \mathrm{H}), 7.01(\mathrm{dd}, J=8.3,1.2 \mathrm{~Hz}, 1 \mathrm{H}), 7.24$ $\left(\mathrm{m}_{\mathrm{C}}, 1 \mathrm{H}\right), 7.27(\mathrm{ddd}, J=8.4,7.7,1.3 \mathrm{~Hz}, 1 \mathrm{H}), 7.49\left(\mathrm{~m}_{\mathrm{C}}, 1 \mathrm{H}\right), 7.54(\mathrm{ddd}, J=8.1,7.3,1.6 \mathrm{~Hz}, 1 \mathrm{H})$, $7.70(\mathrm{dd}, J=7.9,1.6 \mathrm{~Hz}, 1 \mathrm{H}), 7.98(\mathrm{dd}, J=8.2,1.6 \mathrm{~Hz}, 1 \mathrm{H})$.

${ }^{13}$ C-NMR $\left(126 \mathrm{MHz}, \mathrm{CDCl}_{3}\right): \delta(\mathrm{ppm})=119.0,121.4,121.7(\mathrm{q}, J=31.8 \mathrm{~Hz}), 123.0(\mathrm{q}, J=272.8 \mathrm{~Hz})$, 124.0, 124.4, 125.8, 127.5 (q, $J=4.9 \mathrm{~Hz}), 133.5,134.4,141.8,149.4,153.9$ (q, $J=1.7 \mathrm{~Hz})$.

${ }^{19}$ F-NMR $\left(282 \mathrm{MHz}, \mathrm{CDCl}_{3}\right): \delta(\mathrm{ppm})=-61.8(\mathrm{~s})$.

MS (ESI): $m / z(\%)=306.0(100)[\mathrm{M}+\mathrm{Na}]^{+}$.

HRMS (ESI): $m / z=$ gef.: 306.0355 , ber.: $306.0348[\mathrm{M}+\mathrm{Na}]^{+}$.

$\mathrm{C}_{13} \mathrm{H}_{8} \mathrm{~F}_{3} \mathrm{NO}_{3}(283.20)$.

${ }^{\mathrm{r}}$ Für Originalvorschrift siehe: D. R. Polisetti, PCT Int. Appl. 2004, 2004002481. 


\section{2-(2-(Trifluormethyl)phenoxy)anilin (149d)}<smiles>Nc1ccccc1Oc1ccccc1C(F)(F)F</smiles>

Konz. $\mathrm{HCl}(50 \mathrm{~mL})$ und anschließend konz. AcOH $(50 \mathrm{~mL})$ wurden bei $0{ }^{\circ} \mathrm{C}$ tropfenweise zu einer Lösung von 1-Nitro-2-(2-(trifluormethyl)phenoxy)benzol (148d) (3.79 g, 13.4 mmol, 1.00 Äq.) in EtOAc $(100 \mathrm{~mL})$ gegeben. Zinkpulver $(19.8 \mathrm{~g}, 456 \mathrm{mmol}, 30.0$ Äq.) wurde portionsweise zugegeben und die Lösung auf RT erwärmt und $1 \mathrm{~h}$ bei dieser Temperatur gerührt. Die Reaktionsmischung wurde auf $0{ }^{\circ} \mathrm{C}$ abgekühlt und 33\%ige wässr. $\mathrm{NH}_{3}$-Lösung $(200 \mathrm{~mL})$ hinzugetropft. Die Mischung wurde mit $\mathrm{CH}_{2} \mathrm{Cl}_{2}$ extrahiert $(3 \times 300 \mathrm{~mL})$ und die vereinigten organischen Phasen über $\mathrm{Na}_{2} \mathrm{SO}_{4}$ getrocknet. Entfernung des Lösungsmittels in vacuo lieferte Amin 149d als braunes Öl (3.21 g, 12.7 mmol, 95\%) in leicht verunreinigter Form.

DC: $R_{f}=0.49(n-$ Pentan/EtOAc, 20:1).

UV/Vis $\left(\mathrm{CH}_{3} \mathrm{CN}\right): \lambda_{\max }(\mathrm{nm})(\lg \varepsilon)=281(3.738)$.

IR (ATR): $\tilde{v}\left(\mathrm{~cm}^{-1}\right)=1621,1607,1584,1500,1489,1456,1318,1268,1229,1190,1164,1156,1124$, $1109,1053,1034,884,756,740,648,597,521$.

${ }^{1} \mathbf{H}-\mathbf{N M R}\left(600 \mathrm{MHz}, \mathrm{CDCl}_{3}\right): \delta(\mathrm{ppm})=3.81\left(\mathrm{~s}_{\mathrm{br}}, 2 \mathrm{H}, \mathrm{NH}_{2}\right), 6.74(\mathrm{td}, J=7.7,1.5 \mathrm{~Hz}, 1 \mathrm{H}, 4-\mathrm{H})$, 6.82-6.87 (m, 2 H, 6-H, 6'-H), $6.91(\mathrm{dd}, J=8.0,1.3 \mathrm{~Hz}, 1 \mathrm{H}, 3-\mathrm{H}), 7.02(\mathrm{td}, J=7.7,1.4 \mathrm{~Hz}, 1 \mathrm{H}$, 5-H), $7.10\left(\mathrm{~m}_{\mathrm{C}}, 1 \mathrm{H}, 4^{\prime}-\mathrm{H}\right), 7.39$ (m $\left.\mathrm{m}_{\mathrm{C}}, 1 \mathrm{H}, 5^{\prime}-\mathrm{H}\right), 7.64$ (dd, J = 7.8, $\left.1.8 \mathrm{~Hz}, 1 \mathrm{H}, 3^{\prime}-\mathrm{H}\right)$.

${ }^{13}$ C-NMR (126 MHz, CDCl $): \delta(\mathrm{ppm})=116.3,116.8(\mathrm{C}-6, \mathrm{C}-6$ ') 118.9 (C-4), 119.7 (q, $J=31.2 \mathrm{~Hz}$, C-2'), 121.0 (C-3), 121.9 (C-4'), 123.6 (q, $J=272.4 \mathrm{~Hz}, \mathrm{CF}_{3}$ ), 125.8 (C-5), 127.1 (q, $J=5.1 \mathrm{~Hz}$, C-3'), 133.3 (C-5'), 138.8 (C-1), 141.8 (C-2), 155.6 (C-1').

${ }^{19}$ F-NMR $\left(283 \mathrm{MHz}, \mathrm{CDCl}_{3}\right): \delta(\mathrm{ppm})=-62.0\left(\mathrm{~s}, \mathrm{CF}_{3}\right)$.

MS (ESI): $m / z(\%)=254.1(100)[\mathrm{M}+\mathrm{H}]^{+}$.

HRMS (ESI): $m / z=$ gef.: 254.0792 , ber.: $254.0787[\mathrm{M}+\mathrm{H}]^{+}$.

$\mathrm{C}_{\mathbf{1 3}} \mathrm{H}_{\mathbf{1 0}} \mathbf{F}_{\mathbf{3}} \mathbf{N O}$ (253.22).

${ }^{\text {s }}$ Für Originalvorschrift siehe: D. R. Polisetti, PCT Int. Appl. 2004, 2004002481. 


\section{1-Iod-2-(2-(trifluormethyl)phenoxy)benzol (102d) ${ }^{\mathrm{t}}$}<smiles>FC(F)(F)c1ccccc1Oc1ccccc1I</smiles>

Eine Lösung von KI (4.18 g, 25.2 mmol, 2.00 Äq.) und $\mathrm{NaNO}_{2}\left(1.74 \mathrm{~g}, 25.2 \mathrm{mmol}, 2.00\right.$ Äq.) in $\mathrm{H}_{2} \mathrm{O}$ $(50 \mathrm{~mL})$ wurde zu einer Lösung von 2-(2-(Trifluormethyl)phenoxy)anilin (149d) (3.20 g, $12.6 \mathrm{mmol}$, 1.00 Äq.) und $p$-TsOH$\cdot \mathrm{H}_{2} \mathrm{O}\left(7.19 \mathrm{~g}, 37.8 \mathrm{mmol}, 3.00\right.$ Äq.) in $\mathrm{CH}_{3} \mathrm{CN}(100 \mathrm{~mL})$ gegeben und $2 \mathrm{~h}$ bei RT gerührt. Die Reaktionsmischung wurde mit ges. wässr. $\mathrm{NaHCO}_{3}$ Lösung $(100 \mathrm{~mL})$ und ges. wässr. $\mathrm{Na}_{2} \mathrm{~S}_{2} \mathrm{O}_{3}$ Lösung $(100 \mathrm{~mL})$ gewaschen und mit $\mathrm{CH}_{2} \mathrm{Cl}_{2}$ extrahiert $(3 \times 300 \mathrm{~mL})$. Die vereinigten organischen Phasen wurden über $\mathrm{Na}_{2} \mathrm{SO}_{4}$ getrocknet, filtriert und das Lösungsmittel in vacuo entfernt Säulenchromatographische Reinigung $\left(\mathrm{SiO}_{2}, n\right.$-Pentan) lieferte Aryliodid 102d als gelbes Öl (3.58 g, $9.83 \mathrm{mmol}, 78 \%)$.

DC: $R_{f}=0.33(n$-Pentan).

UV/Vis $\left(\mathrm{CH}_{3} \mathrm{CN}\right): \lambda_{\max }(\mathrm{nm})(\lg \varepsilon)=274(3.577)$.

IR (ATR): $\tilde{v}\left(\mathrm{~cm}^{-1}\right)=1609,1594,1574,1491,1457,1438,1318,1271,1164,1129,1111,1054,1035$, $1020,947,881,800,774,745,714,647$.

${ }^{1} \mathbf{H}-\mathbf{N M R}\left(600 \mathrm{MHz}, \mathrm{CDCl}_{3}\right): \delta(\mathrm{ppm})=6.74(\mathrm{~d}, J=8.3 \mathrm{~Hz}, 1 \mathrm{H}, 3-\mathrm{H}), 6.88-6.96\left(\mathrm{~m}, 2 \mathrm{H}, 4{ }^{\prime}-\mathrm{H}\right.$, 6'-H), 7.15 (m $\left.\mathrm{m}_{\mathrm{C}}, 1 \mathrm{H}, 5-\mathrm{H}\right), 7.31$ (ddd, $\left.J=8.3,7.4,1.5 \mathrm{~Hz}, 1 \mathrm{H}, 5^{\prime}-\mathrm{H}\right), 7.42$ (ddd, $J=8.4,7.7,1.6 \mathrm{~Hz}$, $1 \mathrm{H}, 4-\mathrm{H}), 7.68$ (dd, $J=7.8,1.7 \mathrm{~Hz}, 1 \mathrm{H}, 3$ '-H), 7.87 (dd, $J=8.3,1.5 \mathrm{~Hz}, 1 \mathrm{H}, 6-\mathrm{H})$.

${ }^{13}$ C-NMR (126 MHz, $\left.\mathrm{CDCl}_{3}\right): \delta(\mathrm{ppm})=89.2(\mathrm{C}-1), 117.7(\mathrm{C}-3), 120.2(\mathrm{C}-4$ '), 120.7 (q, $J=31.8 \mathrm{~Hz}$, C-2'), 122.7 (C-5), 123.3 (d, $J=273.0 \mathrm{~Hz}, \mathrm{CF}_{3}$ ), 126.2 (C-6'), 127.4 (q, $J=4.9 \mathrm{~Hz}, \mathrm{C}-3$ ') 129.8 (C-5'), 133.1 (C-4), 140.1 (C-6), 154.7 (C-2), 155.5 (C-1').

${ }^{19}$ F-NMR $\left(282 \mathrm{MHz}, \mathrm{CDCl}_{3}\right): \delta(\mathrm{ppm})=-61.8\left(\mathrm{~s}, \mathrm{CF}_{3}\right)$.

MS (ESI): $m / z(\%)=386.9(12)[\mathrm{M}+\mathrm{Na}]^{+}$.

HRMS (ESI): $m / z=$ gef.: 386.9455 , ber.: $386.9464[\mathrm{M}+\mathrm{Na}]^{+}$.

$\mathbf{C}_{13} \mathbf{H}_{8} \mathbf{F}_{3} \mathbf{I O}$ (364.10).

${ }^{\mathrm{t}}$ Allgemeine Methode aus: E. A. Krasnokutskaya, N. I. Semenischeva, V. D. Filimonov, P. Knochel, Synthesis 2007, 81-84. 


\section{1-(Hepta-1,6-diin-1-yl)-2-(2-(trifluormethyl)phenoxy)benzol (112d)}

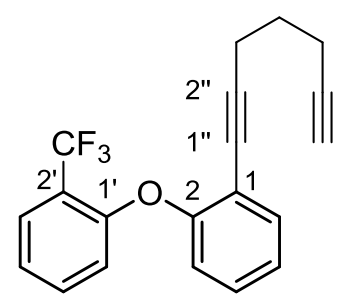

Eine Mischung aus 1-Iod-2-(2-(trifluormethyl)phenoxy)benzol (102d) (318 mg, 874 mmol, 1.00 Äq.), 1,6-Heptadiin (150b) (700 $\mu \mathrm{L}, 564$ mg, 6.12 mmol, 7.00 Äq.), $\mathrm{PdCl}_{2}\left(\mathrm{PPh}_{3}\right)_{2}$ (30.7 mg, $43.7 \mu \mathrm{mol}$, 0.05 Äq.) und CuI (10.4 mg, $87.4 \mu \mathrm{mol}, 0.10$ Äq.) in entgastem $\mathrm{NEt}_{3}(8 \mathrm{~mL})$ wurde $17 \mathrm{~h}$ bei $60^{\circ} \mathrm{C}$ gerührt. Die Reaktionsmischung wurde durch $\mathrm{SiO}_{2}$ filtriert, mit EtOAc gespült und das Lösungsmittel des Filtrats in vacuo entfernt. Säulenchromatographische Reinigung $\left(\mathrm{SiO}_{2}, n\right.$-Pentan/Toluol, 5:1) lieferte Dialkin 112d als gelbes Öl (237 mg, $722 \mu \mathrm{mol}, 83 \%)$ in leicht verunreinigter Form.

DC: $R_{f}=0.42(n$-Pentan/Toluol, 5:1).

UV/Vis $\left(\mathrm{CH}_{3} \mathrm{CN}\right): \lambda_{\max }(\mathrm{nm})(\lg \varepsilon)=241(4.205), 252$ (4.205), 275 (3.588).

IR (ATR): $\tilde{v}\left(\mathrm{~cm}^{-1}\right)=1583,1556,1486,1454,1367,1350,1319,1255,1223,1202,1165,1074,979$, $864,831,803,759,747,727,687,670,638,519$.

${ }^{1} \mathbf{H}-\mathbf{N M R}\left(600 \mathrm{MHz}, \mathrm{CDCl}_{3}\right): \delta(\mathrm{ppm})=1.56\left(\mathrm{p}, J=7.0 \mathrm{~Hz}, 2 \mathrm{H}, 4{ }^{\prime}{ }^{-}-\mathrm{H}_{2}\right), 1.89(\mathrm{t}, J=2.6 \mathrm{~Hz}, 1 \mathrm{H}$, 7' '-H), 2.08 (td, $J=7.1,2.6 \mathrm{~Hz}, 2 \mathrm{H}, 5$ ''- $\left.\mathrm{H}_{2}\right), 2.36$ (t, $J=6.9 \mathrm{~Hz}, 2 \mathrm{H}, 3$ ''- $\left.\mathrm{H}_{2}\right), 6.71$ (d, $J=8.3 \mathrm{~Hz}$, $1 \mathrm{H}, 3-\mathrm{H}), 7.01$ (dd, $\left.J=8.1,1.2 \mathrm{~Hz}, 1 \mathrm{H}, 6^{\prime}-\mathrm{H}\right), 7.09$ (t, $\left.J=7.7 \mathrm{~Hz}, 1 \mathrm{H}, 4^{\prime}-\mathrm{H}\right), 7.13$ (td, $J=7.6$, $1.2 \mathrm{~Hz}, 1 \mathrm{H}, 5-\mathrm{H}), 7.28(\mathrm{td}, J=8.1,1.6 \mathrm{~Hz}, 1 \mathrm{H}, 4-\mathrm{H}), 7.38\left(\mathrm{~m}_{\mathrm{C}}, 1 \mathrm{H}, 5^{\prime}-\mathrm{H}\right), 7.44$ (dd, $J=7.7,1.6 \mathrm{~Hz}$, $1 \mathrm{H}, 6-\mathrm{H}), 7.64$ (dd, $\left.J=7.6,1.5 \mathrm{~Hz}, 3^{\prime}-\mathrm{H}\right)$.

${ }^{13}$ C-NMR (126 MHz, $\left.\mathrm{CDCl}_{3}\right): \delta(\mathrm{ppm})=17.4$ (C-5'), 18.5 (C-3'), 27.3 (C-4'”), 68.6 (C-7''), 76.1 (C-6’), 83.5 (C-2'), 94.9 (C-1'), 116.8 (C-3), 117.3 (C-1), 119.8 (q, J=31.3 Hz, C-2'), 120.8 (C-6'), 121.8 (C-4'), 123.4 (q, $J=272.2 \mathrm{~Hz}, \mathrm{CF}_{3}$ ), 124.7 (C-5), 127.0 (q, $J=5.0 \mathrm{~Hz}, \mathrm{C}-3$ ') 129.1 (C-4), 132.9 (C-5'), 133.6 (C-6), 155.5, 155.6 (C-2, C-1').

${ }^{19}$ F-NMR $\left(282 \mathrm{MHz}, \mathrm{CDCl}_{3}\right): \delta(\mathrm{ppm})=-62.0\left(\mathrm{~s}, \mathrm{CF}_{3}\right)$.

MS (ESI): $m / z(\%)=329.1(48)[\mathrm{M}+\mathrm{H}]^{+}, 351.1(100)[\mathrm{M}+\mathrm{Na}]^{+}$.

HRMS (ESI): $m / z=$ gef.: 329.1148 , ber.: $329.1148[\mathrm{M}+\mathrm{H}]^{+}$.

$\mathbf{C}_{20} \mathbf{H}_{15} \mathbf{F}_{3} \mathbf{O}$ (328.33). 


\section{1-Methoxy-2-(2-nitrophenoxy)benzol (148e $)^{\mathrm{u}}$}<smiles>COc1ccccc1Oc1ccccc1[N+](=O)[O-]</smiles>

Eine Mischung aus 2-Methoxyphenol (147e) $(1.80 \mathrm{~mL}, \quad 2.00 \mathrm{~g}, \quad 16.1 \mathrm{mmol}, \quad 1.00$ Äq.), 2-Fluornitrobenzol (121a) (1.70 mL, $2.27 \mathrm{~g}, 16.1 \mathrm{mmol}, 1.00$ Äq.) und $\mathrm{K}_{2} \mathrm{CO}_{3}(4.45 \mathrm{~g}, 32.2 \mathrm{mmol}$, 2.00 Äq.) in DMSO (40 mL) wurde $20 \mathrm{~h}$ bei $95{ }^{\circ} \mathrm{C}$ gerührt. Nach Abkühlen auf RT wurde die Mischung mit $\mathrm{H}_{2} \mathrm{O}(100 \mathrm{~mL})$ versetzt, filtriert und der abfiltrierte Feststoff in $\mathrm{CH}_{2} \mathrm{Cl}_{2}$ gelöst. Das Filtrat wurde mit MTBE extrahiert $(3 \times 100 \mathrm{~mL})$. Die vereinigten organischen Phasen wurden mit ges. wässr. NaCl-Lösung (200 mL) gewaschen, über $\mathrm{Na}_{2} \mathrm{SO}_{4}$ getrocknet, filtriert und das Lösungsmittel in vacuo entfernt, um Biarylether 148e als gelbes Öl (3.96 g, 16.1 mmol, quant.) in leicht verunreinigter Form zu erhalten.

DC: $R_{f}=0.35(n-$ Pentan/EtOAc, 10:1).

UV/Vis $\left(\mathrm{CH}_{3} \mathrm{CN}\right): \lambda_{\max }(\mathrm{nm})(\lg \varepsilon)=194$ (4.720), 264 (3.749), 319 (3.463).

IR (ATR): $\tilde{v}\left(\mathrm{~cm}^{-1}\right)=1606,1584,1523,1496,1480,1471,1455,1439,1349,1305,1263,1231,1197$, $1175,1163,1147,1111,1089,1043,1018,883,846,801,770,746,738,694,665$.

${ }^{1} \mathbf{H}-\mathrm{NMR}\left(600 \mathrm{MHz}, \mathrm{CDCl}_{3}\right): \delta(\mathrm{ppm})=3.75\left(\mathrm{~s}, 3 \mathrm{H}, \mathrm{OCH}_{3}\right), 6.80(\mathrm{dd}, J=8.5,1.2 \mathrm{~Hz}, 1 \mathrm{H}), 6.94(\mathrm{td}$, $J=7.7,1.4 \mathrm{~Hz}, 1 \mathrm{H}), 6.99(\mathrm{dd}, J=8.2,1.4 \mathrm{~Hz}, 1 \mathrm{H}), 7.06(\mathrm{dd}, J=8.0,1.7 \mathrm{~Hz}, 1 \mathrm{H}), 7.08(\mathrm{td}, J=7.9$, $1.2 \mathrm{~Hz}, 1 \mathrm{H}), 7.18(\mathrm{td}, J=7.8,1.6 \mathrm{~Hz}, 1 \mathrm{H}), 7.39(\mathrm{ddd}, J=8.7,7.3,1.7 \mathrm{~Hz}, 1 \mathrm{H}) 7.92(\mathrm{dd}, J=8.2$, $1.7 \mathrm{~Hz}, 1 \mathrm{H})$.

${ }^{13} \mathbf{C}-\mathrm{NMR}\left(126 \mathrm{MHz}, \mathrm{CDCl}_{3}\right): \delta(\mathrm{ppm})=55.9\left(\mathrm{OCH}_{3}\right), 113.2,118.1,121.3,121.8,122.0,125.6$, $126.3,133.9,140.0,143.3,151.3,151.7$.

MS (ESI): $m / z(\%)=246.1(100)[\mathrm{M}+\mathrm{H}]^{+}, 263.1(94)\left[\mathrm{M}+\mathrm{NH}_{4}\right]^{+}, 268.1(92)[\mathrm{M}+\mathrm{Na}]^{+}$.

HRMS (ESI): $m / z=$ gef.: 246.0763 , ber.: $246.0761[\mathrm{M}+\mathrm{H}]^{+}$.

$\mathrm{C}_{13} \mathrm{H}_{11} \mathrm{NO}_{4}$ (245.23).

u J. A. De la Fuente, J. Med. Chem. 2003, 46, 5208-5221. 


\section{2-(2-Methoxyphenoxy)anilin (149e) $)^{\mathrm{v}}$}<smiles>COc1ccccc1Oc1ccccc1N</smiles>

Konz. $\mathrm{HCl}(50 \mathrm{~mL})$ und anschließend konz. AcOH $(50 \mathrm{~mL})$ wurden bei $0{ }^{\circ} \mathrm{C}$ tropfenweise zu einer Lösung von 1-Methoxy-2-(2-nitrophenoxy)benzol (148e) (3.95 g, $17.0 \mathrm{mmol}, 1.00$ Äq.) in EtOAc (100 mL) hinzugefügt. Zinkpulver (33.3 g, $510 \mathrm{mmol}, 30.0$ Äq.) wurde portionsweise zugegeben, die Lösung auf RT erwärmt und 30 min bei dieser Temperatur gerührt. Die Reaktionsmischung wurde auf $0{ }^{\circ} \mathrm{C}$ abgekühlt und 33\%ige wässr. $\mathrm{NH}_{3}$-Lösung $(200 \mathrm{~mL})$ wurde hinzugetropft. Die Mischung wurde mit $\mathrm{CH}_{2} \mathrm{Cl}_{2}(3 \times 300 \mathrm{~mL})$ extrahiert und die vereinigten organischen Phasen wurden über $\mathrm{Na}_{2} \mathrm{SO}_{4}$ getrocknet und filtriert. Entfernung des Lösungsmittels in vacuo lieferte Amin 149e als braunen Feststoff (3.39 g, 15.7 mmol, 92\%) in leicht verunreinigter Form.

DC: $R_{f}=0.20(n-\mathrm{Pentan} / \mathrm{EtOAc}, 10: 1)$.

UV/Vis $\left(\mathrm{CH}_{3} \mathrm{CN}\right): \lambda_{\max }(\mathrm{nm})(\lg \varepsilon)=196$ (4.681), 279 (3.612).

IR (ATR): $\tilde{v}\left(\mathrm{~cm}^{-1}\right)=3446,3360,1627,1592,1580,1493,1452,1438,1329,1299,1249,1210,1190$, $1181,1162,1149,1113,1042,1024,883,785,745,722,681,671,586,572,551,530$.

${ }^{1} \mathrm{H}-\mathrm{NMR}\left(600 \mathrm{MHz}, \mathrm{CDCl}_{3}\right): \delta(\mathrm{ppm})=3.71\left(\mathrm{~s}_{\mathrm{br}}, 2 \mathrm{H}, \mathrm{NH}_{2}\right), 3.86\left(\mathrm{~s}, 3 \mathrm{H}, \mathrm{OCH}_{3}\right), 6.67(\mathrm{ddd}, J=7.9$, 7.4, $1.5 \mathrm{~Hz}, 1 \mathrm{H}, 4-\mathrm{H}), 6.76$ (dd, $J=8.1,1.4 \mathrm{~Hz}, 1 \mathrm{H}, 3-\mathrm{H}), 6.81$ (dd, $J=7.9,1.5 \mathrm{~Hz}, 1 \mathrm{H}, 6-\mathrm{H}), 6.84$ 6.87 (m, 2 H, 5'-H, 6'-H), 6.92 (td, J = 7.6, 1.4 Hz, 1 H, 5-H), 6.97 (d, J = 8.1 Hz, 1 H, 3'-H), 7.05 (dt, $\left.J=8.0,4.4 \mathrm{~Hz}, 1 \mathrm{H}, 4^{\prime}-\mathrm{H}\right)$.

${ }^{13} \mathbf{C}-\mathrm{NMR}\left(126 \mathrm{MHz}, \mathrm{CDCl}_{3}\right): \delta(\mathrm{ppm})=56.0\left(\mathrm{OCH}_{3}\right), 112.6\left(\mathrm{C}-{ }^{\prime}{ }^{\prime}\right), 116.4(\mathrm{C}-6), 118.5,118.8,118.8$ (C-3, C-4, C-5'/C-6'), 121.0 (C-5’/C-6'), 123.9 (C-4'), 124.1 (C-5), 137.6 (C-1), 144.3 (C-2), 145.8 (C-1'), 150.5 (C-2').

MS (ESI): $m / z(\%)=216.1(100)[\mathrm{M}+\mathrm{H}]^{+}, 238.1(13)[\mathrm{M}+\mathrm{Na}]^{+}$.

HRMS (ESI): $m / z=$ gef.: 216.1018 , ber.: $216.1019[\mathrm{M}+\mathrm{Na}]^{+}$. $\mathrm{C}_{13} \mathrm{H}_{13} \mathrm{NO}_{2}$ (215.25).

\footnotetext{
${ }^{v}$ Für Originalvorschrift siehe: F. Wen, H. Zhang, Z. Yu, H. Jin, Q. Yang, T. Hou, Pestic. Biochem. Physiol.
} 2010, 98, 248-253. 


\section{1-Iod-2-(2-methoxyphenoxy)benzol (102e) ${ }^{\mathrm{w}}$}<smiles>COc1ccccc1Oc1ccccc1I</smiles>

Eine Lösung von KI (5.18 g, 31.2 mmol, 2.00 Äq.) und $\mathrm{NaNO}_{2}$ (2.15 g, 31.2 mmol, 2.00 Äq.) in $\mathrm{H}_{2} \mathrm{O}$ $(50 \mathrm{~mL})$ wurde zu einer Lösung von 2-(2-Methoxyphenoxy)anilin (149e) (3.11 g, $15.6 \mathrm{mmol}$, 1.00 Äq.) und $p$-TsOH$\cdot \mathrm{H}_{2} \mathrm{O}\left(8.90 \mathrm{~g}, 46.8 \mathrm{mmol}, 3.00\right.$ Äq.) in $\mathrm{CH}_{3} \mathrm{CN}(100 \mathrm{~mL})$ getropft und $2 \mathrm{~h}$ bei RT gerührt. Die Reaktionsmischung wurde mit ges. wässr. $\mathrm{NaHCO}_{3}$-Lösung (100 mL) gewaschen und mit $\mathrm{CH}_{2} \mathrm{Cl}_{2}$ extrahiert $(3 \times 300 \mathrm{~mL})$. Die vereinigten organischen Phasen wurden in vacuo eingeengt, in $\mathrm{CH}_{2} \mathrm{Cl}_{2}(200 \mathrm{~mL})$ gelöst, mit ges. wässr. $\mathrm{Na}_{2} \mathrm{~S}_{2} \mathrm{O}_{3}$-Lösung $(100 \mathrm{~mL})$ gewaschen, über $\mathrm{Na}_{2} \mathrm{SO}_{4}$ getrocknet, filtriert und das Lösungsmittel in vacuo entfernt. Säulenchromatographische Reinigung $\left(\mathrm{SiO}_{2}, n\right.$-Pentan/EtOAc, 100:1 $\left.\rightarrow 50: 1\right)$ lieferte Aryliodid 102e als gelbes Öl (4.54 g, 13.9 mmol, 89\%).

DC: $R_{f}=0.33(n$-Pentan/EtOAc, 100:1).

UV/Vis $\left(\mathrm{CH}_{3} \mathrm{CN}\right): \lambda_{\max }(\mathrm{nm})(\lg \varepsilon)=275$ (3.697).

IR (ATR): $\tilde{v}\left(\mathrm{~cm}^{-1}\right)=1600,1572,1497,1462,1437,1304,1258,1222,1197,1174,1158,1109,1040$, $1017,878,802,770,741,712,646$.

${ }^{1} \mathbf{H}-\mathrm{NMR}\left(600 \mathrm{MHz}, \mathrm{CDCl}_{3}\right): \delta(\mathrm{ppm})=3.82\left(\mathrm{~s}, 3 \mathrm{H}, \mathrm{OCH}_{3}\right), 6.66(\mathrm{dd}, J=8.2,1.4 \mathrm{~Hz}, 1 \mathrm{H}, 3-\mathrm{H})$, 6.75-6.82 (m, 1 H, 5-H), 6.88-6.93 (m, 2 H, 5'-H, 6'-H), 6.97-7.02 (m, 1 H, 3'-H), 7.13 (ddd, $J=8.1$, 5.2, 3.9 Hz, $1 \mathrm{H}, 4$ '-H), 7.19 (ddd, $J=8.1,7.3,1.5 \mathrm{~Hz}, 1 \mathrm{H}, 4-\mathrm{H}), 7.81$ (dd, $J=7.8,1.5 \mathrm{~Hz}, 1 \mathrm{H}, 6-\mathrm{H}$ ).

${ }^{13}$ C-NMR $\left(126 \mathrm{MHz}, \mathrm{CDCl}_{3}\right): \delta(\mathrm{ppm})=56.2\left(\mathrm{OCH}_{3}\right), 87.1(\mathrm{C}-1), 113.2(\mathrm{C}-3$ ') $116.8(\mathrm{C}-3), 120.7$, 121.1 (C-5', C-6'), 124.3 (C-5), 125.1 (C-4'), 129.3 (C-4), 139.6 (C-6), 144.9 (C-1'), 151.2 (C-2'), $157.1(\mathrm{C}-2)$.

MS (ESI): $m / z(\%)=349.0(100)[\mathrm{M}+\mathrm{Na}]^{+}$.

HRMS (ESI): $m / z=$ gef.: 348.9699 , ber.: $348.9696[\mathrm{M}+\mathrm{Na}]^{+}$.

$\mathrm{C}_{13} \mathrm{H}_{11} \mathrm{IO}_{2}$ (326.13).

${ }^{w}$ Allgemeine Methode aus: E. A. Krasnokutskaya, N. I. Semenischeva, V. D. Filimonov, P. Knochel, Synthesis 2007, 81-84. 
1-(Hepta-1,6-diin-1-yl)-2-(2-methoxyphenoxy)benzol (112e)

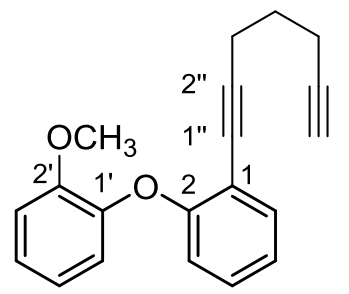

Eine Mischung aus 1-Iod-2-(2-methoxyphenoxy)benzol (102e) (285 mg, $874 \mu \mathrm{mol}, 1.00$ Äq.), 1,6-Heptadiin (150b) (700 $\mu \mathrm{L}, 564 \mathrm{mg}, 6.12 \mathrm{mmol}, 7.00$ Äq.), $\mathrm{PdCl}_{2}\left(\mathrm{PPh}_{3}\right)_{2}(30.7 \mathrm{mg}, 43.7 \mu \mathrm{mol}$, 0.05 Äq.) und CuI (10.4 mg, $87.4 \mu \mathrm{mol}, 0.10$ Äq.) in entgastem $\mathrm{NEt}_{3}(8 \mathrm{~mL})$ wurde $17 \mathrm{~h}$ bei $60^{\circ} \mathrm{C}$ gerührt. Die Reaktionsmischung wurde durch $\mathrm{SiO}_{2}$ filtriert, mit EtOAc gespült und das Lösungsmittel des Filtrats in vacuo entfernt. Säulenchromatographische Reinigung $\left(\mathrm{SiO}_{2}, n\right.$-Pentan/Toluol, 1:1) lieferte Dialkin 112e als gelbes Öl (193 mg, 669 mol, 77\%).

DC: $R_{f}=0.25(n$-Pentan/Toluol, 1:1).

UV/Vis $\left(\mathrm{CH}_{3} \mathrm{CN}\right): \lambda_{\max }(\mathrm{nm})(\lg \varepsilon)=252(4.142), 279(3.630), 297$ (3.505).

IR (ATR): $\tilde{v}\left(\mathrm{~cm}^{-1}\right)=1498,1485,1443,1300,1260,1216,1189,1175,1158,1115,1041,1024,929$, $874,842,745,636,554$.

${ }^{1} \mathbf{H}-\mathbf{N M R}\left(600 \mathrm{MHz}, \mathrm{CDCl}_{3}\right): \delta(\mathrm{ppm})=1.69\left(\mathrm{p}, J=7.0 \mathrm{~Hz}, 2 \mathrm{H}, 4{ }^{\prime}{ }^{-}-\mathrm{H}_{2}\right), 1.92(\mathrm{t}, J=2.7 \mathrm{~Hz}, 1 \mathrm{H}$,

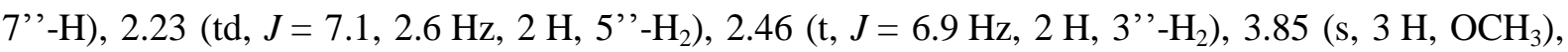
$6.77(\mathrm{dd}, J=8.4,1.1 \mathrm{~Hz}, 1 \mathrm{H}, 3-\mathrm{H}), 6.82(\mathrm{dd}, J=8.0,1.7 \mathrm{~Hz}, 1 \mathrm{H}, 6$ '-H), 6.87 (td, $J=7.7,1.4 \mathrm{~Hz}$, $\left.1 \mathrm{H}, 5^{\prime}-\mathrm{H}\right), 6.97$ (dd, $\left.J=8.0,1.5 \mathrm{~Hz}, 1 \mathrm{H}, 3^{\prime}-\mathrm{H}\right), 7.00$ (dd, $\left.J=7.5,1.2 \mathrm{~Hz}, 1 \mathrm{H}, 5-\mathrm{H}\right), 7.06$ (ddd, $J=$ 8.2, 7.3, $\left.1.7 \mathrm{~Hz}, 1 \mathrm{H}, 4^{\prime}-\mathrm{H}\right), 7.16$ (ddd, $\left.J=8.2,7.4,1.7 \mathrm{~Hz}, 1 \mathrm{H}, 4-\mathrm{H}\right), 7.41(\mathrm{dd}, J=7.6,1.7 \mathrm{~Hz}, 1 \mathrm{H}$, 6-H).

${ }^{13}$ C-NMR $\left(126 \mathrm{MHz}, \mathrm{CDCl}_{3}\right): \delta(\mathrm{ppm})=17.4(\mathrm{C}-5$ ') $), 18.8$ (C-3'”), $27.5(\mathrm{C}-4$ ') $), 56.1\left(\mathrm{OCH}_{3}\right), 68.6$ (C-7'), 76.9 (C-6'), 83.8 (C-2'”), 93.9 (C-1'’), 112.9 (C-3'), 115.4 (C-1), 117.8 (C-3), 119.5 (C-6'), 121.0 (C-5'), 122.8 (C-5), 124.2 (C-4'), 128.8 (C-4), 133.5 (C-6), 145.9 (C-1'), 150.7 (C-2'), 157.7 $(\mathrm{C}-2)$.

MS (ESI): $m / z(\%)=291.1(100)[\mathrm{M}+\mathrm{H}]^{+}, 308.2(100)\left[\mathrm{M}+\mathrm{NH}_{4}\right]^{+}, 313.1(100)[\mathrm{M}+\mathrm{Na}]^{+}$

HRMS (ESI): $m / z=$ gef.: 291.1380, ber.: $291.1380[\mathrm{M}+\mathrm{H}]^{+}$.

$\mathbf{C}_{20} \mathbf{H}_{18} \mathbf{O}_{2}$ (290.36). 


\section{4-Phenyl-1-(2-nitrophenoxy)benzol (148f $)^{x}$}

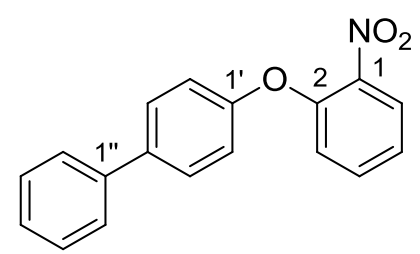

Eine Mischung aus 4-Phenylphenol (147f) (2.42 g, 14.2 mmol, 1.00 Äq.), 2-Fluornitrobenzol (121a) (1.49 mL, 2.00 g, 14.2 mmol, 1.00 Äq.) und $\mathrm{K}_{2} \mathrm{CO}_{3}$ (3.93 g, 28.4 mmol, 2.00 Äq.) in DMSO (40 mL) wurde $20 \mathrm{~h}$ bei $95^{\circ} \mathrm{C}$ gerührt. Nach Abkühlen auf RT wurde die Mischung in Eiswasser $(200 \mathrm{~mL})$ gegeben und mit MTBE extrahiert $(3 \times 200 \mathrm{~mL})$. Die vereinigten organischen Phasen wurden über $\mathrm{Na}_{2} \mathrm{SO}_{4}$ getrocknet und das Lösungsmittel in vacuo entfernt, um Biarylether $148 \mathrm{f}$ als gelben Feststoff (3.98 g, $13.7 \mathrm{mmol}, 96 \%)$ zu erhalten.

DC: $R_{f}=0.52(n-$ Pentan/EtOAc, $10: 1)$.

UV/Vis $\left(\mathrm{CH}_{3} \mathrm{CN}\right): \lambda_{\max }(\mathrm{nm})(\lg \varepsilon)=202$ (4.745), 256 (4.417).

IR (ATR): $\tilde{v}\left(\mathrm{~cm}^{-1}\right)=1589,1527,1477,1449,1357,1264,1240,883,826,764,699,659$.

${ }^{1} \mathbf{H}-\mathbf{N M R}\left(600 \mathrm{MHz}, \mathrm{CDCl}_{3}\right): \delta(\mathrm{ppm})=7.08(\mathrm{dd}, J=8.4,1.1 \mathrm{~Hz}, 1 \mathrm{H}, 6-\mathrm{H}), 7.09-7.12(\mathrm{~m}, 2 \mathrm{H}, 3$ '-H, 5'-H), 7.20 (ddd, $J=8.3,7.5,1.2 \mathrm{~Hz}, 1 \mathrm{H}, 4-\mathrm{H}), 7.31-7.36$ (m, $1 \mathrm{H}, 4$ ''-H), 7.40-7.45 (m, $2 \mathrm{H}, 2$ ''-H, 6' '-H), 7.51 (ddd, J = 8.5, 7.4, 1.7 Hz, 1 H, 5-H), 7.54-7.57 (m, 2 H, 3''-H, 5'-H), 7.56-7.61 (m, 2 H, 2'-H, 6'-H), 7.95 (dd, $J=8.2,1.7 \mathrm{~Hz}, 1 \mathrm{H}, 3-\mathrm{H})$.

${ }^{13}$ C-NMR $\left(126 \mathrm{MHz}, \mathrm{CDCl}_{3}\right): \delta(\mathrm{ppm})=119.4\left(\mathrm{C}^{3}{ }^{\prime}, \mathrm{C}-5\right.$ '), 120.7 (C-6), 123.3 (C-4), 125.8 (C-3), 127.0 (C-3', C-5'), 127.4 (C-4'), 128.7 (C-2', C-6'), 128.9 (C-2', C-6’'), 134.2 (C-5), 137.8 (C-1'’), 140.3 (C-4'), 141.5 (C-2), 150.7 (C-1), 155.4 (C-1').

MS (ESI): $m / z(\%)=314.1(66)[\mathrm{M}+\mathrm{Na}]^{+}, 605.2(100)[2 \mathrm{M}+\mathrm{Na}]^{+}$.

HRMS (ESI): $m / z=$ gef.: 314.0790 , ber.: $314.0788[\mathrm{M}+\mathrm{Na}]^{+}$.

$\mathrm{C}_{18} \mathrm{H}_{13} \mathrm{NO}_{3}$ (291.13).

${ }^{\mathrm{x}}$ Für Originalvorschrift siehe: K. Gao, C.-B. Yu, W. Li, Y.-G. Zhou, X. Zhang, Chem. Commun. 2011, 47, 7845-7847. 


\section{4-Phenyl-1-(2-aminophenoxy)benzol (149f)}<smiles>Nc1ccccc1O[14c]1ccc(-[14c]2ccccc2)cc1</smiles>

Konz. AcOH $(75 \mathrm{~mL})$ und anschließend konz. $\mathrm{HCl}(75 \mathrm{~mL})$ wurden bei $0{ }^{\circ} \mathrm{C}$ tropfenweise zu einer Lösung von 4-Phenyl-1-(2-nitrophenoxy)benzol (148f) (3.97 g, $13.6 \mathrm{mmol}, 1.00$ Äq.) in EtOAc $(50 \mathrm{~mL})$ hinzugefügt. Zinkpulver (26.7 g, $408 \mathrm{mmol}, 30.0$ Äq.) wurde portionsweise zugegeben, die Lösung wurde auf RT erwärmt und $17.5 \mathrm{~h}$ bei dieser Temperatur gerührt. Die Reaktionsmischung wurde auf $0{ }^{\circ} \mathrm{C}$ abgekühlt und 33\%ige wässr. $\mathrm{NH}_{3}$-Lösung $(300 \mathrm{~mL})$ hinzugetropft. Die Mischung wurde mit $\mathrm{CH}_{2} \mathrm{Cl}_{2}$ extrahiert $(3 \times 300 \mathrm{~mL})$, die vereinigten organischen Phasen über $\mathrm{Na}_{2} \mathrm{SO}_{4}$ getrocknet und filtriert. Entfernung des Lösungsmittels in vacuo lieferte Amin 149f als gelben Feststoff (3.29 g, $12.6 \mathrm{mmol}, 93 \%)$.

DC: $R_{f}=0.43(n-$ Pentan/EtOAc, $10: 1)$.

UV/Vis $\left(\mathrm{CH}_{3} \mathrm{CN}\right): \lambda_{\max }(\mathrm{nm})(\lg \varepsilon)=201$ (4.799), $258(4.332)$.

IR (ATR): $\tilde{v}\left(\mathrm{~cm}^{-1}\right)=1617,1503,1481,1229,1196,1166,834,762,748,736,689$.

${ }^{1} \mathrm{H}-\mathrm{NMR}\left(600 \mathrm{MHz}, \mathrm{CDCl}_{3}\right): \delta(\mathrm{ppm})=3.96\left(\mathrm{~s}_{\mathrm{br}}, 2 \mathrm{H}, \mathrm{NH}_{2}\right), 6.76(\mathrm{ddd}, J=8.0,7.4,1.1 \mathrm{~Hz}, 1 \mathrm{H}$, 5-H), $6.86(\mathrm{dd}, J=7.9,1.6 \mathrm{~Hz}, 1 \mathrm{H}, 3-\mathrm{H}), 6.93(\mathrm{dd}, J=8.0,1.4 \mathrm{~Hz}, 1 \mathrm{H}, 6-\mathrm{H}), 7.00$ (ddd, $J=7.9,7.4$, $1.4 \mathrm{~Hz}, 1 \mathrm{H}, 4-\mathrm{H}), 7.02-7.06$ (m, 2 H, 3'-H, 5'-H), 7.30-7.33 (m, $1 \mathrm{H}, 4$ '’-H), 7.40-7.44 (m, 2 H, 2'’-H, 6'-H), 7.50-7.57 (m, 4 H, 2'-H, 6'-H, 3''-H, 5''-H).

${ }^{13}$ C-NMR (126 MHz, $\left.\mathrm{CDCl}_{3}\right): \delta(\mathrm{ppm})=116.7(\mathrm{C}-3), 117.3$ (C-3', C-5'), 119.1 (C-5), 120.2 (C-6), 124.9 (C-4), 126.8 (C-3'”, C-5'), 126.9 (C-4'”), 128.3 (C-2', C-6'), 128.7 (C-2', C-6’'), 135.7 (C-1'’), 138.2 (C-2), 140.5 (C-4'), 143.1 (C-1), 156.9 (C-1').

MS (ESI): $m / z(\%)=262.1(100)[\mathrm{M}+\mathrm{H}]^{+}$.

HRMS (ESI): $m / z=$ gef.: 262.1233, ber.: $262.1226[\mathrm{M}+\mathrm{H}]^{+}$.

$\mathbf{C}_{18} \mathrm{H}_{15} \mathrm{NO}$ (261.32). 


\section{4-Phenyl-1-(2-iod)benzol (102f) ${ }^{y}$}<smiles></smiles>

Eine Lösung von KI (5.22 g, 31.4 mmol, 2.50 Äq.) und $\mathrm{NaNO}_{2}$ (1.76 g, $25.2 \mathrm{mmol}, 2.00$ Äq.) in $\mathrm{H}_{2} \mathrm{O}$ $(50 \mathrm{~mL})$ wurde zu einer Lösung von 4-Phenyl-1-(2-aminophenoxy)benzol (149f) (3.28 g, $12.6 \mathrm{mmol}$, 1.00 Äq.) und $p$-TsOH$\cdot \mathrm{H}_{2} \mathrm{O}\left(7.16 \mathrm{~g}, 37.7 \mathrm{mmol}, 3.00\right.$ Äq.) in $\mathrm{CH}_{3} \mathrm{CN}(300 \mathrm{~mL})$ gegeben und $2 \mathrm{~h}$ bei RT gerührt. Die Reaktionsmischung wurde mit ges. wässr. $\mathrm{NaHCO}_{3}$-Lösung (300 mL) versetzt und mit $\mathrm{CH}_{2} \mathrm{Cl}_{2}$ extrahiert $(3 \times 300 \mathrm{~mL})$. Die vereinigten organischen Phasen wurden in vacuo eingeengt, in $\mathrm{CH}_{2} \mathrm{Cl}_{2}(200 \mathrm{~mL})$ gelöst, mit ges. wässr. $\mathrm{Na}_{2} \mathrm{~S}_{2} \mathrm{O}_{3}$-Lösung $(100 \mathrm{~mL})$ gewaschen, über $\mathrm{Na}_{2} \mathrm{SO}_{4}$ getrocknet, filtriert und das Lösungsmittel in vacuo entfernt. Säulenchromatographische Reinigung ( $\mathrm{SiO}_{2}, n$-Pentan) lieferte Aryliodid $\mathbf{1 0 2 f}$ als gelben Feststoff (3.56 g, $\left.9.60 \mathrm{mmol}, 76 \%\right)$.

DC: $R_{f}=0.38(n-$ Pentan)

UV/Vis $\left(\mathrm{CH}_{3} \mathrm{CN}\right): \lambda_{\max }(\mathrm{nm})(\lg \varepsilon)=201$ (4.797), 260 (4.399).

IR (ATR): $\tilde{v}\left(\mathrm{~cm}^{-1}\right)=1511,1483,1461,1436,1229,1167,1018,835,758,695$.

${ }^{1} \mathbf{H}-\mathrm{NMR}\left(600 \mathrm{MHz}, \mathrm{CDCl}_{3}\right): \delta(\mathrm{ppm})=6.89(\mathrm{ddd}, J=8.7,7.6,1.4 \mathrm{~Hz}, 1 \mathrm{H}, 4-\mathrm{H}), 6.96(\mathrm{dd}, J=8.1$, 1.4 Hz, 1 H, 6-H), 7.01-7.05 (m, 2 H, 3'-H, 5'-H), 7.27-7.35 (m, 2 H, 5-H, 4'--H), 7.40-7.44 (m, 2 H, 2'’-H, 6'-H), 7.54-7.57 (m, 4 H, 2'-H, 6'-H, 3'”-H, 5'’-H), 7.87 (dd, J = 7.9, 1.4 Hz, 1 H, 3-H).

${ }^{13}$ C-NMR (126 MHz, $\left.\mathrm{CDCl}_{3}\right): \delta(\mathrm{ppm})=89.0(\mathrm{C}-2), 118.5(\mathrm{C}-3$ ', C-5'), $119.6(\mathrm{C}-6), 125.4$ (C-4), 126.8 (C-3', C-5’'), 127.0 (C-4’'), 128.4 (C-2', C-6'), 128.7 (C-2', C-6’'), 129.6 (C-5), 136.4 (C-1'’), 139.8 (C-3), 140.4 (C-4'), 156.3 (C-1', C-1).

MS (EI): $m / z(\%)=372.0(100)[\mathrm{M}+\mathrm{H}]^{+}$.

HRMS (EI): $m / z=$ gef.: 372.0020 , ber.: $372.0011[\mathrm{M}]^{+}$.

$\mathrm{C}_{18} \mathrm{H}_{13} \mathrm{IO}(372.21)$.

${ }^{\text {y }}$ Allgemeine Methode aus: E. A. Krasnokutskaya, N. I. Semenischeva, V. D. Filimonov, P. Knochel, Synthesis 2007, 81-84. 


\section{4-(2-(Hepta-1,6-diin-1-yl)phenoxy)-1,1'-biphenyl (112f)}

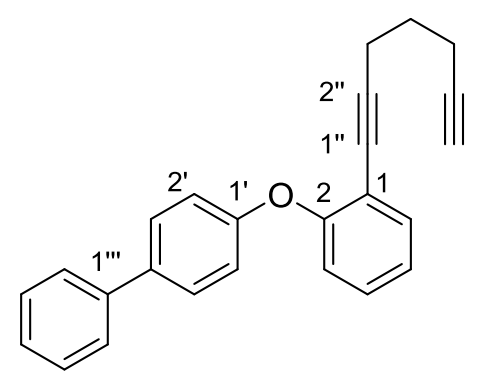

Eine Mischung aus 4-Phenyl-1-(2-iod)benzol (102f) (325 mg, $874 \mu$ mol, 1.00 Äq.), 1,6-Heptadiin (150b) (500 $\mu \mathrm{L}, 403$ mg, 4.37 mmol, 5.00 Äq.), $\mathrm{PdCl}_{2}\left(\mathrm{PPh}_{3}\right)_{2}$ (30.7 mg, $43.7 \mu \mathrm{mol}, 0.05$ Äq.) und CuI (10.4 mg, $87.4 \mu \mathrm{mol}, \quad 0.10$ Äq.) in entgastem $\mathrm{NEt}_{3}(8 \mathrm{~mL})$ wurde $18 \mathrm{~h}$ bei RT gerührt. Die Reaktionsmischung wurde durch $\mathrm{SiO}_{2}$ filtriert, mit EtOAc gespült und das Lösungsmittel des Filtrats in vacuo entfernt. Säulenchromatographische Reinigung $\left(\mathrm{SiO}_{2}, n\right.$-Pentan/Toluol, 5:1) lieferte Dialkin 112f als gelbes Öl (203 mg, $603 \mu \mathrm{mol}, 69 \%)$.

DC: $R_{f}=0.18(n-$ Pentan/Toluol, 5:1).

UV/Vis $\left(\mathrm{CH}_{3} \mathrm{CN}\right): \lambda_{\max }(\mathrm{nm})(\lg \varepsilon)=205$ (4.765), 252 (4.507).

IR (ATR): $\tilde{v}\left(\mathrm{~cm}^{-1}\right)=1514,1481,1444,1266,1252,1224,1168,1105,871,835,760,697,635$.

${ }^{1} \mathbf{H}-\mathrm{NMR}\left(600 \mathrm{MHz}, \mathrm{CDCl}_{3}\right): \delta(\mathrm{ppm})=1.65\left(\mathrm{p}, J=7.0 \mathrm{~Hz}, 2 \mathrm{H}, 4{ }^{\prime}{ }^{-}-\mathrm{H}_{2}\right), 1.90(\mathrm{t}, J=2.7 \mathrm{~Hz}, 1 \mathrm{H}$, 7' '-H), 2.19 (td, $J=7.1,2.7 \mathrm{~Hz}, 2 \mathrm{H}, 5$ ' '- $\left.\mathrm{H}_{2}\right), 2.45$ (t, $J=6.9 \mathrm{~Hz}, 2 \mathrm{H}, 3$ ''- $\left.\mathrm{H}_{2}\right), 6.95-7.04$ (m, $3 \mathrm{H}, 3-\mathrm{H}$, 2'-H, 6'-H), 7.09 (td, $J=7.6,1.2 \mathrm{~Hz}, 1 \mathrm{H}, 5-\mathrm{H}), 7.26$ (ddd, $J=8.4,7.4,1.7 \mathrm{~Hz}, 1 \mathrm{H}, 4-\mathrm{H}), 7.31$ (t, $J=$ $7.4 \mathrm{~Hz}, 1 \mathrm{H}, 4$ '”'-H), 7.41 (t, $J=7.7 \mathrm{~Hz}, 2 \mathrm{H}, 3$ '"'-H, 5','-H), $7.46(\mathrm{dd}, J=7.7,1.7 \mathrm{~Hz}, 1 \mathrm{H}, 6-\mathrm{H})$, 7.50-7.59 (m, 4 H, 3'-H, 5'-H, 2','-H, 6','-H).

${ }^{13}$ C-NMR (126 MHz, $\left.\mathrm{CDCl}_{3}\right): \delta(\mathrm{ppm})=17.5$ (C-5'), 18.7 (C-3'), 27.5 (C-4'”), 68.7 (C-7''), 76.8 (C-6”), 83.6 (C-2'”), 94.4 (C-1'), 116.6 (C-1), 117.9 (C-2', C-6'), 119.9 (C-3), 123.7 (C-5), 126.8 (C-4"', C-2'”, C-6"'), 128.2 (C-3', C-5'), 128.6 (C-3'”, C-5'”'), 129.0 (C-4), 133.6 (C-6), 135.7 (C-4'), 140.5 (C-1'’'), 156.8, 157.1 (C-2, C-1').

MS (ESI): $m / z(\%)=337.2(58)[\mathrm{M}+\mathrm{H}]^{+}, 359.1(79)[\mathrm{M}+\mathrm{Na}]^{+}$.

HRMS (ESI): $m / z=$ gef.: 337.1580 , ber.: $337.1587[\mathrm{M}+\mathrm{H}]^{+}$.

$\mathbf{C}_{25} \mathbf{H}_{20} \mathbf{O}$ (336.43). 


\section{4-tert-Butyl-1-(2-nitrophenoxy)benzol $(148 g)^{z}$}<smiles>CC(C)(C)c1ccc(Oc2ccccc2[N+](=O)[O-])cc1</smiles>

Eine Mischung aus 4-(tert-Butyl)phenol (147g) (2.13 g, $14.2 \mathrm{mmol}, 1.00$ Äq.), 2-Fluornitrobenzol (121a) (1.49 mL, $2.00 \mathrm{~g}, 14.2 \mathrm{mmol}, 1.00$ Äq.) und $\mathrm{K}_{2} \mathrm{CO}_{3}$ (3.93 g, $28.4 \mathrm{mmol}, 2.00$ Äq.) in DMSO $(40 \mathrm{~mL})$ wurde $20 \mathrm{~h}$ bei $95^{\circ} \mathrm{C}$ gerührt. Nach Abkühlen auf RT wurde die Mischung in Eiswasser $(200 \mathrm{~mL})$ gegeben und mit MTBE extrahiert $(3 \times 200 \mathrm{~mL})$. Die vereinigten organischen Phasen wurden über $\mathrm{Na}_{2} \mathrm{SO}_{4}$ getrocknet und das Lösungsmittel in vacuo entfernt, um Biarylether 148g als gelben Feststoff ( $3.40 \mathrm{~g}, 12.5 \mathrm{mmol}, 88 \%$ ) zu erhalten.

DC: $R_{f}=0.58(n-$ Pentan/EtOAc, 10:1).

UV/Vis $\left(\mathrm{CH}_{3} \mathrm{CN}\right): \lambda_{\max }(\mathrm{nm})(\lg \varepsilon)=319(3.370)$.

IR (ATR): $\tilde{v}\left(\mathrm{~cm}^{-1}\right)=1595,1525,1506,1477,1409,1348,1242,1170,884,833,546$.

${ }^{1} \mathbf{H}-\mathrm{NMR}\left(600 \mathrm{MHz}, \mathrm{CDCl}_{3}\right): \delta(\mathrm{ppm})=1.31\left(\mathrm{~s}, 9 \mathrm{H}, \mathrm{C}\left(\mathrm{CH}_{3}\right)_{3}\right), 6.92-7.02(\mathrm{~m}, 3 \mathrm{H}, 6-\mathrm{H}, 2$ '- $\mathrm{H}, 6$ '-H), 7.10-7.18 (m, 1 H, 4-H), 7.33-7.41 (m, 2 H, 3'-H, 5'-H), 7.46 (ddd, J=8.3, 7.4, 1.7 Hz, 1 H, 5-H), $7.91(\mathrm{dd}, J=8.3,1.7 \mathrm{~Hz}, 1 \mathrm{H}, 3-\mathrm{H})$.

$\left.\left.{ }^{13} \mathbf{C}-\mathrm{NMR}\left(126 \mathrm{MHz}, \mathrm{CDCl}_{3}\right): \delta(\mathrm{ppm})=31.5\left(\mathrm{C}_{(\underline{\mathrm{C}}}\right)_{3}\right)_{3}\right), 34.5\left(\underline{\mathrm{C}}\left(\mathrm{CH}_{3}\right)_{3}\right), 118.8\left(\mathrm{C}-2^{\prime}, \mathrm{C}^{\prime} 6\right.$ '), 120.0 (C-6), 122.6 (C-4), 125.5 (C-3), 126.8 (C-3', C-5'), 133.9 (C-5), 141.1 (C-2), 147.5 (C-4'), 151.1 (C-1), 153.1 (C-1').

MS (ESI): $m / z(\%)=289.2(64)\left[\mathrm{M}+\mathrm{NH}_{4}\right]^{+}, 565.2(100)[2 \mathrm{M}+\mathrm{Na}]^{+}$.

HRMS (ESI): $m / z=$ gef.: 294.1107, ber.: $294.1101[\mathrm{M}+\mathrm{Na}]^{+}$.

$\mathbf{C}_{16} \mathbf{H}_{17} \mathrm{NO}_{3}$ (271.31).

\footnotetext{
${ }^{\text {z }}$ Für Originalvorschrift siehe: J. C. Lee, J.-H. Choi, J.-S. Lee, Bull. Korean. Chem. Soc. 2004, 25, 1117-1118.
} 


\section{4-tert-Butyl-1-(2-aminophenoxy)benzol (149g $)^{\text {aa }}$}<smiles>CC(C)(C)c1ccc(Oc2ccccc2N)cc1</smiles>

Konz. AcOH $(75 \mathrm{~mL})$ und anschließend konz. $\mathrm{HCl}(75 \mathrm{~mL})$ wurden bei $0{ }^{\circ} \mathrm{C}$ tropfenweise zu einer Lösung von 4-tert-Butyl-1-(2-nitrophenoxy)benzol (148g) (3.97 g, $13.6 \mathrm{mmol}, 1.00$ Äq.) in EtOAc (50 mL) hinzugefügt. Zinkpulver (26.7 g, $408 \mathrm{mmol}, 30.0$ Äq.) wurde portionsweise zugegeben, die Lösung auf RT erwärmt und $17.5 \mathrm{~h}$ bei dieser Temperatur gerührt. Die Reaktionsmischung wurde auf $0{ }^{\circ} \mathrm{C}$ abgekühlt und 33\%ige wässr. $\mathrm{NH}_{3}$-Lösung (300 mL) hinzugetropft. Die Mischung wurde mit $\mathrm{CH}_{2} \mathrm{Cl}_{2}$ extrahiert $(3 \times 300 \mathrm{~mL})$, die vereinigten organischen Phasen über $\mathrm{Na}_{2} \mathrm{SO}_{4}$ getrocknet und filtriert. Entfernung des Lösungsmittels in vacuo lieferte Amin 149g als gelben Feststoff $(2.96 \mathrm{~g}$, $12.3 \mathrm{mmol}, 98 \%)$.

DC $R_{f}=0.43(n-$ Pentan/EtOAc, 10:1).

UV/Vis $\left(\mathrm{CH}_{3} \mathrm{CN}\right): \lambda_{\max }(\mathrm{nm})(\lg \varepsilon)=282(3.561)$.

IR (ATR): $\tilde{v}\left(\mathrm{~cm}^{-1}\right)=1618,1498,1220,1195,831,742,578$.

${ }^{1} \mathrm{H}-\mathrm{NMR}\left(600 \mathrm{MHz}, \mathrm{CDCl}_{3}\right): \delta(\mathrm{ppm})=1.31\left(\mathrm{~s}, 9 \mathrm{H}, \mathrm{C}\left(\mathrm{CH}_{3}\right)_{3}\right), 3.99\left(\mathrm{~s}_{\mathrm{br}}, 2 \mathrm{H}, \mathrm{NH}_{2}\right), 6.71(\mathrm{ddd}$, $J=8.0,7.4,1.6 \mathrm{~Hz}, 1 \mathrm{H}, 3-\mathrm{H}), 6.84(\mathrm{dd}, J=7.9,1.6 \mathrm{~Hz}, 1 \mathrm{H}, 5-\mathrm{H}), 6.85(\mathrm{dd}, J=7.9,1.4 \mathrm{~Hz}, 1 \mathrm{H}$, 4-H), 6.88-6.93 (m, 2 H, 2'-H, 6'-H), 6.95 (ddd, J = 7.9, 7.4, 1.4 Hz, 1 H, 6-H), 7.28-7.35 (m, 2 H, 3'-H, 5'-H).

$\left.\left.{ }^{13} \mathrm{C}-\mathrm{NMR}\left(126 \mathrm{MHz}, \mathrm{CDCl}_{3}\right): \delta(\mathrm{ppm})=31.6\left(\mathrm{C}_{(\underline{\mathrm{C}}}\right)_{3}\right)_{3}\right), 34.3\left(\underline{\mathrm{C}}\left(\mathrm{CH}_{3}\right)_{3}\right), 116.6(\mathrm{C}-5), 116.7(\mathrm{C}-2$ ', C-6'), 119.0 (C-3), 119.8 (C-4), 124.5 (C-6), 126.4 (C-3', C-5'), 137.9 (C-2), 143.6 (C-1), 145.4 (C-4'), 154.8 (C-1').

MS (ESI): $m / z(\%)=242.2(100)[\mathrm{M}+\mathrm{H}]^{+}$.

HRMS (ESI): $m / z=$ gef.: 242.1544, ber.: $242.1539[\mathrm{M}+\mathrm{Na}]^{+}$.

$\mathbf{C}_{16} \mathrm{H}_{19} \mathrm{NO}$ (241.33).

\footnotetext{
${ }^{\text {aa }}$ Für Originalvorschrift siehe: F. Wen, H. Zhang, Z. Yu, H. Jin, Q. Yang, T. Hou, Pestic. Biochem. Physiol.
} 2010, 98, 248-253. 


\section{4-tert-Butyl-1-(2-iod)benzol (102g) ${ }^{\mathrm{bb}}$}<smiles>CC(C)(C)c1ccc(Oc2ccccc2I)cc1</smiles>

Eine Lösung von KI (5.06 g, 30.5 mmol, 2.50 Äq.) und $\mathrm{NaNO}_{2}$ (1.68 g, 24.4 mmol, 2.00 Äq.) in $\mathrm{H}_{2} \mathrm{O}$ $(50 \mathrm{~mL})$ wurde zu einer Lösung von 4-tert-Butyl-1-(2-aminophenoxy)benzol (149g) (2.95 g, $12.2 \mathrm{mmol}, 1.00$ Äq.) und $p$ - $\mathrm{TsOH} \cdot \mathrm{H}_{2} \mathrm{O}\left(6.96 \mathrm{~g}, 36.6 \mathrm{mmol}, 3.00\right.$ Äq.) in $\mathrm{CH}_{3} \mathrm{CN}$ (300 mL) gegeben und $2.5 \mathrm{~h}$ bei RT gerührt. Die Reaktionsmischung wurde mit ges. wässr. $\mathrm{NaHCO}_{3}$-Lösung $(200 \mathrm{~mL})$ versetzt und mit $\mathrm{CH}_{2} \mathrm{Cl}_{2}$ extrahiert $(3 \times 300 \mathrm{~mL})$. Die vereinigten organischen Phasen wurden in vacuo eingeengt, in $\mathrm{CH}_{2} \mathrm{Cl}_{2}$ (200 mL) gelöst, mit ges. wässr. $\mathrm{Na}_{2} \mathrm{~S}_{2} \mathrm{O}_{3}$-Lösung (100 mL) gewaschen, über $\mathrm{Na}_{2} \mathrm{SO}_{4}$ getrocknet, filtriert und das Lösungsmittel in vacuo entfernt. Säulenchromatographische Reinigung ( $\mathrm{SiO}_{2}, n$-Pentan) lieferte Aryliodid 102g als farbloses Öl (2.81 g, 7.99 mmol, 66\%).

DC $R_{f}=0.27(n$-Pentan/EtOAc, 100:1).

UV/Vis $\left(\mathrm{CH}_{3} \mathrm{CN}\right): \lambda_{\max }(\mathrm{nm})(\lg \varepsilon)=275(3.432)$.

IR (ATR): $\tilde{v}\left(\mathrm{~cm}^{-1}\right)=1506,1462,1232,1171,1017,830,750,544$.

${ }^{1} \mathbf{H}-\mathrm{NMR}\left(600 \mathrm{MHz}, \mathrm{CDCl}_{3}\right): \delta(\mathrm{ppm})=1.31\left(\mathrm{~s}, 9 \mathrm{H}, \mathrm{C}\left(\mathrm{CH}_{3}\right)_{3}\right), 6.83(\mathrm{td}, J=7.8,1.4 \mathrm{~Hz}, 1 \mathrm{H}, 4-\mathrm{H})$ 6.86 (dd, $J=8.2,1.4 \mathrm{~Hz}, 1 \mathrm{H}, 6-\mathrm{H}), 6.88-6.93$ (m, 2 H, 2'-H, 6'-H), 7.22-7.27 (m, 1 H, 5-H), 7.317.36 (m, 2 H, 3'-H, 5'-H), 7.83 (dd, J = 7.9, 1.4 Hz, 1 H, 3-H).

$\left.{ }^{13} \mathbf{C}-N M R\left(126 \mathrm{MHz}, \mathrm{CDCl}_{3}\right): \delta(\mathrm{ppm})=31.5\left(\mathrm{C}_{(\mathrm{C}} \mathrm{C}_{3}\right)_{3}\right), 34.4\left(\underline{\mathrm{C}}\left(\mathrm{CH}_{3}\right)_{3}\right), 88.5(\mathrm{C}-2), 118.0(\mathrm{C}-2$ ', C-6'), 118.8 (C-6), 124.9 (C-4), 126.5 (C-3', C-5'), 129.4 (C-5), 139.7 (C-3), 146.3 (C-4'), 154.2 (C-1'), 156.8 (C-1).

MS (EI): $m / z(\%)=337.0(100)\left[\mathrm{M}-\mathrm{CH}_{3}\right]^{+}, 352.0(57)[\mathrm{M}]^{+}$.

HRMS (EI): $m / z=$ gef.: 352.0322 , ber.: $352.0324[\mathrm{M}]^{+}$.

$\mathrm{C}_{16} \mathrm{H}_{17} \mathrm{IO}$ (352.22).

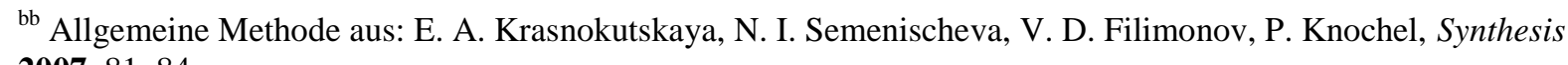
2007, 81-84. 


\section{1-(4-(tert-Butyl)phenoxy)-2-(hepta-1,6-diin-1-yl)benzol (112g)}

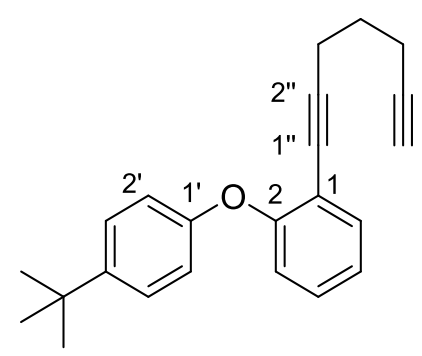

Eine Mischung aus 4-tert-Butyl-1-(2-iod)benzol (102g) (308 mg, $874 \mu \mathrm{mol}, 1.00$ Äq.), 1,6-Heptadiin (150b) (500 $\mu \mathrm{L}, 403$ mg, 4.37 mmol, 5.00 Äq.), $\mathrm{PdCl}_{2}\left(\mathrm{PPh}_{3}\right)_{2}$ (30.7 mg, $43.7 \mu \mathrm{mol}, 0.05$ Äq.) und CuI (10.4 mg, $87.4 \mu \mathrm{mol}, 0.10$ Äq.) in entgastem $\mathrm{NEt}_{3}(8 \mathrm{~mL})$ wurde $18 \mathrm{~h}$ bei RT gerührt. Die Reaktionsmischung wurde durch $\mathrm{SiO}_{2}$ filtriert, mit EtOAc gespült und das Lösungsmittel des Filtrats in vacuo entfernt. Säulenchromatographische Reinigung $\left(\mathrm{SiO}_{2}, n\right.$-Pentan/Toluol, 5:1) lieferte Dialkin 112g als gelbes Öl (162 mg, $512 \mu \mathrm{mol}, 59 \%)$.

DC: $R_{f}=0.34(n-$ Pentan/Toluol, 5:1).

UV/Vis $\left(\mathrm{CH}_{3} \mathrm{CN}\right): \lambda_{\max }(\mathrm{nm})(\lg \varepsilon)=205$ (4.589), 251 (4.252), 287 (3.525).

IR (ATR): $\tilde{v}\left(\mathrm{~cm}^{-1}\right)=2957,1507,1485,1444,1253,1227,1172,1105,875,831,755,635,546$.

${ }^{1} \mathbf{H}-\mathrm{NMR}\left(600 \mathrm{MHz}, \mathrm{CDCl}_{3}\right): \delta(\mathrm{ppm})=1.30\left(\mathrm{~s}, 9 \mathrm{H}, \mathrm{C}\left(\mathrm{CH}_{3}\right)_{3}\right), 1.65\left(\mathrm{p}, J=7.1 \mathrm{~Hz}, 2 \mathrm{H}, 4\right.$ ' '- $\left.\mathrm{H}_{2}\right), 1.92$ (t, $J=2.7 \mathrm{~Hz}, 1 \mathrm{H}, 7^{\prime}$ '-H), 2.19 (td, $J=7.1,2.6 \mathrm{~Hz}, 2 \mathrm{H}, 5^{\prime}$ '- $\left.-\mathrm{H}_{2}\right), 2.45$ (t, $J=6.9 \mathrm{~Hz}, 2 \mathrm{H}, 3$ ' '- $\mathrm{H}_{2}$ ), 6.88 (d, $\left.J=8.8 \mathrm{~Hz}, 2 \mathrm{H}, 2^{\prime}-\mathrm{H}, 6{ }^{\prime}-\mathrm{H}\right), 6.90(\mathrm{~d}, J=8.8 \mathrm{~Hz}, 1 \mathrm{H}, 3-\mathrm{H}), 7.03(\mathrm{td}, J=7.6,1.1 \mathrm{~Hz}, 1 \mathrm{H}, 5-\mathrm{H})$, 7.21 (ddd, $J=8.4,7.5,1.7 \mathrm{~Hz}, 1 \mathrm{H}, 4-\mathrm{H}), 7.31$ (d, $\left.J=8.7 \mathrm{~Hz}, 2 \mathrm{H}, 3^{\prime}-\mathrm{H}, 5^{\prime}-\mathrm{H}\right), 7.42$ (dd, $J=7.6$, $1.6 \mathrm{~Hz}, 1 \mathrm{H}, 6-\mathrm{H})$.

${ }^{13}$ C-NMR $\left(126 \mathrm{MHz}, \mathrm{CDCl}_{3}\right): \delta(\mathrm{ppm})=17.5\left(\mathrm{C}-5\right.$ '”), 18.8 (C-3"'), 27.5 (C-4"'), $31.6\left(\mathrm{C}\left(\mathrm{CH}_{3}\right)_{3}\right), 34.3$ ( $\left.\underline{\mathrm{C}}\left(\mathrm{CH}_{3}\right)_{3}\right), 68.7$ (C-7' '), 76.9 (C-6'), 83.7 (C-2'”), 94.0 (C-1')), 116.2 (C-1), 117.5 (C-2', C-6'), 119.2 (C-3), 123.2 (C-5), 126.3 (C-3', C-5'), 128.9 (C-4), 133.5 (C-6), 145.6 (C-4'), 155.0 (C-1'), 157.4 (C-2).

MS (ESI): $m / z(\%)=317.2(38)[\mathrm{M}+\mathrm{H}]^{+}, 339.2(100)[\mathrm{M}+\mathrm{Na}]^{+}$.

HRMS (ESI): $m / z=$ gef.: 317.1896 , ber.: $317.1900[\mathrm{M}+\mathrm{H}]^{+}$.

$\mathbf{C}_{23} \mathbf{H}_{24} \mathbf{O}$ (316.44). 


\section{1-(Hexa-1,5-diin-1-yl)-2-phenoxybenzol (112h)}

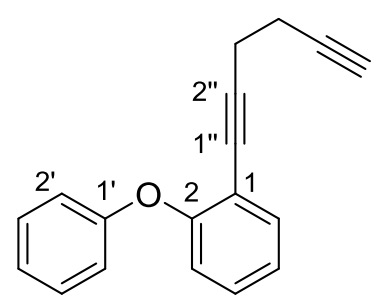

Eine Mischung aus 1-Iod-2-phenoxybenzol (102a) (200 mg, 675 mol, 1.00 Äq.), 1,5-Hexadiin (150a) (50\% in $n$-Pentan, $656 \mu \mathrm{L}, 3.38 \mathrm{mmol}, 5.00$ Äq.), $\mathrm{PdCl}_{2}\left(\mathrm{PPh}_{3}\right)_{2}$ (23.7 mg, $33.8 \mu \mathrm{mol}, 0.05$ Äq.) und $\mathrm{CuI}\left(8.06 \mathrm{mg}, 6.75 \mu \mathrm{mol}, 0.10 \mathrm{Äq}\right.$.) in entgastem $\mathrm{NEt}_{3}(6 \mathrm{~mL})$ wurde $19 \mathrm{~h}$ bei RT gerührt. Die Reaktionsmischung wurde durch $\mathrm{SiO}_{2}$ filtriert, mit EtOAc gespült und das Lösungsmittel des Filtrats in vacuo entfernt. Säulenchromatographische Reinigung $\left(\mathrm{SiO}_{2}, n\right.$-Pentan/Toluol, 5:1) lieferte Dialkin 112h als gelbes Öl (102 mg, $414 \mu \mathrm{mol}, 61 \%)$.

DC: $R_{f}=0.37(n-$ Pentan/Toluol, 5:1).

UV/Vis $\left(\mathrm{CH}_{3} \mathrm{CN}\right): \lambda_{\max }(\mathrm{nm})(\lg \varepsilon)=195$ (4.754), 240 (4.234), 252 (4.223), 271 (3.324), 279 (3.362), 289 (3.408), 297 (3.308).

IR (ATR): $\tilde{v}\left(\mathrm{~cm}^{-1}\right)=3291,1589,1572,1482,1443,1249,1221,1162,1105,1072,1022,883,870$, $854,798,749,689,635$.

${ }^{1} \mathbf{H}-\mathrm{NMR}\left(600 \mathrm{MHz}, \mathrm{CDCl}_{3}\right): \delta(\mathrm{ppm})=1.95\left(\mathrm{t}, J=2.6 \mathrm{~Hz}, 1 \mathrm{H}, 6\right.$ 6'-H), 2.29-2.35 (m, $2 \mathrm{H}, 3$ ''- $\left.\mathrm{H}_{2}\right)$, 2.54 (dd, $J=8.4,6.7 \mathrm{~Hz}, 2 \mathrm{H}, 4$ ' '- $\left.\mathrm{H}_{2}\right), 6.91$ (dd, $\left.J=8.2,1.0 \mathrm{~Hz}, 1 \mathrm{H}, 3-\mathrm{H}\right), 6.96$ (dd, $J=8.7,1.0 \mathrm{~Hz}$, 2 H, 2'-H, 6'-H), 7.02-7.08 (m, 2 H, 5-H, 4'-H), 7.20-7.25 (m, 1 H, 4-H), 7.30 (dd, J = 8.6, 7.4 Hz, 2 H, 3'-H, 5-'H), 7.44 (dd, $J=7.7,1.7 \mathrm{~Hz}, 1 \mathrm{H}, 6-\mathrm{H})$.

${ }^{13}$ C-NMR (126 MHz, $\left.\mathrm{CDCl}_{3}\right): \delta(\mathrm{ppm})=18.6$ (C-4"'), 19.7 (C-3’'), 69.2 (C-6’'), 77.1 (C-5’'), 82.7 (C-2’'), 93.1 (C-1'”), 116.2 (C-1), 118.1 (C-2', C-6'), 119.5 (C-3), 122.8, 123.5 (C-5, C-4'), 129.2 (C-4), 129.5 (C-3', C-5'), 133.8 (C-6), 157.3 (C-1'), 157.5 (C-2).

MS (ESI): $m / z(\%)=247.1(100)[\mathrm{M}+\mathrm{H}]^{+}, 269.1(47)[\mathrm{M}+\mathrm{Na}]^{+}$.

HRMS (ESI): $m / z=$ gef.: 247.1118 , ber.: $247.1117[\mathrm{M}+\mathrm{H}]^{+}$.

$\mathbf{C}_{18} \mathbf{H}_{14} \mathbf{O}(246.30)$. 


\section{1-(Octa-1,7-diin-1-yl)-2-phenoxybenzol (112i)}

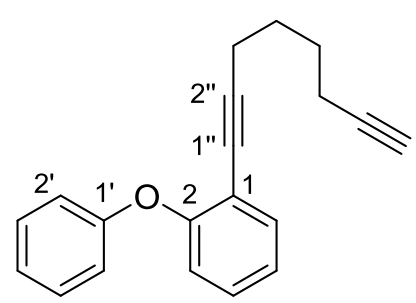

Eine Mischung aus 1-Iod-2-phenoxybenzol (102a) (1.00 g, 3.38 mmol, 1.00 Äq.), 1,7-Octadiin (150c) (1.78 mL, $1.42 \mathrm{~g}, 13.4 \mathrm{mmol}, 3.96$ Äq.), $\mathrm{PdCl}_{2}\left(\mathrm{PPh}_{3}\right)_{2}$ (94.1 mg, $134 \mu \mathrm{mol}, 0.04$ Äq.) und CuI (31.9 mg, $134 \mu \mathrm{mol}, 0.06$ Äq.) in entgastem $\mathrm{NEt}_{3}(20 \mathrm{~mL})$ wurde $16 \mathrm{~h}$ bei RT gerührt. Die Reaktionsmischung wurde durch $\mathrm{SiO}_{2}$ filtriert, mit EtOAc gespült und das Lösungsmittel des Filtrats in vacuo entfernt. Säulenchromatographische Reinigung $\left(\mathrm{SiO}_{2}, n\right.$-Pentan/Toluol, 5:1) lieferte Dialkin 112i als gelbes Öl (629 mg, 2.29 mmol, 68\%) in verunreinigter Form.

DC: $R_{f}=0.21(n-$ Pentan/Toluol, 5:1).

UV/Vis $\left(\mathrm{CH}_{3} \mathrm{CN}\right): \lambda_{\max }(\mathrm{nm})(\lg \varepsilon)=205$ (4.514), 241 (4.202), 252 (4.200), 271 (3.337), 279 (3.359), $288(3.371)$.

IR (ATR): $\tilde{v}\left(\mathrm{~cm}^{-1}\right)=3293,2942,1589,1572,1482,1444,1251,1221,1162,1105,871,798,749$, 689,631 .

${ }^{1} \mathbf{H}-\mathrm{NMR}\left(600 \mathrm{MHz}, \mathrm{CDCl}_{3}\right): \delta(\mathrm{ppm})=1.49-1.59\left(\mathrm{~m}, 4 \mathrm{H}, 4\right.$ ' $-\mathrm{H}_{2}, 5$ ' $\left.-\mathrm{H}_{2}\right), 1.91(\mathrm{t}, J=2.7 \mathrm{~Hz}, 1 \mathrm{H}$,

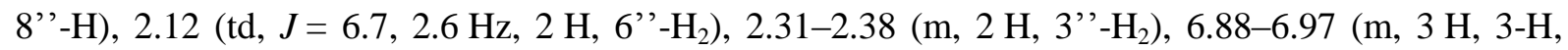
2'-H, 6'-H), 7.01-7.11 (m, 2 H, 5-H, 4'-H), 7.22 (ddd, $J=8.3,7.4,1.7$ Hz, 1 H, 4-H), 7.29 (dd, $J=$ 8.7, 7.4 Hz, 2 H, 3'-H, 5'-H), 7.43 (dd, $J=7.7,1.7$ Hz, 1 H, 6-H).

${ }^{13}$ C-NMR (126 MHz, CDCl $): \delta(\mathrm{ppm})=18.0$ (C-6'), 19.1 (C-3'”), 27.3, 27.4 (C-4', C-5'), 68.4 (C-8'), 76.5 (C-7'”), 84.2 (C-2'), 94.9 (C-1'’), 116.8 (C-1), 117.7 (C-2', C-6'), 119.7 (C-3), 122.6, 123.6 (C-5, C-4'), 128.9 (C-4), 129.4 (C-3', C-5'), 133.5 (C-6), 156.9 (C-1'), 157.5 (C-2).

MS (ESI): $m / z(\%)=275.1(100)[\mathrm{M}+\mathrm{H}]^{+}, 297.1(59)[\mathrm{M}+\mathrm{Na}]^{+}$.

HRMS (ESI): $m / z=$ gef.: 275.1435 , ber.: $275.1430[\mathrm{M}+\mathrm{H}]^{+}$.

$\mathbf{C}_{20} \mathrm{H}_{18} \mathrm{O}(274.36)$. 


\section{1-(Nona-1,8-diin-1-yl)-2-phenoxybenzol (112j)}

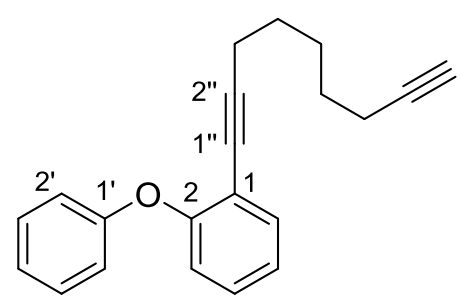

Eine Mischung aus 1-Iod-2-phenoxybenzol (102a) (1.00 g, 3.38 mmol, 1.00 Äq.), 1,8-Nonadiin (2.02 mL, $1.61 \mathrm{~g}, 13.4 \mathrm{mmol}, 3.96$ Äq.), $\mathrm{PdCl}_{2}\left(\mathrm{PPh}_{3}\right)_{2}$ (94.1 mg, $134 \mu \mathrm{mol}, 0.04$ Äq.) und CuI (31.9 mg, $134 \mu \mathrm{mol}, 0.06$ Äq.) in entgastem $\mathrm{NEt}_{3}(20 \mathrm{~mL})$ wurde $16 \mathrm{~h}$ bei RT gerührt. Die Reaktionsmischung wurde durch $\mathrm{SiO}_{2}$ filtriert, mit EtOAc gespült und das Lösungsmittel des Filtrats in vacuo entfernt. Säulenchromatographische Reinigung $\left(\mathrm{SiO}_{2}, n\right.$-Pentan/Toluol, 5:1) lieferte Dialkin 112j als gelbes Öl (649 mg, $2.25 \mathrm{mmol}, 67 \%$ ).

DC: $R_{f}=0.31(n-$ Pentan/Toluol, 5:1).

UV/Vis $\left(\mathrm{CH}_{3} \mathrm{CN}\right): \lambda_{\max }(\mathrm{nm})(\lg \varepsilon)=204$ (4.486), 241 (4.170), 252 (4.276), 271 (3.332), 279 (3.347), $288(3.351)$.

IR $\left(\right.$ ATR): $\tilde{v}\left(\mathrm{~cm}^{-1}\right)=3293,2937,1589,1482,1444,1251,1222,1162,1105,868,798,750,689,630$.

${ }^{1} \mathbf{H}-\mathrm{NMR}\left(600 \mathrm{MHz}, \mathrm{CDCl}_{3}\right): \delta(\mathrm{ppm})=1.39-1.52\left(\mathrm{~m}, 6 \mathrm{H}, 4\right.$ '”- $\mathrm{H}_{2}, 5$ '”- $\mathrm{H}_{2}, 6$ '”- $\left.\mathrm{H}_{2}\right), 1.92$ (t, $J=$ $2.6 \mathrm{~Hz}, 1 \mathrm{H}, 9$ '’-H), 2.12 (td, $J=7.0,2.7 \mathrm{~Hz}, 2 \mathrm{H}, 7$ ' '- $\mathrm{H}_{2}$ ), 2.33 (t, $J=6.6 \mathrm{~Hz}, 2 \mathrm{H}, 3$ '’- $\mathrm{H}_{2}$ ), 6.92 (dd, $J=8.2,1.1 \mathrm{~Hz}, 1 \mathrm{H}, 3-\mathrm{H}), 6.94-6.97$ (m, 2 H, 2'-H, 6'-H), 7.06 (tt, $\left.J=7.4,1.1 \mathrm{~Hz}, 2 \mathrm{H}, 5-\mathrm{H}, 4^{\prime}-\mathrm{H}\right)$, 7.22 (ddd, $J=8.1,7.4,1.7 \mathrm{~Hz}, 1 \mathrm{H}, 4-\mathrm{H}), 7.27-7.33$ (m, $\left.2 \mathrm{H}, 3^{\prime}-\mathrm{H}, 5^{\prime}-\mathrm{H}\right), 7.44$ (dd, $J=7.7,1.7 \mathrm{~Hz}$, $1 \mathrm{H}, 6-\mathrm{H})$.

${ }^{13}$ C-NMR (126 MHz, CDCl $): \delta(\mathrm{ppm})=18.3$ (C-7' '), 19.5 (C-3'”), 27.9, 28.0, 28.1 (C-4'”, C-5'”, C-6”), 68.2 (C-9'), 76.3 (C-8'”), 84.5 (C-2"'), 95.3 (C-1'”), 116.8 (C-1), 117.8 (C-2', C-6'), 119.6 (C-3), 122.6, 123.5 (C-5, C-4'), 128.9 (C-4), 129.4 (C-3', C-5'), 133.5 (C-6), 156.9 (C-1'), 157.5 (C-2).

MS (ESI): $m / z(\%)=289.2(94)[\mathrm{M}+\mathrm{H}]^{+}, 311.1(100)[\mathrm{M}+\mathrm{Na}]^{+}$.

HRMS (ESI): $m / z=$ gef.: 289.1590 , ber.: $289.1587[\mathrm{M}+\mathrm{H}]^{+}$.

$\mathbf{C}_{21} \mathbf{H}_{20} \mathbf{O}(288.38)$. 


\subsubsection{Synthese der Dialkine 151b-k durch die Sonogashira-Reaktion}

\section{Allgemeine Vorschrift für die Synthese der Dialkine 151b-k (Sonogashira-Reaktion):}

Eine Mischung aus Aryliodid 111 (1.00 Äq.), Dialkin 112 (1.10 Äq.), $\mathrm{PdCl}_{2}\left(\mathrm{PPh}_{3}\right)_{2}$ (0.05 Äq.) und $\mathrm{CuI}(0.10$ Äq. $)$ in entgastem $\mathrm{NEt}_{3}(c=0.04 \mathrm{~mol} / \mathrm{L})$ wurde $18-20.5 \mathrm{~h}$ bei $60{ }^{\circ} \mathrm{C}$ gerührt. Nach Abkühlen auf RT wurde die Reaktionsmischung durch $\mathrm{SiO}_{2}$ filtriert, mit EtOAc gespült, das Lösungsmittel des Filtrats in vacuo entfernt und der Rückstand säulenchromatographisch gereinigt, um Dialkin 151 als gelbes Öl zu erhalten.

Tabelle 12: Synthese der Sonogashira-Produkte 151b-k.

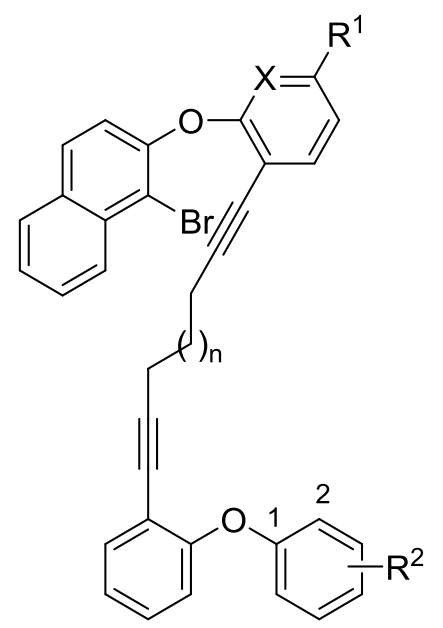

\begin{tabular}{ccccccccc}
\hline Eintrag & Substrate & $\mathbf{R}^{\mathbf{1}}$ & $\mathbf{R}^{\mathbf{2}}$ & $\mathbf{X}$ & $\mathbf{n}$ & Produkt & Ausbeute [mg] & Ausbeute [\%] \\
\hline $1^{[\mathrm{a}, \mathrm{c}]}$ & $\mathbf{1 1 1 c}, \mathbf{1 1 2 a}$ & $\mathrm{H}$ & $\mathrm{H}$ & $\mathrm{N}$ & 1 & $\mathbf{1 5 1 b}$ & 151 & 79 \\
$2^{[\mathrm{a}, \mathrm{b}]}$ & $\mathbf{1 1 1 b}, \mathbf{1 1 2 a}$ & $\mathrm{OMe}$ & $\mathrm{H}$ & $\mathrm{CH}$ & 1 & $\mathbf{1 5 1 c}$ & 342 & 67 \\
3 & $\mathbf{1 1 1 a}, \mathbf{1 1 2 b}$ & $\mathrm{H}$ & $2-\mathrm{F}$ & $\mathrm{CH}$ & 1 & $\mathbf{1 5 1 d}$ & 95.2 & 77 \\
4 & $\mathbf{1 1 1 a}, \mathbf{1 1 2 c}$ & $\mathrm{H}$ & $2-\mathrm{Me}$ & $\mathrm{CH}$ & 1 & $\mathbf{1 5 1 e}$ & 81.4 & 66 \\
5 & $\mathbf{1 1 1 a}, \mathbf{1 1 2 d}$ & $\mathrm{H}$ & $2-\mathrm{CF}_{3}$ & $\mathrm{CH}$ & 1 & $\mathbf{1 5 1 f}$ & 93.5 & 69 \\
6 & $\mathbf{1 1 1 a}, \mathbf{1 1 2 e}$ & $\mathrm{H}$ & $2-\mathrm{OMe}$ & $\mathrm{CH}$ & 1 & $\mathbf{1 5 1 g}$ & 89.2 & 71 \\
$7^{[\mathrm{d}]}$ & $\mathbf{1 1 1 a}, \mathbf{1 1 2 f}$ & $\mathrm{H}$ & $4-\mathrm{Ph}$ & $\mathrm{CH}$ & 1 & $\mathbf{1 5 1 h}$ & 57.0 & 96 \\
$8^{[\mathrm{d}, \mathrm{e}]}$ & $\mathbf{1 1 1 a}, \mathbf{1 1 2 g}$ & $\mathrm{H}$ & $4-\mathrm{Bu}$ & $\mathrm{CH}$ & 1 & $\mathbf{1 5 1 i}$ & 31.9 & 90 \\
$9^{[\mathrm{c}, \mathrm{e}]}$ & $\mathbf{1 1 1 a}, \mathbf{1 1 2 h}$ & $\mathrm{H}$ & $\mathrm{H}$ & $\mathrm{CH}$ & 0 & $\mathbf{1 5 1 j}$ & 132 & 76 \\
$10^{[\mathrm{c}]}$ & $\mathbf{1 1 1 a}, \mathbf{1 1 2 i}$ & $\mathrm{H}$ & $\mathrm{H}$ & $\mathrm{CH}$ & 2 & $\mathbf{1 5 1 k}$ & 153 & 85 \\
\hline
\end{tabular}

[a] 112 (1.25 Äq.); [b] $c=0.1 \mathrm{~mol} / \mathrm{L}$; [c] $c=0.05 \mathrm{~mol} / \mathrm{L}$; [d] $c=0.02 \mathrm{~mol} / \mathrm{L}$; [e] Reinheit laut ${ }^{1} \mathrm{H}-\mathrm{NMR}$ ca. $90 \%$. 


\section{2-((1-Bromnaphthalin-2-yl)oxy)-3-(7-(2-phenoxyphenyl)hepta-1,6-diin-1-yl)pyridin (151b)}

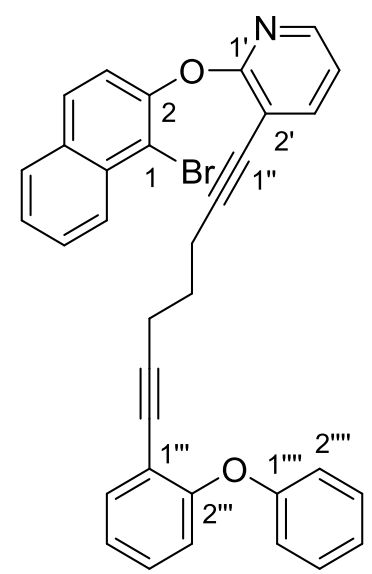

DC: $R_{f}=0.24\left(n-\mathrm{Pentan} / \mathrm{CH}_{2} \mathrm{Cl}_{2}, 2: 1\right)$.

UV/Vis $\left(\mathrm{CH}_{3} \mathrm{CN}\right): \lambda_{\max }(\mathrm{nm})(\lg \varepsilon)=208(4.781), 226$ (4.936), 287 (4.216).

IR (ATR): $\tilde{v}\left(\mathrm{~cm}^{-1}\right)=1567,1483,1414,1248,1224,797,751,690$.

${ }^{1} \mathbf{H}-\mathbf{N M R}\left(600 \mathrm{MHz}, \mathrm{CDCl}_{3}\right): \delta(\mathrm{ppm})=1.75\left(\mathrm{p}, J=7.0 \mathrm{~Hz}, 2 \mathrm{H}, 4{ }^{\prime}{ }^{-} \mathrm{H}_{2}\right), 2.48(\mathrm{t}, J=7.0 \mathrm{~Hz}, 2 \mathrm{H}$,

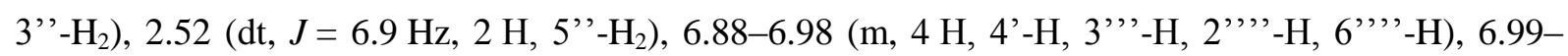
7.08 (m, 2 H, 5','-H, 4",' '-H), 7.21 (ddd, $\left.J=8.1,7.5,1.7 \mathrm{~Hz}, 1 \mathrm{H}, 4{ }^{\prime \prime}{ }^{-}-\mathrm{H}\right), 7.28$ (dd, $J=8.6,7.3 \mathrm{~Hz}$, 2 H, 3" "'-H, 5" "'-H), 7.35 (d, $J=8.8 \mathrm{~Hz}, 1 \mathrm{H}, 3-\mathrm{H}), 7.42$ (dd, $J=7.7,1.6 \mathrm{~Hz}, 1 \mathrm{H}, 6$ " ' -H), 7.48 (ddd, $J=8.0,6.9,1.1 \mathrm{~Hz}, 1 \mathrm{H}, 6-\mathrm{H}), 7.54-7.63(\mathrm{~m}, 1 \mathrm{H}, 7-\mathrm{H}), 7.75\left(\mathrm{dd}, J=7.5,1.9 \mathrm{~Hz}, 1 \mathrm{H}, 3^{\prime}-\mathrm{H}\right), 7.84$ (dd, $J=8.6,1.9 \mathrm{~Hz}, 2 \mathrm{H}, 4-\mathrm{H}, 5-\mathrm{H}), 7.95\left(\mathrm{dd}, J=4.9,1.9 \mathrm{~Hz}, 1 \mathrm{H}, 5^{\prime}-\mathrm{H}\right), 8.26(\mathrm{~d}, J=8.5 \mathrm{~Hz}, 1 \mathrm{H}$, $8-\mathrm{H})$.

${ }^{13}$ C-NMR (126 MHz, $\left.\mathrm{CDCl}_{3}\right): \delta(\mathrm{ppm})=18.7$ (C-3"', C-5"'), 27.5 (C-4”'), 75.1 (C-2"'), 76.7 (C-6"'), 94.4 (C-7’'), 96.1 (C-1’'), 108.9 (C-2'), 115.0 (C-1), 116.6 (C-1'’'), 117.7 (C-2',', C-6”','), 118.1 (C-4'), 119.7, 122.6, 122.7 (C-3, C-3"', C-5'”/C-4",'), 123.5 (C-5'”/C-4','), 125.8 (C-6), 126.9 (C-8), 127.4 (C-7), 128.1, 128.5 (C-4, C-5), 128.9 (C-4'”'), 129.5 (C-3',', C-5',',), 132.0 (C-4a), 133.0 (C-8a), 133.6 (C-6'”), 142.3 (C-3'), 145.6 (C-5'), 149.1 (C-2), 156.9 (C-2','), 157.5 (C-1',''), $162.6\left(\mathrm{C}-1^{\prime}\right)$.

MS (ESI): $m / z(\%)=560.1(64)\left[\mathrm{M}\left({ }^{81} \mathrm{Br}\right)+\mathrm{H}\right]^{+}$.

HRMS (ESI): $m / z=$ gef.: $558.1066\left[\mathrm{M}\left({ }^{79} \mathrm{Br}\right)+\mathrm{H}\right]^{+}$, ber.: $558.1063\left[\mathrm{M}\left({ }^{79} \mathrm{Br}\right)+\mathrm{H}\right]^{+}$; gef.: 560.1050 $\left[\mathrm{M}\left({ }^{81} \mathrm{Br}\right)+\mathrm{H}\right]^{+}$, ber.: $560.1045\left[\mathrm{M}\left({ }^{81} \mathrm{Br}\right)+\mathrm{H}\right]^{+}$.

$\mathrm{C}_{34} \mathrm{H}_{24} \mathrm{BrNO}_{2}$ (558.46). 


\section{1-Brom-2-(5-methoxy-2-(7-(2-phenoxyphenyl)hepta-1,6-diin-1-yl)phenoxy)naphthalin (151c)}

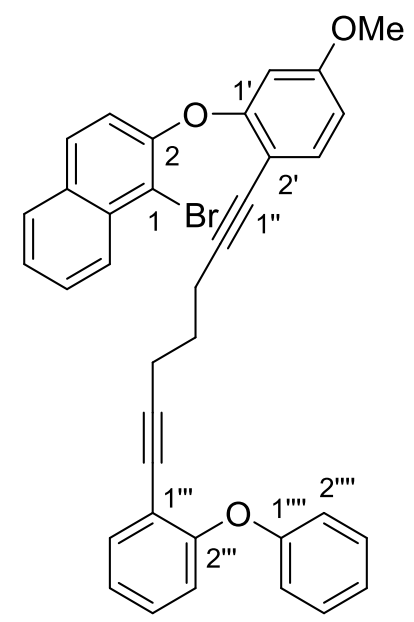

DC: $R_{f}=0.33(n-$ Pentan/EtOAc, 50:1).

UV/Vis $\left(\mathrm{CH}_{3} \mathrm{CN}\right): \lambda_{\max }(\mathrm{nm})(\lg \varepsilon)=194$ (5.122), 232 (4.923), 283 (4.074), 318 (3.227), 332 (3.221), 402 (1.991), 502 (1.451).

IR (ATR): $\tilde{v}\left(\mathrm{~cm}^{-1}\right)=1735,1609,1594,1562,1498,1482,1459,1441,1425,1371,1354,1329,1310$, 1289, 1272, 1252, 1221, 1191, 1118, 1103, 1072, 1035, 993, 958, 801, 749, 689, 671, 644, 527.

${ }^{1} \mathbf{H}-\mathbf{N M R}\left(600 \mathrm{MHz}, \mathrm{CDCl}_{3}\right): \delta(\mathrm{ppm})=1.44\left(\mathrm{p}, J=7.0 \mathrm{~Hz}, 2 \mathrm{H}, 4\right.$ '' $\left.-\mathrm{H}_{2}\right), 2.22(\mathrm{td}, J=6.9,4.7 \mathrm{~Hz}$, $4 \mathrm{H}, 3^{\prime}$ '- $-\mathrm{H}_{2}, 5$ ' '- $\mathrm{H}_{2}$ ), 3.71 (s, $\left.3 \mathrm{H}, \mathrm{OMe}\right), 6.42$ (d, $J=2.4 \mathrm{~Hz}, 1 \mathrm{H}, 6$ ' $\left.-\mathrm{H}\right), 6.62$ (dd, $J=8.6,2.5 \mathrm{~Hz}, 1 \mathrm{H}$, 4'-H), 6.90 (dd, $J=8.0,2.0 \mathrm{~Hz}, 3 \mathrm{H}, 3$ '"'-H, 3'"''-H, 5',' '-H), 7.02 (dt, $J=11.8,7.5 \mathrm{~Hz}, 2 \mathrm{H}, 5$ '"'-H, 4,",'-H), $7.06(\mathrm{~d}, J=8.9 \mathrm{~Hz}, 1 \mathrm{H}, 3-\mathrm{H}), 7.20(\mathrm{td}, J=7.8,1.7 \mathrm{~Hz}, 1 \mathrm{H}, 4$,' $-\mathrm{H}), 7.25$ (dd, $J=8.6$, $7.3 \mathrm{~Hz}, 2 \mathrm{H}, 2$ '" '-H, 6" '”-H), 7.33-7.40 (m, 2 H, 3'-H, 6'"'-H), 7.43 (td, J = 7.3, $1.1 \mathrm{~Hz}, 1 \mathrm{H}, 6-\mathrm{H})$, 7.56 (ddd, $J=8.4,6.9,1.2 \mathrm{~Hz}, 1 \mathrm{H}, 7-\mathrm{H}), 7.72(\mathrm{~d}, J=8.9 \mathrm{~Hz}, 1 \mathrm{H}, 4-\mathrm{H}), 7.78(\mathrm{~d}, J=8.1 \mathrm{~Hz}, 1 \mathrm{H}$, $5-\mathrm{H}), 8.26(\mathrm{~d}, J=8.5 \mathrm{~Hz}, 1 \mathrm{H}, 8-\mathrm{H})$.

${ }^{13}$ C-NMR (126 MHz, CDCl 3 ): $\delta(\mathrm{ppm})=18.6$ (C-3'”, C-5'), 27.5 (C-4'”), 55.5 (OMe), 76.0 (C-1'’), 76.5 (C-7’'), 93.3, 94.7 (C-2’', C-6’'), 105.1 (C-6'), 108.4 (C-2'), 109.4 (C-4'), 112.0 (C-1), 116.8

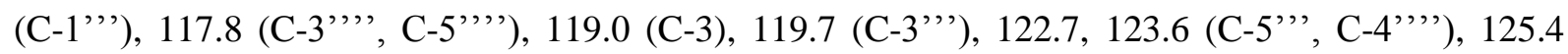
(C-6), 126.6 (C-8), 127.7 (C-7), 128.1 (C-4), 128.9 (C-5, C-4"”), 129.5 (C-2",', C-6”,'), 131.1 (C-4a), 133.1 (C-8a), 133.7, 134.4 (C-3', C-6'”), 151.6 (C-2), 157.0 (C-2'”'), 157.6 (C-1','’), 158.1 (C-1'), 160.3 (C-5').

MS (ESI): $m / z(\%)=611.1(100)[\mathrm{M}+\mathrm{Na}]^{+}$.

HRMS (ESI): $m / z=$ gef.: 587.1217 , ber.: $587.1216\left[\mathrm{M}\left({ }^{79} \mathrm{Br}\right)+\mathrm{H}\right]^{+}$; gef.: 589.1207 , ber.: 589.1198 $\left[\mathrm{M}\left({ }^{81} \mathrm{Br}\right)+\mathrm{H}\right]^{+}$.

$\mathrm{C}_{36} \mathrm{H}_{27} \mathrm{BrO}_{3}$ (587.50). 


\section{1-Brom-2-(2-(7-(2-(2-fluorphenoxy)phenyl)hepta-1,6-diin-1-yl)phenoxy)naphthalin (151d)}

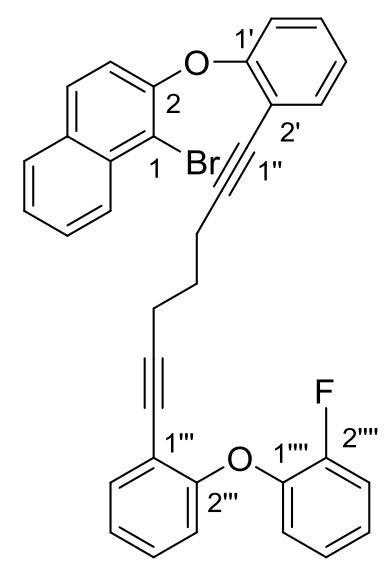

DC: $R_{f}=0.26\left(n-\mathrm{Pentan} / \mathrm{CH}_{2} \mathrm{Cl}_{2}, 5: 1\right)$.

UV/Vis $\left(\mathrm{CH}_{3} \mathrm{CN}\right): \lambda_{\max }(\mathrm{nm})(\lg \varepsilon)=206$ (4.835), 230 (4.929), 253 (4.604), 285 (4.071), 333 (3.210).

IR (ATR): $\tilde{v}\left(\mathrm{~cm}^{-1}\right)=1594,1572,1498,1484,1457,1443,1427,1371,1354,1328,1255,1230,1201$, 1156, 1106, 1034, 879, 864, 826, 808, 794, 776, 744, 642, 527.

${ }^{1} \mathbf{H}-\mathbf{N M R}\left(300 \mathrm{MHz}, \mathrm{CDCl}_{3}\right): \delta(\mathrm{ppm})=1.52\left(\mathrm{p}, J=7.0 \mathrm{~Hz}, 2 \mathrm{H}, 4{ }^{\prime \prime}-\mathrm{H}_{2}\right), 2.26(\mathrm{dt}, J=7.0 \mathrm{~Hz}, 2 \mathrm{H})$, $2.30(\mathrm{t}, J=7.0 \mathrm{~Hz}, 2 \mathrm{H})\left(3\right.$ ' '- $\mathrm{H}_{2}, 5$ ' '- $\left.\mathrm{H}_{2}\right), 6.79-6.92(\mathrm{~m}, 3 \mathrm{H}), 6.95-7.23(\mathrm{~m}, 8 \mathrm{H}, 3-\mathrm{H}), 7.33-7.47$ (m, $3 \mathrm{H}, 6-\mathrm{H}), 7.57$ (ddd, $J=8.4,6.9,1.3 \mathrm{~Hz}, 1 \mathrm{H}, 7-\mathrm{H}), 7.72(\mathrm{~d}, J=8.9 \mathrm{~Hz}, 1 \mathrm{H}, 4-\mathrm{H}), 7.78(\mathrm{~d}, J=$ $8.1 \mathrm{~Hz}, 1 \mathrm{H}, 5-\mathrm{H}), 8.26(\mathrm{dd}, J=8.6,1.0 \mathrm{~Hz}, 1 \mathrm{H}, 8-\mathrm{H})$.

${ }^{13}$ C-NMR (126 MHz, $\left.\mathrm{CDCl}_{3}\right): \delta(\mathrm{ppm})=18.6,18.6$ (C-3"', C-5”), 27.4 (C-4"'), 76.2, 76.3 (C-2"', C-6”), 94.8, 95.1 (C-1', C-7'”), 112.0 (C-1), 115.9, 116.2 (C-2', C-1'”'), 116.8 (d, J=18.2 Hz, 3"','-H), 118.1, 118.8, 119.0, 120.3, 123.5, 123.7, 124.0 (d, $J=6.8 \mathrm{~Hz}, \mathrm{C}-4$ '”'), 124.4 (d, J = 3.9 Hz, C-6”"'), 125.3, 126.6 (C-8), 127.8 (C-7), 128.1 (C-5), 128.8 (C-4), 128.9, 128.9, 131.1, 133.2, 133.8, 133.8, 144.5 (d, $\left.J=11.1 \mathrm{~Hz}, \mathrm{C}-1{ }^{\prime \prime}, '\right), 151.8$ (C-2), 153.6 (d, $J=248.2 \mathrm{~Hz}, \mathrm{C}-2{ }^{\prime \prime}, '$ ), 156.9, 157.0.

Weitere Signale konnten nicht zugeordnet werden.

${ }^{19} \mathbf{F}-\mathbf{N M R}\left(282 \mathrm{MHz}, \mathrm{CDCl}_{3}\right): \delta(\mathrm{ppm})=-131.73--131.84(\mathrm{~m})$.

MS (ESI): $m / z(\%)=594.1(18)\left[\mathrm{M}+\mathrm{NH}_{4}\right]^{+}$.

HRMS (ESI): $m / z=$ gef.: 592.1273, ber.: $592.1282\left[\mathrm{M}\left({ }^{79} \mathrm{Br}\right)+\mathrm{NH}_{4}\right]^{+}$; gef.: 594.1268, ber.: 594.1264 $\left[\mathrm{M}\left({ }^{81} \mathrm{Br}\right)+\mathrm{NH}_{4}\right]^{+}$.

$\mathrm{C}_{35} \mathrm{H}_{24} \mathrm{BrFO}_{2}$ (575.47). 


\section{1-Brom-2-(2-(7-(2-(2-tolyloxy)phenyl)hepta-1,6-diin-1-yl)phenoxy)naphthalin (151e)}

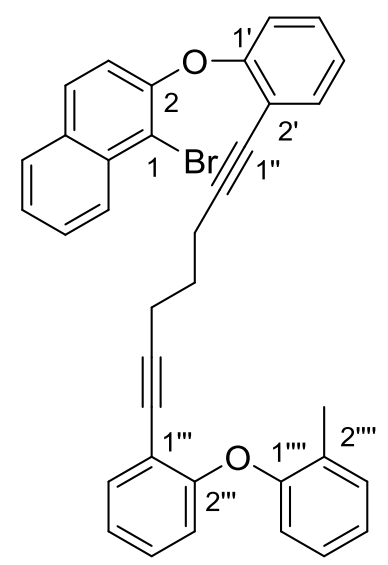

DC: $R_{f}=0.31(n-$ Pentan/EtOAc, 50:1).

UV/Vis $\left(\mathrm{CH}_{3} \mathrm{CN}\right): \lambda_{\max }(\mathrm{nm})(\lg \varepsilon)=195$ (5.025), 230 (4.883), 252 (4.551), 286 (4.007), 341 (2.004), 426 (2.009).

IR (ATR): $\tilde{v}\left(\mathrm{~cm}^{-1}\right)=1624,1594,1483,1459,1443,1428,1354,1252,1228,1201,1180,1156,1128$, 1039, 989, 937, 875, 826, 807, 776, 745, 642, 527.

${ }^{1} \mathbf{H}-\mathbf{N M R}\left(600 \mathrm{MHz}, \mathrm{CDCl}_{3}\right): \delta(\mathrm{ppm})=1.54\left(\mathrm{p}, J=6.9 \mathrm{~Hz}, 2 \mathrm{H}, 4{ }^{\prime}{ }^{-} \mathrm{H}_{2}\right), 2.25\left(\mathrm{~s}, 3 \mathrm{H}, \mathrm{CH}_{3}\right), 2.28-$ $2.33\left(\mathrm{~m}, 4 \mathrm{H}, 3\right.$ ' '- $\mathrm{H}_{2}, 5$ ' '- $\left.\mathrm{H}_{2}\right), 6.73(\mathrm{dd}, J=8.4,1.1 \mathrm{~Hz}, 1 \mathrm{H}), 6.76(\mathrm{dd}, J=8.1,1.2 \mathrm{~Hz}, 1 \mathrm{H}, 3$ ', ' '-H), $6.87(\mathrm{dd}, J=8.3,1.1 \mathrm{~Hz}, 1 \mathrm{H}), 6.98\left(\mathrm{qd}, J=7.5,1.1 \mathrm{~Hz}, 2 \mathrm{H}, 5{ }^{\prime \prime}{ }^{\prime}-\mathrm{H}\right), 7.04-7.12$ (m, $3 \mathrm{H}, 3-\mathrm{H}$, 4", ' $-\mathrm{H}), 7.13-7.24$ (m, 3 H), 7.38 (dd, $J=7.7,1.6 \mathrm{~Hz}, 1 \mathrm{H}, 6$,",'-H), 7.42-7.49 (m, $2 \mathrm{H}, 6-\mathrm{H}), 7.58$ (ddd, $J=8.3,6.9,1.2 \mathrm{~Hz}, 1 \mathrm{H}, 7-\mathrm{H}), 7.73(\mathrm{~d}, J=8.9 \mathrm{~Hz}, 1 \mathrm{H}, 4-\mathrm{H}), 7.79(\mathrm{dd}, J=8.2,1.4 \mathrm{~Hz}, 1 \mathrm{H}$, $5-\mathrm{H}), 8.28(\mathrm{~d}, J=8.6 \mathrm{~Hz}, 1 \mathrm{H}, 8-\mathrm{H})$.

${ }^{13}$ C-NMR (126 MHz, CDCl $): \delta(\mathrm{ppm})=16.2\left(\mathrm{CH}_{3}\right), 18.6\left(\mathrm{C}-3{ }^{\prime},, \mathrm{C}-5{ }^{\prime \prime}\right), 27.5\left(\mathrm{C}-4{ }^{\prime \prime}\right), 76.3,76.6$ (C-2", C-6”), 94.1, 95.1 (C-1", C-7'), 112.0 (C-1), 115.6, 116.2, 117.7, 118.3 (C-3"'”), 118.7, 119.0, 122.6, 123.4, 123.7, 125.3, 126.6, 126.9, 127.7 (C-8), 128.1 (C-5), 128.8, 128.9, 128.9, 129.1, 131.1, 131.2, 133.2 (C-4a), 133.7, 133.8, 151.8 (C-2), 155.0 (C-1 ',', ), 157.0, 157.8.

Weitere Signale konnten nicht zugeordnet werden.

MS (ESI): $m / z(\%)=590.2(6)\left[\mathrm{M}+\mathrm{NH}_{4}\right]^{+}$.

HRMS (ESI): $m / z=$ gef.: 588.1524 , ber.: $588.1533\left[\mathrm{M}\left({ }^{79} \mathrm{Br}\right)+\mathrm{NH}_{4}\right]^{+}$; gef.: 590.1506 , ber.: 590.1515 $\left[\mathrm{M}\left({ }^{81} \mathrm{Br}\right)+\mathrm{NH}_{4}\right]^{+}$.

$\mathrm{C}_{36} \mathrm{H}_{27} \mathrm{BrO}_{2}$ (571.50). 


\section{1-Brom-2-(2-(7-(2-(2-(trifluormethyl)phenoxy)phenyl)hepta-1,6-diin-1-yl)phenoxy)naphthalin} (151f)

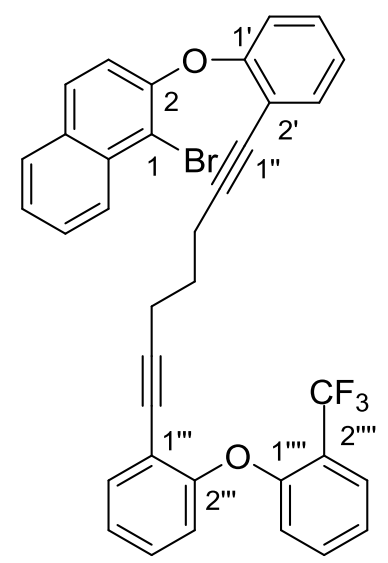

DC: $R_{f}=0.25(n-$ Pentan/EtOAc, 50:1).

UV/Vis $\left(\mathrm{CH}_{3} \mathrm{CN}\right): \lambda_{\max }(\mathrm{nm})(\lg \varepsilon)=194$ (5.200), 230 (4.913), 252 (4.568), 283 (4.074), 319 (3.217), 333 (3.197).

IR (ATR): $\tilde{v}\left(\mathrm{~cm}^{-1}\right)=1609,1593,1484,1458,1443,1428,1355,1319,1268,1253,1230,1199,1157$, $1130,1112,1054,1035,989,938,876,825,804,775,748,648,525$.

${ }^{1} \mathbf{H}-\mathbf{N M R}\left(600 \mathrm{MHz}, \mathrm{CDCl}_{3}\right): \delta(\mathrm{ppm})=1.39\left(\mathrm{p}, J=6.9 \mathrm{~Hz}, 2 \mathrm{H}, 4{ }^{\prime}{ }^{\prime}-\mathrm{H}_{2}\right), 2.14(\mathrm{t}, J=6.9 \mathrm{~Hz}, 2 \mathrm{H}$, 5' $\left.-\mathrm{H}_{2}\right), 2.16$ (t, $J=6.9 \mathrm{~Hz}, 2 \mathrm{H}, 3$ ' '- $\left.\mathrm{H}_{2}\right), 6.65$ (d, $J=8.4 \mathrm{~Hz}, 1 \mathrm{H}, 3$ ', '’-H), 6.85 (dd, $J=8.2,1.1 \mathrm{~Hz}$,

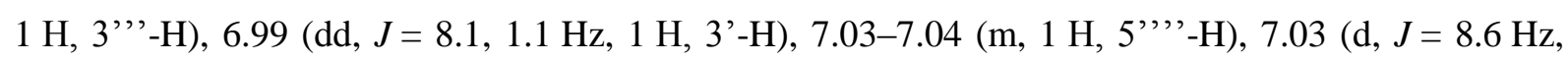
$1 \mathrm{H}, 3-\mathrm{H}), 7.07$ (td, $J=7.6,1.1 \mathrm{~Hz}, 1 \mathrm{H}, 5$ '”' $-\mathrm{H}), 7.10$ (td, $J=7.6,1.2 \mathrm{~Hz}, 1 \mathrm{H}, 5$ '-H), 7.21 (ddd, $J=$ 8.4, 7.5, 1.7 Hz, $1 \mathrm{H}, 4$ ', '-H), 7.26 (ddd, $\left.J=8.4,7.5,1.7 \mathrm{~Hz}, 1 \mathrm{H}, 4^{\prime}-\mathrm{H}\right), 7.32$ (td, $J=7.7,1.6 \mathrm{~Hz}, 1 \mathrm{H}$, 4",' '-H), 7.38 (dd, $J=7.7,1.7 \mathrm{~Hz}, 1 \mathrm{H}, 6$ '-H), 7.41-7.48 (m, $2 \mathrm{H}, 6-\mathrm{H}, 6$ '”' $-\mathrm{H}), 7.54-7.63$ (m, $2 \mathrm{H}$, 7-H, 6" ', '-H), 7.72 (d, J=8.9 Hz, $1 \mathrm{H}, 4-\mathrm{H}), 7.78$ (d, $J=8.1 \mathrm{~Hz}, 1 \mathrm{H}, 5-\mathrm{H}), 8.26$ (dd, $J=8.6,0.9 \mathrm{~Hz}$, $1 \mathrm{H}, 8-\mathrm{H})$.

${ }^{13}$ C-NMR (126 MHz, CDCl 3 ): $\delta(\mathrm{ppm})=18.4$ (C-3"', C-5'”), 27.2 (C-4"'), 75.9 (C-2"'), 76.2 (C-6"'), 95.0 (C-7'”), 95.3 (C-1'), 112.0 (C-1), 116.2 (C-1'”'), 116.7 (C-3','’), 117.5 (C-2'), 118.7 (C-6'”'), 119.0 (C-3), 119.7 (q, $J=31.6 \mathrm{~Hz}, \mathrm{C}-2^{\prime \prime}{ }^{\prime \prime}$ ), 120.9 (C-3'), 121.8 (C-5','), 123.5 (q, $J=272.8 \mathrm{~Hz}$, $\mathrm{CF}_{3}$ ), 123.7 (C-5','), 124.7 (C-5'), 125.4 (C-6), 126.5 (C-8), 127.1 (q, J=5.1 Hz, C-6','), 127.8 (C-7), 128.1 (C-5), 128.9, 128.9, 129.1 (C-4, C-4', C-4'”), 131.1 (C-4a), 133.0 (C-4'”,'), 133.2 (C-8a), 133.7, 133.8 (C-6', C-3'”'), 151.8 (C-2), 155.5 (C-1'), 155.8 (q, J=1.8 Hz, C-1',',), 157.0 $(\mathrm{C}-2, \cdots)$.

${ }^{19}$ F-NMR $\left(282 \mathrm{MHz}, \mathrm{CDCl}_{3}\right): \delta(\mathrm{ppm})=61.9(\mathrm{~s})$.

MS (ESI): $m / z(\%)=644.1(9)\left[\mathrm{M}+\mathrm{NH}_{4}\right]^{+}$. 
HRMS (ESI): $\mathrm{m} / z=$ gef.: 642.1240 , ber.: $642.1250\left[\mathrm{M}\left({ }^{79} \mathrm{Br}\right)+\mathrm{NH}_{4}\right]^{+}$; gef.: 644.1233 , ber.: 644.1232 $\left[\mathrm{M}\left({ }^{81} \mathrm{Br}\right)+\mathrm{NH}_{4}\right]^{+}$.

$\mathrm{C}_{36} \mathrm{H}_{24} \mathrm{BrF}_{3} \mathrm{O}_{2}$ (625.47). 


\section{1-Brom-2-(2-(7-(2-(2-methoxyphenoxy)phenyl)hepta-1,6-diin-1-yl)phenoxy)naphthalin (151g)}

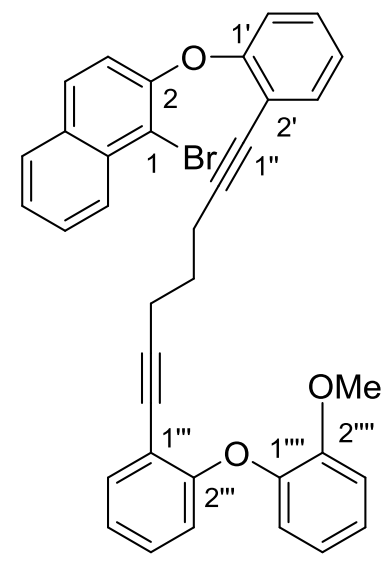

DC: $R_{f}=0.25(n-$ Pentan/MTBE, 20:1).

UV/Vis $\left(\mathrm{CH}_{3} \mathrm{CN}\right): \lambda_{\max }(\mathrm{nm})(\lg \varepsilon)=208$ (4.891), 231 (4.959), 252 (4.6280), 282 (4.155), 319 (3.244), 333 (3.239).

IR (ATR): $\tilde{v}\left(\mathrm{~cm}^{-1}\right)=1594,1572,1498,1484,1458,1442,1428,1354,1253,1229,1216,1200,1175$, 1157, 1113, 1040, 1025, 989, 936, 874, 826, 775, 743, 641, 527.

${ }^{1} \mathbf{H}-\mathrm{NMR}\left(600 \mathrm{MHz}, \mathrm{CDCl}_{3}\right): \delta(\mathrm{ppm})=1.52\left(\mathrm{p}, J=7.0 \mathrm{~Hz}, 2 \mathrm{H}, 4{ }^{\prime} \cdot \mathrm{H}_{2}\right), 2.29(\mathrm{dt}, J=14.1,7.0 \mathrm{~Hz}$, $4 \mathrm{H}, 3$ ' '- $-\mathrm{H}_{2}, 5$ ' '- $\left.\mathrm{H}_{2}\right), 3.81$ (s, $\left.3 \mathrm{H}, \mathrm{OMe}\right), 6.78$ (td, $J=7.9,1.4 \mathrm{~Hz}, 2 \mathrm{H}, 6$ '-H, 6',' '-H), 6.83 (dd, $J=$ 7.6, $1.4 \mathrm{~Hz}, 1 \mathrm{H}, 5$ "','-H), $6.85\left(\mathrm{dd}, J=8.0,1.2 \mathrm{~Hz}, 1 \mathrm{H}, 3^{\prime \prime}{ }^{-}-\mathrm{H}\right), 6.94(\mathrm{dd}, J=8.2,1.4 \mathrm{~Hz}, 1 \mathrm{H}$, 3', ' '-H), 6.97 (td, $\left.J=7.5,1.1 \mathrm{~Hz}, 1 \mathrm{H}, 4^{\prime}-\mathrm{H}\right), 7.01-7.04\left(\mathrm{~m}, 1 \mathrm{H}, 4{ }^{\prime \prime},{ }^{-}-\mathrm{H}\right), 7.05$ (d, $J=8.7 \mathrm{~Hz}, 1 \mathrm{H}$, 3-H), 7.07 (dd, $\left.J=7.7,1.1 \mathrm{~Hz}, 1 \mathrm{H}, 5^{\prime}{ }^{\prime}{ }^{-H}\right), 7.14-7.17$ (m, $\left.1 \mathrm{H}, 5^{\prime}-\mathrm{H}\right), 7.21$ (td, $J=7.8,1.7 \mathrm{~Hz}, 1 \mathrm{H}$, 4'"'-H), 7.36 (dd, $J=7.6,1.7 \mathrm{~Hz}, 1 \mathrm{H}, 3$ '-H), 7.44 (td, $J=7.4,1.3 \mathrm{~Hz}, 2 \mathrm{H}, 6-\mathrm{H}, 6$ ','-H), 7.58 (ddd, $J=8.3,6.9,1.2 \mathrm{~Hz}, 1 \mathrm{H}, 7-\mathrm{H}), 7.73(\mathrm{~d}, J=8.9 \mathrm{~Hz}, 1 \mathrm{H}, 4-\mathrm{H}), 7.78(\mathrm{dd}, J=8.2,1.2 \mathrm{~Hz}, 1 \mathrm{H}, 5-\mathrm{H})$, $8.27(\mathrm{dd}, J=8.5,1.1 \mathrm{~Hz}, 1 \mathrm{H}, 8-\mathrm{H})$.

${ }^{13}$ C-NMR (126 MHz, CDCl $): \delta(\mathrm{ppm})=18.6,18.7$ (C-3"', C-5"'), 27.5 (C-4”'), $56.1(\mathrm{OMe}), 76.2$ (C-2'), 76.7 (C-6'), 94.2, 95.2 (C-1', C-7'’), 112.1 (C-1), 112.9 (C-3','), 115.6 (C-2'), 116.2

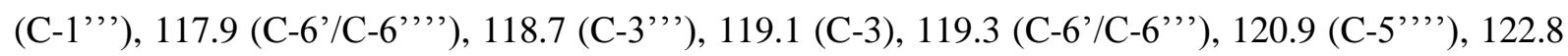
(C-4'), 123.7 (C-5'”), 124.0 (C-4”,'), 125.4 (C-6), 126.5 (C-8), 127.8 (C-7), 128.1 (C-5), 128.7 (C-5'), 128.8 (C-4','), 128.9 (C-4), 131.1 (C-4a), 133.1 (C-8a), 133.6 (C-3'), 133.8 (C-6'/C-6','), 145.9 (C-1','), 150.6 (C-2',’'), 151.7 (C-2), 157.0 (C-2'”'), 157.6 (C-1').

MS (ESI): $m / z(\%)=606.1(32)\left[\mathrm{M}+\mathrm{NH}_{4}\right]^{+}$.

HRMS (ESI): $m / z=$ gef.: 587.1205 , ber.: $587.1216\left[\mathrm{M}\left({ }^{79} \mathrm{Br}\right)+\mathrm{H}\right]^{+}$; gef.: 589.1174 , ber.: 589.1198 $\left[\mathrm{M}\left({ }^{81} \mathrm{Br}\right)+\mathrm{H}\right]^{+}$.

$\mathrm{C}_{36} \mathrm{H}_{27} \mathrm{BrO}_{3}$ (587.50). 


\section{2-(2-(7-(2-([1,1'-Biphenyl]-4-yloxy)phenyl)hepta-1,6-diin-1-yl)phenoxy)-1-bromnaphthalin} (151h)

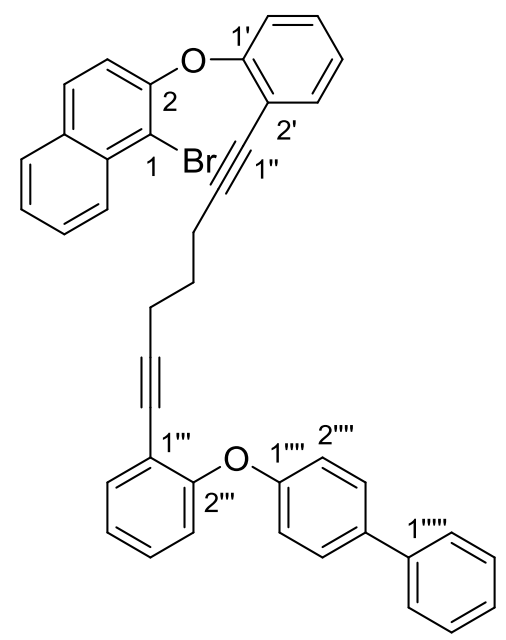

DC: $R_{f}=0.14\left(n-\mathrm{Pentan} / \mathrm{CH}_{2} \mathrm{Cl}_{2}, 3: 1\right)$

UV/Vis $\left(\mathrm{CH}_{3} \mathrm{CN}\right): \lambda_{\max }(\mathrm{nm})(\lg \varepsilon)=194$ (5.075), 231 (4.744), 252 (4.540), 333 (3.063).

IR (ATR): $\tilde{v}\left(\mathrm{~cm}^{-1}\right)=1596,1571,1513,1499,1481,1459,1443,1428,1354,1329,1252,1226,1199$, 1167, 1105, 1038, 1007, 989, 937, 872, 832, 805, 759, 750, 736, 697, 642, 627.

${ }^{1}$ H-NMR $\left(600 \mathrm{MHz}, \mathrm{CDCl}_{3}\right): \delta(\mathrm{ppm})=1.46\left(\mathrm{p}, J=6.9 \mathrm{~Hz}, 2 \mathrm{H}, 4{ }^{\prime}{ }^{-} \mathrm{H}_{2}\right), 2.25(\mathrm{q}, J=6.7 \mathrm{~Hz}, 4 \mathrm{H}$,

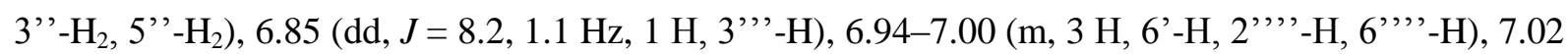
(d, $J=9.0 \mathrm{~Hz}, 1 \mathrm{H}, 3-\mathrm{H}$ ), 7.06 (ddd, $\left.J=8.9,7.6,1.2 \mathrm{~Hz}, 2 \mathrm{H}, 4^{\prime}-\mathrm{H}, 5^{\prime}{ }^{\prime}-\mathrm{H}\right), 7.20$ (ddd, $J=8.3,7.5$, $1.7 \mathrm{~Hz}, 1 \mathrm{H}, 4$ '”'-H), 7.22-7.26 (m, $1 \mathrm{H}$, 5'-H), 7.26-7.30 (m, $1 \mathrm{H}, 4$ ', ', '-H), 7.37 (t, J= 7.7 Hz, $2 \mathrm{H}$, 3",'”-H, 5',',-H), 7.40 (ddd, $J=7.6,3.9,1.5 \mathrm{~Hz}, 2 \mathrm{H}, 3$ '-H, 6"' '-H), 7.43 (ddd, $J=8.1,6.9,1.1 \mathrm{~Hz}$,

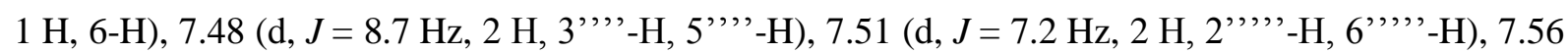
(ddd, $J=8.4,6.9,1.2 \mathrm{~Hz}, 1 \mathrm{H}, 7-\mathrm{H}), 7.70(\mathrm{~d}, J=8.9 \mathrm{~Hz}, 1 \mathrm{H}, 4-\mathrm{H}), 7.77(\mathrm{~d}, J=8.1 \mathrm{~Hz}, 1 \mathrm{H}, 5-\mathrm{H})$, $8.25(\mathrm{dd}, J=8.7,1.1 \mathrm{~Hz}, 1 \mathrm{H}, 8-\mathrm{H})$.

${ }^{13}$ C-NMR (126 MHz, $\left.\mathrm{CDCl}_{3}\right): \delta(\mathrm{ppm})=18.5$ (C-3', C-5'), 27.3 (C-4'”), 76.3, 76.5 (C-2', C-6”),

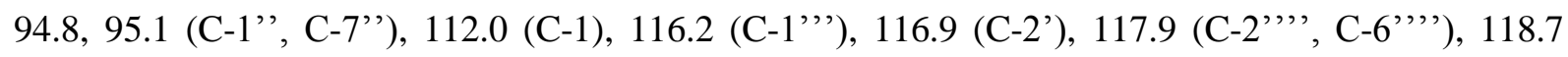
(C-3'”'), 119.0 (C-3), 120.0 (C-6'), 123.7, 123.8 (C-4', C-5'”'), 125.3 (C-6), 126.6 (C-8), 126.9

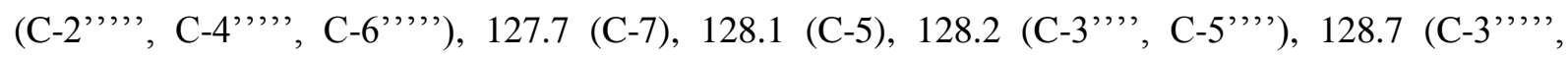
C-5",','), 128.8, 128.9, 129.0 (C-4, C-5', C-4"'), 131.0 (C-8a), 133.2 (C-4a), 133.7, 133.8 (C-3', C-6"''), 135.7 (C-4','’), 140.6 (C-1',','), 151.8 (C-2), 156.8 (C-1'), 157.0 (C-2'”'), 157.3 (C-1',').

MS (ESI): $m / z(\%)=652.2(3)\left[\mathrm{M}+\mathrm{NH}_{4}\right]^{+}$

HRMS (ESI): $m / z=$ gef.: 650.1671 , ber.: $650.1689\left[\mathrm{M}\left({ }^{79} \mathrm{Br}\right)+\mathrm{NH}_{4}\right]^{+}$; gef.: 652.1656 , ber.: 652.1671 $\left[\mathrm{M}\left({ }^{81} \mathrm{Br}\right)+\mathrm{NH}_{4}\right]^{+}$. 
$\mathrm{C}_{41} \mathrm{H}_{29} \mathrm{BrO}_{2}$ (633.57). 


\section{1-Brom-2-(2-(7-(2-(4-(tert-butyl)phenoxy)phenyl)hepta-1,6-diin-1-yl)phenoxy)naphthalin (151i)}

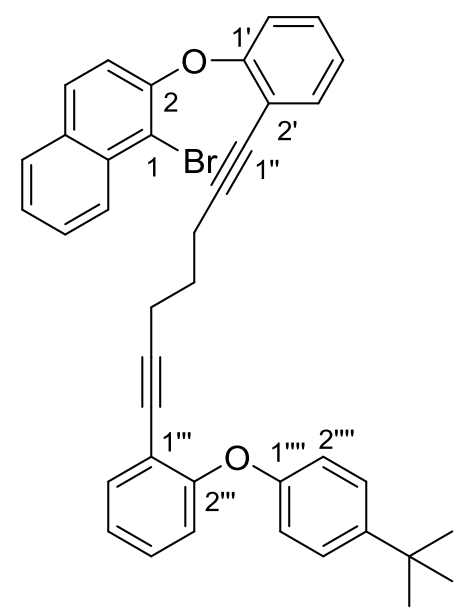

DC: $R_{f}=0.40(n-$ Pentan/EtOAc, 50:1).

UV/Vis $\left(\mathrm{CH}_{3} \mathrm{CN}\right): \lambda_{\max }(\mathrm{nm})(\lg \varepsilon)=195$ (5.510), 230 (4.854), 284 (3.966), 318 (3.206), 333 (3.208).

IR $(\mathrm{ATR}): \tilde{v}\left(\mathrm{~cm}^{-1}\right)=1596,1507,1484,1460,1443,1428,1356,1252,1227,1200,1157,1104,1012$, $990,938,875,750,546,527$.

${ }^{1} \mathbf{H}-\mathbf{N M R}\left(600 \mathrm{MHz}, \mathrm{CDCl}_{3}\right): \delta(\mathrm{ppm})=1.27\left(\mathrm{~s}, 9 \mathrm{H}, \mathrm{C}\left(\mathrm{CH}_{3}\right)_{3}\right), 1.47(\mathrm{p}, J=7.0 \mathrm{~Hz}, 2 \mathrm{H}, 4 \text { '’-H })_{2}, 2.25$

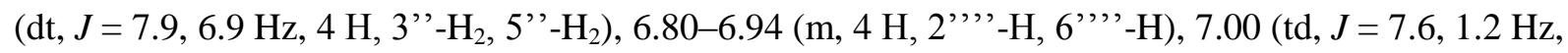
$1 \mathrm{H}), 7.04(\mathrm{~d}, J=8.9 \mathrm{~Hz}, 1 \mathrm{H}, 3-\mathrm{H}), 7.06(\mathrm{td}, J=7.6,1.1 \mathrm{~Hz}, 1 \mathrm{H}), 7.16-7.23(\mathrm{~m}, 2 \mathrm{H}), 7.27(\mathrm{~d}, J=$ 8.8 Hz, 2 H, 3',', '-H, 5',', '-H), 7.36 (dd, J = 7.7, $1.7 \mathrm{~Hz}, 1 \mathrm{H}), 7.39-7.47$ (m, 2 H, 6-H), 7.56 (ddd, J= 8.4, 6.9, $1.3 \mathrm{~Hz}, 1 \mathrm{H}, 7-\mathrm{H}), 7.71$ (d, J=8.9 Hz, $1 \mathrm{H}, 4-\mathrm{H}), 7.77$ (d, J=8.1 Hz, $1 \mathrm{H}, 5-\mathrm{H}), 8.26$ (dd, $J=$ 8.6, 1.1 Hz, $1 \mathrm{H}, 8-\mathrm{H})$.

${ }^{13}$ C-NMR $\left(126 \mathrm{MHz}, \mathrm{CDCl}_{3}\right): \delta(\mathrm{ppm})=18.6\left(\mathrm{C}-3{ }^{\prime},\right.$, C-5 $\left.{ }^{\prime \prime}\right), 27.4\left(\mathrm{C}-4\right.$ '”), $31.5\left(\mathrm{C}\left(\underline{\mathrm{CH}}_{3}\right)_{3}\right), 34.2$

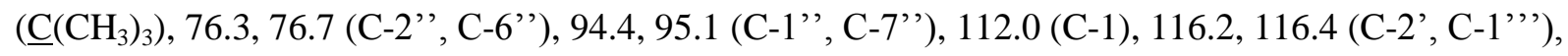
117.5, 118.8, 119.0, 119.2, 119.4, 123.2, 123.7, 125.3, 126.3, 126.4 (C-3',', , C-5',,'), 126.6 (C-6), 127.7 (C-7), 128.1 (C-5), 128.8, 128.9 (C-4), 131.1 (C-4a), 133.2 (C-8a), 133.6, 133.8, 145.6 (C-4',,'), 151.8 (C-2), 155.1 (C-1','’), 157.0, 157.4 (C-1', C-2',').

Weitere Signale konnten nicht zugeordnet werden.

MS (ESI): $m / z(\%)=632.2(3)\left[\mathrm{M}+\mathrm{NH}_{4}\right]^{+}$.

HRMS (ESI): $m / z=$ gef.: 630.1992, ber.: $630.2002\left[\mathrm{M}\left({ }^{79} \mathrm{Br}\right)+\mathrm{NH}_{4}\right]^{+}$; gef.: 632.1996, ber.: 632.1984 $\left[\mathrm{M}\left({ }^{81} \mathrm{Br}\right)+\mathrm{NH}_{4}\right]^{+}$.

$\mathrm{C}_{39} \mathrm{H}_{33} \mathrm{BrO}_{2}(613.58)$. 


\section{1-Brom-2-(2-(6-(2-phenoxyphenyl)hexa-1,5-diin-1-yl)phenoxy)naphthalin (151j)}

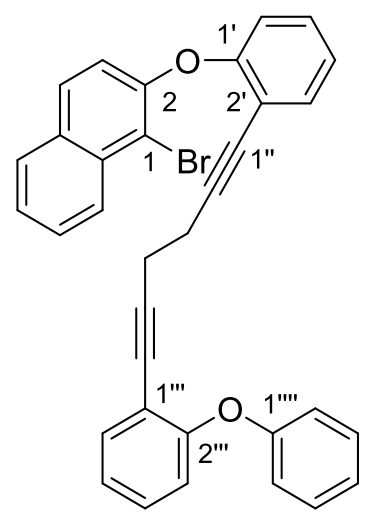

DC: $R_{f}=0.34(n-$ Pentan/EtOAc, 50:1).

UV/Vis $\left(\mathrm{CH}_{3} \mathrm{CN}\right): \lambda_{\max }(\mathrm{nm})(\lg \varepsilon)=194$ (5.138), 230 (4.897), 253 (4.558), 286 (4.021), 332 (3.092).

IR (ATR): $\tilde{v}\left(\mathrm{~cm}^{-1}\right)=1590,1572,1483,1459,1443,1428,1355,1253,1224,1200,1160,1132,1105$, 990, 937, 803, 748, 690, 527.

${ }^{1} \mathbf{H}-\mathrm{NMR}\left(600 \mathrm{MHz}, \mathrm{CDCl}_{3}\right): \delta(\mathrm{ppm})=2.41\left(\mathrm{~s}, 4 \mathrm{H}, 3{ }^{\prime}{ }^{-}-\mathrm{H}_{2}, 4{ }^{\prime}{ }^{\prime}-\mathrm{H}_{2}\right), 6.82(\mathrm{dd}, J=8.3,1.1 \mathrm{~Hz}, 1 \mathrm{H})$, $6.87(\mathrm{dd}, J=8.2,1.1 \mathrm{~Hz}, 1 \mathrm{H}), 6.89-6.94\left(\mathrm{~m}, 2 \mathrm{H}, 2^{\prime \prime}{ }^{\prime \prime}-\mathrm{H}, 6{ }^{\prime \prime}{ }^{\prime}{ }_{-}-\mathrm{H}\right), 6.98$ (td, $\left.J=7.5,1.2 \mathrm{~Hz}, 1 \mathrm{H}\right)$,

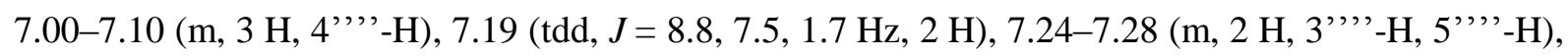
$7.37(\mathrm{dd}, J=7.7,1.7 \mathrm{~Hz}, 1 \mathrm{H}), 7.42-7.48(\mathrm{~m}, 2 \mathrm{H}, 6-\mathrm{H}), 7.59$ (ddd, $J=8.3,6.8,1.2 \mathrm{~Hz}, 1 \mathrm{H}, 7-\mathrm{H})$, $7.70(\mathrm{~d}, J=8.9 \mathrm{~Hz}, 1 \mathrm{H}, 4-\mathrm{H}), 7.78(\mathrm{~d}, J=8.1 \mathrm{~Hz}, 1 \mathrm{H}, 5-\mathrm{H}), 8.27$ (dd, $J=8.6,1.0 \mathrm{~Hz}, 1 \mathrm{H}, 8-\mathrm{H})$.

${ }^{13}$ C-NMR (126 MHz, CDCl $): \delta(\mathrm{ppm})=19.7$ (C-3', C-5’'), 76.6, 76.9 (C-2”, C-5’'), 93.6, 93.9 (C-1'”, C-6'), 112.4 (C-1), 115.8, 116.3 (C-2', C-1'”), 118.1 (C-3',', C-5','), 118.4, 119.4, 119.4, 122.8, 123.4, 123.5, 125.5, 126.6 (C-8), 127.8 (C-7), 128.1 (C-5), 128.9 (C-4), 129.0, 129.1, 129.5 (C-2',', C-6','), 131.1 (C-4a), 133.1 (C-8a), 133.8, 133.9, 151.7 (C-2), 157.2 (C-1', C-2'”'), 157.5 $(\mathrm{C}-1, \cdots)$.

Weitere Signale konnten nicht zugeordnet werden.

MS (ESI): $m / z(\%)=562.1(17)\left[\mathrm{M}+\mathrm{NH}_{4}\right]^{+}$.

HRMS (ESI): $m / z=$ gef.: 543.0946, ber.: $543.0954\left[\mathrm{M}\left({ }^{79} \mathrm{Br}\right)+\mathrm{H}\right]^{+}$; gef.: 545.0933 , ber.: 545.0936 $\left[\mathrm{M}\left({ }^{81} \mathrm{Br}\right)+\mathrm{H}\right]^{+}$.

$\mathrm{C}_{34} \mathrm{H}_{23} \mathrm{BrO}_{2}$ (543.45). 


\section{1-Brom-2-(2-(8-(2-phenoxyphenyl)octa-1,7-diin-1-yl)phenoxy)naphthalin (151k)}

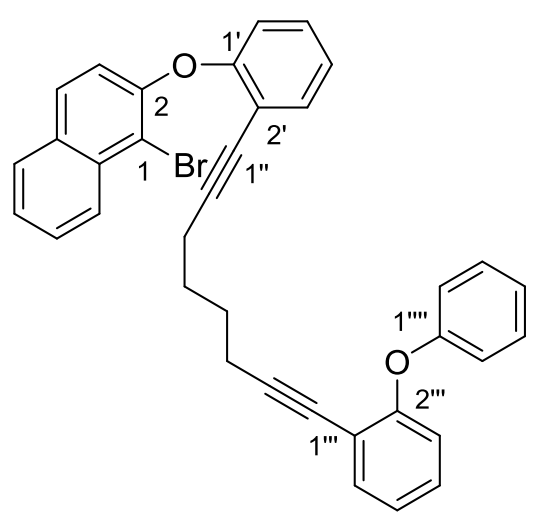

DC: $R_{f}=0.15(n-$ Pentan/EtOAc, 50:1).

UV/Vis $\left(\mathrm{CH}_{3} \mathrm{CN}\right): \lambda_{\max }(\mathrm{nm})(\lg \varepsilon)=193$ (5.375), 231 (4.881), 272 (3.943), 284 (3.995), 319 (3.256), 333 (3.244).

IR (ATR): $\tilde{v}\left(\mathrm{~cm}^{-1}\right)=1590,1572,1482,1458,1443,1428,1355,1329,1251,1223,1199,1161,1105$, $990,938,867,827,802,776,748,689,527$.

${ }^{1} \mathbf{H}-\mathbf{N M R}\left(600 \mathrm{MHz}, \mathrm{CDCl}_{3}\right): \delta(\mathrm{ppm})=1.31\left(\mathrm{t}, J=3.4 \mathrm{~Hz}, 4 \mathrm{H}, 4{ }^{\prime}{ }^{\prime}-\mathrm{H}_{2}, 5\right.$ '”- $\left.\mathrm{H}_{2}\right), 2.05-2.11(\mathrm{~m}, 2 \mathrm{H})$, 2.15-2.22 (m, 2 H) (3'”- $\mathrm{H}_{2}, 6$ 6'- $\left.\mathrm{H}_{2}\right), 6.85-6.94$ (m, 4 H, 2',' '-H, 6','-H), 6.98-7.05 (m, 3 H, 3-H, 4",' $-\mathrm{H}), 7.07$ (td, $J=7.6,1.2 \mathrm{~Hz}, 1 \mathrm{H}), 7.21$ (dtd, $J=9.1,7.7,1.8 \mathrm{~Hz}, 2 \mathrm{H}), 7.24-7.31(\mathrm{~m}, 2 \mathrm{H}$, 3",' -H, 5,"'-H), 7.39 (dd, $J=7.7,1.7 \mathrm{~Hz}, 1 \mathrm{H}), 7.41-7.47$ (m, $2 \mathrm{H}, 6-\mathrm{H}), 7.58$ (ddd, $J=8.4,6.8$, $1.3 \mathrm{~Hz}, 1 \mathrm{H}, 7-\mathrm{H}), 7.70(\mathrm{~d}, J=8.9 \mathrm{~Hz}, 1 \mathrm{H}, 4-\mathrm{H}), 7.77(\mathrm{dd}, J=8.2,1.2 \mathrm{~Hz}, 1 \mathrm{H}, 5-\mathrm{H}), 8.26(\mathrm{~d}, J=$ $8.7 \mathrm{~Hz}, 1 \mathrm{H}, 8-\mathrm{H})$.

${ }^{13}$ C-NMR (126 MHz, $\left.\mathrm{CDCl}_{3}\right): \delta(\mathrm{ppm})=18.9,19.0$ (C-3"', C-6"'), 27.2, 27.3 (C-4", C-5'”), 76.0, 76.3 (C-2', C-7'), 95.2, 95.8 (C-1', C-8'), 111.7 (C-1), 116.4, 116.9 (C-2', C-1','), 117.7 (C-3',', C-5",'), 118.8, 119.0, 119.8, 122.6, 123.6, 123.8, 125.3, 126.5 (C-8), 127.8 (C-7), 128.1, 128.8, 128.9, 129.5 (C-2',', C-6', '), 131.0 (C-4a), 133.2 (C-8a), 133.6, 133.8, 151.9 (C-2), 156.9 (C-1', $\mathrm{C}-2$ ','), $157.6\left(\mathrm{C}-1{ }^{, \prime \prime \prime}\right)$.

Weitere Signale konnten nicht zugeordnet werden.

MS (ESI): $m / z(\%)=590.2(100)\left[\mathrm{M}+\mathrm{NH}_{4}\right]^{+}$.

HRMS (ESI): $m / z=$ gef.: 571.1247 , ber.: $571.1267\left[\mathrm{M}\left({ }^{79} \mathrm{Br}\right)+\mathrm{H}\right]^{+}$; gef.: 573.1230 , ber.: 573.1249 $\left[\mathrm{M}\left({ }^{81} \mathrm{Br}\right)+\mathrm{H}\right]^{+}$.

$\mathrm{C}_{36} \mathrm{H}_{27} \mathrm{BrO}_{2}(571.50)$. 


\subsubsection{Synthese der Polyzyklen 110a-i durch die Domino-Reaktion}

\section{Allgemeine Vorschrift für die Synthese der Produkte 110a-i (Vierfach-Domino-Reaktion):}

Eine Mischung aus Dialkin 112 (1.0 Äq.), Aryliodid 111 (1.1 Äq.), Pd(OAc) 2 (0.01 Äq.), $\mathrm{PPh}_{3}$ (0.05 Äq.) und ( $n \mathrm{Bu})_{4} \mathrm{NOAc}\left(5.0\right.$ Äq.) in entgastem DMF $(3 \mathrm{~mL})$ wurde $14-20 \mathrm{~h}$ bei $100{ }^{\circ} \mathrm{C}$ gerührt. Nach dem Abkühlen auf RT wurde die Lösung durch $\mathrm{SiO}_{2}$ filtriert und das Lösungsmittel des Filtrats in vacuo entfernt. Säulenchromatographische Reinigung $\left(\mathrm{SiO}_{2}\right)$ lieferte Domino-Produkt 110 als gelben Feststoff.

Tabelle 13: Synthese der Domino-Produkte 110a-i.

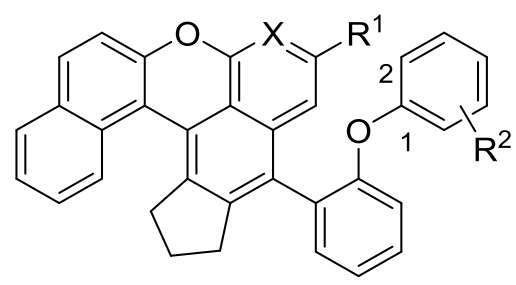

\begin{tabular}{cccccccc}
\hline Eintrag & Substrate & $\mathbf{R}^{\mathbf{1}}$ & $\mathbf{R}^{\mathbf{2}}$ & $\mathbf{X}$ & Produkt & Ausbeute [mg] & Ausbeute [\%] \\
\hline 1 & 111a, 112a & $\mathrm{H}$ & $\mathrm{H}$ & $\mathrm{CH}$ & $\mathbf{1 1 0 a}$ & 38.9 & 73 \\
2 & $\mathbf{1 1 1 c}, \mathbf{1 1 2 a}$ & $\mathrm{H}$ & $\mathrm{H}$ & $\mathrm{N}$ & $\mathbf{1 1 0 b}$ & 30.0 & 89 \\
$3^{[\mathrm{a}]}$ & $\mathbf{1 1 1 b}, \mathbf{1 1 2 a}$ & $\mathrm{OMe}$ & $\mathrm{H}$ & $\mathrm{CH}$ & $\mathbf{1 1 0 c}$ & 157 & 29 \\
4 & $\mathbf{1 1 1 a}, \mathbf{1 1 2 b}$ & $\mathrm{H}$ & $2-\mathrm{F}$ & $\mathrm{CH}$ & $\mathbf{1 1 0 d}$ & 23.5 & 67 \\
5 & $\mathbf{1 1 1 a}, \mathbf{1 1 2}$ & $\mathrm{H}$ & $2-\mathrm{Me}$ & $\mathrm{CH}$ & $\mathbf{1 1 0 e}$ & 57.0 & 40 \\
$6^{[\mathrm{b}]}$ & $\mathbf{1 1 1 a}, \mathbf{1 1 2 d}$ & $\mathrm{H}$ & $2-\mathrm{CF}_{3}$ & $\mathrm{CH}$ & $\mathbf{1 1 0 f}$ & 13.3 & 35 \\
$7^{[\mathrm{b}]}$ & $\mathbf{1 1 1 a}, \mathbf{1 1 2}$ & $\mathrm{H}$ & $2-\mathrm{OMe}$ & $\mathrm{CH}$ & $\mathbf{1 1 0 g}$ & 8.0 & 22 \\
8 & $\mathbf{1 1 1 a}, \mathbf{1 1 2 f}$ & $\mathrm{H}$ & $4-\mathrm{Ph}$ & $\mathrm{CH}$ & $\mathbf{1 1 0 h}$ & 15.7 & 40 \\
9 & $\mathbf{1 1 1 a}, \mathbf{1 1 2 g}$ & $\mathrm{H}$ & $4-\mathrm{Bu}$ & $\mathrm{CH}$ & $\mathbf{1 1 0 i}$ & 14.0 & 37 \\
\hline
\end{tabular}

[a] 111 (1.0 Äq.), Pd(OAc) 2 (0.2 Äq.), PPh $_{3}$ (1.0 Äq.), ( $n$ Bu $)_{4} \operatorname{NOAc}(5.2$ Äq.), DMF (8 mL); [b] 111 (1.0 Äq.). 


\section{7-(2-Phenoxyphenyl)-9,10-dihydro-8H-benzo $[a]$ inden $[6,5,4-k l]$ xanthen (110a)}

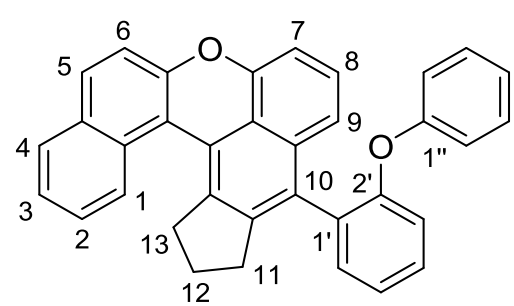

Eine Mischung aus Dialkin 112a (29.1 mg, $112 \mu \mathrm{mol}, 1.00$ Äq.), Aryliodid 111a (50.0 mg, $118 \mu \mathrm{mol}$, 1.05 Äq.), $\mathrm{Pd}(\mathrm{OAc})_{2}\left(251 \mu \mathrm{g}, 1.12 \mu \mathrm{mol}, 0.01\right.$ Äq.), $\mathrm{PPh}_{3}$ (1.47 mg, $5.60 \mu \mathrm{mol}, 0.05$ Äq.) und $(n \mathrm{Bu})_{4} \mathrm{NOAc}\left(169 \mathrm{mg}, 560 \mu \mathrm{mol}, 5.00\right.$ Äq.) in entgastem DMF $(3 \mathrm{~mL})$ wurde $14.5 \mathrm{~h}$ bei $100{ }^{\circ} \mathrm{C}$ gerührt. Nach Abkühlen auf RT wurde die Lösung durch $\mathrm{SiO}_{2}$ filtriert, mit EtOAc gespült und das Lösungsmittel des Filtrats in vacuo entfernt. Säulenchromatographische Reinigung $\left(\mathrm{SiO}_{2}\right.$, $n$-Pentan/ $\mathrm{CH}_{2} \mathrm{Cl}_{2}$ 5:1) lieferte Domino-Produkt 110a (38.9 mg, $81.6 \mu \mathrm{mol}, 73 \%$ ) als gelben Feststoff.

DC: $R_{f}=0.48\left(n-\right.$ Pentan $\left./ \mathrm{CH}_{2} \mathrm{Cl}_{2}, 5: 1\right)$.

UV/Vis $\left(\mathrm{CH}_{3} \mathrm{CN}\right): \lambda_{\max }(\mathrm{nm})(\lg \varepsilon)=214$ (4.886), 266 (4.416), 326 (3.980), 353 (3.845), 397 (3.969).

IR (ATR): $\tilde{v}\left(\mathrm{~cm}^{-1}\right)=1572,1481,1444,1432,1383,1373,1251,1263,1251,1236,1217,810,747$, 735,690 .

${ }^{1} \mathbf{H}-\mathbf{N M R}\left(600 \mathrm{MHz}, \mathrm{C}_{2} \mathrm{D}_{2} \mathrm{Cl}_{4}, 100{ }^{\circ} \mathrm{C}\right): \delta(\mathrm{ppm})=1.90-2.05\left(\mathrm{~m}, 2 \mathrm{H}, 12-\mathrm{H}_{2}\right), 2.70-2.89\left(\mathrm{~m}_{\mathrm{br}}, 2 \mathrm{H}\right.$, 13- $\left.\mathrm{H}_{2}\right), 2.88$ (dd, $\left.J=16.0,8.8 \mathrm{~Hz}, 2 \mathrm{H}, 11-\mathrm{H}_{2}\right), 6.82$ (d, $J=7.0 \mathrm{~Hz}, 2 \mathrm{H}, 2$ ''-H, 6'-H), 6.95 (t, $J=$ $7.3 \mathrm{~Hz}, 1 \mathrm{H}, 4$ ' '-H), 6.99 (d, J = 7.5 Hz, 1 H, 9-H), 7.11-7.20 (m, 3 H, 7-H, 3' '-H, 5' '-H), 7.24 (d, J = $\left.8.0 \mathrm{~Hz}, 1 \mathrm{H}, 3^{\prime}-\mathrm{H}\right), 7.28$ (dd, $\left.J=10.9,5.0 \mathrm{~Hz}, 1 \mathrm{H}, 8-\mathrm{H}\right), 7.34$ (t, $J=7.4 \mathrm{~Hz}, 1 \mathrm{H}, 5$ '-H), 7.40 (dd, $J=$ 8.8, 2.5 Hz, $1 \mathrm{H}, 6-\mathrm{H}), 7.48$ (dd, $J=14.5,7.1 \mathrm{~Hz}, 3 \mathrm{H}, 2-\mathrm{H}, 4^{\prime}-\mathrm{H}, 6$ '-H), 7.55 (t, J = 7.6 Hz, $1 \mathrm{H}, 3-\mathrm{H}$ ), $7.77(\mathrm{~d}, J=8.5 \mathrm{~Hz}, 1 \mathrm{H}, 4-\mathrm{H}), 7.84(\mathrm{dd}, J=8.7 \mathrm{~Hz}, 1 \mathrm{H}, 5-\mathrm{H}), 7.88(\mathrm{~d}, J=8.0 \mathrm{~Hz}, 1 \mathrm{H}, 1-\mathrm{H})$.

${ }^{13} \mathrm{C}-\mathrm{NMR}\left(126 \mathrm{MHz}, \mathrm{C}_{2} \mathrm{D}_{2} \mathrm{Cl}_{4}, 100{ }^{\circ} \mathrm{C}\right): \delta(\mathrm{ppm})=26.3(\mathrm{C}-12), 32.6(\mathrm{C}-11), 36.5(\mathrm{C}-13), 106.6(\mathrm{C}-9)$, 115.8 (C-13d), 116.8 (C-6), 117.7 (C-7), 118.3 (C-2’, C-6’), 119.5 (C-3'), 121.8 (C-4a), 122.4 (C-4”), 123.4 (C-5'), 124.0 (C-2), 124.8 (C-3, C-13b), 125.3 (C-8), 125.5 (C-4), 127.6 (C-1), 128.3 (C-6c), 128.5 (C-4'/C-6'), 128.8 (C-3', C-5'”), 129.5 (C-5, C-13c), 130.5 (C-1'), 131.0 (C-10), 132.0 (C-4'/C-6'), 133.1 (C-9a), 135.4, 145.4 (C-10a, C-13a), 150.7 (C-6b), 153.3 (C-6a), 155.0 (C-2'), $157.2(\mathrm{C}-1$ ' ').

MS (ESI): $m / z(\%)=476.2(100)[\mathrm{M}]^{+}, 499.2(76)[\mathrm{M}+\mathrm{Na}]^{+}$.

HRMS (ESI): $m / z=$ gef.: 476.1767 , ber.: $476.1771[\mathrm{M}]^{+}$.

$\mathbf{C}_{35} \mathbf{H}_{24} \mathbf{O}_{2}$ (476.56). 


\section{Allgemeine Vorschrift für die Synthese der Produkte 110b-i (Dreifach-Domino-Reaktion):}

Eine Mischung aus 151 (1.00 Äq.), $\mathrm{Pd}(\mathrm{OAc})_{2}\left(0.20\right.$ Äq.), $\mathrm{PPh}_{3}(1.00 \text { Äq.) und ( } n \mathrm{Bu})_{4} \mathrm{NOAc}(3.00$ Äq.) in entgastem DMF ( $c=0.01 \mathrm{~mol} / \mathrm{L}$ ) wurde $18-20 \mathrm{~h}$ bei $100{ }^{\circ} \mathrm{C}$ gerührt. Nach Abkühlen auf RT wurde die Reaktionsmischung durch $\mathrm{SiO}_{2}$ filtriert, mit EtOAc gespült und das Lösungsmittel in vacuo entfernt. Säulenchromatographische Reinigung lieferte Domino-Produkt 110 als gelben Feststoff.

Tabelle 14: Synthese der Domino-Produkte 110b-i.<smiles></smiles>

\begin{tabular}{cccccccc}
\hline Eintrag & Substrat & $\mathbf{R}^{\mathbf{1}}$ & $\mathbf{R}^{\mathbf{2}}$ & $\mathbf{X}$ & Produkt & Ausbeute [mg] & Ausbeute [\%] \\
\hline $1^{[\mathrm{a}]}$ & $\mathbf{1 5 1 b}$ & $\mathrm{H}$ & $\mathrm{H}$ & $\mathrm{N}$ & $\mathbf{1 1 0 b}$ & 20.2 & 79 \\
2 & $\mathbf{1 5 1 c}$ & $\mathrm{OMe}$ & $\mathrm{H}$ & $\mathrm{CH}$ & $\mathbf{1 1 0 c}$ & 140 & 54 \\
3 & $\mathbf{1 5 1 d}$ & $\mathrm{H}$ & $2-\mathrm{F}$ & $\mathrm{CH}$ & $\mathbf{1 1 0 d}$ & 14.7 & 57 \\
4 & $\mathbf{1 5 1 e}$ & $\mathrm{H}$ & $2-\mathrm{Me}$ & $\mathrm{CH}$ & $\mathbf{1 1 0 e}$ & 21.1 & 81 \\
$5^{[\mathrm{b}]}$ & $\mathbf{1 5 1 f}$ & $\mathrm{H}$ & $2-\mathrm{CF}_{3}$ & $\mathrm{CH}$ & $\mathbf{1 1 0 f}$ & 44.9 & 53 \\
6 & $\mathbf{1 5 1}$ & $\mathrm{H}$ & $2-\mathrm{OMe}$ & $\mathrm{CH}$ & $\mathbf{1 1 0 g}$ & 13.5 & 53 \\
7 & $\mathbf{1 5 1 h}$ & $\mathrm{H}$ & $4-\mathrm{Ph}$ & $\mathrm{CH}$ & $\mathbf{1 1 0 h}$ & 11.4 & 53 \\
$8^{[\mathrm{c}]}$ & $\mathbf{1 5 1 i}$ & $\mathrm{H}$ & $4-t \mathrm{Bu}$ & $\mathrm{CH}$ & $\mathbf{1 1 0 i}$ & 19.0 & 99 \\
\hline
\end{tabular}

[a] $c=0.09 \mathrm{~mol} / \mathrm{L} ;[\mathrm{b}] c=0.05 \mathrm{~mol} / \mathrm{L} ;$ [c] $\mathrm{Pd}(\mathrm{OAc})_{2}(0.01$ Äq. $), \mathrm{PPh}_{3}(0.05$ Äq.). 


\section{7-(2-Phenoxyphenyl)-9,10-dihydro-8H-benzo[5,6]chromen[4,3,2-ij]cyclopenta[g]isochinolin} (110b)

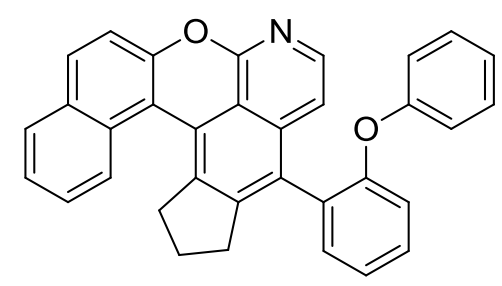

DC: $R_{f}=0.30\left(\mathrm{CH}_{2} \mathrm{Cl}_{2}\right)$.

UV/Vis $\left(\mathrm{CH}_{3} \mathrm{CN}\right): \lambda_{\max }(\mathrm{nm})(\lg \varepsilon)=194$ (5.042), 213 (4.763), 247 (4.345), 315 (3.940), 342 (3.788), 385 (3.971).

IR (ATR): $\tilde{v}\left(\mathrm{~cm}^{-1}\right)=1564,1488,1474,1444,1399,1366,1347,1263,1235,1219,813,797,750$, $733,691$.

${ }^{1} \mathrm{H}-\mathrm{NMR}\left(600 \mathrm{MHz}, \mathrm{C}_{2} \mathrm{D}_{2} \mathrm{Cl}_{4}, 100{ }^{\circ} \mathrm{C}\right): \delta(\mathrm{ppm})=1.95\left(\mathrm{~s}_{\mathrm{br}}, 2 \mathrm{H}\right), 2.81\left(\mathrm{~s}_{\mathrm{br}}, 1 \mathrm{H}\right), 2.88\left(\mathrm{~s}_{\mathrm{br}}, 1 \mathrm{H}\right), 2.93$ (t, $J=7.0 \mathrm{~Hz}, 2 \mathrm{H}), 6.78(\mathrm{~d}, J=8.2 \mathrm{~Hz}, 2 \mathrm{H}), 6.93(\mathrm{t}, J=7.1 \mathrm{~Hz}, 1 \mathrm{H}), 7.01(\mathrm{~d}, J=5.9 \mathrm{~Hz}, 1 \mathrm{H}), 7.14$ (t, $J=7.4 \mathrm{~Hz}, 2 \mathrm{H}), 7.21(\mathrm{~d}, J=8.2 \mathrm{~Hz}, 1 \mathrm{H}), 7.33(\mathrm{t}, J=7.4 \mathrm{~Hz}, 1 \mathrm{H}), 7.42(\mathrm{~d}, J=7.5 \mathrm{~Hz}, 1 \mathrm{H}), 7.45-$ $7.55(\mathrm{~m}, 3 \mathrm{H}), 7.58(\mathrm{t}, J=7.6 \mathrm{~Hz}, 1 \mathrm{H}), 7.79(\mathrm{~d}, J=8.4 \mathrm{~Hz}, 1 \mathrm{H}), 7.89-7.93$ (m, $2 \mathrm{H}), 7.96$ (d, $J=$ $5.9 \mathrm{~Hz}, 1 \mathrm{H})$.

${ }^{13} \mathrm{C}-\mathrm{NMR}\left(126 \mathrm{MHz}, \mathrm{C}_{2} \mathrm{D}_{2} \mathrm{Cl}_{4}, 50{ }^{\circ} \mathrm{C}\right): \delta(\mathrm{ppm})=26.4,33.1,36.8,74.0,113.3,114.7,117.0,117.9$, 118.3, 119.4, 122.8, 123.6, 124.3, 124.8, 125.4, 125.8, 127.3, 127.9, 128.8, 129.0, 129.2, 129.3, 130.7, $130.9,131.8,137.0,140.5,150.6,152.3,154.8,156.8,157.4$.

MS (ESI): $m / z(\%)=478.2(13)[\mathrm{M}+\mathrm{H}]^{+}$.

HRMS (ESI): $m / z=$ gef.: 478.1802, ber.: $478.1802[\mathrm{M}+\mathrm{H}]^{+}$.

$\mathrm{C}_{34} \mathrm{H}_{23} \mathrm{NO}_{2}$ (477.55). 


\section{5-Methoxy-7-(2-phenoxyphenyl)-9,10-dihydro-8H-benzo $[a]$ inden $[6,5,4-k l]$ xanthen $(110 c)$}

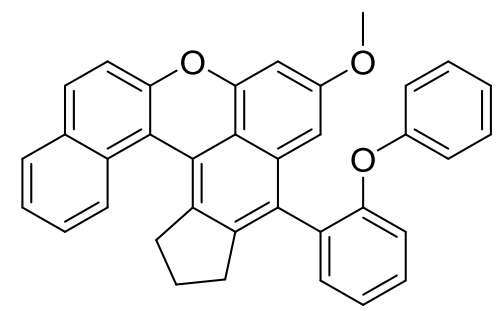

DC: $R_{f}=0.18(n$-Pentan/EtOAc, 100:1).

UV/Vis $\left(\mathrm{CH}_{3} \mathrm{CN}\right): \lambda_{\max }(\mathrm{nm})(\lg \varepsilon)=194$ (4.996), 215 (4.640), 322 (3.773), 398 (3.728).

IR (ATR): $\tilde{v}\left(\mathrm{~cm}^{-1}\right)=1628,1574,1485,1454,1444,1423,1373,1340,1263,1229,1194,1146,1115$, $1067,1034,831,812,734,703,691,670,639$.

${ }^{1} \mathrm{H}-\mathrm{NMR}\left(600 \mathrm{MHz}, \mathrm{C}_{2} \mathrm{D}_{2} \mathrm{Cl}_{4}, 100{ }^{\circ} \mathrm{C}\right): 1.92\left(\mathrm{~s}_{\mathrm{br}}, 2 \mathrm{H}\right), 2.71\left(\mathrm{~s}_{\mathrm{br}}, 2 \mathrm{H}\right), 2.82(\mathrm{t}, J=6.9 \mathrm{~Hz}, 2 \mathrm{H}), 3.80$ (s, $3 \mathrm{H}), 6.54(\mathrm{~s}, 1 \mathrm{H}), 6.69(\mathrm{~s}, 1 \mathrm{H}), 6.82(\mathrm{~d}, J=7.9 \mathrm{~Hz}, 2 \mathrm{H}), 6.94(\mathrm{t}, J=7.4 \mathrm{~Hz}, 1 \mathrm{H}), 7.15$ (t, $J=$ $7.2 \mathrm{~Hz}, 2 \mathrm{H}), 7.21(\mathrm{~d}, J=8.0 \mathrm{~Hz}, 1 \mathrm{H}), 7.31(\mathrm{t}, J=7.4 \mathrm{~Hz}, 1 \mathrm{H}), 7.37(\mathrm{~d}, J=8.8 \mathrm{~Hz}, 1 \mathrm{H}), 7.41-7.48$ $(\mathrm{m}, 3 \mathrm{H}), 7.53(\mathrm{t}, J=7.5 \mathrm{~Hz}, 1 \mathrm{H}), 7.77(\mathrm{~d}, J=8.5 \mathrm{~Hz}, 1 \mathrm{H}), 7.81(\mathrm{~d}, J=8.7 \mathrm{~Hz}, 1 \mathrm{H}), 7.85(\mathrm{~d}, J=$ $8.0 \mathrm{~Hz}, 1 \mathrm{H})$.

${ }^{13} \mathrm{C}-\mathrm{NMR}\left(126 \mathrm{MHz}, \mathrm{C}_{2} \mathrm{D}_{2} \mathrm{Cl}_{4}, 50{ }^{\circ} \mathrm{C}\right): \delta(\mathrm{ppm})=26.5,32.8,36.4,55.4,97.5,99.0,115.7,116.8$, 118.5, 119.4, 120.0, 121.9, 122.7, 123.6, 124.3, 125.0, 125.8, 127.4, 127.7, 128.7, 129.1, 129.4, 129.7, 130.2, 130.8, 132.0, 133.5, 133.8, 146.5, 151.6, 153.0, 155.0, 157.1, 157.7.

MS (ESI): $m / z(\%)=507.2(11)[\mathrm{M}+\mathrm{H}]^{+}$.

HRMS (ESI): $m / z=$ gef.: 507.1943, ber.: $507.1955[\mathrm{M}+\mathrm{H}]^{+}$.

$\mathbf{C}_{36} \mathbf{H}_{26} \mathbf{O}_{3}$ (506.59). 


\section{7-(2-(2-Fluorphenoxy)phenyl)-9,10-dihydro-8H-benzo[a]inden[6,5,4-kl]xanthen (110d)}

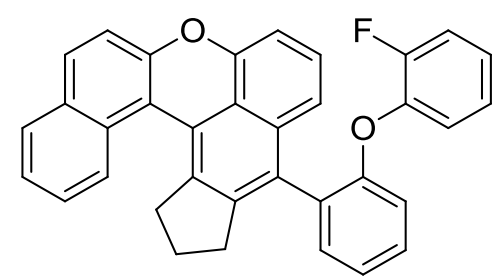

DC: $R_{f}=0.18\left(n-\mathrm{Pentan} / \mathrm{CH}_{2} \mathrm{Cl}_{2}, 10: 1\right)$.

UV/Vis $\left(\mathrm{CH}_{3} \mathrm{CN}\right): \lambda_{\max }(\mathrm{nm})(\lg \varepsilon)=194(5.124), 215$ (4.882), $266(4.414), 326$ (3.974), 353 (3.839), 396 (3.959).

IR (ATR): $\tilde{v}\left(\mathrm{~cm}^{-1}\right)=3391,3061,2953,1623,1596,1573,1498,1484,1456,1444,1433,1384,1374$, $1352,1260,1252,1239,1202,1103,982,882,811,801,777,746$.

${ }^{1} \mathbf{H}-\mathrm{NMR}\left(600 \mathrm{MHz}, \mathrm{C}_{2} \mathrm{D}_{2} \mathrm{Cl}_{4}, 100{ }^{\circ} \mathrm{C}\right): \delta(\mathrm{ppm})=1.94(\mathrm{~s}, 1 \mathrm{H}), 2.00(\mathrm{~s}, 1 \mathrm{H}), 2.73(\mathrm{~s}, 2 \mathrm{H}), 2.83(\mathrm{dt}$, $J=14.5,6.2 \mathrm{~Hz}, 1 \mathrm{H}), 2.96(\mathrm{dt}, J=14.8,7.2 \mathrm{~Hz}, 1 \mathrm{H}), 6.84-7.00(\mathrm{~m}, 5 \mathrm{H}), 7.11(\mathrm{~d}, J=8.2 \mathrm{~Hz}, 2 \mathrm{H})$, 7.24 (t, $J=7.9 \mathrm{~Hz}, 1 \mathrm{H}), 7.30$ (t, $J=7.4 \mathrm{~Hz}, 1 \mathrm{H}), 7.37$ (d, $J=8.7 \mathrm{~Hz}, 1 \mathrm{H}), 7.40-7.44$ (m, $2 \mathrm{H}), 7.44-$ $7.49(\mathrm{~m}, 1 \mathrm{H}), 7.54(\mathrm{t}, J=7.5 \mathrm{~Hz}, 1 \mathrm{H}), 7.78(\mathrm{~d}, J=8.3 \mathrm{~Hz}, 1 \mathrm{H}), 7.82(\mathrm{~d}, J=8.7 \mathrm{~Hz}, 1 \mathrm{H}), 7.86(\mathrm{~d}, J=$ $8.0 \mathrm{~Hz}, 1 \mathrm{H})$.

${ }^{13} \mathrm{C}-\mathrm{NMR}\left(126 \mathrm{MHz}, \mathrm{C}_{2} \mathrm{D}_{2} \mathrm{Cl}_{4}, 50{ }^{\circ} \mathrm{C}\right): \delta(\mathrm{ppm})=26.5,32.5,36.6,99.3,106.7,115.7,116.3,116.5$, 116.8, 117.6, 121.9, 123.4, 124.1, 124.2, 124.5, 125.1, 125.5, 127.8, 128.8, 129.1, 129.4, 129.8, 130.8, $132.0,132.9,135.6,145.9,150.6,153.3,155.0$.

MS (ESI): $m / z(\%)=495.2(3)[\mathrm{M}+\mathrm{H}]^{+}$.

HRMS (ESI): $m / z=$ gef.: 495.1742, ber.: $495.1755[\mathrm{M}+\mathrm{H}]^{+}$.

$\mathbf{C}_{35} \mathbf{H}_{23} \mathbf{F O}_{2}$ (494.55). 


\section{7-(2-(2-Tolyloxy)phenyl)-9,10-dihydro-8H-benzo $[a]$ inden $[6,5,4-k l]$ xanthen $(110 \mathrm{e})$}

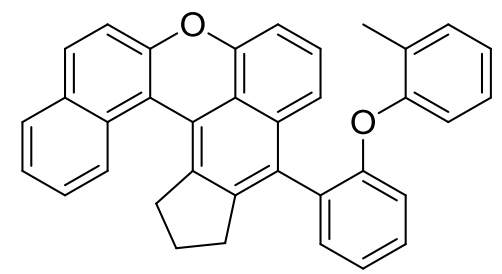

DC: $R_{f}=0.20\left(n-\mathrm{Pentan} / \mathrm{CH}_{2} \mathrm{Cl}_{2}, 10: 1\right)$.

UV/Vis $\left(\mathrm{CH}_{3} \mathrm{CN}\right): \lambda_{\max }(\mathrm{nm})(\lg \varepsilon)=195$ (5.099), 213 (4.882), 266 (4.386), 326 (3.963), 353 (3.824), 396 (3.935).

IR (ATR): $\tilde{v}\left(\mathrm{~cm}^{-1}\right)=1623,1573,1508,1481,1459,1444,1382,1373,1351,1263,1251,1237,1201$, 1181, 1155, 1111, 1096, 1070, 981, 900, 877, 811, 777, 735, 704, 677, 666, 605, 557, 539.

${ }^{1} \mathrm{H}-\mathrm{NMR}\left(600 \mathrm{MHz}, \mathrm{C}_{2} \mathrm{D}_{2} \mathrm{Cl}_{4}, 100{ }^{\circ} \mathrm{C}\right): 2.02(\mathrm{~s}, 3 \mathrm{H})\left(\mathrm{s}_{\mathrm{br}}, 2 \mathrm{H}\right), 2.85(\mathrm{~s}, 2 \mathrm{H}), 2.93(\mathrm{t}, J=7.1 \mathrm{~Hz}, 2 \mathrm{H})$, $6.88(\mathrm{~d}, J=8.1 \mathrm{~Hz}, 1 \mathrm{H}), 6.96(\mathrm{t}, J=7.4 \mathrm{~Hz}, 2 \mathrm{H}), 7.01(\mathrm{~d}, J=7.4 \mathrm{~Hz}, 1 \mathrm{H}), 7.09$ (dt, $J=17.5,8.6 \mathrm{~Hz}$, $3 \mathrm{H}), 7.20(\mathrm{~d}, J=8.3 \mathrm{~Hz}, 1 \mathrm{H}), 7.31(\mathrm{q}, J=7.4 \mathrm{~Hz}, 2 \mathrm{H}), 7.41(\mathrm{~d}, J=8.8 \mathrm{~Hz}, 1 \mathrm{H}), 7.47$ (dq, $J=15.8$, $7.8 \mathrm{~Hz}, 3 \mathrm{H}), 7.56(\mathrm{t}, J=7.6 \mathrm{~Hz}, 1 \mathrm{H}), 7.80(\mathrm{~d}, J=8.4 \mathrm{~Hz}, 1 \mathrm{H}), 7.84(\mathrm{~d}, J=8.8 \mathrm{~Hz}, 1 \mathrm{H}), 7.88(\mathrm{~d}, J=$ $8.0 \mathrm{~Hz}, 1 \mathrm{H})$.

${ }^{13} \mathrm{C}-\mathrm{NMR}\left(126 \mathrm{MHz}, \mathrm{C}_{2} \mathrm{D}_{2} \mathrm{Cl}_{4}, 50{ }^{\circ} \mathrm{C}\right): \delta(\mathrm{ppm})=15.6,26.6,32.7,36.6,53.4,106.8,115.7,116.9$, $117.8,118.5,121.8,122.9,123.1,124.2$, 124.6, 125.1, 125.5, 126.6, 127.8, 128.4, 128.7, 129.1, 129.4, $129.5,129.7,130.8,130.9,131.9,133.2,135.6,145.6,150.7,153.3,154.6,155.1$.

MS (ESI): $m / z(\%)=491.2(100)[\mathrm{M}+\mathrm{H}]^{+}, 513.2(22)[\mathrm{M}+\mathrm{Na}]^{+}$.

HRMS (ESI): $m / z=$ gef.: 491.1995, ber.: $491.2006[\mathrm{M}+\mathrm{H}]^{+}$.

$\mathbf{C}_{36} \mathbf{H}_{26} \mathbf{O}_{2}$ (490.59). 
7-(2-(2-(Trifluormethyl)phenoxy)phenyl)-9,10-dihydro-8H-benzo[a]inden[6,5,4- $k l]$ xanthen (110f)<smiles>Fc1ccccc1Oc1ccccc1-c1c2c(c3c4c(cccc14)Oc1ccc4ccccc4c1-3)CCC2</smiles>

DC: $R_{f}=0.22\left(n-\mathrm{Pentan} / \mathrm{CH}_{2} \mathrm{Cl}_{2}, 20: 1\right)$.

UV/Vis $\left(\mathrm{CH}_{3} \mathrm{CN}\right): \lambda_{\max }(\mathrm{nm})(\lg \varepsilon)=193$ (5.425), 215 (4.892), 266 (4.436), 326 (4.000), 353 (3.859), 396 (3.964).

IR (ATR): $\tilde{v}\left(\mathrm{~cm}^{-1}\right)=2954,2926,1623,1612,1591,1574,1508,1482,1458,1446,1434,1385,1375$, 1352, 1321, 1249, 1164, 1133, 1116, 1054, 1036, 880, 812, 750, 650.

${ }^{1} \mathrm{H}-\mathrm{NMR}\left(600 \mathrm{MHz}, \mathrm{C}_{2} \mathrm{D}_{2} \mathrm{Cl}_{4}, 100{ }^{\circ} \mathrm{C}\right): \delta(\mathrm{ppm})=1.95\left(\mathrm{~s}_{\mathrm{br}}, 1 \mathrm{H}\right), 2.03\left(\mathrm{~s}_{\mathrm{br}}, 1 \mathrm{H}\right), 2.78\left(\mathrm{~s}_{\mathrm{br}}, 1 \mathrm{H}\right), 2.80$ $(\mathrm{dt}, J=14.3,5.9 \mathrm{~Hz}, 1 \mathrm{H}), 2.87\left(\mathrm{~s}_{\mathrm{br}}, 1 \mathrm{H}\right), 3.03(\mathrm{dt}, J=15.2,7.4 \mathrm{~Hz}, 1 \mathrm{H}), 1.91-1.99$ (m, $\left.1 \mathrm{H}\right), 1.99$ $2.13(\mathrm{~m}, 1 \mathrm{H}), 6.88(\mathrm{~d}, J=8.3 \mathrm{~Hz}, 1 \mathrm{H}), 6.93(\mathrm{~d}, J=7.5 \mathrm{~Hz}, 1 \mathrm{H}), 6.98(\mathrm{t}, J=7.5 \mathrm{~Hz}, 1 \mathrm{H}), 7.14$ (dd, $J=12.4,8.4 \mathrm{~Hz}, 2 \mathrm{H}), 7.24(\mathrm{t}, J=7.9 \mathrm{~Hz}, 1 \mathrm{H}), 7.30(\mathrm{t}, J=7.8 \mathrm{~Hz}, 1 \mathrm{H}), 7.36(\mathrm{~d}, J=8.8 \mathrm{~Hz}, 1 \mathrm{H})$, 7.39 (t, $J=7.4 \mathrm{~Hz}, 1 \mathrm{H}), 7.44-7.51(\mathrm{~m}, 4 \mathrm{H}), 7.53$ (t, $J=7.6 \mathrm{~Hz}, 1 \mathrm{H}), 7.73$ (d, $J=8.4 \mathrm{~Hz}, 1 \mathrm{H}), 7.81$ $(\mathrm{d}, J=8.7 \mathrm{~Hz}, 1 \mathrm{H}), 7.84(\mathrm{~d}, J=8.0 \mathrm{~Hz}, 1 \mathrm{H})$.

${ }^{13} \mathrm{C}-\mathrm{NMR}\left(126 \mathrm{MHz}, \mathrm{C}_{2} \mathrm{D}_{2} \mathrm{Cl}_{4}, 50{ }^{\circ} \mathrm{C}\right): \delta(\mathrm{ppm})=28.0,34.0,38.1,108.2,117.2,118.3,118.8,123.2$, 123.5, 123.6, 125.8, 126.1, 126.7, 127.0, 127.0, 128.2, 128.3, 129.3, 130.6, 130.9, 131.3, 132.3, 132.6, $133.6,134.3,137.2,147.9,152.2,154.8,156.6$.

Weitere Signale konnten nicht beobachtet werden.

MS (ESI): $m / z(\%)=545.2(100)[\mathrm{M}+\mathrm{H}]^{+}, 567.2(71)[\mathrm{M}+\mathrm{Na}]^{+}$.

HRMS (ESI): $m / z=$ gef.: 545.1702 , ber.: $545.1723[\mathrm{M}+\mathrm{H}]^{+}$.

$\mathrm{C}_{36} \mathrm{H}_{23} \mathrm{~F}_{3} \mathrm{O}_{2}$ (544.56). 


\section{7-(2-(2-Methoxyphenoxy)phenyl)-9,10-dihydro-8H-benzo[a]inden[6,5,4-kl]xanthen $(110 \mathrm{~g})$}

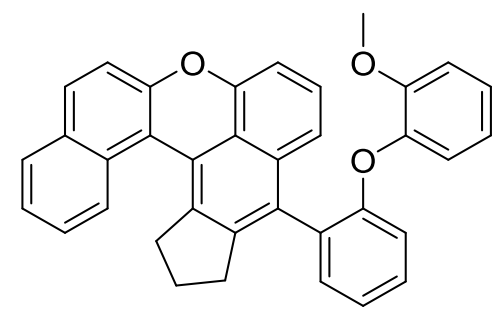

DC: $R_{f}=0.29(n-$ Pentan/EtOAc, 50:1).

UV/Vis $\left(\mathrm{CH}_{3} \mathrm{CN}\right): \lambda_{\max }(\mathrm{nm})(\lg \varepsilon)=194$ (5.046), 214 (4.759), 267 (4.262), 326 (3.823), 353 (3.678), 397 (3.782).

IR (ATR): $\tilde{v}\left(\mathrm{~cm}^{-1}\right)=1623,1573,1498,1483,1455,1444,1433,1384,1373,1351,1253,1237,1210$, 1175, 1157, 1111, 1097, 1070, 1041, 1026, 981, 901, 877, 811, 797, 776, 733, 702, 637, 605, 557, $540,530$.

${ }^{1} \mathrm{H}-\mathrm{NMR}\left(600 \mathrm{MHz}, \mathrm{C}_{2} \mathrm{D}_{2} \mathrm{Cl}_{4}, 100{ }^{\circ} \mathrm{C}\right): \delta(\mathrm{ppm})=1.97\left(\mathrm{~s}_{\mathrm{br}}, 2 \mathrm{H}\right), 2.81\left(\mathrm{~s}_{\mathrm{br}}, 2 \mathrm{H}\right), 2.85(\mathrm{dt}, J=15.4$, $6.9 \mathrm{~Hz}, 1 \mathrm{H}), 2.97(\mathrm{dt}, J=15.1,7.1 \mathrm{~Hz}, 1 \mathrm{H}), 3.59$ (s, $3 \mathrm{H}), 6.82$ (d, $J=6.5 \mathrm{~Hz}, 2 \mathrm{H}), 6.87-6.91(\mathrm{~m}$, $1 \mathrm{H}), 6.93-7.04(\mathrm{~m}, 3 \mathrm{H}), 7.16(\mathrm{~d}, J=8.4 \mathrm{~Hz}, 1 \mathrm{H}), 7.24$ (dt, $J=13.7,7.7 \mathrm{~Hz}, 2 \mathrm{H}), 7.34-7.41$ (m, $3 \mathrm{H}), 7.46(\mathrm{t}, J=7.5 \mathrm{~Hz}, 1 \mathrm{H}), 7.53(\mathrm{t}, J=7.7 \mathrm{~Hz}, 1 \mathrm{H}), 7.78(\mathrm{~d}, J=8.4 \mathrm{~Hz}, 1 \mathrm{H}), 7.81(\mathrm{~d}, J=8.7 \mathrm{~Hz}$, $1 \mathrm{H}), 7.85(\mathrm{~d}, J=8.1 \mathrm{~Hz}, 1 \mathrm{H})$.

${ }^{13} \mathbf{C}-\mathbf{N M R}\left(126 \mathrm{MHz}, \mathrm{C}_{2} \mathrm{D}_{2} \mathrm{Cl}_{4}, 50{ }^{\circ} \mathrm{C}\right): \delta(\mathrm{ppm})=26.6,32.6,36.6,55.7,106.7,113.1,115.7$, $116.9,118.0,120.8,121.7,122.5,124.2,124.5,125.0,125.3,125.6,127.8,128.5,128.6,129.4,129.7$, $130.8,131.7,133.2,135.6,145.9,150.6,151.0,153.3,155.7$.

MS (ESI): $m / z(\%)=507.2(9)[\mathrm{M}+\mathrm{H}]^{+}, 529.2(4)[\mathrm{M}+\mathrm{Na}]^{+}$.

HRMS (ESI): $m / z=$ gef.: 507.1947, ber.: $507.1955[\mathrm{M}+\mathrm{H}]^{+}$.

$\mathbf{C}_{36} \mathbf{H}_{26} \mathbf{O}_{3}$ (506.59). 
7-(2-([1,1'-Biphenyl]-4-yloxy)phenyl)-9,10-dihydro-8H-benzo[a]inden[6,5,4-kl]xanthen (110h)<smiles></smiles>

DC: $R_{f}=0.11\left(n-\mathrm{Pentan} / \mathrm{CH}_{2} \mathrm{Cl}_{2}, 10: 1\right)$

UV/Vis $\left(\mathrm{CH}_{3} \mathrm{CN}\right): \lambda_{\max }(\mathrm{nm})(\lg \varepsilon)=194$ (5.085), 248 (4.285), 263 (4.258), 326 (3.593), 397 (3.570).

IR (ATR): $\tilde{v}\left(\mathrm{~cm}^{-1}\right)=3379,2950,2922,2851,1620,1600,1571,1509,1476,1459,1440,1432,1396$, 1380, 1370, 1351, 1249, 1234, 1219, 1162, 1127, 1111, 1103, 1069, 1048, 1020, 961, 944, 907, 898, $864,844,816,804,792,759,750,728,719,699,684,649,634,605,584,559,542$, 531, 524, 502.

${ }^{1} \mathrm{H}-\mathrm{NMR}\left(600 \mathrm{MHz}, \mathrm{C}_{2} \mathrm{D}_{2} \mathrm{Cl}_{4}, 100{ }^{\circ} \mathrm{C}\right): \delta(\mathrm{ppm})=1.93\left(\mathrm{~s}_{\mathrm{br}}, 2 \mathrm{H}\right), 2.67\left(\mathrm{~s}_{\mathrm{br}}, 2 \mathrm{H}\right), 2.81(\mathrm{dt}, J=15.2$, $7.2 \mathrm{~Hz}, 1 \mathrm{H}), 2.87(\mathrm{dt}, J=13.5,6.7 \mathrm{~Hz}, 1 \mathrm{H}), 6.81(\mathrm{~d}, J=8.4 \mathrm{~Hz}, 2 \mathrm{H}), 6.96(\mathrm{~d}, J=7.5 \mathrm{~Hz}, 1 \mathrm{H}), 7.12$ $(\mathrm{d}, J=8.3 \mathrm{~Hz}, 1 \mathrm{H}), 7.24-7.53(\mathrm{~m}, 17 \mathrm{H}), 7.65(\mathrm{~d}, J=6.6 \mathrm{~Hz}, 1 \mathrm{H}), 7.82(\mathrm{dd}, J=14.0,8.5 \mathrm{~Hz}, 2 \mathrm{H})$.

${ }^{13}$ C-NMR $\left(126 \mathrm{MHz}, \mathrm{C}_{2} \mathrm{D}_{2} \mathrm{Cl}_{4}, 50{ }^{\circ} \mathrm{C}\right): \delta(\mathrm{ppm})=26.5,35.5,36.4,74.0,106.8,115.6,116.8,117.6$, 118.5, 120.2, 121.8, 123.9, 124.2, 124.6, 125.1, 125.4, 125.6, 126.6, 126.8, 127.6, 127.7, 128.1, 128.6, $128.8,129.3,129.7,130.5,130.8,132.1,132.9,135.3,135.5,145.6,150.6,153.3,154.9$.

MS (ESI): $m / z(\%)=575.2(2)[\mathrm{M}+\mathrm{Na}]^{+}$.

HRMS (ESI): $m / z=$ gef.: 575.1989 , ber.: $575.1982[\mathrm{M}+\mathrm{H}]^{+}$.

$\mathrm{C}_{41} \mathrm{H}_{28} \mathrm{O}_{2}$ (552.66). 
7-(2-(4-(tert-Butyl)phenoxy)phenyl)-9,10-dihydro-8H-benzo[a]inden[6,5,4-kl]xanthen (110i)

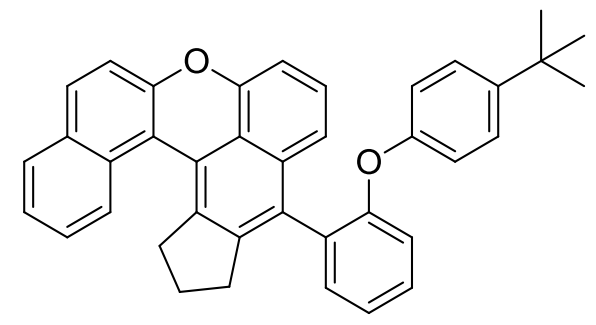

DC: $R_{f}=0.36\left(n-\mathrm{Pentan} / \mathrm{CH}_{2} \mathrm{Cl}_{2}, 5: 1\right)$.

UV/Vis $\left(\mathrm{CH}_{3} \mathrm{CN}\right): \lambda_{\max }(\mathrm{nm})(\lg \varepsilon)=194$ (5.287), 213 (4.615), 267 (4.124), 326 (3.700), 397 (3.659).

IR (ATR): $\tilde{v}\left(\mathrm{~cm}^{-1}\right)=3344,3057,2956,2925,2866,1621,1600,1574,1506,1481,1458,1445,1433$, 1373, 1264, 1237, 1171, 1141, 1109, 1098, 1070, 1049, 1032, 1013, 1003, 982, 948, 923, 900, 877, 846, 832, 812, 784, 751, 736, 702, 665, 648, 637, 629, 606, 546.

${ }^{1} \mathrm{H}-\mathrm{NMR}\left(600 \mathrm{MHz}, \mathrm{C}_{2} \mathrm{D}_{2} \mathrm{Cl}_{4}, 100{ }^{\circ} \mathrm{C}\right): \delta(\mathrm{ppm})=1.25(\mathrm{~s}, 9 \mathrm{H}), 1.94\left(\mathrm{~s}_{\mathrm{br}}, 2 \mathrm{H}\right), 2.74\left(\mathrm{~s}_{\mathrm{br}}, 2 \mathrm{H}\right), 2.84(\mathrm{t}$, $J=6.8 \mathrm{~Hz}, 2 \mathrm{H}), 6.73(\mathrm{~d}, J=8.7 \mathrm{~Hz}, 1 \mathrm{H}), 6.96(\mathrm{~d}, J=7.5 \mathrm{~Hz}, 1 \mathrm{H}), 7.12(\mathrm{~d}, J=8.4 \mathrm{~Hz}, 1 \mathrm{H}), 7.15(\mathrm{~d}$, $J=8.7 \mathrm{~Hz}, 1 \mathrm{H}), 7.20(\mathrm{~d}, J=8.1 \mathrm{~Hz}, 1 \mathrm{H}), 7.26(\mathrm{t}, J=8.0 \mathrm{~Hz}, 1 \mathrm{H}), 7.30(\mathrm{t}, J=7.4 \mathrm{~Hz}, 1 \mathrm{H}), 7.38(\mathrm{~d}$, $J=8.7 \mathrm{~Hz}, 1 \mathrm{H}), 7.41-7.48(\mathrm{~m}, 3 \mathrm{H}), 7.53(\mathrm{t}, J=7.6 \mathrm{~Hz}, 1 \mathrm{H}), 7.77(\mathrm{~d}, J=8.4 \mathrm{~Hz}, 1 \mathrm{H}), 7.82(\mathrm{~d}, J=$ $8.7 \mathrm{~Hz}, 1 \mathrm{H}), 7.86(\mathrm{~d}, J=8.0 \mathrm{~Hz}, 1 \mathrm{H})$.

${ }^{13} \mathbf{C}-\mathrm{NMR}\left(126 \mathrm{MHz}, \mathrm{C}_{2} \mathrm{D}_{2} \mathrm{Cl}_{4}, 50{ }^{\circ} \mathrm{C}\right): \delta(\mathrm{ppm})=26.5,31.2,32.6,33.8,36.6,74.0,106.7,115.7$, $116.9,117.7,118.3,119.1,121.8,123.2,124.2,124.6,125.0,125.5,125.5,125.8,127.8,128.3,128.7$, $129.4,129.7,130.0,130.8,132.0,133.0,135.5,145.7,145.8,150.6,153.3,154.5,155.5$.

MS (ESI): $m / z(\%)=532.2(100)[\mathrm{M}+\mathrm{H}]^{+}$.

HRMS (ESI): $m / z=$ gef.: 532.2402 , ber.: $532.2397[\mathrm{M}+\mathrm{H}]^{+}$.

$\mathrm{C}_{39} \mathrm{H}_{32} \mathrm{O}_{2}$ (532.68). 


\subsubsection{Synthese weiterer Aryliodide vom Typ 111}

\section{0-Bromphenanthren-9-ol (156) ${ }^{\mathrm{cc}}$}

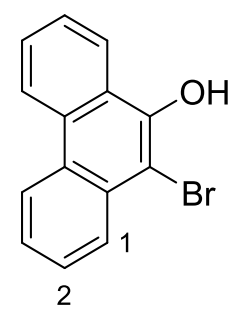

Ein Gemisch von 9-Phenanthrol (155) (1.23 g, 6.33 mmol, 1.00 Äq.), N-Bromsuccinimid (1.13 g, $6.33 \mathrm{mmol}, 1.00$ Äq.) und $i \mathrm{PrNH}_{2}\left(64.1 \mathrm{mg}, 633 \mu \mathrm{mol}, 0.10\right.$ Äq.) in $\mathrm{CH}_{2} \mathrm{Cl}_{2}(80 \mathrm{~mL})$ wurde $45 \mathrm{~min}$ bei RT gerührt und das Lösungsmittel in vacuo entfernt. Säulenchromatographische Reinigung $\left(\mathrm{SiO}_{2}\right.$, $n$-Pentan/ $\left.\mathrm{CH}_{2} \mathrm{Cl}_{2}, 5: 1\right)$ und Umkristallisation aus $n$-Hexan $(70 \mathrm{~mL})$ lieferten Bromid 156 als gelben Feststoff (1.10 g, $4.03 \mathrm{mmol}, 64 \%)$.

DC: $R_{f}=0.52\left(n-\mathrm{Pentan} / \mathrm{CH}_{2} \mathrm{Cl}_{2}, 5: 1\right)$.

UV/Vis $\left(\mathrm{CH}_{3} \mathrm{CN}\right): \lambda_{\max }(\mathrm{nm})(\lg \varepsilon)=213$ (4.456), 223 (4.369), 255 (4.663), 274 (4.107), 297 (3.948), 307 (3.917), 339 (3.140), 356 (3.174).

IR (ATR): $\tilde{v}\left(\mathrm{~cm}^{-1}\right)=3492,3477,1612,1596,1492,1449,1429,1313,1272,1252,1238,1213,1157$, $1112,1079,1045,924,820,760,745,719,660,642,621,615,527$.

${ }^{1} \mathbf{H}-\mathbf{N M R}\left(600 \mathrm{MHz}, \mathrm{CDCl}_{3}\right): \delta(\mathrm{ppm})=6.28(\mathrm{~s}, 1 \mathrm{H}, \mathrm{OH}), 7.54(\mathrm{ddd}, J=8.2,7.0,1.2 \mathrm{~Hz}, 1 \mathrm{H}, 3-\mathrm{H})$, 7.64 (dddd, $J=8.2,7.0,3.3,1.2 \mathrm{~Hz}, 2 \mathrm{H}, 2-\mathrm{H}, 7-\mathrm{H}$ ), 7.70 (ddd, $J=8.3,7.0,1.4 \mathrm{~Hz}, 1 \mathrm{H}, 6-\mathrm{H}$ ), 8.108.14 (m, $1 \mathrm{H}, 1-\mathrm{H}), 8.35-8.38$ (m, $1 \mathrm{H}, 8-\mathrm{H}), 8.59$ (dd, $J=8.2,0.5 \mathrm{~Hz}, 1 \mathrm{H}, 4-\mathrm{H}), 8.63$ (d, $J=8.3 \mathrm{~Hz}$, $1 \mathrm{H}, 5-\mathrm{H})$.

${ }^{13}$ C-NMR $\left(126 \mathrm{MHz}, \mathrm{CDCl}_{3}\right): \delta(\mathrm{ppm})=103.6(\mathrm{C}-10), 122.7(\mathrm{C}-4, \mathrm{C}-5), 123.2(\mathrm{C}-8), 124.8(\mathrm{C}-8 \mathrm{a})$, 124.9 (C-3), 126.2 (C-1), 127.0 (C-7), 127.3 (C-4a), 127.8 (C-2, C-6), 130.3 (C-10a), 130.4 (C-4b), $147.0(\mathrm{C}-9)$.

HRMS (ESI): $m / z=$ gef.: 270.9767, ber.: $270.9764\left[\mathrm{M}\left({ }^{79} \mathrm{Br}\right)+\mathrm{H}\right]^{+}$; gef.: 272.9741 , ber.: 272.9744 $\left[\mathrm{M}\left({ }^{81} \mathrm{Br}\right)+\mathrm{H}\right]^{+}$.

$\mathbf{C}_{14} \mathrm{H}_{9} \mathrm{BrO}$ (273.13).

${ }^{c c}$ Für Originalvorschrift siehe: D. Peña, D. Pérez, E. Guitián, L. Castedo, J. Org. Chem. 2000, 65, 6944-6950. 


\section{1-Brom-2-(brommethyl)naphthalin (158) $)^{\text {dd }}$}<smiles>BrCc1ccc2ccccc2c1Br</smiles>

Ein Gemisch von 1-Brom-2-methylnaphthalin (157) (9.92 g, $7.00 \mathrm{~mL}, 44.9 \mathrm{mmol}, 1.00$ Äq.), $N$-Bromsuccinimid (7.99 g, 44.9 mmol, 1.00 Äq.) und AIBN (73.7 mg, $449 \mu \mathrm{mol}, 0.01$ Äq.) in $\mathrm{CCl}_{4}$ $(60 \mathrm{~mL})$ wurde $15 \mathrm{~h}$ bei $80^{\circ} \mathrm{C}$ gerührt. Die Reaktionsmischung wurde heiß filtriert und der Rückstand mit $\mathrm{CCl}_{4}$ gewaschen. Das Lösungsmittel des Filtrats wurde in vacuo entfernt. Säulenchromatographische Reinigung $\left(\mathrm{SiO}_{2}, n\right.$-Pentan) lieferte Dibromid 158 als gelben Feststoff (11.4 g, $38.0 \mathrm{mmol}, 85 \%)$.

DC: $R_{f}=0.34(\mathrm{PE})$.

UV/Vis $\left(\mathrm{CH}_{3} \mathrm{CN}\right): \lambda_{\max }(\mathrm{nm})(\lg \varepsilon)=236$ (4.671), 287 (3.833), 330 (2.852).

IR (ATR): $\tilde{v}\left(\mathrm{~cm}^{-1}\right)=1498,1327,1259,1223,1202,978,867,856,807,771,752,724,669,643,579$, 528.

${ }^{1}$ H-NMR $\left(300 \mathrm{MHz}, \mathrm{CDCl}_{3}\right): \delta(\mathrm{ppm})=4.87\left(\mathrm{~s}, 2 \mathrm{H}, \mathrm{CH}_{2}\right), 7.52(\mathrm{~d}, J=8.5 \mathrm{~Hz}, 1 \mathrm{H}, 3-\mathrm{H}), 7.54(\mathrm{ddd}$, $J=8.0,6.9,1.3 \mathrm{~Hz}, 1 \mathrm{H}, 6-\mathrm{H}), 7.62(\mathrm{ddd}, J=8.3,6.9,1.4 \mathrm{~Hz}, 1 \mathrm{H}, 7-\mathrm{H}), 7.79(\mathrm{~d}, J=6.1 \mathrm{~Hz}, 1 \mathrm{H}$, 4-H), 7.82 (d, $J=7.6 \mathrm{~Hz}, 1 \mathrm{H}, 5-\mathrm{H}), 8.34(\mathrm{~d}, J=8.7 \mathrm{~Hz}, 1 \mathrm{H}, 8-\mathrm{H})$.

${ }^{13}$ C-NMR $\left(126 \mathrm{MHz}, \mathrm{CDCl}_{3}\right): \delta(\mathrm{ppm})=34.8\left(\mathrm{CH}_{2}\right), 124.9(\mathrm{C}-1), 127.2,127.6,127.7,127.8,128.1$, 128.3 (C-3, C-4, C-5, C-6, C-7, C-8), 132.5 (C-8a), 134.1 (C-4a), 134.9 (C-2).

MS (EI): $m / z(\%)=139.2(85)[\mathrm{M}-2 \mathrm{Br}]^{+}, 221.1(100)[\mathrm{M}-\mathrm{Br}]^{+}, 300.1(19)[\mathrm{M}]^{+}$.

HRMS (EI): $m / z=$ gef.: 297.8997, ber.: 297.8993 [M] ${ }^{+}$.

$\mathbf{C}_{11} \mathbf{H}_{8} \mathrm{Br}_{2}$ (299.99).

\footnotetext{
${ }^{\text {dd }}$ Für Originalvorschrift siehe: M. S. Newman, A. I. Kosak, J. Org. Chem. 1949, 14, 375-378.
} 


\section{1-Brom-2-((2-iodphenoxy)methyl)naphthalin (111e)}

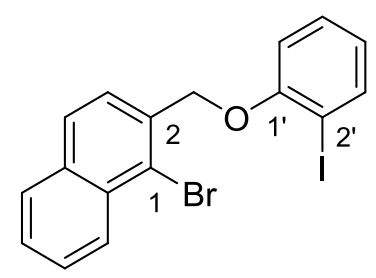

Ein Gemisch von 1-Brom-2-(brommethyl)naphthalin (158) (2.00 g, $6.67 \mathrm{mmol}, \quad 1.00$ Äq.), 2-Iodphenol (159) (1.47 g, 6.67 mmol, 1.00 Äq.) und $\mathrm{K}_{2} \mathrm{CO}_{3}$ (1.84 g, $13.3 \mathrm{~mol}, 2.00$ Äq.) in $\mathrm{CH}_{3} \mathrm{CN}$ (60 mL) wurde $18 \mathrm{~h}$ bei $60{ }^{\circ} \mathrm{C}$ gerührt. Nach Abkühlen auf RT wurde $\mathrm{H}_{2} \mathrm{O}(200 \mathrm{~mL})$ hinzugefügt und die Mischung mit $\mathrm{CH}_{2} \mathrm{Cl}_{2}$ extrahiert $(3 \times 200 \mathrm{~mL})$. Die vereingten organischen Phasen wurden über $\mathrm{Na}_{2} \mathrm{SO}_{4}$ getrocknet, filtriert und das Lösungsmittel in vacuo entfernt. Ether 111e wurde als weißer Feststoff (2.75 g, $6.26 \mathrm{mmol}, 94 \%)$ erhalten.

DC: $R_{f}=0.24$ (n-Pentan).

UV/Vis $\left(\mathrm{CH}_{3} \mathrm{CN}\right): \lambda_{\max }(\mathrm{nm})(\lg \varepsilon)=229$ (4.952), 277 (3.940), 285 (3.946).

IR (ATR): $\tilde{v}\left(\mathrm{~cm}^{-1}\right)=1582,1568,1558,1502,1475,1462,1436,1427,1329,1280,1250,1226,1121$, 1064, 1016, 964, 861, 841, 812, 765, 743, 644, 527.

${ }^{1} \mathbf{H}-\mathrm{NMR}\left(600 \mathrm{MHz}, \mathrm{CDCl}_{3}\right): \delta(\mathrm{ppm})=5.42\left(\mathrm{~s}, 2 \mathrm{H}, \mathrm{OCH}_{2}\right), 6.70-6.78\left(\mathrm{~m}, 1 \mathrm{H}, 4{ }^{\prime}-\mathrm{H}\right), 6.89$ (dd, $J=$ 8.2, $1.2 \mathrm{~Hz}, 1 \mathrm{H}, 6^{\prime}-\mathrm{H}$ ), 7.27 (tdd, $J=7.4,1.6,0.8 \mathrm{~Hz}, 1 \mathrm{H}, 5^{\prime}-\mathrm{H}$ ), 7.52 (ddd, $J=8.0,6.9,1.1 \mathrm{~Hz}, 1 \mathrm{H}$, 6-H), 7.60 (ddd, $J=8.5,6.8,1.3 \mathrm{~Hz}, 1 \mathrm{H}, 7-\mathrm{H}), 7.80-7.84$ (m, $2 \mathrm{H}, 5-\mathrm{H}, 3$ '-H), 7.84-7.88 (m, $2 \mathrm{H}$, 3-H, 4-H), 8.32 (d, J = 8.5 Hz, $1 \mathrm{H}, 8-\mathrm{H})$.

${ }^{13}$ C-NMR $\left(126 \mathrm{MHz}, \mathrm{CDCl}_{3}\right): \delta(\mathrm{ppm})=70.9\left(\mathrm{OCH}_{2}\right), 86.5(\mathrm{C}-2$ ') $112.7(\mathrm{C}-6$ ') $121.4(\mathrm{C}-1), 123.0$ (C-4'), 125.4 (C-3), 126.6, 126.7 (C-6, C-8), 127.5 (C-7), 128.0 (C-4), 128.2 (C-5), 129.5 (C-5'), 132.0 (C-8a), 134.0 (C-2, C-4a), 139.6 (C-3'), 156.8 (C-1').

MS (ESI): $m / z(\%)=457.9(78)\left[\mathrm{M}+\mathrm{NH}_{4}\right]^{+}$.

HRMS (ESI): $m / z=$ gef.: 455.9451 , ber.: $455.9454\left[\mathrm{M}\left({ }^{79} \mathrm{Br}\right)+\mathrm{NH}_{4}\right]^{+}$; gef.: 457.9434 , ber.: 457.9435 $\left[\mathrm{M}\left({ }^{81} \mathrm{Br}\right)+\mathrm{NH}_{4}\right]^{+}$.

$\mathbf{C}_{17} \mathbf{H}_{12}$ BrIO (439.09). 


\subsubsection{Synthese funktionalisierter Polyzyklen vom Typ 161}

\section{7-(2-Phenoxyphenyl)-9,10-dihydro-8H-benzo $[a]$ inden[6,5,4-kl]xanthen-5-yltrifluormethan-} sulfonat (161)

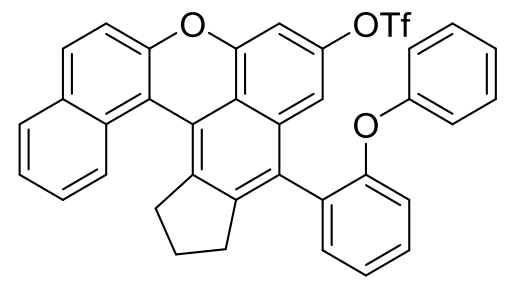

Eine Lösung von Methylether 110c (209 mg, 414 mol, 1.00 Äq.) und NaSEt (174 mg, 2.07 mmol, 5.00 Äq.) in DMF (12 mL) wurde $12 \mathrm{~h}$ bei $150{ }^{\circ} \mathrm{C}$ in der Mikrowelle erhitzt. Nach Abkühlen auf RT wurde die Reaktionsmischung mit ges. wässr. NaCl-Lösung $(50 \mathrm{~mL})$ und $\mathrm{H}_{2} \mathrm{O}(10 \mathrm{~mL})$ versetzt und mit MTBE extrahiert $(3 \times 30 \mathrm{~mL})$. Die vereinigten organischen Phasen wurden mit ges. wässr. $\mathrm{NaCl}$-Lösung $(60 \mathrm{~mL})$ gewaschen, über $\mathrm{Na}_{2} \mathrm{SO}_{4}$ getrocknet, filtriert und das Lösungsmittel in vacuo entfernt. Säulenchromatographische Reinigung $\left(\mathrm{SiO}_{2}, n\right.$-Pentan/EtOAc, 10:1) lieferte Arylalkohol 160 (111 mg), der ohne weitere Reinigung umgesetzt wurde.

Eine Lösung von Alkohol 160 (49.5 mg, $100 \mu \mathrm{mol}, 1.00$ Äq.) NEt 3 (150 $\mu \mathrm{L}, 20.8 \mathrm{mg}, 150 \mu \mathrm{mol}$, 1.50 Äq.) und $\mathrm{Tf}_{2} \mathrm{O}\left(1 \mathrm{M}\right.$ in $\mathrm{CH}_{2} \mathrm{Cl}_{2}, 150 \mu \mathrm{L}, 150 \mu \mathrm{mol}, 1.50$ Äq. $)$ in $\mathrm{CH}_{2} \mathrm{Cl}_{2}(6 \mathrm{~mL})$ wurde $4 \mathrm{~h}$ bei $0{ }^{\circ} \mathrm{C}$ und $2.5 \mathrm{~h}$ bei RT gerührt. $\mathrm{H}_{2} \mathrm{O}(10 \mathrm{~mL})$ wurde hinzugefügt und die Mischung mit $\mathrm{CH}_{2} \mathrm{Cl}_{2}$ extrahiert $(3 \times 10 \mathrm{~mL})$. Die vereinigten organischen Phasen wurden über $\mathrm{Na}_{2} \mathrm{SO}_{4}$ getrocknet, filtriert und das Lösungsmittel in vacuo entfernt. Säulenchromatographische Reinigung $\left(\mathrm{SiO}_{2}\right.$, n-Pentan/EtOAc, 50:1) lieferte Ester 161 als grünes Öl (53.0 mg, $84.9 \mu \mathrm{mol}, 46 \%$ über 2 Stufen).

DC: $R_{f}=0.41(n-$ Pentan/EtOAc, 50:1).

UV/Vis $\left(\mathrm{CH}_{3} \mathrm{CN}\right): \lambda_{\max }(\mathrm{nm})(\lg \varepsilon)=194$ (5.160), 212 (4.890), 249 (4.582), 324 (4.026), 354 (4.001), 389 (4.081), 501 (1.633).

IR (ATR): $\tilde{v}\left(\mathrm{~cm}^{-1}\right)=3060,2955,2360,2342,1624,1590,1574,1483,1446,1420,1388,1374,1352$, 1256, 1241, 1207, 1162, 1138, 1097, 1069, 1045, 1003, 992, 964, 898, 872, 859, 834, 811, 749, 731, $692,630,603,592,582,506$.

${ }^{1} \mathrm{H}-\mathrm{NMR}\left(600 \mathrm{MHz}, \mathrm{C}_{2} \mathrm{D}_{2} \mathrm{Cl}_{4}, 100{ }^{\circ} \mathrm{C}\right): \delta(\mathrm{ppm})=1.96\left(\mathrm{~s}_{\mathrm{br}}, 2 \mathrm{H}\right), 2.79\left(\mathrm{~s}_{\mathrm{br}}, 2 \mathrm{H}\right), 2.99-2.85(\mathrm{~m}, 2 \mathrm{H})$, $6.78(\mathrm{~d}, J=7.9 \mathrm{~Hz}, 2 \mathrm{H}), 6.90(\mathrm{~s}, 1 \mathrm{H}), 6.92(\mathrm{t}, J=7.4 \mathrm{~Hz}, 1 \mathrm{H}), 7.01(\mathrm{~s}, 1 \mathrm{H}), 7.13$ (t, $J=8.0 \mathrm{~Hz}$, $2 \mathrm{H}), 7.20$ (t, $J=7.0 \mathrm{~Hz}, 2 \mathrm{H}), 7.27$ (t, $J=7.5 \mathrm{~Hz}, 1 \mathrm{H}), 7.33$ (t, $J=7.4 \mathrm{~Hz}, 1 \mathrm{H}), 7.40$ (t, $J=7.5 \mathrm{~Hz}$, $2 \mathrm{H}), 7.49$ (dd, $J=16.4,8.2 \mathrm{~Hz}, 2 \mathrm{H}), 7.56$ (t, $J=7.5 \mathrm{~Hz}, 1 \mathrm{H}), 7.72(\mathrm{~d}, J=8.4 \mathrm{~Hz}, 1 \mathrm{H}), 7.88$ (t, $J=$ $8.3 \mathrm{~Hz}, 2 \mathrm{H})$. 
${ }^{13} \mathrm{C}$-NMR $\left(126 \mathrm{MHz}, \mathrm{C}_{2} \mathrm{D}_{2} \mathrm{Cl}_{4}, 50{ }^{\circ} \mathrm{C}\right): \delta(\mathrm{ppm})=26.4,32.8,36.6,99.4,100.6,109.0,115.1,116.5$, 118.4, 119.6, 120.1, 122.1, 122.8, 123.7, 124.7, 125.4, 125.5, 127.9, 128.1, 128.8, 128.9, 129.1, 129.2, $129.4,130.4,131.0,131.7,132.8,137.0,147.0,148.0,151.8,152.6$.

MS (ESI): $m / z(\%)=647.1(28)[\mathrm{M}+\mathrm{Na}]^{+}$.

HRMS (ESI): $m / z=$ gef.: 647.1080 , ber.: $647.1111[\mathrm{M}+\mathrm{Na}]^{+}$.

$\mathrm{C}_{36} \mathrm{H}_{23} \mathrm{~F}_{3} \mathrm{O}_{5} \mathrm{~S}(624.63)$. 


\subsubsection{Synthese des Dimers 162}

\section{1-Brom-2-(2-(hepta-1,6-diin-1-yl)phenoxy)naphthalin (163)}

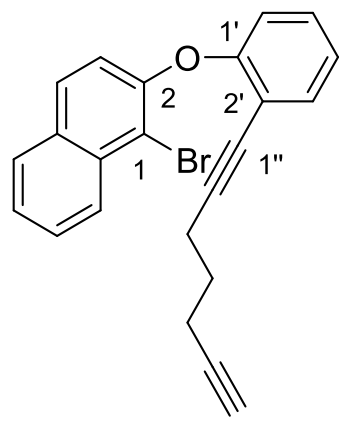

Eine Mischung aus 1-Brom-2-(2-iodphenoxy)naphthalin (111a) (2.22 g, $5.22 \mathrm{mmol}, 1.00$ Äq.), 1,6-Heptadiin (4.19 mL, $3.37 \mathrm{~g}, 36.6 \mathrm{mmol}, 7.00$ Äq.), $\mathrm{PdCl}_{2}\left(\mathrm{PPh}_{3}\right)_{2}$ (183 mg, $261 \mu \mathrm{mol}, 0.05$ Äq.) und CuI (99.4 mg, $522 \mu \mathrm{mol}, 0.10$ Äq.) in entgastem $\mathrm{NEt}_{3}(50 \mathrm{~mL})$ wurde $18 \mathrm{~h}$ bei RT gerührt. Die Reaktionsmischung wurde durch $\mathrm{SiO}_{2}$ filtriert, mit EtOAc gespült und das Lösungsmittel des Filtrats in vacuo entfernt. Säulenchromatographische Reinigung ( $\mathrm{SiO}_{2}, n$-Pentan/EtOAc, 100:1) lieferte Dialkin 163 als gelbes Öl (2.90 g, quant.) in verunreinigter Form.

DC: $R_{f}=0.42(n-$ Pentan/EtOAc, 100:1).

UV/Vis $\left(\mathrm{CH}_{3} \mathrm{CN}\right): \lambda_{\max }(\mathrm{nm})(\lg \varepsilon)=207$ (4.555), 230 (4.858), 283 (3.943), 294 (3.850).

IR (ATR): $\tilde{v}\left(\mathrm{~cm}^{-1}\right)=3294,1624,1594,1573,1562,1499,1484,1459,1443,1428,1371,1354,1328$, $1253,1230,1199,1157,1105,1039,989,936,825,806,776,748,632,527$.

${ }^{1} \mathbf{H}-\mathrm{NMR}\left(600 \mathrm{MHz}, \mathrm{CDCl}_{3}\right): \delta(\mathrm{ppm})=1.56\left(\mathrm{p}, J=7.0 \mathrm{~Hz}, 2 \mathrm{H}, 4{ }^{\prime}{ }^{\prime}-\mathrm{H}_{2}\right), 1.84(\mathrm{t}, J=2.6 \mathrm{~Hz}, 1 \mathrm{H}$, 7' '-H), 2.11 (td, $J=7.1,2.6 \mathrm{~Hz}, 2 \mathrm{H}, 5$ ''- $\left.\mathrm{H}_{2}\right), 2.39$ (t, $J=6.9 \mathrm{~Hz}, 2 \mathrm{H}, 3$ ''- $\left.-\mathrm{H}_{2}\right), 6.87$ (dd, $J=8.2$, $\left.1.0 \mathrm{~Hz}, 1 \mathrm{H}, 4^{\prime}-\mathrm{H}\right), 7.04$ (d, J = 8.9 Hz, $\left.1 \mathrm{H}, 3-\mathrm{H}\right), 7.07$ (td, J = 7.6, 1.1 Hz, 1 H, 6'-H), 7.20-7.23 (m, $\left.1 \mathrm{H}, 5^{\prime}-\mathrm{H}\right), 7.44-7.48$ (m, $2 \mathrm{H}, 6-\mathrm{H}, 3$ '-H), 7.59 (ddd, $\left.J=8.4,6.9,1.3 \mathrm{~Hz}, 1 \mathrm{H}, 7-\mathrm{H}\right), 7.73$ (d, $J=$ $8.9 \mathrm{~Hz}, 1 \mathrm{H}, 4-\mathrm{H}), 7.80(\mathrm{dd}, J=8.1,0.5 \mathrm{~Hz}, 1 \mathrm{H}, 5-\mathrm{H}), 8.28(\mathrm{dd}, J=8.5,0.7 \mathrm{~Hz}, 1 \mathrm{H}, 8-\mathrm{H})$.

${ }^{13}$ C-NMR (126 MHz, C $\left.\mathrm{C}_{6}\right): \delta(\mathrm{ppm})=17.3$ (C-5'), 18.6 (C-3'”), 27.3 (C-4'”), 68.6 (C-7'), 76.4 (C-6”), 83.6 (C-2'”), 94.7 (C-1'), 112.0 (C-1), 116.1 (C-2'), 118.8, 119.0 (C-3, C-6'), 123.7, 125.4 (C-6, C-4'), 126.6 (C-8), 127.8 (C-7), 128.1 (C-5), 128.9, 129.0 (C-4, C-5'), 131.1 (C-4a), 133.2 (C-8a), 133.8 (C-3'), 151.8 (C-1'), 157.0 (C-2).

MS (ESI): $m / z(\%)=411.1(87)[\mathrm{M}+\mathrm{Na}]^{+}$.

HRMS (ESI): $m / z=$ gef.: 411.0346, ber.: $411.0355\left[\mathrm{M}\left({ }^{79} \mathrm{Br}\right)+\mathrm{Na}\right]^{+}$; gef.: 413.0322, ber.: 413.0336 $\left[\mathrm{M}\left({ }^{81} \mathrm{Br}\right)+\mathrm{Na}\right]^{+}$. 
$\mathbf{C}_{23} \mathbf{H}_{17} \mathbf{B r O}$ (389.29). 


\section{1,4-Bis $(9,10$-dihydro-8H-benzo $[a]$ inden $[6,5,4-k l]$ xanthen-7-yl)benzol (162)}

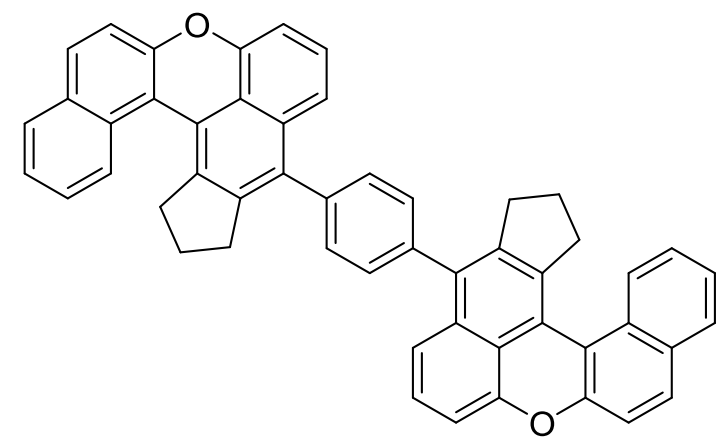

Ein Gemisch aus 1,4-Diiodbenzol (138) (21.3 mg, 64.6 mol, 1.00 Äq.), 1-Brom-2-(2-(hepta-1,6-diin1-yl)phenoxy)naphthalin (163) (60.2 mg, $155 \mu \mathrm{mol}, 2.40$ Äq.), Pd(OAc) 2 (1.45 mg, $6.46 \mu \mathrm{mol}$, 0.10 Äq.), $\mathrm{PPh}_{3}$ (8.47 mg, $32.3 \mu \mathrm{mol}, 0.50$ Äq.) und ( $\left.n \mathrm{Bu}\right)_{4} \mathrm{NOAc}(117 \mathrm{mg}, 388 \mu \mathrm{mol}, 6.00 \mathrm{Äq}$.) in entgastem DMF ( $3 \mathrm{~mL}$ ) wurde $18.5 \mathrm{~h}$ bei $100{ }^{\circ} \mathrm{C}$ gerührt. Nach Abkühlen auf RT wurde die Mischung mit ges. wässr. $\mathrm{NH}_{4} \mathrm{Cl}$-Lsg. $(10 \mathrm{~mL})$ versetzt und mit MTBE extrahiert $(3 \times 10 \mathrm{~mL})$. Die vereinigten organischen Phasen wurden mit ges. wässr. NaCl-Lsg. $(20 \mathrm{~mL})$ gewaschen, über $\mathrm{Na}_{2} \mathrm{SO}_{4}$ getrocknet, filtriert und das Lösungsmittel in vacuo entfernt. Säulenchromatographische Reinigung $\left(\mathrm{SiO}_{2}\right.$, $n$-Pentan/EtOAc, 100:1) lieferte Domino-Produkt 162 als gelben Feststoff (22.6 mg, 32.7 mol, 51\%).

DC: $R_{f}=0.28\left(n-\mathrm{Pentan} / \mathrm{CH}_{2} \mathrm{Cl}_{2}, 5: 1\right)$.

MS (EI): $m / z(\%)=690.3(100)[\mathrm{M}]^{+}$.

HRMS (ESI): $\mathrm{m} / z=$ gef.: 690.2555 , ber.: $690.2559[\mathrm{M}+\mathrm{Na}]^{+}$.

$\mathbf{C}_{52} \mathbf{H}_{34} \mathbf{O}_{2}$ (690.84). 


\subsection{Synthese der Polyzyklen des Typs 114}

\subsubsection{Synthese der Dialkine 115a-g}

\section{2-((Trimethylsilyl)ethinyl)benzaldehyd $(\mathbf{1 6 5})^{\mathrm{ee}}$}

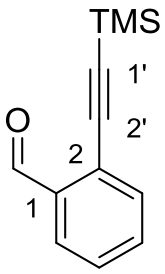

Ein Gemisch aus 2-Brombenzaldehyd (164) (15.7 mL, 25.0 g, 135 mmol, 1.00 Äq.), TMS-Acetylen (19.6 mL, 13.9 g, 142 mmol, 1.50 Äq.), $\mathrm{PdCl}_{2}\left(\mathrm{PPh}_{3}\right)_{2}$ (2.00 g, 2.85 mmol, 0.02 Äq.) und CuI (515 mg, $2.70 \mathrm{mmol}, 0.02 \mathrm{Äq}$.) in entgastem $\mathrm{NEt}_{3}(500 \mathrm{~mL})$ wurde $17 \mathrm{~h}$ bei $100{ }^{\circ} \mathrm{C}$ gerührt. Nach Abkühlen auf RT wurde die Reaktionsmischung durch Celite ${ }^{\circledR}$ filtriert, mit EtOAc gespült und das Lösungsmittel in vасио entfernt. Säulenchromatographische Reinigung $\left(\mathrm{SiO}_{2}, \quad \mathrm{PE} / \mathrm{EtOAc}, \quad 80: 1\right)$ und Umkristallisation aus $n$-Hexan/EtOAc (50:1) lieferten Alkin 165 als gelben Feststoff (18.5 g, $91.4 \mathrm{mmol}, 68 \%)$.

DC: $R_{f}=0.33(\mathrm{PE} / \mathrm{EtOAc}, 80: 1)$.

UV/Vis $\left(\mathrm{CH}_{3} \mathrm{CN}\right): \lambda_{\max }(\mathrm{nm})(\lg \varepsilon)=235$ (1.572), $262(1.081), 269(1.040), 312(0.554)$.

IR (ATR): $\tilde{v}\left(\mathrm{~cm}^{-1}\right)=2150,1693,1592,1473,1393,1260,1245,1216,1191,1090,862,840,809$, $756,717,704,653,637,595,566,544$.

${ }^{1} \mathbf{H}-\mathbf{N M R}\left(300 \mathrm{MHz}, \mathrm{CDCl}_{3}\right): \delta(\mathrm{ppm})=0.26\left(\mathrm{~s}, 9 \mathrm{H}, \mathrm{Si}\left(\mathrm{CH}_{3}\right)_{3}\right), 7.36-7.47(\mathrm{~m}, 1 \mathrm{H}, 3-\mathrm{H}), 7.47-7.61$ (m, 2 H, 4-H, 5-H), 7.88 (ddd, $J=7.8,1.4,0.7 \mathrm{~Hz}, 1 \mathrm{H}, 6-\mathrm{H}), 10.53$ (d, $J=0.8 \mathrm{~Hz}, 1 \mathrm{H}, \mathrm{CHO}$ ).

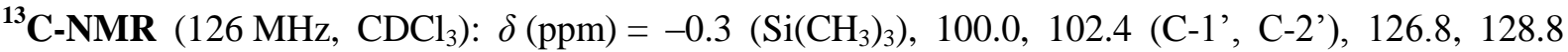
(C-2, C-6, C-3), 133.5, 133.6 (C-4, C-5), 136.2 (C-1), 191.8 (CHO).

MS (ESI): $m / z(\%)=225.1(40)[\mathrm{M}+\mathrm{Na}]^{+}, 257.1(100)[\mathrm{M}+\mathrm{Cs}]^{+}$.

HRMS (EI): $m / z=$ gef.: 225.0709, ber.: $225.0706[\mathrm{M}+\mathrm{Na}]^{+}$.

$\mathrm{C}_{12} \mathrm{H}_{14} \mathrm{OSi}$ (202.32).

\footnotetext{
${ }^{\text {ee }}$ Für Originalvorschrift siehe: T. Sakamoto, Y. Kondo, N. Miura, K. Hayashi, H. Yamanaka, Heterocycles 1986, 24, 2311-2314.
} 


\section{Allgemeine Vorschrift für die Synthese der Propargylakohole 167a-e (Addition):}

$n$-Butyllithium (2.5 M in $n$-Hexan, 1.50 Äq.) wurde bei $-78^{\circ} \mathrm{C}$ zu einer Lösung von 166 (1.50 Äq.) in THF ( $c=0.8 \mathrm{~mol} / \mathrm{L}$ ) hinzugefügt. Die Lösung wurde $15 \mathrm{~min}$ bei $-78{ }^{\circ} \mathrm{C}$ gerührt, über $45 \mathrm{~min}$ auf RT erwärmt und $1 \mathrm{~h}$ bei dieser Temperatur gerührt. Die Lösung wurde bei $-78^{\circ} \mathrm{C}$ zu einer Lösung von 165 (1.00 Äq.) in THF ( $c=0.5 \mathrm{~mol} / \mathrm{L}$ ) gegeben und über Nacht auf RT erwärmt. Nach Zugabe von ges. wässr. $\mathrm{NH}_{4} \mathrm{Cl}$-Lösung und $\mathrm{H}_{2} \mathrm{O}$ wurde die Reaktionsmischung mit $\mathrm{CH}_{2} \mathrm{Cl}_{2}$ extrahiert $(3 \times)$, die vereinigten organischen Phasen über $\mathrm{Na}_{2} \mathrm{SO}_{4}$ getrocknet, filtriert und das Lösungsmittel in vacuo entfernt. Säulenchromatographische Reinigung lieferte Dialkin 167 als braunes Öl, welches ohne weitere Reinigung im nächsten Schritt eingesetzt wurde.

Tabelle 15: Synthese der Dialkine 167a-e.

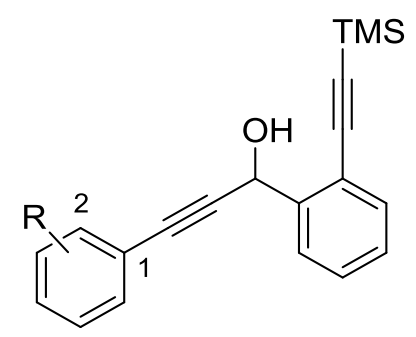

\begin{tabular}{cccccc}
\hline Eintrag & Alkin & R & Produkt & Ausbeute [g] & Ausbeute [\%] \\
\hline 1 & $\mathbf{1 6 6 a}$ & $\mathrm{H}$ & $\mathbf{1 6 7 a}$ & 2.64 & 88 \\
2 & $\mathbf{1 6 6 b}$ & $4-\mathrm{Me}$ & $\mathbf{1 6 7 b}$ & 3.01 & 96 \\
3 & $\mathbf{1 6 6 c}$ & $4-\mathrm{F}$ & $\mathbf{1 6 7 c}$ & 1.38 & 93 \\
4 & $\mathbf{1 6 6 d}$ & $4-\mathrm{OMe}$ & $\mathbf{1 6 7 d}$ & 1.37 & 96 \\
$5^{\text {[a] }}$ & $\mathbf{1 6 6 e}$ & $2-\mathrm{OPh}$ & $\mathbf{1 6 7 e}$ & 0.397 & 67 \\
\hline
\end{tabular}

[a] $c(\mathbf{1 6 6})=1.0 \mathrm{~mol} / \mathrm{L}, 30 \mathrm{~min}$ bei $-78^{\circ} \mathrm{C}, 1.5 \mathrm{~h}$ bei $\mathrm{RT}$. 


\section{3-Phenyl-1-(2-((trimethylsilyl)ethinyl)phenyl)prop-2-in-1-ol (167a)}

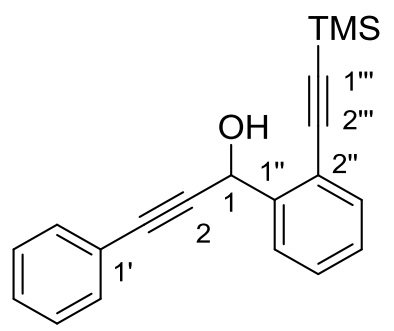

DC: $R_{f}=0.48(\mathrm{PE} / \mathrm{EtOAc}, 10: 1)$.

UV/Vis $\left(\mathrm{CH}_{3} \mathrm{CN}\right): \lambda_{\max }(\mathrm{nm})(\lg \varepsilon)=203$ (4.670), 244 (4.534), 249 (4.532).

IR (ATR): $\tilde{v}\left(\mathrm{~cm}^{-1}\right)=2154,1489,1445,1249,1031,862,839,689,647$.

${ }^{1} \mathrm{H}-\mathrm{NMR}\left(300 \mathrm{MHz}, \mathrm{CDCl}_{3}\right): \delta(\mathrm{ppm})=0.27\left(\mathrm{~s}, 9 \mathrm{H}, \mathrm{Si}\left(\mathrm{CH}_{3}\right)_{3}\right), 2.97(\mathrm{dd}, J=6.0,2.2 \mathrm{~Hz}, 1 \mathrm{H}, \mathrm{OH})$, $6.04(\mathrm{~d}, J=6.0 \mathrm{~Hz}, 1 \mathrm{H}, 1-\mathrm{H}), 7.27-7.32$ (m, 4 H, 3'-H, 4'-H, 5'-H, 4' '-H), 7.37 (dt, J= 7.7, $1.5 \mathrm{~Hz}$, 1 H, 5' -H), 7.42-7.48 (m, 2 H, 2'-H, 6'-H), 7.50 (dd, $J=7.6,1.5$ Hz, 1 H, 3''-H), 7.72 (ddd, $J=7.7$, $1.3,0.5 \mathrm{~Hz}, 1 \mathrm{H}, 6$ '"-H).

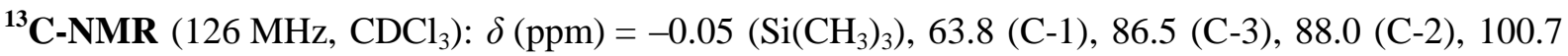
(C-1'”'), 102.3 (C-2'”), 121.1 (C-2'”), 122.5 (C-1'), 126.6 (C-6'), 128.0, 128.1, 128.4 (C-3', C-4', C-5', C-4'’), 129.0 (C-5'’), 131.7 (C-2', C-6'), 132.7 (C-3'’), 142.8 (C-1'’).

MS (EI): $m / z(\%)=73.1(100)\left[\mathrm{Si}\left(\mathrm{CH}_{3}\right)_{3}\right]^{+}, 215.1(36)\left[\mathrm{M}-\mathrm{Si}\left(\mathrm{CH}_{3}\right)_{3}-\mathrm{O}\right]^{+}, 231.1$ (21) $\left[\mathrm{M}-\mathrm{Si}\left(\mathrm{CH}_{3}\right)_{3}\right]^{+}$, $287.1(61)[\mathrm{M}-\mathrm{OH}]^{+}, 304.1(7)[\mathrm{M}]^{+}$.

HRMS (EI): $m / z=$ gef.: 304.1272 , ber.: $304.1283[\mathrm{M}]^{+}$.

$\mathrm{C}_{20} \mathrm{H}_{20} \mathrm{OSi}$ (304.46). 


\section{3-(p-Tolyl)-1-(2-((trimethylsilyl)ethinyl)phenyl)prop-2-in-1-ol (167b)}

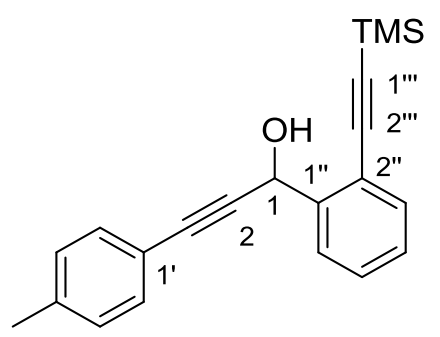

DC: $R_{f}=0.41(\mathrm{PE} / \mathrm{EtOAc}, 10: 1)$.

UV/Vis $\left(\mathrm{CH}_{3} \mathrm{CN}\right): \lambda_{\max }(\mathrm{nm})(\lg \varepsilon)=205$ (4.676), 249 (4.598).

IR (ATR): $\tilde{v}\left(\mathrm{~cm}^{-1}\right)=2155,1509,1478,1447,1249,1023,962,863,841,815,757,699,646,525$.

${ }^{1} \mathbf{H}-\mathrm{NMR}\left(300 \mathrm{MHz}, \mathrm{CDCl}_{3}\right): \delta(\mathrm{ppm})=0.26\left(\mathrm{~s}, 9 \mathrm{H}, \mathrm{Si}\left(\mathrm{CH}_{3}\right)_{3}\right), 2.33\left(\mathrm{~s}, 3 \mathrm{H}, \mathrm{CH}_{3}\right), 2.91(\mathrm{~d}$, $J=6.0 \mathrm{~Hz}, 1 \mathrm{H}, \mathrm{OH}), 6.03(\mathrm{~d}, J=6.0 \mathrm{~Hz}, 1 \mathrm{H}, 1-\mathrm{H}), 7.10$ (d, $J=7.9 \mathrm{~Hz}, 2 \mathrm{H}, 3$ '-H, 5'-H), 7.37 (dt, $J=7.5,1.4 \mathrm{~Hz}, 1 \mathrm{H}, 4$ ''-H), 7.33-7.39 (m, 3 H, 2'-H, 6'-H, 5''-H), 7.49 (dd, J = 7.5, $1.4 \mathrm{~Hz}, 1 \mathrm{H}$, 3''-H), 7.72 (ddd, $J=7.7,1.2,0.5 \mathrm{~Hz}, 1 \mathrm{H}, 6$ ''-H).

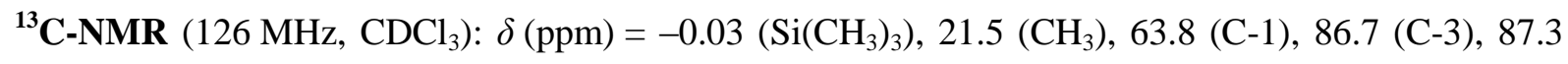
(C-2), 100.6 (C-1','), 102.3 (C-2'”), 119.4 (C-1'), 121.2 (C-2'”), 126.6 (C-6'”), 128.0 (C-4'”), 128.9, 129.0 (C-3', C-5', C-5'), 131.6 (C-2', C-6'), 132.7 (C-3'”), 138.5 (C-4'), 142.8 (C-1'’).

MS (ESI): $m / z(\%)=341.2(13)[\mathrm{M}+\mathrm{Na}]^{+}, 659.3(100)[2 \mathrm{M}+\mathrm{Na}]^{+}$.

HRMS (ESI): $m / z=$ gef.: 341.1333 , ber.: $341.1332[\mathrm{M}+\mathrm{Na}]^{+}$.

$\mathbf{C}_{21} \mathbf{H}_{22} \mathbf{O S i}$ (318.48). 


\section{3-(4-Fluorphenyl)-1-(2-((trimethylsilyl)ethinyl)phenyl)prop-2-in-1-ol (167c)}

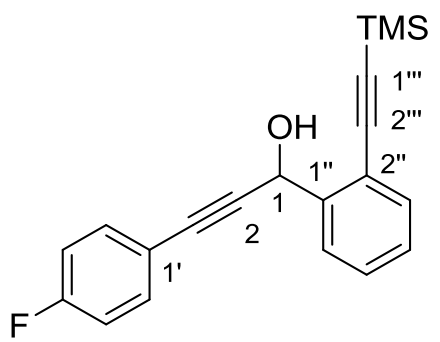

DC: $R_{f}=0.35(\mathrm{PE} / \mathrm{EtOAc}, 10: 1)$.

UV/Vis $\left(\mathrm{CH}_{3} \mathrm{CN}\right): \lambda_{\max }(\mathrm{nm})(\lg \varepsilon)=202(2.774), 244$ (2.619), 250 (2.625).

IR (ATR): $\tilde{v}\left(\mathrm{~cm}^{-1}\right)=3376,2958,2155,1505,1478,1447,1249,1229,1155,1093,1015,963,863$, $832,756,699,646,527,517$.

${ }^{1}$ H-NMR $\left(300 \mathrm{MHz}, \mathrm{CDCl}_{3}\right): \delta(\mathrm{ppm})=0.25\left(\mathrm{~s}, 9 \mathrm{H}, \mathrm{Si}\left(\mathrm{CH}_{3}\right)_{3}\right), 2.93(\mathrm{~d}, J=6.1 \mathrm{~Hz}, 1 \mathrm{H}, \mathrm{OH}), 6.01$ (d, $J=6.1 \mathrm{~Hz}, 1 \mathrm{H}, 1-\mathrm{H}), 6.98$ (t, $J=8.7 \mathrm{~Hz}, 2 \mathrm{H}, 3$ '-H, 5'-H), 7.28 (dt, $J=7.7,1.4 \mathrm{~Hz}, 1 \mathrm{H}, 4$ ' '-H), 7.37 (dt, $J=7.7,1.5 \mathrm{~Hz}, 1 \mathrm{H}, 5$ '’-H), 7.40-7.46 (m, 2 H, 2'-H, 6'-H), 7.51 (dd, $J=7.7,1.5 \mathrm{~Hz}, 1 \mathrm{H}$, 3''-H), 7.68 (ddd, $J=7.7,1.4,0.6 \mathrm{~Hz}, 1 \mathrm{H}, 6$ ''-H).

${ }^{13}$ C-NMR $\left(126 \mathrm{MHz}, \mathrm{CDCl}_{3}\right): \delta(\mathrm{ppm})=-0.04\left(\mathrm{Si}\left(\mathrm{CH}_{3}\right)_{3}\right), 63.8(\mathrm{C}-1), 85.4(\mathrm{C}-3), 87.8(\mathrm{C}-2), 100.8$ (C-1'”'), 102.2 (C-2'”'), 115.5 (d, J=22.0 Hz, C-3', C-5'), 118.6 (C-1'), 121.1 (C-2'”), 126.6 (C-6'), 128.1 (C-4'), 129.1 (C-5'), 132.8 (C-3'), 133.6 (d, J = 8.4 Hz, C-2', C-6'), 142.7 (C-1'), 162.5 (d, $\left.J=249.5 \mathrm{~Hz}, \mathrm{C}-4^{\prime}\right)$.

${ }^{19}$ F-NMR $\left(282 \mathrm{MHz}, \mathrm{CDCl}_{3}\right): \delta(\mathrm{ppm})=-110.6\left(\mathrm{tt}, J=8.5,5.4 \mathrm{~Hz}, 4^{\prime}-\mathrm{F}\right)$.

MS (ESI): $m / z(\%)=345.1(23)[\mathrm{M}+\mathrm{Na}]^{+}, 667.3(100)[2 \mathrm{M}+\mathrm{Na}]^{+}$.

HRMS (ESI): $m / z=$ gef.: 345.1081 , ber.: $345.1081[\mathrm{M}+\mathrm{Na}]^{+}$.

$\mathrm{C}_{20} \mathrm{H}_{19} \mathrm{FOSi}$ (322.45). 


\section{3-(4-Methoxyphenyl)-1-(2-((trimethylsilyl)ethinyl)phenyl)prop-2-in-1-ol (167d)}

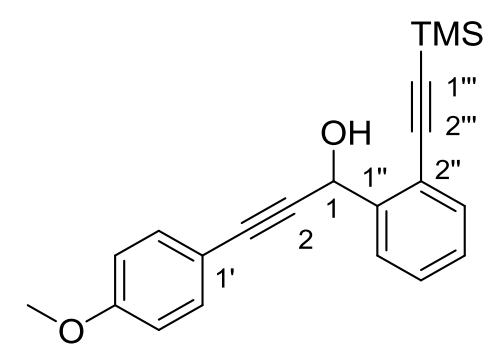

DC: $R_{f}=0.46(n-\mathrm{Hexan} / \mathrm{EtOAc}, 5: 1)$.

UV/Vis $\left(\mathrm{CH}_{3} \mathrm{CN}\right): \lambda_{\max }(\mathrm{nm})(\lg \varepsilon)=204$ (4.676), 251 (4.593), 260 (4.579).

IR (ATR): $\tilde{v}\left(\mathrm{~cm}^{-1}\right)=3422,2956,2154,1605,1569,1508,1478,1465,1445,1290,1246,1173,1105$, $1031,961,862,830,805,757,699,645,624,553,532$.

${ }^{1}$ H-NMR $\left(300 \mathrm{MHz}, \mathrm{CDCl}_{3}\right): \delta(\mathrm{ppm})=0.25\left(\mathrm{~s}, 9 \mathrm{H}, \mathrm{Si}\left(\mathrm{CH}_{3}\right)_{3}\right), 2.90(\mathrm{~d}, J=6.0 \mathrm{~Hz}, 1 \mathrm{H}, \mathrm{OH}), 3.79(\mathrm{~s}$, $\left.3 \mathrm{H}, \mathrm{OCH}_{3}\right), 6.02(\mathrm{~d}, J=6.0 \mathrm{~Hz}, 1 \mathrm{H}, 1-\mathrm{H}), 6.82\left(\mathrm{~d}, J=8.7 \mathrm{~Hz}, 2 \mathrm{H}, 3^{\prime}-\mathrm{H}, 5\right.$ '-H), 7.27 (dt, $J=7.6$, $1.2 \mathrm{~Hz}, 1 \mathrm{H}, 4$ '”-H), 7.36 (dt, J= 7.6, 1.4 Hz, 1 H, 5' '-H), 7.39 (d, $J=8.7 \mathrm{~Hz}, 2$ H, 2'-H, 6'-H), 7.49 (dd, $J=7.6,1.3 \mathrm{~Hz}, 1 \mathrm{H}, 3$ '”-H), 7.72 (ddd, $J=7.6,1.3,0.4 \mathrm{~Hz}, 1 \mathrm{H}, 6$ ' '-H).

${ }^{13}$ C-NMR $\left(126 \mathrm{MHz}, \mathrm{CDCl}_{3}\right): \delta(\mathrm{ppm})=-0.03\left(\mathrm{Si}\left(\mathrm{CH}_{3}\right)_{3}\right), 55.3\left(\mathrm{OCH}_{3}\right), 63.9(\mathrm{C}-1), 86.5,86.6(\mathrm{C}-2$, C-3), 100.6 (C-1'”'), 102.3 (C-2'”'), 113.8 (C-3', C-5'), 114.6 (C-1'), 121.1 (C-2'”), 126.6 (C-6’'), 128.0 (C-4’'), 129.0 (C-5'), 132.7 (C-3'’), 133.2 (C-2', C-6'), 142.8 (C-1'’), 159.6 (C-4').

MS (ESI): $m / z(\%)=357.1(37)[\mathrm{M}+\mathrm{Na}]^{+}, 691.3(100)[2 \mathrm{M}+\mathrm{Na}]^{+}$.

HRMS (ESI): $m / z=$ gef.: 357.1272 , ber.: $357.1281[\mathrm{M}+\mathrm{Na}]^{+}$.

$\mathbf{C}_{21} \mathbf{H}_{22} \mathbf{O}_{2} \mathbf{S i}$ (334.48). 


\section{3-(2-Phenoxyphenyl)-1-(2-((trimethylsilyl)ethinyl)phenyl)prop-2-in-1-ol (167e)}

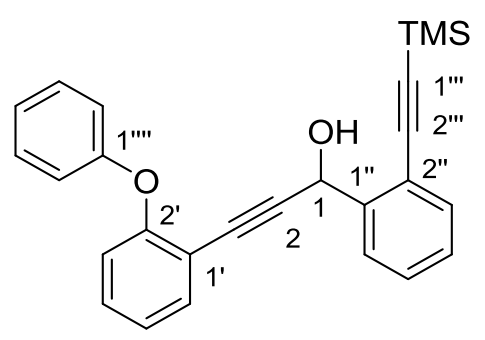

DC: $R_{f}=0.35(\mathrm{PE} / \mathrm{EtOAc}, 10: 1)$.

UV/Vis $\left(\mathrm{CH}_{3} \mathrm{CN}\right): \lambda_{\max }(\mathrm{nm})(\lg \varepsilon)=207$ (4.727), 250 (4.512), 279 (3.538), 290 (3.570).

IR (ATR): $\tilde{v}\left(\mathrm{~cm}^{-1}\right)=2155,1589,1483,1444,1249,1222,1162,1037,1021,862,841,800,751,690$, 648.

${ }^{1} \mathrm{H}-\mathrm{NMR}\left(300 \mathrm{MHz}, \mathrm{CDCl}_{3}\right): \delta(\mathrm{ppm})=0.25(\mathrm{~s}, 9 \mathrm{H}), 2.68\left(\mathrm{~s}_{\mathrm{br}}, 1 \mathrm{H}\right), 5.98(\mathrm{~s}, 1 \mathrm{H}), 6.90-7.00(\mathrm{~m}$, $3 \mathrm{H}), 7.04-7.11$ (m, $2 \mathrm{H}), 7.20-7.25$ (m, $2 \mathrm{H}), 7.26-7.32$ (m, $3 \mathrm{H}), 7.42-7.46$ (m, $1 \mathrm{H}), 7.52$ (dd, $J=$ 7.7, $1.7 \mathrm{~Hz}, 1 \mathrm{H}), 7.54-7.58(\mathrm{~m}, 1 \mathrm{H})$.

${ }^{13}$ C-NMR $\left(126 \mathrm{MHz}, \mathrm{CDCl}_{3}\right): \delta(\mathrm{ppm})=-0.1,26.9,63.6,82.4,93.0,100.3,102.3,115.3,118.1$, 119.5, 121.3, 123.0, 123.5, 126.9, 128.0, 129.1, 129.6, 130.0, 132.6, 133.9, 142.5, 157.4, 157.5.

MS (ESI): $m / z(\%)=419.2(30)[\mathrm{M}+\mathrm{Na}]^{+}, 815.3(100)[2 \mathrm{M}+\mathrm{Na}]^{+}$.

HRMS (ESI): $m / z=$ gef.: 419.1439 , ber.: $419.1438[\mathrm{M}+\mathrm{Na}]^{+}$.

$\mathbf{C}_{26} \mathbf{H}_{24} \mathbf{O}_{2} \mathrm{Si}$ (396.56). 


\section{Allgemeine Vorschrift für die Synthese der TBS-Ether 168a-e (TBS-Schützung):}

Eine Lösung von TBSCl (1.50 Äq.) in $\mathrm{CH}_{2} \mathrm{Cl}_{2}(c=1.5 \mathrm{~mol} / \mathrm{L})$ wurde zu einer Lösung von 167 (1.00 Äq.) und Imidazol (1.50 Äq.) in $\mathrm{CH}_{2} \mathrm{Cl}_{2}(c=0.4 \mathrm{~mol} / \mathrm{L})$ gegeben und 16-22 h bei RT gerührt. Nach Zugabe von ges. wässr. $\mathrm{NH}_{4} \mathrm{Cl}$-Lösung wurde die Reaktionsmischung mit $\mathrm{CH}_{2} \mathrm{Cl}_{2}$ extrahiert $(3 \times)$, die vereinigten organischen Phasen über $\mathrm{Na}_{2} \mathrm{SO}_{4}$ getrocknet, filtriert und das Lösungsmittel in vacuo entfernt. Säulenchromatographische Reinigung lieferte Dialkin 168 als farbloses Öl.

Tabelle 16: Synthese der Dialkine 168a-e.

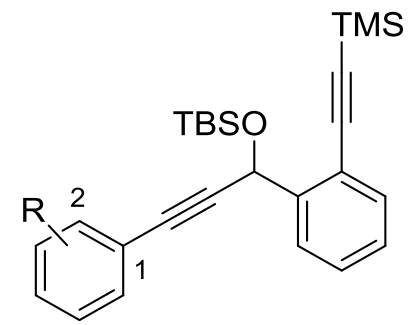

\begin{tabular}{cccccc}
\hline Eintrag & Substrat & R & Produkt & Ausbeute [g] & Ausbeute [\%] \\
\hline 1 & $\mathbf{1 6 7 a}$ & H & $\mathbf{1 6 8 a}$ & 3.50 & 98 \\
2 & $\mathbf{1 6 7 b}$ & $4-\mathrm{Me}$ & $\mathbf{1 6 8 b}$ & 3.93 & 96 \\
3 & $\mathbf{1 6 7 c}$ & $4-\mathrm{F}$ & $\mathbf{1 6 8 c}$ & 1.72 & 97 \\
4 & $\mathbf{1 6 7 d}$ & $4-\mathrm{OMe}$ & $\mathbf{1 6 8 d}$ & 1.69 & 95 \\
$5^{[\mathrm{a}]}$ & $\mathbf{1 6 7 e}$ & $2-\mathrm{OPh}$ & $\mathbf{1 6 8 e}$ & 2.96 & 86 \\
\hline
\end{tabular}

[a] $c(\mathbf{1 6 7})=0.1 \mathrm{~mol} / \mathrm{L}, c(\mathrm{TBSCl})=1.0 \mathrm{~mol} / \mathrm{L}$. 
tert-Butyldimethyl((3-phenyl-1-(2-((trimethylsilyl)ethinyl)phenyl)prop-2-in-1-yl)oxy)silan (168a)

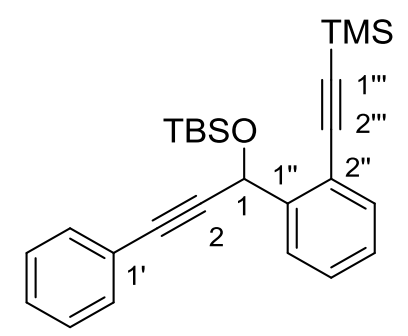

DC: $R_{f}=0.38\left(\mathrm{PE} / \mathrm{CH}_{2} \mathrm{Cl}_{2}, 10: 1\right)$.

UV/Vis $\left(\mathrm{CH}_{3} \mathrm{CN}\right): \lambda_{\max }(\mathrm{nm})(\lg \varepsilon)=203(4.671), 245(4.536)$.

IR (ATR): $\tilde{v}\left(\mathrm{~cm}^{-1}\right)=1490,1472,1445,1249,1105,1063,833,776,753,689,646$.

${ }^{1} \mathbf{H}-\mathrm{NMR}\left(300 \mathrm{MHz}, \mathrm{CDCl}_{3}\right): \delta(\mathrm{ppm})=0.18,0.23\left(2 \times \mathrm{s}, 2 \times 3 \mathrm{H}, \mathrm{Si}\left(\mathrm{CH}_{3}\right)_{2}\right), 0.27\left(\mathrm{~s}, 9 \mathrm{H}, \mathrm{Si}\left(\mathrm{CH}_{3}\right)_{3}\right)$, 0.96 (s, 9 H, $\left.\mathrm{SiC}\left(\mathrm{CH}_{3}\right)_{3}\right), 6.13$ (s, 1 H, 1-H), 7.20-7.29 (m, 4 H, 3'-H, 4'-H, 5'-H, 4'’-H), 7.35-7.42 (m, 3 H, 2'-H, 6'-H, 5' '-H), 7.45 (dd, $J=7.7,1.3 \mathrm{~Hz}, 1 \mathrm{H}, 3$ '’-H), 7.79 (ddd, $J=7.9,1.3,0.6 \mathrm{~Hz}, 1 \mathrm{H}$, 6"'-H).

${ }^{13} \mathbf{C}-\mathbf{N M R}\left(126 \mathrm{MHz}, \mathrm{CDCl}_{3}\right): \delta(\mathrm{ppm})=-4.7,-4.4\left(\mathrm{Si}\left(\mathrm{CH}_{3}\right)_{2}\right), 0.1\left(\mathrm{Si}\left(\mathrm{CH}_{3}\right)_{3}\right), 18.5\left(\mathrm{SiC}\left(\mathrm{CH}_{3}\right)_{3}\right), 25.9$ $\left(\mathrm{SiC}\left(\mathrm{CH}_{3}\right)_{3}\right), 63.4$ (C-1), 84.7 (C-3), 90.0 (C-2), 99.7 (C-1'”'), 102.5 (C-2'”'), 120.6 (C-2'”), 123.0 (C-1'), 126.3 (C-6'), 127.3 (C-4'’), 128.0 (C-3', C-4', C-5'), 128.9 (C-5' '), 131.5 (C-2', C-6'), 132.0 (C-3'), $144.1\left(\mathrm{C}-1{ }^{\prime \prime}\right)$.

MS (ESI): $m / z(\%)=441.2(36)[\mathrm{M}+\mathrm{Na}]^{+}$.

HRMS (ESI): $m / z=$ gef.: 441.2041 , ber.: $441.2040[\mathrm{M}+\mathrm{Na}]^{+}$.

$\mathbf{C}_{26} \mathbf{H}_{34} \mathrm{OSi}_{2}$ (418.72). 
tert-Butyldimethyl((3-(p-tolyl)-1-(2-((trimethylsilyl)ethinyl)phenyl)prop-2-in-1-yl)oxy)silan (168b)

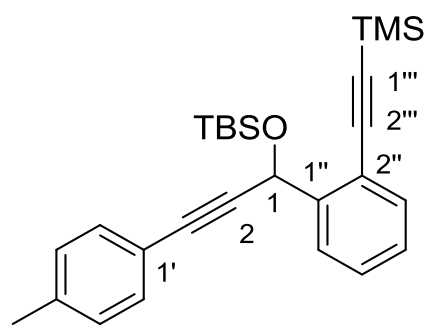

DC: $R_{f}=0.44\left(\mathrm{PE} / \mathrm{CH}_{2} \mathrm{Cl}_{2}, 10: 1\right)$.

UV/Vis $\left(\mathrm{CH}_{3} \mathrm{CN}\right): \lambda_{\max }(\mathrm{nm})(\lg \varepsilon)=202$ (4.713), 249 (4.611), 259 (4.532), 327 (3.021).

IR (ATR): $\tilde{v}\left(\mathrm{~cm}^{-1}\right)=2156,1509,1472,1249,1105,1060,833,814,776,756,646,526$.

${ }^{1} \mathbf{H}-\mathbf{N M R}\left(300 \mathrm{MHz}, \mathrm{CDCl}_{3}\right): \delta(\mathrm{ppm})=0.16,0.21\left(2 \times \mathrm{s}, 2 \times 3 \mathrm{H}, \mathrm{Si}\left(\mathrm{CH}_{3}\right)_{2}\right), 0.25\left(\mathrm{~s}, 9 \mathrm{H}, \mathrm{Si}\left(\mathrm{CH}_{3}\right)_{3}\right)$, 0.93 (s, $\left.9 \mathrm{H}, \mathrm{SiC}\left(\mathrm{CH}_{3}\right)_{3}\right), 2.31$ (s, $\left.3 \mathrm{H}, \mathrm{CH}_{3}\right), 6.09$ (s, $\left.1 \mathrm{H}, 1-\mathrm{H}\right), 7.07$ (d, J = 8.0 Hz, $2 \mathrm{H}, 3$ '- $\left.\mathrm{H}, 5^{\prime}-\mathrm{H}\right)$, $7.21\left(\mathrm{dt}, J=7.5,1.3 \mathrm{~Hz}, 1 \mathrm{H}, 4^{\prime}\right.$ ' $\left.-\mathrm{H}\right), 7.27$ (d, $\left.J=8.0 \mathrm{~Hz}, 2 \mathrm{H}, 2^{\prime}-\mathrm{H}, 6^{\prime}-\mathrm{H}\right), 7.36$ (dt, $J=7.8,1.5 \mathrm{~Hz}$, $1 \mathrm{H}, 5$ '’-H), 7.43 (dd, $J=7.7,1.5 \mathrm{~Hz}, 1 \mathrm{H}, 3$ ''-H), 7.75 (ddd, $J=7.8,1.3,0.6 \mathrm{~Hz}, 1 \mathrm{H}, 6$ ' '-H).

${ }^{13} \mathbf{C}-\mathbf{N M R}\left(126 \mathrm{MHz}, \mathrm{CDCl}_{3}\right): \delta(\mathrm{ppm})=-4.7,-4.4\left(\mathrm{Si}\left(\mathrm{CH}_{3}\right)_{2}\right), 0.1\left(\mathrm{Si}\left(\mathrm{CH}_{3}\right)_{3}\right), 18.5\left(\mathrm{SiC}\left(\mathrm{CH}_{3}\right)_{3}\right), 21.5$ $\left(\mathrm{CH}_{3}\right), 25.9\left(\mathrm{SiC}\left(\mathrm{CH}_{3}\right)_{3}\right), 63.4$ (C-1), 84.9 (C-3), 89.3 (C-2), 99.7 (C-1"’'), 102.0 (C-2'”), 120.6 (C-2'), 123.0 (C-1'), 126.3 (C-6'”), 127.2 (C-4'’), 128.8, 128.9 (C-3', C-5', C-5'), 131.4 (C-2', C-6'), 132.0 (C-3'), 138.0 (C-4'), 144.2 (C-1'”).

MS (ESI): $m / z(\%)=455.2(100)[\mathrm{M}+\mathrm{Na}]^{+}$.

HRMS (ESI): $m / z=$ gef.: 455.2198 , ber.: $455.2197[\mathrm{M}+\mathrm{Na}]^{+}$.

$\mathrm{C}_{27} \mathrm{H}_{36} \mathrm{OSi}_{2}$ (432.75). 


\section{tert-Butyldimethyl((3-(4-fluorphenyl)-1-(2-((trimethylsilyl)ethinyl)phenyl)prop-2-in-1-}

yl)oxy)silan (168c)

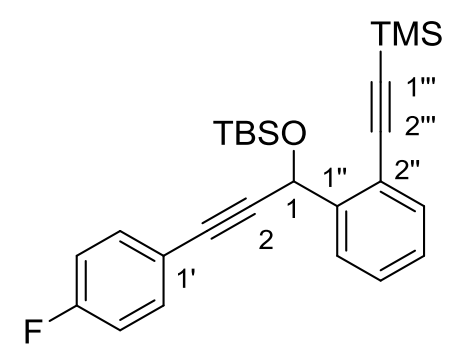

DC: $R_{f}=0.45\left(\mathrm{PE} / \mathrm{CH}_{2} \mathrm{Cl}_{2}, 10: 1\right)$.

UV/Vis $\left(\mathrm{CH}_{3} \mathrm{CN}\right): \lambda_{\max }(\mathrm{nm})(\lg \varepsilon)=244(4.511), 249(4.514)$.

IR (ATR): $\tilde{v}\left(\mathrm{~cm}^{-1}\right)=2955,2929,2856,2157,1601,1506,1250,1230,1155,1105,1061,831,776$, $756,646,527,510$.

${ }^{1} \mathbf{H}-\mathrm{NMR}\left(300 \mathrm{MHz}, \mathrm{CDCl}_{3}\right): \delta(\mathrm{ppm})=0.15,0.20\left(2 \times \mathrm{s}, 2 \times 3 \mathrm{H}, \mathrm{Si}\left(\mathrm{CH}_{3}\right)_{2}\right), 0.25\left(\mathrm{~s}, 9 \mathrm{H}, \mathrm{Si}\left(\mathrm{CH}_{3}\right)_{3}\right)$, 0.93 (s, $\left.9 \mathrm{H}, \mathrm{SiC}\left(\mathrm{CH}_{3}\right)_{3}\right), 6.08(\mathrm{~s}, 1 \mathrm{H}, 1-\mathrm{H}), 6.95$ (t, $J=8.7 \mathrm{~Hz}, 2 \mathrm{H}, 3^{\prime}-\mathrm{H}, 5$ '- $\left.-\mathrm{H}\right), 7.22$ (dt, $J=7.6$, $1.3 \mathrm{~Hz}, 1 \mathrm{H}, 4$ "' $-\mathrm{H}$ ), 7.32-7.39 (m, $3 \mathrm{H}, 2$ '-H, 6'-H, 5' -H), 7.43 (ddd, J= 7.6, 1.4, 0.6 Hz, $1 \mathrm{H}$, 3''-H), 7.73 (ddd, $J=7.9,1.3,0.6 \mathrm{~Hz}, 1 \mathrm{H}, 6$ ''-H).

${ }^{13} \mathbf{C}-\mathbf{N M R}\left(126 \mathrm{MHz}, \mathrm{CDCl}_{3}\right): \delta(\mathrm{ppm})=-4.7,-4.4\left(\mathrm{Si}\left(\mathrm{CH}_{3}\right)_{2}\right), 0.1\left(\mathrm{Si}\left(\mathrm{CH}_{3}\right)_{3}\right), 18.5\left(\mathrm{SiC}\left(\mathrm{CH}_{3}\right)_{3}\right), 25.9$ ( $\left.\mathrm{SiC}\left(\mathrm{CH}_{3}\right)_{3}\right), 63.4$ (C-1), 83.6 (C-3), 89.7 (C-2), 99.8 (C-1','), 102.5 (C-2'”), 115.4 (d, J=22.0 Hz, C-3', C-5'), 119.5 (d, J=3.5 Hz, C-1'), 120.5 (C-2'), 126.2 (C-6'), 127.3 (C-4'’), 129.0 (C-5')), 132.0 (C-3'’), 133.4 (d, J= 8.3 Hz, C-2', C-6'), 144.0 (C-1'’), 162.3 (d, J=248.7 Hz, C-4').

${ }^{19}$ F-NMR $\left(282 \mathrm{MHz}, \mathrm{CDCl}_{3}\right): \delta(\mathrm{ppm})=-111.3\left(\mathrm{tt}, J=8.7,5.4 \mathrm{~Hz}, 4^{\prime}-\mathrm{F}\right)$.

MS (ESI): $m / z(\%)=459.2(100)[\mathrm{M}+\mathrm{Na}]^{+}$.

HRMS (ESI): $m / z=$ gef.: 459.1945, ber.: $459.1946[\mathrm{M}+\mathrm{Na}]^{+}$.

$\mathbf{C}_{26} \mathbf{H}_{33} \mathrm{FOSi}_{2}$ (436.71). 
tert-Butyldimethyl((3-(4-methoxyphenyl)-1-(2-((trimethylsilyl)ethinyl)phenyl)prop-2-in-1yl)oxy)silan (168d)

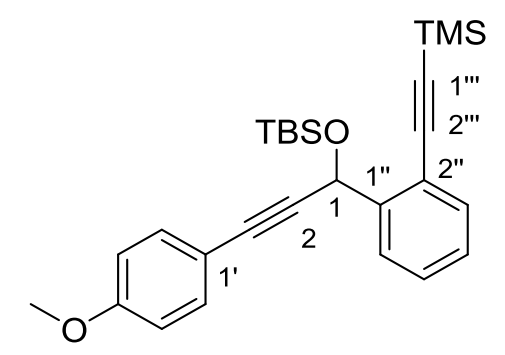

DC: $R_{f}=0.32(n-\mathrm{Hexan} / \mathrm{EtOAc}, 50: 1)$.

UV/Vis $\left(\mathrm{CH}_{3} \mathrm{CN}\right): \lambda_{\max }(\mathrm{nm})(\lg \varepsilon)=204$ (4.670), 251 (4.581), 260 (4.575).

IR (ATR): $\tilde{v}\left(\mathrm{~cm}^{-1}\right)=2955,2928,2856,2156,1605,1508,1471,1464,1444,1290,1247,1180,1172$, $1105,1059,1035,830,776,757,646,534$.

${ }^{1} \mathbf{H}-\mathrm{NMR}\left(300 \mathrm{MHz}, \mathrm{CDCl}_{3}\right): \delta(\mathrm{ppm})=0.16,0.21\left(2 \times \mathrm{s}, 2 \times 3 \mathrm{H}, \mathrm{Si}\left(\mathrm{CH}_{3}\right)_{2}\right), 0.25\left(\mathrm{~s}, 9 \mathrm{H}, \mathrm{Si}\left(\mathrm{CH}_{3}\right)_{3}\right)$, 0.93 (s, $\left.9 \mathrm{H}, \mathrm{SiC}\left(\mathrm{CH}_{3}\right)_{3}\right), 3.77$ (s, $\left.3 \mathrm{H}, \mathrm{OCH}_{3}\right), 6.09$ (s, $\left.1 \mathrm{H}, 1-\mathrm{H}\right), 6.79$ (d, J = 8.9 Hz, $2 \mathrm{H}, 3$ '-H, 5'-H), 7.21 (dt, $\left.J=7.6,1.4 \mathrm{~Hz}, 1 \mathrm{H}, 4^{\prime}{ }^{\prime}-\mathrm{H}\right), 7.32$ (d, $\left.J=8.9 \mathrm{~Hz}, 2 \mathrm{H}, 2^{\prime}-\mathrm{H}, 6^{\prime}-\mathrm{H}\right), 7.36$ (dt, $J=7.6,1.4 \mathrm{~Hz}$, $1 \mathrm{H}, 5$ '’-H), 7.43 (dd, J=7.7, 1.4 Hz, $1 \mathrm{H}, 3$ '’-H), 7.75 (dd, J= 7.9, 0.9 Hz, $1 \mathrm{H}, 6$ ''-H).

${ }^{13} \mathbf{C}-\mathrm{NMR}\left(126 \mathrm{MHz}, \mathrm{CDCl}_{3}\right): \delta(\mathrm{ppm})=-4.7,-4.4\left(\mathrm{Si}\left(\mathrm{CH}_{3}\right)_{2}\right), 0.1\left(\mathrm{Si}\left(\mathrm{CH}_{3}\right)_{3}\right), 18.5\left(\mathrm{SiC}\left(\mathrm{CH}_{3}\right)_{3}\right), 26.0$ $\left(\mathrm{SiC}\left(\mathrm{CH}_{3}\right)_{3}\right), 55.3\left(\mathrm{OCH}_{3}\right), 63.5(\mathrm{C}-1), 84.6(\mathrm{C}-3), 88.6$ (C-2), $99.6\left(\mathrm{C}-1{ }^{\prime \prime \prime}\right), 102.6\left(\mathrm{C}-2{ }^{\prime \prime \prime}\right), 113.7$ (C-3', C-5'), 115.2 (C-1'), 120.6 (C-2'), 126.3 (C-6'), 127.2 (C-4'”), 128.9 (C-5'), 132.0 (C-3'”), 132.9 (C-2', C-6'), 144.3 (C-1'’), 159.3 (C-4').

MS (ESI): $m / z(\%)=471.2(100)[\mathrm{M}+\mathrm{Na}]^{+}$.

HRMS (ESI): $m / z=$ gef.: 471.2146, ber.: $471.2146[\mathrm{M}+\mathrm{Na}]^{+}$.

$\mathbf{C}_{27} \mathbf{H}_{36} \mathbf{O}_{2} \mathrm{Si}_{2}$ (448.74). 
tert-Butyldimethyl((3-(2-phenoxyphenyl)-1-(2-((trimethylsilyl)ethinyl)phenyl)prop-2-in-1yl)oxy)silan (168e)

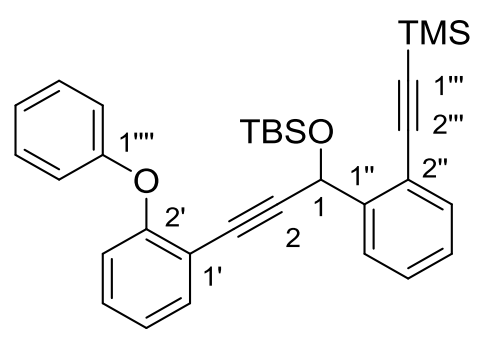

DC: $R_{f}=0.43(n-\mathrm{Hexan} / \mathrm{EtOAc}, 50: 1)$.

UV/Vis $\left(\mathrm{CH}_{3} \mathrm{CN}\right): \lambda_{\max }(\mathrm{nm})(\lg \varepsilon)=207$ (4.717), 249 (4.506), 279 (3.516), 290 (3.556).

IR (ATR): $\tilde{v}\left(\mathrm{~cm}^{-1}\right)=1484,1446,1250,1227,1106,1063,841,801,777,752,689$.

${ }^{1} \mathbf{H}-\mathbf{N M R}\left(600 \mathrm{MHz}, \mathrm{CDCl}_{3}\right): \delta(\mathrm{ppm})=0.08(\mathrm{~s}, 3 \mathrm{H}), 0.12(\mathrm{~s}, 3 \mathrm{H}), 0.22(\mathrm{~s}, 9 \mathrm{H}), 0.86(\mathrm{~s}, 9 \mathrm{H}), 6.05$ (s, $1 \mathrm{H}), 6.90(\mathrm{td}, J=8.6,1.1 \mathrm{~Hz}, 3 \mathrm{H}), 6.99-7.10(\mathrm{~m}, 2 \mathrm{H}), 7.18(\mathrm{td}, J=7.6,1.3 \mathrm{~Hz}, 1 \mathrm{H}), 7.21-7.29$ (m, $4 \mathrm{H}), 7.39(\mathrm{ddd}, J=7.6,1.4,0.5 \mathrm{~Hz}, 1 \mathrm{H}), 7.44(\mathrm{dd}, J=7.7,1.7 \mathrm{~Hz}, 1 \mathrm{H}), 7.59$ (dd, $J=7.9$, $1.3 \mathrm{~Hz}, 1 \mathrm{H})$.

${ }^{13} \mathbf{C}-N M R\left(126 \mathrm{MHz}, \mathrm{CDCl}_{3}\right): \delta(\mathrm{ppm})=-4.9,-4.6,-0.1,18.3,25.8,63.4,80.7,94.7,99.3,102.7$, 115.9, 118.2, 119.2, 120.8, 122.8, 123.3, 126.9, 127.3, 128.9, 129.5, 132.0, 133.8, 143.9, 157.4.

MS (ESI): $m / z(\%)=533.3(100)[\mathrm{M}+\mathrm{Na}]^{+}$.

HRMS (ESI): $m / z=$ gef.: 533.2305, ber.: $533.2303[\mathrm{M}+\mathrm{Na}]^{+}$.

$\mathbf{C}_{32} \mathbf{H}_{38} \mathbf{O}_{2} \mathbf{S i}_{2}$ (510.81). 
Allgemeine Vorschrift für die Synthese der terminalen Alkine 169a-e (TMS-Entschützung):

Eine Lösung von 168 (1.00 Äq.) und $\mathrm{K}_{2} \mathrm{CO}_{3}$ (0.50 Äq.) in $\mathrm{CH}_{3} \mathrm{OH}(c=0.1 \mathrm{~mol} / \mathrm{L}$ ) wurde $17-19 \mathrm{~h}$ bei RT gerührt. Nach Zugabe von ges. wässr. $\mathrm{NH}_{4} \mathrm{Cl}$-Lösung wurde die Reaktionsmischung mit $\mathrm{CH}_{2} \mathrm{Cl}_{2}$ extrahiert $(3 \times)$, die vereinigten organischen Phasen über $\mathrm{Na}_{2} \mathrm{SO}_{4}$ getrocknet, filtriert und das Lösungsmittel in vacuo entfernt. Säulenchromatographische Reinigung lieferte Dialkin 169 als farbloses Öl.

Tabelle 17: Synthese der Dialkine 169a-e.

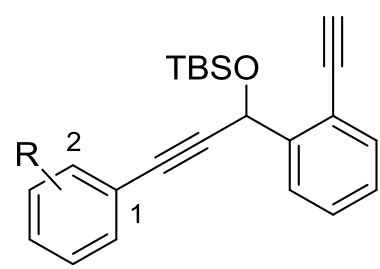

\begin{tabular}{cccccc}
\hline Eintrag & Substrat & $\mathbf{R}$ & Produkt & Ausbeute [g] & Ausbeute [\%] \\
\hline 1 & $\mathbf{1 6 8 a}$ & $\mathrm{H}$ & $\mathbf{1 6 9 a}$ & 2.69 & 93 \\
2 & $\mathbf{1 6 8 b}$ & $4-\mathrm{Me}$ & $\mathbf{1 6 9 b}$ & 3.05 & 94 \\
3 & $\mathbf{1 6 8 c}$ & $4-\mathrm{F}$ & $\mathbf{1 6 9 c}$ & 1.28 & 90 \\
4 & $\mathbf{1 6 8 d}$ & $4-\mathrm{OMe}$ & $\mathbf{1 6 9 d}$ & 1.28 & 80 \\
$5^{[\mathrm{a}]}$ & $\mathbf{1 6 8 e}$ & $2-\mathrm{OPh}$ & $\mathbf{1 6 9 e}$ & 2.46 & 97 \\
\hline
\end{tabular}

[a] $c(\mathbf{1 6 8})=0.06 \mathrm{~mol} / \mathrm{L}$. 
tert-Butyldimethyl((1-(2-ethinylphenyl)-3-phenylprop-2-in-1-yl)oxy)silan (169a)

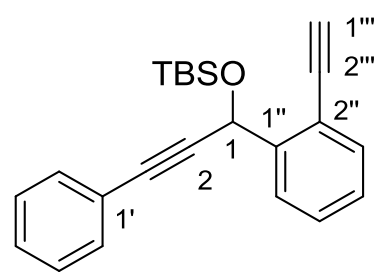

DC: $R_{f}=0.38\left(\mathrm{PE} / \mathrm{CH}_{2} \mathrm{Cl}_{2}, 10: 1\right)$.

UV/Vis $\left(\mathrm{CH}_{3} \mathrm{CN}\right): \lambda_{\max }(\mathrm{nm})(\lg \varepsilon)=204$ (4.726), 243 (4.486), 279 (3.004).

IR (ATR): $\tilde{v}\left(\mathrm{~cm}^{-1}\right)=1489,1471,1462,1444,1251,1059,832,776,753,689,668,652,612$.

${ }^{1} \mathrm{H}-\mathrm{NMR}\left(300 \mathrm{MHz}, \mathrm{CDCl}_{3}\right): \delta(\mathrm{ppm})=0.16,0.23\left(2 \times \mathrm{s}, 2 \times 3 \mathrm{H}, \quad \mathrm{Si}\left(\mathrm{CH}_{3}\right)_{2}\right), 0.94(\mathrm{~s}, 9 \mathrm{H}$, $\left.\mathrm{SiC}\left(\mathrm{CH}_{3}\right)_{3}\right), 3.35$ (s, $1 \mathrm{H}, 1$ '’'-H), 6.10 (s, $\left.1 \mathrm{H}, 1-\mathrm{H}\right), 7.22-7.30$ (m, 4 H, 3'-H, 4'-H, 5'-H, 4' '-H), 7.36-7.43 (m, 3 H, 2'-H, 6'-H, 5' '-H), 7.48 (dd, $J=7.6,1.3 \mathrm{~Hz}, 1 \mathrm{H}, 3$ ''-H), 7.79 (dd, $J=7.8,1.2 \mathrm{~Hz}$, $1 \mathrm{H}, 6$ '”-H).

${ }^{13}$ C-NMR $\left(126 \mathrm{MHz}, \mathrm{CDCl}_{3}\right): \delta(\mathrm{ppm})=-4.7,-4.3\left(\mathrm{Si}\left(\mathrm{CH}_{3}\right)_{2}\right), 18.4\left(\mathrm{SiC}\left(\mathrm{CH}_{3}\right)_{3}\right), 25.9\left(\mathrm{SiC}\left(\mathrm{CH}_{3}\right)_{3}\right)$, 63.3 (C-1), 81.2, (C-1'’'), 82.3 (C-2'”'), 85.1 (C-3), 89.8 (C-2), 119.6 (C-2’’), 122.9 (C-1'), 126.5 (C-6'), 127.4 (C-4'’), 128.1 (C-3', C-4', C-5'), 129.2 (C-5'”), 131.5 (C-2', C-6'), 132.6 (C-3'”), 144.2 $(\mathrm{C}-1$ '”).

MS (ESI): $m / z(\%)=368.2(23)[\mathrm{M}+\mathrm{Na}]^{+}, 487.2(100)\left[\mathrm{M}+\mathrm{C}_{5} \mathrm{H}_{14} \mathrm{OSi}+\mathrm{Na}\right]^{+}$.

HRMS (ESI): $m / z=$ gef.: 369.1640 , ber.: $369.1645[\mathrm{M}+\mathrm{Na}]^{+}$.

$\mathrm{C}_{23} \mathrm{H}_{26} \mathrm{OSi}$ (346.54). 


\section{tert-Butyldimethyl((1-(2-ethinylphenyl)-3-(p-tolyl)prop-2-in-1-yl)oxy)silan (169b)}

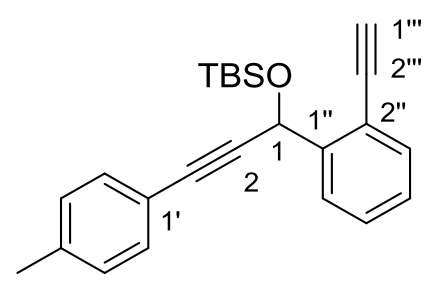

DC: $R_{f}=0.36\left(\mathrm{PE} / \mathrm{CH}_{2} \mathrm{Cl}_{2}, 10: 1\right)$.

UV/Vis $\left(\mathrm{CH}_{3} \mathrm{CN}\right): \lambda_{\max }(\mathrm{nm})(\lg \varepsilon)=205$ (4.739), 248 (4.517).

IR (ATR): $\tilde{v}\left(\mathrm{~cm}^{-1}\right)=3293,2927,2855,1509,1471,1462,1251,1103,1057,1005,984,832,815$, $776,755,664,649,621,527$.

${ }^{1} \mathbf{H}-\mathbf{N M R}\left(600 \mathrm{MHz}, \mathrm{CDCl}_{3}\right): \delta(\mathrm{ppm})=0.16,0.22\left(2 \times \mathrm{s}, 2 \times 3 \mathrm{H}, \quad \mathrm{Si}\left(\mathrm{CH}_{3}\right)_{2}\right), 0.93 \quad(\mathrm{~s}, 9 \mathrm{H}$, $\left.\mathrm{SiC}\left(\mathrm{CH}_{3}\right)_{3}\right), 2.31$ (s, $\left.3 \mathrm{H}, \mathrm{CH}_{3}\right), 3.34$ (s, $1 \mathrm{H}, 1$ ' ',-H), 6.09 (s, $\left.1 \mathrm{H}, 1-\mathrm{H}\right), 7.09$ (d, J = 8.0 Hz, $2 \mathrm{H}, 3$ '-H, 5'-H), 7.25 (dt, $J=7.7,1.2 \mathrm{~Hz}, 1 \mathrm{H}, 4$ ''-H), 7.29 (d, $J=8.0 \mathrm{~Hz}, 2 \mathrm{H}, 2^{\prime}-\mathrm{H}, 6$ '-H), 7.40 (dt, $J=7.7$, $1.3 \mathrm{~Hz}, 1 \mathrm{H}, 5$ '’-H), 7.40 (dd, $J=7.7,1.1 \mathrm{~Hz}, 1 \mathrm{H}, 3$ ''-H), 7.79 (ddd, $J=7.7,1.2,0.6 \mathrm{~Hz}, 1 \mathrm{H}, 6$ ' '-H).

${ }^{13}$ C-NMR $\left(126 \mathrm{MHz}, \mathrm{CDCl}_{3}\right): \delta(\mathrm{ppm})=-4.7,-4.3\left(\mathrm{Si}\left(\mathrm{CH}_{3}\right)_{2}\right), 18.4\left(\mathrm{SiC}\left(\mathrm{CH}_{3}\right)_{3}\right), 21.5\left(\mathrm{CH}_{3}\right), 25.9$ $\left(\mathrm{SiC}\left(\mathrm{CH}_{3}\right)_{3}\right), 63.3$ (C-1), 81.3, (C-1'”'), 82.1 (C-2'”'), 85.3 (C-3), 89.1 (C-2), 119.6 (C-2’'), 119.9 (C-1'), 126.5 (C-6'”), 127.4 (C-4'’), 128.8 (C-3', C-5'), 129.2 (C-5'), 131.4 (C-2', C-6'), 132.6 (C-3'), 138.1 (C-4'), 144.2 (C-1'”).

MS (ESI): $m / z(\%)=383.2(100)[\mathrm{M}+\mathrm{Na}]^{+}$.

HRMS (ESI): $m / z=$ gef.: 383.1798 , ber.: $383.1802[\mathrm{M}+\mathrm{Na}]^{+}$.

$\mathrm{C}_{24} \mathrm{H}_{28} \mathrm{OSi}$ (360.56). 


\section{tert-Butyldimethyl((1-(2-ethinylphenyl)-3-(4-fluorphenyl)prop-2-in-1-yl)oxy)silan (169c)}

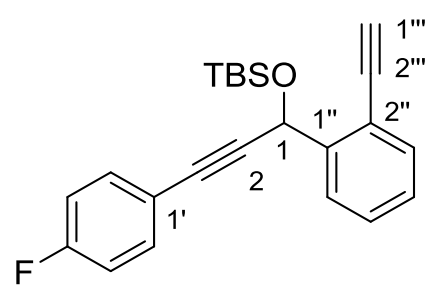

DC: $R_{f}=0.32\left(\mathrm{PE} / \mathrm{CH}_{2} \mathrm{Cl}_{2}, 10: 1\right)$.

UV/Vis $\left(\mathrm{CH}_{3} \mathrm{CN}\right): \lambda_{\max }(\mathrm{nm})(\lg \varepsilon)=203$ (4.726), 241 (4.479), 285 (3.165), 326 (2.731).

IR (ATR): $\tilde{v}\left(\mathrm{~cm}^{-1}\right)=3301,2954,2928,2856,1602,1506,1471,1252,1230,1155,1093,1059,1006$, $984,832,776,756,653,616,527$.

${ }^{1}$ H-NMR $\left(300 \mathrm{MHz}, \mathrm{CDCl}_{3}\right): \delta(\mathrm{ppm})=0.15,0.21\left(2 \times \mathrm{s}, 2 \times 3 \mathrm{H}, \quad \mathrm{Si}\left(\mathrm{CH}_{3}\right)_{2}\right), 0.93(\mathrm{~s}, 9 \mathrm{H}$, $\left.\mathrm{SiC}\left(\mathrm{CH}_{3}\right)_{3}\right), 3.34$ (s, $1 \mathrm{H}, 1$ ',' $\left.-\mathrm{H}\right), 6.07$ (s, $\left.1 \mathrm{H}, 1-\mathrm{H}\right), 6.95$ (t, J = 8.7 Hz, $2 \mathrm{H}, 3$ '-H, 5'-H), 7.25 (dt, $J=7.6,1.3 \mathrm{~Hz}, 1 \mathrm{H}, 4$ ''-H), 7.34-7.39 (m, 2 H, 2'-H, 6'-H), 7.40 (dt, $J=7.6,1.3 \mathrm{~Hz}, 1 \mathrm{H}, 5$ '’-H), 7.48 (dd, $J=7.6,1.3 \mathrm{~Hz}, 1 \mathrm{H}, 3$ '"-H), 7.48 (ddd, $J=7.9,1.3,0.8 \mathrm{~Hz}, 1 \mathrm{H}, 6$ ' '-H).

${ }^{13} \mathrm{C}-\mathrm{NMR}\left(126 \mathrm{MHz}, \mathrm{CDCl}_{3}\right): \delta(\mathrm{ppm})=-4.7,-4.4\left(\mathrm{Si}\left(\mathrm{CH}_{3}\right)_{2}\right), 18.4\left(\mathrm{SiC}\left(\mathrm{CH}_{3}\right)_{3}\right), 25.9\left(\mathrm{SiC}\left(\mathrm{CH}_{3}\right)_{3}\right)$, 63.3 (C-1), 81.2 (C-2'”'), 82.3 (C-1'”'), 84.0 (C-3), 89.5 (C-2), 115.4 (d, J=22.0 Hz, C-3', C-5'), 119.0 (C-1'), 119.5 (C-2'), 126.4 (C-6’), 127.5 (C-4’'), 129.2 (C-5'), 132.6 (C-3'”), 133.4 (d, $J=8.4 \mathrm{~Hz}, \mathrm{C}-2^{\prime}$, C-6'), 144.1 (C-1' '), 162.3 (d, $J=249.0 \mathrm{~Hz}, \mathrm{C}-4$ ').

${ }^{19} \mathbf{F}-\mathbf{N M R}\left(282 \mathrm{MHz}, \mathrm{CDCl}_{3}\right): \delta(\mathrm{ppm})=-111.2\left(\mathrm{tt}, J=8.6,5.4 \mathrm{~Hz}, 4^{\prime}-\mathrm{F}\right)$.

MS (ESI): $m / z(\%)=383.2(100)[\mathrm{M}+\mathrm{Na}]^{+}$.

HRMS (ESI): $m / z=$ gef.: 383.1798 , ber.: $383.1802[\mathrm{M}+\mathrm{Na}]^{+}$.

$\mathbf{C}_{23} \mathbf{H}_{25}$ FOSi (364.53). 
tert-Butyldimethyl((1-(2-ethinylphenyl)-3-(4-methoxyphenyl)prop-2-in-1-yl)oxy)silan (169d)

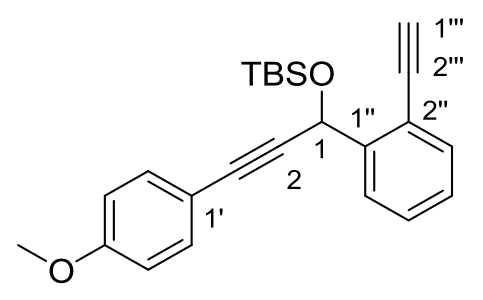

DC: $R_{f}=0.30(n-\mathrm{Hexan} / \mathrm{EtOAc}, 30: 1)$.

UV/Vis $\left(\mathrm{CH}_{3} \mathrm{CN}\right): \lambda_{\max }(\mathrm{nm})(\lg \varepsilon)=203$ (4.777), 249 (4.470), 258 (4.443).

IR (ATR): $\tilde{v}\left(\mathrm{~cm}^{-1}\right)=3290,2953,2928,2855,2219,1605,1508,1471,1463,1443,1290,1246,1172$, $1105,1056,1033,1006,983,829,776,756,665,621,533$.

${ }^{1}$ H-NMR $\left(300 \mathrm{MHz}, \mathrm{CDCl}_{3}\right): \delta(\mathrm{ppm})=0.16,0.22\left(2 \times \mathrm{s}, 2 \times 3 \mathrm{H}, \quad \mathrm{Si}\left(\mathrm{CH}_{3}\right)_{2}\right), 0.93 \quad(\mathrm{~s}, 9 \mathrm{H}$, $\left.\mathrm{SiC}\left(\mathrm{CH}_{3}\right)_{3}\right), 3.34\left(\mathrm{~s}, 1 \mathrm{H}, 1\right.$ ','-H), $3.77\left(\mathrm{~s}, 3 \mathrm{H}, \mathrm{OCH}_{3}\right), 6.08(\mathrm{~s}, 1 \mathrm{H}, 1-\mathrm{H}), 6.79(\mathrm{~d}, J=9.0 \mathrm{~Hz}, 2 \mathrm{H}$, 3'-H, 5'-H), 7.24 (dt, $J=7.6,1.3 \mathrm{~Hz}, 1 \mathrm{H}, 4$ '’-H), 7.33 (d, J=9.0 Hz, 2 H, 2'-H, 6'-H), 7.39 (dt, $J=7.7,1.4 \mathrm{~Hz}, 1 \mathrm{H}, 5$ '’-H), 7.47 (dd, $J=7.6,1.4 \mathrm{~Hz}, 1 \mathrm{H}, 3$ '’-H), 7.78 (ddd, $J=7.8,1.3,0.6 \mathrm{~Hz}, 1 \mathrm{H}$, 6' $-\mathrm{H})$.

${ }^{13} \mathbf{C}-\mathbf{N M R}\left(126 \mathrm{MHz}, \mathrm{CDCl}_{3}\right): \delta(\mathrm{ppm})=-4.7,-4.3\left(\mathrm{Si}\left(\mathrm{CH}_{3}\right)_{2}\right), 18.4\left(\mathrm{SiC}\left(\mathrm{CH}_{3}\right)_{3}\right), 25.9\left(\mathrm{SiC}\left(\underline{\mathrm{CH}}_{3}\right)_{3}\right)$, $55.3\left(\mathrm{OCH}_{3}\right), 63.4$ (C-1), 81.3 (C-2’’'), 82.2 (C-1'’'), 85.1 (C-3), 88.6 (C-2), 113.7 (C-3', C5'), 115.1 (C-1'), 119.6 (C-2'), 126.5 (C-6’'), 127.4 (C-4'’), 129.2 (C-5'), 132.6 (C-3'’), 132.9 (C-2', C-6'), 144.3 (C-1'"), 159.4 (C-4').

MS (ESI): $m / z(\%)=399.2(100)[\mathrm{M}+\mathrm{Na}]^{+}$.

HRMS (ESI): $m / z=$ gef.: 399.1741, ber.: $399.1751[\mathrm{M}+\mathrm{Na}]^{+}$.

$\mathrm{C}_{24} \mathrm{H}_{28} \mathrm{O}_{2} \mathrm{Si}$ (376.56). 
tert-Butyldimethyl((1-(2-ethinylphenyl)-3-(2-phenoxyphenyl)prop-2-in-1-yl)oxy)silan (169e)

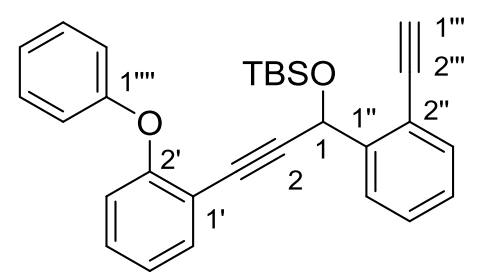

DC: $R_{f}=0.42(\mathrm{PE} / \mathrm{EtOAc}, 50: 1)$.

UV/Vis $\left(\mathrm{CH}_{3} \mathrm{CN}\right): \lambda_{\max }(\mathrm{nm})(\lg \varepsilon)=205$ (4.753), 238 (4.428), 280 (3.468), 289 (3.515).

IR (ATR): $\tilde{v}\left(\mathrm{~cm}^{-1}\right)=1483,1445,1250,1223,1105,1059,985,872,833,777,749,580,552$.

${ }^{1}$ H-NMR $\left(300 \mathrm{MHz}, \mathrm{CDCl}_{3}\right): \delta(\mathrm{ppm})=0.09(\mathrm{~s}, 3 \mathrm{H}), 0.14(\mathrm{~s}, 3 \mathrm{H}), 0.86(\mathrm{~s}, 9 \mathrm{H}), 3.21(\mathrm{~s}, 1 \mathrm{H}), 6.01$ (s, $1 \mathrm{H}), 6.90$ (ddd, $J=8.4,3.6,1.1 \mathrm{~Hz}, 3 \mathrm{H}), 7.04(\mathrm{td}, J=7.7,1.1 \mathrm{~Hz}, 2 \mathrm{H}), 7.20$ (td, $J=7.6,1.3 \mathrm{~Hz}$, $1 \mathrm{H}), 7.22-7.31(\mathrm{~m}, 4 \mathrm{H}), 7.42(\mathrm{dd}, J=7.6,1.3 \mathrm{~Hz}, 1 \mathrm{H}), 7.44(\mathrm{dd}, J=7.7,1.7 \mathrm{~Hz}, 1 \mathrm{H}), 7.62(\mathrm{dd}, J=$ 8.0, $1.2 \mathrm{~Hz}, 1 \mathrm{H})$.

${ }^{13} \mathbf{C}-\mathbf{N M R}\left(126 \mathrm{MHz}, \mathrm{CDCl}_{3}\right): \delta(\mathrm{ppm})=-4.9,-4.6,-0.1,18.3,25.8,63.4,80.7,94.7,99.3,102.7$, $115.9,118.2,119.2,120.8,122.8,123.3,126.9,127.3,128.9,129.5,132.0,133.8,143.9,157.4$.

MS (ESI): $m / z(\%)=461.2(100)[\mathrm{M}+\mathrm{Na}]^{+}$.

HRMS (ESI): $m / z=$ gef.: 461.1907, ber.: $461.1907[\mathrm{M}+\mathrm{Na}]^{+}$.

$\mathbf{C}_{29} \mathbf{H}_{30} \mathbf{O}_{2} \mathrm{Si}$ (438.63). 


\section{Allgemeine Vorschrift für die Synthese der Dialkine 171a-g (Addition): ${ }^{\mathrm{ff}}$}

Eine Lösung von LiHMDS ( $1 \mathrm{M}$ in Toluol, 2.00 Äq.) und $\mathrm{NEt}_{3}(10.0$ Äq.) in THF ( $c=0.1 \mathrm{~mol} / \mathrm{L})$ wurde auf $-78{ }^{\circ} \mathrm{C}$ gekühlt und mit einer Lösung von 169 (2.00 Äq.) in Toluol ( $\left.c=0.1 \mathrm{~mol} / \mathrm{L}\right)$ versetzt. Die Reaktionsmischung wurde $15 \mathrm{~min}$ bei $-78{ }^{\circ} \mathrm{C}$ und $15 \mathrm{~min}$ bei $-60^{\circ} \mathrm{C}$ gerührt, über $30 \mathrm{~min}$ auf RT erwärmt und $1 \mathrm{~h}$ bei dieser Temperatur gerührt. Nach dem Abkühlen auf $-78{ }^{\circ} \mathrm{C}$ wurde eine Lösung von 170 (1.00 Äq.) in Toluol $(c=0.05 \mathrm{~mol} / \mathrm{L})$ via Spritzenpumpe über $5 \mathrm{~h}$ hinzugefügt und die Reaktion über Nacht auf RT erwärmt. Nach Zugabe von ges. wässr. $\mathrm{NH}_{4} \mathrm{Cl}$-Lösung und $\mathrm{H}_{2} \mathrm{O}$ wurde die Reaktionsmischung mit $\mathrm{CH}_{2} \mathrm{Cl}_{2}$ extrahiert $(3 \times)$, die vereinigten organischen Phasen über $\mathrm{Na}_{2} \mathrm{SO}_{4}$ getrocknet, filtriert und das Lösungsmittel in vacuo entfernt. Säulenchromatographische Reinigung lieferte Additionsprodukt 171 als gelbes Öl, welches ohne weitere Reinigung im nächsten Schritt eingesetzt wurde.

Tabelle 18: Synthese der Dialkine 171a-g.<smiles>[R]c1cccc(C#CC(O[AsH3])c2ccccc2C#CC(O)CCc2ccccc2Br)c1</smiles>

\begin{tabular}{ccccccc}
\hline Eintrag & Substrate & $\mathbf{R}^{\mathbf{1}}$ & $\mathbf{R}^{\mathbf{2}}$ & Produkt & Ausbeute [mg] & Ausbeute [\%] \\
\hline 1 & $\mathbf{1 6 9 a}, \mathbf{1 7 0 a}$ & $\mathrm{H}$ & $\mathrm{H}$ & $\mathbf{1 7 1 a}$ & 348 & 86 \\
2 & $\mathbf{1 6 9 b}, \mathbf{1 7 0 a}$ & $\mathrm{H}$ & $4-\mathrm{Me}$ & $\mathbf{1 7 1 b}$ & 377 & 81 \\
3 & $\mathbf{1 6 9 c}, \mathbf{1 7 0 a}$ & $\mathrm{H}$ & $4-\mathrm{F}$ & $\mathbf{1 7 1}$ & 215 & 79 \\
$4^{[\mathrm{a}]}$ & $\mathbf{1 6 9 d}, \mathbf{1 7 0 a}$ & $\mathrm{H}$ & $4-\mathrm{OMe}$ & $\mathbf{1 7 1 d}$ & 290 & 70 \\
$5^{[\mathrm{b}]}$ & $\mathbf{1 6 9 e}, \mathbf{1 7 0 a}$ & $\mathrm{H}$ & $2-\mathrm{OPh}$ & $\mathbf{1 7 1 e}$ & 421 & 73 \\
$6^{[\mathrm{c}]}$ & $\mathbf{1 6 9 a}, \mathbf{1 7 0 b}$ & $\mathrm{Me}$ & $\mathrm{H}$ & $\mathbf{1 7 1 f}$ & 94.5 & - \\
$7^{[\mathrm{b}]}$ & $\mathbf{1 6 9 a}, \mathbf{1 7 0 c}$ & $\mathrm{F}$ & $\mathrm{H}$ & $\mathbf{1 7 1 g}$ & 270 & - \\
\hline
\end{tabular}

[a] Zugabe über $7 \mathrm{~h}$; [b] Zugabe über $6 \mathrm{~h}$; [c] Zugabe über $2.5 \mathrm{~h}$.

\footnotetext{
${ }^{\mathrm{ff}}$ Für eine Synthesevorschrift für 170a-c siehe u.a.: T. Hungerland, Dissertation, Georg-August-Universität, Göttingen, 2013.
} 


\section{5-(2-Bromphenyl)-1-(2-(1-((tert-butyldimethylsilyl)oxy)-3-phenylprop-2-in-1-yl)phenyl)pent-1-} in-3-ol (171a)

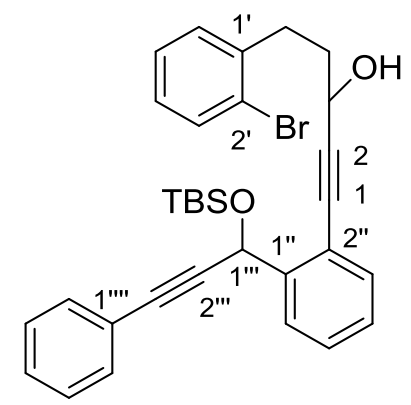

Für Diastereomerengemisch.

DC: $R_{f}=0.34\left(\mathrm{PE} / \mathrm{CH}_{2} \mathrm{Cl}_{2}, 1: 1\right)$.

UV/Vis $\left(\mathrm{CH}_{3} \mathrm{CN}\right): \lambda_{\max }(\mathrm{nm})(\lg \varepsilon)=244$ (4.564), 254 (4.496).

IR (ATR): $\tilde{v}\left(\mathrm{~cm}^{-1}\right)=3375,2953,2927,2855,1598,1568,1489,1471,1443,1326,1251,1109,1060$, 1022, 1002, 980, 836, 777, 750, 689, 670, 659, 527.

${ }^{1} \mathbf{H}-\mathrm{NMR}\left(300 \mathrm{MHz}, \mathrm{CDCl}_{3}\right): \delta(\mathrm{ppm})=0.15,0.21\left(2 \times \mathrm{s}, 2 \times 6 \mathrm{H}, 2 \times \mathrm{Si}\left(\mathrm{CH}_{3}\right)_{2}\right), 0.93(\mathrm{~s}, 2 \times 9 \mathrm{H}$, $\left.2 \times \mathrm{SiC}\left(\mathrm{CH}_{3}\right)_{3}\right), 1.98(\mathrm{~d}, J=5.3 \mathrm{~Hz}, 2 \times 1 \mathrm{H}, 2 \times \mathrm{OH}), 2.14\left(\mathrm{dd}, J=9.2,6.5 \mathrm{~Hz}, 2 \times 2 \mathrm{H}, 2 \times 4-\mathrm{H}_{2}\right)$, $2.99\left(\mathrm{dd}, J=9.4,6.3 \mathrm{~Hz}, 2 \times 2 \mathrm{H}, 2 \times 5-\mathrm{H}_{2}\right), 4.67(\mathrm{q}, J=6.1 \mathrm{~Hz}, 2 \times 1 \mathrm{H}, 2 \times 3-\mathrm{H}), 6.06(\mathrm{~d}$, $\left.J=2.7 \mathrm{~Hz}, 2 \times 1 \mathrm{H}, 2 \times 1^{\prime \prime}{ }^{\prime}-\mathrm{H}\right), 7.04\left(\mathrm{tt}, J=7.5,1.5 \mathrm{~Hz}, 2 \times 1 \mathrm{H}, 2 \times 5^{\prime}-\mathrm{H}\right), 7.14-7.27(\mathrm{~m}, 2 \times 6 \mathrm{H}$,

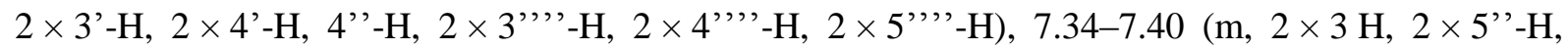
$2 \times 2$ ",' $-\mathrm{H}, 2 \times 6$ " "' $-\mathrm{H}), 7.43\left(\mathrm{dd}, J=7.6,1.3 \mathrm{~Hz}, 2 \times 1 \mathrm{H}, 2 \times 3{ }^{\prime}-\mathrm{H}\right), 7.51(\mathrm{dt}, J=7.8,1.2 \mathrm{~Hz}$, $\left.2 \times 1 \mathrm{H}, 2 \times 6^{\prime}-\mathrm{H}\right), 7.76\left(\mathrm{~d}, J=7.8 \mathrm{~Hz}, 2 \times 1 \mathrm{H}, 2 \times 6^{\prime}{ }^{\prime}-\mathrm{H}\right)$.

${ }^{13} \mathrm{C}-\mathrm{NMR}\left(126 \mathrm{MHz}, \mathrm{CDCl}_{3}\right): \delta(\mathrm{ppm})=-4.6,-4.3\left(\mathrm{Si}\left(\mathrm{CH}_{3}\right)_{2}\right), 18.4\left(\mathrm{SiC}\left(\mathrm{CH}_{3}\right)_{3}\right), 25.9\left(\mathrm{SiC}\left(\mathrm{CH}_{3}\right)_{3}\right)$, 32.0 (C-5), 37.8 (C-4), 62.4 (C-3), 63.5 (C-1'”'), 83.0 (C-1), 85.0 (C-3"'”), 89.9 (C-2"'”), 95.0 (C-2), 119.9 (C-2'”), 122.8 (C-1'”,'), 124.4 (C-2'), 126.2 (C-6’'), 127.4, 127.7 (C-4', C-5', C-4’'), 128.1

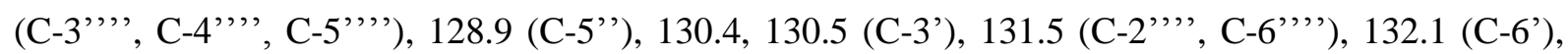
132.8 (C-3'”), 140.5 (C-1'), 143.6, 143.7 (C-1'').

MS (ESI): $m / z(\%)=595.2(100)[\mathrm{M}+\mathrm{Na}]^{+}$.

HRMS (ESI): $m / z=$ gef.: 581.1476 , ber.: $581.1476\left[\mathrm{M}\left({ }^{79} \mathrm{Br}\right)+\mathrm{Na}\right]^{+}$; gef.: 583.1460 , ber.: 583.1463 $\left[\mathrm{M}\left({ }^{81} \mathrm{Br}\right)+\mathrm{Na}\right]^{+}$.

$\mathrm{C}_{32} \mathrm{H}_{35} \mathrm{BrO}_{2} \mathrm{Si}$ (559.61). 
5-(2-Bromphenyl)-1-(2-(1-((tert-butyldimethylsilyl)oxy)-3-(p-tolyl)prop-2-in-1-yl)phenyl)pent-1in-3-ol (171b)

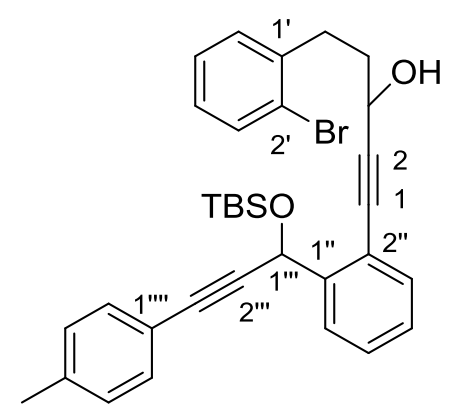

Für Diastereomerengemisch.

DC: $R_{f}=0.27\left(\mathrm{PE} / \mathrm{CH}_{2} \mathrm{Cl}_{2}, 1: 1\right)$.

UV/Vis $\left(\mathrm{CH}_{3} \mathrm{CN}\right): \lambda_{\max }(\mathrm{nm})(\lg \varepsilon)=197$ (4.945), 247 (4.572).

IR (ATR): $\tilde{v}\left(\mathrm{~cm}^{-1}\right)=3375,2953,2926,2855,1508,1471,1446,1324,1251,1184,1108,1058,1021$, $1006,983,836,815,777,750,682,659,623,579,527$.

${ }^{1} \mathrm{H}-\mathrm{NMR}\left(600 \mathrm{MHz}, \mathrm{CDCl}_{3}\right): \delta(\mathrm{ppm})=0.15,0.20\left(2 \times \mathrm{s}, 2 \times 6 \mathrm{H}, 2 \times \mathrm{Si}\left(\mathrm{CH}_{3}\right)_{2}\right), 0.92(\mathrm{~s}, 2 \times 9 \mathrm{H}$, $\left.2 \times \mathrm{SiC}\left(\mathrm{CH}_{3}\right)_{3}\right), 1.97(\mathrm{~d}, J=5.5 \mathrm{~Hz}, 2 \times 1 \mathrm{H}, 2 \times \mathrm{OH}), 2.10-2.15\left(\mathrm{~m}, 2 \times 2 \mathrm{H}, 2 \times 4-\mathrm{H}_{2}\right), 2.29(\mathrm{~s}$, $\left.2 \times 3 \mathrm{H}, 2 \times \mathrm{CH}_{3}\right), 2.95-3.02\left(\mathrm{~m}, 2 \times 2 \mathrm{H}, 2 \times 5-\mathrm{H}_{2}\right), 4.66(\mathrm{q}, J=6.0 \mathrm{~Hz}, 2 \times 1 \mathrm{H}, 2 \times 3-\mathrm{H}), 6.05(\mathrm{~d}$,

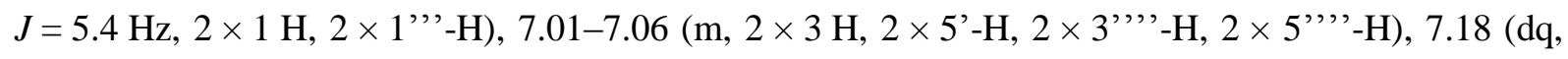
$\left.J=8.0, \quad 1.3 \mathrm{~Hz}, 2 \times 1 \mathrm{H}, 2 \times 4^{\prime}-\mathrm{H}\right), 7.22-7.26\left(\mathrm{~m}, 2 \times 4 \mathrm{H}, 2 \times 3^{\prime}-\mathrm{H}, 2 \times 4^{\prime \prime}-\mathrm{H}, 2 \times 2^{\prime \prime \prime},-\mathrm{H}\right.$, $2 \times 6$ ', '-H), $7.38(\mathrm{t}, J=7.6 \mathrm{~Hz}, 2 \times 1 \mathrm{H}, 2 \times 5$ ',-H), $7.43(\mathrm{dd}, J=7.7,1.1 \mathrm{~Hz}, 2 \times 1 \mathrm{H}, 2 \times 3$ '”-H), $7.51\left(\mathrm{dt}, J=8.0,1.5 \mathrm{~Hz}, 2 \times 1 \mathrm{H}, 2 \times 6^{\prime}-\mathrm{H}\right), 7.76\left(\mathrm{ddt}, J=7.8,1.3,0.7 \mathrm{~Hz}, 2 \times 1 \mathrm{H}, 2 \times 6^{\prime}{ }^{-}-\mathrm{H}\right)$.

${ }^{13} \mathrm{C}-\mathrm{NMR}\left(126 \mathrm{MHz}, \mathrm{CDCl}_{3}\right): \delta(\mathrm{ppm})=-4.6,-4.3\left(\mathrm{SiC}\left(\mathrm{CH}_{3}\right)_{3}\left(\mathrm{CH}_{3}\right)_{2}\right), 18.4\left(\mathrm{SiC}\left(\mathrm{CH}_{3}\right)_{3}\left(\mathrm{CH}_{3}\right)_{2}\right), 21.5$ $\left(\mathrm{CH}_{3}\right), 25.9\left(\mathrm{SiC}\left(\mathrm{CH}_{3}\right)_{3}\left(\mathrm{CH}_{3}\right)_{2}\right), 32.0(\mathrm{C}-5), 37.8(\mathrm{C}-4), 62.4,62.5(\mathrm{C}-3), 63.5,63.6\left(\mathrm{C}-1{ }^{\prime \prime \prime}\right), 83.1$ (C-1), 85.2 (C-3"'’), 89.2 (C-2'”), 95.0 (C-2), 119.7 (C-2'”), 119.9 (C-1','), 124.4 (C-2'), 126.2 (C-6'), 127.4, (C-4', C-4'”), 127.7 (C-5'), 128.9 (C-5', C-3',', C-5',',), 130.5 (C-3'), 131.4 (C-2',',, C-6"','), 132.0, 132.1 (C-6'), 132.8 (C-3'”), 138.2 (C-4','), 140.5 (C-1'), 143.8 (C-1'”).

MS (ESI): $m / z(\%)=581.2(46)[\mathrm{M}+\mathrm{Na}]^{+}$.

HRMS (ESI): $m / z=$ gef.: 595.1628 , ber.: $595.1638\left[\mathrm{M}\left({ }^{79} \mathrm{Br}\right)+\mathrm{Na}\right]^{+}$; gef.: 597.1618 , ber.: 597.1620 $\left[\mathrm{M}\left({ }^{81} \mathrm{Br}\right)+\mathrm{Na}\right]^{+}$.

$\mathrm{C}_{33} \mathrm{H}_{37} \mathrm{BrO}{ }_{2} \mathrm{Si}$ (573.64) . 


\section{5-(2-Bromphenyl)-1-(2-(1-((tert-butyldimethylsilyl)oxy)-3-(4-fluorphenyl)prop-2-in-1-}

\section{yl)phenyl)pent-1-in-3-ol (171c)}

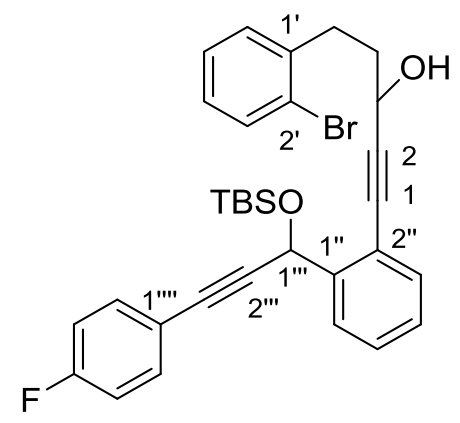

Für Diastereomerengemisch.

DC: $R_{f}=0.25\left(\mathrm{PE} / \mathrm{CH}_{2} \mathrm{Cl}_{2}, 1: 1\right)$.

UV/Vis $\left(\mathrm{CH}_{3} \mathrm{CN}\right): \lambda_{\max }(\mathrm{nm})(\lg \varepsilon)=197$ (4.966), 243 (4.557), 311 (2.993), 326 (2.974).

IR (ATR): $\tilde{v}\left(\mathrm{~cm}^{-1}\right)=3363,2953,2927,2855,1601,1505,1471,1446,1252,1230,1155,1110,1060$, 1022, 1006, 983, 833, 777, 750, 659, 623, 579, 528.

${ }^{1} \mathbf{H}-\mathbf{N M R}\left(600 \mathrm{MHz}, \mathrm{CDCl}_{3}\right): \delta(\mathrm{ppm})=0.15,0.20\left(2 \times \mathrm{s}, 2 \times 6 \mathrm{H}, 2 \times \mathrm{Si}\left(\mathrm{CH}_{3}\right)_{2}\right), 0.92(\mathrm{~s}, 2 \times 9 \mathrm{H}$, $\left.2 \times \mathrm{SiC}\left(\mathrm{CH}_{3}\right)_{3}\right), 1.97(\mathrm{~d}, J=5.3 \mathrm{~Hz}, 2 \times 1 \mathrm{H}, 2 \times \mathrm{OH}), 2.09-2.16\left(\mathrm{~m}, 2 \times 2 \mathrm{H}, 2 \times 4-\mathrm{H}_{2}\right), 2.99(\mathrm{dd}$, $\left.J=9.5,6.4,2 \times 2 \mathrm{H}, 2 \times 5-\mathrm{H}_{2}\right), 4.67(\mathrm{q}, J=6.1 \mathrm{~Hz}, 2 \times 1 \mathrm{H}, 2 \times 3-\mathrm{H}), 6.04(\mathrm{~d}, J=3.4 \mathrm{~Hz}, 2 \times 1 \mathrm{H}$, $\left.2 \times 1{ }^{\prime \prime \prime}-\mathrm{H}\right), 6.91\left(\mathrm{t}, J=8.8 \mathrm{~Hz}, 2 \times 2 \mathrm{H}, 2 \times 3{ }^{\prime \prime}{ }^{\prime}-\mathrm{H}, 2 \times 5{ }^{\prime \prime}{ }^{\prime}-\mathrm{H}\right), 7.05$ (ddt, $J=7.2,2.0,1.1 \mathrm{~Hz}$, $\left.2 \times 1 \mathrm{H}, 2 \times 5^{\prime}-\mathrm{H}\right), 7.18\left(\mathrm{ddt}, J=7.6,3.5,1.3 \mathrm{~Hz}, 2 \times 1 \mathrm{H}, 2 \times 4^{\prime}-\mathrm{H}\right), 7.24(\mathrm{dd}, J=7.5,1.5 \mathrm{~Hz}$, $\left.2 \times 1 \mathrm{H}, 2 \times 3^{\prime}-\mathrm{H}\right), 7.24-7.35\left(\mathrm{~m}, 2 \times 3 \mathrm{H}, 2 \times 4{ }^{\prime}{ }^{-} \mathrm{H}, 2 \times 2{ }^{\prime}{ }^{\prime \prime}{ }^{-}-\mathrm{H}, 2 \times 6{ }^{\prime \prime}{ }^{\prime}-\mathrm{H}\right), 7.38(\mathrm{dt}, J=7.8$, $1.4 \mathrm{~Hz}, 2 \times 1 \mathrm{H}, 2 \times 5$ ' $-\mathrm{H}), 7.43\left(\mathrm{ddd}, J=7.7,1.4,0.9 \mathrm{~Hz}, 2 \times 1 \mathrm{H}, 2 \times 3{ }^{\prime}-\mathrm{H}\right), 7.51(\mathrm{dt}, J=7.9$, $1.1 \mathrm{~Hz}, 2 \times 1 \mathrm{H}, 2 \times 6$ '-H), 7.74 (ddt, $J=7.8,1.3,0.6 \mathrm{~Hz}, 2 \times 1 \mathrm{H}, 2 \times 6$ '”-H).

${ }^{13} \mathrm{C}-\mathrm{NMR}\left(126 \mathrm{MHz}, \mathrm{CDCl}_{3}\right): \delta(\mathrm{ppm})=-4.7,-4.3\left(\mathrm{Si}\left(\mathrm{CH}_{3}\right)_{2}\right), 18.4\left(\mathrm{SiC}\left(\mathrm{CH}_{3}\right)_{3}\right), 25.9\left(\mathrm{SiC}\left(\mathrm{CH}_{3}\right)_{3}\right)$, 32.0 (C-5), 37.8 (C-4), 62.4 (C-3), 63.4 (C-1'”'), 82.9 (C-1), 84.9 (C-3'”'), 89.6 (C-2'”'), 95.1 (C-2), 115.5 (d, $J=22.0 \mathrm{~Hz}, \mathrm{C}-3$ ', ', C-5','), 118.9 (C-1','), 119.7 (C-2'”), 124.4 (C-2'), 126.1 (C-6'”), 127.4, 127.5 (C-4', C-4'), 127.7 (C-5'), 128.9, 129.0 (C-5'’), 130.4 (C-3'), 132.1 (C-6'), 132.8 (C-3'), 133.4 (d, J=8.4 Hz, C-2'”,', C-6"',), 140.5 (C-1'), 143.5, 143.6 (C-1'”), 162.3 (d, $J=249.0 \mathrm{~Hz}, \mathrm{C}-4$, ',').

${ }^{19}$ F-NMR $\left(282 \mathrm{MHz}, \mathrm{CDCl}_{3}\right): \delta(\mathrm{ppm})=-111.6\left(\mathrm{ddq}, J=10.9,5.4,2.2 \mathrm{~Hz}, 4{ }^{,,},{ }_{-F}\right)$.

MS (ESI): $m / z(\%)=601.2(100)[\mathrm{M}+\mathrm{Na}]^{+}$.

HRMS (ESI): $m / z=$ gef.: 599.1387 , ber.: $599.1388\left[\mathrm{M}\left({ }^{79} \mathrm{Br}\right)+\mathrm{Na}\right]^{+}$; gef.: 601.1370 , ber.: 601.1369 $\left[\mathrm{M}\left({ }^{81} \mathrm{Br}\right)+\mathrm{Na}\right]^{+}$. 
$\mathrm{C}_{32} \mathrm{H}_{34} \mathrm{BrFO}_{2} \mathrm{Si}$ (577.60). 


\section{5-(2-Bromphenyl)-1-(2-(1-((tert-butyldimethylsilyl)oxy)-3-(4-methoxyphenyl)prop-2-in-1-}

\section{yl)phenyl)pent-1-in-3-ol (171d)}

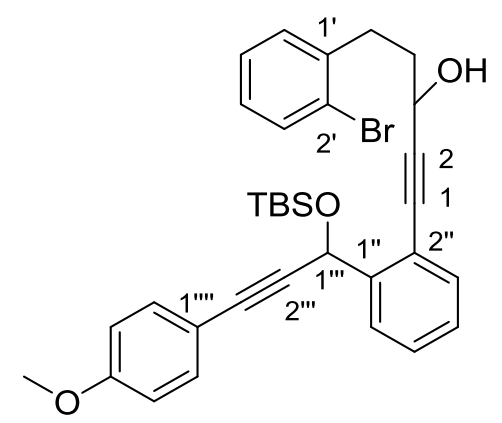

Für Diastereomerengemisch.

DC: $R_{f}=0.28(n-\mathrm{Hexan} / \mathrm{EtOAc}, 10: 1)$.

UV/Vis $\left(\mathrm{CH}_{3} \mathrm{CN}\right): \lambda_{\max }(\mathrm{nm})(\lg \varepsilon)=196(4.946), 255$ (4.546).

IR (ATR): $\tilde{v}\left(\mathrm{~cm}^{-1}\right)=3409,2953,2927,2854,1605,1508,1471,1441,1290,1247,1172,1107,1055$, 1032, 1006, 982, 830, 777, 750, 666, 659, 623, 578, 533.

${ }^{1} \mathbf{H}-\mathrm{NMR}\left(600 \mathrm{MHz}, \mathrm{CDCl}_{3}\right): \delta(\mathrm{ppm})=0.15,0.20\left(2 \times \mathrm{s}, 2 \times 6 \mathrm{H}, 2 \times \mathrm{Si}\left(\mathrm{CH}_{3}\right)_{2}\right), 0.93(\mathrm{~s}, 2 \times 9 \mathrm{H}$, $\left.2 \times \mathrm{SiC}\left(\mathrm{CH}_{3}\right)_{3}\right), 2.03\left(\mathrm{~s}_{\mathrm{br}}, J=4.0 \mathrm{~Hz}, 2 \times 1 \mathrm{H}, 2 \times \mathrm{OH}\right), 2.11-2.16\left(\mathrm{~m}, 2 \times 2 \mathrm{H}, 2 \times 4-\mathrm{H}_{2}\right), 2.95-3.03$ $\left(\mathrm{m}, 2 \times 2 \mathrm{H}, 2 \times 5-\mathrm{H}_{2}\right), 3.76\left(2 \times \mathrm{s}, 2 \times 3 \mathrm{H}, 2 \times \mathrm{OCH}_{3}\right), 4.66\left(\mathrm{~m}_{\mathrm{C}}, 2 \times 1 \mathrm{H}, 2 \times 3-\mathrm{H}\right), 6.05(\mathrm{~d}$, $J=5.8 \mathrm{~Hz}, 2 \times 1 \mathrm{H}, 2 \times 1$ '"'-H), 6.75 (dd, $\left.J=9.0,0.9 \mathrm{~Hz}, 2 \times 2 \mathrm{H}, 2 \times 3{ }^{\prime \prime},{ }^{-H}, 2 \times 5{ }^{\prime \prime}, '-\mathrm{H}\right), 7.04$ (tt, $\left.J=7.7,2.0 \mathrm{~Hz}, 2 \times 1 \mathrm{H}, 2 \times 5^{\prime}-\mathrm{H}\right), 7.18\left(\mathrm{dq}, J=7.4,1.2 \mathrm{~Hz}, 2 \times 1 \mathrm{H}, 2 \times 4^{\prime}-\mathrm{H}\right), 7.23-7.26(\mathrm{~m}$,

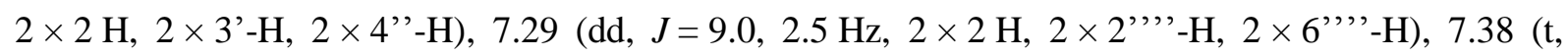
$\left.J=7.7 \mathrm{~Hz}, 2 \times 1 \mathrm{H}, 2 \times 5{ }^{\prime} '-\mathrm{H}\right), 7.43(\mathrm{dd}, J=7.7,1.1 \mathrm{~Hz}, 2 \times 1 \mathrm{H}, 2 \times 3$ ' $-\mathrm{H}), 7.52(\mathrm{dt}, J=8.0,1.5 \mathrm{~Hz}$, $\left.2 \times 1 \mathrm{H}, 2 \times 6{ }^{\prime}-\mathrm{H}\right), 7.76\left(\mathrm{~d}, J=7.9 \mathrm{~Hz}, 2 \times 1 \mathrm{H}, 2 \times 6^{\prime}{ }^{\prime}-\mathrm{H}\right)$.

${ }^{13} \mathbf{C}-\mathrm{NMR}\left(126 \mathrm{MHz}, \mathrm{CDCl}_{3}\right): \delta(\mathrm{ppm})=-4.6,-4.3\left(\mathrm{Si}\left(\mathrm{CH}_{3}\right)_{2}\right), 18.4\left(\mathrm{SiC}\left(\mathrm{CH}_{3}\right)_{3}\right), 25.9\left(\mathrm{SiC}\left(\mathrm{CH}_{3}\right)_{3}\right)$, 32.0 (C-5), 37.7, 37.8 (C-4), $55.5\left(\mathrm{OCH}_{3}\right), 62.4,62.5$ (C-3), 63.6 (C-1"'”), 83.1 (C-1), 84.9, 85.0

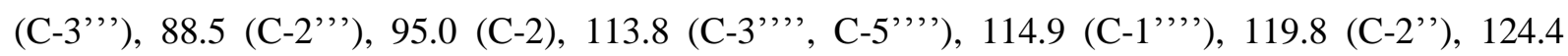
(C-2'), 126.2 (C-6'), 127.3, 127.4 (C-4', C-4'”), 127.7 (C-5'), 128.9 (C-5'), 130.5 (C-3'), 132.0, 132.1 (C-6'), 132.8 (C-3”'), 132.9 (C-2',', C-6"'”), 140.5 (C-1'), 143.8, 143.9 (C-1’'), 159.4 $(\mathrm{C}-4,,, ')$.

MS (ESI): $m / z(\%)=613.2(100)[\mathrm{M}+\mathrm{Na}]^{+}$.

HRMS (ESI): $m / z=$ gef.: 611.1578 , ber.: $611.1588\left[\mathrm{M}\left({ }^{79} \mathrm{Br}\right)+\mathrm{Na}\right]^{+}$; gef.: 613.1558 , ber.: 613.1569 $\left[\mathrm{M}\left({ }^{81} \mathrm{Br}\right)+\mathrm{Na}\right]^{+}$.

$\mathrm{C}_{33} \mathrm{H}_{37} \mathrm{BrO}_{3} \mathrm{Si}$ (589.63). 


\section{5-(2-Bromphenyl)-1-(2-(1-((tert-butyldimethylsilyl)oxy)-3-(2-phenoxyphenyl)prop-2-in-1-}

\section{yl)phenyl)pent-1-in-3-ol (171e)}

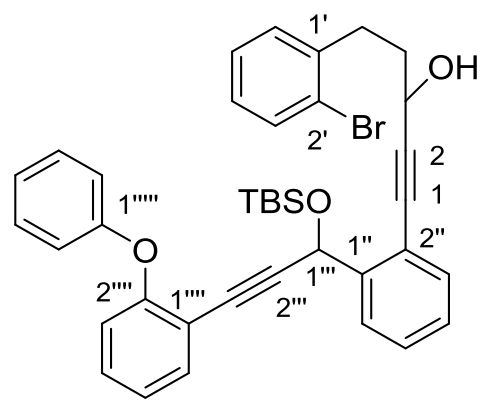

Für Diastereomerengemisch.

DC: $R_{f}=0.21(\mathrm{PE} / \mathrm{EtOAc}, 15: 1)$.

UV/Vis $\left(\mathrm{CH}_{3} \mathrm{CN}\right): \lambda_{\max }(\mathrm{nm})(\lg \varepsilon)=194$ (4.978), 245 (4.497), 255 (4.457), 290 (3.552).

IR (ATR): $\tilde{v}\left(\mathrm{~cm}^{-1}\right)=1483,1471,1444,1251,1222,1107,1057,1022,1005,985,837,800,777,747$, $689,670,658$.

${ }^{1} \mathbf{H}-\mathbf{N M R}\left(600 \mathrm{MHz}, \mathrm{CDCl}_{3}\right): \delta(\mathrm{ppm})=0.09(\mathrm{~s}, 2 \times 3 \mathrm{H}), 0.13(\mathrm{~s}, 2 \times 3 \mathrm{H}), 0.87(\mathrm{~s}, 2 \times 9 \mathrm{H}), 2.05-$ $2.11(\mathrm{~m}, 2 \times 2 \mathrm{H}), 2.13\left(\mathrm{~s}_{\mathrm{br}}, 2 \times 1 \mathrm{H}\right), 2.85-3.03(\mathrm{~m}, 2 \times 2 \mathrm{H}), 4.59(\mathrm{q}, J=6.7 \mathrm{~Hz}, 2 \times 1 \mathrm{H}), 6.01(\mathrm{~s}$, $2 \times 1 \mathrm{H}), 6.86(\mathrm{ddd}, J=8.3,2.4,1.1 \mathrm{~Hz}, 2 \times 1 \mathrm{H}), 6.89(\mathrm{ddd}, J=8.7,7.0,1.0 \mathrm{~Hz}, 2 \times 2 \mathrm{H}), 6.98-7.07$ $(\mathrm{m}, 2 \times 3 \mathrm{H}), 7.17(\mathrm{tdd}, J=7.3,5.6,1.3 \mathrm{~Hz}, 2 \times 1 \mathrm{H}), 7.20-7.28(\mathrm{~m}, 2 \times 5 \mathrm{H}), 7.30(\mathrm{td}, J=7.6,1.4 \mathrm{~Hz}$, $2 \times 1 \mathrm{H}), 7.38(\mathrm{ddd}, J=7.7,4.4,1.3 \mathrm{~Hz}, 2 \times 1 \mathrm{H}), 7.43(\mathrm{dt}, J=7.7,1.9 \mathrm{~Hz}, 2 \times 1 \mathrm{H}), 7.51(\mathrm{dt}, J=8.0$, $1.1 \mathrm{~Hz}, 2 \times 1 \mathrm{H}), 7.60-7.66(\mathrm{~m}, 2 \times 1 \mathrm{H})$.

${ }^{13}$ C-NMR $\left(126 \mathrm{MHz}, \mathrm{CDCl}_{3}\right): \delta(\mathrm{ppm})=-4.9,-4.5,18.3,25.8,26.9,31.9,37.6,62.3,63.6,80.8,83.0$, 94.5, 95.1, 115.6, 118.2, 119.2, 120.1, 123.4, 124.5, 126.3, 127.4, 127.7, 128.8, 129.6, 130.5, 131.9, $132.8,133.8,140.6,143.6,157.3,157.4$.

MS (ESI): $m / z(\%)=675.2(100)[\mathrm{M}+\mathrm{Na}]^{+}$.

HRMS (ESI): $m / z=$ gef.: 673.1745 , ber.: $673.1744\left[\mathrm{M}\left({ }^{79} \mathrm{Br}\right)+\mathrm{Na}\right]^{+}$; gef.: 675.1731 , ber.: 675.1726 $\left[\mathrm{M}\left({ }^{81} \mathrm{Br}\right)+\mathrm{Na}\right]^{+}$.

$\mathrm{C}_{38} \mathrm{H}_{39} \mathrm{BrO}_{3} \mathrm{Si}(651.70)$. 


\section{Allgemeine Vorschrift für die Synthese der Diole 115a-g (TBS-Entschützung):}

Eine Lösung von 171 (1.00 Äq.) und $3 \mathrm{M} \mathrm{HCl}$ in THF ( $c=0.04 \mathrm{~mol} / \mathrm{L}$ ) wurde für $5 \mathrm{~h}$ bei $60{ }^{\circ} \mathrm{C}$ gerührt. Nach Zugabe von ges. wässr. $\mathrm{NaHCO}_{3}$-Lösung wurde die Reaktionsmischung mit $\mathrm{CH}_{2} \mathrm{Cl}_{2}$ extrahiert $(3 \times)$, die vereinigten organischen Phasen über $\mathrm{Na}_{2} \mathrm{SO}_{4}$ getrocknet, filtriert und das Lösungsmittel in vacuo entfernt. Säulenchromatographische Reinigung lieferte Diol 115 als gelbes Harz.

Tabelle 19: Synthese der Dialkine 115a-g.<smiles>[R]c1cccc(C#CC(O)c2ccccc2C#CC(O)CCc2ccccc2Br)c1</smiles>

\begin{tabular}{cccccccc}
\hline Eintrag & Substrat & $\mathbf{R}^{\mathbf{1}}$ & $\mathbf{R}^{\mathbf{2}}$ & Produkt & $\boldsymbol{V}(\mathbf{H C l})[\mathbf{m L}]$ & Ausbeute [mg] & Ausbeute [\%] \\
\hline 1 & $\mathbf{1 7 1 a}$ & H & H & $\mathbf{1 1 5 a}$ & 8 & 123 & 78 \\
2 & $\mathbf{1 7 1 b}$ & H & $4-\mathrm{Me}$ & $\mathbf{1 1 5 b}$ & 13 & 90.1 & 59 \\
3 & $\mathbf{1 7 1 c}$ & H & $4-\mathrm{F}$ & $\mathbf{1 1 5 c}$ & 6 & 120 & 84 \\
4 & $\mathbf{1 7 1 f}$ & Me & H & $\mathbf{1 1 5 f}$ & 5 & 57.3 & $69^{[\mathrm{a}]}$ \\
5 & $\mathbf{1 7 1 g}$ & F & H & $\mathbf{1 1 5 g}$ & 5 & 61.6 & $64^{[\mathrm{a}]}$ \\
\hline
\end{tabular}

[a] über zwei Stufen. 


\section{5-(2-Bromphenyl)-1-(2-(1-hydroxy-3-phenylprop-2-in-1-yl)phenyl)pent-1-in-3-ol (115a)}

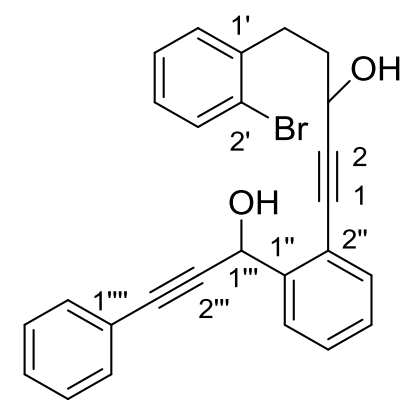

Für Diastereomerengemisch.

DC: $R_{f}=0.34\left(\mathrm{CH}_{2} \mathrm{Cl}_{2} / \mathrm{MTBE}, 50: 1\right)$.

UV/Vis $\left(\mathrm{CH}_{3} \mathrm{CN}\right): \lambda_{\max }(\mathrm{nm})(\lg \varepsilon)=197(4.941), 244$ (4.533), 253 (4.467).

IR (ATR): $\tilde{v}\left(\mathrm{~cm}^{-1}\right)=3312,1597,1567,1488,1471,1441,1328,1258,1181,1018,997,962,749$, $689,657,634,581,525$.

${ }^{1} \mathbf{H}-\mathrm{NMR}\left(600 \mathrm{MHz}, \mathrm{CDCl}_{3}\right): \delta(\mathrm{ppm})=2.07-2.16\left(\mathrm{~m}, 2 \times 2 \mathrm{H}, 2 \times 4-\mathrm{H}_{2}\right), 2.93-3.00(\mathrm{~m}, 2 \times 2 \mathrm{H}$, $\left.2 \times 5-\mathrm{H}_{2}\right), 3.31,3.43\left(2 \times \mathrm{s}_{\mathrm{br}}, 2 \times 2 \mathrm{H}, 4 \times \mathrm{OH}\right), 4.65(\mathrm{dt}, J=6.5,4.6 \mathrm{~Hz}, 2 \times 1 \mathrm{H}, 2 \times 3-\mathrm{H}), 6.10(\mathrm{~d}$, $\left.J=10.1 \mathrm{~Hz}, 2 \times 1 \mathrm{H}, 2 \times 1^{\prime \prime}{ }^{\prime}-\mathrm{H}\right), 7.03$ (tt, $\left.J=7.6,2.0 \mathrm{~Hz}, 2 \times 1 \mathrm{H}, 2 \times 5^{\prime}-\mathrm{H}\right), 7.17$ (ddt, $J=7.6,2.5$,

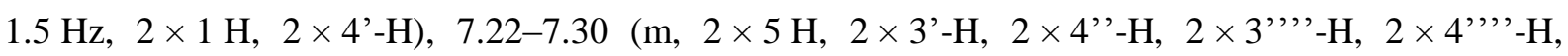
$2 \times 5 ", "-\mathrm{H}), 7.36\left(\mathrm{dt}, J=7.7,1.3 \mathrm{~Hz}, 2 \times 1 \mathrm{H}, 2 \times 5{ }^{\prime}-\mathrm{H}\right), 7.42(\mathrm{dd}, J=7.9,1.2 \mathrm{~Hz}, 2 \times 2 \mathrm{H}$, $2 \times 2$ ',' $-\mathrm{H}, 2 \times 6$ ',' $-\mathrm{H}), 7.45\left(\mathrm{ddd}, J=7.7,3.6,1.3 \mathrm{~Hz}, 2 \times 1 \mathrm{H}, 2 \times 3{ }^{\prime},-\mathrm{H}\right), 7.50(\mathrm{dt}, J=7.9,1.5 \mathrm{~Hz}$, $2 \times 1 \mathrm{H}, 2 \times 6 '-\mathrm{H}), 7.78\left(\mathrm{ddd}, J=7.7,4.5,1.2 \mathrm{~Hz}, 2 \times 1 \mathrm{H}, 2 \times 6{ }^{\prime}-\mathrm{H}\right)$.

${ }^{13}$ C-NMR $\left(126 \mathrm{MHz}, \mathrm{CDCl}_{3}\right): \delta(\mathrm{ppm})=32.0(\mathrm{C}-5), 37.5,37.6$ (C-4), 62.2 (C-3), 63.4, 63.5 (C-1','), 82.5, 82.6 (C-1), 86.7 (C-3'”), 88.1 (C-2'”), 95.3, 95.4 (C-2), 121.1, 121.2 (C-2'), 122.3 (C-1',',), 124.4 (C-2'), 126.8 (C-6'), 127.4, 127.7 (C-4', C-5', C-4'”), 128.2 (C-4'”,', C-3',', C-5'”,'), 128.4 (C-5'), 128.9 (C-3'), 130.5 (C-3'), 131.6 (C-2',', C-6','), 132.5, 132.6 (C-6'), 132.7 (C-3’'), 140.4 $(\mathrm{C}-1$ ') 142.0 (C-1'”).

MS (ESI): $m / z(\%)=467.1(24)[\mathrm{M}+\mathrm{Na}]^{+}, 913.2(100)[2 \mathrm{M}+\mathrm{Na}]^{+}$

HRMS (ESI): $m / z=$ gef.: 467.0629 , ber.: $467.0617\left[\mathrm{M}\left({ }^{79} \mathrm{Br}\right)+\mathrm{Na}\right]^{+}$; gef.: 469.0610 , ber.: 469.0598 $\left[\mathrm{M}\left({ }^{81} \mathrm{Br}\right)+\mathrm{Na}\right]^{+}$.

$\mathrm{C}_{26} \mathrm{H}_{21} \mathrm{BrO}_{2}$ (445.35). 


\section{5-(2-Bromphenyl)-1-(2-(1-hydroxy-3-(p-tolyl)prop-2-in-1-yl)phenyl)pent-1-in-3-ol (115b)}

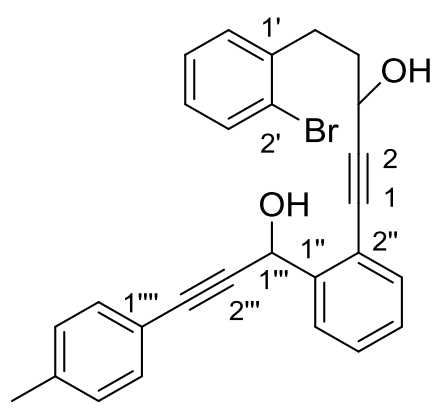

Für Diastereomerengemisch.

DC: $R_{f}=0.13\left(\mathrm{CH}_{2} \mathrm{Cl}_{2} / \mathrm{MTBE}, 50: 1\right)$.

UV/Vis $\left(\mathrm{CH}_{3} \mathrm{CN}\right): \lambda_{\max }(\mathrm{nm})(\lg \varepsilon)=197$ (4.926), 247 (4.554).

IR (ATR): $\tilde{v}\left(\mathrm{~cm}^{-1}\right)=3316,2922,2855,1508,1480,1488,1471,1326,1258,1043,1021,1012,966$, $948,816,748,658,624,524$.

${ }^{1} \mathbf{H}-\mathbf{N M R}\left(600 \mathrm{MHz} \mathrm{CDCl}_{3}\right): \delta(\mathrm{ppm})=2.09-2.15\left(\mathrm{~m}, 2 \times 2 \mathrm{H}, 2 \times 4-\mathrm{H}_{2}\right), 2.31\left(\mathrm{~s}, 2 \times 3 \mathrm{H}, 2 \times \mathrm{CH}_{3}\right)$, 2.93, $3.14\left(2 \times \mathrm{s}_{\mathrm{br}}, 2 \times 2 \mathrm{H}, 4 \times \mathrm{OH}\right), 2.93-3.00\left(\mathrm{~m}, 2 \times 2 \mathrm{H}, 2 \times 5-\mathrm{H}_{2}\right), 4.66(\mathrm{dt}, J=6.4,3.0 \mathrm{~Hz}$, $2 \times 1 \mathrm{H}, 2 \times 3-\mathrm{H}), 6.07\left(\mathrm{~d}, J=5.4 \mathrm{~Hz}, 2 \times 1 \mathrm{H}, 2 \times 1^{\prime},{ }^{\prime}-\mathrm{H}\right), 7.03\left(\mathrm{tt}, J=7.5,1.6 \mathrm{~Hz}, 2 \times 1 \mathrm{H}, 5^{\prime}-\mathrm{H}\right)$, $7.06\left(\mathrm{~d}, J=7.9 \mathrm{~Hz}, 2 \times 2 \mathrm{H}, 2 \times 3^{\prime}{ }^{\prime},{ }^{-H}, 2 \times 5^{\prime}{ }^{\prime},{ }^{-H}\right), 7.18\left(\mathrm{tt}, J=7.5,1.3 \mathrm{~Hz}, 2 \times 1 \mathrm{H}, 2 \times 4^{\prime}-\mathrm{H}\right), 7.24$ $\left(\mathrm{tt}, J=7.7,1.9 \mathrm{~Hz}, 2 \times 1 \mathrm{H}, 2 \times 3^{\prime}-\mathrm{H}\right), 7.27$ (tt, $\left.J=7.6,1.1 \mathrm{~Hz}, 2 \times 1 \mathrm{H}, 2 \times 4{ }^{\prime}-\mathrm{H}\right), 7.31$ (d, $J=8.1 \mathrm{~Hz}, 2 \times 2 \mathrm{H}, 2 \times 2$ ', ' $\left.-\mathrm{H}, 2 \times 6{ }^{\prime},{ }^{\prime}-\mathrm{H}\right), 7.36(\mathrm{dt}, J=7.6,1.2 \mathrm{~Hz}, 2 \times 1 \mathrm{H}, 2 \times 5$ ' $-\mathrm{H}), 7.46$ (ddd, $\left.J=7.6,2.7,1.2 \mathrm{~Hz}, 2 \times 1 \mathrm{H}, 2 \times 3^{\prime}{ }^{\prime}-\mathrm{H}\right), 7.50\left(\mathrm{dt}, J=8.0,1.2 \mathrm{~Hz}, 2 \times 1 \mathrm{H}, 2 \times 6{ }^{\prime}-\mathrm{H}\right), 7.77$ (ddd, $J=7.9,3.7,1.2 \mathrm{~Hz}, 2 \times 1 \mathrm{H}, 2 \times 6$ ' $-\mathrm{H})$.

${ }^{13}$ C-NMR $\left(126 \mathrm{MHz}_{\mathrm{CDCl}}\right): \delta(\mathrm{ppm})=21.5\left(\mathrm{CH}_{3}\right), 32.0(\mathrm{C}-5), 37.6(\mathrm{C}-4), 62.3,(\mathrm{C}-3), 63.5,63.6$

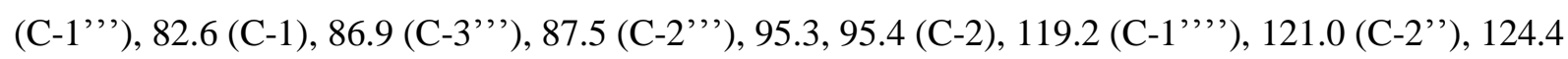
(C-2’), 126.8 (C-6’’), 127.4 (C-4'), 127.7 (C-5'), 128.2 (C-4’'), 128.9 (C-5',, C-3',,', C-5',,'), 130.5 (C-3'), 131.6 (C-2',',, C-6',',), 132.6 (C-3'’), 132.8 (C-6') 138.6 (C-4',,'), 140.5 (C-1'), 142.1 $\left(\mathrm{C}-1{ }^{\prime},\right)$.

MS (ESI): $m / z(\%)=481.1(36)[\mathrm{M}+\mathrm{Na}]^{+}, 941.1(100)[2 \mathrm{M}+\mathrm{Na}]^{+}$.

HRMS (ESI): $m / z=$ gef.: 481.0761 , ber.: $481.0774\left[\mathrm{M}\left({ }^{79} \mathrm{Br}\right)+\mathrm{Na}\right]^{+}$; gef.: 483.0754, ber.: 483.0755 $\left[\mathrm{M}\left({ }^{81} \mathrm{Br}\right)+\mathrm{Na}\right]^{+}$.

$\mathrm{C}_{27} \mathrm{H}_{23} \mathrm{BrO}_{2}$ (459.37). 


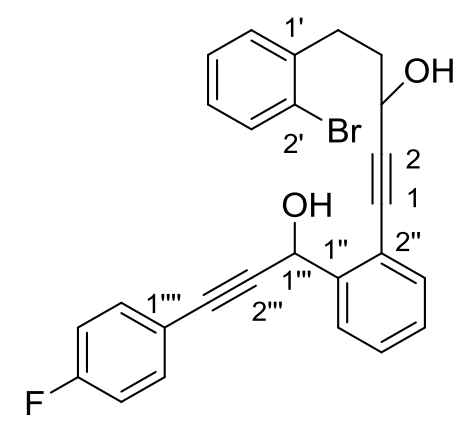

Für Diastereomerengemisch.

DC: $R_{f}=0.22\left(\mathrm{CH}_{2} \mathrm{Cl}_{2} / \mathrm{MTBE}, 50: 1\right)$.

UV/Vis $\left(\mathrm{CH}_{3} \mathrm{CN}\right): \lambda_{\max }(\mathrm{nm})(\lg \varepsilon)=197$ (4.955), 243 (4.539), 303 (2.867), 326 (2.777).

IR (ATR): $\tilde{v}\left(\mathrm{~cm}^{-1}\right)=3323,2934,2863,1600,1504,1471,1447,1439,1234,1220,1156,1062,1036$, $1016,964,905,837,819,751,659,623,532$.

${ }^{1} \mathbf{H}-\mathrm{NMR}\left(600 \mathrm{MHz}, \mathrm{CDCl}_{3}\right): \delta(\mathrm{ppm})=2.07-2.15\left(\mathrm{~m}, 2 \times 2 \mathrm{H}, 4-\mathrm{H}_{2}\right), 2.69,2.72\left(2 \times \mathrm{s}_{\mathrm{br}}, 2 \times 1 \mathrm{H}\right.$, $2 \times \mathrm{OH}), 2.93-3.01\left(\mathrm{~m}, 2 \times 2 \mathrm{H}, 2 \times 5-\mathrm{H}_{2}\right), 3.04,3.06\left(2 \times \mathrm{s}_{\mathrm{br}}, 2 \times 1 \mathrm{H}, 2 \times \mathrm{OH}\right), 4.67\left(\mathrm{~m}_{\mathrm{C}}, 2 \times 1 \mathrm{H}\right.$, $2 \times 3-\mathrm{H}), 6.06\left(\mathrm{~d}, J=9.4 \mathrm{~Hz}, 2 \times 1 \mathrm{H}, 2 \times 1{ }^{\prime \prime} \cdot-\mathrm{H}\right), 6.94\left(\mathrm{dt}, J=8.7,1.6 \mathrm{~Hz}, 2 \times 2 \mathrm{H}, 2 \times 3{ }^{\prime \prime \prime} \cdot-\mathrm{H}\right.$, $\left.2 \times 5^{\prime \prime \prime \prime}-\mathrm{H}\right), 7.04\left(\mathrm{dt}, J=7.3,1.2 \mathrm{~Hz}, 2 \times 1 \mathrm{H}, 2 \times 5^{\prime}-\mathrm{H}\right), 7.19$ (tt, $J=7.4,1.4 \mathrm{~Hz}, 2 \times 1 \mathrm{H}, 2 \times 4^{\prime}-\mathrm{H}$ ), $7.23\left(\mathrm{dt}, J=7.7,2.1 \mathrm{~Hz}, 2 \times 1 \mathrm{H}, 2 \times 3{ }^{\prime}-\mathrm{H}\right), 7.28\left(\mathrm{dt}, J=7.8,1.3 \mathrm{~Hz}, 2 \times 1 \mathrm{H}, 2 \times 4{ }^{\prime \prime}-\mathrm{H}\right), 7.36-7.40$ (m, $2 \times 3 \mathrm{H}, 2 \times 5$ ' $-\mathrm{H}, 2 \times 2$ ', ' $-\mathrm{H}, 2 \times 6$ ',' $-\mathrm{H}), 7.46$ (ddd, J=7.8, 2.5, $1.3 \mathrm{~Hz}, 2 \times 1 \mathrm{H}, 2 \times 3$ '”-H), $7.51\left(\mathrm{dt}, J=8.1,1.1 \mathrm{~Hz}, 2 \times 1 \mathrm{H}, 2 \times 6^{\prime}-\mathrm{H}\right), 7.74\left(\mathrm{ddd}, J=7.8,4.6,1.2 \mathrm{~Hz}, 2 \times 1 \mathrm{H}, 2 \times 6{ }^{\prime} '-\mathrm{H}\right)$.

${ }^{13} \mathrm{C}-\mathrm{NMR}\left(126 \mathrm{MHz}, \mathrm{CDCl}_{3}\right): \delta(\mathrm{ppm})=32.0(\mathrm{C}-5), 37.6,37.7$ (C-4), 62.3 (C-3), 63.5 (C-1 '”), 82.6 (C-1), 85.6 (C-2','), 87.9 (C-3'”'), 95.4 (C-2), 115.5 (d, J=22.1 Hz, C-3",', C-5','), 118.4 (d, $J=3.8 \mathrm{~Hz}, \mathrm{C}-1^{\prime \prime \prime \prime ')}$, 120.9, 121.0 (C-2'’), 124.4 (C-2'), 126.7 (C-6’'), 127.4 (C-4'), 127.7 (C-5'), 128.3 (C-4’'), 129.0 (C-5'), 130.4 (C-3'), 132.6 (C-3’'), 132.8 (C-6'), 133.6 (d, J = 8.4 Hz, C-2',', C-6’,'), 140.4 (C-1'), 142.0 (C-1'’), 162.5 (d, J=249.7 Hz, C-4',',).

${ }^{19}$ F-NMR $\left(282 \mathrm{MHz}, \mathrm{CDCl}_{3}\right): \delta(\mathrm{ppm})=-110.4\left(\mathrm{tdd}, J=9.0,4.3,2.7 \mathrm{~Hz}, 4,{ }^{\prime \prime}{ }^{-}-\mathrm{F}\right)$.

MS (ESI): $m / z(\%)=487.1(33)[\mathrm{M}+\mathrm{Na}]^{+}, 949.1(100)[2 \mathrm{M}+\mathrm{Na}]^{+}$.

HRMS (ESI): $m / z=$ gef.: 485.0520 , ber.: $485.0523\left[\mathrm{M}\left({ }^{79} \mathrm{Br}\right)+\mathrm{Na}\right]^{+}$; gef.: 487.0497 , ber.: 487.0504 $\left[\mathrm{M}\left({ }^{81} \mathrm{Br}\right)+\mathrm{Na}\right]^{+}$.

$\mathrm{C}_{26} \mathrm{H}_{20} \mathrm{BrFO}_{2}$ (463.34). 


\section{5-(2-Bromphenyl)-1-(2-(1-hydroxy-3-(4-methoxyphenyl)prop-2-in-1-yl)phenyl)pent-1-in-3-ol} (115d)

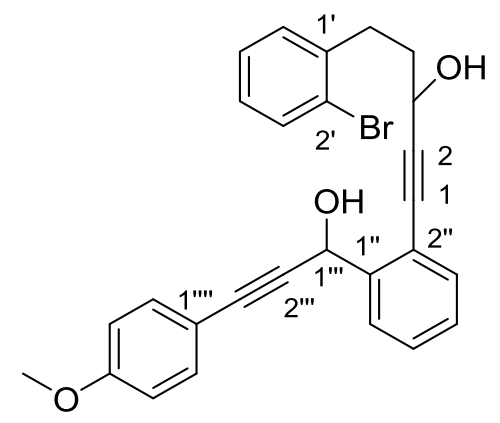

Eine Lösung von 171d (320 mg, $543 \mu \mathrm{mol}, 1.00$ Äq.), TBAF (1.0 M in THF, $814 \mu \mathrm{L}, 814 \mu \mathrm{mmol}$, 1.50 Äq.) und $\mathrm{NH}_{4} \mathrm{Cl}(43.5 \mathrm{mg}, 814 \mu \mathrm{mol}, 1.50$ Äq.) in THF $(10 \mathrm{~mL})$ wurde $4 \mathrm{~h}$ bei RT gerührt. Nach Zugabe von ges. wässr. $\mathrm{NaHCO}_{3}$-Lösung $(10 \mathrm{~mL})$ wurde die Reaktionsmischung mit $\mathrm{CH}_{2} \mathrm{Cl}_{2}$ extrahiert $(3 \times 10 \mathrm{~mL})$. Die vereinigten organischen Phasen wurden über $\mathrm{Na}_{2} \mathrm{SO}_{4}$ getrocknet und das Lösungsmittel in vacuo entfernt. Säulenchromatographische Reinigung $\left(\mathrm{SiO}_{2}, \mathrm{CH}_{2} \mathrm{Cl}_{2} / \mathrm{MTBE} 20: 1\right)$ lieferte Diol 115d als gelbes Harz (225 mg, $473 \mu \mathrm{mol}, 87 \%)$.

Für Diastereomerengemisch.

DC: $R_{f}=0.20\left(\mathrm{CH}_{2} \mathrm{Cl}_{2} / \mathrm{Et}_{2} \mathrm{O}, 10: 1\right)$.

UV/Vis $\left(\mathrm{CH}_{3} \mathrm{CN}\right): \lambda_{\max }(\mathrm{nm})(\lg \varepsilon)=197$ (4.922), 255 (4.531).

IR (ATR): $\tilde{v}\left(\mathrm{~cm}^{-1}\right)=3321,2955,2931,2836,2224,1605,1568,1508,1470,1450,1290,1245,1173$, $1020,961,951,830,751,702,658,625,574,534$.

${ }^{1} \mathbf{H}-\mathrm{NMR}\left(600 \mathrm{MHz}, \mathrm{CDCl}_{3}\right): \delta(\mathrm{ppm})=2.06-2.15\left(\mathrm{~m}, 2 \times 2 \mathrm{H}, 4-\mathrm{H}_{2}\right), 2.92-3.00\left(\mathrm{~m}, 2 \times 2 \mathrm{H}, 5-\mathrm{H}_{2}\right)$, $3.28,3.33,3.38,3.42\left(4 \times \mathrm{s}_{\mathrm{br}}, 4 \times 1 \mathrm{H}, 4 \times \mathrm{OH}\right), 3.76,3.77\left(2 \times \mathrm{s}, 2 \times 3 \mathrm{H}, 2 \times \mathrm{OCH}_{3}\right), 4.65(\mathrm{q}$, $J=5.3 \mathrm{~Hz}, 2 \times 1 \mathrm{H}, 2 \times 3-\mathrm{H}), 6.08\left(\mathrm{~d}, J=10.9 \mathrm{~Hz}, 2 \times 1 \mathrm{H}, 2 \times 1{ }^{\prime \prime}{ }^{-}-\mathrm{H}\right), 6.77(\mathrm{dd}, J=8.9,1.4 \mathrm{~Hz}$, $\left.2 \times 2 \mathrm{H}, 2 \times 3{ }^{\prime},{ }^{\prime}{ }^{-H}, 2 \times 5^{\prime},{ }^{\prime}-\mathrm{H}\right), 7.03\left(\mathrm{tt}, J=7.8,1.8 \mathrm{~Hz}, 2 \times 1 \mathrm{H}, 2 \times 5^{\prime}-\mathrm{H}\right), 7.17$ (tt, $J=7.5,1.5 \mathrm{~Hz}$, $\left.2 \times 1 \mathrm{H}, 2 \times 4^{\prime}-\mathrm{H}\right), 7.23\left(\mathrm{ddd}, J=7.6,2.4,1.9 \mathrm{~Hz}, 2 \times 1 \mathrm{H}, 2 \times 3^{\prime}-\mathrm{H}\right), 7.26(\mathrm{dt}, J=7.6,1.1 \mathrm{~Hz}$,

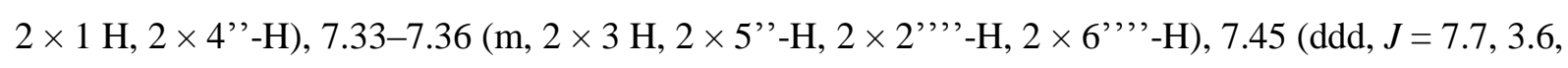
$\left.1.2 \mathrm{~Hz}, 2 \times 1 \mathrm{H}, 2 \times 3^{\prime}{ }^{-}-\mathrm{H}\right), 7.50\left(\mathrm{dt}, J=8.0,1.4 \mathrm{~Hz}, 2 \times 1 \mathrm{H}, 2 \times 6^{\prime}-\mathrm{H}\right), 7.77(\mathrm{~d}, J=7.7,4.9 \mathrm{~Hz}$, $2 \times 1 \mathrm{H}, 2 \times 6 " '-\mathrm{H})$.

${ }^{13} \mathrm{C}-\mathrm{NMR}\left(126 \mathrm{MHz}, \mathrm{CDCl}_{3}\right): \delta(\mathrm{ppm})=32.0(\mathrm{C}-5), 37.5,37.6(\mathrm{C}-4), 55.3\left(\mathrm{OCH}_{3}\right), 62.2(\mathrm{C}-3), 63.5$ (C-1','), 82.6 (C-1), 86.7, 86.8 (C-3',', C-2','), 95.3 (C-2), 113.8 (C-3',', C-5',',), 114.4 (C-1',',), 121.1 (C-2'”), 124.4 (C-2'), 126.8 (C-6’'), 127.4 (C-4'), 127.6 (C-5'), 128.1 (C-4'), 128.9 (C-5'), 130.5 (C-3'), 132.5, 132.6 (C-3'’), 132.7 (C-6'), 133.1 (C-2',', C-6','’), 140.5 (C-1'), 142.2 (C-1'’), $159.6(\mathrm{C}-4,, ')$. 
MS (ESI): $m / z(\%)=497.1(37)[\mathrm{M}+\mathrm{Na}]^{+}, 973.1(100)[2 \mathrm{M}+\mathrm{Na}]^{+}$.

HRMS (ESI): $m / z=$ gef.: 497.0709 , ber.: $497.0723\left[\mathrm{M}\left({ }^{79} \mathrm{Br}\right)+\mathrm{Na}\right]^{+}$; gef.: 499.0694 , ber.: 499.0704 $\left[\mathrm{M}\left({ }^{81} \mathrm{Br}\right)+\mathrm{Na}\right]^{+}$.

$\mathrm{C}_{27} \mathrm{H}_{23} \mathrm{BrO}_{3}$ (475.37). 


\section{5-(2-Bromphenyl)-1-(2-(1-hydroxy-3-(2-phenoxyphenyl)prop-2-in-1-yl)phenyl)pent-1-in-3-ol} (115e)

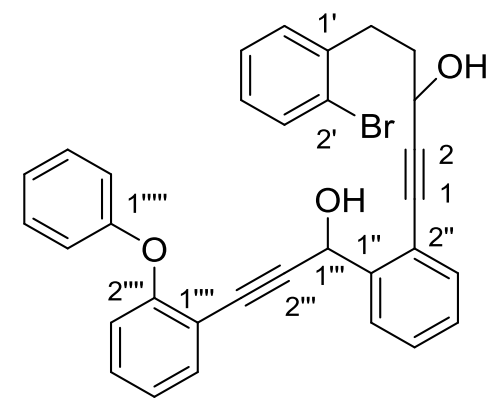

Eine Lösung von 171e (341 mg, $523 \mu \mathrm{mol}, 1.00$ Äq.) und TBAF ( $1 \mathrm{M}$ in THF, $785 \mu \mathrm{L}, 1.50$ Äq.) in THF $(10 \mathrm{~mL})$ wurde $1.5 \mathrm{~h}$ bei RT gerührt. Nach Zugabe von ges. wässr. $\mathrm{NH}_{4}$ Cl-Lösung $(20 \mathrm{~mL})$ wurde die Reaktionsmischung mit $\mathrm{CH}_{2} \mathrm{Cl}_{2}$ extrahiert $(3 \times 20 \mathrm{~mL})$, die vereinigten organischen Phasen über $\mathrm{Na}_{2} \mathrm{SO}_{4}$ getrocknet, filtriert und das Lösungsmittel in vacuo entfernt. Säulenchromatographische Reinigung ( $\mathrm{SiO}_{2}$, PE/EtOAc 3:1) lieferte Diol 115e als gelbes Harz (246 mg, $\left.458 \mu \mathrm{mol}, 87 \%\right)$ in leicht verunreinigter Form.

Für Diastereomerengemisch.

DC: $R_{f}=0.46(\mathrm{PE} / \mathrm{EtOAc}, 3: 1)$.

UV/Vis $\left(\mathrm{CH}_{3} \mathrm{CN}\right): \lambda_{\max }(\mathrm{nm})(\lg \varepsilon)=194$ (4.949), 245 (4.469), 255 (4.433), 289 (3.527).

IR (ATR): $\tilde{v}\left(\mathrm{~cm}^{-1}\right)=3318,1589,1482,1443,1252,1221,1181,1161,1106,1039,1018,964,950$, 906, 872, 799, 747, 689, 658, 634, 589, 518.

${ }^{1} \mathbf{H}-\mathbf{N M R}\left(600 \mathrm{MHz}, \mathrm{CDCl}_{3}\right): \delta(\mathrm{ppm})=2.04-2.12(\mathrm{~m}, 2 \times 2 \mathrm{H}), 2.87-3.00(\mathrm{~m}, 2 \times 2 \mathrm{H}), 3.14\left(\mathrm{~s}_{\mathrm{br}}\right.$, $2 \times 2 \mathrm{H}), 4.60(\mathrm{td}, J=6.5,3.7 \mathrm{~Hz}, 2 \times 1 \mathrm{H}), 5.98(\mathrm{~d}, J=4.7 \mathrm{~Hz}, 2 \times 1 \mathrm{H}), 6.91(\mathrm{~d}, J=8.3 \mathrm{~Hz}$, $2 \times 1 \mathrm{H}), 6.92-6.95(\mathrm{~m}, 2 \times 2 \mathrm{H}), 6.99-7.08(\mathrm{~m}, 2 \times 3 \mathrm{H}), 7.14-7.30(\mathrm{~m}, 2 \times 7 \mathrm{H}), 7.37-7.42(\mathrm{~m}$, $2 \times 1 \mathrm{H}), 7.47(\mathrm{dd}, J=7.7,1.7 \mathrm{~Hz}, 2 \times 1 \mathrm{H}), 7.50(\mathrm{ddd}, J=8.0,2.8,1.2 \mathrm{~Hz}, 2 \times 1 \mathrm{H}), 7.55-7.59(\mathrm{~m}$, $2 \times 1 \mathrm{H})$.

${ }^{13} \mathbf{C}-\mathrm{NMR}\left(126 \mathrm{MHz}, \mathrm{CDCl}_{3}\right): \delta(\mathrm{ppm})=31.9,37.4,62.1,63.4,82.5,93.1,95.3,115.1,118.1,119.4$, 121.2, 123.1, 123.6, 124.4, 126.9, 127.5, 127.7, 128.1, 128.9, 129.7, 130.0, 130.6, 132.4, 132.8, 133.8, 140.6, 141.9, 157.3, 157.4.

Weitere Signale wurden nicht beobachtet.

MS (ESI): $m / z(\%)=561.1(8)[\mathrm{M}+\mathrm{Na}]^{+}$. 
HRMS (ESI): $m / z=$ gef.: 559.0869 , ber.: $559.0879\left[\mathrm{M}\left({ }^{79} \mathrm{Br}\right)+\mathrm{Na}\right]^{+}$; gef.: 561.0854 , ber.: 561.0861 $\left[\mathrm{M}\left({ }^{81} \mathrm{Br}\right)+\mathrm{Na}\right]^{+}$.

$\mathrm{C}_{32} \mathrm{H}_{25} \mathrm{BrO}_{3}$ (537.44). 
5-(2-Brom-4-methylphenyl)-1-(2-(1-hydroxy-3-phenylprop-2-in-1-yl)phenyl)pent-1-in-3-ol (115f)

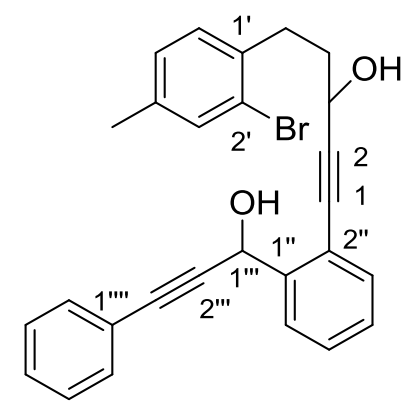

Für Diastereomerengemisch.

DC: $R_{f}=0.19\left(\mathrm{CH}_{2} \mathrm{Cl}_{2} / \mathrm{Et}_{2} \mathrm{O}, 50: 1\right)$.

UV/Vis $\left(\mathrm{CH}_{3} \mathrm{CN}\right): \lambda_{\max }(\mathrm{nm})(\lg \varepsilon)=200$ (4.942), 244 (4.529), 253 (4.464), 278 (3.201).

IR (ATR): $\tilde{v}\left(\mathrm{~cm}^{-1}\right)=3314,2922,2863,2226,1598,1489,1444,1390,1263,1181,1036,1012,997$, 962, 950, 865, 818, 753, 737, 689, 673, 634, 576, 525.

${ }^{1} \mathbf{H}-\mathbf{N M R}\left(600 \mathrm{MHz}, \mathrm{CDCl}_{3}\right): \delta(\mathrm{ppm})=2.05-2.16\left(\mathrm{~m}, 2 \times 2 \mathrm{H}, 2 \times 4-\mathrm{H}_{2}\right), 2.26(2 \times \mathrm{s}, 2 \times 3 \mathrm{H}$, $\left.2 \times \mathrm{CH}_{3}\right), 2.92\left(\mathrm{ddd}, J=9.6,6.9,3.3 \mathrm{~Hz}, 2 \times 2 \mathrm{H}, 2 \times 5-\mathrm{H}_{2}\right), 2.98,3.03,3.23,3.27\left(4 \times \mathrm{s}_{\mathrm{br}}, 4 \times 1 \mathrm{H}\right.$, $\mathrm{OH}), 4.64(\mathrm{dt}, J=6.5,3.6 \mathrm{~Hz}, 2 \times 1 \mathrm{H}, 2 \times 3-\mathrm{H}), 6.09\left(\mathrm{~d}, J=7.8 \mathrm{~Hz}, 2 \times 1 \mathrm{H}, 2 \times 1{ }^{\prime \prime}{ }^{-\mathrm{H}}\right), 6.98(\mathrm{dt}$, $\left.J=7.9,2.0 \mathrm{~Hz}, 2 \times 1 \mathrm{H}, 2 \times 5^{\prime}-\mathrm{H}\right), 7.11$ (dd, $\left.J=7.7,1.9 \mathrm{~Hz}, 2 \times 1 \mathrm{H}, 2 \times 6^{\prime}-\mathrm{H}\right), 7.25-7.31$ (m, $\left.2 \times 4 \mathrm{H}, 2 \times 4{ }^{\prime \prime}-\mathrm{H}, 2 \times 3^{\prime \prime \prime \prime}-\mathrm{H}, 2 \times 4{ }^{\prime \prime \prime \prime}-\mathrm{H}, 2 \times 5^{\prime \prime \prime}-\mathrm{H}\right), 7.33$ (s, $2 \times 1 \mathrm{H}, 2 \times 3$ '-H), 7.36 (t, $J=7.6 \mathrm{~Hz}, 2 \times 1 \mathrm{H}, 2 \times 5{ }^{\prime}$ '-H), $7.43\left(\mathrm{dd}, J=8.1,1.5 \mathrm{~Hz}, 2 \times 2 \mathrm{H}, 2 \times 2\right.$ ',' $\left.-\mathrm{H}, 2 \times 6{ }^{\prime \prime},{ }^{\prime}-\mathrm{H}\right), 7.45$ (ddd, $J=7.7,2.9,1.3 \mathrm{~Hz}, 2 \times 1 \mathrm{H}, 2 \times 3$ '’-H), 7.77 (ddd, $J=7.8,3.6,1.2 \mathrm{~Hz}, 2 \times 1 \mathrm{H}, 2 \times 6$ '”-H).

${ }^{13} \mathbf{C}-N M R\left(126 \mathrm{MHz}, \mathrm{CDCl}_{3}\right): \delta(\mathrm{ppm})=20.6\left(\mathrm{CH}_{3}\right), 31.5(\mathrm{C}-5), 37.7,37.8(\mathrm{C}-4), 62.3(\mathrm{C}-3), 63.5$

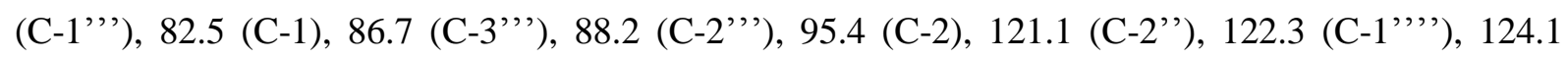
(C-2'), 126.8 (C-6'), 128.2, 128.4 (C-5', C-4', C-3',', C-4',', C-5','), 128.9 (C-5'), 130.2 (C-6'), 131.7 (C-2',', C-6’,'), 132.6 (C-3'’), 133.1 (C-3'), 137.2 (C-4'), 137.6 (C-1'), 142.0 (C-1'”).

MS (ESI): $m / z(\%)=483.1(45)[\mathrm{M}+\mathrm{Na}]^{+}, 941.3(100)[2 \mathrm{M}+\mathrm{Na}]^{+}$.

HRMS (ESI): $m / z=$ gef.: 481.0761 , ber.: $481.0774\left[\mathrm{M}\left({ }^{79} \mathrm{Br}\right)+\mathrm{Na}\right]^{+}$; gef.: 483.0754 , ber.: 483.0755 $\left[\mathrm{M}\left({ }^{81} \mathrm{Br}\right)+\mathrm{Na}\right]^{+}$.

$\mathbf{C}_{27} \mathbf{H}_{23} \mathrm{BrO}_{2}$ (459.37). 


\section{5-(2-Brom-4-fluorphenyl)-1-(2-(1-hydroxy-3-phenylprop-2-in-1-yl)phenyl)pent-1-in-3-ol (115g)}

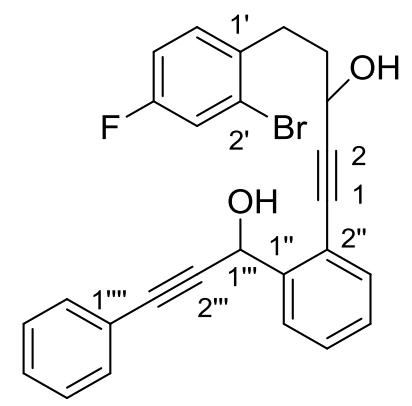

Für Diastereomerengemisch.

DC: $R_{f}=0.33\left(\mathrm{CH}_{2} \mathrm{Cl}_{2} / \mathrm{Et}_{2} \mathrm{O}, 50: 1\right)$.

UV/Vis $\left(\mathrm{CH}_{3} \mathrm{CN}\right): \lambda_{\max }(\mathrm{nm})(\lg \varepsilon)=195$ (4.890), 244 (4.530), 253 (4.466), 276 (3.361).

IR (ATR): $\tilde{v}\left(\mathrm{~cm}^{-1}\right)=3313,1598,1585,1484,1448,1392,1335,1260,1225,1179,1045,1032,1008$, 998, 962, 947, 862, 815, 752, 689, 672, 633, 581, 513.

${ }^{1} \mathbf{H}-\mathbf{N M R}\left(600 \mathrm{MHz}, \mathrm{CDCl}_{3}\right): \delta(\mathrm{ppm})=2.04-2.12\left(\mathrm{~m}, 2 \times 2 \mathrm{H}, 2 \times 4-\mathrm{H}_{2}\right), 2.93(\mathrm{ddt}, J=7.9,5.6$, $\left.2.6 \mathrm{~Hz}, 2 \times 2 \mathrm{H}, 2 \times 5-\mathrm{H}_{2}\right), 3.00,3.06,3.21,3.25\left(4 \times \mathrm{s}_{\mathrm{br}}, 4 \times 1 \mathrm{H}, 4 \times \mathrm{OH}\right), 4.64(\mathrm{dt}, J=6.5,4.5 \mathrm{~Hz}$, $2 \times 1 \mathrm{H}, 2 \times 3-\mathrm{H}), 6.08\left(\mathrm{~d}, J=9.3 \mathrm{~Hz}, 2 \times 1 \mathrm{H}, 2 \times 1^{\prime \prime}{ }^{-}-\mathrm{H}\right), 6.89(\mathrm{tt}, J=8.3,2.6 \mathrm{~Hz}, 2 \times 1 \mathrm{H}, 5$ '-H), 7.18 (ddd, $J=8.6,6.0,2.7 \mathrm{~Hz}, 2 \times 1 \mathrm{H}, 2 \times 6$ ' $-\mathrm{H}), 7.22-7.32\left(\mathrm{~m}, 2 \times 5 \mathrm{H}, 2 \times 3^{\prime}-\mathrm{H}, 2 \times 4\right.$ ' '-H,

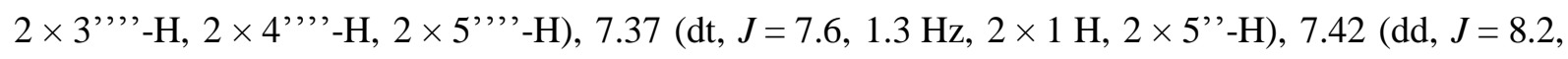
$\left.1.5 \mathrm{~Hz}, 2 \times 2 \mathrm{H}, 2 \times 2{ }^{\prime \prime \prime \prime}-\mathrm{H}, 2 \times 6{ }^{\prime \prime}, '-\mathrm{H}\right), 7.45\left(\mathrm{ddd}, J=7.7,2.6,1.4 \mathrm{~Hz}, 2 \times 1 \mathrm{H}, 2 \times 3{ }^{\prime}-\mathrm{H}\right), 7.77$ (ddd, $J=7.8,4.4,1.2 \mathrm{~Hz}, 2 \times 1 \mathrm{H}, 2 \times 6$ ''-H).

${ }^{13}$ C-NMR (126 MHz, $\left.\mathrm{CDCl}_{3}\right): \delta(\mathrm{ppm})=31.2(\mathrm{C}-5), 37.6$ (C-4), $62.1(\mathrm{C}-3), 63.5$ (C-1"’'), $82.6(\mathrm{C}-1)$, 86.7 (C-3'”'), 88.1 (C-2','), 95.2 (C-2), 114.5 (d, $J=20.7$ Hz, C-5'), 119.8 (d, J=24.2 Hz, C-3'), 121.0 (C-2"'), 122.2 (C-1','), 124.0 (d, J=9.4 Hz, C-2'), 126.8 (C-6'), 128.2 (C-3',', C-5','),

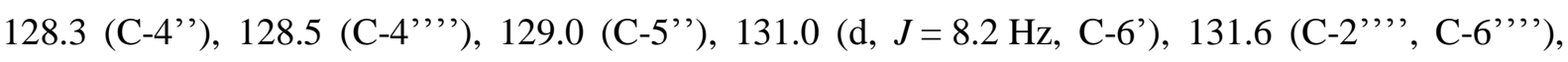
132.5, 132.6 (C-3'”), 136.2 (d, J=3.5 Hz, C-1'), 142.0 (C-1'’), 160.7 (d, J = 248.5 Hz, C-4').

${ }^{19}$ F-NMR $\left(282 \mathrm{MHz}, \mathrm{CDCl}_{3}\right): \delta(\mathrm{ppm})=-115.0\left(\mathrm{dt}, J=8.2,6.1 \mathrm{~Hz}, 4^{\prime}-\mathrm{F}\right)$.

MS (ESI): $m / z(\%)=485.1(100)[\mathrm{M}+\mathrm{Na}]^{+}, 949.2(92)[2 \mathrm{M}+\mathrm{Na}]^{+}$.

HRMS (ESI): $m / z=$ gef.: 485.0520 , ber.: $485.0523\left[\mathrm{M}\left({ }^{79} \mathrm{Br}\right)+\mathrm{Na}\right]^{+}$; gef.: 487.0497 , ber.: 487.0504 $\left[\mathrm{M}\left({ }^{81} \mathrm{Br}\right)+\mathrm{Na}\right]^{+}$.

$\mathrm{C}_{26} \mathrm{H}_{20} \mathrm{BrFO}_{2}$ (462.06). 


\subsubsection{Synthese der Poylyzyklen 114a-g durch die Domino-Reaktion}

Allgemeine Vorschrift für die Synthese der Produkte 114a-g (Dreifach-Domino-Reaktion):

Eine Mischung aus 115 (1.00 Äq.), Pd(TFA) 2 (0.20 Äq.), PCy (0.40 Äq.) und $\mathrm{K}_{2} \mathrm{CO}_{3}$ (3.00 Äq.) in entgastem DME ( $c=0.02 \mathrm{~mol} / \mathrm{L})$ wurde bei $80{ }^{\circ} \mathrm{C}$ gerührt. Die Reaktionsmischung wurde durch $\mathrm{SiO}_{2}$ filtriert, mit EtOAc gespült und das Lösungsmittel des Filtrats in vacuo entfernt. Säulenchromatographische Reinigung lieferte Domino-Produkt 114 als braunroten Feststoff in Form zweier Diastereomere.

Tabelle 20: Synthese der Domino-Produkte 114a-f.<smiles></smiles>

\begin{tabular}{ccccccccc}
\hline Eintrag & Substrat & $\mathbf{R}^{\mathbf{1}}$ & $\mathbf{R}^{\mathbf{2}}$ & Produkt & $\boldsymbol{t}[\mathbf{h}]$ & Ausbeute [mg] & Ausbeute [\%] & $\boldsymbol{d} . \boldsymbol{r}$. \\
\hline 1 & $\mathbf{1 1 5 a}$ & $\mathrm{H}$ & $\mathrm{H}$ & $\mathbf{1 1 4 a}$ & 6 & 16.1 & 52 & $1: 1.7$ \\
2 & $\mathbf{1 1 5 b}$ & $\mathrm{H}$ & $4-\mathrm{Me}$ & $\mathbf{1 1 4 b}$ & 9 & 23.7 & $29^{[\mathrm{a}]}$ & $1: 1.2$ \\
3 & $\mathbf{1 1 5 c}$ & $\mathrm{H}$ & $4-\mathrm{F}$ & $\mathbf{1 1 4 c}$ & 4 & 18.5 & 25 & $1: 1.3$ \\
4 & $\mathbf{1 1 5 d}$ & $\mathrm{H}$ & $4-\mathrm{OMe}$ & $\mathbf{1 1 4 d}$ & 9 & 23.5 & 26 & $1: 3.6$ \\
5 & $\mathbf{1 1 5 f}$ & $\mathrm{Me}$ & $\mathrm{H}$ & $\mathbf{1 1 4 f}$ & 8 & 33.8 & 44 & $1.5: 1$ \\
6 & $\mathbf{1 1 5 g}$ & $\mathrm{F}$ & $\mathrm{H}$ & $\mathbf{1 1 4 g}$ & 8 & 21.3 & 31 & $1: 1.2$ \\
\hline
\end{tabular}

[a] $33 \%$ brsm. 


\section{7-Phenyl-1,2,3,8-tetrahydroinden[1,2-a]phenalen-1,8-diol (114a)}

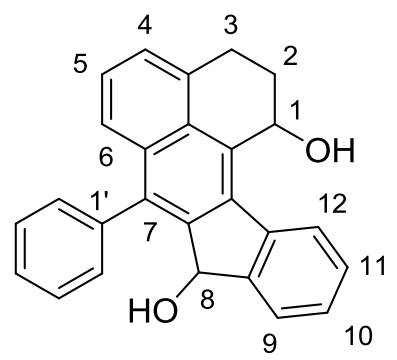

DC: $R_{f}=0.62$ (1. Diastereomer), 0.38 (2. Diastereomer) $\left(\mathrm{CH}_{2} \mathrm{Cl}_{2} / \mathrm{Et}_{2} \mathrm{O}, 50: 1\right)$.

UV/Vis $\left(\mathrm{CH}_{3} \mathrm{CN}\right): \lambda_{\max }(\mathrm{nm})(\lg \varepsilon)=257$ (4.422), 277 (4.514), 312 (4.101), 325 (4.083).

IR (ATR): $\tilde{v}\left(\mathrm{~cm}^{-1}\right)=3359,2922,1701,1606,1491,1429,1406,1312,1280,1245,1171,1081,1060$, 1037, 1028, 973, 940, 902, 891, 754, 743, 726, 711, 699, 634, 608, 582, 543, 521.

\section{Diastereomer:}

${ }^{1} \mathbf{H}-\mathbf{N M R}\left(600 \mathrm{MHz}\right.$, Aceton- $\left.\mathrm{d}_{6}\right): \delta(\mathrm{ppm})=2.08\left(\mathrm{tt}, J=13.9,3.6 \mathrm{~Hz}, 1 \mathrm{H}, 2-\mathrm{H}_{\mathrm{a}}\right), 2.43(\mathrm{dq}, J=13.3$, $\left.3.1 \mathrm{~Hz}, 1 \mathrm{H}, 2-\mathrm{H}_{\mathrm{b}}\right), 3.00\left(\mathrm{dt}, J=15.7,3.4,1 \mathrm{H}, 3-\mathrm{H}_{\mathrm{a}}\right), 3.55\left(\mathrm{dt}, J=15.3,3.9,1 \mathrm{H}, 3-\mathrm{H}_{\mathrm{b}}\right), 3.96(\mathrm{~d}$, $J=7.9 \mathrm{~Hz}, 1 \mathrm{H}, 8-\mathrm{OH}), 4.36(\mathrm{~d}, J=5.2 \mathrm{~Hz}, 1 \mathrm{H}, 1-\mathrm{OH}), 5.63(\mathrm{~d}, J=7.0 \mathrm{~Hz}, 1 \mathrm{H}, 8-\mathrm{H}), 5.71(\mathrm{~s}, 1 \mathrm{H}$, 1-H), 7.28 (dd, $J=8.2,6.9 \mathrm{~Hz}, 1 \mathrm{H}, 5-\mathrm{H}), 7.30-7.34(\mathrm{~m}, 2 \mathrm{H}, 4-\mathrm{H}, 6-\mathrm{H}), 7.35$ (dt, 7.4, 0.6 Hz, $1 \mathrm{H}$, 10-H), 7.42-7.49 (m, 4 H, 11-H, 3'-H, 4'-H, 5'-H), 7.51-7.56 (m, 2 H, 2'-H, 6'-H), 7.57 (d, $J=7.4 \mathrm{~Hz}, 1 \mathrm{H}, 9-\mathrm{H}), 8.32(\mathrm{~d}, J=7.8 \mathrm{~Hz}, 1 \mathrm{H}, 12-\mathrm{H})$.

${ }^{13}$ C-NMR $\left(126 \mathrm{MHz}\right.$, Aceton- $\left.\mathrm{d}_{6}\right): \delta(\mathrm{ppm})=25.4(\mathrm{C}-3), 32.0(\mathrm{C}-2), 64.7(\mathrm{C}-1), 73.6(\mathrm{C}-8), 124.9$ (C-4), 125.4 (C-5), 125.8, 125.9 (C-6, C-9, C-12), 127.9 (C-6'), 128.5, 128.6 (C-10, C-3'/C-5'), 129.1 (C-11), 129.5, 129.8 (C-3'/C-5', C-4'), 130.0 (C-12c), 130.6 (C-3b), 132.0 (C-2'), 133.5 (C-6a), 136.7 (C-12b), 137.0 (C-3a), 138.2 (C-7), 139.1 (C-1'), 140.0 (C-12a), 142.3 (C-7a), 148.7 (C-8a).

\section{Diastereomer:}

${ }^{1}$ H-NMR $\left(600 \mathrm{MHz}\right.$, DMSO-d $\left.{ }_{6}\right): \delta(\mathrm{ppm})=1.98\left(\mathrm{tt}, J=13.5,3.5 \mathrm{~Hz}, 1 \mathrm{H}, 2-\mathrm{H}_{\mathrm{a}}\right), 2.31(\mathrm{dd}, J=13.2$, $\left.3.0 \mathrm{~Hz}, 1 \mathrm{H}, 2-\mathrm{H}_{\mathrm{b}}\right), 3.00\left(\mathrm{dt}, J=15.4,3.2,1 \mathrm{H}, 3-\mathrm{H}_{\mathrm{a}}\right), 3.46\left(\mathrm{dt}, J=14.4,3.8 \mathrm{~Hz}, 1 \mathrm{H}, 3-\mathrm{H}_{\mathrm{b}}\right), 5.16(\mathrm{~d}$, $J=7.8 \mathrm{~Hz}, 1 \mathrm{H}, 8-\mathrm{OH}), 5.31(\mathrm{~d}, J=5.5 \mathrm{~Hz}, 1 \mathrm{H}, 1-\mathrm{OH}), 5.51(\mathrm{~d}, J=7.8 \mathrm{~Hz}, 1 \mathrm{H}, 8-\mathrm{H}), 5.54-5.58(\mathrm{~m}$, $1 \mathrm{H}, 1-\mathrm{H}), 7.24$ (d, J= 8.0 Hz, $1 \mathrm{H}, 6-\mathrm{H}), 7.27-7.35$ (m, $3 \mathrm{H}, 4-\mathrm{H}, 5-\mathrm{H}, 11-\mathrm{H}), 7.36$ (t, $J=7.3 \mathrm{~Hz}, 1 \mathrm{H}$, 10-H), 7.43-7.47 (m, 3 H, 3'-H, 4'-H, 5'-H), 7.49-7.55 (m, 3 H, 9-H, 2'-H, 6'-H), 8.26 (d, J = 7.8 Hz, $1 \mathrm{H}, 12-\mathrm{H})$. 
${ }^{13}$ C-NMR (126 MHz, DMSO-d $\left.{ }_{6}\right): \delta(\mathrm{ppm})=24.3(\mathrm{C}-3), 31.2(\mathrm{C}-2), 62.9$ (C-1), 71.9 (C-8), 123.5 (C-6), 124.2 (C-4), 124.7 (C-12), 124.9 (C-5, C-2'), 126.7 (C-9), 127.3 (C-3'/C-5'), 127.6 (C-10), 128.0 (C-3'/C-5'), 128.3 (C-11), 128.6 (C-6'), 129.2 (C-3b, C-12c), 131.1 (C-4'), 131.8 (C-6a), 135.4 (C-12b), 136.0 (C-3a), 136.7 (C-7), 137.9 (C-1'), 138.7 (C-12a), 141.5 (C-7a), 148.0 (C-8a).

MS (ESI): $m / z(\%)=387.2(100)[\mathrm{M}+\mathrm{Na}]^{+}$.

HRMS (ESI): $m / z=$ gef.: 387.1355 , ber.: $387.1356[\mathrm{M}+\mathrm{Na}]^{+}$.

$\mathrm{C}_{26} \mathrm{H}_{20} \mathrm{BrO}_{2}$ (364.44). 


\section{7-(p-Tolyl)-1,2,3,8-tetrahydroinden[1,2-a]phenalen-1,8-diol (114b)}

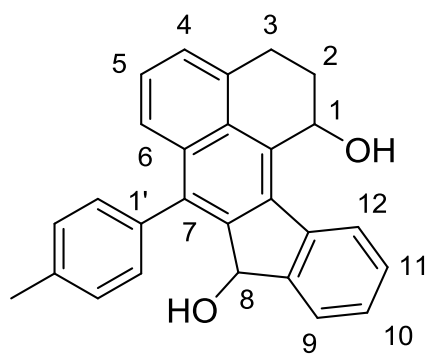

SC: $\mathrm{SiO}_{2}, \mathrm{CH}_{2} \mathrm{Cl}_{2} / \mathrm{Et}_{2} \mathrm{O}, 50: 1 ; \mathrm{RP}-\mathrm{SiO}_{2}, \mathrm{CH}_{3} \mathrm{OH} / \mathrm{H}_{2} \mathrm{O}, 5: 1$ (1. Diastereomer).

DC: $R_{f}=0.47$ (1. Diastereomer), 0.17 (2. Diastereomer) $\left(\mathrm{CH}_{2} \mathrm{Cl}_{2} / \mathrm{Et}_{2} \mathrm{O}, 50: 1\right)$.

UV/Vis $\left(\mathrm{CH}_{3} \mathrm{CN}\right): \lambda_{\max }(\mathrm{nm})(\lg \varepsilon)=257$ (4.449), 277 (4.557), 314 (4.139), 326 (4.125).

IR (ATR): $\tilde{v}\left(\mathrm{~cm}^{-1}\right)=3564,3321,2922,1499,1440,1395,1286,1261,1158,1104,1083,1031,944$, $885,823,807,764,737,638,619,594,525,502$.

\section{Diastereomer:}

${ }^{1}$ H-NMR $\left(600 \mathrm{MHz}\right.$, Aceton- $\left.\mathrm{d}_{6}\right): \delta(\mathrm{ppm})=2.08\left(\mathrm{ddt}, J=14.0,4.1,3.2 \mathrm{~Hz}, 1 \mathrm{H}, 2-\mathrm{H}_{\mathrm{a}}\right), 2.43$ (ddt, $\left.J=13.3,4.2,2.8 \mathrm{~Hz}, 1 \mathrm{H}, 2-\mathrm{H}_{\mathrm{b}}\right), 2.45\left(\mathrm{~s}, 3 \mathrm{H}, \mathrm{CH}_{3}\right), 3.00\left(\mathrm{dt}, J=15.6,3.3 \mathrm{~Hz}, 1 \mathrm{H}, 3-\mathrm{H}_{\mathrm{a}}\right), 3.54(\mathrm{dt}$, $\left.J=15.4,4.1 \mathrm{~Hz}, 1 \mathrm{H}, 3-\mathrm{H}_{\mathrm{b}}\right), 3.88,4.33\left(2 \times \mathrm{s}_{\mathrm{br}}, 2 \mathrm{H}, 1-\mathrm{OH}, 8-\mathrm{OH}\right), 5.63(\mathrm{~s}, 1 \mathrm{H}, 8-\mathrm{H}), 5.70(\mathrm{~s}, 1 \mathrm{H}$, 1-H), 7.27 (dd, $J=8.1,6.9 \mathrm{~Hz}, 1 \mathrm{H}, 5-\mathrm{H}), 7.29-7.32$ (m, $2 \mathrm{H}, 4-\mathrm{H}, 6$ '-H), 7.33-7.39 (m, 5 H, 6-H, 10-H, 2'-H, 3'-H, 5'-H), 7.44 (dt, $J=7.6,1.2 \mathrm{~Hz}, 1 \mathrm{H}, 11-\mathrm{H}), 7.57$ (dd, $J=7.4,1.0 \mathrm{~Hz}, 1 \mathrm{H}, 9-\mathrm{H})$, $8.31(\mathrm{~d}, J=7.8 \mathrm{~Hz}, 1 \mathrm{H}, 12-\mathrm{H})$.

${ }^{13}$ C-NMR $\left(126 \mathrm{MHz}\right.$, Aceton- $\left.\mathrm{d}_{6}\right): \delta(\mathrm{ppm})=21.3\left(\mathrm{CH}_{3}\right), 25.4(\mathrm{C}-3), 32.0(\mathrm{C}-2), 64.7(\mathrm{C}-1), 73.6$ (C-8), 124.9 (C-6), 125.3 (C-5), 125.7, 125.8, 125.9 (C-4, C-9, C-12), 128.6 (C-10), 129.2 (C-11), 129.5 (C-3'/C-5'), 129.7, 129.8 (C-3'/C-5', C-6'), 129.9 (C-12c), 130.6 (C-3b), 131.9 (C-2'), 133.6 (C-6a), 136.0 (C-1'), 136.7 (C-12b), 137.0 (C-3a), 137.3 (C-4'), 138.2 (C-7), 140.1 (C-12a), 142.3 (C-7a), $148.6(\mathrm{C}-8 \mathrm{a})$.

\section{Diastereomer:}

${ }^{1}$ H-NMR $\left(600 \mathrm{MHz}\right.$, Aceton- $\left.\mathrm{d}_{6}\right): \delta(\mathrm{ppm})=2.06\left(\mathrm{ddd}, J=14.0,4.0,3.1 \mathrm{~Hz}, 1 \mathrm{H}, 2-\mathrm{H}_{\mathrm{a}}\right), 2.42(\mathrm{ddt}$, $\left.J=13.5,4.1,2.7 \mathrm{~Hz}, 1 \mathrm{H}, 2-\mathrm{H}_{\mathrm{b}}\right), 2.45\left(\mathrm{~s}, 3 \mathrm{H}, \mathrm{CH}_{3}\right), 2.98\left(\mathrm{ddd}, J=15.4,4.0,2.7 \mathrm{~Hz}, 1 \mathrm{H}, 3-\mathrm{H}_{\mathrm{a}}\right), 3.55$ (dt, $\left.J=14.9,4.0 \mathrm{~Hz}, 1 \mathrm{H}, 3-\mathrm{H}_{\mathrm{b}}\right), 3.88(\mathrm{~d}, J=7.5 \mathrm{~Hz}, 1 \mathrm{H}, 8-\mathrm{OH}), 4.35(\mathrm{~d}, J=5.2 \mathrm{~Hz}, 1 \mathrm{H}, 1-\mathrm{OH})$, $5.62(\mathrm{~d}, J=7.5 \mathrm{~Hz}, 1 \mathrm{H}, 8-\mathrm{H}), 5.68-5.74(\mathrm{~m}, 1 \mathrm{H}, 1-\mathrm{H}), 7.25(\mathrm{dd}, J=7.5,1.6 \mathrm{~Hz}, 1 \mathrm{H}, 5-\mathrm{H}), 7.26$ (dd, $\left.J=8.2,1.4 \mathrm{~Hz}, 1 \mathrm{H}, 6^{\prime}-\mathrm{H}\right), 7.30$ (dt, $\left.J=7.6,1.6 \mathrm{~Hz}, 1 \mathrm{H}, 4-\mathrm{H}\right), 7.33-7.36$ (m, $4 \mathrm{H}, 6-\mathrm{H}, 10-\mathrm{H}, 3^{\prime}-\mathrm{H}$, 
5'-H), 7.41 (dd, $\left.J=7.9,2.0 \mathrm{~Hz}, 1 \mathrm{H}, 2^{\prime}-\mathrm{H}\right), 7.43$ (tt, $\left.J=7.4,0.6 \mathrm{~Hz}, 1 \mathrm{H}, 11-\mathrm{H}\right), 7.55$ (dd, $J=7.2$, $1.0 \mathrm{~Hz}, 1 \mathrm{H}, 9-\mathrm{H}), 8.32$ (d, $J=7.8 \mathrm{~Hz}, 1 \mathrm{H}, 12-\mathrm{H})$.

${ }^{13}$ C-NMR (126 MHz, Aceton-d $\left.\mathrm{d}_{6}\right): \delta(\mathrm{ppm})=21.3\left(\mathrm{CH}_{3}\right), 25.5(\mathrm{C}-3), 32.1$ (C-2), 64.7 (C-1), 73.7 (C-8), 124.9 (C-6), 125.3 (C-5), 125.7, 125.8, 125.9 (C-4, C-9, C-12), 128.6 (C-10), 129.1 (C-11), 129.3 (C-3'/C-5'), 129.6, 129.8 (C-3'/C-5', C-6'), 130.0 (C-12c), 130.6 (C-3b), 131.9 (C-2'), 133.6 (C-6a), 136.1 (C-1'), 136.8 (C-12b), 137.0 (C-3a), 137.3 (C-4'), 138.3 (C-7), 140.1 (C-12a), 142.7 (C-7a), 148.5 (C-8a).

MS (ESI): $m / z(\%)=401.2(78)[\mathrm{M}+\mathrm{Na}]^{+}, 779.4(100)[2 \mathrm{M}+\mathrm{Na}]^{+}$.

HRMS (ESI): $m / z=$ gef.: 401.1512 , ber.: $401.1512[\mathrm{M}+\mathrm{Na}]^{+}$.

$\mathbf{C}_{27} \mathbf{H}_{22} \mathbf{O}_{2}$ (378.46). 


\section{7-(4-Fluorphenyl)-1,2,3,8-tetrahydroinden[1,2-a]phenalen-1,8-diol (114c)}

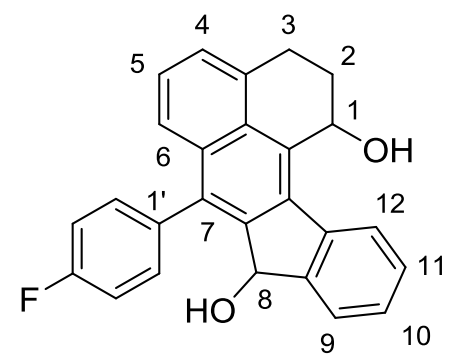

SC: $\mathrm{SiO}_{2}, \mathrm{CH}_{2} \mathrm{Cl}_{2} \rightarrow \mathrm{CH}_{2} \mathrm{Cl}_{2} / \mathrm{Et}_{2} \mathrm{O}, 5: 1 ; \mathrm{SiO}_{2}, n$-Hexan/EtOAc, 3:1 (2. Diastereomer).

DC: $R_{f}=0.19$ (1. Diastereomer) $\left(\mathrm{CH}_{2} \mathrm{Cl}_{2}\right), 0.56$ (1. Diastereomer) $\left(\mathrm{CH}_{2} \mathrm{Cl}_{2} / \mathrm{Et} 2 \mathrm{O}, 5: 1\right)$.

UV/Vis $\left(\mathrm{CH}_{3} \mathrm{CN}\right): \lambda_{\max }(\mathrm{nm})(\lg \varepsilon)=257$ (4.455), 272 (4.528), 277 (4.540), 312 (4.130), 325 (4.113), 351 (2.963).

IR (ATR): $\tilde{v}\left(\mathrm{~cm}^{-1}\right)=3354,2930,2852,1702,1602,1499,1465,1434,1400,1218,1153,1087,1060$, 1038, 1013, 974, 940, 903, 892, 837, 818, 754, 739, 653, 623, 603, 555, 523, 513.

\section{Diastereomer:}

${ }^{1} \mathbf{H}-\mathrm{NMR}\left(600 \mathrm{MHz}\right.$, Aceton- $\left.\mathrm{d}_{6}\right): \delta(\mathrm{ppm})=2.03-2.06\left(\mathrm{~m}, 1 \mathrm{H}, 2-\mathrm{H}_{\mathrm{a}}\right), 2.43(\mathrm{ddt}, J=13.6,4.1,2.7 \mathrm{~Hz}$, $\left.1 \mathrm{H}, 2-\mathrm{H}_{\mathrm{b}}\right), 2.99\left(\mathrm{dt}, J=15.5,3.4 \mathrm{~Hz}, 1 \mathrm{H}, 3-\mathrm{H}_{\mathrm{a}}\right), 3.56\left(\mathrm{dt}, J=15.3,4.1 \mathrm{~Hz}, 1 \mathrm{H}, 3-\mathrm{H}_{\mathrm{b}}\right), 4.12(\mathrm{~d}$, $J=8.1 \mathrm{~Hz}, 1 \mathrm{H}, 8-\mathrm{OH}), 4.35(\mathrm{~d}, J=5.3 \mathrm{~Hz}, 1 \mathrm{H}, 1-\mathrm{OH}), 5.61(\mathrm{~d}, J=8.1 \mathrm{~Hz}, 1 \mathrm{H}, 8-\mathrm{H}), 5.71$ (dt, $J=5.5,2.9 \mathrm{~Hz}, 1 \mathrm{H}, 1-\mathrm{H}), 7.26-7.33$ (m, $5 \mathrm{H}, 4-\mathrm{H}, 5-\mathrm{H}, 6-\mathrm{H}, 3$ '-H, 5'-H), 7.35 (dt, $J=7.4,1.0 \mathrm{~Hz}$, $1 \mathrm{H}, 10-\mathrm{H}), 7.40$ (ddd, $J=8.1,5.5,2.1 \mathrm{~Hz}, 1 \mathrm{H}, 6$ '-H), 7.44 (dt, $J=7.6,1.2 \mathrm{~Hz}, 1 \mathrm{H}, 11-\mathrm{H}), 7.52-7.59$ (m, 2 H, 9-H, 2'-H), 8.33 (d, $J=7.8 \mathrm{~Hz}, 1 \mathrm{H}, 12-\mathrm{H})$.

${ }^{13}$ C-NMR (126 MHz, Aceton- $\left.\mathrm{d}_{6}\right): \delta(\mathrm{ppm})=25.4(\mathrm{C}-3), 32.1(\mathrm{C}-2), 64.7$ (C-1), 73.7 (C-8), $115.2(\mathrm{~d}$, $J=21.4, \mathrm{C}^{3} 3^{\prime} / \mathrm{C}-5$ ') 115.7 (d, $J=21.4, \mathrm{C}^{\prime} 3^{\prime} / \mathrm{C}-5$ ') 124.7 (C-6), 125.4 (C-5), 125.8, 126.0 (C-4, C-9, C-12), 128.7 (C-10), 129.4 (C-11), 130.3 (C-12c), 130.6 (C-3b), 131.6 (d, J=8.0 Hz, C-6'), 133.5 (C-6a), 134.1 (d, J=8.0 Hz, C-2'), 135.3 (d, J=3.5 Hz, C-1'), 136.8 (C-12b), 137.2 (C-3a, C-7), 140.0 (C-12a), 143.0 (C-7a), 148.6 (C-8a), 162.8 (d, $J=243.2$ Hz, C-4').

${ }^{19}$ F-NMR $\left(282 \mathrm{MHz}\right.$, Aceton- $\left.\mathrm{d}_{6}\right): \delta(\mathrm{ppm})=-117.2\left(\mathrm{tt}, J=9.1,5.7 \mathrm{~Hz}, 4^{\prime}-\mathrm{F}\right)$.

\section{Diastereomer:}

${ }^{1}$ H-NMR $\left(600 \mathrm{MHz}\right.$, Aceton- $\left.\mathrm{d}_{6}\right): \delta(\mathrm{ppm})=2.09\left(\mathrm{ddt}, J=14.1,4.3,3.0 \mathrm{~Hz}, 1 \mathrm{H}, 2-\mathrm{H}_{\mathrm{a}}\right), 2.43$ (ddt, $\left.J=13.5,4.2,2.7 \mathrm{~Hz}, 1 \mathrm{H}, 2-\mathrm{H}_{\mathrm{b}}\right), 3.01\left(\mathrm{dt}, J=15.6,3.3 \mathrm{~Hz}, 1 \mathrm{H}, 3-\mathrm{H}_{\mathrm{a}}\right), 3.55(\mathrm{dt}, J=14.4,4.2 \mathrm{~Hz}, 1 \mathrm{H}$, $\left.3-\mathrm{H}_{\mathrm{b}}\right), 4.10(\mathrm{~d}, J=8.2 \mathrm{~Hz}, 1 \mathrm{H}, 8-\mathrm{OH}), 4.32(\mathrm{~d}, J=5.3 \mathrm{~Hz}, 1 \mathrm{H}, 1-\mathrm{OH}), 5.62(\mathrm{~d}, J=8.2 \mathrm{~Hz}, 1 \mathrm{H}, 8-\mathrm{H})$, 
$5.71(\mathrm{dt}, J=5.1,2.7 \mathrm{~Hz}, 1 \mathrm{H}, 1-\mathrm{H}), 7.25-7.34$ (m, $\left.5 \mathrm{H}, 4-\mathrm{H}, 5-\mathrm{H}, 6-\mathrm{H}, 3^{\prime}-\mathrm{H}, 5^{\prime}-\mathrm{H}\right), 7.35$ (dt, $J=7.4$, $1.1 \mathrm{~Hz}, 1 \mathrm{H}, 10-\mathrm{H}), 7.43-7.50$ (m, 3 H, 11 H, 2'-H, 6'-H), 7.57 (ddt, $J=7.4,1.3,0.7 \mathrm{~Hz}, 1 \mathrm{H}, 9-\mathrm{H}$ ), $8.32(\mathrm{~d}, J=7.8 \mathrm{~Hz}, 1 \mathrm{H}, 12-\mathrm{H})$.

${ }^{13}$ C-NMR (126 MHz, Aceton- $\left.\mathrm{d}_{6}\right): \delta(\mathrm{ppm})=25.3(\mathrm{C}-3), 32.0(\mathrm{C}-2), 64.6(\mathrm{C}-1), 73.5(\mathrm{C}-8), 115.3(\mathrm{~d}$, $J=21.4$, C-3'/C-5'), 115.8 (d, $J=21.3$, C-3'/C-5'), 124.7 (C-6), 125.4 (C-5), 125.8, 125.9, 126.0 (C-4, C-9, C-12), 128.7 (C-10), 129.5 (C-11), 130.2 (C-12c), 130.6 (C-3b), 131.7 (d, J = 8.0 Hz, C-6'), 133.5 (C-6a), 134.0, (d, J=8.0 Hz, C-2'), 135.2 (d, $J=3.4$ Hz, C-1'), 136.7 (C-12b), 137.1, 137.2 (C-3a, C-7), 140.0 (C-12a), 142.6 (C-7a), 148.7 (C-8a), 162.8 (d, J=243.2 Hz, C-4').

${ }^{19}$ F-NMR $\left(282 \mathrm{MHz}\right.$, Aceton- $\left.\mathrm{d}_{6}\right): \delta(\mathrm{ppm})=-117.2\left(\mathrm{tt}, J=9.2,5.6 \mathrm{~Hz}, 4^{\prime}-\mathrm{F}\right)$.

MS (ESI): $m / z(\%)=405.2(52)[\mathrm{M}+\mathrm{Na}]^{+}, 787.3(100)[2 \mathrm{M}+\mathrm{Na}]^{+}$.

HRMS (ESI): $m / z=$ gef.: 405.1261, ber.: $405.1258[\mathrm{M}+\mathrm{Na}]^{+}$.

$\mathbf{C}_{26} \mathrm{H}_{19} \mathrm{FO}_{2}$ (382.43). 
7-(4-Methoxyphenyl)-1,2,3,8-tetrahydroinden[1,2-a]phenalen-1,8-diol (114d)

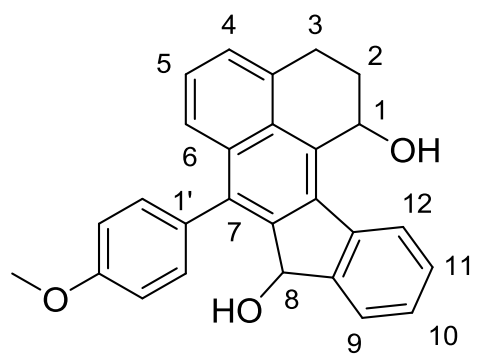

SC: $\mathrm{SiO}_{2}, \mathrm{CH}_{2} \mathrm{Cl}_{2} / \mathrm{Et}_{2} \mathrm{O}, 50: 1 ; \mathrm{RP}-\mathrm{SiO}_{2}, \mathrm{CH}_{3} \mathrm{OH} / \mathrm{H}_{2} \mathrm{O}, 5: 1$ (1. Diastereomer).

DC: $R_{f}=0.37$ (1. Diastereomer), 0.12 (2. Diastereomer) $\left(\mathrm{CH}_{2} \mathrm{Cl}_{2} / \mathrm{Et}_{2} \mathrm{O}, 50: 1\right)$.

UV/Vis $\left(\mathrm{CH}_{3} \mathrm{CN}\right): \lambda_{\max }(\mathrm{nm})(\lg \varepsilon)=194$ (4.656), 257 (4.235), 273 (4.289), 312 (3.930), 325 (3.881).

IR (ATR): $\tilde{v}\left(\mathrm{~cm}^{-1}\right)=3347,2926,2851,1707,1602,1508,1463,1442,1286,1244,1174,1103,1080$, $1060,1029,943,832,757,735,701,656,633,621,602,566,528$.

\section{Diastereomer:}

${ }^{1}$ H-NMR $\left(600 \mathrm{MHz}\right.$, Aceton- $\left.\mathrm{d}_{6}\right): \delta(\mathrm{ppm})=2.08\left(\mathrm{ddt}, J=13.9,4.1,3.1 \mathrm{~Hz}, 1 \mathrm{H}, 2-\mathrm{H}_{\mathrm{a}}\right), 2.42$ (ddt, $\left.J=13.5,4.1,2.7 \mathrm{~Hz}, 1 \mathrm{H}, 2-\mathrm{H}_{\mathrm{b}}\right), 3.00\left(\mathrm{dt}, J=15.5,3.1 \mathrm{~Hz}, 1 \mathrm{H}, 3-\mathrm{H}_{\mathrm{a}}\right), 3.54(\mathrm{dt}, J=15.4,4.1 \mathrm{~Hz}, 1 \mathrm{H}$, 3- $\left.\mathrm{H}_{\mathrm{b}}\right), 3.89$ (s, $\left.3 \mathrm{H}, \mathrm{OCH}_{3}\right), 3.91\left(\mathrm{~s}_{\mathrm{br}}, 1 \mathrm{H}, 8-\mathrm{OH}\right), 4.31(\mathrm{~d}, J=5.3 \mathrm{~Hz}, 1 \mathrm{H}, 1-\mathrm{OH}), 5.64(\mathrm{~d}, J=7.1 \mathrm{~Hz}$, $1 \mathrm{H}, 8-\mathrm{H}), 5.70(\mathrm{~d}, J=4.4 \mathrm{~Hz}, 1 \mathrm{H}, 1-\mathrm{H}), 7.09$ (d, $J=8.9 \mathrm{~Hz}, 2 \mathrm{H}, 3^{\prime}-\mathrm{H}, 5$ '-H), 7.28 (dd, $J=8.0$, $6.9 \mathrm{~Hz}, 1 \mathrm{H}, 5-\mathrm{H}), 7.29-7.32$ (m, $1 \mathrm{H}, 4-\mathrm{H}), 7.33-7.36$ (m, $2 \mathrm{H}, 10-\mathrm{H}, 6$ '-H), 7.37-7.41 (m, $2 \mathrm{H}, 6-\mathrm{H}$, 2'-H), $7.44(\mathrm{dt}, J=7.6,1.2 \mathrm{~Hz}, 1 \mathrm{H}, 11-\mathrm{H}), 7.57(\mathrm{dd}, J=7.3,1.1 \mathrm{~Hz}, 1 \mathrm{H}, 9-\mathrm{H}), 8.31(\mathrm{~d}, J=7.7 \mathrm{~Hz}$, $1 \mathrm{H}, 12-\mathrm{H})$.

${ }^{13}$ C-NMR $\left(126 \mathrm{MHz}\right.$, Aceton- $\left.\mathrm{d}_{6}\right): \delta(\mathrm{ppm})=25.4(\mathrm{C}-3), 32.0(\mathrm{C}-2), 55.5\left(\mathrm{OCH}_{3}\right), 64.7(\mathrm{C}-1), 73.6$ (C-8), 114.1, 114.5 (C-3', C-5'), 124.9 (C-6), 125.3 (C-5), 125.7, 125.8, 125.9 (C-4, C-9, C-12), 128.6 (C-10), 129.4 (C-11), 129.8 (C-12c), 130.6 (C-3b), 130.9 (C-6'), 131.0 (C-2'), 133.0 (C-1'), 133.8 (C-6a), 136.7 (C-12b), 137.0 (C-3a, C-4'), 138.0 (C-7), 140.1 (C-12a), 142.5 (C-7a), 148.6 (C-8a).

\section{Diastereomer:}

${ }^{1}$ H-NMR $\left(600 \mathrm{MHz}\right.$, Aceton- $\left.\mathrm{d}_{6}\right): \delta(\mathrm{ppm})=2.07\left(\mathrm{ddd}, J=13.5,4.1,3.1 \mathrm{~Hz}, 1 \mathrm{H}, 2-\mathrm{H}_{\mathrm{a}}\right), 2.42$ (ddt, $\left.J=13.5,4.0,2.7 \mathrm{~Hz}, 1 \mathrm{H}, 2-\mathrm{H}_{\mathrm{b}}\right), 2.99\left(\mathrm{ddt}, J=15.6,3.3 \mathrm{~Hz}, 1 \mathrm{H}, 3-\mathrm{H}_{\mathrm{a}}\right), 3.55$ (ddd, $J=15.6,4.0 \mathrm{~Hz}$, 1.5, $\left.1 \mathrm{H}, 3-\mathrm{H}_{\mathrm{b}}\right), 3.90\left(\mathrm{~s}, 3 \mathrm{H}, \mathrm{OCH}_{3}\right), 3.92(\mathrm{~s}, 1 \mathrm{H}, 8-\mathrm{OH}), 4.33$ (d, J = 5.1 Hz, $\left.1 \mathrm{H}, 1-\mathrm{OH}\right), 5.63(\mathrm{~d}$, $J=7.2 \mathrm{~Hz}, 1 \mathrm{H}, 8-\mathrm{H}), 5.68-5.73$ (m, $1 \mathrm{H}, 1-\mathrm{H}), 7.09$ (ddd, $J=9.1,7.1,2.6 \mathrm{~Hz}, 2 \mathrm{H}, 3$ '-H, 5'-H), 7.25$7.32\left(\mathrm{~m}, 3 \mathrm{H}, 4-\mathrm{H}, 5-\mathrm{H}, 6{ }^{\prime}-\mathrm{H}\right), 7.35(\mathrm{dt}, J=7.4,1.0 \mathrm{~Hz}, 1 \mathrm{H}, 10-\mathrm{H}), 7.38(\mathrm{dt}, J=8.1,1.3 \mathrm{~Hz}, 1 \mathrm{H}$, 
6-H), 7.41-7.47 (m, 2 H, 11-H, 2'-H), 7.56 (dd, $J=7.4,1.0 \mathrm{~Hz}, 1 \mathrm{H}, 9-\mathrm{H}), 8.33$ (d, $J=7.8 \mathrm{~Hz}, 1 \mathrm{H}$, $12-\mathrm{H})$.

${ }^{13}$ C-NMR $\left(126 \mathrm{MHz}\right.$, Aceton- $\left.\mathrm{d}_{6}\right): \delta(\mathrm{ppm})=25.5(\mathrm{C}-3), 32.1(\mathrm{C}-2), 55.5\left(\mathrm{OCH}_{3}\right), 64.7(\mathrm{C}-1), 73.8$ (C-8), 114.1, 114.5 (C-3', C-5'), 125.0 (C-6), 125.3 (C-5), 125.7, 125.8, 125.9 (C-4, C-9, C-12), 128.6 (C-10), 129.3 (C-11), 130.0 (C-12c), 130.6 (C-3b), 130.8 (C-6'), 131.1 (C-2'), 133.1 (C-1'), 133.8 (C-6a), 136.8 (C-12b), 137.1 (C-3a, C-4'), 138.1 (C-7), 140.2 (C-12a), 142.9 (C-7a), 148.6 (C-8a).

MS (ESI): $m / z(\%)=417.2(100)[\mathrm{M}+\mathrm{Na}]^{+}, 811.4(72)[2 \mathrm{M}+\mathrm{Na}]^{+}$.

HRMS (ESI): $m / z=$ gef.: 417.1449 , ber.: $417.1461[\mathrm{M}+\mathrm{Na}]^{+}$.

$\mathbf{C}_{27} \mathbf{H}_{22} \mathbf{O}_{2}$ (394.46). 


\section{6-Methyl-7-phenyl-1,2,3,8-tetrahydroinden[1,2-a]phenalen-1,8-diol (114f)}

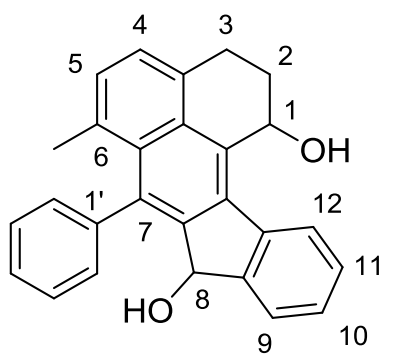

SC: $\mathrm{SiO}_{2}, \mathrm{CH}_{2} \mathrm{Cl}_{2} / \mathrm{Et}_{2} \mathrm{O}, 50: 1 ; \mathrm{RP}-\mathrm{SiO}_{2}, \mathrm{CH}_{3} \mathrm{OH} / \mathrm{H}_{2} \mathrm{O}, 4: 1$ (1. Diastereomer).

DC: $R_{f}=0.52$ (1. Diastereomer), 0.38 (2. Diastereomer) $\left(\mathrm{CH}_{2} \mathrm{Cl}_{2} / \mathrm{Et}_{2} \mathrm{O}, 50: 1\right)$.

UV/Vis $\left(\mathrm{CH}_{3} \mathrm{CN}\right): \lambda_{\max }(\mathrm{nm})(\lg \varepsilon)=263$ (4.164), 274 (4.241), 283 (4.262), 316 (3.791).

IR (ATR): $\tilde{v}\left(\mathrm{~cm}^{-1}\right)=3306,2921,2851,1710,1664,1589,1458,1444,1376,1058,1029,1017,970$, $950,917,890,821,735,721,701,668,651,578,554,531,508$.

\section{Diastereomer:}

${ }^{1} \mathbf{H}-\mathbf{N M R}\left(600 \mathrm{MHz}\right.$, Aceton-d $\left.\mathrm{d}_{6}\right): \delta(\mathrm{ppm})=1.91\left(\mathrm{~s}, 3 \mathrm{H}, \mathrm{CH}_{3}\right), 2.01-2.08\left(\mathrm{~m}, 1 \mathrm{H}, 2-\mathrm{H}_{\mathrm{a}}\right), 2.39$ (ddt, $\left.J=13.4,4.2,2.7 \mathrm{~Hz}, 1 \mathrm{H}, 2-\mathrm{H}_{\mathrm{b}}\right), 2.97\left(\mathrm{dt}, J=15.6,3.4 \mathrm{~Hz}, 1 \mathrm{H}, 3-\mathrm{H}_{\mathrm{a}}\right), 3.44\left(\mathrm{~d}_{\mathrm{br}}, J=6.9 \mathrm{~Hz}, 1 \mathrm{H}\right.$, 8-OH), $3.56\left(\mathrm{dt}, J=15.7,4.1 \mathrm{~Hz}, 1 \mathrm{H}, 3-\mathrm{H}_{\mathrm{b}}\right), 4.26\left(\mathrm{~s}_{\mathrm{br}}, 1 \mathrm{H}, 1-\mathrm{OH}\right), 5.47(\mathrm{~d}, J=4.9 \mathrm{~Hz}, 1 \mathrm{H}, 8-\mathrm{H})$, $5.70(\mathrm{~s}, 1 \mathrm{H}, 1-\mathrm{H}), 7.10(\mathrm{dd}, J=7.0,1.1 \mathrm{~Hz}, 1 \mathrm{H}, 5-\mathrm{H}), 7.20(\mathrm{dd}, J=7.1,1.5 \mathrm{~Hz}, 4-\mathrm{H}), 7.33$ (dt, $J=7.4,1.0 \mathrm{~Hz}, 1 \mathrm{H}, 10-\mathrm{H}), 7.41-7.46$ (m, 5 H, 11-H, 3'-H, 4'-H, 5'-H, 6'-H), 7.46-7.50 (m, 1 H, 2'-H), $7.52(\mathrm{dd}, J=7.3 \mathrm{~Hz}, 1.0 \mathrm{~Hz}, 1 \mathrm{H}, 9-\mathrm{H}), 8.32$ (d, $J=7.8 \mathrm{~Hz}, 1 \mathrm{H}, 12-\mathrm{H})$.

${ }^{13}$ C-NMR $\left(126 \mathrm{MHz}\right.$, Aceton- $\left.\mathrm{d}_{6}\right): \delta(\mathrm{ppm})=25.4\left(\mathrm{CH}_{3}\right), 25.8(\mathrm{C}-3), 31.6(\mathrm{C}-2), 65.0(\mathrm{C}-1), 74.0$ (C-8), 125.6 (C-4), 125.8, 125.9 (C-9, C-12), 127.8, 127.9 (C-3', C-5'), 128.5, 128.7 (C-10, C-2'), 129.5, 129.8 (C-11, C-6'), 130.2 (C-5), 130.7 (C-12c), 131.9, 132.0 (C-3a, C-3b), 132.5 (C-4'), 134.2 (C-6), 135.4 (C-6a), 136.2 (C-12b), 138.6 (C-7), 139.9 (C-12a), 142.5 (C-1'), 143.4 (C-7a), 148.4 (C-8a).

\section{Diastereomer:}

${ }^{1}$ H-NMR $\left(600 \mathrm{MHz}\right.$, Aceton- $\left.\mathrm{d}_{6}\right): \delta(\mathrm{ppm})=1.90\left(\mathrm{~s}, 3 \mathrm{H}, \mathrm{CH}_{3}\right), 2.02\left(\mathrm{tt}, J=13.5,3.5 \mathrm{~Hz}, 1 \mathrm{H}, 2-\mathrm{H}_{\mathrm{a}}\right)$, 2.39 (ddt, $\left.J=13.4,4.0,2.6 \mathrm{~Hz}, 1 \mathrm{H}, 2-\mathrm{H}_{\mathrm{b}}\right), 2.96\left(\mathrm{dd}, J=15.3,3.4 \mathrm{~Hz}, 1 \mathrm{H}, 3-\mathrm{H}_{\mathrm{a}}\right), 3.55\left(\mathrm{~m}_{\mathrm{C}}, 1 \mathrm{H}\right.$, $\left.3-\mathrm{H}_{\mathrm{b}}\right), 3.65(\mathrm{~d}, J=7.3 \mathrm{~Hz}, 1 \mathrm{H}, 8-\mathrm{OH}), 4.31(\mathrm{~d}, J=5.2 \mathrm{~Hz}, 1 \mathrm{H}, 1-\mathrm{OH}), 5.37(\mathrm{~d}, J=7.0 \mathrm{~Hz}, 1 \mathrm{H}, 8-\mathrm{H})$, 5.62-5.69 (m, $1 \mathrm{H}, 1-\mathrm{H}), 7.10(\mathrm{~d}, J=7.1 \mathrm{~Hz}, 1 \mathrm{H}, 5-\mathrm{H}), 7.20(\mathrm{~d}, J=7.2 \mathrm{~Hz}, 4-\mathrm{H}), 7.24-7.26(\mathrm{~m}, 1 \mathrm{H}$, 6'-H), 7.33 (dt, $J=7.4,0.9 \mathrm{~Hz}, 1 \mathrm{H}, 10-\mathrm{H}), 7.41-7.49$ (m, $4 \mathrm{H}, 11-\mathrm{H}, 3^{\prime}-\mathrm{H}, 4$ '-H, 5'-H), 7.51 (d, $J=7.5 \mathrm{~Hz}, 1 \mathrm{H}, 9-\mathrm{H}), 7.67\left(\mathrm{~d}, J=7.4 \mathrm{~Hz}, 1 \mathrm{H}, 2^{\prime}-\mathrm{H}\right), 8.34(\mathrm{~d}, J=7.8 \mathrm{~Hz}, 1 \mathrm{H}, 12-\mathrm{H})$. 
${ }^{13}$ C-NMR (126 MHz, Aceton-d $\left.\mathrm{d}_{6}\right): \delta(\mathrm{ppm})=25.4\left(\mathrm{CH}_{3}\right), 26.0(\mathrm{C}-3), 31.9(\mathrm{C}-2), 65.1(\mathrm{C}-1), 74.1$ (C-8), 125.6 (C-4), 125.8, 125.9 (C-9, C-12), 127.8 (C-3', C-5'), 128.5, 128.6 (C-10, C-2'), 129.3 (C-11), 129.9 (C-6'), 130.2 (C-5), 130.9 (C-12c), 131.9, 132.0 (C-3a, C-3b), 132.7 (C-4'), 134.3 (C-6), 135.5 (C-6a), 136.6 (C-12b), 138.9 (C-7), 139.9 (C-12a), 142.5 (C-1'), 143.9 (C-7a), 148.5 (C-8a).

MS (ESI): $m / z(\%)=401.2(100)[\mathrm{M}+\mathrm{Na}]^{+}, 779.4(63)[2 \mathrm{M}+\mathrm{Na}]^{+}$.

HRMS (ESI): $m / z=$ gef.: 401.1510 , ber.: $401.1512[\mathrm{M}+\mathrm{Na}]^{+}$.

$\mathbf{C}_{27} \mathbf{H}_{22} \mathbf{O}_{2}$ (378.46). 


\section{6-Fluor-7-phenyl-1,2,3,8-tetrahydroinden[1,2-a]phenalen-1,8-diol (114g)}

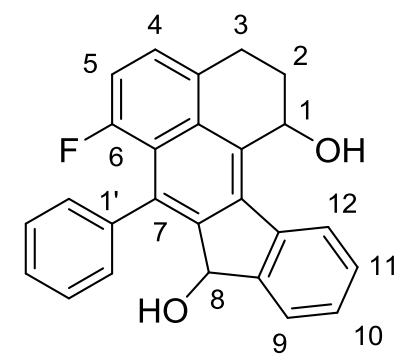

SC: $\mathrm{SiO}_{2}, \mathrm{CH}_{2} \mathrm{Cl}_{2} / \mathrm{Et}_{2} \mathrm{O}, 50: 1 ; \mathrm{SiO}_{2}, n$-Hexan/EtOAc, 3:1 (1. und 2. Diastereomer).

DC: $R_{f}=0.29$ (1. Diastereomer), 0.13 (2. Diastereomer) $\left(\mathrm{CH}_{2} \mathrm{Cl}_{2} / \mathrm{Et}_{2} \mathrm{O}, 50: 1\right)$.

UV/Vis $\left(\mathrm{CH}_{3} \mathrm{CN}\right): \lambda_{\max }(\mathrm{nm})(\lg \varepsilon)=260$ (4.439), 272 (4.516), 282 (4.513), 315 (4.063), 323 (4.023), $356(3.110)$.

IR (ATR): $\tilde{v}\left(\mathrm{~cm}^{-1}\right)=3342,2923,2849,1698,1613,1592,1488,1465,1451,1441,1356,1238,1185$, 1154, 1058, 1029, 1018, 975, 894, 824, 788, 767, 734, 724, 700, 665, 655, 635, 613, 579, 560, 533, 516.

\section{Diastereomer:}

${ }^{1} \mathbf{H}-\mathrm{NMR}\left(600 \mathrm{MHz}\right.$, Aceton- $\left.\mathrm{d}_{6}\right): \delta(\mathrm{ppm})=2.06\left(\mathrm{~m}_{\mathrm{C}}, 1 \mathrm{H}, 2-\mathrm{H}_{\mathrm{a}}\right), 2.42(\mathrm{ddt}, J=13.5,3.9,2.8 \mathrm{~Hz}, 1 \mathrm{H}$, $\left.2-\mathrm{H}_{\mathrm{b}}\right), 3.00\left(\mathrm{dt}, J=15.4,3.2 \mathrm{~Hz}, 1 \mathrm{H}, 3-\mathrm{H}_{\mathrm{a}}\right), 3.51\left(\mathrm{~m}_{\mathrm{C}}, 1 \mathrm{H}, 3-\mathrm{H}_{\mathrm{b}}\right), 3.70(\mathrm{~d}, J=7.3 \mathrm{~Hz}, 1 \mathrm{H}, 8-\mathrm{OH})$, $4.41(\mathrm{~d}, J=5.1 \mathrm{~Hz}, 1 \mathrm{H}, 1-\mathrm{OH}), 5.55(\mathrm{~d}, J=7.1 \mathrm{~Hz}, 1 \mathrm{H}, 8-\mathrm{H}), 5.66-5.67$ (m, $1 \mathrm{H}, 1-\mathrm{H}), 6.98$ (dd, $J=13.0,7.7 \mathrm{~Hz}, 1 \mathrm{H}, 5-\mathrm{H}), 7.29$ (m $\mathrm{C}, 1 \mathrm{H}, 4-\mathrm{H}), 7.34-7.47$ (m, $7 \mathrm{H}, 10-\mathrm{H}, 11-\mathrm{H}, 2^{\prime}-\mathrm{H}, 3^{\prime}-\mathrm{H}, 4^{\prime}-\mathrm{H}$, 5'-H, 6'-H), 7.56 (d, J = 7.5 Hz, $1 \mathrm{H}, 9-\mathrm{H}), 8.34$ (d, $J=7.8 \mathrm{~Hz}, 1 \mathrm{H}, 12-\mathrm{H})$.

${ }^{13}$ C-NMR (126 MHz, Aceton- $\left.\mathrm{d}_{6}\right): \delta(\mathrm{ppm})=25.1(\mathrm{C}-3), 32.8(\mathrm{C}-2), 64.3(\mathrm{C}-1), 73.6(\mathrm{C}-8), 111.3(\mathrm{~d}$, $J=22.0 \mathrm{~Hz}, \mathrm{C}-5), 122.5$ (d, $J=9.0 \mathrm{~Hz}, \mathrm{C}-6 \mathrm{a}), 125.5$ (d, $J=8.3 \mathrm{~Hz}, \mathrm{C}-4), 125.9,126.1$ (C-9, C-12), 127.3, 127.8 (C-3', C-5'), 128.3 (d, J=2.8 Hz, C-2'/C-6'), 128.4 (C-4'), 129.0 (C-10), 129.6 (C-11), 130.2 (d, $J=2.5 \mathrm{~Hz}, \mathrm{C}-12 \mathrm{c}$ ), 131.0 (d, $J=4.9 \mathrm{~Hz}, \mathrm{C}-2^{\prime} / \mathrm{C}^{-6}$ '), 132.5 (d, $J=2.8 \mathrm{~Hz}, \mathrm{C}-3 \mathrm{~b}$ ), 133.0 (d, $J=4.5 \mathrm{~Hz}, \mathrm{C}-3 \mathrm{a}), 135.1$ (d, $J=1.0 \mathrm{~Hz}, \mathrm{C}-7), 137.7$ (d, $J=1.6 \mathrm{~Hz}, \mathrm{C}-12 \mathrm{~b}), 139.5$ (C-12a), 141.2 (d, $\left.J=4.0 \mathrm{~Hz}, \mathrm{C}-1^{\prime}\right), 143.9$ (C-7a), 148.6 (C-8a), 159.1 (d, $\left.J=251.4 \mathrm{~Hz}, \mathrm{C}-6\right)$.

${ }^{19}$ F-NMR $\left(282 \mathrm{MHz}\right.$, Aceton-d $\left.\mathrm{d}_{6}\right): \delta(\mathrm{ppm})=-110.6(\mathrm{ddd}, J=12.9,5.0,2.4 \mathrm{~Hz}, 6-\mathrm{F})$.

\section{Diastereomer:}

${ }^{1}$ H-NMR $\left(600 \mathrm{MHz}\right.$, Aceton- $\left.\mathrm{d}_{6}\right): \delta(\mathrm{ppm})=2.02\left(\mathrm{~m}_{\mathrm{C}}, 1 \mathrm{H}, 2-\mathrm{H}_{\mathrm{a}}\right), 2.42(\mathrm{ddt}, J=13.6,4.1,2.7 \mathrm{~Hz}, 1 \mathrm{H}$, 2- $\left.\mathrm{H}_{\mathrm{b}}\right), 2.99\left(\mathrm{dt}, J=15.5,3.3 \mathrm{~Hz}, 1 \mathrm{H}, 3-\mathrm{H}_{\mathrm{a}}\right), 3.50\left(\mathrm{~m}_{\mathrm{C}}, 1 \mathrm{H}, 3-\mathrm{H}_{\mathrm{b}}\right), 3.73(\mathrm{~d}, J=7.3 \mathrm{~Hz}, 1 \mathrm{H}, 8-\mathrm{OH})$, 
$4.42(\mathrm{~d}, J=5.2 \mathrm{~Hz}, 1 \mathrm{H}, 1-\mathrm{OH}), 5.51(\mathrm{~d}, J=7.3 \mathrm{~Hz}, 1 \mathrm{H}, 8-\mathrm{H}), 5.68-5.71(\mathrm{~m}, 1 \mathrm{H}, 1-\mathrm{H}), 6.98(\mathrm{dd}$, $J=13.0,7.7 \mathrm{~Hz}, 1 \mathrm{H}, 5-\mathrm{H}), 7.28-7.32$ (m, 2 H, 4-H, 2'-H/6'-H), 7.37 (dt, $J=7.4,1.0 \mathrm{~Hz}, 1 \mathrm{H}, 10-\mathrm{H})$, 7.37-7.47 (m, 4 H, 11-H, 3'-H, 4'-H, 5'-H), 7.54-7.57 (m, 2 H, 9-H, 2'-H/6'-H), 8.35 (d, J = 7.9 Hz, $1 \mathrm{H}, 12-\mathrm{H})$.

${ }^{13}$ C-NMR (126 MHz, Aceton- $\left.\mathrm{d}_{6}\right): \delta(\mathrm{ppm})=25.3(\mathrm{C}-3), 31.9(\mathrm{C}-2), 64.4(\mathrm{C}-1), 73.7$ (C-8), $111.3(\mathrm{~d}$, $J=22.0 \mathrm{~Hz}, \mathrm{C}-5), 122.5$ (d, $J=9.1 \mathrm{~Hz}, \mathrm{C}-6 \mathrm{a}), 125.5$ (d, $J=8.3, \mathrm{C}-4), 125.9,126.1$ (C-9, C-12), 127.3, 127.8 (C-3', C-5'), 128.3 (d, J=3.1 Hz, C2'/C-6'), 128.3 (C-4'), 129.1 (C-10), 129.5 (C-11), 130.4 (d, $J=2.5 \mathrm{~Hz}, \mathrm{C}-12 \mathrm{c}$ ), 131.1 (d, $J=4.6 \mathrm{~Hz}, \mathrm{C}-2^{\prime} / \mathrm{C}^{-6}$ ') 132.5 (d, $\left.J=2.9 \mathrm{~Hz}, \mathrm{C}-3 \mathrm{~b}\right), 133.1$ (d, $J=4.5 \mathrm{~Hz}$, C-3a), 135.3 (d, $J=1.0 \mathrm{~Hz}, \mathrm{C}-7), 137.9$ (d, $J=1.6 \mathrm{~Hz}, \mathrm{C}-12 \mathrm{~b}), 139.6$ (C-12a), 141.3 (d, $J=4.1 \mathrm{~Hz}$, C-1'), 144.3 (C-7a), 148.5 (C-8a), 159.1 (d, $J=251.4 \mathrm{~Hz}, \mathrm{C}-6)$.

${ }^{19}$ F-NMR (282 MHz, Aceton-d $\left.\mathrm{d}_{6}\right): \delta(\mathrm{ppm})=-110.6(\mathrm{ddd}, J=12.9,4.9,2.5 \mathrm{~Hz}, 6-\mathrm{F})$.

MS (ESI): $m / z(\%)=405.2(100)[\mathrm{M}+\mathrm{Na}]^{+}, 787.3(79)[2 \mathrm{M}+\mathrm{Na}]^{+}$.

HRMS (ESI): $m / z=$ gef.: 405.1261, ber.: $405.1251[\mathrm{M}+\mathrm{Na}]^{+}$.

$\mathbf{C}_{26} \mathrm{H}_{19} \mathrm{FO}_{2}$ (382.43). 


\subsubsection{Synthese des Ketons 172}

\section{3-Phenyl-1-(2-((trimethylsilyl)ethinyl)phenyl)prop-2-in-1-on (172)}

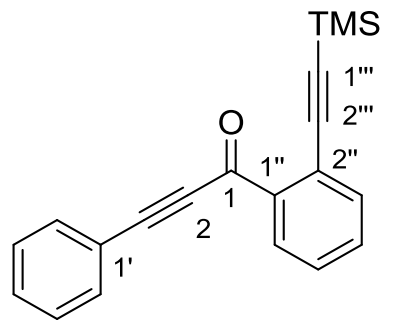

Eine Lösung von Alkohol 167a (5.25 g, 17.2 mmol, 1.00 Äq.) in $\mathrm{CH}_{2} \mathrm{Cl}_{2}$ (100 mL) wurde über $30 \mathrm{~min}$ portionsweise mit $\mathrm{MnO}_{2}(29.9 \mathrm{~g}, 344 \mathrm{mmol}, 20.0$ Äq.) versetzt und $17 \mathrm{~h}$ bei RT gerührt. Säulenchromatographische Reinigung $\left(\mathrm{SiO}_{2}, n\right.$-Pentan/EtOAc, 20:1) lieferte Keton 172 als gelbes Öl (4.41 g, $14.6 \mathrm{mmol}, 85 \%)$.

DC: $R_{f}=0.61(n-$ Pentan/EtOAc, 20:1).

UV/Vis $\left(\mathrm{CH}_{3} \mathrm{CN}\right): \lambda_{\max }(\mathrm{nm})(\lg \varepsilon)=194(4.540), 242$ (4.365), 306 (4.171).

IR (ATR): $\tilde{v}\left(\mathrm{~cm}^{-1}\right)=2194,2156,1646,1590,1561,1489,1476,1442,1409,1300,1277,1261,1248$, 1217, 1202, 1176, 1162, 1027, 1009, 993, 862, 837, 786, 752, 684, 643, 626, 619, 595, 576, 545, 533, 518.

${ }^{1} \mathbf{H}-\mathbf{N M R}\left(600 \mathrm{MHz}, \mathrm{CDCl}_{3}\right): \delta(\mathrm{ppm})=0.19\left(\mathrm{~s}, 9 \mathrm{H}, \mathrm{Si}\left(\mathrm{CH}_{3}\right)_{3}\right), 7.38(\mathrm{dd}, J=10.4,4.6 \mathrm{~Hz}, 2 \mathrm{H}, 3$ '- $\mathrm{H}$, 5'-H), 7.40-7.47 (m, 2 H, 4'-H, 5''-H), 7.48 (td, $J=7.6,1.4$ Hz, 1 H, 4''-H), 7.60 (dd, $J=7.7,0.8 \mathrm{~Hz}$, $1 \mathrm{H}, 3$ '’-H), 7.63 (dd, $J=5.2,3.2 \mathrm{~Hz}, 2$ H, 2'-H, 6'-H), 8.09 (dd, $\left.J=7.8,0.9 \mathrm{~Hz}, 1 \mathrm{H}, 6^{\prime}{ }^{\prime}-\mathrm{H}\right)$.

${ }^{13} \mathbf{C}-\mathrm{NMR}\left(126 \mathrm{MHz}, \mathrm{CDCl}_{3}\right): \delta(\mathrm{ppm})=-0.27\left(\mathrm{Si}\left(\mathrm{CH}_{3}\right)_{3}\right), 88.0(\mathrm{C}-2), 93.3$ (C-3), 101.4 (C-1"’'), 102.9 (C-2’’'), 120.3 (C-1'), 122.7 (C-2’'), 128.2 (C-5’'), 128.6 (C-3', C-5'), 130.7 (C-4'), 131.1 (C-6’'), 132.2 (C-4’'), 133.2 (C-2', C-6'), 135.0 (C-3'’), 139.0 (C-1'’), 177.5 (C=O).

MS (EI): $m / z(\%)=303.1(100)[\mathrm{M}+\mathrm{H}]^{+}$.

HRMS (ESI): $m / z=$ gef.: 303.1202 , ber.: $303.1200[\mathrm{M}+\mathrm{H}]^{+}$.

$\mathrm{C}_{20} \mathrm{H}_{18} \mathrm{OSi}$ (302.44). 


\subsection{Untersuchungen zur Synthese der tetrasubstituierten Polyalkene 116-120}

\subsubsection{Untersuchungen zur Synthese von Dialkenen des Typs 116}

1-Iod-2-(3-methoxyphenoxy)-4-methylbenzol (173)

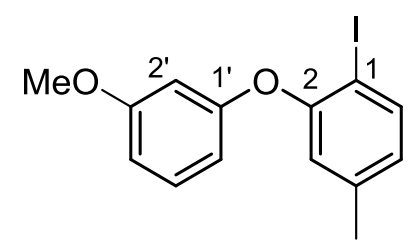

Eine Mischung aus 3-Methoxyphenol (147h) (647 $\mu \mathrm{L}, \quad 738 \mathrm{mg}, \quad 5.94 \mathrm{mmol}, \quad 1.00$ Äq.), 2-Fluor-4-methyl-nitrobenzol (121d) $\left(922 \mathrm{mg}, 5.94 \mathrm{mmol}, 1.00\right.$ Äq.) und $\mathrm{K}_{2} \mathrm{CO}_{3}$ (1.64 g, 11.9 mmol, 2.00 Äq.) in DMSO $(20 \mathrm{~mL})$ wurde $15 \mathrm{~h}$ bei $95^{\circ} \mathrm{C}$ gerührt. Nach Abkühlen auf RT wurde die Mischung in $\mathrm{H}_{2} \mathrm{O}(100 \mathrm{~mL})$ gegeben und mit MTBE extrahiert $(3 \times 100 \mathrm{~mL})$. Die vereinigten organischen Phasen wurden mit ges. wässr. $\mathrm{NaCl}$-Lösung $(150 \mathrm{~mL})$ gewaschen, über $\mathrm{Na}_{2} \mathrm{SO}_{4}$ getrocknet und das Lösungsmittel in vacuo entfernt, um Biarylether 148h als gelbes Öl zu erhalten.

Konz. $\mathrm{HCl}(50 \mathrm{~mL})$ und anschließend konz. AcOH $(50 \mathrm{~mL})$ wurden bei $0{ }^{\circ} \mathrm{C}$ tropfenweise zu einer Lösung des Rückstands bestehend aus $\mathbf{1 4 8 h}$ (1.00 Äq.) in EtOAc $(50 \mathrm{~mL})$ hinzugefügt. Zinkpulver (11.6 g, 178 mmol, 30.0 Äq.) wurde portionsweise zugegeben, die Lösung auf RT erwärmt und 15 min bei dieser Temperatur gerührt. Die Reaktionsmischung wurde auf $0{ }^{\circ} \mathrm{C}$ abgekühlt und $33 \%$ ige wässr. $\mathrm{NH}_{3}$-Lösung $(100 \mathrm{~mL})$ hinzugetropft. Die Mischung wurde mit $\mathrm{CH}_{2} \mathrm{Cl}_{2}$ extrahiert $(3 \times 200 \mathrm{~mL})$, die vereinigten organischen Phasen über $\mathrm{Na}_{2} \mathrm{SO}_{4}$ getrocknet und filtriert. Entfernung des Lösungsmittels in vacuo lieferte Amin 149h als braunes Öl.

${ }^{\mathrm{gg}}$ Eine Lösung von KI (1.98 g, 11.9 mmol, 2.00 Äq.) und $\mathrm{NaNO}_{2}$ (821 mg, 11.9 mmol, 2.00 Äq.) in $\mathrm{H}_{2} \mathrm{O}(50 \mathrm{~mL})$ wurde zu einer Lösung des Rückstands bestehend aus $149 \mathbf{h}$ (1.00 Äq.) und $p$-TsOH$\cdot \mathrm{H}_{2} \mathrm{O}$ (3.39 g, $17.8 \mathrm{mmol}, 3.00 \mathrm{Äq}$ ) in $\mathrm{CH}_{3} \mathrm{CN}(100 \mathrm{~mL})$ getropft und $45 \mathrm{~min}$ bei RT gerührt. Die Reaktionsmischung wurde mit ges. wässr. $\mathrm{NaHCO}_{3}$-Lösung $(100 \mathrm{~mL})$ versetzt und mit $\mathrm{CH}_{2} \mathrm{Cl}_{2}$ extrahiert $(3 \times 200 \mathrm{~mL})$. Die vereinigten organischen Phasen wurden mit ges. wässr. $\mathrm{Na}_{2} \mathrm{~S}_{2} \mathrm{O}_{3}$-Lösung $(200 \mathrm{~mL})$ gewaschen, über $\mathrm{Na}_{2} \mathrm{SO}_{4}$ getrocknet, filtriert und das Lösungsmittel in vacuo entfernt. Säulenchromatographische Reinigung $\left(\mathrm{SiO}_{2}, \quad n\right.$-Pentan $\rightarrow n$-Pentan/EtOAc, 50:1 $\rightarrow$ 20:1) lieferte Aryliodid 173 als gelbes Öl (803 mg, $2.36 \mathrm{mmol}$, $40 \%$ über 3 Stufen), welches ohne weitere Reinigung weiterverwendet wurde.

\footnotetext{
${ }^{\text {gg }}$ Allgemeine Methode aus: E. A. Krasnokutskaya, N. I. Semenischeva, V. D. Filimonov, P. Knochel, Synthesis
} 2007, 81-84. 
DC: $R_{f}=0.44(n-$ Pentan/EtOAc, 50:1).

MS (ESI): $m / z(\%)=341.0(100)[\mathrm{M}+\mathrm{H}]^{+}$.

HRMS (ESI): $m / z=$ gef.: 341.0028 , ber.: $341.0033[\mathrm{M}+\mathrm{H}]^{+}$.

$\mathbf{C}_{14} \mathrm{H}_{13} \mathrm{IO}_{2}$ (340.16). 


\section{3-(2-Iod-5-methylphenoxy)phenol (174)}<smiles>Cc1ccc(I)c(Oc2cccc(O)c2)c1</smiles>

Eine Lösung von 1-Iod-2-(3-methoxyphenoxy)-4-methylbenzol (173) (229 mg, 673 mol, 1.00 Äq.) in

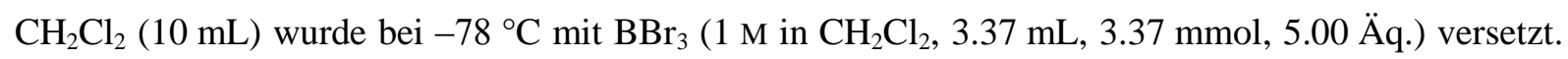
Die Lösung wurde über $21 \mathrm{H}$ auf RT erwärmt, mit $\mathrm{H}_{2} \mathrm{O}(20 \mathrm{~mL})$ versetzt und mit $\mathrm{CH}_{2} \mathrm{Cl}_{2}$ extrahiert $(3 \times 20 \mathrm{~mL})$. Die vereinigten organischen Phasen wurden über $\mathrm{Na}_{2} \mathrm{SO}_{4}$ getrocknet, filtriert und das Lösungsmittel in vacuo entfernt. Säulenchromatographische Reinigung $\left(\mathrm{SiO}_{2}, \mathrm{n}\right.$-Pentan/EtOAc, 10:1) lieferte Phenol 174 als gelbes Öl (142 mg, $435 \mu \mathrm{mol}, 65 \%$ ).

DC: $R_{f}=0.29(n-$ Pentan/EtOAc, $10: 1)$.

UV/Vis $\left(\mathrm{CH}_{3} \mathrm{CN}\right): \lambda_{\max }(\mathrm{nm})(\lg \varepsilon)=198(4.720), 275$ (3.607), 280 (3.574).

IR (ATR): $\tilde{v}\left(\mathrm{~cm}^{-1}\right)=1597,1567,1481,1472,1395,1325,1304,1274,1236,1167,1127,1021,1000$, $969,932,846,805,768,683,576,528$.

${ }^{1} \mathbf{H}-\mathrm{NMR}\left(600 \mathrm{MHz}, \mathrm{C}_{6} \mathrm{D}_{6}\right): \delta(\mathrm{ppm})=1.75\left(\mathrm{~s}, 3 \mathrm{H}, \mathrm{CH}_{3}\right), 4.03\left(\mathrm{~s}_{\mathrm{br}}, 1 \mathrm{H}, \mathrm{OH}\right), 6.23(\mathrm{t}, J=2.3 \mathrm{~Hz}, 1 \mathrm{H}$, 2'-H), 6.25-6.30 (m, 2 H, 5-H, 5'-H), 6.49 (ddd, $J=8.2,2.2,0.7$ Hz, 1 H, 4'-H), 6.60 (d, $J=1.4$ Hz, $1 \mathrm{H}, 3-\mathrm{H}), 6.86\left(\mathrm{t}, J=8.2 \mathrm{~Hz}, 1 \mathrm{H}, 6^{\prime}-\mathrm{H}\right), 7.55$ (d, $\left.J=8.0 \mathrm{~Hz}, 1 \mathrm{H}, 6-\mathrm{H}\right)$.

${ }^{13} \mathbf{C}-N M R\left(126 \mathrm{MHz}, \mathrm{C}_{6} \mathrm{D}_{6}\right): \delta(\mathrm{ppm})=20.6\left(\mathrm{CH}_{3}\right), 85.6(\mathrm{C}-1), 105.7,110.4,110.5,121.2,126.8$, 130.7 (C-3, C-5, C-2', C-4', C-5', C-6'), 139.7 (C-6), 140.2 (C-4), 156.6, 157.7, 158.8 (C-2, C-1', $\left.\mathrm{C}-3^{\prime}\right)$.

MS (ESI): $m / z(\%)=327.0(100)[\mathrm{M}+\mathrm{H}]^{+}$.

HRMS (ESI): $m / z=$ gef.: 326.9880 , ber.: $326.9876[\mathrm{M}+\mathrm{H}]^{+}$.

$\mathrm{C}_{13} \mathrm{H}_{11} \mathrm{IO}_{2}$ (326.13). 


\section{3-(2-Iod-5-methylphenoxy)phenyldimethylcarbamat (102h)}

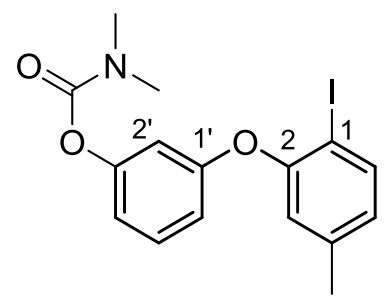

Eine Mischung aus 3-(2-Iod-5-methylphenoxy)phenol (174) (130 mg, $399 \mu \mathrm{mol}, 1.00$ Äq.), Dimethylcarbamoylchlorid (175) $\left(55.1 \mu \mathrm{L}, 599 \mu \mathrm{mol}, 1.50\right.$ Äq.) und $\mathrm{K}_{2} \mathrm{CO}_{3}$ in $\mathrm{CH}_{3} \mathrm{CN}$ (4 mL) wurde $14 \mathrm{~h}$ bei $60^{\circ} \mathrm{C}$ gerührt. Das Lösungsmittel wurde in vacuo entfernt und säulenchromatographische Reinigung $\left(\mathrm{SiO}_{2}, n\right.$-Pentan/EtOAc, 5:1) lieferte Carbamat 102h als farbloses Öl (103 mg, $259 \mu$ mol, $65 \%)$.

DC: $R_{f}=0.22(n-$ Pentan/EtOAc, $5: 1)$.

UV/Vis $\left(\mathrm{CH}_{3} \mathrm{CN}\right): \lambda_{\max }(\mathrm{nm})(\lg \varepsilon)=270(3.450), 278(3.404)$.

IR (ATR): $\tilde{v}\left(\mathrm{~cm}^{-1}\right)=1717,1596,1567,1472,1443,1382,1253,1233,1153,1122,1073,1018,1002$, $961,929,876,862,828,805,770,750,683,608,573$.

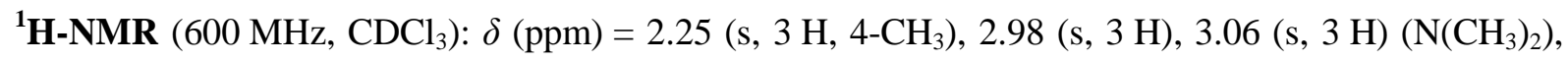
6.69-6.71 (m, $1 \mathrm{H}, 5-\mathrm{H}), 6.72(\mathrm{t}, J=2.3 \mathrm{~Hz}, 1 \mathrm{H}, 3-\mathrm{H}), 6.75$ (ddd, $\left.J=8.2,2.4,0.9 \mathrm{~Hz}, 1 \mathrm{H}, 4^{\prime}-\mathrm{H}\right)$, 6.77 (dd, $\left.J=1.9,0.6 \mathrm{~Hz}, 1 \mathrm{H}, 2^{\prime}-\mathrm{H}\right), 6.87$ (ddd, $\left.J=8.1,2.2,0.9 \mathrm{~Hz}, 1 \mathrm{H}, 6^{\prime}-\mathrm{H}\right), 7.27$ (t, $J=8.2 \mathrm{~Hz}$, $\left.1 \mathrm{H}, 5^{\prime}-\mathrm{H}\right), 7.68$ (d, J = 8.0 Hz, $\left.1 \mathrm{H}, 6-\mathrm{H}\right)$.

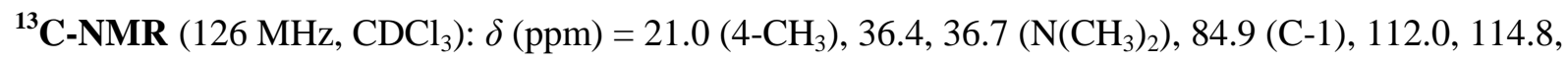
116.7, 120.8, 126.8, 129.8, 139.3 (C-3, C-5, C-6, C-2', C-4', C-5', C-6'), 140.3 (C-4), 152.6, 154.5, $155.8,157.6$ (C-2, C-1', C-3', C=O).

MS (ESI): $m / z(\%)=398.0(100)[\mathrm{M}+\mathrm{H}]^{+}$.

HRMS (ESI): $m / z=$ gef.: 398.0252 , ber.: $398.0248[\mathrm{M}+\mathrm{H}]^{+}$.

$\mathrm{C}_{16} \mathrm{H}_{16} \mathrm{INO}_{3}$ (397.21). 


\subsubsection{Untersuchungen zur Synthese von Dialkenen des Typs 117}

Octa-2,7-diin-1-ol (140b) ${ }^{\text {hh }}$

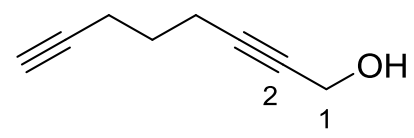

Eine Lösung von 1,6-Heptadiin (150b) $(3.20 \mathrm{~mL}, 2.58 \mathrm{~g}, 28.0 \mathrm{mmol}, 1.50$ Äq.) und $n$-Butyllithium (2.5 M in $n$-Hexan, $7.45 \mathrm{~mL}, 18.6 \mathrm{mmol}, 1.00$ Äq.) in THF $(20 \mathrm{~mL})$ wurde $75 \mathrm{~min}$ bei $-78{ }^{\circ} \mathrm{C}$ gerührt. Paraformaldehyd (566 mg, $18.6 \mathrm{mmol}, 1.00$ Äq.) wurde hinzugefügt und die Reaktionsmischung über $19 \mathrm{~h}$ auf RT erwärmt. Nach Zugabe von ges. wässr. $\mathrm{NH}_{4} \mathrm{Cl}$-Lösung $(20 \mathrm{~mL})$ wurde die Mischung mit $\mathrm{CH}_{2} \mathrm{Cl}_{2}$ extrahiert $(3 \times 20 \mathrm{~mL})$. Die vereinigten organischen Phasen wurden über $\mathrm{Na}_{2} \mathrm{SO}_{4}$ getrocknet, filtriert und das Lösungsmittel in vасиo entfernt. Säulenchromatographische Reinigung $\left(\mathrm{SiO}_{2}\right.$, n-Pentan/MTBE, 2:1) lieferte Alkohol 140b als farbloses Öl (1.02 g, $8.37 \mathrm{mmol}, 45 \%)$.

DC: $R_{f}=0.38(n-$ Pentan/MTBE, 2:1).

UV/Vis $\left(\mathrm{CH}_{3} \mathrm{CN}\right): \lambda_{\max }(\mathrm{nm})(\lg \varepsilon)=$ keine Aktivität nachgewiesen.

IR (ATR): $\tilde{v}\left(\mathrm{~cm}^{-1}\right)=3290,2937,2910,2868,2852,1453,1432,1390,1345,1329,1311,1292,1227$, $1133,1007,978,785,631,567,555,520$.

${ }^{1} \mathbf{H}-\mathrm{NMR}\left(300 \mathrm{MHz}, \mathrm{CDCl}_{3}\right): \delta(\mathrm{ppm})=1.68\left(\mathrm{p}, J=6.9 \mathrm{~Hz}, 2 \mathrm{H}, 5-\mathrm{H}_{2}\right), 1.94(\mathrm{t}, J=3.0 \mathrm{~Hz}, 1 \mathrm{H}, 8-\mathrm{H})$, 2.05-2.18 (m, $1 \mathrm{H}, \mathrm{OH}), 2.23-2.37$ (m, 4 H, 4- $\left.\mathrm{H}_{2}, 6-\mathrm{H}_{2}\right), 4.20$ (t, $\left.J=2.2 \mathrm{~Hz}, 2 \mathrm{H}, 1-\mathrm{H}_{2}\right)$.

${ }^{13}$ C-NMR $\left(126 \mathrm{MHz}, \mathrm{CDCl}_{3}\right): \delta(\mathrm{ppm})=17.4,17.7$ (C-4, C-6), $27.3(\mathrm{C}-5), 51.1(\mathrm{C}-1), 68.9(\mathrm{C}-8)$, 79.0 (C-7), 83.4, 85.0 (C-2, C-3).

HRMS (ESI): $m / z=$ gef.: 123.0801 , ber.: $123.0804[\mathrm{M}+\mathrm{H}]^{+}$.

$\mathbf{C}_{8} \mathbf{H}_{10} \mathbf{O}$ (122.17).

${ }^{\text {hh }}$ Für Originalvorschrift siehe: S. López, F. Fernández-Trillo, L. Castedo, C. Saá, Org. Lett. 2003, 5, 3725-3728. 


\section{Nona-2,8-diin-1-ol (140c) $)^{\text {ii }}$}

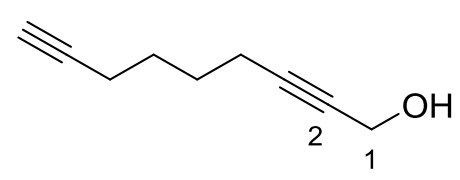

Eine Lösung von 1,7-Octadiin (150c) $(2.00 \mathrm{~mL}, 1.60 \mathrm{~g}, 15.1 \mathrm{mmol}, 1.50$ Äq.) und $n$-Butyllithium (2.5 M in $n$-Hexan, $4.02 \mathrm{~mL}, 10.0 \mathrm{mmol}, 1.00$ Äq.) in THF $(20 \mathrm{~mL})$ wurde $75 \mathrm{~min}$ bei $-78^{\circ} \mathrm{C}$ gerührt. Paraformaldehyd (305 mg, 10.0 mmol, 1.00 Äq.) wurde hinzugefügt und die Reaktionsmischung über $19 \mathrm{~h}$ auf RT erwärmt. Nach Zugabe von ges. wässr. $\mathrm{NH}_{4} \mathrm{Cl}$-Lösung $(20 \mathrm{~mL})$ wurde die Mischung mit $\mathrm{CH}_{2} \mathrm{Cl}_{2}$ extrahiert $(3 \times 20 \mathrm{~mL})$. Die vereinigten organischen Phasen wurden über $\mathrm{Na}_{2} \mathrm{SO}_{4}$ getrocknet, filtriert und das Lösungsmittel in vacuo entfernt. Säulenchromatographische Reinigung $\left(\mathrm{SiO}_{2}\right.$, n-Pentan/MTBE, 2:1) lieferte Alkohol 140c als farbloses Öl (592 mg, $8.37 \mathrm{mmol}, 43 \%$ ).

DC: $R_{f}=0.38(n-$ Pentan/MTBE, 2:1).

UV/Vis $\left(\mathrm{CH}_{3} \mathrm{CN}\right): \lambda_{\max }(\mathrm{nm})(\lg \varepsilon)=$ keine Aktivität nachgewiesen.

IR (ATR): $\tilde{v}\left(\mathrm{~cm}^{-1}\right)=3290,2939,2864,1458,1431,1390,1363,1329,1309,1276,1226,1133,1007$, $975,776,630,557,502$.

${ }^{1} \mathbf{H}-\mathbf{N M R}\left(300 \mathrm{MHz}, \mathrm{CDCl}_{3}\right): \delta(\mathrm{ppm})=1.49-1.66\left(\mathrm{~m}, 4 \mathrm{H}, 5-\mathrm{H}_{2}, 6-\mathrm{H}_{2}\right), 1.92(\mathrm{t}, J=2.7 \mathrm{~Hz}, 1 \mathrm{H}$, 9-H), 2.12-2.27 (m, $\left.5 \mathrm{H}, 4-\mathrm{H}_{2}, 7-\mathrm{H}_{2}, \mathrm{OH}\right), 4.18\left(\mathrm{td}, J=2.2,0.9 \mathrm{~Hz}, 2 \mathrm{H}, 1-\mathrm{H}_{2}\right)$.

${ }^{13} \mathbf{C}-\mathbf{N M R}\left(126 \mathrm{MHz}, \mathrm{CDCl}_{3}\right): \delta(\mathrm{ppm})=17.8,18.1(\mathrm{C}-5, \mathrm{C}-6), 27.3,27.4(\mathrm{C}-4, \mathrm{C}-7), 51.1(\mathrm{C}-1), 68.5$ (C-9), 78.7, 84.0, 85.6 (C-2, C-3, C-8).

HRMS (ESI): $m / z=$ gef.: 137.0954, ber.: $137.0961[\mathrm{M}+\mathrm{H}]^{+}$.

$\mathbf{C}_{9} \mathbf{H}_{12} \mathbf{O}$ (136.09).

\footnotetext{
${ }^{\text {ii }}$ Für Originalvorschrift siehe: C. Mukai, I. Nomura, S. Kitagaki, J. Org. Chem. 2003, 68, 1376-1485.
} 


\section{1-Brom-2-((octa-2,7-diin-1-yloxy)methyl)naphthalin (176a)}

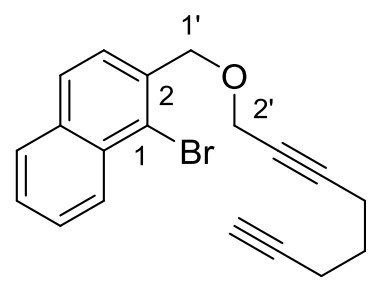

Eine Lösung von Octa-2,7-diin-1-ol (140b) (136 mg, $1.11 \mu \mathrm{mol}, 1.50$ Äq.) in THF (5 mL) wurde bei $0{ }^{\circ} \mathrm{C}$ zu einer Suspension von $\mathrm{NaH}(60 \%$ in Mineralöl, $89.0 \mathrm{mg}, 2.22 \mu \mathrm{mol}, 3.00 \mathrm{Äq}$.) hinzugefügt. Die Reaktionsmischung wurde mit einer Lösung von 1-Brom-2-(brommethyl)naphthalin (158) (223 mg, $742 \mu \mathrm{mol}, 1.00$ Äq.) in THF (10 mL) versetzt und über $20 \mathrm{~h}$ auf RT erwärmt. Nach Zugabe von ges. wässr. $\mathrm{NH}_{4} \mathrm{Cl}$-Lösung $(40 \mathrm{~mL})$ wurde die Mischung mit $\mathrm{CH}_{2} \mathrm{Cl}_{2}$ extrahiert $(3 \times 20 \mathrm{~mL})$. Die vereinigten organischen Phasen wurden über $\mathrm{Na}_{2} \mathrm{SO}_{4}$ getrocknet, filtriert und das Lösungsmittel in vacuo entfernt. Säulenchromatographische Reinigung $\left(\mathrm{SiO}_{2}, n\right.$-Pentan/EtOAc, 50:1) lieferte Ether 176a als gelbes Öl (239 mg, 8.37 mmol, 94\%).

DC: $R_{f}=0.46(n-$ Pentan/EtOAc, 50:1).

UV/Vis $\left(\mathrm{CH}_{3} \mathrm{CN}\right): \lambda_{\max }(\mathrm{nm})(\lg \varepsilon)=228$ (4.910), 285 (3.818).

IR (ATR): $\tilde{v}\left(\mathrm{~cm}^{-1}\right)=3295,2936,2905,2845,1556,1501,1451,1430,1353,1343,1326,1292,1257$, 1225, 1132, 1095, 1073, 1023, 986, 955, 930, 863, 812, 766, 754, 743, 631, 529.

${ }^{1} \mathbf{H}-\mathbf{N M R}\left(600 \mathrm{MHz}, \mathrm{CDCl}_{3}\right): \delta(\mathrm{ppm})=1.69-1.79\left(\mathrm{~m}, 2 \mathrm{H}, 6^{\prime}-\mathrm{H}_{2}\right), 1.95(\mathrm{dd}, J=3.3,2.0 \mathrm{~Hz}, 1 \mathrm{H}$, 9'-H), 2.31 (td, $\left.J=7.0,2.1 \mathrm{~Hz}, 2 \mathrm{H}, 7^{\prime}-\mathrm{H}_{2}\right), 2.38$ (tt, $\left.J=7.0,2.6 \mathrm{~Hz}, 2 \mathrm{H}, 5^{\prime}-\mathrm{H}_{2}\right), 4.27$ (t, $J=2.1 \mathrm{~Hz}$, $\left.2 \mathrm{H}, 2^{\prime}-\mathrm{H}_{2}\right), 4.90\left(\mathrm{~s}, 2 \mathrm{H}, 1\right.$ ' $-\mathrm{H}_{2}$ ), 7.50 (ddd, $\left.J=8.0,6.8,1.1 \mathrm{~Hz}, 1 \mathrm{H}, 6-\mathrm{H}\right), 7.57$ (ddd, $J=8.4,6.8$, $1.3 \mathrm{~Hz}, 1 \mathrm{H}, 7-\mathrm{H}), 7.61$ (d, $J=8.4 \mathrm{~Hz}, 1 \mathrm{H}, 3-\mathrm{H}), 7.80$ (d, $J=8.0 \mathrm{~Hz}, 1 \mathrm{H}, 4-\mathrm{H}), 7.80-7.81$ (m, $1 \mathrm{H}$, 5-H), $8.31(\mathrm{dd}, J=8.5,0.7 \mathrm{~Hz}, 1 \mathrm{H}, 8-\mathrm{H})$.

${ }^{13}$ C-NMR (126 MHz, CDCl $): \delta(\mathrm{ppm})=17.6,17.9$ (C-5', C-7'), 27.4 (C-6'), 58.4 (C-2'), 68.9 (C-9'), 71.6 (C-1'), 76.5 (C-8'), 83.4, 86.3 (C-3', C-4'), 122.8 (C-1), 126.2, 126.5, 127.1, 127.4, 127.7, 128.1 (C-3, C-4, C-5, C-6, C-7, C-8), 132.2 (C-8a), 134.0 (C-4a), 135.4 (C-2).

MS (ESI): $m / z=340.0(11)[\mathrm{M}]^{+}$.

HRMS (EI): $m / z=$ gef.: 340.0469 , ber.: $340.0463 \quad\left[\mathrm{M}\left({ }^{79} \mathrm{Br}\right)\right]^{+}$; gef.: 342.0525 , ber.: 342.0442 $\left[\mathrm{M}\left({ }^{81} \mathrm{Br}\right)\right]^{+}$.

$\mathrm{C}_{19} \mathrm{H}_{17} \mathrm{BrO}(341.25)$. 


\section{1-Brom-2-((nona-2,8-diin-1-yloxy)methyl)naphthalin (176b)}

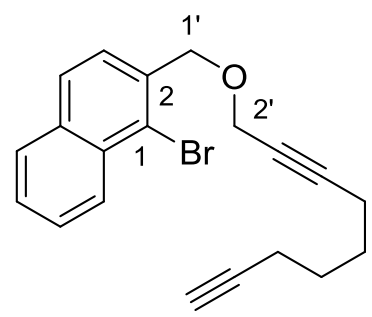

Eine Lösung von 1-Brom-2-(brommethyl)naphthalin (158) (870 mg, $2.90 \mathrm{mmol}, 1.00$ Äq.) und Nona-2,8-diin-1-ol (140c) (592 mg, $4.35 \mathrm{mmol}, 1.50$ Äq.) in THF (50 mL) wurde bei $0{ }^{\circ} \mathrm{C}$ mit $\mathrm{NaH}$ (60\% in Mineralöl, $348 \mathrm{mg}, 8.70 \mathrm{mmol}, 3.00$ Äq.) versetzt und über $20 \mathrm{~h}$ auf RT erwärmt. Nach Zugabe von ges. wässr. $\mathrm{NH}_{4} \mathrm{Cl}$-Lösung $(50 \mathrm{~mL})$ wurde die Mischung mit $\mathrm{CH}_{2} \mathrm{Cl}_{2}$ extrahiert $(3 \times 50 \mathrm{~mL})$. Die vereinigten organischen Phasen wurden über $\mathrm{Na}_{2} \mathrm{SO}_{4}$ getrocknet, filtriert und das Lösungsmittel in vacuo entfernt. Säulenchromatographische Reinigung $\left(\mathrm{SiO}_{2}, n\right.$-Pentan/EtOAc, 50:1) lieferte Ether 176b als gelbes Öl (967 mg, 2.72 mmol, 94\%).

DC: $R_{f}=0.46(n-$ Pentan/EtOAc, $50: 1)$.

UV/Vis $\left(\mathrm{CH}_{3} \mathrm{CN}\right): \lambda_{\max }(\mathrm{nm})(\lg \varepsilon)=228$ (4.902), 285 (3.806).

IR (ATR): $\tilde{v}\left(\mathrm{~cm}^{-1}\right)=3297,2941,2860,1557,1502,1458,1444,1429,1354,1341,1327,1257,1225$, $1133,1096,1075,1024,965,955,931,863,813,767,753,744,655,635,530$.

${ }^{1} \mathbf{H}-\mathbf{N M R}\left(600 \mathrm{MHz}, \mathrm{CDCl}_{3}\right): \delta(\mathrm{ppm})=1.62-1.67\left(\mathrm{~m}, 4 \mathrm{H}, 6^{\prime}-\mathrm{H}_{2}, 7^{\prime}-\mathrm{H}_{2}\right), 1.95(\mathrm{t}, J=2.6 \mathrm{~Hz}, 1 \mathrm{H}$, $\left.10^{\prime}-\mathrm{H}\right), 2.17-2.23\left(\mathrm{~m}, 2 \mathrm{H}, 8^{\prime}-\mathrm{H}_{2}\right), 2.25-2.31\left(\mathrm{~m}, J=6.5,4.3,2.2 \mathrm{~Hz}, 2 \mathrm{H}, 5^{\prime}-\mathrm{H}_{2}\right), 4.28(\mathrm{t}, J=2.2 \mathrm{~Hz}$, $\left.2 \mathrm{H}, 2^{\prime}-\mathrm{H}_{2}\right), 4.90\left(\mathrm{~s}, 2 \mathrm{H}, 1^{\prime}-\mathrm{H}_{2}\right), 7.50$ (ddd, $\left.J=8.0,6.8,1.1 \mathrm{~Hz}, 1 \mathrm{H}, 6-\mathrm{H}\right), 7.57$ (ddd, $J=8.4,6.8$, $1.3 \mathrm{~Hz}, 1 \mathrm{H}, 7-\mathrm{H}), 7.62(\mathrm{~d}, J=8.4 \mathrm{~Hz}, 1 \mathrm{H}, 3-\mathrm{H}), 7.77-7.82(\mathrm{~m}, 2 \mathrm{H}, 4-\mathrm{H}, 5-\mathrm{H}), 8.31$ (dd, $J=8.5$, $0.6 \mathrm{~Hz}, 1 \mathrm{H}, 8-\mathrm{H})$.

${ }^{13}$ C-NMR $\left(126 \mathrm{MHz}, \mathrm{CDCl}_{3}\right): \delta(\mathrm{ppm})=17.9,18.3\left(\mathrm{C}-5^{\prime}, \mathrm{C}^{\prime} 8^{\prime}\right), 27.4,27.5$ (C-6', C-7'), 58.4 (C-2'), 68.5 (C-10'), 71.5 (C-1'), 76.1 (C-9'), 84.0, 86.9 (C-3', C-4'), 122.7 (C-1), 126.1 (C-3), 126.4 (C-6), 127.0 (C-8), 127.3 (C-7), 127.6, 128.0 (C-4, C-5), 132.1 (C-8a), 134.0 (C-4a), 135.4 (C-2).

MS (ESI): $m / z=354.1(10)[\mathrm{M}]^{+}$.

HRMS (EI): $m / z=$ gef.: 354.0621, ber.: 354.0619 [M( $\left.\left({ }^{79} \mathrm{Br}\right)\right]^{+}$; gef.: 356.0544 , ber.: 356.0599 $\left[\mathrm{M}\left({ }^{81} \mathrm{Br}\right)\right]^{+}$.

$\mathrm{C}_{20} \mathrm{H}_{19} \mathrm{BrO}$ (355.28). 


\section{1-(5-(2-Phenoxybenzyliden)cyclopent-1-en-1-yl)-4H-benzo[f]isochromen (177a)}

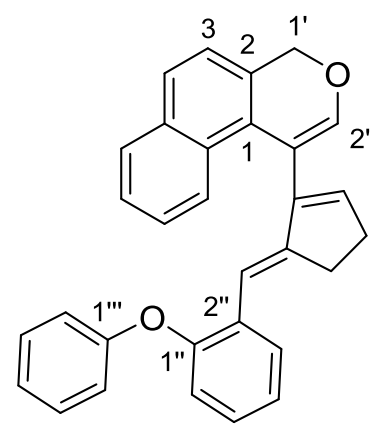

Eine Mischung aus Aryliodid 102a (50.0 mg, $169 \mu$ mol, 1.00 Äq.), Dialkin 176a (86.4 mg, $254 \mu \mathrm{mol}$, 1.50 Äq.), $\mathrm{Pd}(\mathrm{OAc})_{2}$ (7.50 mg, $33.8 \mu \mathrm{mol}, 0.20$ Äq.), $\mathrm{PPh}_{3}$ (44.9 mg, $169 \mu \mathrm{mol}, 1.00$ Äq.) und $(n \mathrm{Bu})_{4} \mathrm{NOAc}\left(255 \mathrm{mg}, 845 \mu \mathrm{mol}, 5.00 \mathrm{Äq}\right.$.) wurde $5 \mathrm{~h}$ bei $100{ }^{\circ} \mathrm{C}$ in der Mikrowelle gerührt. Die Mischung wurde durch $\mathrm{SiO}_{2}$ filtriert, mit EtOAc gespült und das Lösungsmittel in vacuo entfernt. Säulenchromatographische Reinigung $\left(\mathrm{SiO}_{2}, n-\mathrm{Pentan} / \mathrm{CH}_{2} \mathrm{Cl}_{2}, 3: 1\right)$ lieferte Trialken 177a als farbloses Öl (35.4 mg, $82.6 \mu \mathrm{mol}, 52 \%)$.

DC: $R_{f}=0.26\left(n-\mathrm{Pentan} / \mathrm{CH}_{2} \mathrm{Cl}_{2}, 3: 1\right)$.

UV/Vis $\left(\mathrm{CH}_{3} \mathrm{CN}\right): \lambda_{\max }(\mathrm{nm})(\lg \varepsilon)=227(4.571), 296(4.232)$.

IR (ATR): $\tilde{v}\left(\mathrm{~cm}^{-1}\right)=1598,1587,1490,1473,1446,1393,1236,1206,1187,1164,1152,1130,1107$, 1088, 1037, 1023, 952, 935, 871, 855, 818, 807, 791, 751, 743, 724, 689, 680, 595, 589, 501.

${ }^{1} \mathbf{H}-\mathrm{NMR}\left(600 \mathrm{MHz}, \mathrm{C}_{6} \mathrm{D}_{6}\right): \delta(\mathrm{ppm})=2.20-2.37\left(\mathrm{~m}, 2 \mathrm{H}, 6^{\prime}-\mathrm{H}_{2}\right), 2.80\left(\mathrm{t}, J=4.2 \mathrm{~Hz}, 2 \mathrm{H}, 7^{\prime}-\mathrm{H}_{2}\right), 4.80$ (s, 2 H, 1'-H $), 5.91$ (t, J=2.8 Hz, 1 H, 5'-H), 6.42-6.56 (m, 2 H, 2' ' $-\mathrm{H}, 6$ ','-H), 6.67-6.71 (m, $2 \mathrm{H}$, 9'-H, 4'”'-H), 6.71-6.75 (m, 2 H, 3-H, 6'-H), 6.78 (s, 1 H, 2'-H), 6.80-6.87 (m, 3 H, 5'”-H, 3','-H, 5"'--H), 6.90 (td, $J=7.5,1.2 \mathrm{~Hz}, 1 \mathrm{H}, 4$ ',-H), 7.12 (ddd, $J=7.9,6.6,1.0 \mathrm{~Hz}, 6-\mathrm{H}), 7.19$ (ddd, $J=8.5$, 6.7, 1.4 Hz, $1 \mathrm{H}, 7-\mathrm{H}), 7.40$ (d, $J=8.2 \mathrm{~Hz}, 1 \mathrm{H}, 4-\mathrm{H}), 7.44$ (dd, $J=7.9,1.6 \mathrm{~Hz}, 1 \mathrm{H}, 3$ '’-H), 7.52 (dd, $J=8.1,1.3 \mathrm{~Hz}, 1 \mathrm{H}, 5-\mathrm{H}), 8.43(\mathrm{dd}, J=8.7,1.0 \mathrm{~Hz}, 1 \mathrm{H}, 8-\mathrm{H})$.

${ }^{13}$ C-NMR (126 MHz, C $\left.{ }_{6} \mathrm{D}_{6}\right): \delta(\mathrm{ppm})=30.0\left(\mathrm{C}^{\prime} 7^{\prime}\right), 31.5$ (C-6'), 70.1 (C-1'), 114.4 (C-9'), 115.5

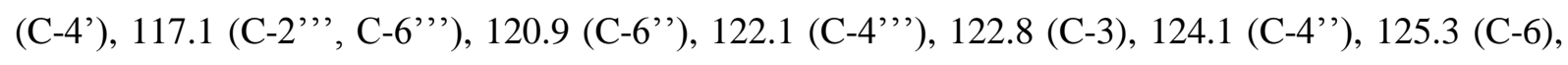
125.6, 125.7 (C-7, C-8), 127.0 (C-2), 127.5 (C-5'), 127.6 (C-1), 127.9 (C-4), 128.8 (C-5, C-3'”), 129.0 (C-8a), 129.5 (C-3”, , C-5'”'), 131.6 (C-2'”), 134.6 (C-4a), 134.9 (C-5'), 146.6 (C-8'), 147.5 (C-2'), 149.1 (C-3'), $153.8\left(\mathrm{C}-1\right.$ ''), $158.6\left(\mathrm{C}-1{ }^{\prime \prime \prime}{ }^{\prime}\right)$.

MS (EI): $m / z(\%)=428.2(100)[\mathrm{M}]^{+}$.

HRMS (EI): $m / z=$ gef.: 428.1769 , ber.: $428.1776[\mathrm{M}]^{+}$.

$\mathbf{C}_{31} \mathbf{H}_{24} \mathbf{O}_{2}$ (428.53). 


\subsubsection{Untersuchungen zur Synthese von Dialkenen des Typs 119}

\section{2-Brom-4-methyl-1-(2-nitrophenoxy)benzol (122b $)^{\mathrm{jj}}$}<smiles>Cc1ccc(Oc2ccccc2[N+](=O)[O-])c(Br)c1</smiles>

Ein Gemisch aus 2-Brom-4-methyl-phenol (120b) (1.94 mL, $3.00 \mathrm{~g}, \quad 16.0 \mathrm{mmol}, 1.00$ Äq.), 2-Fluornitrobenzol (121a) (1.68 mL, 2.26 g, $16.0 \mathrm{mmol}, 1.00$ Äq.) und $\mathrm{K}_{2} \mathrm{CO}_{3}$ (4.42 g, $32.0 \mathrm{mmol}$, 2.00 Äq.) in DMSO $(30 \mathrm{~mL})$ wurde $22 \mathrm{~h}$ bei $95^{\circ} \mathrm{C}$ gerührt. Nach Abkühlen auf RT wurde die Mischung in $\mathrm{H}_{2} \mathrm{O}(100 \mathrm{~mL})$ gegeben und mit MTBE extrahiert $(3 \times 100 \mathrm{~mL})$. Die vereingten organischen Phasen wurden über $\mathrm{Na}_{2} \mathrm{SO}_{4}$ getrocknet, filtriert und das Lösungsmittel in vacuo entfernt. Biarylether 122b wurde als gelbes Öl ( $9.86 \mathrm{~g}, 32.0 \mathrm{mmol}$, quant.) erhalten.

DC: $R_{f}=0.31(\mathrm{PE} / \mathrm{EtOAc}, 20: 1)$.

UV/Vis $\left(\mathrm{CH}_{3} \mathrm{CN}\right): \lambda_{\max }(\mathrm{nm})(\lg \varepsilon)=195$ (4.742), $313(3.401)$.

IR (Film): $\tilde{v}\left(\mathrm{~cm}^{-1}\right)=3351,2924,2868,1608,1595,1527,1479,1350,1251,1046,820,776,740$.

${ }^{1}$ H-NMR $\left(300 \mathrm{MHz}, \mathrm{CDCl}_{3}\right): \delta(\mathrm{ppm})=2.33\left(\mathrm{~s}, 3 \mathrm{H}, \mathrm{CH}_{3}\right), 6.78(\mathrm{dd}, J=8.4,1.1 \mathrm{~Hz}, 1 \mathrm{H}, 6-\mathrm{H}), 6.95$ (d, $J=8.2 \mathrm{~Hz}, 1 \mathrm{H}, 6$ '-H), 7.07-7.18 (m, 2 H, 4-H, 5'-H), 7.40-7.48 (m, 2 H, 6-H, 3'-H), 7.94 (dd, $\left.J=8.1,1.7 \mathrm{~Hz}, 1 \mathrm{H}, 3^{\prime}-\mathrm{H}\right)$.

${ }^{13}$ C-NMR $\left(125 \mathrm{MHz}, \mathrm{CDCl}_{3}\right): \delta(\mathrm{ppm})=20.6\left(\mathrm{CH}_{3}\right), 114.9(\mathrm{C}-2$ ') $), 118.4(\mathrm{C}-6), 121.4(\mathrm{C}-6$ ') 122.6 (C-5'), 125.7 (C-3), 129.5 (C-4), 134.0 (C-3'), 134.3 (C-5, C-4'), 136.7 (C-1), 149.4 (C-2), 150.5 $(\mathrm{C}-1$ ').

MS (ESI): $m / z(\%)=330.0(72)[\mathrm{M}+\mathrm{Na}]^{+}, 639.0(100)[2 \mathrm{M}+\mathrm{Na}]^{+}$.

HRMS (ESI): $m / z=$ gef.: 329.9738 , ber.: $329.9736[\mathrm{M}+\mathrm{Na}]^{+}$.

$\mathrm{C}_{13} \mathrm{H}_{10} \mathrm{BrNO}_{3}$ (308.13).

\footnotetext{
jj Originalvorschrift und experimentelle Daten aus: M. A. Düfert, Disseration, Georg-August-Universität, Göttingen, 2010.
} 


\section{2-(2-Brom-4-methylphenoxy)anilin (123b $)^{\mathrm{kk}}$}<smiles>Cc1ccc(Oc2ccccc2N)c(Br)c1</smiles>

Konz. $\mathrm{HCl}(240 \mathrm{~mL})$ und anschließend konz. AcOH $(240 \mathrm{~mL})$ wurden bei $0{ }^{\circ} \mathrm{C}$ zu einer Lösung von Biarylether 122b $(5.99 \mathrm{~g}, 19.0 \mathrm{mmol}, 1.00$ Äq.) in EtOAc $(120 \mathrm{~mL})$ getropft. Zinkpulver $(37.5 \mathrm{~g}$, $574 \mathrm{mmol}, 30.0$ Äq.) wurde portionsweise hinzugefügt, die Lösung auf RT erwärmt und 15 min bei dieser Temperatur gerührt. Die Reaktionsmischung wurde auf $0{ }^{\circ} \mathrm{C}$ abgekühlt und 33\%ige wässr. $\mathrm{NH}_{3}$-Lösung $(600 \mathrm{~mL})$ hinzugetropft. Die Mischung wurde mit $\mathrm{CH}_{2} \mathrm{Cl}_{2}$ extrahiert $(3 \times 600 \mathrm{~mL})$ und die vereinigten organischen Phasen über $\mathrm{Na}_{2} \mathrm{SO}_{4}$ getrocknet, filtriert und das Lösungsmittel in vacuo entfernt. Amin 123b wurde als gelbes Öl (4.67 g, 16.8 mmol, 88\%) erhalten.

DC: $R_{f=} 0.63(\mathrm{PE} / \mathrm{EtOAc}, 5: 1)$.

UV/Vis $\left(\mathrm{CH}_{3} \mathrm{CN}\right): \lambda_{\max }(\mathrm{nm})(\lg \varepsilon)=197$ (4.724), 287 (3.648).

IR (Film): $\tilde{v}\left(\mathrm{~cm}^{-1}\right)=3466,3379,3033,2921,1619,1501,1486,1296,1239,1190,1044,743$.

${ }^{1}$ H-NMR $\left(300 \mathrm{MHz}, \mathrm{CDCl}_{3}\right): \delta(\mathrm{ppm})=2.29\left(\mathrm{~s}, 3 \mathrm{H}, \mathrm{CH}_{3}\right), 3.84\left(\mathrm{~s}, 2 \mathrm{H}, \mathrm{NH}_{2}\right), 6.67(\mathrm{ddd}, J=8.6,7.1$, $1.5 \mathrm{~Hz}, 1 \mathrm{H}, 4-\mathrm{H}), 6.74\left(\mathrm{dd}, J=7.8,1.6 \mathrm{~Hz}, 1 \mathrm{H}, 5^{\prime}-\mathrm{H}\right), 6.76$ (d, $\left.J=8.2 \mathrm{~Hz}, 1 \mathrm{H}, 6^{\prime}-\mathrm{H}\right), 6.80$ (dd, $J=7.9,1.5 \mathrm{~Hz}, 1 \mathrm{H}, 6-\mathrm{H}), 6.91-7.03$ (m, $2 \mathrm{H}, 3-\mathrm{H}, 5-\mathrm{H}), 7.42$ (d, $\left.J=1.5 \mathrm{~Hz}, 1 \mathrm{H}, 3^{\prime}-\mathrm{H}\right)$.

${ }^{13}$ C-NMR (125 MHz, $\left.\mathrm{CDCl}_{3}\right): \delta(\mathrm{ppm})=20.4\left(\mathrm{CH}_{3}\right), 113.2(\mathrm{C}-2$ '), 116.3 (C-6), 118.4 (C-6'), 118.5 (C-4), 118.8 (C-5'), 124.6 (C-3), 129.1 (C-5), 133.8 (C-3'), 134.2 (C-4'), 137.9 (C-1), 143.4 (C-2), $151.3\left(\mathrm{C}-1^{\prime}\right)$.

MS (ESI): $m / z(\%)=300.0(58)[\mathrm{M}+\mathrm{Na}]^{+}, 579.0(100)[2 \mathrm{M}+\mathrm{Na}]^{+}$.

HRMS (ESI): $m / z(\%)=$ gef.: 278.0173 , ber.: $278.0175[\mathrm{M}+\mathrm{H}]^{+}$.

$\mathrm{C}_{13} \mathrm{H}_{12} \mathrm{BrNO}$ (278.14).

\footnotetext{
${ }^{k k}$ Originalvorschrift und experimentelle Daten aus: M. A. Düfert, Disseration, Georg-August-Universität, Göttingen, 2010.
} 


\section{2-Brom-1-(2-iodphenoxy)-4-methylbenzol (124b)}<smiles>Cc1ccc(Oc2ccccc2I)c(Br)c1</smiles>

Eine Lösung von KI (6.97 g, 42.0 mmol, 2.50 Äq.) und $\mathrm{NaNO}_{2}$ (2.32 g, 33.6 mmol, 2.00 Äq.) in $\mathrm{H}_{2} \mathrm{O}$ (34 mL) wurde zu einer Lösung von Amin 123b (4.67 g, $16.8 \mathrm{mmol}, 1.00$ Äq.) und $p$ - Ts $\mathrm{OH} \cdot \mathrm{H}_{2} \mathrm{O}$ (9.58 g, $50.4 \mathrm{mmol}, 3.00$ Äq.) in $\mathrm{CH}_{3} \mathrm{CN}(68 \mathrm{~mL})$ getropft und $45 \mathrm{~min}$ bei RT gerührt. Die Reaktionsmischung wurde mit ges. wässr. $\mathrm{NaHCO}_{3}$-Lösung $(230 \mathrm{~mL})$ versetzt und mit $\mathrm{CH}_{2} \mathrm{Cl}_{2}$ extrahiert $(3 \times 300 \mathrm{~mL})$. Die vereinigten organischen Phasen wurden mit ges. wässr. $\mathrm{Na}_{2} \mathrm{~S}_{2} \mathrm{O}_{3}$-Lösung $(500 \mathrm{~mL})$ gewaschen, über $\mathrm{Na}_{2} \mathrm{SO}_{4}$ getrocknet, filtriert und das Lösungsmittel in vacuo entfernt. Säulenchromatographische Reinigung $\left(\mathrm{SiO}_{2}, n\right.$-Pentan) lieferte Aryliodid 124b als gelbes Öl (3.50 g, $8.77 \mathrm{mmol}, 54 \%)$.

DC: $R_{f}=0.35$ (n-Pentan).

UV/Vis $\left(\mathrm{CH}_{3} \mathrm{CN}\right): \lambda_{\max }(\mathrm{nm})(\lg \varepsilon)=195$ (4.810), 277 (3.549).

IR (Film): $\tilde{v}\left(\mathrm{~cm}^{-1}\right)=3350,3060,2920,1576,1487,1464,1438,1246,1208,1045,1020$.

${ }^{1}$ H-NMR $\left(300 \mathrm{MHz}, \mathrm{CDCl}_{3}\right): \delta(\mathrm{ppm})=2.32\left(\mathrm{~s}, 3 \mathrm{H}, \mathrm{CH}_{3}\right), 6.67(\mathrm{dd}, J=8.2,1.4 \mathrm{~Hz}, 1 \mathrm{H}, 3-\mathrm{H}), 6.78$ (d, $J=8.2 \mathrm{~Hz}, 1 \mathrm{H}, 6$ '-H), 6.84 (dd, $J=7.7,1.4 \mathrm{~Hz}, 1 \mathrm{H}, 5-\mathrm{H}), 7.05$ (ddd, $J=8.2,2.0,0.6 \mathrm{~Hz}, 1 \mathrm{H}$, 5'-H), 7.22 (ddd, $J=8.2,7.4,1.6 \mathrm{~Hz}, 1 \mathrm{H}, 4-\mathrm{H}), 7.44$ (d, $J=1.4 \mathrm{~Hz}, 1 \mathrm{H}, 3$ '-H), 7.84 (dd, $J=7.9$, $1.6 \mathrm{~Hz}, 1 \mathrm{H}, 6-\mathrm{H})$.

${ }^{13}$ C-NMR (125 MHz, $\left.\mathrm{CDCl}_{3}\right): \delta(\mathrm{ppm})=20.5\left(\mathrm{CH}_{3}\right), 87.4(\mathrm{C}-1), 114.4(\mathrm{C}-2$ ') $117.4(\mathrm{C}-3), 120.2$ (C-6’), 124.8 (C-4), 129.2 (C-5'), 129.4 (C-4), 134.1 (C-3'), 135.4 (C-4'), 139.8 (C-6), 150.6 (C-1'), $156.2(\mathrm{C}-2)$.

MS (ESI): $m / z(\%)=410.9(42)[\mathrm{M}+\mathrm{Na}]^{+}, 800.8(100)[2 \mathrm{M}+\mathrm{Na}]^{+}$.

HRMS (ESI): $m / z(\%)=$ gef.: 410.8852 , ber.: $410.8852[\mathrm{M}+\mathrm{Na}]^{+}$.

$\mathbf{C}_{13} \mathbf{H}_{10}$ BrIO (389.03).

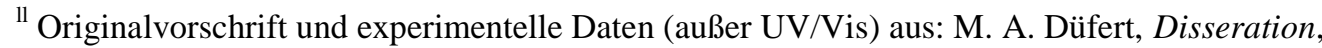
Georg-August-Universität, Göttingen, 2010.
} 


\section{2-Brom-1-(2-(hepta-1,6-diin-1-yl)phenoxy)-4-methylbenzol (109b)}

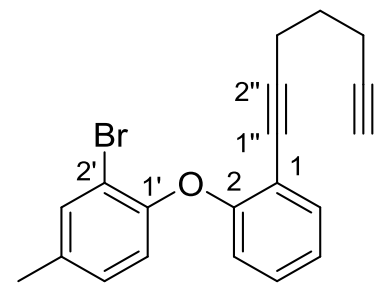

Ein Gemisch von 2-Brom-1-(2-iodphenoxy)-4-methylbenzol (124b) (342 mg, $879 \mu$ mol, 1.00 Äq.), 1,6-Heptadiin (500 $\mu \mathrm{L}, 405$ mg, $4.40 \mathrm{mmol}, 5.00$ Äq.), $\mathrm{PdCl}_{2}\left(\mathrm{PPh}_{3}\right)_{2}$ (30.9 mg, $44.0 \mu$ mol, 0.05 Äq.) und $\mathrm{CuI}\left(10.5 \mathrm{mg}, 87.9 \mu \mathrm{mol}, 0.10 \mathrm{Äq}\right.$.) in $\mathrm{NEt}_{3}(8 \mathrm{~mL})$ wurde $16 \mathrm{~h}$ bei RT gerührt. Die Reaktionsmischung wurde durch $\mathrm{SiO}_{2}$ filtriert und das Lösungsmittel des Filtrats in vacuo entfernt. Säulenchromatographische Reinigung $\left(\mathrm{SiO}_{2}, n\right.$-Pentan/ $\left./ \mathrm{CH}_{2} \mathrm{Cl}_{2}, 5: 1\right)$ lieferte Dialkin 109b als gelbes Öl (211 mg, $599 \mu \mathrm{mol}, 68 \%)$.

DC: $R_{f}=0.21\left(n-\mathrm{Pentan} / \mathrm{CH}_{2} \mathrm{Cl}_{2}, 5: 1\right)$.

UV/Vis $\left(\mathrm{CH}_{3} \mathrm{CN}\right): \lambda_{\max }(\mathrm{nm})(\lg \varepsilon)=197$ (4.740), 252 (4.264), 282 (3.581), 288 (3.609), 294 (3.485).

IR (Film): $\tilde{v}\left(\mathrm{~cm}^{-1}\right)=3293,1480,1444,1255,1236,1201,1105,1044,881,873,839,822,811,783$, $749,726,669,631$.

${ }^{1} \mathbf{H}-\mathrm{NMR}\left(600 \mathrm{MHz}, \mathrm{CDCl}_{3}\right): \delta(\mathrm{ppm})=1.69\left(\mathrm{p}, J=7.0 \mathrm{~Hz}, 2 \mathrm{H}, 4{ }^{\prime} \cdot-\mathrm{H}_{2}\right), 1.92(\mathrm{t}, J=2.7 \mathrm{~Hz}, 1 \mathrm{H}$, 7' '-H), 2.21 (td, $J=7.1,2.7 \mathrm{~Hz}, 2 \mathrm{H}, 5$ ' '- $\left.\mathrm{H}_{2}\right), 2.29$ (s, $\left.3 \mathrm{H}, \mathrm{CH}_{3}\right), 2.46\left(\mathrm{t}, J=6.9 \mathrm{~Hz}, 2 \mathrm{H}, 3\right.$ '”- $\left.\mathrm{H}_{2}\right), 6.69$ $(\mathrm{d}, J=8.4 \mathrm{~Hz}, 1 \mathrm{H}, 3-\mathrm{H}), 6.82\left(\mathrm{dd}, J=8.2,1.1 \mathrm{~Hz}, 1 \mathrm{H}, 6^{\prime}-\mathrm{H}\right), 7.00(\mathrm{dd}, J=8.3,2.1 \mathrm{~Hz}, 1 \mathrm{H}, 5-\mathrm{H})$, $7.04(\mathrm{td}, J=7.5,1.1 \mathrm{~Hz}, 1 \mathrm{H}, 5$ '-H), 7.20 (ddd, $J=8.2,7.5,1.6 \mathrm{~Hz}, 1 \mathrm{H}, 4-\mathrm{H}), 7.40-7.44$ (m, $2 \mathrm{H}$, 3'-H, 6-H).

${ }^{13}$ C-NMR (125 MHz, CDCl 3 ): $\delta(\mathrm{ppm})=17.5$ (C-5’'), 18.7 (C-3’’), $20.4\left(\mathrm{CH}_{3}\right), 27.5$ (C-4’'), 68.6 (C-7'), 76.5 (C-6’'), 83.7 (C-2'’), 94.4 (C-1'), 113.6 (C-2'), 116.0 (C-1), 118.6 (C-6'), 119.0 (C-3), 123.5 (C-5), 128.9, 129.0 (C-4, C-5'), 133.7, 133.9 (C-6, C-3'), 134.3 (C-4'), 151.8 (C-1'), 157.1 $(\mathrm{C}-2)$.

MS (ESI): $m / z(\%)=353.1(64)[\mathrm{M}+\mathrm{H}]^{+}, 375.00(100)[\mathrm{M}+\mathrm{Na}]^{+}$.

HRMS (ESI): $m / z=$ gef.: 353.0525, ber.: $353.0536\left[\mathrm{M}\left({ }^{79} \mathrm{Br}\right)+\mathrm{H}\right]^{+}$; gef.: 355.0509 , ber.: 355.0516 $\left[\mathrm{M}\left({ }^{81} \mathrm{Br}\right)+\mathrm{H}\right]^{+}$.

$\mathrm{C}_{20} \mathrm{H}_{17} \mathrm{BrO}$ (352.05). 


\section{2-Brom-4-methyl-1-(2-(7-phenylhepta-1,6-diin-1-yl)phenoxy)benzol (179)}

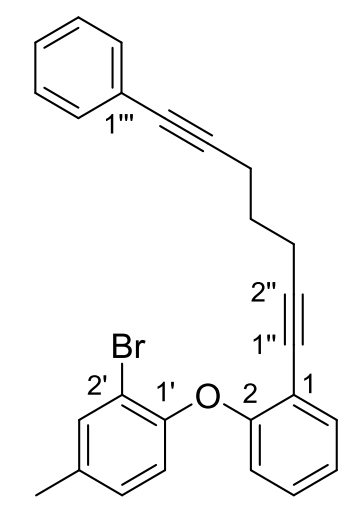

Eine Mischung aus Iodbenzol (85.9 mg, $421 \mu$ mol, 1.00 Äq.), 109b (156 mg, $442 \mu \mathrm{mol}, 1.05$ Äq.), $\mathrm{PdCl}_{2}\left(\mathrm{PPh}_{3}\right)_{2}(14.8 \mathrm{mg}, 21.1 \mu \mathrm{mol}, 0.05$ Äq.) und $\mathrm{CuI}$ (5.03 mg, $42.1 \mu \mathrm{mol}, 0.10$ Äq.) in entgastem $\mathrm{NEt}_{3}(8 \mathrm{~mL})$ und $\mathrm{CH}_{2} \mathrm{Cl}_{2}(4 \mathrm{~mL})$ wurde $16 \mathrm{~h}$ bei RT gerührt. Die Reaktionsmischung wurde durch $\mathrm{SiO}_{2}$ filtriert, mit EtOAc gespült und das Lösungsmittel des Filtrats in vacuo entfernt. Säulenchromatographische Reinigung $\left(\mathrm{SiO}_{2}, n\right.$-Pentan/EtOAc, 50:1) lieferte Dialkin 179 als gelbes Öl (133 mg, $310 \mu \mathrm{mol}, 74 \%$ ).

DC: $R_{f}=0.46(n-$ Pentan/EtOAc, 50:1).

IR (ATR): $\tilde{v}\left(\mathrm{~cm}^{-1}\right)=1481,1444,1255,1238,1205,1106,1045,889,838,811,784,753,691$.

${ }^{1} \mathbf{H}-\mathbf{N M R}\left(600 \mathrm{MHz}, \mathrm{CDCl}_{3}\right): \delta(\mathrm{ppm})=1.77\left(\mathrm{p}, J=7.1 \mathrm{~Hz}, 2 \mathrm{H}, 4\right.$ '”- $\left.\mathrm{H}_{2}\right), 2.28\left(\mathrm{~s}, 3 \mathrm{H}, \mathrm{CH}_{3}\right), 2.43(\mathrm{t}$, $J=7.1 \mathrm{~Hz}, 2 \mathrm{H}), 2.51(\mathrm{t}, J=6.9 \mathrm{~Hz}, 2 \mathrm{H})\left(3^{\prime}{ }^{\prime}-\mathrm{H}_{2}, 5^{\prime} '-\mathrm{H}_{2}\right), 6.70(\mathrm{~d}, J=8.2 \mathrm{~Hz}, 1 \mathrm{H}, 6$ ' $-\mathrm{H}), 6.83$ (dd, $J=8.2,1.0 \mathrm{~Hz}, 1 \mathrm{H}, 5-\mathrm{H}), 6.98-7.02\left(\mathrm{~m}, 1 \mathrm{H}, 4{ }^{\prime \prime}{ }^{-}-\mathrm{H}\right), 7.04(\mathrm{td}, J=7.6,1.2 \mathrm{~Hz}, 1 \mathrm{H}, 5$ '-H), 7.18-7.23 (m, 1 H, 4-H), 7.23-7.29 (m, 3 H), 7.34-7.39 (m, 2 H) (6-H, 2'"'-H, 3','-H, 5'"'-H, 6" '-H), 7.40-7.42 (m, 1 H, 3'-H), 7.43 (dd, $J=7.7,1.7 \mathrm{~Hz}, 1 \mathrm{H}, 3-\mathrm{H})$.

${ }^{13}$ C-NMR (126 MHz, $\left.\mathrm{CDCl}_{3}\right): \delta(\mathrm{ppm})=18.5,18.9\left(\mathrm{C}-3\right.$ ', C-5"'), $20.4\left(\mathrm{CH}_{3}\right), 27.8(\mathrm{C}-4$ '”), 76.5, 81.0 (C-2', C-6'), 89.4, 94.7 (C-1', C-7'), 113.6 (C-2'), 116.1 (C-1), 118.7, 119.0 (C-3, C-6'), 123.6, 123.9 (C-5, C-1','), 127.5 (C-4'”'), 128.1 (C-3',', C-5'”'), 128.9, 129.0 (C-4, C-5'), 131.5 (C-2'”, C-6’'), 133.7, 133.9 (C-6, C-3'), 134.3 (C-4'), 151.8 (C-1'), 157.1 (C-2).

MS (ESI): $m / z(\%)=349.2(100)[\mathrm{M}-\mathrm{Br}]^{+}, 429.1(13)[\mathrm{M}]^{+}$.

HRMS (EI): $m / z=$ gef.: 427.0698 , ber.: $427.0698\left[\mathrm{M}\left({ }^{79} \mathrm{Br}\right)\right]^{+}$.

$\mathbf{C}_{26} \mathbf{H}_{21} \mathbf{B r O}$ (429.36). 


\section{(7-(2-(2-Bromphenoxy)phenyl)hepta-1,6-diin-1-yl)trimethylsilan (183)}

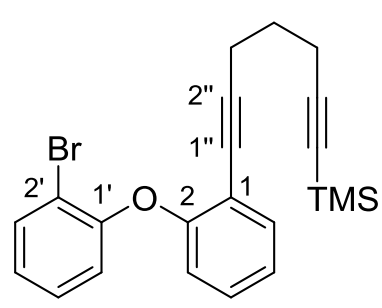

Eine Lösung von 1-Brom-2-(2-(hepta-1,6-diin-1-yl)phenoxy)benzol (109a) (929 mg, 2.74 mmol, 1.00 Äq.) und LiHMDS (1 M, $4.11 \mathrm{~mL}, 4.11 \mathrm{mmol}, 1.50$ Äq.) in THF $(15 \mathrm{~mL})$ wurde $1.5 \mathrm{~h}$ bei $-78{ }^{\circ} \mathrm{C}$ gerührt. TMSCl $(520 \mu \mathrm{L}, \quad 447 \mathrm{mg}, \quad 4.11 \mathrm{mmol}, \quad 1.50$ Äq. $) \quad$ wurde hinzugefügt und das Reaktionsgemisch über $20 \mathrm{~h}$ auf RT erwärmt. Nach Zugabe von ges. wässr. $\mathrm{NH}_{4} \mathrm{Cl}$-Lösung $(50 \mathrm{~mL})$ wurde die Mischung mit $\mathrm{CH}_{2} \mathrm{Cl}_{2}$ extrahiert $(3 \times 50 \mathrm{~mL})$. Die vereinigten organischen Phasen wurden über $\mathrm{Na}_{2} \mathrm{SO}_{4}$ getrocknet, filtriert und das Lösungsmittel in vacuo entfernt. Säulenchromatographische Reinigung $\left(\mathrm{SiO}_{2}, n\right.$-Pentan/ $\left.\mathrm{CH}_{2} \mathrm{Cl}_{2}, 10: 1 \rightarrow 5: 1\right)$ lieferte Dialkin 183 als gelbes Öl (1.05 g, 2.55 mmol, 93\%).

DC: $R_{f}=0.40\left(n-\mathrm{Pentan} / \mathrm{CH}_{2} \mathrm{Cl}_{2}, 5: 1\right)$.

UV/Vis $\left(\mathrm{CH}_{3} \mathrm{CN}\right): \lambda_{\max }(\mathrm{nm})(\lg \varepsilon)=207$ (4.608), 239 (4.230), 252 (4.215), 282 (3.465), 296 (3.307).

IR (ATR): $\tilde{v}\left(\mathrm{~cm}^{-1}\right)=2955,2936,2172,1584,1568,1486,1471,1441,1249,1229,1196,1105,1044$, $1029,837,799,748,697,659,638$.

${ }^{1} \mathbf{H}-\mathrm{NMR}\left(600 \mathrm{MHz}, \mathrm{CDCl}_{3}\right): \delta(\mathrm{ppm})=0.13\left(\mathrm{~s}, 9 \mathrm{H}, \mathrm{Si}\left(\mathrm{CH}_{3}\right)_{3}\right), 1.63\left(\mathrm{p}, J=7.1 \mathrm{~Hz}, 2 \mathrm{H}, 4\right.$ '’- $\left.\mathrm{H}_{2}\right), 2.21$ (t, $J=7.1 \mathrm{~Hz}, 1 \mathrm{H}, 5^{\prime}$ ' $-\mathrm{H}_{2}$ ), 2.43 (t, $J=7.0 \mathrm{~Hz}, 2 \mathrm{H}, 3$ ' '- $\mathrm{H}_{2}$ ), 6.75 (dd, $J=8.2,1.4 \mathrm{~Hz}, 1 \mathrm{H}, 6$ '-H), 6.90 (dd, $\left.J=8.2,1.1 \mathrm{~Hz}, 1 \mathrm{H}, 3^{\prime}-\mathrm{H}\right), 6.92-6.96(\mathrm{~m}, 1 \mathrm{H}, 5-\mathrm{H}), 7.08$ (td, $\left.J=7.6,1.1 \mathrm{~Hz}, 1 \mathrm{H}, 4-\mathrm{H}\right), 7.16-$ 7.20 (m, 1 H), 7.21-7.25 (m, 1 H) (4'-H, 5'-H), 7.44 (dd, $J=7.7,1.7$ Hz, 1 H, 3-H), 7.59 (dd, J = 7.9, $1.5 \mathrm{~Hz}, 1 \mathrm{H}, 6-\mathrm{H})$.

${ }^{13}$ C-NMR $\left(126 \mathrm{MHz}, \mathrm{CDCl}_{3}\right): \delta(\mathrm{ppm})=0.2\left(\mathrm{Si}\left(\mathrm{CH}_{3}\right)_{3}\right), 18.7,19.0(\mathrm{C}-3$ ', C-5" $), 27.6(\mathrm{C}-4$ '”), 76.2 (C-6’), 84.9 (C-2'), 95.0 (C-1'’), 106.5 (C-7'’), 113.6 (C-2'), 116.5 (C-1), 118.5 (C-6'), 119.5 (C-3'), 124.0, 124.1 (C-4, C-5), 128.4, 129.0 (C-4', C-5'), 133.6, 133.7 (C-3, C-6), 154.3 (C-1'), 156.6 (C-2).

MS (ESI): $m / z(\%)=430.1(33)\left[\mathrm{M}+\mathrm{NH}_{4}\right]^{+}$.

HRMS (ESI): $m / z=$ gef.: 411.0762, ber.: $411.0774\left[\mathrm{M}\left({ }^{79} \mathrm{Br}\right)+\mathrm{H}\right]^{+}$; gef.: 413.0751 , ber.: 413.0755 $\left[\mathrm{M}\left({ }^{81} \mathrm{Br}\right)+\mathrm{H}\right]^{+}$.

$\mathbf{C}_{22} \mathbf{H}_{23} \mathbf{B r O S i}$ (411.41). 
Tributyl(2-(2-(hepta-1,6-diin-1-yl)phenoxy)phenyl)stannan (185)

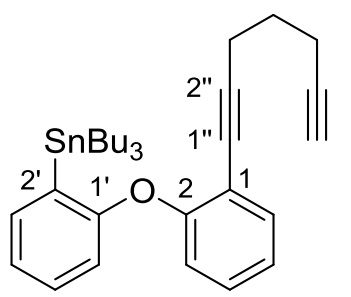

tert-Butyllithium (1.7 M in $n$-Pentan, $665 \mu \mathrm{L}, 1.13 \mathrm{mmol}, 2.50$ Äq.) wurde bei $-78{ }^{\circ} \mathrm{C}$ zu einer Lösung von (7-(2-(2-Bromphenoxy)phenyl)hepta-1,6-diin-1-yl)trimethylsilan (183) (185 mg, $450 \mu \mathrm{mol}$, 1.00 Äq.) in THF ( $8 \mathrm{~mL})$ getropft und $45 \mathrm{~min}$ bei dieser Temperatur gerührt. $\mathrm{Bu}_{3} \mathrm{SnCl}(307 \mu \mathrm{L}$, $368 \mathrm{mg}, 1.13 \mathrm{mmol}, 2.50$ Äq.) wurde hinzugefügt und die Reaktionsmischung über $16 \mathrm{~h}$ auf RT erwärmt. Nach Zugabe von ges. wässr. $\mathrm{NH}_{4} \mathrm{Cl}$-Lösung $(10 \mathrm{~mL})$ wurde die Mischung mit $\mathrm{CH}_{2} \mathrm{Cl}_{2}$ extrahiert $(3 \times 10 \mathrm{~mL})$. Die vereinigten organischen Phasen wurden über $\mathrm{Na}_{2} \mathrm{SO}_{4}$ getrocknet, filtriert und das Lösungsmittel in vacuo entfernt. Säulenchromatographische Reinigung $\left(\mathrm{SiO}_{2}\right.$, $n$-Pentan/ $\left.\mathrm{CH}_{2} \mathrm{Cl}_{2}, 10: 1\right)$ lieferte das TMS-geschützte Stannan als farbloses Öl (134 mg, $216 \mu \mathrm{mol}$, $48 \%)$.

Eine Lösung des TMS-geschützten Stannans (508 mg, $817 \mu$ mol, 1.00 Äq.) und $\mathrm{K}_{2} \mathrm{CO}_{3}$ (67.7 mg, $490 \mu \mathrm{mol}$, $0.60 \mathrm{Äq}$.) in $\mathrm{CH}_{3} \mathrm{OH}(10 \mathrm{~mL})$ und $\mathrm{CH}_{2} \mathrm{Cl}_{2}(10 \mathrm{~mL})$ wurde $24 \mathrm{~h}$ bei $\mathrm{RT}$ gerührt. Säulenchromatographische Reinigung $\left(\mathrm{SiO}_{2}, n\right.$-Pentan/ $\left.\mathrm{CH}_{2} \mathrm{Cl}_{2}, 10: 1\right)$ lieferte Dialkin 185 als farbloses Öl (316 mg, $575 \mu \mathrm{mol}, 70 \%$; 34\% über 2 Stufen).

DC: $R_{f}=0.33\left(n-\mathrm{Pentan} / \mathrm{CH}_{2} \mathrm{Cl}_{2}, 10: 1\right)$.

UV/Vis $\left(\mathrm{CH}_{3} \mathrm{CN}\right): \lambda_{\max }(\mathrm{nm})(\lg \varepsilon)=201$ (4.705), 230 (4.393), 252 (4.237), 289 (3.539), 299 (3.471).

IR (ATR): $\tilde{v}\left(\mathrm{~cm}^{-1}\right)=3310,2953,2922,2870,2850,1564,1486,1460,1446,1429,1249,1217,1188$, 1156, 1071, 1050, 865, 842, 799, 750, 689, 633, 597, 507.

${ }^{1} \mathbf{H}-\mathbf{N M R}\left(600 \mathrm{MHz}, \mathrm{CDCl}_{3}\right): \delta(\mathrm{ppm})=0.82\left(\mathrm{t}, J=7.3 \mathrm{~Hz}, 9 \mathrm{H}, \mathrm{Sn}\left(\left(\mathrm{CH}_{2}\right)_{3} \mathrm{CH}_{3}\right)_{3}\right), 1.03-1.07(\mathrm{~m}, 6 \mathrm{H}$, $\left.\mathrm{Sn}\left(\mathrm{C}_{2}\left(\mathrm{CH}_{2}\right)_{2} \mathrm{CH}_{3}\right)_{3}\right), 1.22-1.30\left(\mathrm{~m}, 6 \mathrm{H}, \mathrm{Sn}\left(\left(\mathrm{CH}_{2}\right)_{2} \mathrm{CH}_{2} \mathrm{CH}_{3}\right)_{3}\right), 1.46-1.52$ (m, $6 \mathrm{H}, \mathrm{Sn}\left(\mathrm{CH}_{2} \mathrm{CH}_{2}\right.$ $\left.\left.\mathrm{CH}_{2} \mathrm{CH}_{3}\right)_{3}\right), 1.67$ (p, $J=7.1 \mathrm{~Hz}, 2 \mathrm{H}, 4$ ' '- $\left.\mathrm{H}_{2}\right), 1.91(\mathrm{t}, J=2.6 \mathrm{~Hz}, 1 \mathrm{H}, 7$ ' '-H), 2.19 (td, $J=7.1,2.6 \mathrm{~Hz}$, $2 \mathrm{H}, 5$ ' '- $\left.\mathrm{H}_{2}\right), 2.43$ (dt, $J=6.0,4.8 \mathrm{~Hz}, 2 \mathrm{H}, 3$ ''- $\left.-\mathrm{H}_{2}\right), 6.70$ (d, $J=7.5 \mathrm{~Hz}, 1 \mathrm{H}, 6$ ' $\left.-\mathrm{H}\right), 6.78$ (dd, $J=8.3$, $\left.0.9 \mathrm{~Hz}, 1 \mathrm{H}, 3^{\prime}-\mathrm{H}\right), 7.00$ (td, $\left.J=7.6,1.1 \mathrm{~Hz}, 1 \mathrm{H}, 5-\mathrm{H}\right), 7.06$ (td, $\left.J=7.2,0.9 \mathrm{~Hz}, 1 \mathrm{H}, 4-\mathrm{H}\right), 7.17$ (ddd, $\left.J=8.2,7.5,1.7 \mathrm{~Hz}, 1 \mathrm{H}, 5^{\prime}-\mathrm{H}\right), 7.22$ (ddd, $\left.J=8.2,7.3,1.7 \mathrm{~Hz}, 1 \mathrm{H}, 4^{\prime}-\mathrm{H}\right), 7.42$ (dd, $J=7.7,1.6 \mathrm{~Hz}$, $1 \mathrm{H}, 3-\mathrm{H}), 7.46(\mathrm{dd}, J=7.1,1.6 \mathrm{~Hz}, 1 \mathrm{H}, 6-\mathrm{H})$.

${ }^{13} \mathbf{C}-\mathbf{N M R}\left(126 \mathrm{MHz}, \mathrm{CDCl}_{3}\right): \delta(\mathrm{ppm})=9.8\left(\mathrm{Sn}\left(\underline{\mathrm{CH}}_{2}\left(\mathrm{CH}_{2}\right)_{2} \mathrm{CH}_{3}\right)_{3}\right), 13.7\left(\mathrm{Sn}\left(\left(\mathrm{CH}_{2}\right)_{3} \underline{\mathrm{CH}}_{3}\right)_{3}\right), 17.5$ (C-5'), 18.8 (C-3'”), 27.4, 27.6 (C-4', $\left.\mathrm{Sn}\left(\left(\mathrm{CH}_{2}\right)_{2} \mathrm{CH}_{2} \mathrm{CH}_{3}\right)_{3}\right), 29.1 \quad\left(\mathrm{Sn}\left(\mathrm{CH}_{2} \mathrm{C}_{2} \mathrm{CH}_{2} \mathrm{CH}_{3}\right)_{3}\right), 68.7$ 
(C-7'), 70.8 (C-6'), 93.7 (C-2'”), 115.9, 116.3 (C-1, C-1')), 118.3 (C-6'), 122.8, 123.1 (C-4, C-5), 128.8, 129.6 (C-3, C-4', C-5'), 132.4 (C-2'), 133.8 (C-6), 137.4 (C-3'), 157.7 (C-1'), 161.7 (C-2).

HRMS (ESI): $m / z=$ gef.: 551.2325 , ber.: $551.2336\left[\mathrm{M}\left({ }^{120} \mathrm{Sn}\right)+\mathrm{H}\right]^{+}$.

$\mathbf{C}_{31} \mathbf{H}_{42} \mathbf{O S n}$ (549.39). 


\subsubsection{Untersuchungen zur Synthese von Trialkenen des Typs 120}

\section{5-(2-(2-Bromphenoxy)phenyl)pent-4-in-1-ol (191) ${ }^{\mathrm{mm}}$}

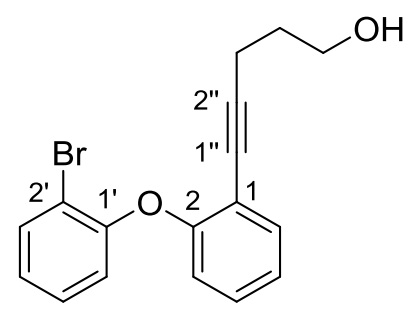

Eine Mischung aus 1-Iod-2-(2-bromphenoxy)benzol (124a) (2.02 g, 5.39 mmol, 1.00 Äq.), 4-Pentin-1-ol (190) (600 $\mu \mathrm{L}, 544$ mg, 6.47 mmol, 1.20 Äq.), PdCl $\left(\mathrm{PPh}_{3}\right)_{2}$ (190 mg, $270 \mu \mathrm{mol}$, 0.05 Äq.) und CuI (64.5 mg, $540 \mu \mathrm{mol}, 0.10$ Äq.) in entgastem $\mathrm{NEt}_{3}(50 \mathrm{~mL})$ wurde $22.5 \mathrm{~h}$ bei RT gerührt. Die Reaktionsmischung wurde durch $\mathrm{SiO}_{2}$ filtriert, mit EtOAc gespült und das Lösungsmittel in vacuo entfernt. Säulenchromatographische Reinigung $\left(\mathrm{SiO}_{2}, \mathrm{CH}_{2} \mathrm{Cl}_{2}\right)$ lieferte Alkin 191 als gelbes Öl (1.58 g, $4.77 \mathrm{mmol}, 88 \%)$.

DC: $R_{f}=0.22\left(\mathrm{CH}_{2} \mathrm{Cl}_{2}\right)$.

UV/Vis $\left(\mathrm{CH}_{3} \mathrm{CN}\right): \lambda_{\max }(\mathrm{nm})(\lg \varepsilon)=206$ (4.591), 239 (4.202), 252 (4.188), 282 (3.435), 296 (3.289).

IR (ATR): $\tilde{v}\left(\mathrm{~cm}^{-1}\right)=3314,2945,2932,2875,1583,1568,1485,1470,1440,1253,1228,1195,1157$, 1120, 1105, 1044, 1029, 873, 799, 748, 658.

${ }^{1} \mathbf{H}-\mathrm{NMR}\left(600 \mathrm{MHz}, \mathrm{CDCl}_{3}\right): \delta(\mathrm{ppm})=1.66(\mathrm{~s}, 1 \mathrm{H}, \mathrm{OH}), 1.68\left(\mathrm{p}, J=6.5 \mathrm{~Hz}, 2 \mathrm{H}, 4{ }^{\prime}{ }^{-}-\mathrm{H}_{2}\right), 2.42(\mathrm{t}$, $J=6.9 \mathrm{~Hz}, 2 \mathrm{H}, 3$ ' '- $\left.\mathrm{H}_{2}\right), 3.62\left(\mathrm{t}, J=6.2 \mathrm{~Hz}, 2 \mathrm{H}, 5^{\prime}{ }^{-}-\mathrm{H}_{2}\right), 6.76(\mathrm{dd}, J=8.2,1.5 \mathrm{~Hz}, 1 \mathrm{H}, 6$ '- $\mathrm{H}), 6.87$ (dd, $J=8.2,1.1 \mathrm{~Hz}, 1 \mathrm{H}, 5-\mathrm{H}), 6.94\left(\mathrm{td}, J=7.7,1.5 \mathrm{~Hz}, 1 \mathrm{H}, 3^{\prime}-\mathrm{H}\right), 7.07$ (td, $J=7.6,1.2 \mathrm{~Hz}, 1 \mathrm{H}$, 4-H), 7.19 (ddd, $J=8.5,7.5,1.6 \mathrm{~Hz}, 1 \mathrm{H}), 7.21-7.25$ (m, 1 H) (4'-H, 5'-H), 7.43 (dd, $J=7.6,1.7 \mathrm{~Hz}$, $1 \mathrm{H}, 3-\mathrm{H}), 7.59$ (dd, $J=8.0,1.5 \mathrm{~Hz}, 1 \mathrm{H}, 6-\mathrm{H})$.

${ }^{13}$ C-NMR $\left(126 \mathrm{MHz}, \mathrm{CDCl}_{3}\right): \delta(\mathrm{ppm})=16.1$ (C-3"'), 30.9 (C-4’'), 61.5 (C-5'”), 76.2 (C-2"'), 95.1 (C-1'), 113.6 (C-2'), 116.3 (C-1), 118.7 (C-6'), 119.2 (C-3'), 124.0, 124.2 (C-4, C-5), 128.4, 129.0 (C-4', C-5'), 133.6 (C-3, C-6), 154.1 (C-1'), 156.5 (C-2).

MS (ESI): $m / z(\%)=331.1(100)[\mathrm{M}+\mathrm{H}]^{+}$.

HRMS (ESI): $m / z=$ gef.: 331.0331, ber.: $331.0328\left[\mathrm{M}\left({ }^{79} \mathrm{Br}\right)+\mathrm{H}\right]^{+}$; gef.: 333.0312, ber.: 333.0308 $\left[\mathrm{M}\left({ }^{81} \mathrm{Br}\right)+\mathrm{H}\right]^{+}$.

$\mathrm{C}_{17} \mathrm{H}_{15} \mathrm{BrO}_{2}(331.21)$

${ }^{\mathrm{mm}}$ Für eine Synthesevorschrift von 124a siehe u.a.: C. Eichhorst, Diplomarbeit, Georg-August-Universität, Göttingen, 2010. 


\section{5-(2-(2-Bromphenoxy)phenyl)pent-4-inal (192)}

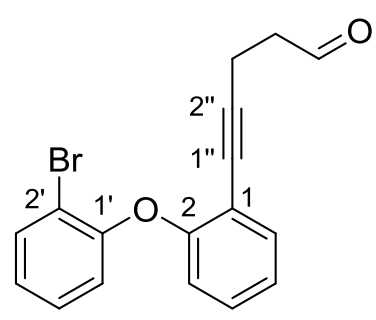

Eine Mischung aus 5-(2-(2-Bromphenoxy)phenyl)pent-4-in-1-ol (191) (240 mg, 725 umol, 1.00 Äq.) und Dess-Martin-Periodinan (DMP) (369 mg, $870 \mu \mathrm{mol}, 1.20 \mathrm{Äq}$.) in $\mathrm{CH}_{2} \mathrm{Cl}_{2}(10 \mathrm{~mL}$ ) wurde $16 \mathrm{~h}$ bei RT gerührt. Das Lösungsmittel wurde in vacuo entfernt und Säulenfiltration $\left(\mathrm{SiO}_{2}, \mathrm{CH}_{2} \mathrm{Cl}_{2}\right)$ lieferte Aldehyd 192 als gelbes Öl (229 mg, $696 \mu \mathrm{mol}, 96 \%)$.

DC: $R_{f}=0.84\left(\mathrm{CH}_{2} \mathrm{Cl}_{2}\right)$.

UV/Vis $\left(\mathrm{CH}_{3} \mathrm{CN}\right): \lambda_{\max }(\mathrm{nm})(\lg \varepsilon)=207$ (4.598), 241 (4.192), 252 (4.165), 282 (3.485), 295 (3.346).

IR (ATR): $\tilde{v}\left(\mathrm{~cm}^{-1}\right)=1722,1582,1568,1486,1470,1440,1408,1252,1228,1196,1157,1120,1105$, 1045, 1029, 942, 871, 799, 749, 658.

${ }^{1} \mathbf{H}-\mathbf{N M R}\left(600 \mathrm{MHz}, \mathrm{CDCl}_{3}\right): \delta(\mathrm{ppm})=2.54-2.58\left(\mathrm{~m}, 2 \mathrm{H}, 3\right.$ ' '- $\left.\mathrm{H}_{2}\right), 2.59-2.64\left(\mathrm{~m}, 2 \mathrm{H}, 4\right.$ '”- $\left.\mathrm{H}_{2}\right), 6.74$ $\left(\mathrm{dd}, J=8.2,1.4 \mathrm{~Hz}, 1 \mathrm{H}, 6^{\prime}-\mathrm{H}\right), 6.90(\mathrm{dd}, J=8.1,1.3 \mathrm{~Hz}, 1 \mathrm{H}, 5-\mathrm{H}), 6.95(\mathrm{td}, J=7.7,1.5 \mathrm{~Hz}, 1 \mathrm{H}$, 3'-H), 7.08 (td, $J=7.6,1.2$ Hz, 1 H, 4-H), 7.16-7.22 (m, 1 H, 4-H), 7.23-7.27 (m, 1 H, 5'-H), 7.42 (dd, $\left.J=7.6,1.7 \mathrm{~Hz}, 1 \mathrm{H}, 4^{\prime}-\mathrm{H}\right), 7.60$ (dd, $\left.J=8.0,1.6 \mathrm{~Hz}, 1 \mathrm{H}, 6-\mathrm{H}\right), 9.68$ (t, $\left.J=1.3 \mathrm{~Hz}, 1 \mathrm{H}, \mathrm{CHO}\right)$.

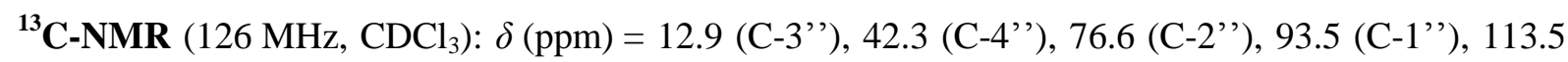
(C-2'), 116.0 (C-1), 118.6 (C-6'), 119.5 (C-3'), 124.1, 124.2 (C-4, C-5), 128.5, 129.3 (C-4', C-5'), 133.6, 133.7 (C-3, C-6), 154.2 (C-1'), 156.6 (C-2), 200.5 (CHO).

MS (ESI): $m / z(\%)=331.0(13)[\mathrm{M}+\mathrm{H}]^{+}$.

HRMS (ESI): $m / z=$ gef.: 329.0165, ber.: $329.0172\left[\mathrm{M}\left({ }^{79} \mathrm{Br}\right)+\mathrm{H}\right]^{+}$; gef.: 331.0148 , ber.: 331.0152 $\left[\mathrm{M}\left({ }^{81} \mathrm{Br}\right)+\mathrm{H}\right]^{+}$.

$\mathrm{C}_{17} \mathrm{H}_{13} \mathrm{BrO}_{2}$ (329.19). 


\section{1-(2-(2-Bromphenoxy)phenyl)dodeca-1,6,11-triin-5-ol (189)}

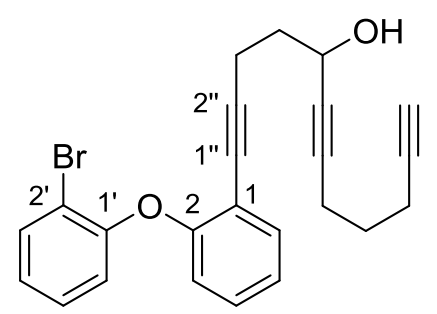

Eine Lösung von $n$-Butyllithium (2.5 M in $n$-Hexan, $293 \mu \mathrm{L}, 733 \mu$ mol, 1.20 Äq.) und 1,6-Heptadiin (150b) $\left(83.3 \mu \mathrm{L}, 67.5 \mathrm{mg}, 733 \mu \mathrm{mol}, 1.20\right.$ Äq.) in THF $(15 \mathrm{~mL})$ wurde $1 \mathrm{~h}$ bei $-78^{\circ} \mathrm{C}$ gerührt. Eine Lösung von 5-(2-(2-Bromphenoxy)phenyl)pent-4-inal (192) (201 mg, $611 \mu \mathrm{mol}, 1.00$ Äq.) in THF $(10 \mathrm{~mL})$ wurde via Spritzenpumpe über $1 \mathrm{H}$ hinzugetropft und die Reaktionsmischung über $18 \mathrm{~h}$ auf RT erwärmt. Die Mischung wurde mit ges. wässr. $\mathrm{NH}_{4} \mathrm{Cl}$-Lösung $(50 \mathrm{~mL})$ versetzt und mit $\mathrm{CH}_{2} \mathrm{Cl}_{2}$ extrahiert $(3 \times 50 \mathrm{~mL})$. Die vereinigten organischen Phasen wurden über $\mathrm{Na}_{2} \mathrm{SO}_{4}$ getrocknet, filtriert und das Lösungsmittel in vacuo entfernt. Säulenchromatographische Reinigung $\left(\mathrm{SiO}_{2}\right.$, n-Pentan/EtOAc, 5:1) lieferte Propargylalkohol 189 als gelbes Öl (133 mg, 316 umol, 52\%).

DC: $R_{f}=0.38(n-$ Pentan/EtOAc, $5: 1)$.

UV/Vis $\left(\mathrm{CH}_{3} \mathrm{CN}\right): \lambda_{\max }(\mathrm{nm})(\lg \varepsilon)=207$ (4.612), 240 (4.227), 252 (4.213), 282 (3.474), 295 (3.321).

IR (ATR): $\tilde{v}\left(\mathrm{~cm}^{-1}\right)=3292,1568,1486,1471,1440,1255,1229,1196,1105,1062,1045,1029,936$, $870,798,750,657,635$.

${ }^{1} \mathbf{H}-\mathbf{N M R}\left(300 \mathrm{MHz}, \mathrm{CDCl}_{3}\right): \delta(\mathrm{ppm})=1.72(\mathrm{t}, J=7.0 \mathrm{~Hz}, 2 \mathrm{H}), 1.75-1.87(\mathrm{~m}, 2 \mathrm{H}), 1.96(\mathrm{t}, J=$ $2.6 \mathrm{~Hz}, 1 \mathrm{H}, 12$ ''-H), 2.23-2.39 (m, $4 \mathrm{H}), 2.39-2.64$ (m, $2 \mathrm{H}), 4.40$ (tt, $J=6.6,2.0 \mathrm{~Hz}, 1 \mathrm{H}, 5$ '’-H), $6.78\left(\mathrm{dd}, J=8.2,1.5 \mathrm{~Hz}, 1 \mathrm{H}, 6^{\prime}-\mathrm{H}\right), 6.90$ (dd, $\left.J=8.2,1.2 \mathrm{~Hz}, 1 \mathrm{H}, 3^{\prime}-\mathrm{H}\right), 6.97$ (ddd, $J=7.9,7.3$, $1.5 \mathrm{~Hz}, 1 \mathrm{H}, 5-\mathrm{H}), 7.09$ (td, $J=7.5,1.2 \mathrm{~Hz}, 1 \mathrm{H}, 4-\mathrm{H}), 7.17-7.35$ (m, 2 H, 4'-H, 5'-H), 7.45 (dd, $J=$ 7.6, $1.8 \mathrm{~Hz}, 1 \mathrm{H}, 3-\mathrm{H}), 7.63$ (dd, $J=7.9,1.6 \mathrm{~Hz}, 1 \mathrm{H}, 6-\mathrm{H})$.

${ }^{13}$ C-NMR (126 MHz, CDCl $): \delta(\mathrm{ppm})=$ 15.7, 17.5, 17.7 (C-8', C-9', C-10'”), 27.4, 36.5 (C-3'”,

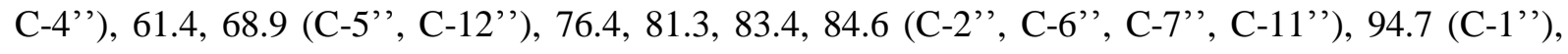
113.6 (C-2'), 116.3 (C-1), 118.7 (C-6'), 119.3 (C-3'), 124.0, 124.2 (C-4, C-5), 128.5, 129.1 (C-4', C-5'), 133.6, 133.7 (C-3, C-6), 154.2 (C-1'), 156.6 (C-2).

MS (ESI): $m / z(\%)=421.3(100)[\mathrm{M}+\mathrm{H}]^{+}$.

HRMS (ESI): $m / z=$ gef.: 421.0782, ber.: $421.0798\left[\mathrm{M}\left({ }^{79} \mathrm{Br}\right)+\mathrm{H}\right]^{+}$; gef.: 423.0770 , ber.: 423.0778 $\left[\mathrm{M}\left({ }^{81} \mathrm{Br}\right)+\mathrm{H}\right]^{+}$.

$\mathbf{C}_{24} \mathbf{H}_{21} \mathrm{BrO}_{2}$ (421.33). 


\section{Anhang}

\subsection{Kristallstrukturdaten}

Tabelle 21: Kristallstrukturparameter von Molekül 110a.

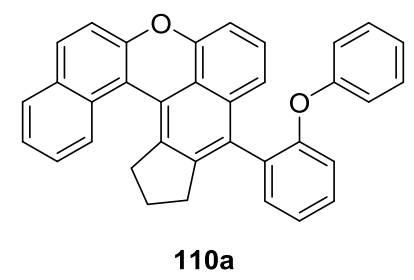

\begin{tabular}{|c|c|c|}
\hline Eintrag & Parameter & Werte für 110a \\
\hline 1 & Empirische Formel & $\mathrm{C}_{35} \mathrm{H}_{24} \mathrm{O}_{2}$ \\
\hline 2 & Molekulare Masse [g/mol] & 476.54 \\
\hline 3 & Temperatur $[\mathrm{K}]$ & $133(2)$ \\
\hline 4 & Wellenlänge $[\AA ̊ 丿$ & $0.71073 \AA$ \\
\hline 5 & Kristallsystem & triklin \\
\hline 6 & Raumgruppe & $\mathrm{P}-1$ \\
\hline 7 & Dimensionen der Elementarzelle & $\begin{array}{c}a=9.3688(4) \AA ; b=15.4443(8) \AA ; c=18.4387(10) \AA \\
\alpha=114.625(4)^{\circ} ; \beta=90.516(4)^{\circ} ; \gamma=99.404(4)^{\circ} .\end{array}$ \\
\hline 8 & Volumen $\left[\AA^{3}\right]$ & $2383.9(2)$ \\
\hline 9 & $\mathrm{Z}$ & 4 \\
\hline 10 & Dichte (ber.) $\left[\mathrm{Mg} / \mathrm{m}^{3}\right]$ & 1.328 \\
\hline 11 & Absorptionskoeffizient $\left[\mathrm{mm}^{-1}\right]$ & 0.081 \\
\hline 12 & $\mathrm{~F}(000)$ & 1000 \\
\hline 13 & Kristallgröße $\left[\mathrm{mm}^{3}\right]$ & $0.500 \times 0.450 \times 0.240$ \\
\hline 14 & $\theta$-Bereich für Datenaufnahme $\left[{ }^{\circ}\right]$ & $1.219-26.099$ \\
\hline 15 & Index-Grenzen & $-11 \leq \mathrm{h} \leq 11 ;-19 \leq \mathrm{k} \leq 18 ;-22 \leq 1 \leq 22$ \\
\hline 16 & Gemessene Reflexe & 44852 \\
\hline 17 & Unabhängige Reflexe [R(int)] & 44852 \\
\hline 18 & Vollständigkeit für $\theta=25.242^{\circ}[\%]$ & 99.9 \\
\hline 19 & Absorptionskorrektur & numerisch \\
\hline 20 & Max. und min. Transmission & 0.9849 und 0.9654 \\
\hline 21 & Verfeinerungsmethode & Volle Matrix und kleinste Quadrate an $\mathrm{F}^{2}$ \\
\hline 22 & Daten/Beschränkungen/Parameter & 44852 / 0 / 668 \\
\hline 23 & Anpassungsgüte an $\mathrm{F}^{2}$ & 0.965 \\
\hline 24 & Finale R-Indizes $[\mathrm{I}>2 \sigma(\mathrm{I})]$ & $\mathrm{R} 1=0.0550, \mathrm{wR} 2=0.1326$ \\
\hline 25 & R-Indizes (Gesamte Daten) & $\mathrm{R} 1=0.0791, \mathrm{wR} 2=0.1429$ \\
\hline 26 & Größte Differenz (Min./Max.) $\left[\mathrm{e} \times \AA^{-3}\right]$ & $0.229 /-0.149$ \\
\hline
\end{tabular}




\subsection{Abkürzungsverzeichnis}

\begin{tabular}{|c|c|c|c|}
\hline$\delta$ & chemische Verschiebung & $\mathrm{DMF}$ & $N, N$-Dimethylformamid \\
\hline$\lambda$ & Wellenlänge & DMP & Dess-Martin-Periodinan \\
\hline$\tilde{v}$ & Wellenzahl & DMSO & Dimethylsulfoxid \\
\hline Ac & Acetyl & DPEN & Diphenylethylendiamin \\
\hline AIBN & Azo-bis-(isobutyronitril) & DPE-Phos & Bis-(2-(diphenylphosphin)- \\
\hline Äq. & Äquivalente & & phenyl)ether \\
\hline $\mathrm{AU}$ & Absorptionseinheiten & dppe & 1,2-Bis(diphenylphosphin)ethan \\
\hline ber. & berechnet & dppp & 1,3-Bis(diphenylphosphin)propan \\
\hline BODIPY & Bordipyrromethen & $d . r$. & Diastereomerenverhältnis \\
\hline BOXAX & Bis(oxazolin)-Ligand & $e e$ & Enantiomerenüberschuss \\
\hline \multirow[t]{2}{*}{ BRET } & Biolumineszenz- Resonanz- & EI & Elektronenstoß-Ionisation \\
\hline & Energie -Transfer & ESI & Elektrospray-Ionisation \\
\hline brsm. & bez. auf reisoliertes Edukt & Et & Ethyl \\
\hline $\mathrm{Bu}$ & Butyl & et al. & und Mitarbeiter \\
\hline $\mathrm{BuLi}$ & Butyllithium & EtOAc & Essigsäureethylester \\
\hline$c$ & Konzentration & FRAP & fluorescence recovery \\
\hline CBS & Corey-Bakshi-Shibata & & after photobleaching \\
\hline COSY & $(\mathrm{H}, \mathrm{H})-$ Korrelationsspektroskopie & FRET & Förster-Resonanzenergietransfer \\
\hline $\mathrm{Cy}$ & Cyclohexyl & gef. & gefunden \\
\hline $\mathrm{d}$ & $\operatorname{Tag}(e)$ & ges. & gesättigt \\
\hline $\mathrm{dba}$ & $(E, E)$-Dibenzylidenaceton & $\mathrm{h}$ & Stunde(n) \\
\hline DC & Dünnschichtchromatographie & Het & Heterozyklus \\
\hline DCE & Dichlorethan & HMBC & (C,H)-Korrelationsspektroskopie \\
\hline DMA & $N, N$-Dimethylacetamid & & (über mehrere Bindungen) \\
\hline DME & 1,2-Dimethoxyethan & HRMS & hochaufgelöste Massenspektromet \\
\hline
\end{tabular}




\begin{tabular}{|c|c|c|c|}
\hline HOMO & höchstes besetztes Molekülorbital & ppm & parts per million \\
\hline HSQC & $(\mathrm{C}, \mathrm{H})$-Korrelationsspektroskopie & Py & Pyridyl \\
\hline $\mathrm{Hz}$ & Hertz & quant. & quantitativ \\
\hline$i, i s o$ & isomer, verzweigt & $\mathrm{RP}$ & Reverse Phase \\
\hline \multirow[t]{2}{*}{$\mathrm{IPr} \cdot \mathrm{HCl}$} & 1,3-Bis(2,6-diisopropyl)- & RT & Raumtemperatur \\
\hline & imidazoliumchlorid & SPhos & 2-(Dicyclohexylphosphin)- \\
\hline$J$ & Kopplungskonstante & & 2',6'-dimethoxybiphenyl \\
\hline$k$ & Geschwindigkeitskonstante & msec & Millisekunde(n) \\
\hline konz. & konzentriert & $t$ & Zeit \\
\hline $\mathrm{L}$ & Ligand & $t$, tert & tertiär \\
\hline LiHMDS & Lithiumhexamethyldisilazid & $T$ & Temperatur \\
\hline Lsg. & Lösung & $t$ BuXPhos & 2-Di-tert-butylphosphin- \\
\hline \multirow[t]{2}{*}{ LUMO } & niedrigstes unbesetztes & & 2',4',6'-triisopropylbiphenyl \\
\hline & Molekülorbital & $\mathrm{Tf}$ & Trifluormethansulfonyl \\
\hline M & Molarität, molar & TFA & Trifluoracetat/ -essigsäure \\
\hline $\mathrm{Me}$ & Methyl & THF & Tetrahydrofuran \\
\hline $\min$ & Minute(n) & TMS & Trimethylsilyl \\
\hline MS & Massenspektrometrie & Tol & Tolyl \\
\hline MW & Mikrowelle & Ts & Toluolsulfonyl \\
\hline$n$ & normal, unverzweigt & ÜM & Übergangsmetall \\
\hline NBS & $N$-Bromsuccinimid & UV & Ultraviolett(-Spektroskopie) \\
\hline NMR & Kernresonanz & $V$ & Volumen \\
\hline \multirow[t]{2}{*}{ NOESY } & $(\mathrm{H}, \mathrm{H})$-Korrelationsspektroskopie & Vis & visueller Bereich (380-750 nm) \\
\hline & (durch den Raum) & wässr. & wässrig \\
\hline$o$ & ortho & XantPhos & 4,5-Bis(diphenylphosphin)- \\
\hline$p$ & para & & 9,9-dimethylxanthen \\
\hline $\mathrm{Ph}$ & Phenyl & & \\
\hline pin & Pinacol(at) & & \\
\hline
\end{tabular}




\subsection{Literaturverzeichnis}

1 L. F. Tietze, T. Hungerland, C. Eichhorst, A. Düfert, C. Maaß, D. Stalke, Angew. Chem. Int. Ed. 2013, 52, 3668-3671.

2 L. F. Tietze, T. Hungerland, M. A. Düfert, I. Objartel, D. Stalke, Chem. Eur. J. 2012, 18, 32863291.

3 L. F. Tietze, T. Hungerland, C. Depken, C. Maaß, D. Stalke, Synlett 2012, 2516-2520.

4 L. F. Tietze, T. Hungerland, J. Ammermann, C. Eichhorst, S. O. Reichmann, D. Stalke, J. Indian Chem. Soc. 2013, 90, 1537-1555.

5 L. F. Tietze, F. Lotz, Eur. J. Org. Chem. 2006, 4676-4684.

6 L. F. Tietze, G. Brasche, K. Gericke, Domino Reactions in Organic Synthesis, Wiley-VCH, Weinheim, 2006.

7 L. F. Tietze, H. Geissler, Angew. Chem. Int. Ed. Engl. 1993, 32, 1038-1040.

8 L. F. Tietze, Domino Reactions, Wiley-VCH, Weinheim, 2014.

9 G. Pattenden, D. A. Stoker, N. M. Thomson, Org. Biomol. Chem. 2007, 5, 1776-1788.

10 L. F. Tietze, S. Jackenkroll, J. Hierold, L. Ma, B. Waldecker, Chem. Eur. J. 2014, 20, 8628-8635.

11 K. Sonogashira, Y. Tohda, N. Hagihara, Tet. Lett. 1975, 50, 4467-4470.

12 L. Kürti, B. Czakó, Strategic Applications of Named Reactions in Organic Synthesis, Elsevier Academic Press, London, 2005.

13 F. Kluklas, A. Grunwald, F. Menschel, T. J. J. Müller, Beilstein J. Org. Chem. 2014, 10, 672679.

14 E. Negishi, C. Copéret, S. Ma, S.-Y. Liou, F. Liu, Chem. Rev. 1996, 96, 365-393.

15 E. Negishi, Handbook of Organopalladium Chemistry for Organic Synthesis: Volume 1, WileyVCH, Weinheim, 2002.

16 S. Ma, E. Negishi, J. Am. Chem. Soc. 1995, 117, 6345-6357.

17 C. M. R. Volla, J.-E. Bäckvall, Angew. Chem. Int. Ed. 2013, 52, 14209-14213.

18 L. Ackermann, Modern Arylation Methods, Wiley-VCH, Weinheim, 2009.

19 I. Moritani, Y. Fujiwara, Tet. Lett. 1967, 1119-1122. 
20 D. Alberico, M. E. Scott, M. Lautens, Chem. Rev. 2007, 107, 174-238.

21 C. C. Hughes, D. Trauner, Angew. Chem. Int. Ed. 2002, 41, 1569-1572.

22 L.-C. Campeau, M. Parisien, A. Jean, K. Fagnou, J. Am. Chem. Soc. 2006, 128, 581-590.

23 D. García-Cuadrado, A. A. C. Braga, F. Maseras, A. M. Echavarren, J. Am. Chem. Soc. 2006, $128,1066-1067$.

24 D. García-Cuadrado, P. de Mendoza, A. A. C. Braga, F. Maseras, A. M. Echavarren, J. Am. Chem. Soc. 2007, 129, 6880-6886.

25 S. Pascual, P. de Mendoza, A. A. C. Braga, F. Maseras, A. M. Echavarren, Tetrahedron 2008, 64, $6021-6029$.

26 H.-Y. Sun, S. I. Gorelsky, D. R. Stuart, L.-C. Campeau, K. Fagnou, J. Org. Chem. 2010, 75, $8180-8189$.

27 D. Lapointe, K. Fagnou, Chem. Lett. 2010, 39, 1118-1126.

28 S. Gorelsky, D. Lapointe, K. Fagnou, J. Org. Chem. 2012, 77, 658-668.

29 M. Lafrance, K. Fagnou, J. Am. Chem. Soc. 2006, 128, 16496-16497.

30 S. R. Neufeldt, M. S. Sanford, Acc. Chem. Res. 2012, 45, 936-946.

31 B.-J. Li, S.-D. Yang, Z.-J. Shi, Synlett 2008, 7, 949-957.

32 N. R. Deprez, M. S. Sanford, J. Am. Chem. Soc. 2009, 131, 11234-11241.

33 Z. Qureshi, H. Weinstabl, M. Suhartono, H. Liu, P. Thesmar, M. Lautens, Eur. J. Org. Chem. 2014, 4053-4069.

34 M. Catellani, F. Frignani, A. Rangoni, Angew. Chem. Int. Ed. Engl. 1997, 36, 119-122.

35 M. Catellani, M. C. Fagnola, Angew. Chem. Int. Ed. Engl. 1994, 33, 2421-2422.

36 D. J. Cárdenas, D. Martín-Matute, A. M. Echavarren, J. Am. Chem. Soc. 2008, 128, 5033-5040.

37 M. Hesse, H. Meier, B. Zeeh, Spektroskopische Methoden in der organischen Chemie, 7. Aufl., Georg-Thieme-Verlag, Stuttgart, 2005.

38 J. R. Lakowicz, Principles of Fluorescence Spectroscopy, Springer Science + Business Media, New York, 2006.

39 L. D. Lavis, R. T. Raines, ACS Chem. Biol. 2014, 9, 855-866.

40 Y. Pu, W. Wang, Y. Yang, R. R. Alfano, Opt. Lett. 2012, 37, 3360-3362. 
41 H. von Pechmann, C. Duisberg, Ber. Dtsch. Chem. Ges. 1883, 6, 2119-2128.

42 X. Jin, C. Uttamapinant, A. Y. Ting, ChemBioChem 2011, 12, 65-70.

43 C. Uttamapinant, K. A. White, H. Baruah, S. Thompson, M. Fernández-Suárez, S. Puthenveetil, A. Y. Ting, Proc. Natl. Acad. Sci. U.S.A. 2010, 107, 10914-10919.

44 L. D. Lavis, T.-Y. Chao, R. T. Raines, ChemBioChem 2006, 7, 1151-1154.

45 E. Vos de Wael, J. A. Pardoen, J. A. van Knoeveringe, J. Lugtenburg, Recl. Trav. Chim. PaysBas 1977, 96, 306-309.

46 R. Kojima, H. Takakura, T. Ozawa, Y. Tada, T. Nagano, Y. Urano, Angew. Chem. Int. Ed. 2013, $52,1175-1179$.

47 A. Baeyer, Ber. Dtsch. Chem. Ges. 1871, 4, 555-558.

48 W.-C. Sun, K. R. Gee, D. H. Klaubert, R. P. Haugland, J. Org. Chem. 1997, 62, 6469-6475.

49 A. Rukavishnikov, K. R. Gee, I. Johnson, S. Corry, Anal. Biochem. 2011, 419, 9-16.

50 M. F. Marmor, J. G. Ravin, Arch. Ophthalmol. 2011, 129, 943-948.

51 M. Ceresole, Verfahren zur Darstellung von Farbstoffen aus der Gruppe des Meta-amidophenolPhthaleïns, D. R. Patent-Nr. 44002, 1887.

52 J. B. Grimm, L. D. Lavis, Org. Lett. 2011, 13, 6354-6357.

53 L. D. Lavis, T.-Y. Chao, R. T. Raines, ACS Chem. Biol. 2006, 1, 252-260.

54 M. N. Levine, R. T. Raines, Anal. Biochem. 2011, 418, 247-252.

55 R. Nietzki, A. Dietze, H. Mäckler, Ber. Dtsch. Chem. Ges. 1889, 22, 3020-3038.

56 A. Sharonov, R. M. Hochstrasser, Proc. Nat. Sci. U.S.A. 2006, 103, 18911-18916.

57 J. Jose, K. Burgess, Tetrahedron 2006, 62, 11021-11037.

58 K. E. Beatty, M. Williams, B. L. Carlson, B. M. Swarts, R. M. Warren, P. D. van Helden, C. R. Bertozzi, Proc. Nat. Sci. U.S.A. 2013, 110, 12911-12916.

59 W. König, Ber. Dtsch. Chem. Ges. 1924, 57, 685-692.

60 A. Waggoner, Annu. Rev. Biophys. Bioeng. 1979, 8, 47-68.

61 H. S. Rye, S. Yue, D. E. Wemmer, M. A. Quesada, R. P. Haugland, R. A. Mathies, A. N. Glazer, Nucleic Acids Res. 1992, 20, 2803-2812.

62 R. P. Haugland, M. T. Z. Spence, I. D. Johnson, A. Basey, The Handbook: A Guide to 
Fluorescent Probes and Labeling, 10. Aufl., Invitrogen Corp., Carlsbad, USA, 2005.

63 X. Lv, J. Liu, Y. L. Liu, Y. Zhao, M. L. Chen, P. Wang, W. Guo, Sens. Actuators B, 2011, 158, $405-410$.

64 F. Lotz, Dissertation, Georg-August-Universität, Göttingen, 2007.

65 W. A. Herrmann, C. Brosmer, K. Ofele, C.-P. Reisinger, T. Priermeier, M. Beller, H. Fischer, Angew. Chem. Int. Ed. Engl. 1995, 34, 1844-1846;

66 M. A. Düfert, Dissertation, Georg-August-Universität, Göttingen, 2010.

67 L. F. Tietze, A. Düfert, F. Lotz, L. Sölter, K. Oum, T. Lenzer, T. Beck, R. Herbst-Irmer, J. Am. Chem. Soc. 2009, 131, 17879-17884.

68 L. F. Tietze, M. A. Düfert, T. Hungerland, K. Oum, T. Lenzer, Chem. Eur. J. 2011, 17, 84528461.

69 T. Hungerland, Dissertation, Georg-August-Universität, Göttingen, 2013

70 J. Ammermann, Masterarbeit, Georg-August-Universität, Göttingen, 2013.

71 E. A. Krasnokutskaya, N. I. Semenischeva, V. D. Filimonov, P. Knochel, Synthesis, 2007, 81-84.

72 T. Kometani, D. S. Watt, T. Ji, T. Fitz, J. Org. Chem. 1985, 50, 5384-5387.

73 W. S. Rapson, R. G. Shuttleworth, J. Chem. Soc. 1941, 487-490.

74 M. R. Acheson, G. C. M. Lee, J. Chem. Soc., Perkin Trans. 1 1987, 11, 2321-2332.

75 D. R. Levine, A. Caruso Jr., M. A. Siegler, J. D. Tovar, Chem. Commun. 2012, 48, 6256-6258.

76 C. Mukai, I. Nomura, S. Kitagaki, J. Org. Chem. 2003, 68, 1376-1385.

77 M. Koppitz, B. Lindenthal, PCT Int. Appl. 2010, 2010012396.

78 D. Peña, D. Pérez, E. Guitián, L. Castedo, J. Org. Chem. 2000, 65, 6944-6950.

79 M. S. Newman, A. I. Kosak, J. Org. Chem. 1949, 14, 375-378.

80 T. Sakamoto, Y. Kondo, N. Miura, K. Hayashi, H. Yamanaka, Heterocycles 1986, 24, 2311 2314.

81 E. J. Corey, R. K. Bakshi, S. Shibata, J. Am. Chem. Soc. 1987, 109, 5551-5553.

82 N. Uematsu, A. Fujii, S. Haghiguchi, T. Ikariya, R. Noyori, J. Am. Chem. Soc. 1996, 118, 49164917.

83 S. López, F. Fernández-Trillo, L. Castedo, C. Saá, Org. Lett. 2003, 5, 3725-3728. 
84 E. Marchal, J.-F. Cupif, P. Uriac, P. van de Weghe, Tetrahedron Lett. 2008, 49, 3713-3715.

85 M. Artuis, R. Pontikis, J.-C. Florent, J. Org. Chem. 2009, 74, 2234-2237.

86 A. F. Littke, G. C. Fu, Angew. Chem. Int. Ed. 1999, 38, 2411-2413.

87 A. F. Littke, L. Schwarz, G. C. Fu, J. Am. Chem. Soc. 2002, 114, 4350-4386.

88 K. L. Billingsley, T. E. Barder, S. L. Buchwald, Angew. Chem. Int. Ed. 2007, 46, 5359-5363.

89 B. Waldecker, Masterarbeit, Georg-August-Universität, Göttingen, 2014.

90 G. M. Sheldrick, Acta Crystallogr. 2008, A64, 112-122. 


\subsection{Danksagungen}

Zum Abschluss dieser Arbeit möchte ich all jenen danken, die auf die eine oder andere Weise Anteil an deren Gelingen hatten.

Zuallererst gilt mein Dank den Mitarbeitern der analytischen Abteilungen der Fakultät für Chemie: Ich danke Reinhard Machinek, Michael John, Christiane Siebert, Martin Weitemeyer, Carola Zolke und Ralf Schöne für das überaus schnelle und zuverlässige Messen von unzähligen NMR-Spektren. Ganz besonders möchte ich Herrn Machinek danken, der bei auch bei schwierigsten NMR-Problemen mit Rat und Tat zur Seite stand.

Des Weiteren danke ich Holm Frauendorf, Frank Hambloch, Györgi Sommer-Udvarnoki, Ulrike Bode und Gabriele Krökel für das zügige Messen der massenhaften Massenspektren.

Evelyn Pfeil und Katja Grube danke ich für das unverzügliche Messen von UV/Vis- und IR-Spektren.

Raphael Hubrich aus der Arbeitsgruppe von Prof. Claudia Steinem sei für das Messen der Fluoreszenzspektren gedankt.

Für die Aufnahme und Berechnung der Röntgenkristallstruktur möchte ich Markus Steinert und Sebastian Dechert aus der Arbeitsgruppe von Prof. Franc Meyer danken.

Martina Pretor gilt mein Dank für ihre schnelle und kompetente Hilfe bei EDV-Problemen jeder Art.

Sabine Schacht möchte ich für die unermüdliche Verwaltungsarbeit danken, die den Arbeitskreis am Laufen hielt.

Gleiches gilt für Isabel Trzeciok und Berta Hampel, die im Dekanat bei Problemen mit etwaigen bürokratischen Hürden stets hilfsbereit waren.

Außerdem danke ich Holger Tucholla und Rupert Schrommek für die prompte Bereitstellung von Chemikalien und anderen Laborutensilien.

Den Materialwärten der Abteilung Tietze, Galina Pestel, Johannes Reiner, Judith Hierold und Simon Biller, danke ich für die Sicherstellung der Versorgung mit Chemikalien und sonstigen Hilfsmitteln.

Für das zügige und überaus gewissenhafte Korrekturlesen dieser Arbeit sei Simon Biller, Judith Hierold und Sven Heidemann gedankt, die jedes noch so unauffällige tiefgestellte Komma bemerkt haben. Alle verbliebenen Fehler sind allein mir zuzuschreiben.

Meinem „Teamkollegen“ Tim Hungerland möchte ich für die gute Zusammenarbeit und seine hilfreichen Ratschläge danken. 
Mein Dank gilt außerdem meinen Masterstudenten Bernd Waldecker und Jonas Ammermann für ihre hervorragenden Leistungen auf diesem Thema.

Des Weiteren danke ich meinen Laborpraktikanten, die mir die Frustration bei der Arbeit an der xten Domino-Vorläufer-Synthese erspart haben. Dank an: Anne Schober, Raphael Hubrich, Natali Dubel, Ruth Linder und Anna Kölpin.

Ich danke außerdem allen Mitarbeitern der Abteilung Tietze, die auch die frustrierenden und ermüdenden Episoden dieser Arbeit zu einem Vergnügen gemacht haben. Besonders hervorheben möchte ich dabei Judith Hierold, Simon Biller, Sven Heidemann, Stefan Jackenkroll, Jerome Clerc, Tobias Nack, Kianga Schmuck, Bernd Waldecker, Jonas Ammermann und Marius Hartmer.

Meinen Labormitarbeitern, Stefan Jackenkroll, Simon Biller und Bernd Waldecker, sei für die überaus entspannte, chaotische, durchgeknallte, ,pfiffige“, idiotische, aber auch intellektuell anspruchsvolle (!) Atmosphäre gedankt. Mir werden insbesondere die sowohl verrückten als auch inhaltsreichen Diskussionen mit Stefan sowie die Wunschkonzerte mit Simon und Bernd in Erinnerung bleiben.

Verschiedene Menschen haben meinen Weg in Göttingen begleitet und diesen zu einer angenehmen Episode in meinem Leben gemacht. Ich möchte besonders Markus Steinert, Dominik Herkt und Benjamin Bollmann danken, die von Anfang an dabei waren. Des Weiteren danke ich Simon Biller, Sven Heidemann, Stefan Jackenkroll, Jerome Clerc, Tobias Nack, Kianga Schmuck, Timo Scheffer, Sebastian „Bob“ Weske, Ulrike Rost, Ruth Linder, Sandra Lange, Natali Dubel, Jasper Ploog, Steffen Eggers, Benjamin Schröder, Jasmine Chamiolo, Markus „Bob Schneider“ Scheibel, Isabel Klopsch, Rene Rieger, Bernd Waldecker und Marten Seeba.

Meinen Eltern danke ich für ihre bedingungslose Unterstützung, die mir dieses Studium ermöglicht hat. Auch meinen Großeltern danke ich für ihren Rückhalt beim Bewältigen dieser Ausbildung.

$\mathrm{Zu}$ guter Letzt danke ich Judith, die mir durch ihre Liebe und Unterstützung die nötige Kraft gegeben hat, diese Arbeit zu bewältigen.

\section{Herzlichsten Dank!}

Meine akademischen Lehrer waren unter anderem:

L. Ackermann, P. Botschwina, A. de Meijere, U. Diederichsen, C. Ducho, G. Eckold, C. Griesinger, F. Güthoff, W. Hack, C. Höbartner, K. Luther, R. Machinek, J. Magull, F. Meyer, G. M. Sheldrick, L. F. Tietze, K. Samwer, S. Schmatz, J. Schröder, C. Schulzke, D. Schwarzer, I. Siewert, D. Stalke, C. Steinem, H. J. Steinfelder, P. Vana, D. B. Werz, P. von Zezschwitz, W.-H. Zimmermann. 


\subsection{Lebenslauf}

\section{Persönliche Daten}

Name: $\quad$ Christoph Eichhorst

Geburtsdatum: $\quad 07.03 .1986$

Geburtsort: $\quad$ Lübeck

Familienstand: $\quad$ ledig

Staatsangehörigkeit: deutsch

\section{Ausbildung}

1992-1996

Gerhart-Hauptmann-Grundschule, Stockelsdorf

1996-2005

Gymnasium am Mühlenberg, Bad Schwartau

2005

Allgemeine Hochschulreife

2006-2014

Studium an der Georg-August-Universität, Göttingen

2008

Vordiplomsprüfung, Note „gut“

2010

Diplomarbeit unter der Leitung von Prof. Dr. Dr. h.c. L. F. Tietze mit dem Thema: „Synthese von Acenaphthylenen und tetrasubstituierten Alkenen durch Palladium-katalysierte Domino-Reaktionen“

Diplomprüfung, Note „sehr gut“

2011-2014

Promotion unter der Leitung von Prof. Dr. Dr. h.c. L. F. Tietze mit dem Thema: „Untersuchungen zur Synthese fluoreszenzaktiver aromatischer Polyzyklen durch Palladium-katalysierte Domino-C-H-Aktivierungen“ 